\title{
Design, Synthesis and Evaluation of WD40-repeat containing protein 5 (WDR5) Degraders
}

\author{
Dissertation \\ zur Erlangung des Doktorgrades \\ der Naturwissenschaften \\ vorgelegt beim Fachbereich 14 \\ Biochemie, Chemie und Pharmazie \\ der Johann Wolfgang Goethe-Universität \\ in Frankfurt am Main
}

von

Anja Dölle

geboren in Würzburg

Frankfurt am Main (2021)

(D30) 
vom Fachbereich Biochemie, Chemie und Pharmazie der

Johann Wolfgang Goethe-Universität Frankfurt als Dissertation angenommen

Dekan: Prof. Dr. Clemens Glaubitz

Gutachter: Prof. Dr. Stefan Knapp

Prof. Dr. Eugen Proschak

Datum der Disputation: 


\section{List of content}

List of content.

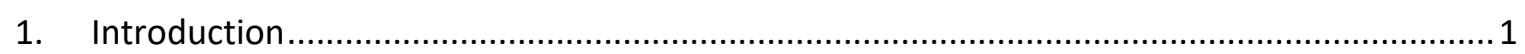

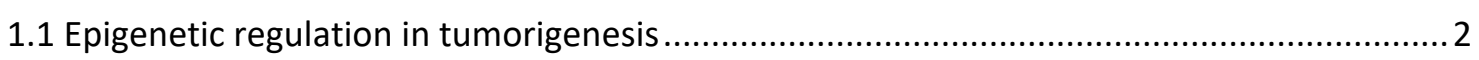

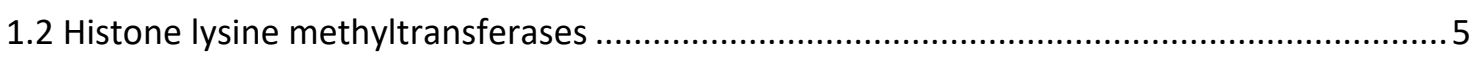

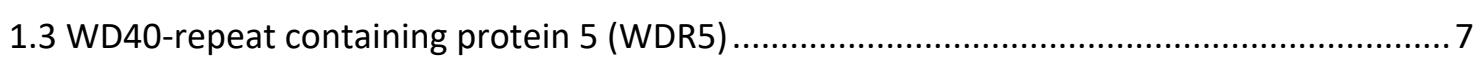

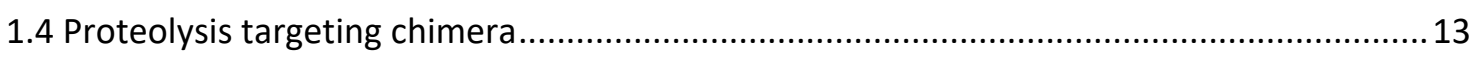

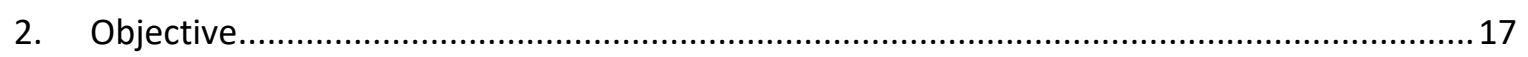

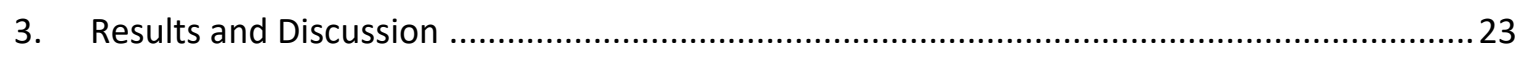

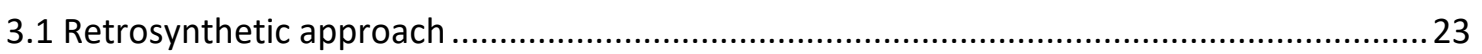

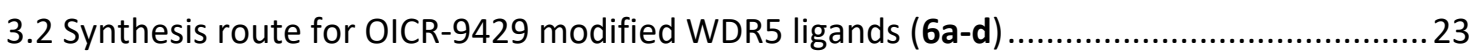

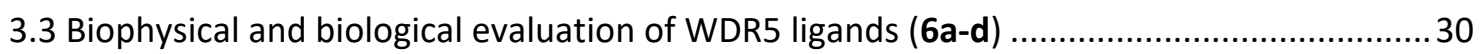

3.4 Synthesis route for OICR-9429 modified WDR5 PROTACs (7a-e), (8a-j) and (9a-c) .............. 31

3.5 Synthesis of pyrroloimidazole derived degraders $(\mathbf{1 6 a}, \mathbf{b})$ and $(\mathbf{2 0 a}-\mathbf{g})$................................. 38

3.6 Biophysical Evaluation of WDR5 PROTACs (7a-e), (8a-j) and (9a-c) .................................. 41

3.7 PROTAC-mediated degradation of cellular WDR5 …........................................................ 51

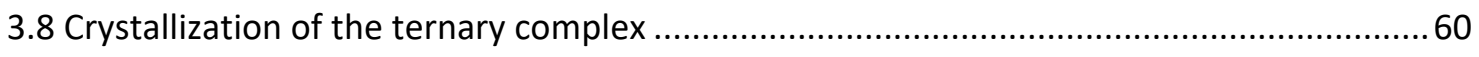

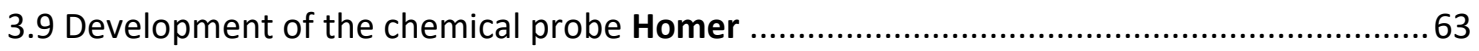

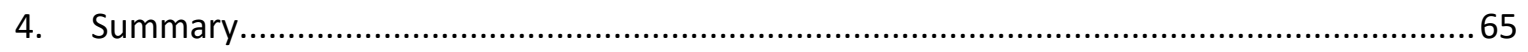

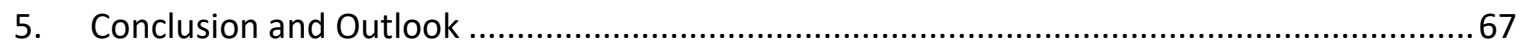

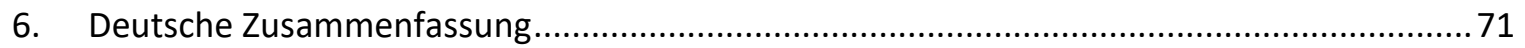

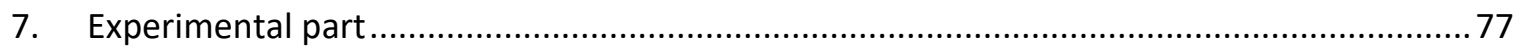

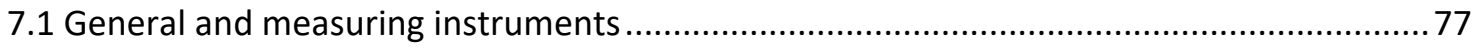

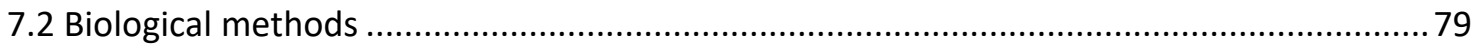

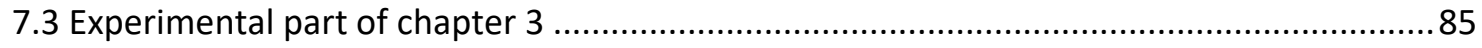

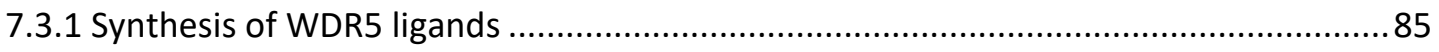

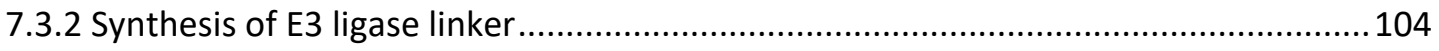

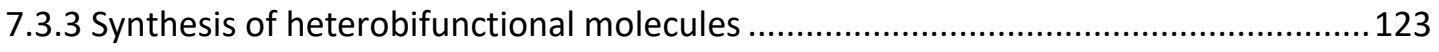

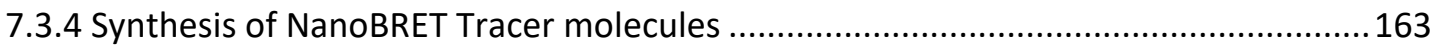

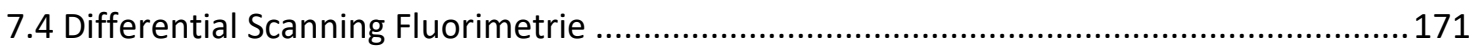

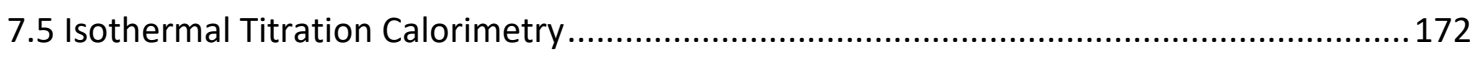

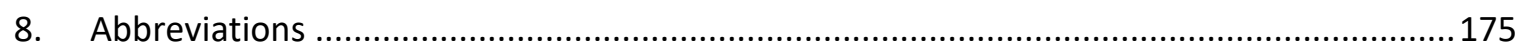

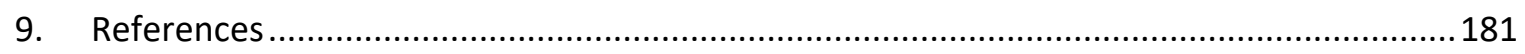

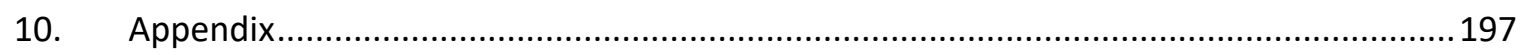

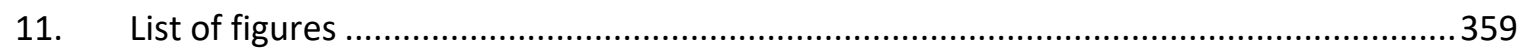




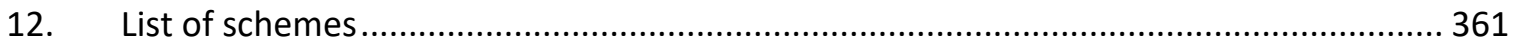

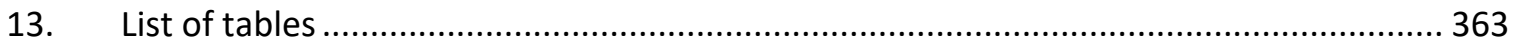

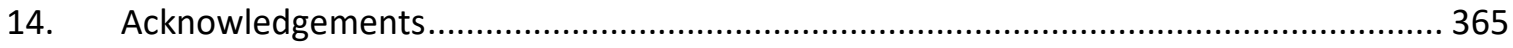

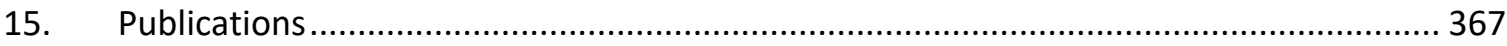

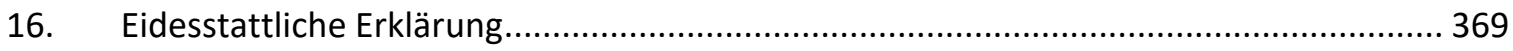

17. Curriculum Vitae ........................................................Fehler! Textmarke nicht definiert. 


\section{Introduction}

In eukaryotic cells, DNA is tightly wrapped around histone proteins to form nucleosomes. ${ }^{1}$ This basic unit of chromatin structure envelops 146 base pair segments of DNA around a histone octamer $^{2}$ and is shown in the Cryo-EM structure in Figure 1. For cell replication, the unwrapping of DNA is crucial and the process is carried out by various protein complexes. The tightly regulated procedure is mediated by histone tail modifications that widen or close the chromatin structure by altering electrostatic interactions. ${ }^{2}$ The post-translational modifications (PTMs), ${ }^{3}$ comprising methylation, phosphorylation, ubiquitylation, sumolation, citrullination or acetylation of histone tail residues, control the so-called epigenome which differs in each cell type of each person, and is strongly influenced by the environment and lifestyle of the individual. ${ }^{2}$ Although there are many post-translational modifications, the exact modified amino acid of the histone protein is highly specific (see Figure 1). ${ }^{3}$

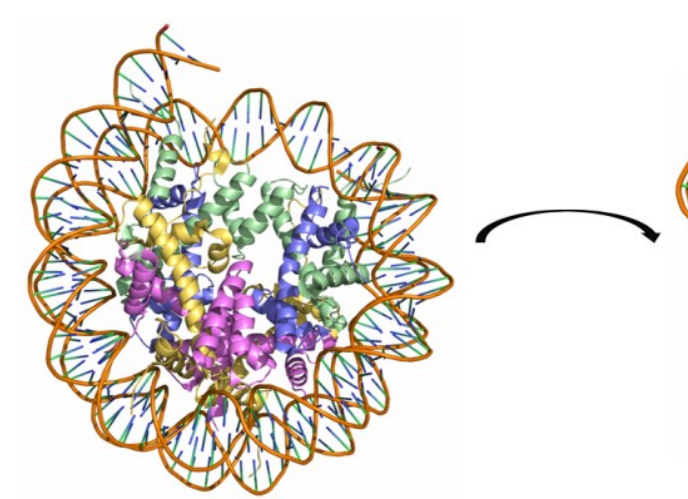

DNA H2A H2B H3 H4

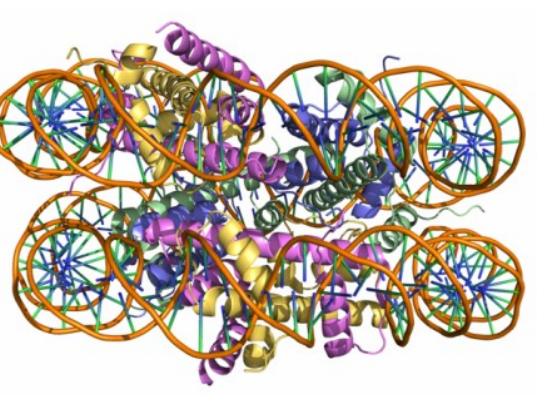

DNA H2A H2B H3 H4

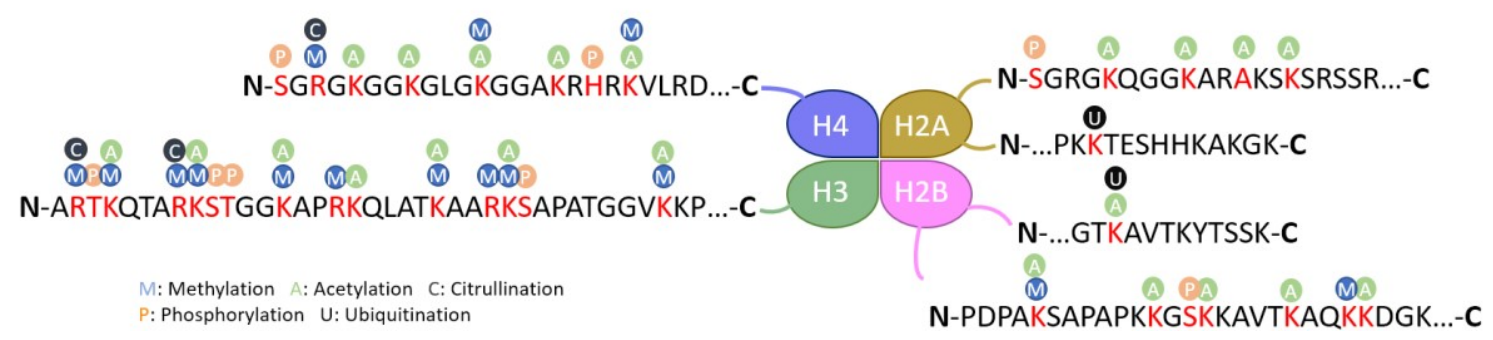

Figure 1: Histone proteins interact with the DNA to form nucleosomes. (top, left) Top view of Cryo-EM structure of a histone octamer (with its proteins H2A (yellow), H2B (violet), H3 (green) and H4 (blue)) in complex with DNA (orange). (top, right) Side view of histone octamer in complex with DNA. (pdb entry: $6 \mathrm{pwv}) .{ }^{4}$ (bottom) Schematic drawing of the main histone modifications patterns on different amino acid residues in normal cells. These modifications are mainly taking place on the $\mathrm{N}$-terminal tails, but they can also occur at C-terminal regions, thereby contributing to an opened or closed chromatin state and driving gene expression. Scheme based on Rodriguez-Paredes and Esteller. ${ }^{5}$ 
As a result of regulating chromatin accessibility and hence driving gene expression, epigenetic modifications play a crucial role in many cellular processes: as a result, a disruption of PTMs can lead to developmental abnormalities in plants, like failure in tomate fruit ripening, ${ }^{6-7}$ and in mammals, e.g. embryo lethality in mice. ${ }^{8}$ Additionally to the coordination of developmental processes, adaption to environmental changes ${ }^{9}$ and relation to alternative RNA processing ${ }^{10}$ are also controlled by epigenetic regulation.

In summary, the influence of the epigenetic status within a cell is enormous, as it regulates the way how the cellular genome manifests itself in different cell types and developmental stages, and if aberrant, how it promotes disease progression and cancer formation. ${ }^{5}$

\subsection{Epigenetic regulation in tumorigenesis}

Initially, the key concept of cancer formation focused on how genetic mutations lead to an activation of oncogenes and/ or an inactivation of tumor-suppressor genes. ${ }^{11}$ Heritable defects of these gene products have been shown to influence essential cellular control pathways that are disturbed in almost all human cancer types. ${ }^{11}$ The gained knowledge of control pathway regulation promoted great progression in cancer research. But in the 1990s, an emerging research area investigated the heritable changes that were regulated by epigenetic alterations and are equally critical for human tumorigenesis. ${ }^{12}$ These epigenetic alterations comprise abnormal DNA methylation patterns, disordered PTM patterns on histones and structural changes in chromatin organization. ${ }^{12}$ Early-stage tumors display global DNA hypomethylation as well as promotorspecific hypomethylation, and demonstrate how epigenetic deregulation leads to the classical transforming events of tumor suppressor or proto-oncogene mutations. ${ }^{13}$ Epigenetic mutations can silence the remaining active allele of a mutated tumor suppressor, ${ }^{14}$ and in this manner overcome the cellular control mechanisms what potentially might lead to cancer initiation. Disruption of the epigenetic machinery can occur through mutation, deletion or altered expression of any of their components and result in modified gene expression patterns and expression states. ${ }^{15}$

As alterations of epigenetic modifications are a hallmark of cancer, chromatin was identified as one of the earliest targets for cancer therapeutics. ${ }^{15-16}$ Early drug development intended altering the chromatin structure with differentiating agents ${ }^{16-17}$ and sequencing studies of different cancer types revealed mutations in numerous genes encoding chromatin regulating proteins ${ }^{18}$, as listed 
in Table 1. These proteins have been divided into distinct epigenetic protein classes, according on how their enzymatic activity affects chromatin accessibility.

Table 1: Examples of epigenetic protein complexes and epigenetic mutations that are now known to be perturbed in cancer and affect gene expression and genomic stability. ${ }^{12,}{ }^{19-21}$ Perturbations can occur in epigenetic modifiers, histones or DNA. The list is not exhaustive.

\begin{tabular}{l|l}
\multicolumn{1}{c|}{ Perturbation } & \multicolumn{1}{c}{ Protein complexes } \\
\hline $\begin{array}{l}\text { Histone-modifying enyzmes } \\
\text { Nucleosome remodeling }\end{array}$ & $\begin{array}{l}\text { MLL1/2/3, CBP/ P300, SETD2, EZH2, JARID1C, UTX, BMI1, LSD1 } \\
\text { BRG1/ BRM, ARID1A/ 1B/ 2, SNF5, ATRX }\end{array}$ \\
Histone PTM readers & BRD4, TRIM33, ING1, BRCA1 \\
& $\begin{array}{l}\text { Mutations } \\
\text { Histones and variants } \\
\text { DNA methylation }\end{array}$ \\
H3K27, H3K34 & DNMT1/ 3A, TET1/ 2, IDH1/ 2, MBDs
\end{tabular}

In total, more than 700 proteins regulate chromatin function ${ }^{18,22-23}$ and they are often part of multi-domain protein complexes. Beside the catalytic subunit that controls chromatin accessibility, also subunits that recognize and interact with epigenetic modifications are crucial components of histone modifying complexes. ${ }^{2}$ Despite the three classes of epigenetic readers, erasers, and writers, also epigenetic movers, shapers and insulators interact with chromatin structure. ${ }^{24-25}$

Proteins that recognize post-translational modifications are classified as epigenetic readers. ${ }^{26}$ Well-studied protein families for epigenetic readers are, e.g., bromodomains (BRDs), which recognize acetylated lysine residues. The BRDs have been extensively studied and successfully drugged in cancer treatment. ${ }^{26}$ In Table 1, the bromodomain BRD4 of the bromodomain and extraterminal domain (BET) family is listed due to its prominent role in super-enhancers (SEs) organization and regulation of oncogene expression in cancer. ${ }^{27}$ Targeting BRD4 by inhibiting the acetyl-lysine binding site with small molecules, e.g., the first BRD targeting inhibitor (JQ1), was shown to be an effective strategy for cancers like the aggressive NUT midline carcinoma (NMC). ${ }^{28-}$ ${ }^{29}$ Beside the outstanding role of BRD4, other BRDs are involved as epigenetic readers in various nucleosome remodeling complexes: in the ATP-dependent human complexes BAF (BRG1/BRMassociated factor) and PBAF (polybromo-associated BAF factor), two bromodomains, SMARCA2/ 4 (SWI/SNF-related, matrix-associated actin-dependent regulator of chromatin, subfamily A2/4), perturbate with the core subunits BRG1/BRM histone-DNA contacts. ${ }^{30-32}$ Mutations in BAF components are one of the most frequently observed genetic alteration in cancer. ${ }^{33-34}$ 
In addition to epigenetic readers, proteins that remove epigenetic marks from histone tails are classified as epigenetic erasers. ${ }^{24}$ Within this epigenetic classification, the Histone Deacetylases (HDACs) and Lysine Demethylases (KMDs) are well-studied drug targets. ${ }^{35-36}$ Each of them is responsible for the removal of one distinct functional chemical group. The last posttranslationalmodifying enzyme class are the epigenetic writers that add post-translational modifications on histone tails. Prominent protein classes are the Histone Acetylases (HATs) and Histone Lysine Methyltransferases (KMTs). ${ }^{37}$ The connection between histone acetylation and cancer has been stated and reviewed in many studies. ${ }^{38-39}$ Current HDAC inhibitors are evaluated as anti-tumor agent in clinical trials for breast cancer, ${ }^{40-41}$ while the development and characterization of HAT inhibitors focusses on other diseases, such as Alzheimer, diabetes and hyperlipidaemia. ${ }^{42}$

The previous stated examples focused on acetylated histone modifications and their influence in cancer. Acetyl groups remove the positive charge of the histones and are generally associated with an opened chromatin structure, while methylation of histone tail residues can turn genes "on" and "off" by granting or denying DNA accessibility. ${ }^{3}$ Histone tail methylation is a complex process and dependent on the modified amino acid residue (lysines, arginines and histidines) and the methylation state (mono-, di- and trimethylation), it can serve different purposes in cellular processes: generally, methylation on the histone lysines H3K9, H3K27 and H4K2O is associated with a repressed chromatin state, while methylation of $\mathrm{H} 3 \mathrm{~K} 4, \mathrm{H} 3 \mathrm{~K} 36$ and $\mathrm{H} 3 \mathrm{~K} 79$ are considered to mark an opened chromatin state and activate gene transcription. ${ }^{43-45}$ Many studies demonstrated how mutations and misregulations of histone lysine methyltransferases (KMTs), demethylases and methyl-lysine-binding proteins are connected to various diseases, thus making them effective therapeutic targets for cancer treatments. ${ }^{46-47}$ The histone demethylation process is carried out by lysine demethylases like LSD $1^{48}$ and the JARID1 familiy ${ }^{49}$ - epigenetic erasers that are known to be perturbed in cancer, as previously listed in Table 1. Equally involved in cancer formation is the class of histone lysine methyltransferases (KMTs) which are categorized as epigenetic writers. KMTs comprise proteins like MLL1-3 and SET1D which are relevant drug targets, as shown in the non-exhaustive list in Table 1. Within histone lysine methylation, H3K4 methylation is an evolutionary conserved motif that marks active gene transcription ${ }^{50-51}$ and is highly enriched at the promotor region and transcription start site. ${ }^{51}$ The family of Histone lysine Methyltransferases and its adaptor proteins are described in the following chapter. 


\subsection{Histone lysine methyltransferases}

The methylation of lysine residues is catalyzed by over 100 existing histone lysine methyltransferases (KMTs) which are able to add methylation marks on six distinct lysine residues at the histone tail $\mathrm{H} 3(\mathrm{~K} 4, \mathrm{~K} 9, \mathrm{~K} 23, \mathrm{~K} 27, \mathrm{~K} 36$ and $\mathrm{K} 79)$ as well as on lysine (K20) of histone H4. ${ }^{52-54}$ The SET1/MLL (mixed lineage leukemia) family of methyltransferases is conserved from yeast to mammals which attach the mono-, di-, or tri-methylation of H3K4 by their catalytic SET (Suppressor of variegation 3-9, Enhancer of Zeste, Trithorax) domain. ${ }^{37,55-56}$ While in yeast, only one KMT is able to catalyze all three states of methylation, six H3K4 methyltransferases are found in human: MLL1 (MLL/KMT2A), MLL2 (KMT2B), MLL3 (KMT2C), MLL4 (KMT2D), SETD1A (KMT2F) and SETD1B (KMT2G). ${ }^{57-58}$ The MLL protein is encoded by the MIl1 gene, located on chromosome 11q23.3. ${ }^{57-58}$ In 1992, the involvement of MII1 in haematological malignancies, including acute lymphoid leukaemia and acute myeloid leukaemia was revealed. ${ }^{59-61}$ The chromosomal translocation with different partner genes ${ }^{62}$ creates a hybrid transcription factor that drives malignancy by reactivating the expression of developmentally important genes, e.g., HOX genes. ${ }^{63}$ HOX gene products are vital for MLL-fusion cells to maintain the malignant, stem-like state and the progression of tumorigenesis. ${ }^{63-64}$

Regarding structural similarity, all MLL family members share an evolutionarily conserved SET domain that catalyzes the transfer of methyl moiety from S-adenosylmethionine to the $\varepsilon$-amine of lysine 4 of Histone $\mathrm{H}^{65}$. The SET1 KMTs represent the active subunit in large chromatin modifying enzyme complexes, as shown in the Cryo-EM in Figure 2, although their enzymatic activity itself is low and association with additional adaptor proteins is required for enhanced methyltransferase activity. ${ }^{37}$ These adaptor proteins are associated in the so-called 'WRAD' complex that consist of four highly conserved protein types: WD40-repeat containing protein 5 (WDR5), Retinoblastoma Binding Protein 5 (RbBP5), A Absent-Small-Homeotic-2-Like protein (ASH2L) and a Dumpy-30 protein (DPY30) homodimer. ${ }^{66-69}$ In vitro experiments showed that in absence of WRAD complex, MLL1 is only a weak mono methyltransferase, while in the presence of WDR5, RbBP5 and ASH2L, MLL1 displays robust di- and moderate tri-methyltransferase activity. ${ }^{66,69-70}$ 
6 Introduction

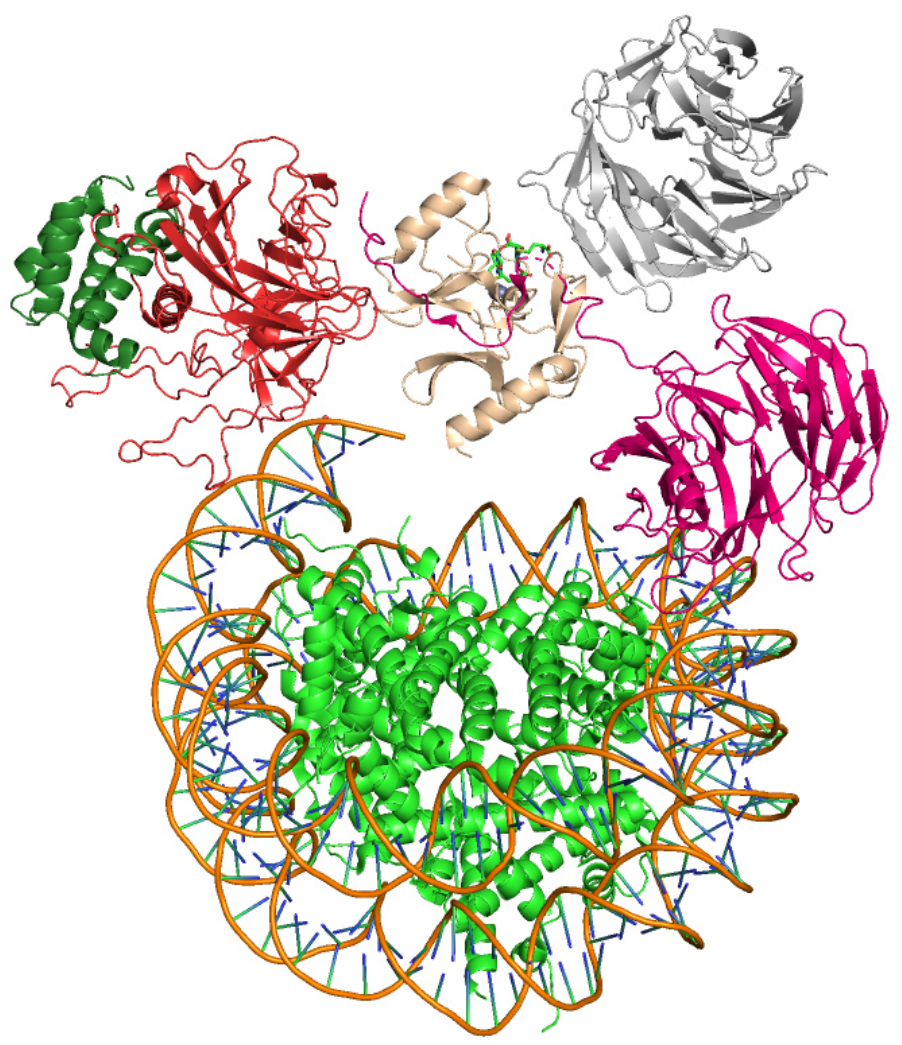

Figure 2: Cryo-EM structure of the MLL1 KMT complex in complex with DNA (orange) and a histone octamer (green) (pdb entry: 6pwv). ${ }^{4}$ The activity of the wheat-colored active MLL1 SET domain with Sadenosylmethionine (light green) is enhanced with the association of the WRAD complex, consisting out of four proteins: WDR5 (grey), RbBP5 (pink), ASH2L (red) and DYP30 homodimer (dark green).

Contary to all other core subunits, the DYP30 homodimer does not interact directly with the histone methyltransferase. ${ }^{71-72}$ Nevertheless, it has been shown to be important for genome-wide H3K4 methylation ${ }^{73-74}$ as it is thought to stimulate methylation activity by structural arrangements, leading to an amplification of ASH2 L protein expression. ${ }^{72} \mathrm{ASH} 2 \mathrm{~L}$ forms a heterodimer with $\mathrm{RbBP} 5$ that directly binds and activates all catalytic subunits and stabilizes the interaction between the SET domain and its substrates. ${ }^{75-76}$ The weak interaction between MLL1 and the RbBP5-ASH2L dimer is further enhanced by WDR5 which interacts with MLL1 and RbBP5 at its two binding sites. $^{50,} 77$ This scaffolding function is crucial for the structural integrity and enhanced methyltransferase activity of the complex. ${ }^{66,78}$ Studies have shown that depletion of WDR5 leads to reduction of H3K4 methylation levels in cells ${ }^{66}$ and in animals. ${ }^{79}$ These observations were not seen when MLL1 was removed from cells. ${ }^{66,79-80}$ Inhibition of the WDR5-MLL1 interaction led to a decrease in MLL1 KMT activity, and resulted in decreased H3K4 methylation at HOX genes. ${ }^{50}$ The results suggested that it may be relevant to treat MLL1-fusion cancers with WDR5 inhibitors, although the exact mechanism of action remained unknown. Further proof-of-concept 
experiments showed that pharmacological inhibition of WDR5 can reduce the growth of cancer cells in vitro, including those derived from KMT2A (MLL1)-rearranged ${ }^{81-82}$ and C/EBPa mutant leukemias, ${ }^{83}$ neuroblastoma, ${ }^{84}$ and breast cancers, ${ }^{85}$ as well as those expressing p53 gain-offunction variants. ${ }^{86}$

A study of mice bearing histone and DNA methylation defects showed the surprising finding that MLL-associated H3K4 methyltransferase activity is not required in vivo, as mice with a homozygous deletion of the MLL SET domain were born with a relatively mild phenotype. ${ }^{87}$ Moreover, MLLdeficient cells showed no global changes in H3K4 methylation. ${ }^{88}$ On the other hand, the expression of many genes is affected by the loss of MLL, indicating that MLL may regulate transcription independently of its active KMT domain. ${ }^{63-64,88}$ Since not all known functions of the adaptor proteins have been investigated, these proteins may be responsible for SET-independent transcriptional regulation. Contrary to the studies that showed the pharmacological relevance of WDR5 inhibitors, no small molecule based ligands have been published for the other WRAD subunits RbBP5, ASH2L and DYP30. ${ }^{89}$ Targeting MLL- $r$ cancers focus thus mostly on inhibiting the MLL-Menin ${ }^{90-92}$ or MLL-WDR5 protein protein interaction (PPI)..$^{50,83,93-96}$

\subsection{WD40-repeat containing protein 5 (WDR5)}

WDR5 most prominent role is in scaffolding the assembly of epigenetic writer complexes such as the previously described MLL/SET histone methyltransferases $(M L L)^{50,66,72,97}$, but also the nonspecific lethal (NSL) ${ }^{98}$ and Ada2-containing (ATAC) histone acetyltransferase (HAT). ${ }^{99}$ The latter listed complex shows that WDR5 also plays an important role in other epigenetic marks, e.g., in the ATAC complex that drives H4K16 acetylation. ${ }^{99}$ Other chromatin remodeling enzymes that interact with WDR5 include HDACs $1-3,{ }^{100}$ and the chromatin remodeling enzyme CHD8, that is involved in H3K4 trimethylation. ${ }^{101}$

The class of WD40-repeat containing proteins typically forms a seven-bladed propeller structure, as shown in Figure 3. Due to its characteristic shape, WDR5 possesses a large surface that allows various protein-protein interactions (PPIS). WDR5 interacts with many different proteins and is thought to indirectly affect intracellular H3K4 methylation as it also binds to long non-coding RNAs (IncRNAs) ${ }^{102}$ which are known to mediate gene transcription. Among all of the protein interaction partners, the most prominent are the MLL-fusion oncoproteins and the transcription factor family MYC. ${ }^{77,97,103}$ As shown in Figure 3, WDR5 has two binding sites, called WDR5-interacting site (Win) and WDR5-binding motif (WBM). The Win site is a deep binding pocket in the center of the 
propeller and recognizes arginine containing motifs (ARA). The motifs are found in several WDR5 interaction partners, including Histone H3, KANSL1 of the NSL complex and all five MLL/ SET family members. ${ }^{97-98}$ In contrast to the Win site, the WBM site is a narrow cleft on WDR5s' surface to which peptide sequences of the WRAD interaction partner RbBP5 or the transcription factor cMYC bind. ${ }^{103}$
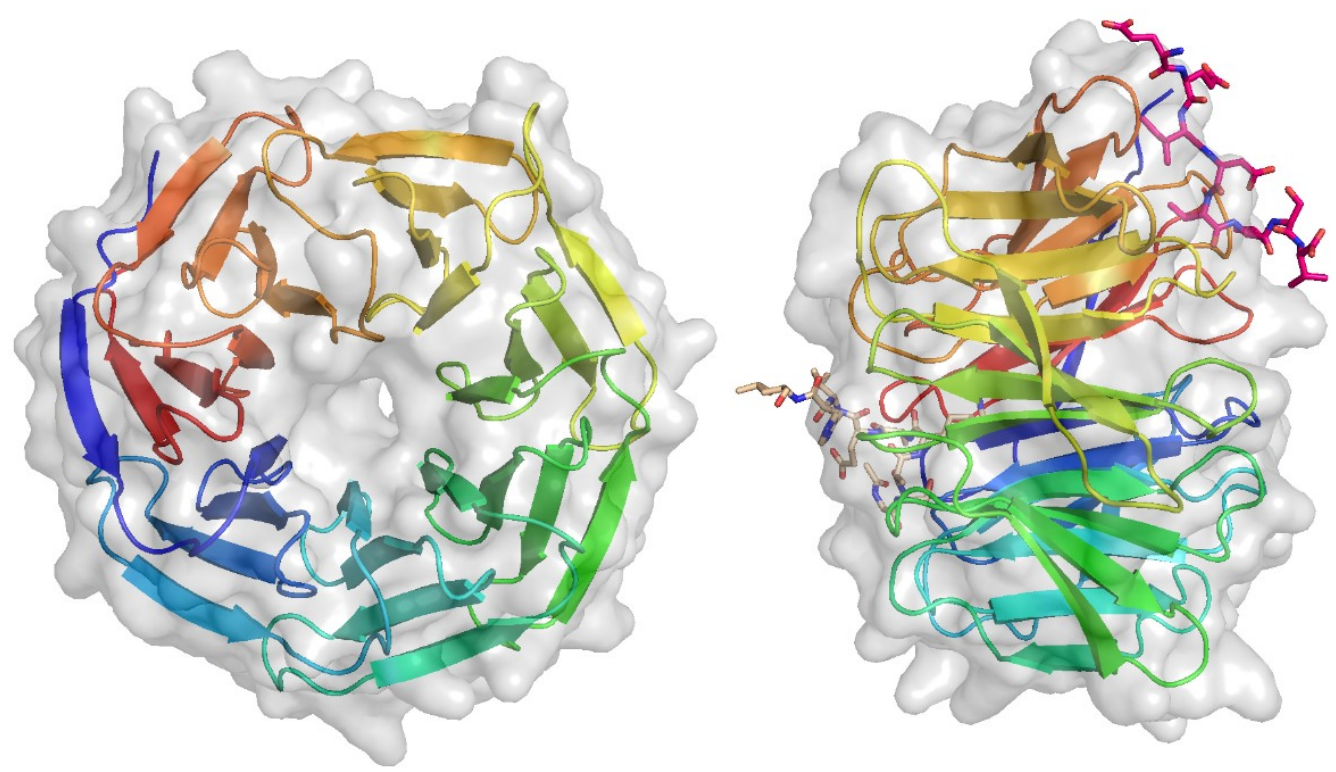

Figure 3: (left) Apo crystal structure of WDR5. Like all WD40 proteins, WDR5 consists out of a seven-bladed propeller of beta sheets (pdb entry: 2h14). (right) Side view of the WDR5 protein with interaction partners onboth binding sites: the wheat colored MLL1 peptide binds on the deep Win site in the center of WDR5, while the pink colored c-Myc peptide Mblllb binds to WDR5 on a shallow cleft on the surface, the so called WBM side (pdb entry: 3eg6 and 4y7r).

WDR5 has emerged as a promising drug target for anti-cancer therapies as it is overexpressed in various cancer typer like leukemia ${ }^{104}$, bladder cancer ${ }^{105}$, hepatocellular carcinoma ${ }^{106}$, and breast cancer. ${ }^{85,107}$ Furthermore, WDR5 is involved in malignant processes such as the epithelial to mesenchymal transition and cell motility 85,100 and it serves as critical cofactor for the retinoic acid receptor. ${ }^{108}$

\section{WDR5 and its interaction with MYC}

Among all listed interaction partners of WDR5, one stands out: the transcription factor family MYC which controls the expression of many distinct genes, ranging from cell cycle progression to DNA replication to cell proliferation in healthy tissue, but is also responsible for tumorigenesis, metastasis and a poor prognosis of survival in various cancer types. ${ }^{109-110}$ Aberrant MYC expression causes one third of all cancer deaths. ${ }^{111}$ MYCs dimerizes with MAX to a heterodimer (as seen in 
Figure 4), which is able to recognize E-box motifs on DNA and starts trancsprition of target genes. ${ }^{111}$

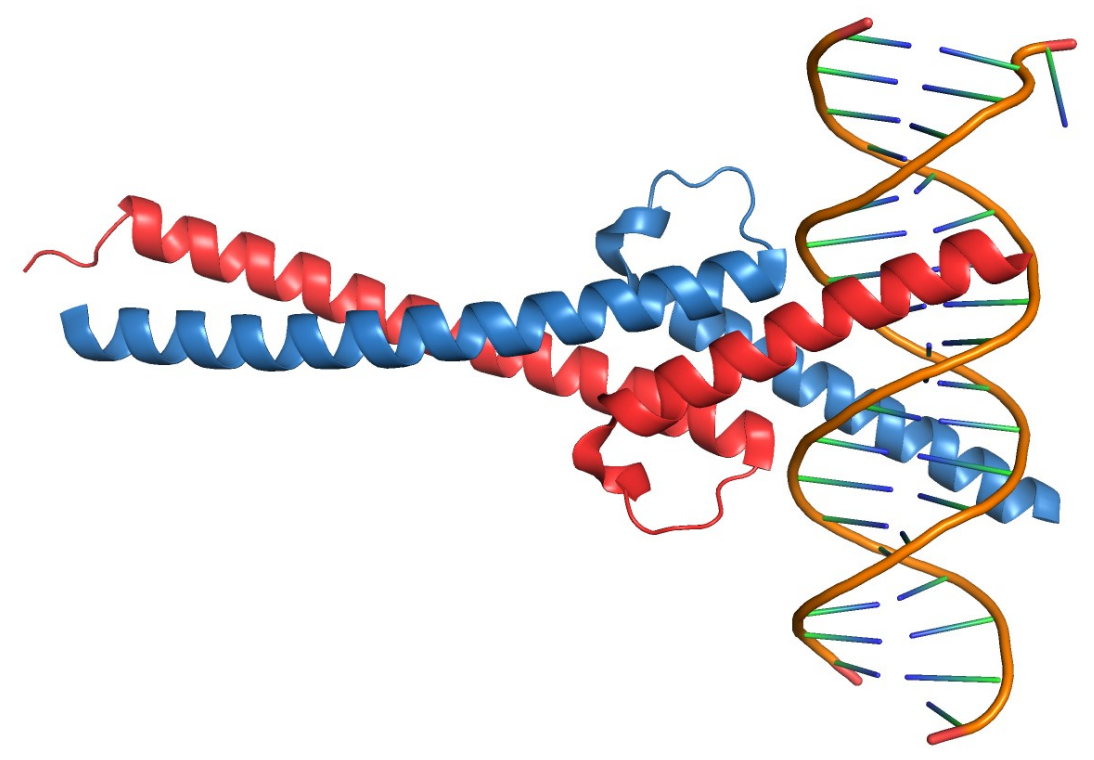

Figure 4: Crystal structure of the MYC-MAX heterodimer in complex with DNA (pdb entry: 1nkp). The crystal structure shows the dependency of the MYC protein structure on the interaction partner.

The shape of the MYC protein depends highly on its interaction partners and does not contain any druggable binding pockets. ${ }^{111}$ Thus, one way to target the protein is through the indirect targeting of MYCs' interaction partners, e.g. MAX, Aurora A kinase and WDR5. ${ }^{111-113}$ The seven interaction sites, called MYC boxes (Mb), are highly conserved and interact with various proteins to perform multiple cellular functions, as shown in Figure 5. These cellular functions reach from chromatin remodeling, modification and association to promotor binding and classify the MYC family as master regulators for gene expression.

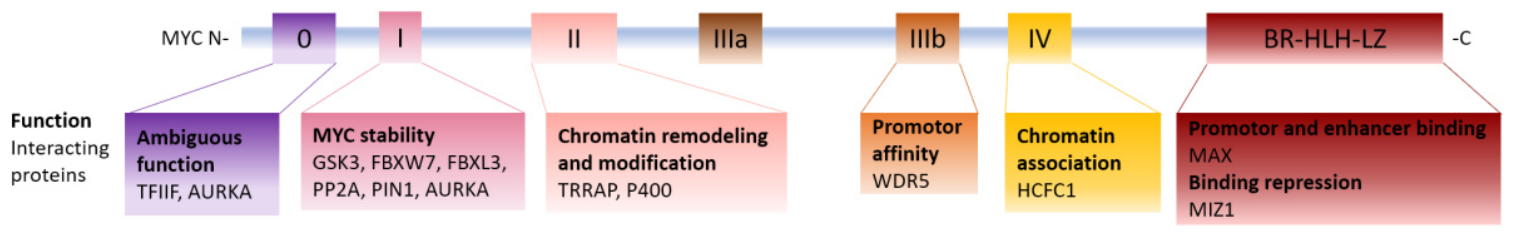

Figure 5: Schematic illustration of the MYC protein structure (blue) with its protein-protein interaction domains (0, I, II, IIIa, III b, IV and BR-HLH-LZ; colored in purple-reddish). Due to the various interaction sites and engagement partners, MYC performs different cellular tasks. Figure taken and modified from Baluapuri et al. ${ }^{111}$ 
As shown in Figure 3, the interacting sequence of MYC (Myc box IIlb peptide, Mblllb) binds to the shallow cleft on WDR5s' surface. A disruption, caused by mutations of amino acids, of the WDR5MYC interaction attenuates MYCs' ability to localize on chromatin. ${ }^{111}$ Inhibiton of WDR5 with a small molecule decreased WDR5/ N-Myc complex formation, resulting in reduced N-Myc target gene expression and diminished cell growth in neuroblastoma cells. ${ }^{114}$ These observations, along with the previous seen effects on cancer cells bearing a MLL-fusion hybrid, repurpose WDR5 as therapeutical drug target in cancer therapy. While the outcome of both studies, the cell death, had been proved, the mechanism of action how WDR5 inhibition leads to the observed cell killing, was not clear. ${ }^{82}$

\section{Mechanism of action and biological relevance of WDR5 inhibition and depletion}

In 2019, Aho et al. investigated for the first time the mechanism of cell inhibition by WDR5 ${ }^{81}$ Using chromatin immunoprecipitation sequencing (ChIP-Seq), the authors found that the WDR5 Win site interacts directly with chromatin. ${ }^{81}$ WDR5 inhibition resulted in changes that were detectable within minutes in the transcription of WDR5-bound protein synthesis genes (PSGs), as shown in Figure 6.

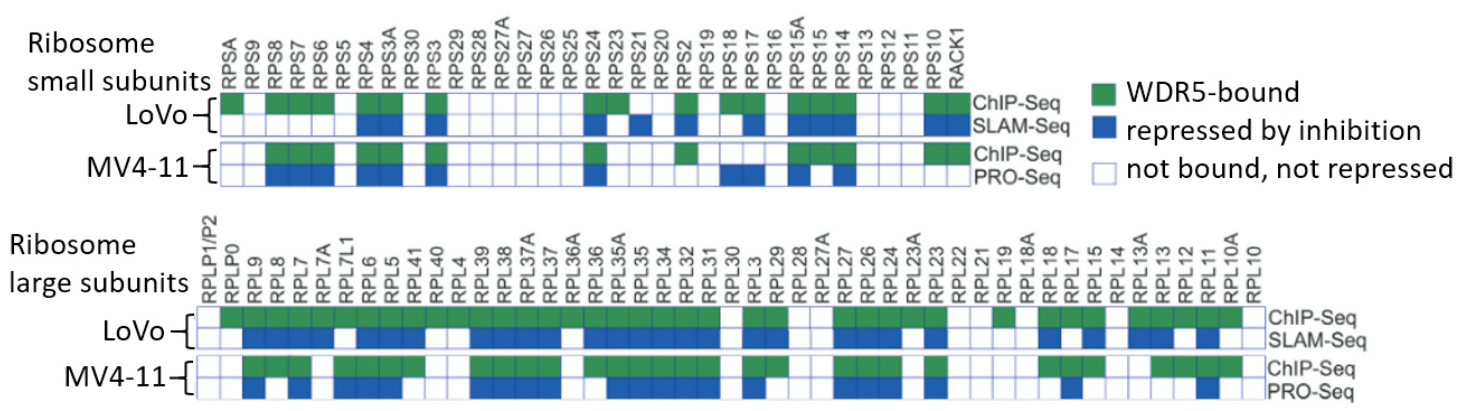

Figure 6: Ribosomogram, displaying ribosome subunits of (top) small and (bottom) large subunit ribosomal protein genes (RPGs). Green-colored boxes indicate that WDR5 is bound to each RPG in the indicated cell type, blue-colored boxes indicate gene repression upon WIN site inhibition. LoVo cell data are from Bryan et al.,${ }^{84}$ MV4-11 data are taken from Aho et al. ${ }^{81}$ Figure taken and modified from Bryan et al. ${ }^{84}$

Thus, the main biological role of WDR5 seems to be non-epigenetic. ${ }^{82}$ The mapping of WDR5s' localization on chromatin in MV4-11 cells (bearing one of the most common MLL fusion AF4) revealed that WDR5 did not bind to loci of expected developmentally important genes, e.g. the previously described HOX genes, ${ }^{79}$ but is associated with genes that are connected to protein synthesis (PSGs). ${ }^{81,84}$ Among these genes, approximately 40 out of 80 ribosomal protein genes (RPGs) are WDR5 targets, as well as genes encoding nucleolar RNAs and translation initiation factors. ${ }^{84}$ The authors investigated the effects of a small molecule Win site inhibitor $\mathbf{C 6}$ (and its 
negative control (6nc) and found that Win site inhibition results in a decreased expression of PSGs, which in turn results in a reduced translational capacity of MV4-11 cells. The imbalance and deficiency of ribosomal subunits was associated with nucleolar stress response and resulted in p53-dependent cell death, as illustrated in the proposed mechanism in Figure 7. So, the sensitivity of MLL-fusion leukaemia cells to Win site inhibition results from their tendency to retain p53. ${ }^{114}$ Also, MLL-fusion oncoproteins tend to drive ectopic protein synthesis as part of their tumorigenic program and are therefore triggered by Win site inhibition. ${ }^{84,115}$

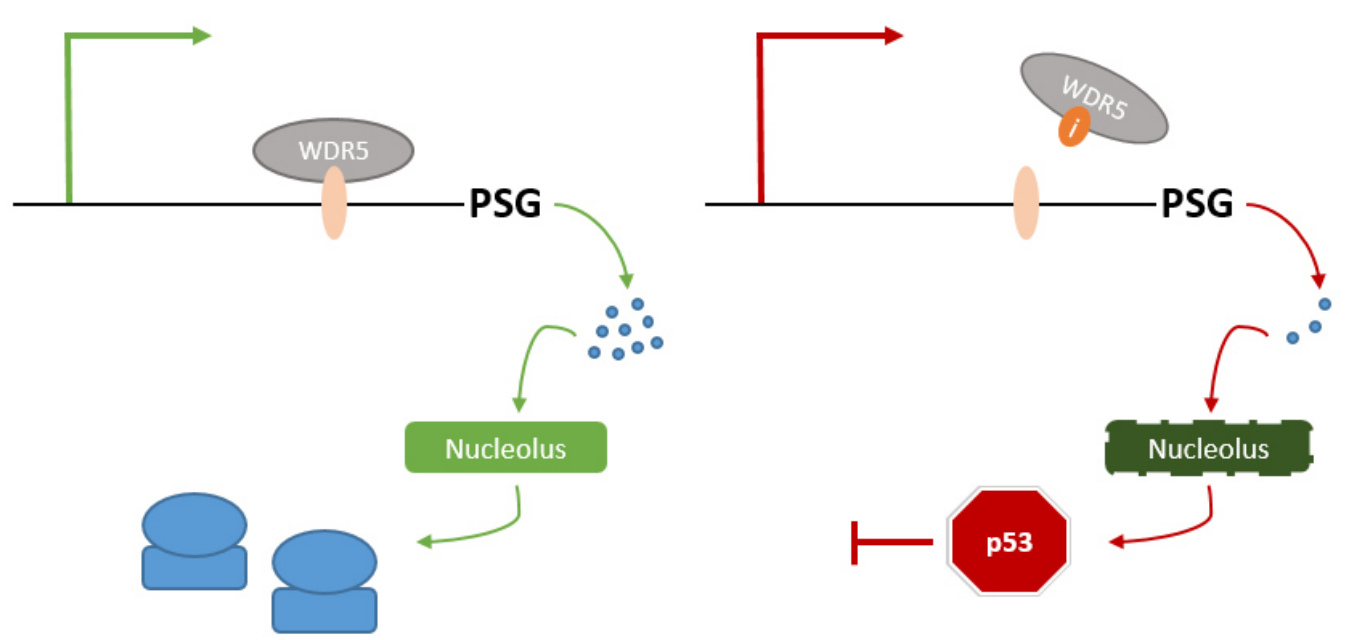

Figure 7: Proposed mechanism of Win site inhibition by Aho et al.: (left) WDR5 binds with its Win interaction site to the transcription start site (green arrow) at genes relevant for protein synthesis to ensure balanced production of ribosomal subunits, nucleolar and translation factors. (right) Inhibition ("i") of the Win site displaces WDR5 from chromatin. This results in reduced PSG expression, causing an imbalance of ribosomal subunits. This failsafe mechanism induces nucleolar stress and p53-dependent cell death. Figure modified from Aho et al. ${ }^{82}$

Bryan et al. continued the investigation of the WDR5 mechanism by examining different mouse and human cancer cell lines. ${ }^{84}$ Their results showed that a conserved set of 94 genes were bound by WDR5 and encoded proteins involved in translation processes, including PSGs and RPGs. ${ }^{84}$ In conclusion, the expression of PSGs was stated as a general regulatory function of WDR5 and can be influenced by Win site inhibition. Furthermore, they found that cell lines that tend to retain p53 ${ }^{114}$ - similar to MLL-fusion oncogenes - are sensitive to Win site inhibition. ${ }^{84}$ Consistent with this, small molecule inhibition reduced MYCN-amplified, p53 wild-type neuroblastoma cells. ${ }^{84,114}$ Beside just inhibiting enzymatic function of WDR5, the authors performed WDR5 depletion experiments to gain insights into the phenotypic consequences of Win site functions by using the dTAG approach in neuroblastoma cells. ${ }^{84}$ Consistent with Win site inhibition, depletion also 
indicated that the predominant role of WDR5 is to regulate expression of PSGs, as shown in Figure 8. However, slight differences in the biological clustering of the two different approaches have been observed, e.g. WDR5 depletion has more widespread effects on neuronal differentiation, while inhibition showed enhanced effects on the expression of genes linked to cell cycle. $^{84}$

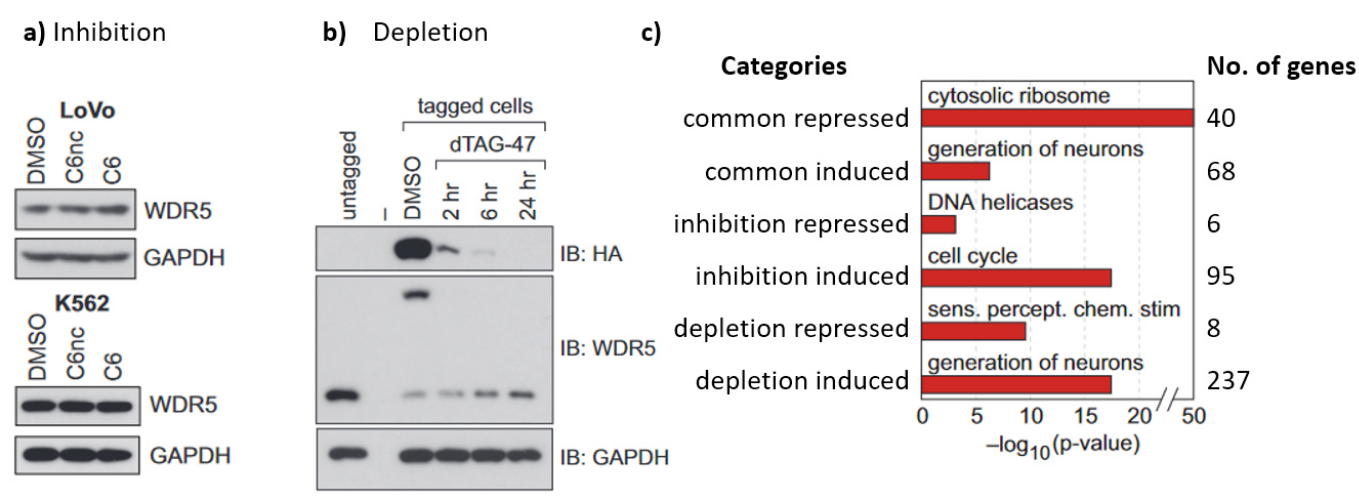

Figure 8: WDR5 phenocopies by WIN site inhibition and WDR5 depletion. (a) Immunoblotting for WDR5 in (top) LoVo or (bottom) K562 cells in which endogenous WDR5 is treated by the Win site inhibitor C6 or negative control C6nc. GAPDH serves as loading control. (b) Immunoblotting for WDR5 in CHP-134 cells in which endogenous WDR5 is depleted by the dTAG (dTAG47) approach. GAPDH serves as loading control. (c) Top GO enrichment categories for genes repressed by inhibtion and depletion (common repressed), induced by inhibtion and depletion (common induced), repressed by inhibition but not by depletion (inhibition repressed), induced by inhibition but not by depletion (inhibition induced), repressed by depletion but inhibtion (depletion repressed) and induced by depletion but inhibtion (depletion induced). Numbers to the right show genes number in each category. Figure taken and modified from Bryan et al. ${ }^{84}$

In addition to the similarities in regulating PSG expression, the differences indicate that not all functions of WDR5 might be disabled by Win site blockade. ${ }^{84} \mathrm{All}$ in all, the surprising findings of the non-epigenetic mechanism of action highlights again the moonlighting abilities of WDR5. ${ }^{102}$ Taking the surprising findings that WDR5 influences non-epigenetic processes as well as the association of oncogenic drivers on both binding sides of WDR5, indicate that a classical inhibitor approach may have limited success in studying the overall effects of WDR5 on cellular processes. Therefore, Proteolysis targeting chimeras (PROTACS) that induce protein degradation instead of inhibiting the enzymatic function, are an elegant way to drug a cellular multitasker like WDR5. ${ }^{102}$ 


\subsection{Proteolysis targeting chimera}

Proteolysis-targeting chimaeras (PROTACs) are heterobifunctional molecules containing two different ligands: one warhead for the protein of interest (POI) and a second ligand that binds to an E3 ubiquitin ligase. ${ }^{116-117}$ Both ligands are bridged by a chemical linker. The heterobifunctional molecule can create proximity between both proteins, ${ }^{116}$ as shown in Figure 9, and induce formation of a ternary complex. The ubiquitin transfer from the E2 enzyme to the protein of interest leads to proteasomal degradation and release of the small molecule which can enter a new cycle of target protein degradation (TPD). ${ }^{116-117}$

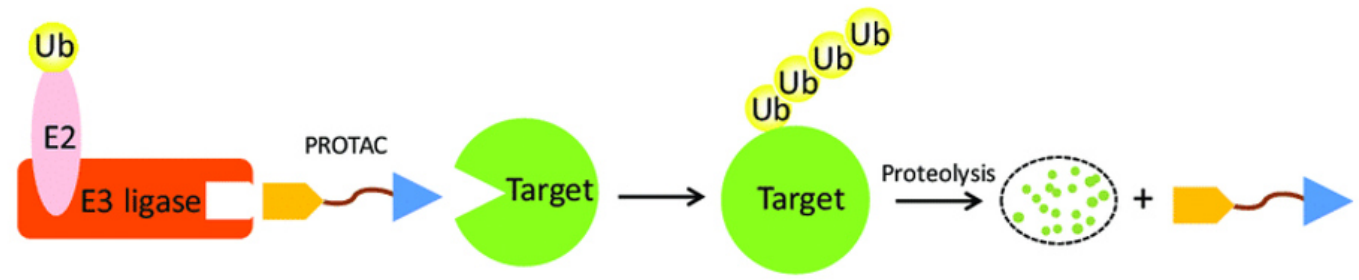

Figure 9: Mechanism of action (MoA) of proteolysis targeting chimeras (PROTACs). (top) Schematic structure of a heterobifunctional molecule, also called PROTAC. (bottom) A PROTAC mediates proximity between an E3 Ligase and the target protein. Ubiquitin transfer results in proteasomal degradation of the target protein and the recovery of the PROTAC molecule. Figure taken from Gu et al. ${ }^{118}$

PROTACs expand the toolbox of chemical matter by redirecting the activity of the UbiquitinProteasome-System. ${ }^{119}$ Contrary to the occupancy-driven binding event of small molecule inhibitors, PROTACs rely on an event-driven mechanism of action, making them attractive for undruggable proteins (like transcription factors) ${ }^{120}$ or proteins with scaffolding functions. ${ }^{112,}{ }^{121}$ Due to the different MOA, PROTACs harboring a weak affinity ligand for the target protein can also result in an effective binder. ${ }^{122-123}$ In comparison to nucleic acid-based approaches that suppress protein synthesis, PROTAC-mediated degradation works independently from protein half-life times. $^{124}$

Ubiquitin transfer to $\varepsilon$-amino function of lysine residues is catalyzed by three enzymes: the ubiquitin-activating enzyme E1, the ubiquitin-conjugating enzyme E2 and the ubiquitin ligase E3. In an ATP-dependend reaction, the E1 enzyme attaches ubiquitin through a reactive thioester bond. ${ }^{125}$ In the next step os the cascade, the E1 transfers the ubiquitin to the E2 enzyme. ${ }^{125}$ The E2 enzymes determine which type of ubiquitin chain is made. ${ }^{125}$ In general, polyubiquitin chains can be linked through seven lysines in ubiquitin (K6, K11, K27, K29, K33, K48 and K63). ${ }^{125}$ Together 
with the E3 enzyme that determines substrate specifity, the E2 transfers the Ubiqutin chains directly or indirectly to the target protein. ${ }^{125}$ The role of each enzyme within the ubiquitination process is reflected in the existence of over 600 different E3 ligases, while there are $30-50$ different E2s and only two E1s and encoded in the human genome..$^{125-126}$ E3 ligases can be divided into HECT, RING and HECT-RING-hybrids. ${ }^{127}$ The difference between HECT and RING E3 ligases lies in the ubiquitin transfer to the substrate: HECT E3s receive ubiquitin from E2 enzymes and catalyze the transfer direct to the target protein, while RING E3s associate with E2s and the target protein to mediate the transfer. ${ }^{127}$ Also RING E3s can function as monomers, dimers or multi-subunit protein complexes. ${ }^{127}$ Among the multi-subunit E3s, the Cullin-RING Ligases (CRLs) are the largest E3 ligase family. Each E3 is composed of a modular E3 core containing specific Cullin proteins (CUL1, CUL2/5, CUL3, CUL4) and RBX1 (RING-box1 proteins) and different substrate specifity modules (e.g., Skp1, EloB/C, and DDB1). ${ }^{127}$ In PROTAC technique, the substrate receptor of the $\mathrm{E} 3$ ligase is addressed by the heterobifunctional molecule. With growing popularity of PROTACs, several E3 ligases have been addressed with small molecules: to date, the E3 ligases MDM2, ${ }^{128}$ IAP, ${ }^{129}$ RNF4, ${ }^{130}$ BTRCP, ${ }^{131}$ parkin ${ }^{132}$ and DCAF16 ${ }^{133}$ are druggable with potent ligands. The most prominent and frequently used E3 ligases are Cereblon (CRBN) and the Von-HippelLindau (VHL) protein. ${ }^{134-137}$ The model of a crystal structure of the CRL4 ${ }^{\text {CRBN }}$ E3 ligase is shown in Figure $10,{ }^{137}$ to get a better visibility of the multi-domain complexes and their druggable receptor subunit. The ligand thalidomide addresses the E3 ligase CRBN and rose tragic prominence by being administered in the 1950s as pharmaceutical drug "Contergan" to pregnant women, but has revived as PROTAC component in cancer treatment to date. ${ }^{138}$ The binary affinity to CRBN is rather moderade $(10 \mu \mathrm{M}){ }^{139}$ but sufficient for inducing degradation on various targets, while literatureknown and optimized ligands of the other popular E3 ligase VHL display nanomolar potencies (185 nM). ${ }^{140}$ Similar to CRL4 ${ }^{\text {CRBN }}$ E3 ligase, the VHL E3 ligase contains a CUL2 and RBX1 core subunit, but differs in the adaptor subunits Elongin B and C (EloB/ C) as well as the VHL receptor. ${ }^{141}$ 


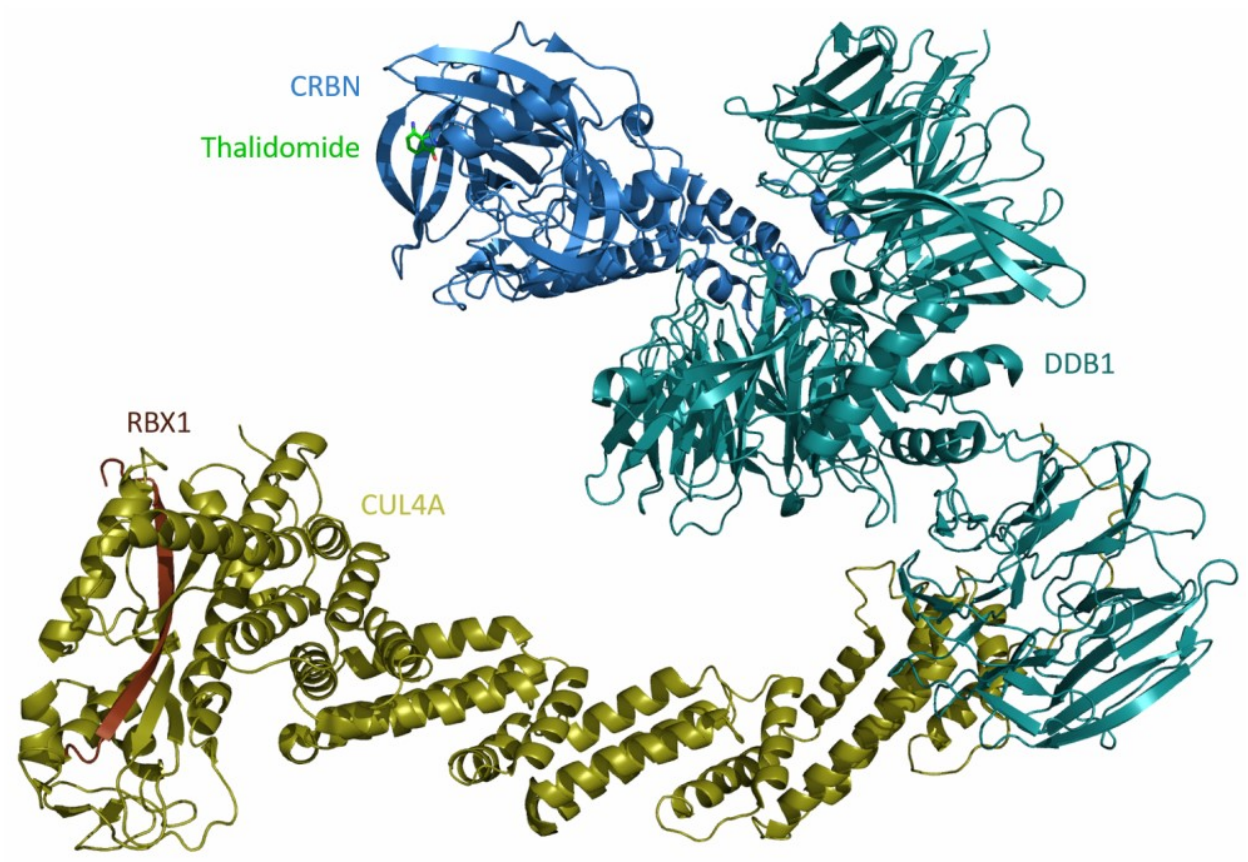

Figure 10: Model of a crystal structure of the CRL4 ${ }^{\text {CRBN }}$ E3 ligase in complex with thalidomide. (pdb entry: 4aOk and 4ci3). ${ }^{137}$ Thalidomide (green) binds to Cereblon (CRBN, blue), the recocnition unit of the CRL4 ${ }^{\text {CRBN }}$ E3 ligase. As substrate specifity module, the DNA-damage binding protein 1 (DDB1, cyan) is associated to CRBN and the core subunits, Cullin 4 (CUL4A, gold) and RING Box protein 1 (RBX1, brown).

Targeted protein degradation (TPD) displays many strengths compared to classical inhibition of the enzymatic activity, as PROTACS do not necessarily need to bind to the active site, thus expanding the sphere of the druggable proteome, ${ }^{119}$ and they can act in a catalytic manner which allows a substoichiometric dose of the PROTAC. ${ }^{122}$ Over the last two decades, TPD has been shown to be a powerful tool for addressing diverse disease-causing proteins. The majority of early studies focused on the development of degraders that utilized well-validated chemical compounds. These PROTACs degraded proteins such as hormone receptors, bromodomains and kinases. ${ }^{117,}$, 142-144 The androgen receptor degrader ARV-110 and the estrogen receptor degrader ARV-471 are currently the first degrader molecules examined in clinical trials phase 1/2. ${ }^{143}$ Apart from hormone receptors, also BET family proteins ${ }^{145-147}$ and protein kinases ${ }^{123,}$ 148-163 - both protein classes involved in oncologic diseases - have been successfully targeted for degradation. Over the past decade growing awareness and popularity of the TPD field led to a broader application system of PROTACs, including multifunctional proteins like SMARCA2 $2^{164}$, TRIM24 ${ }^{165}$ or tau. ${ }^{166}$ 
16 Introduction 


\section{Objective}

WDR5 is a biologically interesting target because it acts as a scaffolding subunit to ensure stability and function of KMT complexes, ${ }^{83}$ it recruits the transcription factor MYC to chromatin ${ }^{103}$ and it regulates the gene expression of ribosomal subunits. ${ }^{84}$ Targeting WDR5 function has been shown to be a promising approach for inhibiting leukemia (MLL-rearranged) and neuroblastoma (MYCdependent) cell growth by inducing p53-dependent apoptosis. WDR5s' role as conserved regulator of protein synthesis genes is also likely to play a role, as increased ribosome activity is common in cancers. ${ }^{82}$

Both interaction sites of WDR5 are associated with oncogenic drivers, so an inhibition of one binding site may have limited success. Instead, molecules that induce degradation of the entire protein offer a perspective to fully understand WDR5s' impact. This work describes the design and synthesis of WDR5 degrader molecules and their biological evaluation.

\section{Literature-known WDR5 ligands}

The aim of targeting WDR5 with small molecules has been successfully achieved by various groups with different chemical scaffolds. To date, most efforts have been made to target the Win site of WDR5. As shown in Scheme 1, several chemical motifs have been developed to bind to the to this site: while the chemical probe OICR-9429 by Grebien et al..$^{83,93}$ and DDO-2117 by Li et al. ${ }^{94}$ incorporated a methyl-piperazine scaffold, the later developed WDR5 ligands by Wang et al. ${ }^{167}$ and $\mathbf{C 6}$ by Aho et al. ${ }^{81}$ used pyrroloimidazole and imidazolimine-based scaffolds. All chemical groups mimic the consensus sequence 'ARA' that is contained in the peptide sequence of the interaction partners of WDR5 such as the SET/MLL family. 
<smiles>CN1CCN(c2ccc(-c3cccc(CN4CCOCC4)c3)cc2NC(=O)c2c[nH]c(=O)cc2C(F)(F)F)CC1</smiles>

OICR-9429

Grebien et al.<smiles>COc1ccc(CC(=O)NC2CCc3ccc(-c4cn5c(n4)CCC5)cc32)cc1OC</smiles>

pyrroloimidazole scaffold Wang et al.<smiles>Cc1c(F)c(N)cc(C(=O)Nc2cc(-c3ccc(NCCCN)cc3)ccc2N2CCN(C)CC2)c1Cl</smiles>

DDO-2117

Li et al.<smiles>Cc1ccc(-c2cc(Cn3cc[nH]c3=N)cc(NC(=O)c3ccc(Cl)c(Cl)c3)c2)c(F)n1</smiles>

C6

Scheme 1: Chemical structures of WDR5 ligands. As arginine mimic, either (top) piperazine-based ${ }^{83,94}$ or (bottom) imidazole-based ${ }^{81,167}$ ligands can be used for WDR5 Win site inhibition.

All published Win site ligands showed excellent binding affinities to the WDR5 protein, with OICR9429 showing the weakest affinity with $98 \mathrm{nM}$ in vitro and $1 \mu \mathrm{M}$ affinity in cellulo. ${ }^{83,93}$ The second piperazine-based WDR5 ligand DDO-2117 displays a cellular affinity of $7.6 \mathrm{nM} .^{94}$ The two pyrroloimidazole based scaffolds displayed a higher affinity ${ }^{167}$ than the piperazine scaffolds and bound slightly deeper into the Win pocket. The recently published ligand C6 showed an even stronger binding affinity in the picomolar range $\left(\mathrm{K}_{\mathrm{d}}: 0.1 \mathrm{nM}\right) .{ }^{81}$ Beside ligands that bind to the Win site of WDR5, several ligands have also been published that show nanomolar affinity to the WBM site. ${ }^{168}$ They were able to disrupt WDR5 and MYC protein protein interaction, although high doses $(50 \mu \mathrm{M})$ were required to observe the desired effect, suggesting off-target toxicity effects. ${ }^{168}$

\section{WDR5 small molecule antagonist OICR-9429}

The small molecule OICR-9429 was developed in 2015 and is the first small molecule to target WDR5. Its pharmacological activity has been shown in various cancer cell lines in vitro, thereby inhibiting cancer growth of MLL1-rearranged and C/EBPa mutant leukemias, neuroblastoma, and breast cancers. ${ }^{83,85,93,106,114}$ 
OICR-9429 interacts with the WDR5 Win site, as depicted in Figure 11: the methyl-piperazine moiety binds deep into the binding pocket and interacts via a waterbridge with the carbonyl function of the Cystein 261 backbone. A second waterbridge-mediated interaction is observed between the nitrogen of the Cystein 261 backbone and the carbonyl oxygen of the (trifluoromethyl)nicotinic acid moiety. Further interactions involve the two nitrogen atoms of the OICR-9429 amide functions which form bonds with the polar amino acids of Serine 91 and Aspartic acid 107. In addition to the observed polar interactions, the Win site contains many non-polar amino acids, e.g. several benzyl systems of Phenylalanine 133 or Tyrosine 191, that facilitate $\pi$ stackings of the biaryl moieties of OICR-9429 and enhance the interaction.

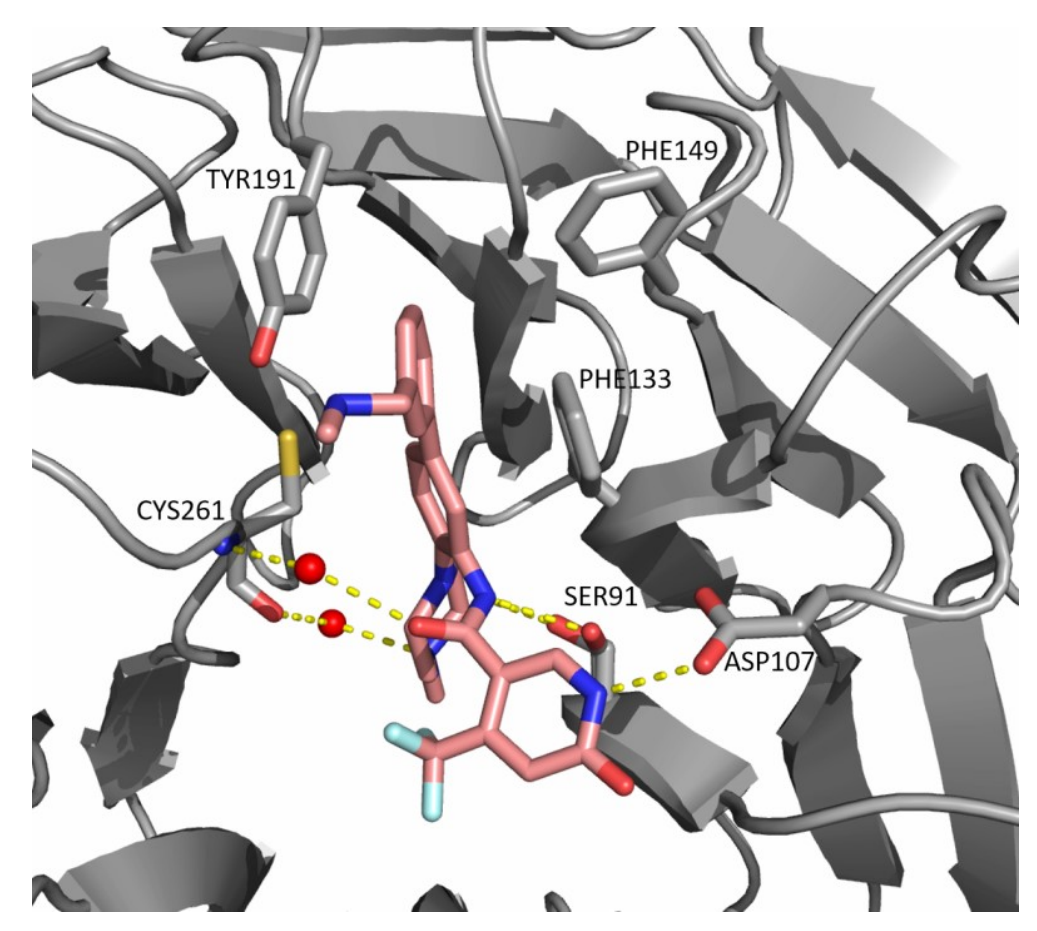

Figure 11: WDR5 Win site (grey) interaction of OICR-9429 (pink): two water bridge-mediated interactions between the CYS261 backbone and the Methylpiperazine moiety, respectively, the carbonyl function of the amide function anchor the small molecule in the Win site binding pocket. Further interactions between the amine of the amide bonds of the (Trifluoromethyl)nicotinic acid and the amino acid residues of SER91 and ASP107 provide additional affinity. The OICR-9429 biaryl system is stabilized by non-polar, aromatic residues like PHE133 and TYR191 (pdb entry: 4ql1). ${ }^{83}$

Summed up, all WDR5 Win site ligands demonstrate therapeutic effects and contribute to the studying of the biological functions of WDR5. The effects of WDR5 Win site inhibition by the OICR$\mathbf{9 4 2 9}$ or $\mathbf{C 6}$ were described in various studies in the previous chapters, so the following chapter will focus on the modification of the the published ligands into heterobifunctional molecules. 


\section{PROTAC design strategy}

At the beginning of the project, only the piperazine and the pyrroloimidazole-based inhibitors were known, while the high affinity imidazolimine inhibitor $\mathbf{C 6}$ was published later by Aho et al. Thus, the picomolar affine ligand C6 was not incorporated into PROTAC development. Furthermore, the arginine-mimicking motif imidazolimine of $\mathbf{C 6}$ hindered the synthesis of PROTAC molecules. The pyrroloimidazole-derived degraders were synthesized and characterized as a side project in the Master theses of Janik Weckesser and Dominic Löw, and are therefore only briefly explained in this work. Taken together, the synthetic work focused on WDR5 PROTACs containing predominantly the OICR-9429 and marginally the DDO-2117 inhibitors. During biological evaluation, both PROTAC types were compared. As shown in Figure 12, the two distinct scaffolds have different exit vectors of the Win binding pocket. This variation of the exit vectors was used to develop two subsets of PROTACs in order to increase the possibility of successful ternary complex formation.
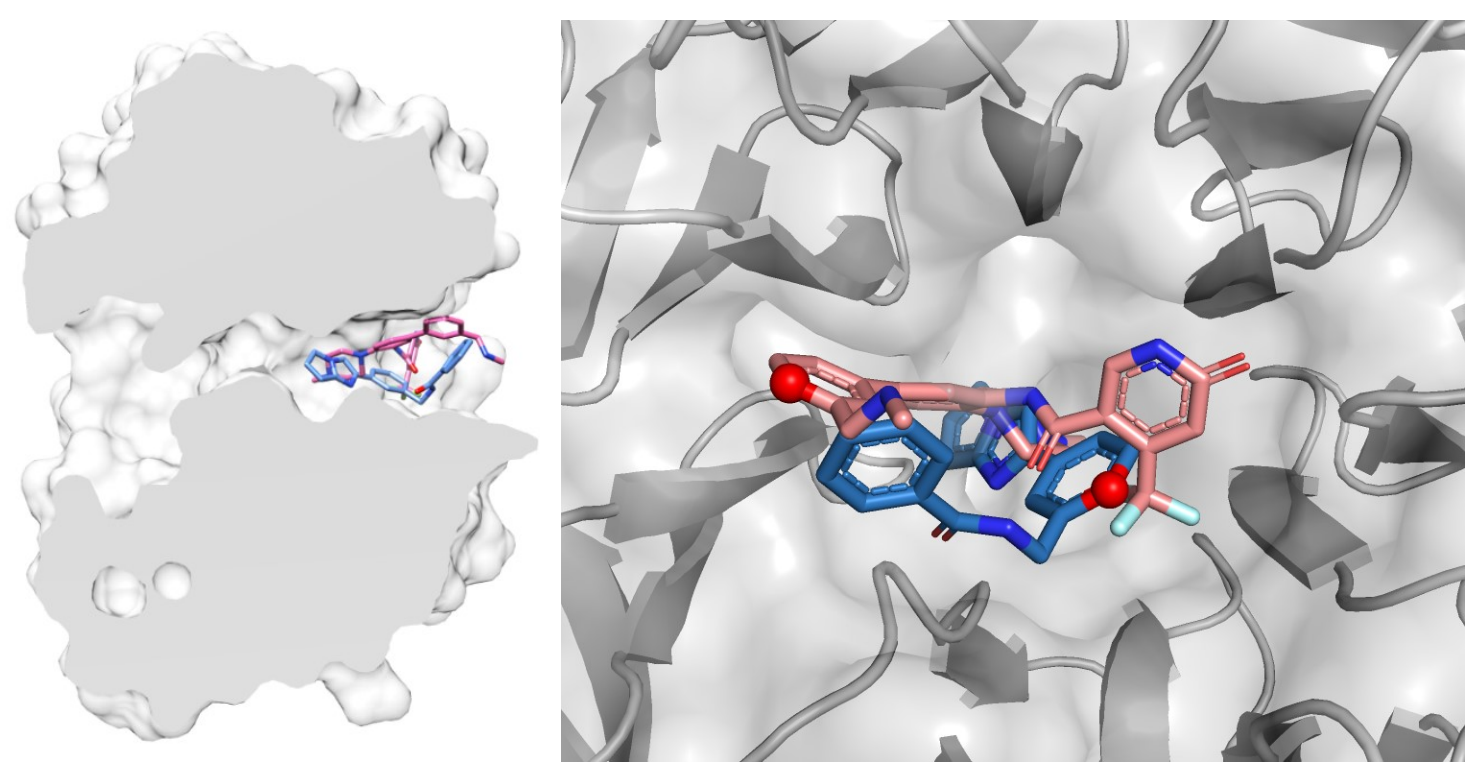<smiles>CN1CCN(c2ccc(-c3cccc(CN4CCOCC4)c3)cc2NC(=O)c2c[nH]c(=O)cc2C(F)(F)F)CC1</smiles>

OICR-9429 piperazine based WDR5 ligand

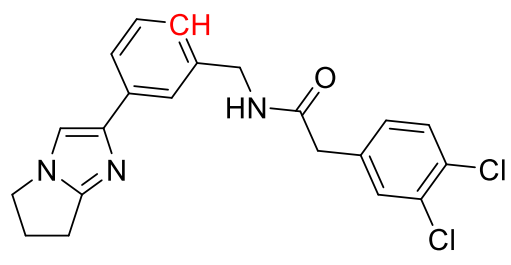

pyrroloimidazole based ligand

Figure 12: Exit vectors out of the WDR5 Win site binding pocket. (top, left) Side view of crystal structure overlay of WDR5 surface (light grey) with WDR5 ligands OICR-9429 (pink; pdb entry: 4ql1) and a 
pyrroloimidazole-based ligand from Wang et al. (blue; pdb entry: 6dak). (top, right) Top view of overlayed crystal structures of pyrroloimidazole and OICR-9429 ligand. The different exit points are marked as red spheres and show the attachment points of the designed degrader series (pdb entries: $4 \mathrm{ql} 1$ and $6 \mathrm{dak}) .{ }^{83,167}$ (bottom) Chemical structures of the exit points of the pyrroloimidazole and the OICR-9429 derived degraders. The red marked carbon atoms indicate the attachment points for PROTAC design.

The chosen exit points differ in the mentioned angle, but also in the relative height: the OICR-9429 was modified to exit the binding pocket, almost at the end of the Win site on top of the surface, while the linker attachment point of the pyrroloimidazole-based inhbitior was set deeper in the binding pocket to create a different exit vector. Scheme 2 shows a schematic drawing on how the PROTAC building blocks need to be connected to form a heterobifunctional molecule.

WDR5 ligand $\cdots \cdots \cdots$ Linker $\cdots \cdots \cdots$ E3 ligase ligand

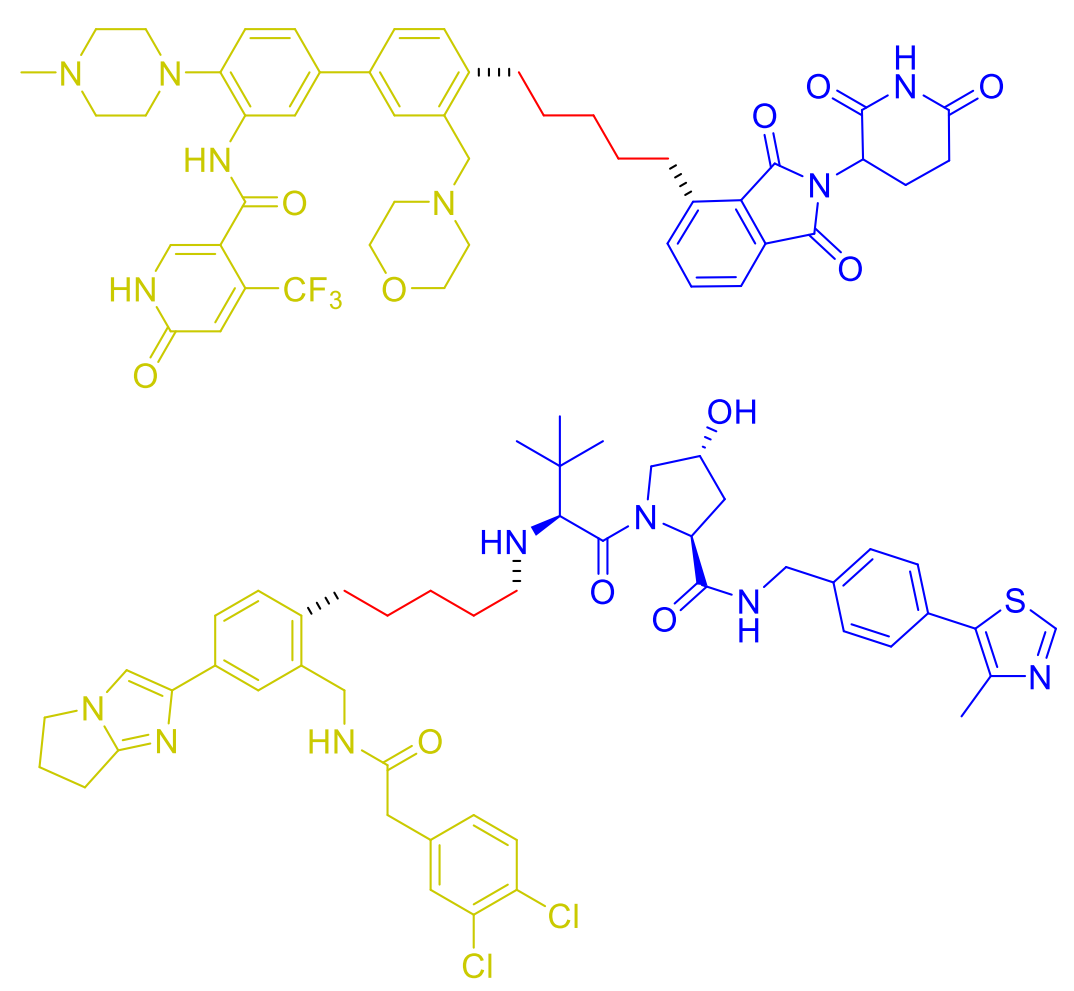

Scheme 2: Schematic drawing of the chemical structures of the heterobifunctional molecules. Both WDR5 ligand scaffolds (yellow) are connected to different linkers (red) and different E3 ligase ligands (blue). As representative E3 ligase ligands, the upper ligand is Pomalidomide that addresses the E3 ligase Cereblon, and the ligand below is VHL that addresses the E3 ligase Von-Hippel-Lindau. The dotted lines represent the bonds that need to be formed between the building blocks. 
22 Objective 


\section{Results and Discussion}

\subsection{Retrosynthetic approach}

Scheme 3 shows the retrosynthetic approach for modifying the OICR-9429 ligand to suitable ligands for linker attachment. In contrast to the pyrroloimidazole scaffold, the $\pi$-stacking of the OICR-9429 Biaryl system is suitable to maintain affinity (as seen in the crystal structure in Figure 11), so the linker was attached on top of the biaryl system, resulting in linker exposure on the WDR5 surface (as indicated in Figure 12). To reduce steric clashes with the linker and to reduce the molecular mass of the target compound, the originally methyl-morpholino ring of OICR-9429 was not incorporated into synthesis.<smiles>CN1CCN(c2ccc(-c3cccc(CN4CCOCC4)c3)cc2NC(=O)c2c[nH]c(=O)cc2C(F)(F)F)CC1</smiles>

OICR-9429

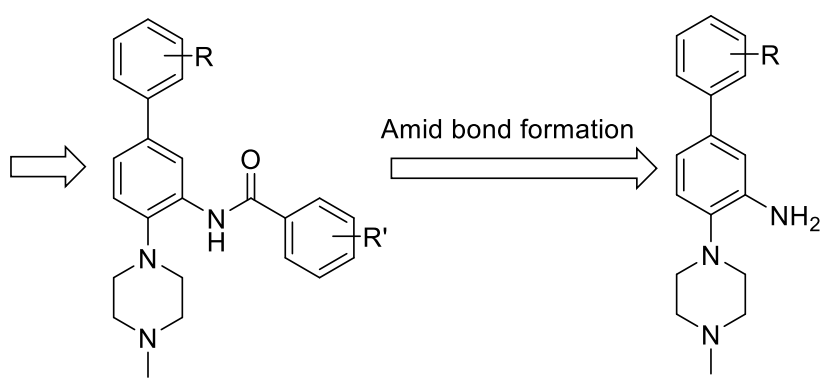

6a-d<smiles></smiles>

Scheme 3: Retrosynthetic approach for addressing WDR5 with OICR-9429 derived scaffolds.

\subsection{Synthesis route for OICR-9429 modified WDR5 ligands (6a-d)}

According to Scheme 3, several WDR5 ligands (6a-d) (with different benzoic acid motifs and aromatic systems at the exit site) were synthesized. The synthesis route is shown in Scheme 4: educts (1) and (2) formed intermediate (3) in a nucleophilic aromatic substitution. The reduction reaction of the aromatic nitro group lead to intermediate (4) which was coupled via a SuzukiMiyaura cross coupling to yield either a biaryl system of a benzene-indole system (5a) or two benzenes (5b). The benzene-indole biaryl system was chosen due to a publication from Getlik et 
al. that showed improved affinity upon using that scaffold. ${ }^{93}$ To generate affinity to WDR5, an amide coupling reactions of the primary amine $(\mathbf{5} \mathbf{a}, \mathbf{b})$ with the various benzoic acids were carried out to form the final modified ligands for PROTAC development (6a-d). The in situ deprotection resulted in a free carboxylic acid or a free amine function of the indole that served as attachment point. The modified ligands were then connected to various E3 ligase linkers.

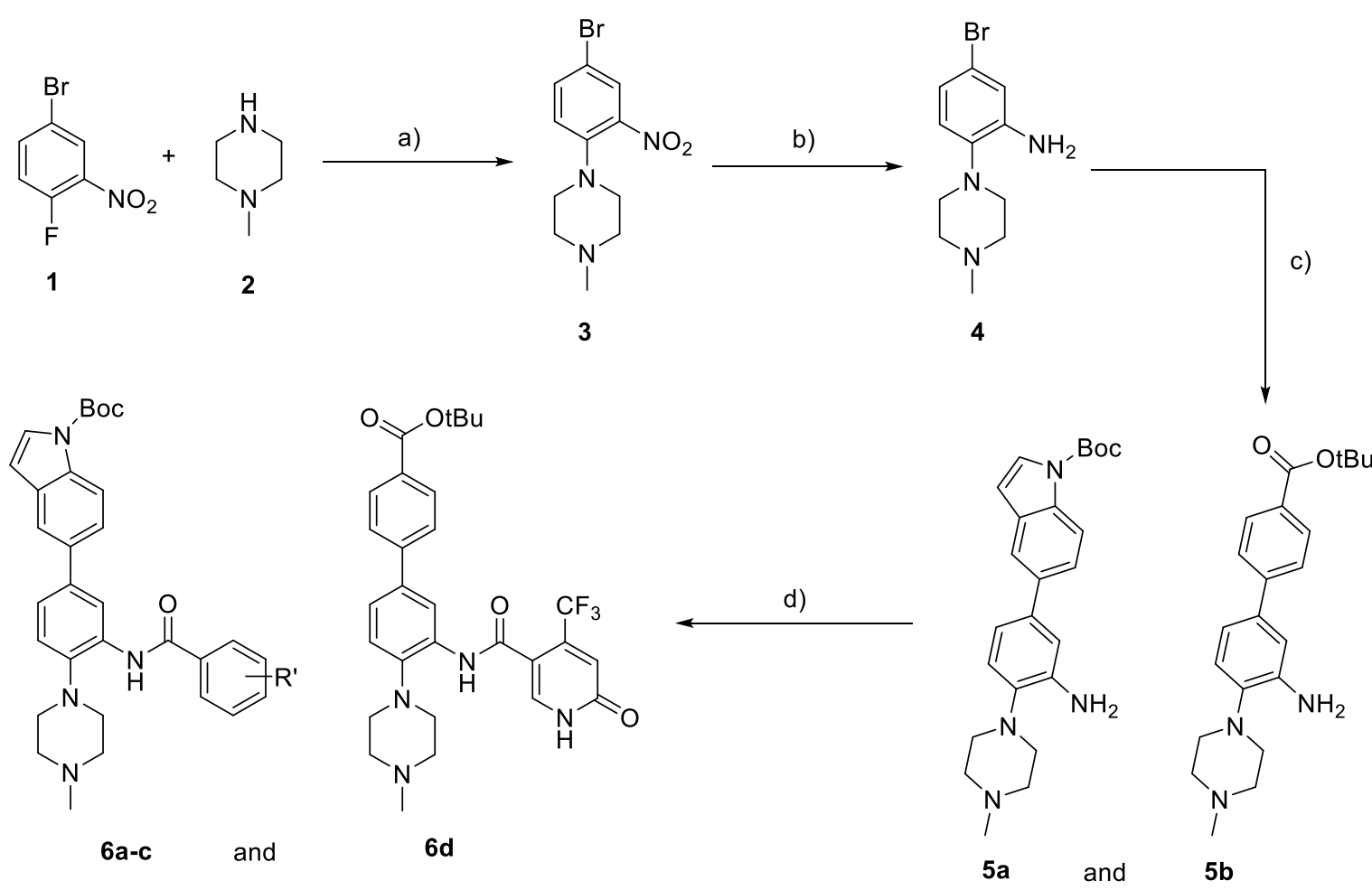

Scheme 4: Synthetic procedure to obtain WDR5 ligands (6a-d). a) DIEA, EtOH, $80^{\circ} \mathrm{C}, 16 \mathrm{~h} ;$ b) $\mathrm{Zn}, \mathrm{NH}_{4} \mathrm{Cl}$, Dioxane/ water (3/1), rt, $30 \mathrm{~min}$; c) Boronic acid, XPhos Pd G3, $\mathrm{NaHCO}_{3}$, Dioxane/ water (3/1), $85{ }^{\circ} \mathrm{C}, 16 \mathrm{~h}$; d) 6a: Carboxylic acid, HATU, DIEA, DMF, rt, $16 \mathrm{~h}$ or 6b-d: 1 . Carboxylic acid, $\mathrm{SOCl}_{2}, \mathrm{CH}_{2} \mathrm{Cl}_{2} / \mathrm{ACN}(1 / 1), 50{ }^{\circ} \mathrm{C}$, $3 \mathrm{~h} ; 2$. pyridine, $\mathrm{CH}_{2} \mathrm{Cl}_{2} / \mathrm{ACN}(1 / 1), 50^{\circ} \mathrm{C}, 16 \mathrm{~h}$.

A detailed synthetic description of the complete process follows below.

\section{Description of synthesis steps $a$ ) and b)}

In the first part of the synthetic route, the precursor 5-bromo-2-(4-methylpiperazin-1-yl)aniline (4) was synthesized. The molecule was obtained in a two-step synthesis as shown in Scheme 5. The two starting materials 4-Bromo-1-Fluoro-2-Nitrobenzene (1) and 1-Methylpiperazine (2) reacted in a nucleophilic aromatic substitution reaction to 1-(4-bromo-2-nitrophenyl)-4methylpiperazine (3). In this reaction, the base DIEA was used to scavenge the resulting hydrofluoric acid. The electron density of the aromatic system (1) is remarkably reduced due to the three $(-\mathrm{I} / \mathrm{-M})$ substituents, so the piperazine easily substitutes the fluoride atom. Contrary to 
nucleophilic substitutions of type 1 or type 2 , the $S_{N} A r$ rate is generally increased when the leaving group is fluorine due to the differences in the energy transition state: the highest energy transition state for this two-step addition-elimination process occurs in the first step which is the ratedetermining step. This results in the formation of a high energy Meisenheimer-like complex. The effect of the greater electron withdrawal capacity of the fluorines stabilizes the resulting negative charge on the complex compared to the other halogens. The displacement of the leaving group occurs rapidly and does not affect the overall rate of reaction. The reaction in this work, as well as described in the literature, was carried out under heating $\left(80^{\circ} \mathrm{C}\right)$ for several hours in ethanol and gave excellent yields of $95+\%$ for 1-(4-bromo-2-nitrophenyl)-4-methylpiperazine (3). These high yields are also observed in the literature. ${ }^{93}$<smiles>O=[N+]([O-])c1cc(Br)ccc1F</smiles>

1<smiles>CN1CCNCC1</smiles>

2

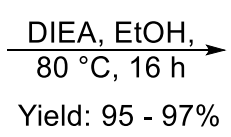

Yield: $95-97 \%$

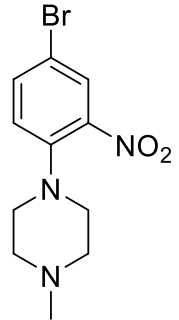

3

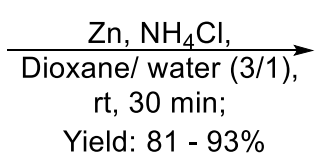

Yield: $81-93 \%$<smiles>CN1CCN(c2ccc(Br)cc2N)CC1</smiles>

4

Scheme 5: Steps a) and b) to synthesise precursor 5-bromo-2-(4-methylpiperazin-1-yl)aniline (4).

In the next step, the aromatic nitro group was reduced to an aniline derivative under mild reduction conditions, using zinc and ammonium chloride in a dioxane/ water mixture. Other reduction attempts that used hydrogen and palladium on carbon failed in terms of product isolation and producd various uncharacterisable by-products. The product (4) could be isolated by a variant of the Béchamp reduction, a mild reduction that does not hydrate the aromatic ring system and aromatic-bound halogens. The reaction was carried out by stirring all reactants at room temperature for thirty minutes. Thereby, a discoloration from the orange nitro compound to the bright rose-colored amine was observed and indicated the reaction progress. Dioxane was removed from the reaction solution and the weak acidic water phase was neutralized with sodium bicarbonate. Extraction of the uncharged amine with an organic solvent led to the desired product (4) in high purity and excellent yields (with an overall yield of 90\%).

\section{Description of synthesis steps $c$ ) and d)}

Next synthesis steps included the attachment of the second aryl system and the final attachment point for the E3 ligase linker molecule. As shown in Scheme 6 and Scheme 7, both intermediates 
(5a) and (5b) were obtained in a Suzuki-Miyaura cross coupling. In initial experiments, (5a) was prepared using Pd XPhos G3 as catalyst and sodium hydroxide in a toluene/ water mixture. Initially, the product was obtained in $28 \%$ yield and in subsequent experiments, the system was optimized by varying the catalyst, the base and the solvent. Here, the system that relied on $\mathrm{Pd}\left(\mathrm{Ph}_{3}\right)_{4}$, potassium carbonate in a toluene/ water mixture achieved the best yield of $43 \%$. The rather modest yield is probably related to the Boc-group of the indole system: by heating at $100{ }^{\circ} \mathrm{C}$ for a long period (16-24 h) in a strongly basic environment, a cleavage of the protecting group is possible. The resulting amine is more difficult to isolate in an aqueous environment and thus might explain the low yield. However, when the solvent system was changed to dimethoxyethane, a yield of $41 \%$ was also isolated. Experiments carried out in a microwave were not successful.

In contrast to the difficulties in isolating the quantitative amounts of (5a), (5b) could be obtained in high yields with different systems. The best system for cross coupling used catalyst Xphos Pd G3, sodium hydroxide as base and a toluene/ water mixture. As tert-butyl esters are also likely to be cleaved under basic conditions, the amount of water was limited to a minimum $(1 \mathrm{~mL})$. However, the other experiments using $\mathrm{Pd}\left(\mathrm{Ph}_{3}\right)_{4}$, potassium carbonate in a toluene/ water mixture gave a yield of $73 \%$, showing clear favor for product formation of the less reactive tert-butylester (5b) over the more reactive Boc-protected indole (5a).

The next step was the derivatization of the amine (5b) to amides (6a-c) as shown in Scheme $\mathbf{6}$. As initial experiment, the inexpensive building block 3-methylbenzoic acid was used for establishing the synthesis route. This simple aryl decoration was used in a publication from Getlik et al. and showed an affinity to WDR5 of $0.37 \mu \mathrm{M}$ in vitro. ${ }^{93}$ For the synthesis of tert-butyl 5-(3-(3methylbenzamido)-4-(4-methylpiperazin-1-yl)phenyl)-1H-indole-1-carboxylate (6a), the amide bond formation was carried out by using the uronium salt 1-[bis(dimethylamino)methylene]-1H1,2,3-triazolo[4,5-b]pyridinium 3-oxide hexafluorophosphate (HATU) in the polar, aprotic solvent $\mathrm{N}, \mathrm{N}$-Dimethylformamide. The coupling reagent HATU has widely been used for various amide bond formations. A pre-activation of the carboxylic acid with the coupling reagent and DIEA has been shown to enhance the overall reaction speed, ${ }^{169-170}$ and resulting in only small amounts of epimers and high yields, even for sterically hindered carboxylic acids/amino acids. ${ }^{171}$ The azabenzotriazole scaffold of HATU enables the formation of a highly reactive NO-ester in situ, and additives such as the thermally unstable and explosive HOBt used in amide bond formation with the popular coupling reagent EDC- $\mathrm{HCl}$, are redundant. The beneficial effect on the enhanced reaction rate can be attributed to the nitrogen atom in 7-position of the azabenzotriazole, which stabilizes the leaving group by its electron withdrawing effect. ${ }^{172}$ The nitrogen atom also stabilizes a seven-centered transition state, in which the neighboring-group effect facilitates the 
deprotonation and release of the final amide. For amide coupling via HATU, 3-methylbenzoic acid was activated for $20 \mathrm{~min}$ with the coupling reagent to form an active ester. Subsequently, amine (5b) was dissolved in DMF with an non-nucleophilic base ( $N, N$-Diisopropylethylamine, DIEA) and added to the pre-activated reaction solution. The obtained yield of the amide bond formation via HATU of tert-butyl 5-(3-(3-methylbenzamido)-4-(4-methylpiperazin-1-yl)phenyl)-1H-indole-1carboxylate (6a) was $74 \%$.

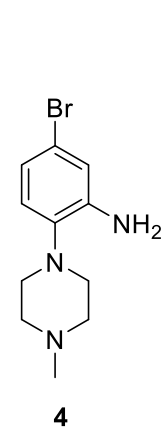

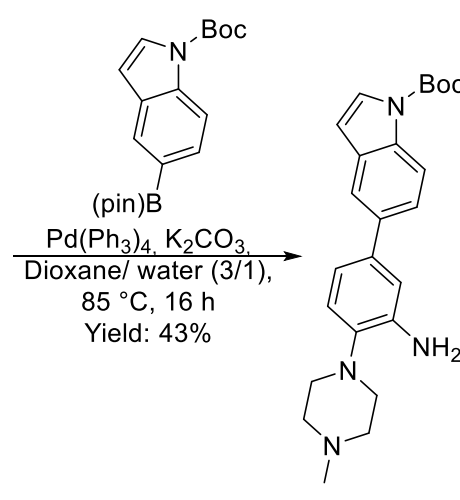

$5 a$

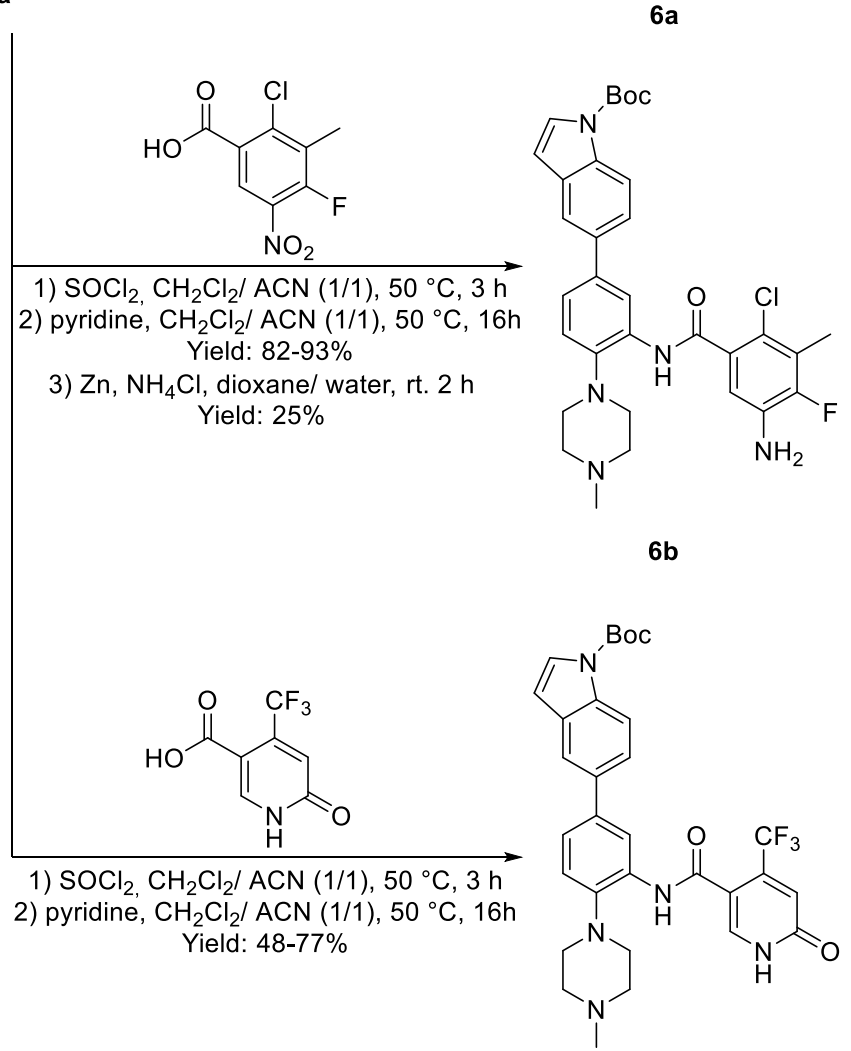

6c

Scheme 6: Steps c) and d) to synthesize piperazine-based WDR5 ligands tert-butyl 5-(3-(3methylbenzamido)-4-(4-methylpiperazin-1-yl)phenyl)-1H-indole-1-carboxylate (6a), tert-butyl 5-(3-(5- 
amino-2-chloro-4-fluoro-3-methylbenzamido)-4-(4-methylpiperazin-1-yl)phenyl)-1H-indole-1-carboxylate (6b) and tert-butyl 5-(4-(4-methylpiperazin-1-yl)-3-(6-oxo-4-(trifluoromethyl)-1,6-dihydropyridine-3carboxamido)phenyl)-1H-indole-1-carboxylate (6c).
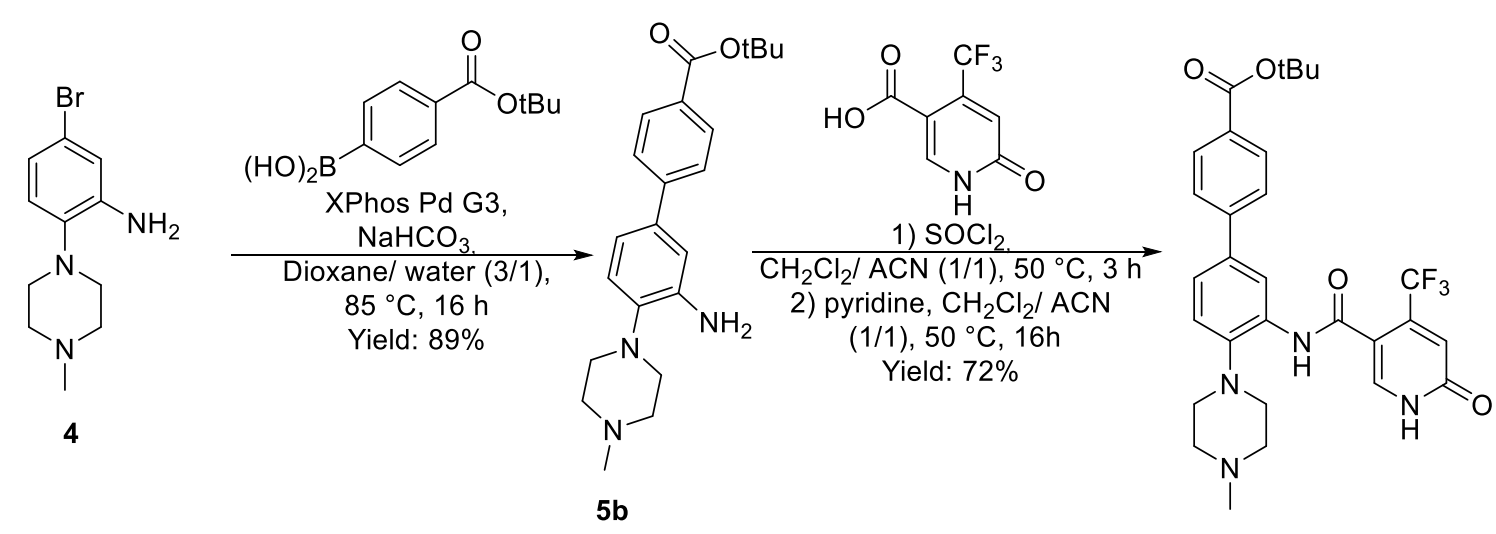

$6 \mathrm{~d}$

Scheme 7: Steps c) and d) to synthesize the piperazine-based WDR5 ligand tert-butyl 4'-(4-methylpiperazin1-yl)-3'-(6-oxo-4-(trifluoromethyl)-1,6-dihydropyridine-3-carboxamido)-[1,1'-biphenyl]-4-carboxylate (6d).

In contrast to WDR5 ligand (6a), the amide bond formation for (6b-d) required more drastic reaction conditions to make the coupling reaction feasible. This is due to the strongly deactivated carboxylic acid on the Trifluormethyl-pyridinone ring. Initially, tert-butyl 5-(4-(4-methylpiperazin1-yl)-3-(6-oxo-4-(trifluoromethyl)-1,6-dihydropyridine-3-carboxamido)phenyl)-1H-indole-1carboxylate (6c) was aimed to be synthesized by using the coupling reagent HATU, but no product formation between the deactivated carboxylic acid and the unreactive aniline (5a) was observed. As shown in Scheme 6 and Scheme 7, activation of the carboxylic acid with an excess of thionyl chloride, to form a more electrophilic acyl chloride, led to successful product formation. Solvents were varied from methylene chloride to mixtures of methylene chloride and acetonitrile to obtain a better solubility of the carboxylic acid, and pyridine was added as a base. Usually, the acyl chloride was prepared first, and the excess of thionyl chloride was removed under vacuum. The crude product was then re-dissolved in a methylene chloride and acetonitrile mixture. Subsequently, amines (5a) or (5b) were dissolved with pyridine in methylene chloride and added to the crude acid chloride. The reaction solution was stirred overnight under heating $\left(50{ }^{\circ} \mathrm{C}\right)$ and product formation was observed by TLC and ESI-MS. The purification was carried out on a reversephase column chromatography system to obtain excellent yields of $80+\%$ for 5 -amino-2-chloro4-fluoro-3-methylbenzene derivate (6b) and high yields for the derivatives (6c) and (6d). For molecules $(\mathbf{6 c})$ and $(\mathbf{6 d})$, two side products were isolated and characterized by ESI and ${ }^{1} \mathrm{H}-$ NMR. Both side products result from a second reaction between the amine and the acyl chloride and were obtained in a yield of approx. $25 \%$. Additionally to the isolated by-products, a small 
fraction of amine $(\mathbf{5 a})$ or $\mathbf{( 5 b}$ ) was isolated after purification, indicating a partly degradation of the acyl chloride due to minimal traces of oxygen. The observation of side product formation occurred in experiments where the solid amines were added to the suspended acyl chloride. Herein, the reaction conditions were modified and amine $(\mathbf{5 a})$ or $(\mathbf{5 b})$ was pre-dissolved in DCM, before being added to the acyl chloride. This resulted in higher yields of the desired product.

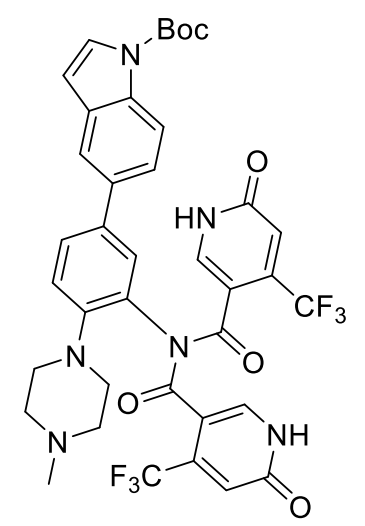

side product of $(\mathbf{6 c})$<smiles>CCC(C)OC(=O)c1ccc(-c2ccc(N3CCN(C)CC3)c(N(C(=O)c3c[nH]c(=O)cc3C(F)(F)F)C(=O)c3c[nH]c(=O)cc3C(F)(F)F)c2)cc1</smiles>

side product of $(\mathbf{6 d})$

Scheme 8: Isolated side products of WDR5 ligands (6c,d), obtained in yields of approx. $25 \%$.

The final step in obtaining the molecule derived from DDO-2117, tert-butyl 5-(3-(5-amino-2chloro-4-fluoro-3-methylbenzamido)-4-(4-methylpiperazin-1-yl)phenyl)-1H-indole-1-carboxylate (6b), was a reduction reaction of a nitro group to a primary amine. While reduction with tin chloride or with Hydrogen gas with palladium on carbon were not successful, WDR5 ligand tertbutyl 5-(3-(5-amino-2-chloro-4-fluoro-3-methylbenzamido)-4-(4-methylpiperazin-1-yl)phenyl)$1 \mathrm{H}$-indole-1-carboxylate (6b) could be obtained in a yield of $25 \%$ using zinc and ammonium chloride.

In summary, the overall yield from starting materials (1) and (2) showed molecule (6d) as most favorable product of all four WDR5 ligands, with a total yield of $64 \%$ over four reaction steps. As the Suzuki-Miyaura cross coupling step for the WDR5 ligands (6a-c) showed a rather moderate yield, the followed-up amide bond formation in good yields could not cover up this reaction step. The resulting overall yields were thus in a signifiable lower range. For molecule (6a), an overall yield of $29 \%$ was obtained. The same range was observed for molecule $(6 c)$ that was isolated with $30 \%$. The yield of WDR5 ligand (6b) decreased to $9 \%$ due to the additional reaction step of the nitro reduction. After the chemical synthesis of these four different WDR5 ligands was achieved successfully, the next step was the biophysical evaluation to determine binding affinity to WDR5. 


\subsection{Biophysical and biological evaluation of WDR5 ligands (6a-d)}

The synthesized ligands (6a-d) were tested in vitro in terms of their binding affinity by the Differential Scanning Flourimetrie (DSF) assay and for their cell permeability and target engagement using the NanoBRET ${ }^{\mathrm{TM}}$ assay. The results of both assays are shown in Table 2.

The DSF assay, also called thermal shift assay (TSA), measures the thermal stabilization $\left(\Delta T_{m}\right)$ of a protein-ligand complex and can serve as indication for a binding interaction between ligand and protein. This method for validating proteins in vitro as well as the NanoBRET ${ }^{\mathrm{TM}}$ assay system will be discussed in more detail in the PROTACs evaluation chapter. The DSF method is simple, inexpensive and allows the prediction of a binding tendency of the investigated WDR5 ligands. The unselective and literature-known ligand (6a) displayed the lowest shift, and surprisingly molecule (6b) also showed a rather moderate thermal stabilization of the WDR5 protein. Ligand (6c) showed a thermal stabilization in the range of the high-affinity binder OICR-9429, known from literature. The highest thermal stabilization was observed for the WDR5 ligand (6d) which showed a significant thermal stabilization $\Delta T_{m}$ of almost $21 \mathrm{~K}$ and suggested improved potency towards WDR5.

As the ligands (6a) and (6b) showed only weak shifts, the NanoBRET ${ }^{\mathrm{TM}}$ assay focused on WDR5 ligands $(\mathbf{6 c})$ and $(\mathbf{6 d})$. BRET measurements monitor the cellular binding affinity $\left({ }^{\prime} C_{50}\right)$ of the ligand to the target protein and can indicate the cellular permeability of a compound (a limiting factor for insoluble molecules with a high molecular weight.) WDR5 ligand (6c) displayed an affinity of $15 \mu \mathrm{M}$, while the OICR-9429 and ligand (6d) showed single-digit micromolar potency towards WDR5. The differences between the parent compound OICR-9429 and its PROTAC-applicable ligands $(\mathbf{6 c}, \mathbf{d})$ are possibly due to the missing morpholine ring system. The missing morpholine might lead to the observed decreased solubility of the compounds in the NanoBRET ${ }^{\mathrm{TM}}$ assay. 
Table 2: DSF and NanoBRET ${ }^{\mathrm{TM}}$ data for WDR5 ligands (6a-d). DMSO and OICR-9429 served as controls. Each measurement was carried out in triplicates to determine the thermal shift $\left(\Delta T_{m}\right)$ and the standard deviation (SD) and in duplicates for the cellular affinity $\left(I_{50}\right)$. n.d: not determined.

\begin{tabular}{c|ccc}
\multicolumn{1}{c}{ ID } & \multicolumn{1}{c}{$\Delta T_{m}[\mathrm{~K}]$} & $\mathrm{SD}[\mathrm{K}]$ & $\mathrm{IC}_{50}[\mu \mathrm{M}]$ \\
\hline DMSO & 0.0 & 0.2 & $\geq 50 \mu \mathrm{M}$ \\
OICR-9429 & 13.3 & 0.1 & 1.7 \\
$\mathbf{6 a}$ & 1.2 & 0.1 & n.d. \\
6b & 3.5 & 0.2 & n.d. \\
6c & 12.7 & 0.3 & 15 \\
6d & 20.8 & 0.6 & 5.4
\end{tabular}

Summed up, the data indicate that ligand (6d) was the preferred ligand for PROTAC modifications, and thus several degraders were developed - based on (6d) with various linker motifs and E3 ligases.

\subsection{Synthesis route for OICR-9429 modified WDR5 PROTACs (7a-e), (8a-j) and $(9 \mathrm{a}-\mathrm{c})$}

Since the WDR5 ligand (6d) was found to be the most promising ligand for a degrader synthesis, the corresponding E3 ligase linker molecules were designed to complement the tert-butyl ester function of $(\mathbf{6 d})$. As a corresponding chemical function, a primary amine was chosen. Analogous to molecules (6a-d), the amine function had to be protected in order to be spared from interactions in previous reaction steps. As seen in Scheme 9 and Scheme 10, the E3 ligase linker products for the two most commonly addressed E3 ligases Cereblon (CRBN) and Von-HippelLindau (VHL) were generated by amide bond formation. As this reaction occurred in a basic environment, linkers that protected the amine with a tert-Butyloxycarbonyl (Boc) group were used as starting material. For the synthesis of the VHL and MDM2 addressing PROTACs, the commercially available ligands were used, while the CRBN-addressing ligand (L0) was synthesized in-house. 4-Flouropomalidomide (LO) was gained by heating 4-fluoroisobenzofuran-1,3-dione and 3-aminopiperidine-2,6-dione hydrochloride in pyridine overnight. Pyridine served in this reaction as solvent and base. After stirring overnight, product formation, which was monitored by TLC, was moderate. After removal of the pyridine, the reaction mixture was dissolved in water and DCM. 
The extraction of the crude product proved to be challenging, as the solution did not separate well in the separator funnel, possibly due to educt residues of 3-aminopiperidine-2,6-dione hydrochloride and a difficult separation of, aqueous and organic layer. In addition, both educts and the product tended to be very polar, which complicated the extraction that had to be carried out at least six times. The organic phases were washed with $1 \mathrm{M} \mathrm{HCl}$ to remove pyridine residues and brine to remove water traces. The repetitive extraction process was successful because only the desired product peak was observed on TLC at $254 \mathrm{~nm}$. However, traces of 3-aminopiperidine2,6-dione hydrochloride were observed when staining the TLC with potassium permanganate. Column chromatography led to isolation of the desired product (L) in high purity as observed by NMR, but with a low yield of $33 \%$. Possible optimizations for this reaction would be a prolonged reaction time with an additional water trap to shift equilibrium towards product formation.

In a subsequent nucleophilic aromatic substitution, the fluorine of (L0) was substituted by the free amine of the different Boc-protected linker chains, as shown in Scheme 9. Mild reaction conditions were chosen as the Boc protecting group can also be cleaved in a basic environment at high temperatures: the used amount of Hünigs' base was limited to two equivalents. N-Methyl-2pyrrolidon (NMP) was chosen as solvent due to its high polarity. Analogous to the synthesis of the educt (L0), the extraction of the very polar molecules had to be repeated frequently to separate the polar products from the aqueous phase. The expectation that the long and thus more hydrophilic [PEG] linker molecules would lead to lower yields, was not observed. E3 ligase linker molecules (L3) and (L4) gave higher yields (around 50\%) than shorter E3 ligase linker products (L1) and (L2) (around 30\%). The best yield was achieved when the aromatic-bridged diamine linker was attached to 4-Flouropomalidomide (LO). A possible change of the solvent to, e.g., DMSO, combined with higher heating temperatures, could optimization the nucleophilic substitution. 
<smiles>O=C1OC(=O)c2c(F)cccc21</smiles><smiles>NC1CCC(=O)NC1=O</smiles><smiles>O=C1CCC(N2C(=O)c3cccc(F)c3C2=O)C(=O)N1</smiles><smiles>CC(C)(C)OC(=O)NCCCOCCN</smiles>

Lo

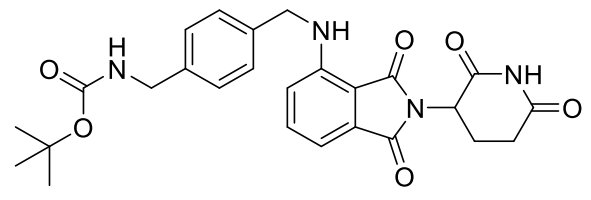

L5, $55 \%$

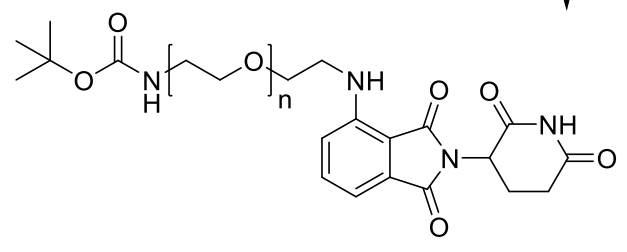

$\mathrm{n}=1 \quad \mathbf{L} \mathbf{1}, 33 \%$

$\mathrm{n}=3 \quad \mathbf{L 2}, 33 \%$

$\mathrm{n}=5 \quad \mathbf{L} 3,46 \%$

$\mathrm{n}=7 \quad$ L4, $52 \%$

Scheme 9: Synthesis of E3 ligase ligand pomalidomide (LO) and E3 ligase linkers (L1-L5) addressing the E3 ligase Cereblon (CRBN).

Beside the immunomodulatory drugs thalidomide, lenalidomide and pomalidomide, which target the E3 ligase Cereblon (CRBN) and are most commonly used for PROTAC design, ligands addressing the E3 ligase Von-Hippel-Lindau (VHL) were also used in this study. The herein used VHL ligand VH032 was commercially available and coupled to different purchasable linker, comprising [PEG] (L6-L8), aliphatic (L9-L13) or aromatic linker (L14) motifs, as shown in Scheme 10. The amide formation was carried out using HATU as coupling reagent and Hünigs' base in DMF, and the desired products were isolated in high purity and yields ranging from $30 \%$ to $87 \%$. The lowest yields were observed when the heaviest linker moieties was attached to the VHL ligand, namely the $[\mathrm{PEG}]_{4}$ moiety. The steric complexity could potentially be compensated by increasing the electrophilicity of the carboxylic acid, e.g. by generating acyl chlorides. When using acyl chlorides, attention should be paid to the protecting groups: the very reactive reagent is able to cleave Boc and tert-butylester groups under certain conditions. ${ }^{173}$ In summary, six of nine E3 ligase linkers were obtained in very good/ satisfying yields and with high purity as determined by NMR and HPLC. 


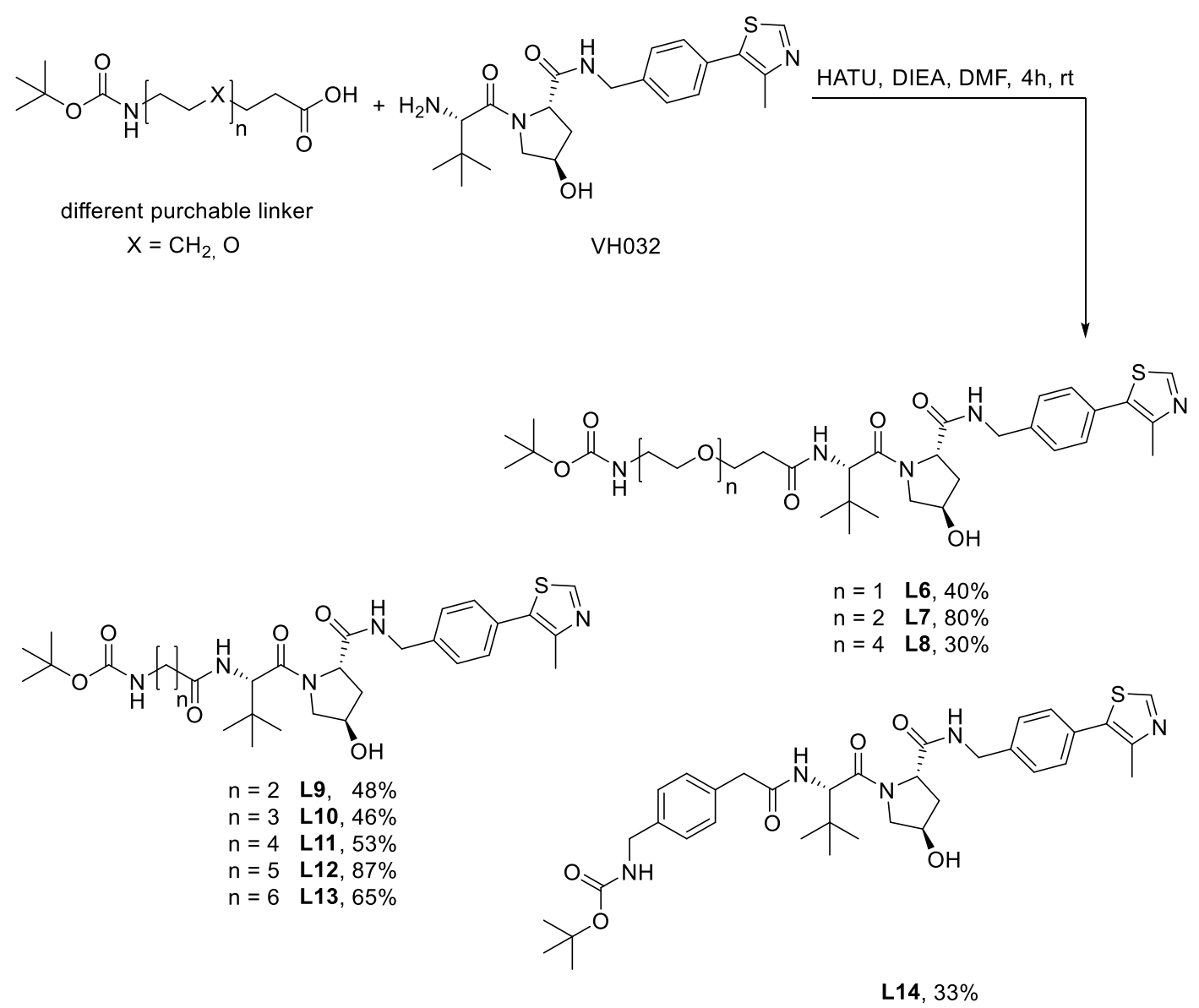

Scheme 10: Synthesis procedure of E3 ligase linkers (L6-L14) addressing the E3 ligase Von-Hippel-Lindau (VHL).

To generate the heterobifunctional molecules, the deprotection of the tert-butylester function of WDR5 ligand (6d) and the deprotection of the Boc-protected amine of the linkers (L1-L14) was carried out in situ with a mixture of trifluoracetic acid (TFA) in dichloromethane. Both components were stirred separately for a period between $30 \mathrm{~min}$ and $2 \mathrm{~h}$, and the deprotection was monitored by TLC. Due to the stable ester bond, the deprotection of the tert-butyl ester of (6d) occurred slower than the deprotection of the amine E3 ligase linkers (L1-L14). After removing the solvent and excess TFA, both active species were transferred in one flask and the amide reaction was carried out by using HATU in DMF. Product formation was observed within a few hours by TLC and TLC-ESI. The reaction was quenched with water. To remove the solvent DMF and the base DIEA, saturated sodium bicarbonate was added, and the solution was extracted with dichloromethane or ethyl acetate. After evaporation, the crude product was purified by a preparative HPLC system. The isolated products were extracted with sodium bicarbonate to remove the TFA that was used as additive in HPLC solvents. In this way, the isolated heterobifunctional molecules (7a-e), (8a-j) and (9a-c) were obtained. The synthetic steps of the OICR-9429 based PROTAC molecules that 
address CRBN are shown in Scheme 11. Scheme 12 shows the chemical structures of the synthesized PROTACs (8a-j) addressing the VHL E3 ligase.<smiles>CCCCOC(=O)c1ccc(-c2ccc(N3CCN(C)CC3)c(NC(=O)c3c[nH]c(=O)cc3C(F)(F)F)c2)cc1</smiles>

$6 d$<smiles>NCCOCCNc1cccc2c1C(=O)N(C1CCC(=O)NC1=O)C2=O</smiles>

PEG E3 ligase linker L1-4 or aromatic E3 ligase linker L5
1) TFA/ DCM (1/1), rt, $1 \mathrm{~h}$ 2) HATU, DIEA, DMF, rt, $3-5 \mathrm{~h}$

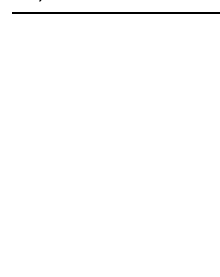

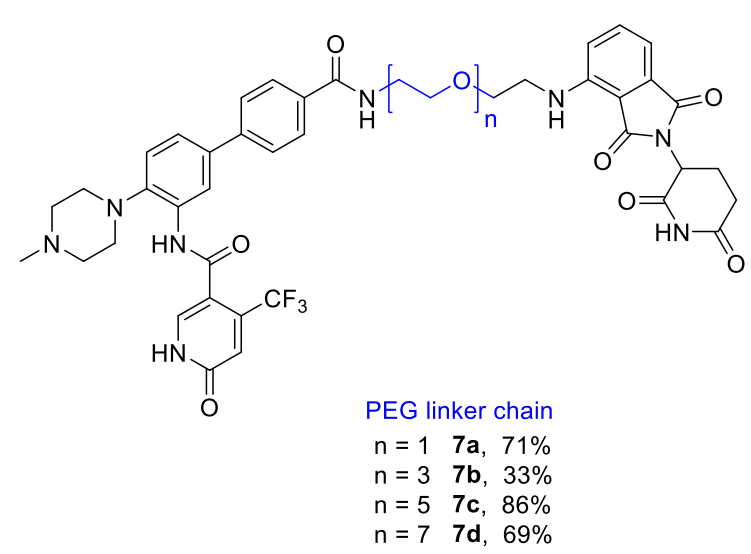

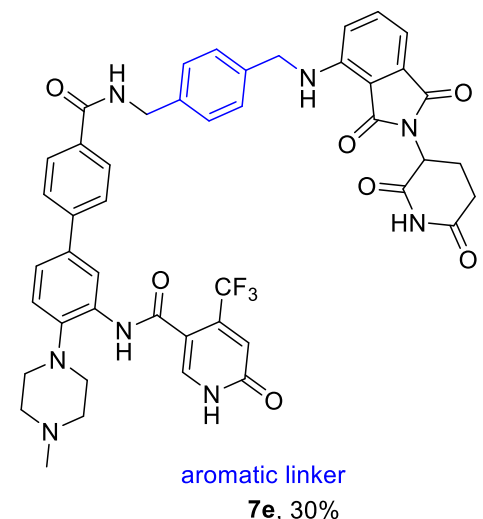

Scheme 11: Synthesis scheme of WDR5 degraders (7a-e) based on ligand (6d) addressing E3 ligase CRBN. The type and nature of the linker is indicated in blue.

The generally higher yields of the CRBN-addressing PROTACs (7a-e) up to $86 \%$ might be due to their lower molecular weight and thus their more small molecule-like character that simplified laboratory handling. Furthermore, these PROTAC possessed a remarkable yellow colour which visually simplified the extraction and purification process. In contrast to the CRBN-addressing PROTACs, the more peptide-like structured VHL PROTACs and MDM2 PROTACS possessed a higher molecular weight and thus less-favored properties. Nevertheless, the highest isolated yields were, respectively, 67\% for the VHL-addressing PROTAC (8d) and 25\% for the MDM2-based degrader (9b). 


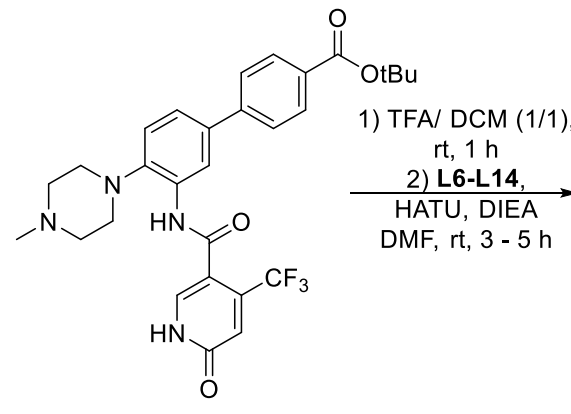

6d<smiles>Cc1ncsc1-c1ccc(CNC(=O)[C@@H]2C[C@H](O)CN2C(=O)[C@H](NC(=O)CCOCCNC(=O)c2ccc(-c3ccc(N4CCN(C)CC4)c(NC(=O)c4c[nH]c(=O)cc4C(F)(F)F)c3)cc2)C(C)(C)C)cc1</smiles>

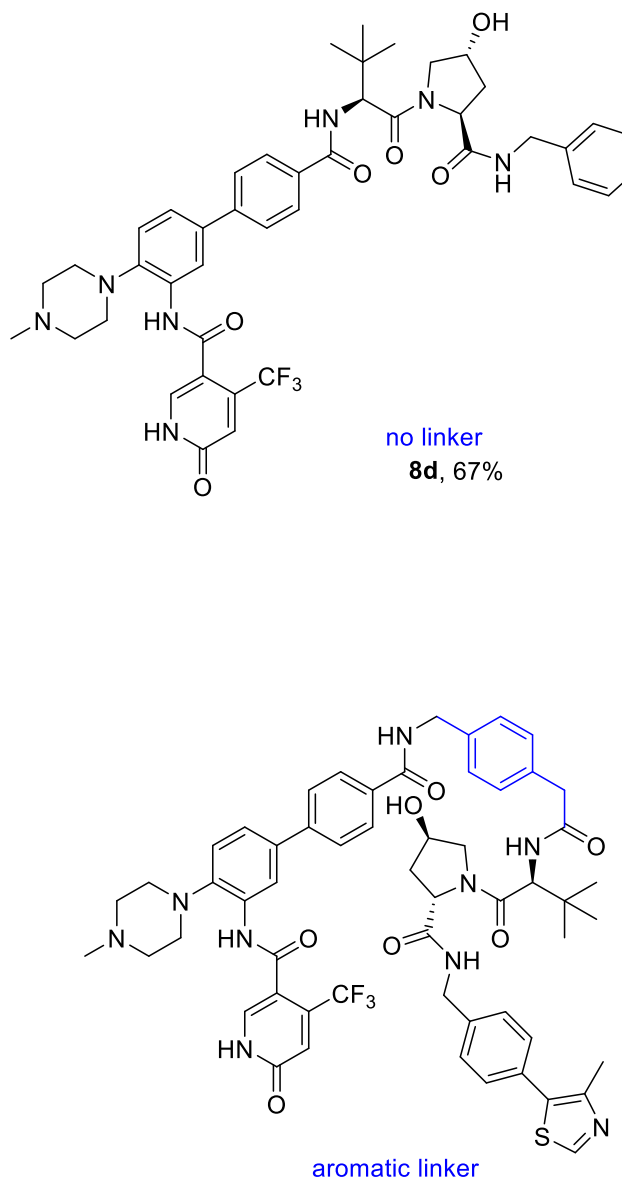<smiles>Cc1ncsc1-c1ccc(CNC(=O)[C@@H]2C[C@H](O)CN2C(=O)[C@H](NC(=O)[C@H](C)NC(=O)c2ccc(-c3ccc(N4CCN(C)CC4)c(NC(=O)c4c[nH]c(=O)cc4C(F)(F)F)c3)cc2)C(C)(C)C)cc1</smiles>

$8 \mathbf{8 j}, 30 \%$

Scheme 12: Chemical structures and obtained yield of WDR5 degraders (8a-j) addressing the E3 ligase VonHippel-Lindau (VHL). The synthesis steps start from intermediate (6d). The type and nature of the linker is indicated in blue.

For the synthesis of the MDM2 targeting PROTACs (9a-c), the synthesis route was changed as the MDM2 ligand Idasanutlin is very expensive. Thus, the linker was directly attached to ligand (6d) and then coupled to idasanutlin in an amide formation reaction, as shown in Scheme 13. The obtained $[\mathrm{PEG}]_{1}$-conjugated intermediate (6e) was obtained in a good yield and high purity. The more polar intermediate $(\mathbf{6 g})$ showed a lower yield. The low yield of intermediate $(\mathbf{6 f})$ is likely due 
to the reduced reactivity due to steric constraints. After isolating the intermediates, all three molecules were coupled to the carboxylic acid function of Idasanutlin. As Idasanutlin itself is a heavyweighted molecule, the coupling reaction was hampered by the steric constraints. Due to the incomplete reaction profile, the three MDM2-addressing PROTACs were isolated in low yields, but showed a satisfying purity (>95\%). Further reaction optimizations should focus on increasing the electrophilicity of Idasanutlin by attempting different coupling reagents.

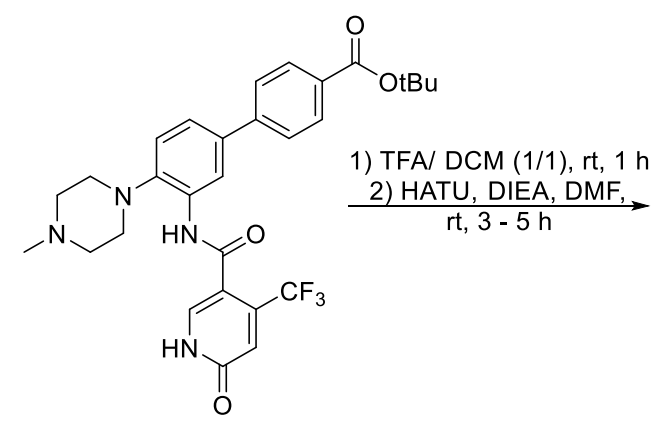

6d<smiles>CN1CCN(c2ccc(-c3ccc(C(=O)NCCOCCNC(=O)OCc4ccccc4)cc3)cc2C(=O)c2c[nH]c(=O)cc2C(F)(F)F)CC1</smiles>

$\mathrm{n}=1$ 6e, $73 \%$ $\mathrm{n}=7 \quad 6 \mathrm{f}, \quad 29 \%$ and<smiles>CN1CCN(c2ccc(-c3ccc(C(=O)NCc4ccc(CNC(=O)OC(C)(C)C)cc4)cc3)cc2NC(=O)c2c[nH]c(=O)cc2C(F)(F)F)CC1</smiles>

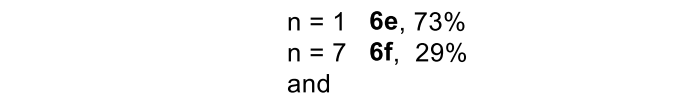

$6 g, 54 \%$

1) $T F A / D C M(1 / 1), r t, 1 \mathrm{~h}$ 2) Idasanutlin,<smiles>COc1cc(C(=O)NCCOCCNC(=O)c2ccc(-c3ccc(N4CCN(C)CC4)c(NC(=O)C(C)C(F)(F)F)c3)cc2)ccc1NC(=O)[C@@H]1N[C@@H](CC(C)(C)C)[C@](C#N)(c2ccc(Cl)cc2F)[C@H]1c1cccc(Cl)c1F</smiles><smiles>Cc1c[nH]c(=O)cc1C(F)(F)F</smiles>

PEG linker chain

$\mathrm{n}=1 \quad 9 \mathrm{a}, 12 \%$

$\mathrm{n}=7 \quad 9 \mathrm{~b}, 25 \%$

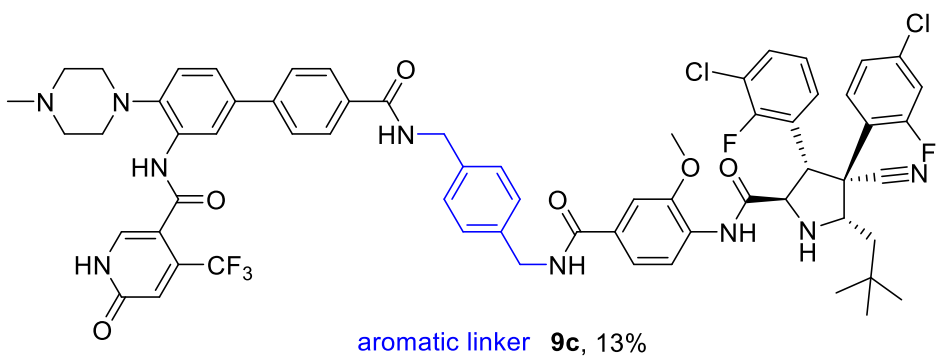

Scheme 13: Synthesis route of WDR5 degraders (9a-c) addressing the E3 ligase MDM2. The synthesis steps start from intermediate (6d). The type and nature of the linker is indicated in blue. 
In adittion to the PROTACs (7a-e), (8a-j) and (9a-c), which used ligand (6d), degraders based on ligands $(\mathbf{6 a})$ and $(\mathbf{6 c})$ were also to be synthesised, but this failed due to difficulties in purification. It is likely that the two reactive position on the indole ring - the nitrogen atom in 1-position as well as the carbon atom in 3-position - performed a nucleophilic attack of the E3 ligase linker active ester, thereby generating two different linked PROTACs in low yields. These experiments have been discarded.

All in all, a subset of heterobifunctional molecules was synthesized successfully. The generated PROTACs covered various linker length and types which is crucial for degradational activity. Also, a variation of addressing E3 ligase ligands was imbedded into the synthesis. Thus, the 18 generated PROTACs allow a structure-activity-relationship (SAR) study on WDR5 degradation.

\subsection{Synthesis of pyrroloimidazole derived degraders $(16 \mathrm{a}, \mathrm{b})$ and $(20 \mathrm{a}-\mathrm{g})$}

This chapter briefly summarizes the synthesis efforts of two master theses by Dominic Löw and Janik Weckesser. The synthetic schemes for generating pyrroloimidazole based molecules addressing either CRBN or VHL were slightly modified from the work of Wang et al., ${ }^{167}$ and are shown in Scheme 14 and Scheme 15. Analogous to the published synthesis, both synthesis routes started with educt (10) that underwent a Vilsmeier-Haack-like intramolecular cyclisation to form aryl bromide (11). Intermediate (11) reacted with (3-cyano-4-methoxyphenyl)boronic acid in a Suzuki-Miyaura cross coupling to give the biaryl system (12). Reduction of the nitrile group led to amine (13) which was then coupled with the according carboxylic acid to yield the amide (14). The mild ether deprotection of intermediate (14) by Borontribromide led to the free phenol (15). To generate the CRBN-addressing PROTACs, the E3 ligase linkers with a free hydroxy moiety were connected via a Mitsunobu reaction, resulting in the products $(\mathbf{1 6 a}, \mathbf{b})$. 


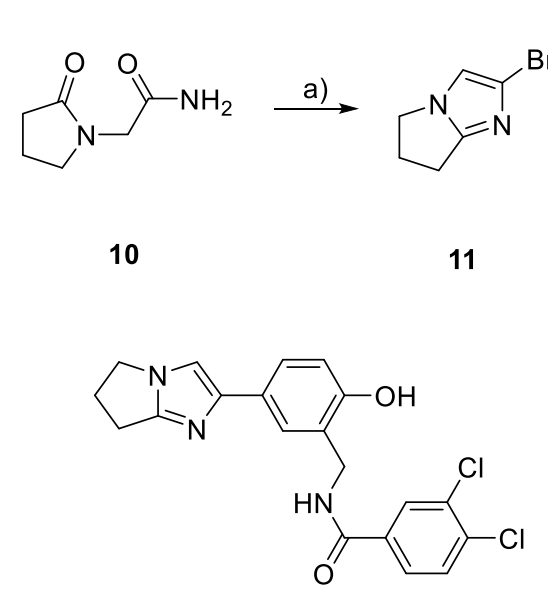

15 $\stackrel{\text { b) }}{\longrightarrow}$<smiles>COc1ccc(-c2cn3c(n2)CCC3)cc1C#N</smiles>

12

14<smiles>COc1ccc(-c2cn3c(n2)CCC3)cc1CNC(=O)c1ccc(Cl)c(Cl)c1</smiles><smiles>COc1ccc(-c2cn3c(n2)CCC3)cc1CN</smiles>

13

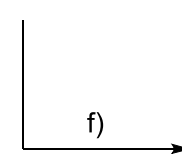<smiles>O=C1CCC2C(=O)C(=O)c3c(NCCCCCCOc4ccc(-c5cn6c(n5)CCC6)cc4CNC(=O)c4ccc(Cl)c(Cl)c4)cccc3C(=O)N2C1</smiles>

$16 a$

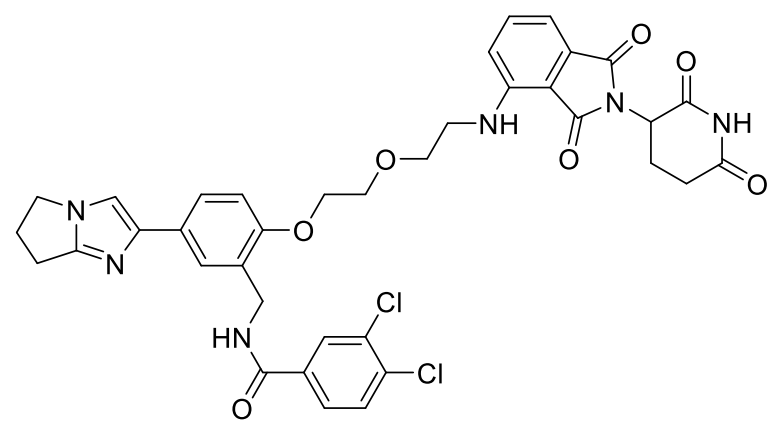

$16 b$

Scheme 14: Synthesis of WDR5 degraders $(\mathbf{1 6 a}, \mathbf{b})$ addressing the $\mathrm{E} 3$ ligase $\mathrm{CRBN}$ : a) $\mathrm{POBr}_{3}, \mathrm{ACN}, \mathrm{MW}, 70^{\circ} \mathrm{C}$, $2 \mathrm{~h}$; b) (3-cyano-4-methoxyphenyl)boronic acid, XPhos PdG3, NaOH, THF/ $\mathrm{H}_{2} \mathrm{O}, 80^{\circ} \mathrm{C}, 21 \mathrm{~h} ; \mathrm{c}$ ) LiAlH $\mathrm{H}_{4} \mathrm{THF}$, $60{ }^{\circ} \mathrm{C}$ - rt, 21 h; d) 3,4-dichlorobenzoic acid, HATU, DIEA, DMF, rt, 16 h; e) 1. $\mathrm{BBr}_{3}, \mathrm{CH}_{2} \mathrm{Cl}_{2},-78{ }^{\circ} \mathrm{C}-\mathrm{rt}, 21 \mathrm{~h} ; 2$. $\mathrm{NaOH} / \mathrm{H}_{2} \mathrm{O}$; f) Linker-OTs, $\mathrm{K}_{2} \mathrm{CO}_{3}, \mathrm{DMF}, 70^{\circ} \mathrm{C}, 16-22.5 \mathrm{~h}$ or DEAD, $\mathrm{PPh}_{3},-10{ }^{\circ} \mathrm{C}$ to $\mathrm{rt}, 16 \mathrm{~h}$.

Beside the two CRBN-addressing PROTACs $(\mathbf{1 6 a , b})$, PROTACs with the pyrroloimidazole-based scaffold addressing the VHL E3 ligase were additionally synthesized. For the synthesis of the VHL addressing PROTACS, the previously described amine (13) was coupled to 2-(3,4dichlorophenyl)acetic acid to form intermediate (17). The methoxy ether of intermediate (17) was cleaved under mild conditions to the corresponding phenol (18) and was then reacted in a nucleophilic substitution reaction with the tosylated linker derivatives to yield intermediates (19a- 
g). The deprotection of the tert-butyl ester (19a-g) was carried out in an acidic environment, and a subsequent amide bond formation with the VH032 yielded the VHL-based PROTACs (20a-g).<smiles>COc1ccc(-c2cn3c(n2)CCC3)cc1CNC(=O)Cc1ccc(Cl)c(Cl)c1</smiles><smiles>CCCCCCCCOCCOc1ccc(-c2cn3c(n2)CCC3)cc1CNC(=O)Cc1ccc(Cl)c(Cl)c1</smiles><smiles>O=C(Cc1ccc(Cl)c(Cl)c1)NCc1cc(-c2cn3c(n2)CCC3)ccc1O</smiles>
18
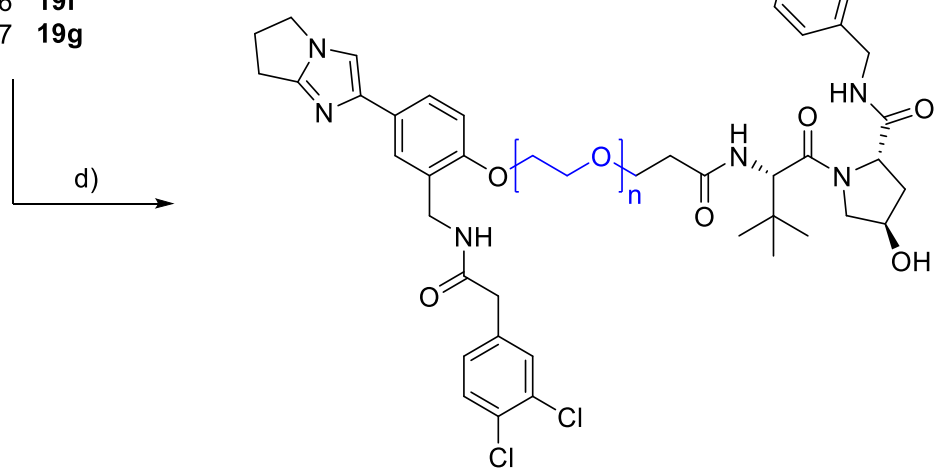

$$
\begin{array}{cl}
\text { PEG linker chain } \\
n=1 & 20 a \\
n=2 & 20 b \\
n=3 & 20 c \\
n=4 & 20 d \\
n=5 & 20 \mathrm{e} \\
n=6 & 20 f \\
n=7 & 20 g
\end{array}
$$

Scheme 15: Synthesis of WDR5 degraders (20a-g) addressing the E3 ligase VHL: a) 2-(3,4dichlorophenyl)acetic acid, EDC, $\mathrm{HOBt}$, DIEA, DMF, rt, 16 h; b) $1 . \mathrm{BBr}_{3}, \mathrm{CH}_{2} \mathrm{Cl}_{2},-78{ }^{\circ} \mathrm{C}-\mathrm{rt}, 21 \mathrm{~h} ; 2 . \mathrm{NaOH} / \mathrm{H}_{2} \mathrm{O}$; c) Linker-OTs, $\mathrm{K}_{2} \mathrm{CO}_{3}, \mathrm{DMF}, 70{ }^{\circ} \mathrm{C}, 16-22.5 \mathrm{~h}$; d) 1 . TFA $/ \mathrm{CH}_{2} \mathrm{Cl}_{2}(1 / 1), \mathrm{rt}, 1.5 \mathrm{~h}$; $2 . \mathrm{HATU}, \mathrm{DIEA}, \mathrm{DMF}, \mathrm{VHL}$ hydrochloride linker, rt, 3-18 h. The type and nature of the linker is indicated in blue.

The chemical and biological results have already been discussed and published in two Master theses. Thus, the pyrroloimidazole scaffold molecules (20a-g) are discussed here only as references. 


\subsection{Biophysical Evaluation of WDR5 PROTACs (7a-e), (8a-j) and (9a-c)}

\section{In vitro characterization of PROTAC molecules}

To gain a first insight into the binding affinity of the synthesized PROTACs to WDR5, differential scanning fluorimetry (DSF) measurements were performed. The obtained temperature shifts $\Delta T_{m}$ are listed in Table 3. The small molecule antagonist (OICR-9429) was used as a reference and assay validation, as previously described in the literature. ${ }^{83}$ Beside DMSO that served as reference compound, the inhibitors Idasanutlin, VH032 and a modified thalidomide were included in the experiments. All E3 ligase ligands alone did not result in significant temperature shifts of WDR5, suggesting that the E3 ligase moieties itself do not bind to the protein-of-interest. The comparison of intermediates of the MDM2 targeting PROTACs (6e-g) showed a substantial decrease in temperature shifts: while the parent compound $(6 \mathrm{~d})$ showed an initial $\Delta T_{m}$ shift of more than $20 \mathrm{~K}$, the $[P E G]$ modified intermediates $(6 \mathrm{e}-\mathrm{g})$ only achieved a $\Delta T_{m}$ value of $4-5 \mathrm{~K}$. It is likely that the bulky tert-Butylester of ligand (6d) performs additional interactions on the hydrophobic surface of WDR5 which in turn gets lost by linker attachment. In general, the $\Delta T_{m}$ data of the measured PROTACs correlated with the nature of the introduced linker: mostly aliphatic-bridged PROTACs (8e-i) showed weaker thermal stabilization compared to more polar and thus more soluble [PEG]bridged PROTACs like (8a) or (8c). Comparison of similar heterobifunctional molecules that have the same linker chain but address different E3 ligases, e.g. (7a), (8a) and (9a), showed that the shortest $[\mathrm{PEG}]$-linker $\left([\mathrm{PEG}]_{1}\right)$ resulted in higher melting temperature shifts than PROTACs containing longer [PEG] chains. Many PROTACs displayed a $\Delta T_{m}$ shift similar to the (OICR-9429) ligand and thus indicated to be potent in vitro binders. Only the MDM2 addressing PROTACs (9ac) lost their affinity towards WDR5, possibly due to their large size and associated less favorable physiochemical properties or steric constrains.

Table 3: Thermal shift experiments of OICR-9429 derived molecules (6d-g), degraders (7a-e), (8a-j), (9a-c) and the negative controls (nc_VHL) and (nc_WDR5). $\Delta T_{m}$ : thermal shift change; SD: standard deviation. As negative controls, DMSO, Thalidomide, Idasanutlin and VH032 were used. As positive control OICR-9429 was used. Table adapted with permission from "Design, Synthesis and Evaluation of WD40-repeat containing protein 5 (WDR5) Degraders by Dölle, Adhikari et al. ${ }^{174}$ Copyright 2021 American Chemical Society.

\begin{tabular}{c|cccc}
\multicolumn{1}{c}{ ID } & Linker & E3 Ligase & $\Delta T_{m}[K]^{\mathrm{a}}$ & SD $[\mathrm{K}]^{\mathrm{a}}$ \\
\hline DMSO & - & - & 0.0 & 0.3 \\
OICR-9429 & - & - & 13.3 & 0.1
\end{tabular}


42 Results and Discussion

Table 3. (continued)

\begin{tabular}{|c|c|c|c|c|}
\hline VH032 & - & VHL & -0.4 & 0.1 \\
\hline Thalidomide & - & CRBN & 0.0 & -0.1 \\
\hline Idasanutlin & - & MDM2 & -0.2 & 0.1 \\
\hline $6 d$ & - & - & 20.8 & 0.6 \\
\hline $6 e$ & & - & 5.7 & 0.4 \\
\hline $6 f$ & & - & 4.1 & 0.6 \\
\hline $6 g$ & & - & 4.2 & 0.7 \\
\hline $7 a$ & & CRBN & 13.6 & 0.2 \\
\hline $7 b$ & & CRBN & 12.7 & 0.3 \\
\hline $7 c$ & & CRBN & 9.0 & 0.5 \\
\hline $7 d$ & & CRBN & 11.9 & 0.3 \\
\hline $7 e$ & & CRBN & 12.5 & 0.6 \\
\hline $8 a$ & & VHL & 15.3 & 0.2 \\
\hline $8 b$ & & VHL & 7.7 & 0.5 \\
\hline $8 c$ & & VHL & 12.5 & 0.2 \\
\hline $8 d$ & & VHL & 15.6 & 0 \\
\hline $8 e$ & & VHL & 11.0 & 0.3 \\
\hline $8 f$ & & VHL & 10.4 & 0.7 \\
\hline $8 g$ & & VHL & 13.2 & 0.1 \\
\hline $8 \mathrm{~h}$ & & VHL & 9.7 & 2.8 \\
\hline $8 i$ & & VHL & 3.5 & 0.4 \\
\hline $8 j$ & & VHL & 14.0 & 0 \\
\hline
\end{tabular}


Table 3. (continued)

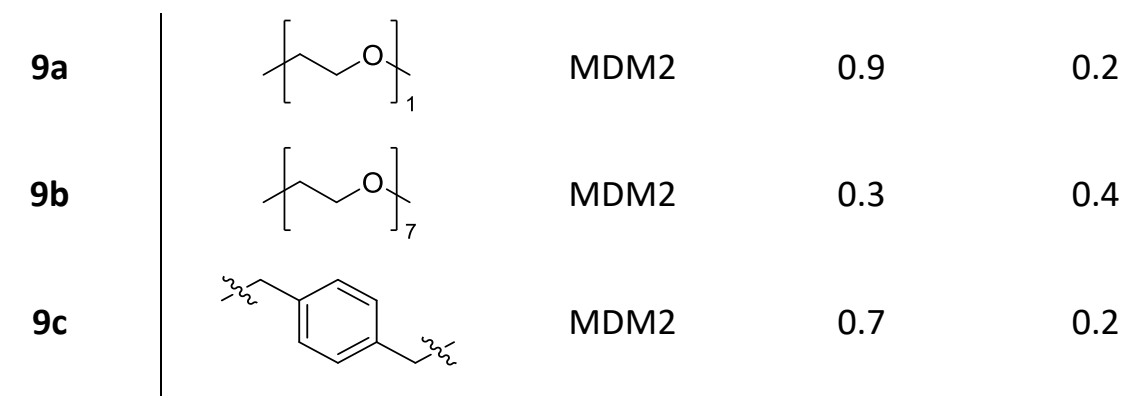

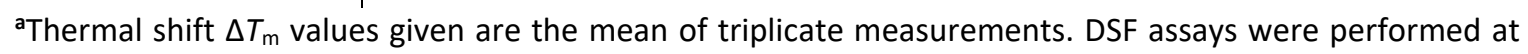
$2 \mu \mathrm{M}$ protein concentration and a final compound concentration of $10 \mu \mathrm{M}$.

Since the DSF assay only indicates a binding tendency and cannot state a correct binding affinity $\left(K_{D}\right)$ value, the binding affinities of the synthesized PROTACs (7a), $(8 \mathrm{a})$ and $(\mathbf{8 e - j})$ to WDR5 were determined by isothermal titration calorimetry (ITC). This biophysical method detects the released or consumed heat along a binding event where the ligand interacts with the protein under a constant temperature. The biophysical properties are listed in Table 4 and titration curves of aliphatic-bridged PROTACs $(\mathbf{8 g}, \mathbf{i})$ are shown in Figure 13. All other binding curves can be found in the Experimental Chapter 7. The CRBN-based PROTAC (7a) as well as the VHL-based PROTAC (8a) were chosen for ITC measurements due to their identical linker structure, their comparably low molecular weight and because they displayed the highest $\Delta T_{m}$ value within their cluster. The comparison showed that binding affinity did not correlate well with $\Delta T_{m}$ shifts, as the CRBNaddressing PROTAC (7a), which showed less thermal stabilization, had a three times higher affinity for WDR5 than the highly thermal stabilized VHL-addressing PROTAC (8a). Also, the DSF measurements revealed that PROTACs (8e-i) showed an unexpected variety of thermal stabilization with $\Delta T_{m}$ shifts ranging from $3.5 \mathrm{~K}$ to $13 \mathrm{~K}$. These ligands were therefore chosen for further characterization by ITC. All examined PROTACs (8e-i) showed binding affinities in a low nanomolar range and did not correlate well with the $\Delta T_{m}$ data. A decrease in solubility was observed for degraders with long aliphatic linker (especially (8h) and (8i)) that made data collection by ITC challenging. This effect also observed in the titration curves that showed nearly an instant saturation (see titration curve of (8i) in Figure 13.) Several experimental parameters were changed to obtain titration curves of a higher quality, but nor the use of higher concentrations of PROTAC, neither the reduced injection volume of WDR5 protein resulted in improved titration curves. The limited solubility of these PROTACs complicated the comparison of thermodynamic parameters, and so the thermodynamic data measured on PROTACs containing longer aliphatic and aromatic linkers such as $(\mathbf{8 h}),(\mathbf{8 i})$ and $(\mathbf{8 j})$ have to be treated carefully. Despite 
all these technical challenges, all heterobifunctional molecules showed excellent affinity to WDR5 in the single- or double-digit nanomolar $K_{d}$ range.
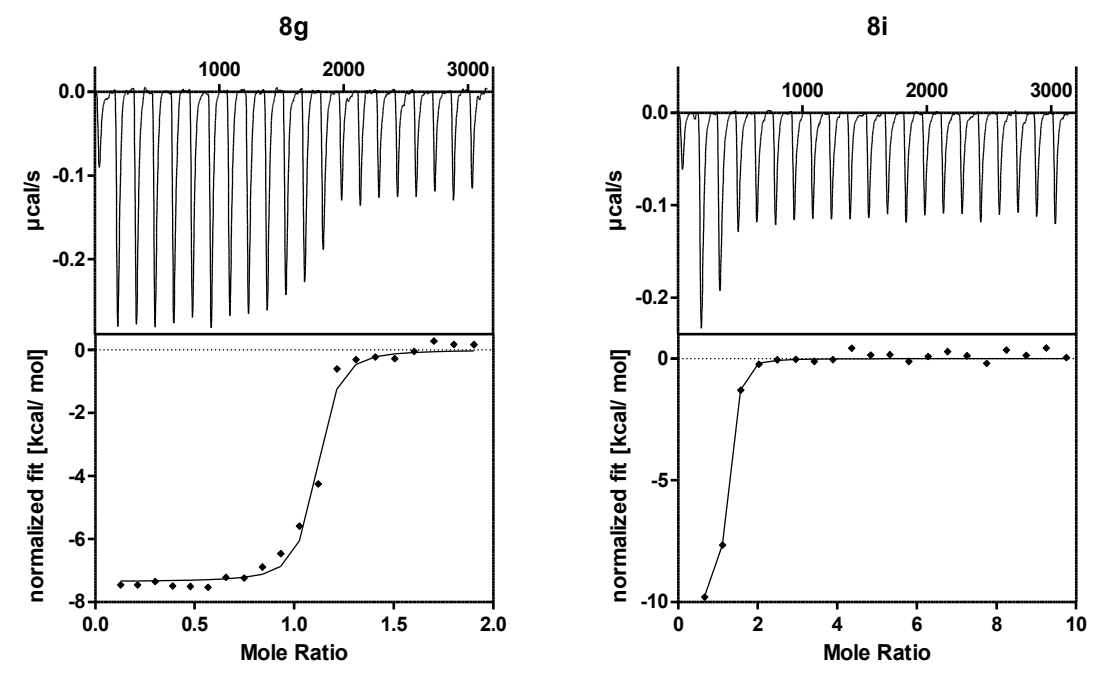

Figure 13: ITC curves for VHL-addressing PROTACs $(\mathbf{8 g})$ and $(\mathbf{8 i})$. The titration curve of $(\mathbf{8 i})$ shows the limiting solubility of the hexyl-bridged PROTAC within the ITC experiments.

The analysis of the thermodynamic properties of the examined PROTACs in Table 4 revealed large negative binding enthalpies (-10 to $-4.9 \mathrm{kcal} / \mathrm{mol})$. Beside PROTAC (8a) that showed a negative entropy change $(T \Delta S)$ of $-0.2 \mathrm{kcal} / \mathrm{mol}$, all other entropy changes were favourable, ranging from +0.7 to $+4.5 \mathrm{kcal} / \mathrm{mol}$. Furthermore, enthalpy-entropy compensation was observed for PROTACs with large favourable $\Delta \mathrm{H}$ values, as shown for molecules (8a), (8f) and (8i): all compounds possessed large negative binding enthalpies of $-10 \mathrm{kcal} / \mathrm{mol}$ with close to zero entropy changes (0.2 to $1.1 \mathrm{kcal} / \mathrm{mol}$ ). On the other hand, compounds like (8e) that displayed large positive entropy changes $(+4.5 \mathrm{kcal} / \mathrm{mol})$ showed small binding enthalpy $(\Delta \mathrm{H}=-6.3 \mathrm{kcal} / \mathrm{mol})$. These thermodynamic compensation mechanisms might be possibly due to water displacement as the polar $[\mathrm{PEG}]_{1}$ linker of PROTAC (8a) showed an unfavourable binding entropy change while the aliphatic linker bridged PROTACs showed an increased binding entropy term. 
Table 4: Thermodynamic properties of OICR-9429 derived molecule (6d) and degraders (7a), (8a), (8e-j). $K_{D}$ : dissociation constant; SD: standard deviation; $\mathrm{n}$ : stoichiometry; $\Delta \mathrm{H}$ : enthalpy change $\Delta \mathrm{S}$ : entropy change. Table adapted with permission from "Design, Synthesis and Evaluation of WD40-repeat containing protein 5 (WDR5) Degraders by Dölle, Adhikari et al. ${ }^{174}$ Copyright 2021 American Chemical Society.

\begin{tabular}{c|ccccc} 
ID & $\mathbf{K}_{\mathrm{D}}[\mathrm{nM}]^{\mathrm{a}}$ & $\mathbf{S D}[\mathrm{nM}]^{\mathrm{a}}$ & $\mathbf{n}$ & $\Delta \mathbf{H}[\mathrm{kcal} / \mathrm{mol}]$ & $\mathbf{T} \Delta \mathbf{S}[\mathrm{cal} / \mathrm{mol} \cdot \mathbf{K}]$ \\
\hline $\mathbf{6 d}$ & 25 & 6 & 1.0 & -9.4 & 0.8 \\
$\mathbf{7 a}$ & 12 & 4 & 1.0 & -8.1 & 2.6 \\
$\mathbf{8 a}$ & 41 & 9 & 1.0 & -10 & -0.2 \\
$\mathbf{8 e}$ & 9 & 2 & 1.1 & -6.3 & 4.5 \\
$\mathbf{8 f}$ & 6 & 2 & 1.0 & -10 & 1.1 \\
$\mathbf{8 g}$ & 18 & 5 & 1.1 & -7.4 & 3.2 \\
$\mathbf{8 h}$ & 12 & 4 & 1.0 & -9.3 & 1.4 \\
$\mathbf{8 i}$ & 11 & 3 & 1.1 & -10 & 0.7 \\
$\mathbf{8 j}$ & 33 & 5 & 1.0 & -7.9 & 2.2
\end{tabular}

${ }^{a} K_{d}$ values were derived from ITC measurements (carried out as duplicate, except for (8i) that was measured in a single measurement) and calculated by assuming a sigmoidal dose-response relationship (four parameters). The errors of the fits were calculated using standard deviation and a confidence interval of $68 \%$.

Summed up, the gained thermodynamic data gave a small insight into the binding mechanism of the different PROTACs and stated the high in vitro binding affinity of these large molecules to WDR5. Beside the determination of the binding affinity, the ITC measurements highlighted the value of using orthogonal assay systems. The two assays showed that similar $\Delta T_{m}$ shifts resulted in some cases in very different binding affinities. Another finding was that PROTACs with high $\Delta T_{m}$ shift were not necessarily more affine than PROTACs with a moderate $\Delta T_{m}$ shift. This observation was also observed for the pyrroloimidazole-based inhibitor (17) and PROTAC (20b) that showed a substantial lower $\Delta T_{m}$ shift of around $4-5 \mathrm{~K}$, but the binding affinity measured by ITC was still in a two- to three-digit nanomolar range.

\section{In cellulo characterization of PROTAC molecules}

Due to the characteristic large molecular weight of heterobifunctional molecules, cellular permeability can be limiting factor for PROTACs. To address this question, BRET (Bioluminescence Resonance Energy Transfer) experiments were performed to determine the cellular permeability 
and potency $\left(\mathrm{IC}_{50}\right)$ of the synthesized PROTACs. The biophysical technique of the proximity-based assay relies on the naturally occurring dipole-dipole energy transfer from a donor enzyme to an acceptor fluorophore. If both interacting partners are in close proximity (less than $10 \mathrm{nM}$ ), light emission from the acceptor results in a quantifable signal. ${ }^{175}$ The herein developed BRET assay monitored the luminescence of an engineered Nanoluciferase-tagged WDR5 system. N-terminally and C-terminally tagged WDR5 NanoLuc fusion constructs were transfected to HEK293 cells, resulting in an overexpression of the luminescence energy donor. A small molecule-based tracer served as flourescence acceptor. To establish this assay, three BODIPY fluorescent tracer molecules (21a-c) based on WDR5 ligand (6d) were synthesized. The synthetic procedures of the tracer molecules (21a-c) via their intermediates $(\mathbf{6 e}, \mathbf{h}, \mathbf{i})$ are shown in Scheme 16 . The amide coupling of the [PEG] linker chains to intermediates resulted in overall good yields, and subsequent coupling with the $\mathrm{N}$-succinimide activated $\mathrm{N}$-succinimide activated BODIPY dye also resulted in good yields of the final tracer molecules. Due to the large molecular weight and the high solubility of the $[\mathrm{PEG}]_{9}$-bridged tracer (21b) in the aqueous phase, a lower yield was obtained. 
<smiles>CN1CCN(c2ccc(-c3ccc(C(=O)OC(C)(C)C)cc3)cc2NC(=O)c2c[nH]c(=O)cc2C(F)(F)F)CC1</smiles>

6d<smiles></smiles>

NanoBRET ${ }^{\mathrm{TM}}$ Tracer SE590<smiles>CCOCCNC(=O)c1ccc(-c2ccc(N3CCN(C)CC3)c(NC(=O)c3c[nH]c(=O)cc3C(F)(F)F)c2)cc1</smiles>

$\mathrm{n}=1 \quad \mathbf{6 e}, 73 \%$ $\mathrm{n}=9$ 6h, $89 \%$ and<smiles>CN1CCN(c2ccc(-c3ccc(C(=O)NCCCOCCCOC(C)(C)C)cc3)cc2NC(=O)c2c[nH]c(=O)cc2C(F)(F)F)CC1</smiles>

6i, $78 \%$

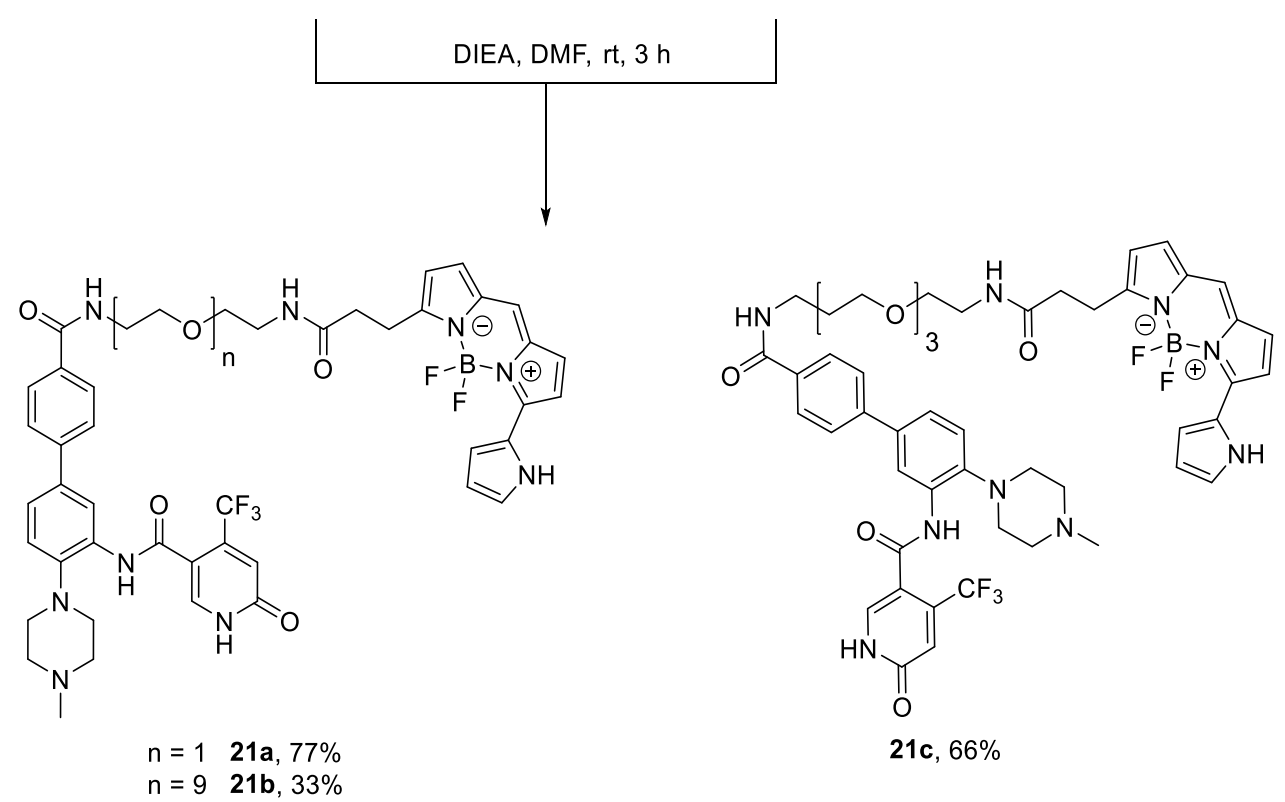

Scheme 16: Synthesis of NanoBRET ${ }^{\mathrm{TM}}$ tracer molecules (21a-c) via intermediates $(\mathbf{6 e}, \mathbf{h}, \mathbf{i})$.

In order to determine the best assay system, the generated tracer molecules (21a-c) were titrated into HEK293 cells containing the engineered WDR5 NanoLuc fusion constructs. These experiments revealed that the $[\mathrm{PEG}]_{3}$ bridged tracer (21c) and the C-terminally tagged Nanoluc-WDR5 construct as most suitable combination for cellular BRET assays, as shown in Figure 14. 
a)

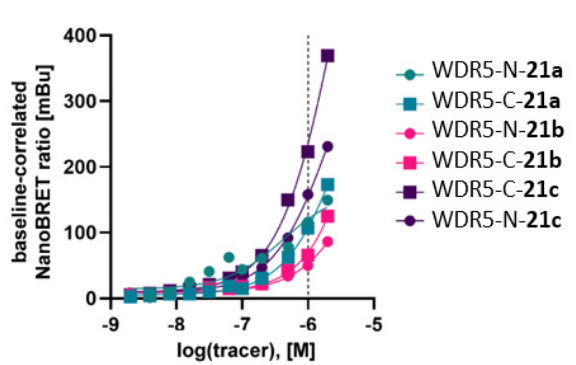

b)

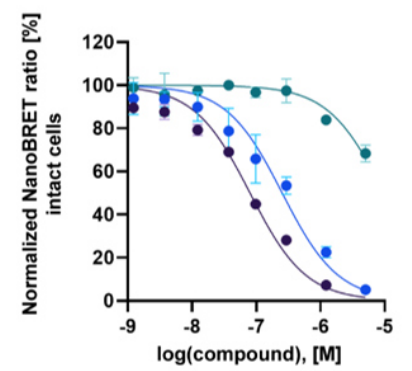

c)

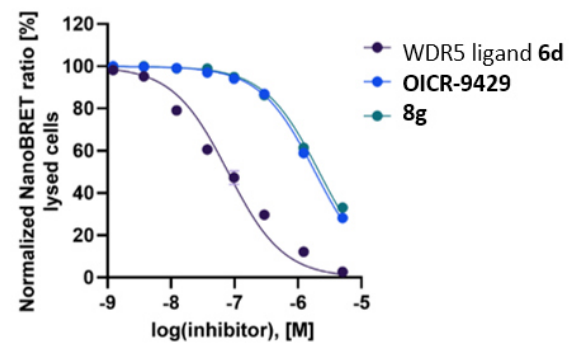

Figure 14: Cellular permeability and target engagement studies were performed with the BRET assay. (a) Tracer titration of all three synthesized tracer molecules and either $\mathrm{C}$-terminal or $\mathrm{N}$-terminal Nanoluctagged WDR5 (WDR5-C or WDR5-N). (b) and (c) NanoBRET ${ }^{\text {TM }}$ dose response curves of PROTAC (8g), WDR5 ligand (6d) and reference compound OICR-9429 in (b) intact cells as well as in (c) lysed cells. Figure reprinted (adapted) with permission from "Design, Synthesis and Evaluation of WD40-repeat containing protein 5 (WDR5) Degraders by Dölle, Adhikari et al. ${ }^{174}$ Copyright 2021 American Chemical Society.

The following BRET measurements of all degraders were performed in lysed as well as in living cells. The different assay formats were used to compare cell penetration as well as cellular affinity towards WDR5, and the obtained data of the BRET measurements are shown in Table 5. The experiments were carried out three times as duplicate measurements, and within the measurements itself, a wide shift/ variety of the binding curves was observed. For example, the reference compound OICR-9429 showed in all three measurements different binding affinities, ranging from three-digit $\mathrm{nM}$ to single-digit $\mu \mathrm{M}$ values, although a cellular potency of $1 \mu \mathrm{M}$ has been reported in literature. ${ }^{83}$ Thus, the obtained data of the PROTACs rather indicate an affinity interval than fixed values. Due to the catalytic mechanism of action of PROTAC molecules, the binary affinity to the protein-of-interest is negligible and efficient degradation for moderate affine PROTACs has been described in literature. ${ }^{122}$

A significant decrease in affinity was observed when parent compound (6d) was elongated to the final PROTACs(7a-e), (8a-j) and (9a-c). Significant changes in affinity could be observed by comparing the attached E3 ligase ligands and the nature of the linker: most Cereblon (CRBN) targeting PROTACs (7a-e) displayed similar cellular potencies in both assay formats. VHL targeting PROTACs (8a-j) showed weaker cellular activity, possibly due to the peptide like nature of the ligand. Interestingly, an affinity determination for all VHL addressing PROTACs, no matter of the linker nature, could be achieved in lysed cells (only the aliphatic linker containing PROTAC (8g) showed a two-digit $\mu \mathrm{M}$ affinity in the intact cellular experiment). Comparing all linker variants of the VHL-addressing PROTACs - the aliphatic-bridged PROTACs (8e-i), the [PEG]-bridged variants $(\mathbf{8 a}-\mathbf{c})$, the directly linked degrader $(\mathbf{8 d})$ and the aromatic linked molecule $(\mathbf{8 j})$ - indicated that the 
weak solubility might be the limiting factor for this observation. Furthermore, the limiting solubility has already been a limiting factor in the ITC experiments. Regarding the MDM2 addressing PROTACs (9a-c), the BRET measurements confirmed the weak in cellulo activity of these molecules to WDR5, as indicated in vitro in the DSF assay. Summed up, the MDM2 targeting degraders were excluded from further experiments.

Table 5. In cellulo data of WDR5 antagonist OICR-9429, the modified inhibitor (6d) and degraders (7a-e), (8a-j) and (9a-c). I $C_{50}$ : cellular binding affinity; SD: Standard deviation. Table adapted with permission from "Design, Synthesis and Evaluation of WD40-repeat containing protein 5 (WDR5) Degraders by Dölle, Adhikari et al. ${ }^{174}$ Copyright 2021 American Chemical Society.

\section{NanoBRET ${ }^{\mathrm{TM}}$}

\begin{tabular}{|c|c|c|c|c|}
\hline ID & Linker & E3 ligase & $\begin{array}{c}I_{50} \pm S D \\
{[\mu \mathrm{M}]^{\mathrm{a}}}\end{array}$ & $\begin{array}{c}I_{50} \text { lysate } \pm \text { SD } \\
{[\mu \mathrm{M}]^{\mathrm{a}}}\end{array}$ \\
\hline OICR-9429 & - & - & $0.31 \pm 0.06$ & $1.85 \pm 0.07$ \\
\hline $6 d$ & & - & $0.14 \pm 0.03$ & $0.08 \pm 0.01$ \\
\hline $7 a$ & & CRBN & $4.30 \pm 1.17$ & $0.99 \pm 0.11$ \\
\hline $7 b$ & & CRBN & $4.18 \pm 0.53$ & $1.22 \pm 0.04$ \\
\hline $7 c$ & & CRBN & $14.1 \pm 0.2$ & $2.87 \pm 0.01$ \\
\hline $7 d$ & & CRBN & $4.92 \pm 0.98$ & $1.05 \pm 0.03$ \\
\hline $7 e$ & & CRBN & $1.18 \pm 0.12$ & $0.85 \pm 0.02$ \\
\hline $8 a$ & & VHL & $8.65 \pm 0.43$ & $0.74 \pm 0.04$ \\
\hline
\end{tabular}


Table 5. (continued)

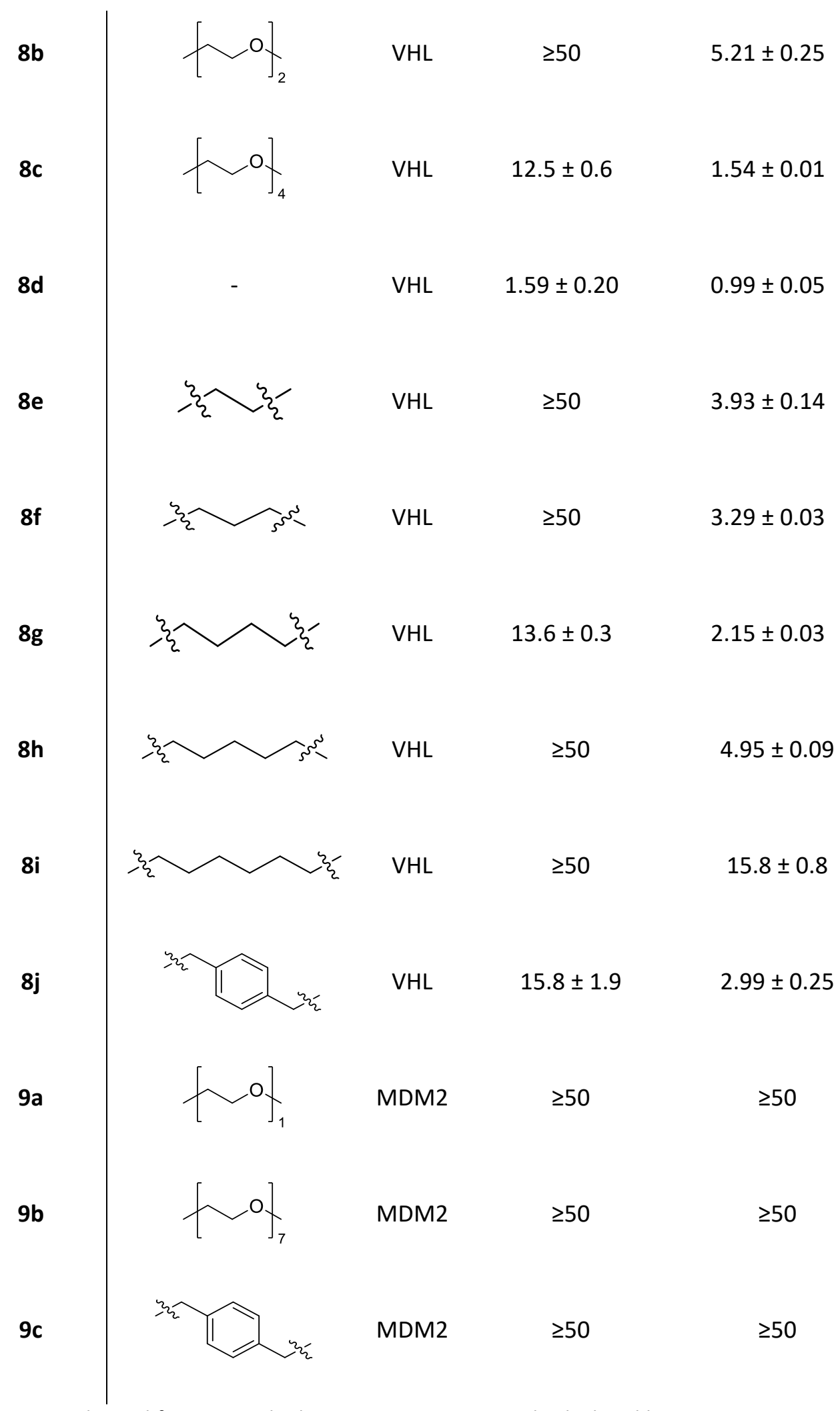

${ }^{\mathrm{a}} \mathrm{C}_{50}$ values were derived from BRET duplicate measurements and calculated by assuming a normalized 3parameter curve fit; n.d.: not determined. 
Another limitation of the herein established BRET assay system was observed for the pyrroloimidazole based degraders (20a-g): the used tracer (21c) could not be displaced by the small molecules and thus, no cellular affinity could be determined. Nevertheless, the heterobifunctional molecule (20b) was able to induce degradation of WDR5, concluding that at least this PROTAC must be able to bind to WDR5 in cellulo and that the BRET assay system retains limitations. A possible hypothesis for the missing tracer displacement might be the different binding modes of the used inhibitor. In summary, the herein established BRET assay for WDR5 indicates that several degrader molecules are cell permeable and bind to WDR5 in cells and lysates, indicating that they might be suitable compounds for cellular usage.

\subsection{PROTAC-mediated degradation of cellular WDR5}

\section{HiBiT assay and Immunoblotting}

Besides cellular permeability and target engagement, PROTAC molecules need to mediate a stable ternary complex to induce protein degradation. The generated proximity between both proteins leads ideally to ubiquitylation of the target protein and subsequent proteasomal degradation. To analyze PROTAC-mediated degradation of WDR5 in cells, the open reading frame of WDR5 was fused with a luciferase peptide, called HiBiT, followed by a transfection into the AML cell line MV411 (MV4-11 ${ }^{\text {WDR5-HiBiT }}$ ) and co-transfected with the second part of the luciferase (Large Bit, LgBit). Similar to the previously described BRET assay, the emitting signal can be used to quantify the degradation rate. ${ }^{176}$ To compare the expression levels of WDR5-HiBiT to endogenously expressed WDR5, Immunoblotting was performed previously to PROTAC treatment and demonstrated similarity of both expression levels, as shown in Figure 15. Afterwards, the engineered MV4$11^{\text {WDRS-HiBiT }}$ cells were treated with various concentrations of the synthesized PROTACs for 24 hours and the depletion of WDR5-HiBiT was determined by measuring luciferase activity. Figure 15 shows that the depletion was achieved for numerous PROTACs. None of the Cereblon addressing PROTACs induced cellular degradation of WDR5, whereas the aliphatic and aromatic linker bridged molecules (8e-j) that recruited VHL E3 ligase resulted in a successful degradation. The observed depletion of WDR5-HiBiT varied greatly between the different degraders, resulting in different degradation efficacies and concentrations, as listed in Table 6. Within the effective PROTAC series, compound $(\mathbf{8 g})$ that linked both functional binding moieties by a butyl chain, showed a maximum depletion of $58 \pm 3 \%$ and $\mathrm{DC}_{50}$-values of $53 \pm 10 \mathrm{nM}$, indicating the catalytic nature that is observed by PROTACs. Within the HiBiT curves that are shown in Figure 15, the Hook effect was observed with increasing PROTAC concentrations. This behavior is due to binding site competition and thus 
less efficient ternary complex formation. Linker shortening or linker elongation lowered degradation efficacy to $20-30 \%$. The substitution of a carbon atom to an oxygen, like in the chemical structures of pentyl-bridged PROTAC (8h) and [PEG] ${ }_{1}$-bridged PROTAC (8a), resulted in a complete loss of degradational activity.
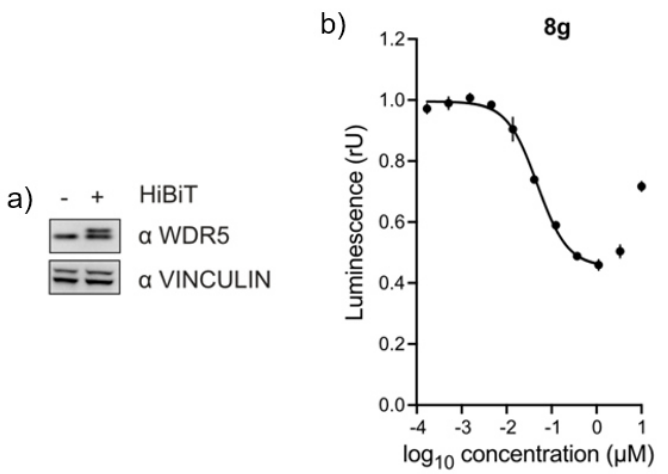
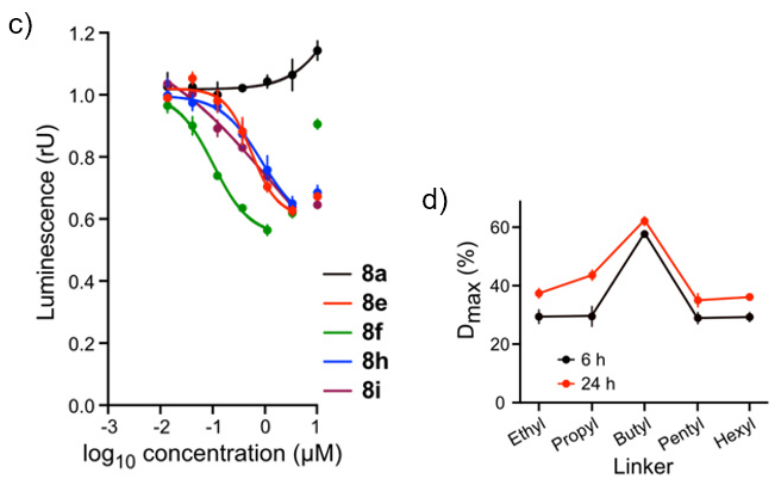

Figure 15: Cellular degradation studies on WDR5. (a) Immunoblot of HiBiT-tagged (+) and endogenous (-) WDR5 in MV4-11 cells. Vinculin was used as loading control. (b) WDR5 levels based on luciferase measurements. MV4-11 ${ }^{\text {WDR5-HiBiT }}$ cells were treated with different concentrations of $(\mathbf{8 g})$ for $24 \mathrm{~h}$. (c) WDR5 levels based on luciferase measurements. MV4-11 ${ }^{\text {WDR5-HiBiT }}$ cells were treated with different concentrations of degraders (8a), (8e), (8f), (8h) and (8i) for 24 h. (d) Quantification of WDR5 $D_{\max }$ (maximal degradation) from HiBiT assay for degraders with different aliphatic linkers. MV4-11 ${ }^{\text {WDR5-HiBiT }}$ cells were treated with different concentrations of degraders for $6 \mathrm{~h}$ or $24 \mathrm{~h}$ and measured for luciferase activity of linkers comprising ethyl to hexyl motifs (8e-8i). Figure reprinted (adapted) with permission from "Design, Synthesis and Evaluation of WD40-repeat containing protein 5 (WDR5) Degraders by Dölle, Adhikari et al. ${ }^{174}$ Copyright 2021 American Chemical Society.

Table 6. HiBiT data of WDR5 ligand (6d) and degraders (7a-e) and (8a-j). Table adapted with permission from "Design, Synthesis and Evaluation of WD40-repeat containing protein 5 (WDR5) Degraders by Dölle, Adhikari et al. ${ }^{174}$ Copyright 2021 American Chemical Society.

HiBit Assay

\begin{tabular}{|c|c|c|c|c|c|}
\hline ID & Linker & E3 ligase & $\begin{array}{c}\mathrm{DC}_{50} \\
{[\mu \mathrm{M}]^{\mathrm{a}}}\end{array}$ & $\begin{array}{l}\mathrm{DC}_{\max } \\
{[\mu \mathrm{M}]^{\mathrm{b}}}\end{array}$ & $\begin{array}{l}D_{\max } \\
{\left[^{c}\right]^{\mathrm{c}}}\end{array}$ \\
\hline $6 d$ & - & - & no & no & no \\
\hline $7 a$ & & CRBN & no & no & no \\
\hline $7 b$ & & CRBN & no & no & no \\
\hline
\end{tabular}


Table 6. (continued)

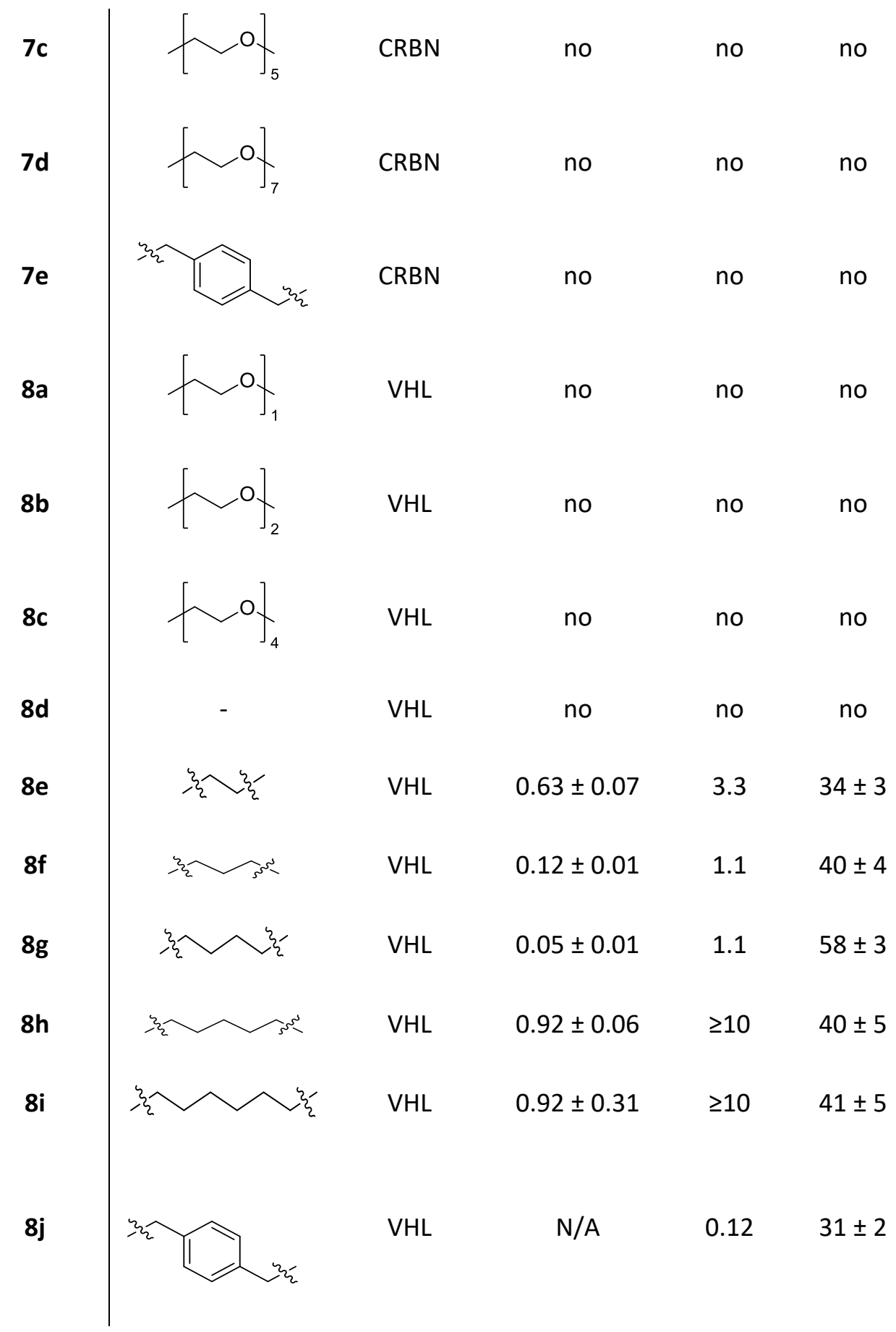

${ }^{a} \mathrm{DC}_{50}$ : half-maximal degradation concentration, calculated with the dose-response (four parameters) equation; ${ }^{b} \mathrm{DC} C_{\max }$ : maximal degradation concentration; ${ }^{c} D_{\max }$ : maximal degradation; no: no degradation; N/A: not applicable.

Two inactive variants of PROTAC $(\mathbf{8 g})$ served as negative controls to validate that WDR5 degradation is caused by PROTAC treatment. The HiBiT assay data and the chemical structures of both negative controls are shown in Figure 16. Both molecules were synthesized analogue to the 
previously described synthesis route, one containing the inactive epimer of the VHL ligand (22), and one containing an inactive WDR5 ligand (23) that uses a morpholine scaffold instead of the methyl piperazine. Alongside to the negative controls, also effects of parent compound (6d) on cellular degradation was tested in the experiments. The increased HiBiT signal of WDR5 levels by ligand (6d) treatment (as observed in Figure 16a) is most likely induced by ligand binding and thus an effect on WDR5 stability.

Additional biophysical data of the negative controls (22) and (23), e.g. DSF results, and cellular data from the BRET and HiBiT assay are listed in Table 7. Comparing the biological data of the negative control (22) to the active PROTAC (8g) shows that the affinity towards WDR5 in the DSF and BRET assay is maintained and in a similar range to the PROTAC molecule, and only the degradational efficacy is lost. The negative control of the WDR5 ligand, compund (23), already showed weak activity in the DSF assay and no degradational effects in the HiBiT assay. With this data, molecule (23) was excluded from BRET measurements.

a)
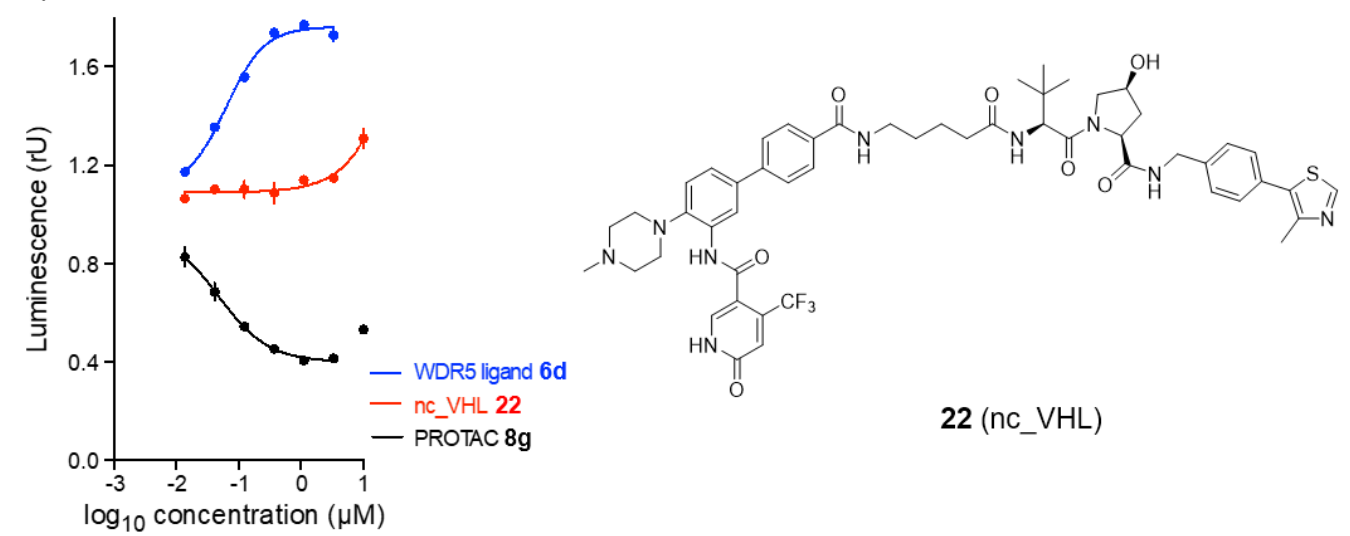

b)
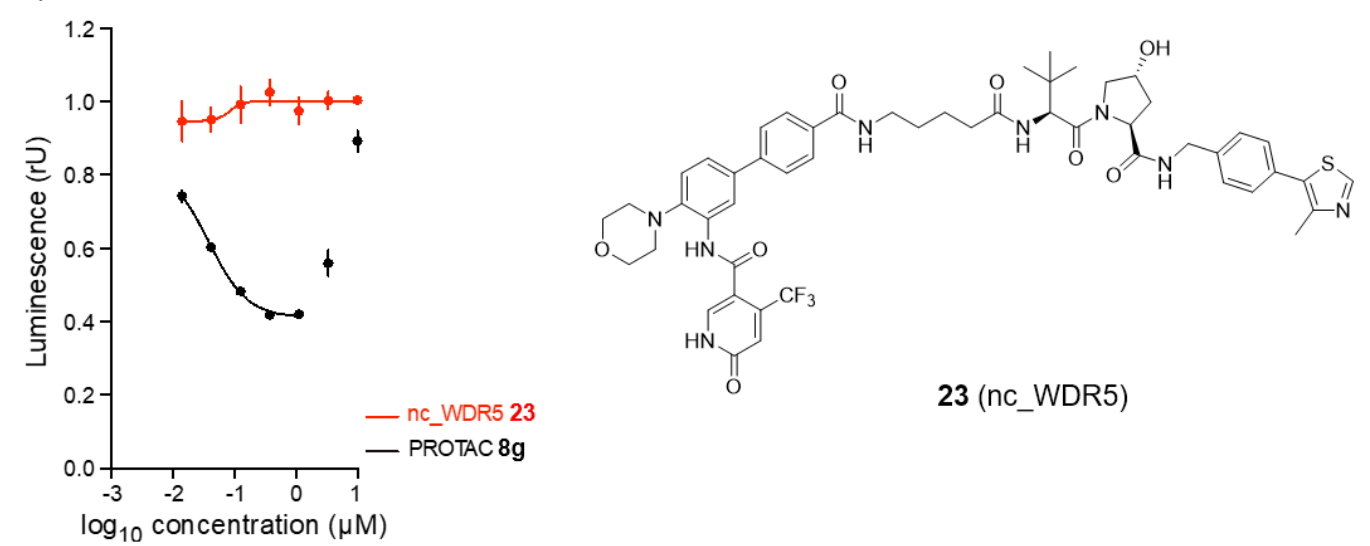

Figure 16: Cellular degradation studies on WDR5. MV4-11WDR5-HiBiT cells were treated with different concentrations of (a) WDR5 ligand (6d), PROTAC (8g) and VHL negative control (22/ nc_VHL) and (b) PROTAC (8g) and WDR5 negative control (23/ nc_WDR5) for $24 \mathrm{~h}$. Figure reprinted (adapted) with permission from 
"Design, Synthesis and Evaluation of WD40-repeat containing protein 5 (WDR5) Degraders by Dölle, Adhikari et al. ${ }^{174}$ Copyright 2021 American Chemical Society.

Table 7. Biophysical and biological data of the negative controls of PROTAC (8g). Negative control (22) inhibits binding to VHL, while negative control (23) inhibits binding to WDR5. Table adapted with permission from “Design, Synthesis and Evaluation of WD40-repeat containing protein 5 (WDR5) Degraders by Dölle, Adhikari et al. ${ }^{174}$ Copyright 2021 American Chemical Society.

\begin{tabular}{|c|c|c|c|c|c|}
\hline \multirow[b]{2}{*}{ ID } & \multirow[b]{2}{*}{$\Delta T_{m} \pm \mathrm{SD}[\mathrm{K}]^{\mathrm{a}}$} & \multicolumn{2}{|c|}{ NanoBRET $^{\mathrm{TM}}$} & \multicolumn{2}{|c|}{ HiBiT Assay } \\
\hline & & $\begin{array}{c}\mathrm{IC}_{50} \pm \mathrm{SD} \\
{[\mu \mathrm{M}]^{\mathrm{b}}}\end{array}$ & $\begin{array}{c}\text { IC }_{50} \text { lysate } \pm \text { SD } \\
{[\mu \mathrm{M}]^{\mathrm{b}}}\end{array}$ & $\begin{array}{c}\mathrm{DC}_{50} \pm \mathrm{SD} \\
{[\mu \mathrm{M}]^{\mathrm{c}}}\end{array}$ & $\begin{array}{c}\mathrm{DC}_{\max } \pm \mathrm{SD} \\
{[\mu \mathrm{M}]^{\mathrm{d}}}\end{array}$ \\
\hline DMSO & $0.0 \pm 0.3$ & $>50$ & $>50$ & no & no \\
\hline OICR-9429 & $13.3 \pm 0.1$ & $0.31 \pm 0.06$ & $1.85 \pm 0.07$ & no & no \\
\hline $8 g$ & $13.2 \pm 0.1$ & $13.6 \pm 0.3$ & $2.15 \pm 0.03$ & $0.05 \pm 0.01$ & 1.1 \\
\hline 22/nc_VHL & $12.2 \pm 0.1$ & $4.26 \pm 0.46$ & $1.12 \pm 0.03$ & no & no \\
\hline 23/nc_WDR5 & $0.0 \pm 0.2$ & n.d. & n.d. & no & no \\
\hline
\end{tabular}

${ }^{\text {aThermal shift }} \Delta T_{\mathrm{m}}$ values given are the mean of triplicate measurements. DSF assays were performed at 2 $\mu \mathrm{M}$ protein concentration and a final compound concentration of $10 \mu \mathrm{M}$.

${ }^{\mathrm{b}} \mathrm{C}_{50}$ values were derived from BRET duplicate measurements and calculated by assuming a normalized 3parameter curve fit; n.d.: not determined.

${ }^{\mathrm{C} D C_{50}}$ : half-maximal degradation concentration, calculated with the dose-response (four parameters) equation

${ }^{\mathrm{d}} \mathrm{DC}$ max: maximal degradation concentration; no: no degradation.

Within the pyrroloimidazole-based PROTAC series, only PROTAC (20b), containing a [PEG $]_{2}$ linker motif, was able to induce degradation of WDR5. The induced depletion by PROTAC (20b) was in a similar range to $(\mathbf{8 g})$ but differentiated in the effective maximal degradation concentration $\left(\mathrm{DC}_{\max }\right)$. In similar manner to the OICR-9429 derived PROTAC (8g), a negative control using the inactive VH032 ligand (24) was synthesized and tested alongside to the active degrader and the parent compound (17). ${ }^{174}$

In addition to the HiBiT results, degradation of untagged WDR5 in MV4-11 ${ }^{\text {WDR5-HiBiT }}$ cells was monitored using immunoblotting for various time points ( 6 hours, 24 hours and 72 hours). The PROTAC induced efficient depletion of WDR5, and the dose-dependency and gratifyingly 
degradation efficacy of the protein resembled the depletion observed in the HiBiT system, as shown in Figure 17. Other immunoblotting studies confirmed the depletion efficacy of the other degraders $(\mathbf{8 e}, \mathbf{f})$ and $(\mathbf{8 h - j})$ in naive MV4-11 cells. As shown in Figure $\mathbf{1 7}$, degradation of WDR5 through (8g) was also shown to be efficient in human leukemia cell line HL-60.

a)

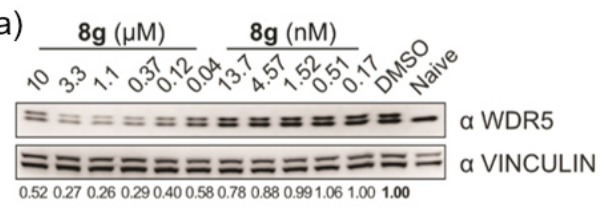

b)

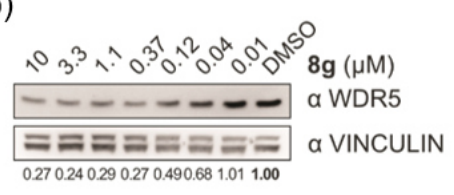

c)

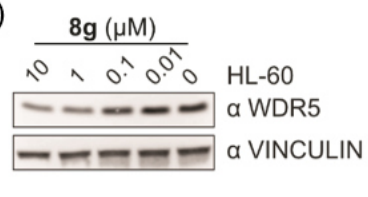

Figure 17: (a) Immunoblot of WDR5. MV4-11 ${ }^{\text {WDR5-HiBiT }}$ cells were treated with different concentration of (8g) for $24 \mathrm{~h}$ and compared with DMSO treated or naive MV4-11 cells. Vinculin was used as loading control (as in all further immunoblot experiments). Quantification is based on both protein bands (endogenous /HiBiT tagged WDR5). (b) Immunoblot of WDR5. Naive MV4-11 cells were treated with different concentration of (8g) for $72 \mathrm{~h}$ and compared with DMSO treated cells. (c) Immunoblot of WDR5. HL-60 cells were treated with different concentrations of $(\mathbf{8 g})$ for $24 \mathrm{~h}$. Figure reprinted (adapted) with permission from "Design, Synthesis and Evaluation of WD40-repeat containing protein 5 (WDR5) Degraders by Dölle, Adhikari et al. ${ }^{174}$ Copyright 2021 American Chemical Society.

\section{Proteasome-dependent mechanism of action studies}

Additional experiments were performed by B. Adhikari to test if the PROTACs induce degradation by inducing ubiquitylation and proteasomal degradation. The results of the experiments are shown in Figure 18. In a protein stability measurement, protein translation was blocked by incubating PROTAC- and vehicle-treated cells with cycloheximide. The WDR5 levels were examined by immunoblotting at several time points and the PROTAC-treated cells showed a decreased protein stability of WDR5 in comparison to the vehicle treated cells.

In a quantitative PCR (qPCR) experiment in MV4-11 cells, effects of the compounds (6d) and (8g) on WDR5 transcription was examined and a transcriptional effect could be excluded as the mRNA levels did not decrease.

An additional rescue experiment showed that WDR5 depletion requires binding of (8g) to WDR5 and that nor WDR5 ligand (6d) neither VH032 are able to induce degradation. WDR5 levels could be restored by excess co-incubation of MV4-11 cells with ligand (6d). 
a)

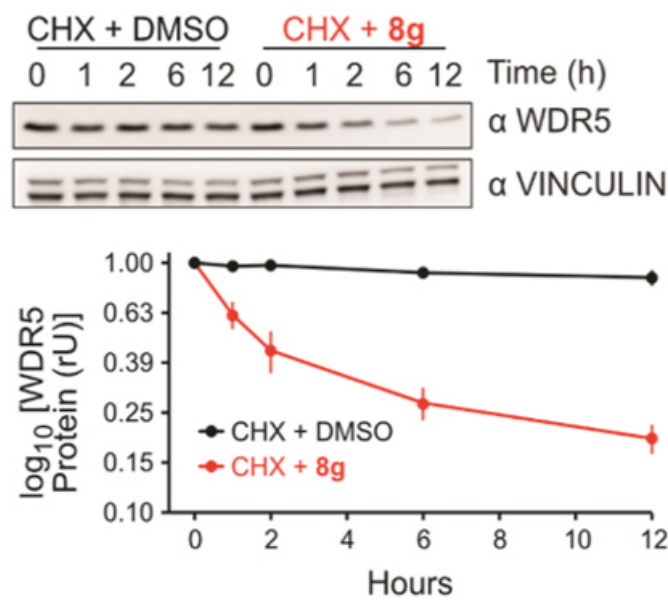

b)

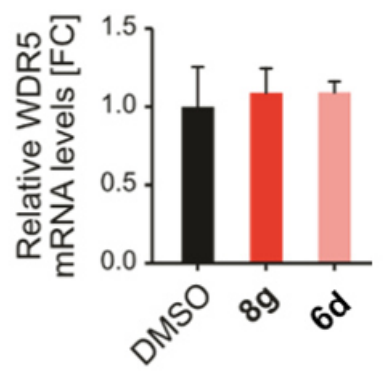

c)

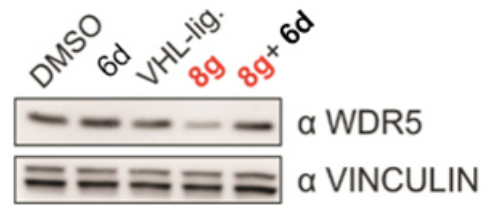

Figure 18: Degrader-induced depletion of WDR5 depends on the ubiquitin system. (a) Immunoblot and quantification of WDR5 levels by the cycloheximide ( $\mathrm{CHX}$ ) chase assay. WDR5 protein stability was evaluated by treating $1 \mu \mathrm{M}(\mathbf{8 g})$ or DMSO incubated MV4-11 cells for $0,1,2,6$ and $12 \mathrm{~h}$ with cycloheximide (CHX). The data is mean $\pm S D$ from $n=2$ biological replicates. (b) Quantitative RT-PCR analysis of WDR5 mRNA levels. RNA was extracted from MV4-11 cells incubated with $1 \mu \mathrm{M}(\mathbf{8 g}), 1 \mu \mathrm{M}(\mathbf{6 d})$ for 24h. WDR5 expression levels were normalized with a reference gene $(B 2 M)$. Bars represents mean $\pm S D$ of $n=3$ technical replicates. (c) Immunoblot of WDR5. MV4-11 cells were treated for $6 \mathrm{~h}$ with $1 \mu \mathrm{M}(\mathbf{8 g})$ or $5 \mu \mathrm{M}(\mathbf{6 d})$ or $10 \mu \mathrm{M}$ VHL-ligand or combination of them. Figure reprinted (adapted) with permission from "Design, Synthesis and Evaluation of WD40-repeat containing protein 5 (WDR5) Degraders by Dölle, Adhikari et al. ${ }^{174}$ Copyright 2021 American Chemical Society.

For the pyrroloimidazole-based PROTAC (20b), other experiments, e.g. proteasomal inhibition with MG132 and neddylation inhibition with MLN4924, to prove a proteasome-mediated mechanism were performed. Similar to PROTAC $(\mathbf{8 g})$, the depletion of endogenous WDR5 was shown in various cancer cell lines by inducing protein ubiquitylation and degradation. ${ }^{174}$

\section{Selectivity profile of PROTAC $(8 \mathrm{~g})$ mediated WDR5 degradation by quantitative proteomics}

Quantitative proteomic studies were performed by B. Adhikari and N. Berner from the TU Munich to get an insight into the selectivity profile of PROTAC-mediated WDR5 degradation. Therefore, cellular protein levels of MV4-11 cells had been treated with PROTAC $(\mathbf{8 g})$ and compared to untreated cells by mass spectrometry. The obtained volcano plots in Figure 19 showed that among 5805 detected proteins, only the WDR5 protein was significantly and substantially depleted ($\left.\log _{10} p>3, \log _{2} F C<-0.5\right)$. Further experiments showed that WDR5 inhibition with ligand $(6 \mathbf{d})$ as well as ligand (17) did not significantly alter WDR5 levels $\left(-\log _{10} \mathrm{p}<1.2, \log _{2} \mathrm{FC}<0.2\right)$. 

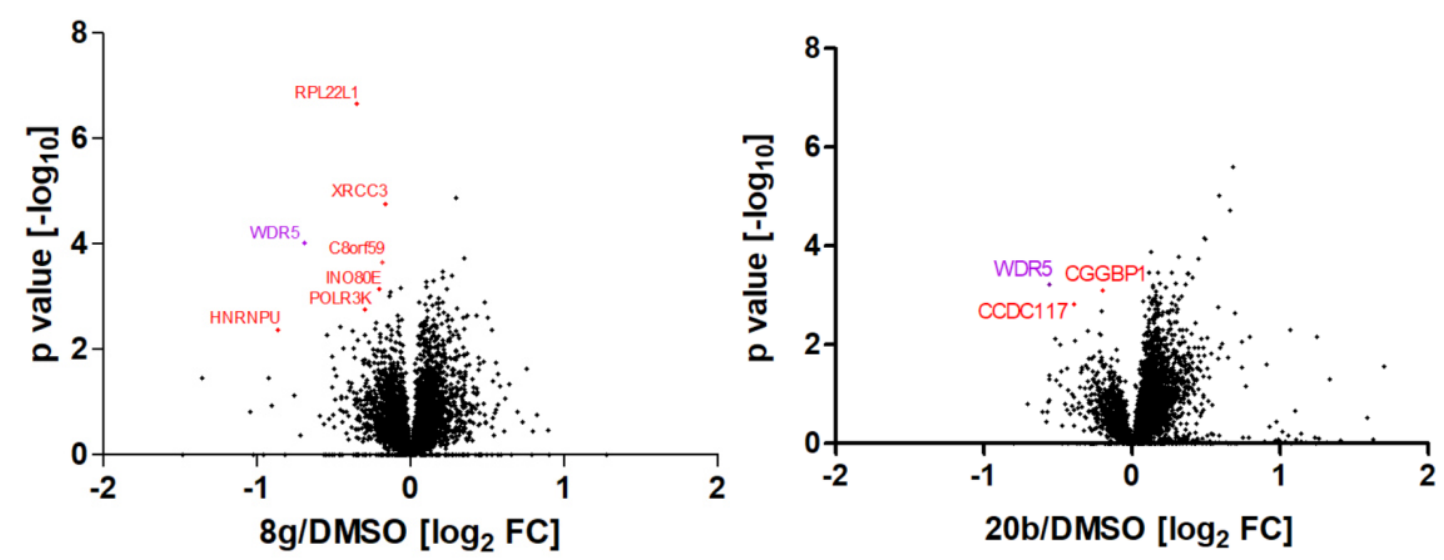

Figure 19: Volcano plot exhibiting global proteomics change upon PROTAC treatment. MV4-11 cells were treated with (left) $1 \mu \mathrm{M}$ (8g) or (right) $5 \mu \mathrm{M}$ (20b) for $9 \mathrm{~h}$ and lysates were analyzed by quantitative proteomics. WDR5 (purple) and depleted proteins (red): 60S Ribosomal protein L22-like 1, RPL22L1; DNA repair protein, XRCC3; Human Ribosomal biogenesis factor, C8orf59; INO80 complex subunit E, INO80E; DNA-directed RNA polymerase III subunit, RPC10, Heterogeneous nuclear ribonucleoprotein U, HNRNPU. Figure reprinted (adapted) with permission from "Design, Synthesis and Evaluation of WD40-repeat containing protein 5 (WDR5) Degraders by Dölle, Adhikari et al. ${ }^{174}$ Copyright 2021 American Chemical Society.

Analysis of the obtained Proteomic data of PROTAC (8g)-treated MV4-11 cells hinted that WDR5 influences transcriptional processes, as also stated by the literature: Bryan et al. depleted endogenous WDR5 via the dTAG approach in neuroblastoma cells and compared degradation versus inhibition. ${ }^{84}$ The authors found slightly differences in the biological clustering of the two distinct approaches: WDR5 depletion had more extensive effects on neuronal differentiation, while inhibition showed enhanced effects on the expression of genes linked to cell cycle. ${ }^{84}$ Further proteomic experiments with the WDR5 Win site ligand C6 from Guarnaccia et al. showed that WDR5 depletion influenced inter alia proteins with links to tRNA synthetases. ${ }^{177}$ In this study, the observed effects of a phenotypic response to WDR5 depletion is moderate, most likely due to the incomplete degradation profile of $\mathbf{( 8 g})$. Nevertheless, WDR5 depletion tends to downregulate ribosomal proteins, e.g. Heterogeneous nuclear ribonucleoprotein $U$ (HNRNPU), Human Ribosomal biogenesis factor (C8orf59), or 60S Ribosomal protein L22-like 1 (RPL22L1) and thus indicate WDR5 is conntected to the expression of genes linked to protein synthesis.

Analogue to PROTAC (8g), Proteomic analysis for PROTAC (20b) has been performed and also showed a significant depletion of WDR5, as displayed in Figure 19. The observed proteomic effect was smaller than for PROTAC $(\mathbf{8 g})$, although the required concentration was adjusted. Beside WDR5 depletion, two other proteins involved in transcription and translational processes were depleted alongside to WDR5: the CGG triplet repeat-binding protein 1 (CGGBP1) and the Coiled- 
coil domain-containing protein 117 (CCDC117). In alignment with PROTAC (8g) mediated WDR5 depletion, the observed effects are weak due to the incomplete WDR5 degradation by PROTAC (20b). Both proteomic experiments with different PROTACs proved the hypothesis of WDR5s' role in non-epigenetic processes.

The enriched proteins upon Win site blockade from Guarnaccia et al. had few connections and represented various biological processes e.g., transcription, ubiquitylation and chromatin remodeling. ${ }^{177}$ The proteomic experiments upon treatment with parent compound $(\mathbf{6 d})$ are displayed in Figure $\mathbf{2 0}$ showed an enriched profile of proteins involved in ferritinophagy (NCOA4), pentose phosphate pathway (PGD) and glycolysis (DLD/ PFKL) and had few connections. Quantitative proteomic analysis of pyrroloimidazole ligand (17) revealed that treatment enriched the structure protein Aktin and the protein Chromosome 7 Open Reading Frame 26 (C7orf26) that is assumed to be involved in transcriptional processes. Both ligands do not cause a decrease in WDR5 levels.
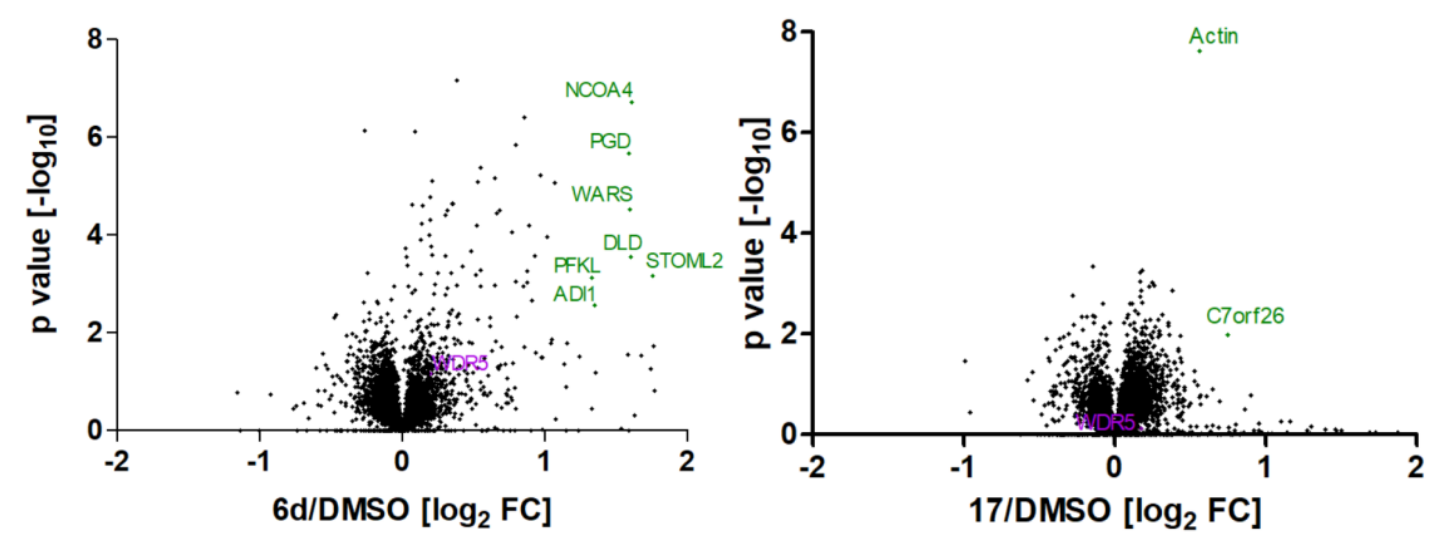

Figure 20: Volcano plot exhibiting global proteomics change upon Win site ligand binding. MV4-11 cells were treated with $1 \mu \mathrm{M}(\mathbf{6 d}) 5 \mu \mathrm{M}(\mathbf{1 7})$ for $9 \mathrm{~h}$ and lysates were analyzed by quantitative proteomics. WDR5 (purple) and depleted proteins (red): CGG triplet repeat-binding protein 1, CGGBP1; Coiled-coil domaincontaining protein 117, CCDC117. The enriched proteins are (green): Nuclear receptor coactivator 4, NCOA4; 6-phosphogluconate dehydrogenase, PGD; Tryptophan-tRNA ligase, WARS; Dihydrolipoyl dehydrogenase, DLD; ATP-dependent 6-phosphofructokinase, PFKL; 2-dihydroxy-3-keto-5methylthiopentene dioxygenase, ADI1; Stomatin-like protein 2, STOML2; Chromosome 7 Open Reading Frame 26 (C7orf26), Actin. Figure reprinted (adapted) with permission from "Design, Synthesis and Evaluation of WD40-repeat containing protein 5 (WDR5) Degraders by Dölle, Adhikari et al. ${ }^{174}$ Copyright 2021 American Chemical Society.

Comparing the proteomic data of ligand (6d) and (17) suggests that further experiments are necessary to evaluate the observed effects. While treatment with PROTAC (8g) affects various 
proteins that are involved in transcriptional and translational processes in the nucleus, the enriched effects of parent compound (6d) as well as (17) relate to proteins located in the cytoplasm. As Figure 19 indicates, these proteins are not influenced by WDR5 degradation and thus the proteomic data upon ligand treatment might be overestimated. Another hypothesis would be that the WDR5 ligands are able to act as molecular glue between the surface of WDR5 and the enriched proteins: as the elongation of the WDR5 ligands to heterobifunctional molecules removed the molecular glue like properties, the observed effect were no longer detectable in the proteomic studies.

To validate the proteomic results, additional qPCR experiments to check the mRNA levels and immunoblots of the enriched and repressed proteins would be a suitable tool to determine the influence of WDR5 on these proteins. As the overall effect is only weak, knockdown studies on WDR5 and subsequent analysis of the interactions could provide more clear results.

All in all, the PROTAC mediated degradation of cellular WDR5 was shown in various assays and control experiments. Various PROTACs with two different WDR5 ligand scaffolds successfully induced degradation of WDR5 upon three days after treatment. The OICR-9429 derived PROTAC $(8 \mathrm{~g})$ was shown to be the most effective degrader in terms of degradation efficacy (58\%) and required concentration $(1 \mu \mathrm{M})$. PROTAC (20b) containing the pyrroloimidazole scaffold also showed favorable properties ( $54 \%$ degradation upon treatment with a dose of $3 \mu \mathrm{M}$ ).

\subsection{Crystallization of the ternary complex}

The formation of a ternary complex between the target protein and the E3 ligase is the main goal of the proximity-generating heterobifunctional molecule. This protein formation was shown to be the crucial step for the success of TPD. ${ }^{160,}{ }^{178}$ Further studies validated that PROTAC-mediated ubiquitination depends on the existence of a ternary complex equilibria and also the dosedependent degradation efficacy is consistent with ternary complex formation. A criterion for measuring ternary complex stability is the so-called cooperativity factor $\alpha$ which is calculated out of the binary and ternary binding constants of a protein complex ${ }^{179}$ and commonly determined by the biophysical methods ITC or SPR. The target protein is preferentially recruited in a positively cooperative ternary complex with the respective E3 ligase. ${ }^{180} \mathrm{~A}$ time-resolved study by Roy and coworkers examined two different PROTACs that addressed the ternary complex formation of BRD4 and CRBN. ${ }^{179}$ The study demonstrated that a long-lasting and stable ternary complex correlates with an enhanced degradation profile. Farnaby and Koegl explained the activity of a 
PROTAC targeting the BAF chromatin remodeling complex by means of the obtained ternary complex crystal structure, making ternary complex characterization critically important in PROTAC development. ${ }^{160,164}$

To understand the molecular mechanism of WDR5 degradation and to further improve the degradation profile of PROTAC (8g), crystallization studies on the WDR5/ VHL system had been performed. As the crystal structure was obtained successfully within a few attempts, timeconsuming cooperativity studies that usually require a huge amount of both proteins, have been postponed. The crystal structure of the ternary complex between WDR5, PROTAC (8g) and VHL/ Elongin $\mathrm{B}$ / Elongin $\mathrm{C}$ was obtained with a resolution of $2.5 \AA$, as shown in Figure 21. In contrast to the predicted model ${ }^{174}$ and the assumption that proteins involved in degradational processes tend to stabilize the ternary complex by large surface interactions ${ }^{164,180-181}$, a rather diminished overlap of the protein surfaces was observed withing the crystal structure. Most of the published ternary complex crystal structures investigated the bromodomain family ${ }^{164,180-181}$ that occur as small subunits (ca. $15 \mathrm{kDa}$ ) in large chromatin modifying enzymes and often interact in a positively cooperative ternary complex with the E3 ligase. For the $37 \mathrm{kDa}$ heavy WDR5 protein, two additional PPIs between the amino acid side chains of Lysine 259 (WDR5) and Aspartic acid 92 (VHL) as well as the carbonyl function of the Tyrosine 191 and Asparagine 214 backbone (both WDR5) with Arginine 69 (VHL) were observed. 


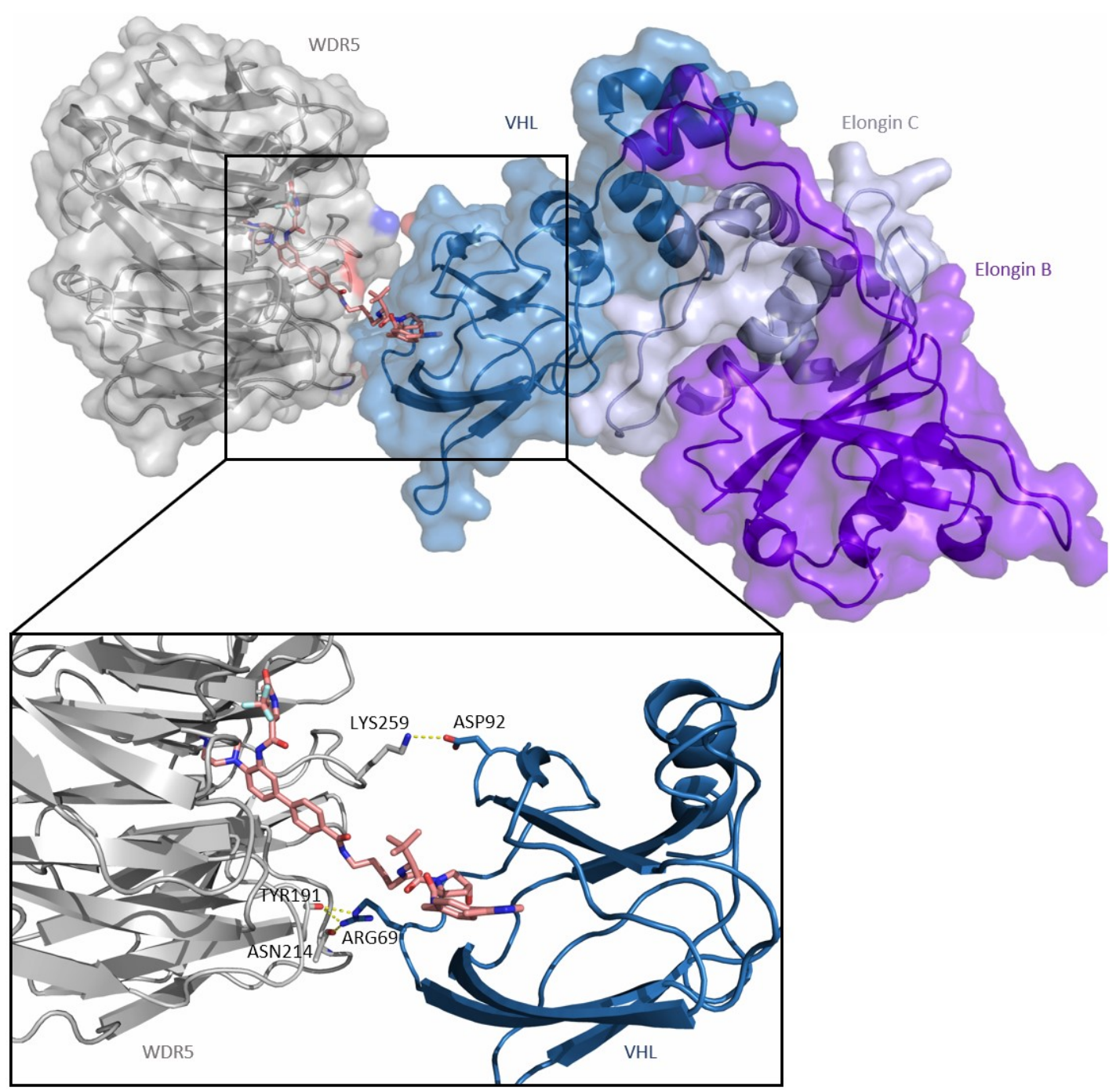

Figure 21: (top) Crystal structure of the WDR5/ (8g)/ VHL ternary complex. Protein surfaces are displayed to show the minimal overlap between WDR5 (grey) and VHL (blue)/ Elonging B (purple)/ Elonging C (lightblue). The red and blue colored surface spots represent the additional PPI interactions. (bottom, outlined) Close-up of the interaction site shows that PROTAC (8g) (pink) is mainly responsible for the PPI. Additional PPIs occur between the side chains of LYS259 and ASP92 as well as the backbone of TYR191, ASN214 and the side chain of ARG69.

The surprising few interactions between both proteins within the crystal structure indicated that the main reason for successful WDR5 degradation lies in the high affinity and chemical stability of PROTAC (8g) towards WDR5 and VHL. The high affinity has already been shown in vitro measurements by ITC (as shown in Figure 10 and Table 3), while the long-lasting chemical stability of the compound was observed by Immunoblotting experiments for a timeframe of up to 72 hours (see Figure 14). Though the crystal structure provided an insight into the binding mode, a structural improvement of PROTAC (8g) maintained challenging due to the linker exposure towards the solvent and non-existing opportunities to stabilize the ternary complex with an 
improved degrader molecule. Nevertheless, short and unipolar motifs like alkines could be implemented into PROTAC design.

\subsection{Development of the chemical probe Homer}

An advantage of the degradation approach is that targeted protein degradation removes a protein entirely from the cell such as for instance the moonlighting multitasker WDR5, while inhibition of WDR5 alone would only show cellular effects of the respective inhibited binding site. Although the degradation profile is not quantitative, the herein observed effects suggested that PROTAC (8g) might be a suitable tool for exploring WDR5 manifold cellular functions. As shown in Table 8, the low $\mathrm{DC}_{50}$ and $\mathrm{DC}_{\max }$ values indicate a catalytic turnover, and the proteomic effects clearly demonstrate the substantial and significant depletion of WDR5 in a cellular environment. The control experiments, e.g. the $\mathrm{CHX}$ chase assay, the qPCR and the rescue experiment showed the proteasome-dependent mechanism of action as well as the necessity of PROTAC (8g) to bind to WDR5. The crystal structure of the ternary complex demonstrates the binding mode of the PROTAC. Thus, the WDR5 PROTAC (8g), alongside with its two negative controls (22/ nc_VHL and 23/ nc_WDR5), was revised successfully as an open-source chemical probe via the donated chemical probes program under the name Homer (https://www.thesgc.org/chemicalprobes/homer).

Table 8: Probe criteria for the WDR5 PROTAC probe Homer.

\begin{tabular}{|c|c|c|}
\hline Probe criteria & Homer & \\
\hline Potency & $\circ$ & $\mathrm{K}_{\mathrm{d}}(\mathrm{ITC}): 18 \mathrm{nM}$ \\
\hline Activity & $\begin{array}{l}\text { Proteas } \\
\text { free } \mathrm{VH}\end{array}$ & $\begin{array}{l}\text { ome-dependent degradation; rescue experiments with } \\
L \text { ligand and WDR5 ligand in addition to Homer }\end{array}$ \\
\hline In cell validation & ○ & $\mathrm{IC}_{50}$ (NanoBRET): $13.6 \mu \mathrm{M}$ \\
\hline Evidence and quantification of target & $\circ$ & $\mathrm{DC}_{50}: 53 \mathrm{nM}$ and $\mathrm{D}_{\max }: 58 \%$ \\
\hline engagement and degradation & ○ & Concentration course for degradation \\
\hline & $\circ$ & Evidence of E3, ubiquitin and proteasome-dependence: \\
\hline & & CHX assay, qPCR \\
\hline Off-target activity in cells & $\circ$ & proteomics: only depletion of WDR5 $\left(-\log _{10} p>3, \log _{2} F C\right.$ \\
\hline & & $<-0.5)$ \\
\hline Control compounds & 2 negat & ive controls: nc_VHL and nc_WDR5 \\
\hline Recommendation on use & $1 \mu \mathrm{M}(\mathrm{e}$ & valuated at $6 \mathrm{~h}, 24 \mathrm{~h}, 72 \mathrm{~h})$ \\
\hline
\end{tabular}


64 Results and Discussion 


\section{Summary}

Cancer is a serious disease for anyone affected, threatening the long-term well-being and life of the patient. For the society and the healthcare system, cancer causes massive economic costs, revealing an urgent need for novel and more effective therapies. Recent progress in cancer therapy came from the field of molecular targeted therapies, which inhibit the function of proteins that drive tumor progression, as well as immunotherapies, which increase the ability of the immune system to recognize and eliminate tumor cells. Both therapeutic approaches aim to overcome the major side effects that occurs in classical chemotherapy, in particular the toxicity to all dividing cells. In addition to the enourmous achievements in cancer diagnosis and treatment, elementary questions about cellular processes remain unsolved which limits therapeutical options.

One class of oncoproteins that drives leukemia growth are the protein class of mixed lineage leukemia (MLL) histone lysine methytransferases. ${ }^{50,59,62}$ The multi-domain protein complexes mark specific histone tails of the chromatin structure by methylation, enabling gene transcription. Genetic fusions of the $\mathrm{m} / \mathrm{l}$ gene, so-called rearragments, result in MLL-fusion products that drive development of acute myeloid lekemia (AML). ${ }^{60,182}$ Another oncoprotein responsible for the aggressive progression of various cancer types is the transcription factor family MYC. ${ }^{109-110}$ Several studies showed that the amplification of neuroblastoma cells depends on MYC and that high cellular MYC levels regulate the expression of genes crucial for transcriptional processes and tumour growth. ${ }^{109-110,114,183-184}$ Due to the instable protein structure of MYC and its lack of binding pockets, MYC is still considered as undruggable. ${ }^{109-110}$ Thus, the pharmacological efforts on targeting the oncogene focusses on indirect targeting strategies, e.g. by inhibiting the interaction partners of the transcription factor.

Both the MLL histone lysine methyltransferase family and the transcription factor family MYC interact with an approximately $37 \mathrm{kDa}$ protein called WD40-repeat containing protein 5 (WDR5). Several studies have shown that this scaffolding protein is a promising drug target. ${ }^{82}$ Due to its propeller-shaped structure, WDR5 has a large surface with two binding sites. Both binding sites are involved in binding MLL complex proteins, thereby ensuring the stability and function of the MLL1 complex. ${ }^{57}$ In the context of MLL misregulation, inhibition of WDR5 emerged as a promising strategy for the treatment of MLL-rearragend leukemia. ${ }^{50,83,93-95,185-186}$ Further studies showed that WDR5 mediates the interaction between MYC and chromatin via its two binding sites and 
thus WDR5 was evaluated as a promising and druggable interaction partner for MYC-dependent cancer types. ${ }^{81,84,86,102,168,177}$

In summary, WDR5 has significant roles in scaffold functions and in engaging multiple binding partners at different binding sites. Thus, inhibition of the individual protein-protein interactions results in only a partial knockdown of the enzymatic function. Although the developed WDR5 anatagonists ${ }^{81,83,167-168,177}$ demonstrated the great potential of this drug target, a classic inhibitory approach of only one binding site limits the studies of investigating WDR5s' overall impact on cellular processes. This limitation could be overcome by the proteasomal degradation of the entire protein removing the essential protein-protein interactions. A new class of small molecules, called PROTACs (Proteolysis targeting chimeras), induce chemically the degradation of the respective target proteins by the cell-own ubiquitin/proteasome symstem ${ }^{117,122}$ PROTACs have already demonstrated the successful degradation of several protein classes. ${ }^{116,118,122}$ Due to the induction of selective and efficient target protein degradation, this class of small molecules offers a novel approach for investigating the effects of individual proteins on cellular mechanisms. First PROTACS have been optimized for therapeutic uptake and are currently undergoing clinical tirals. ${ }^{187}$ This aim of this thesis was the design and synthesis of WDR5 degrader molecules, using two distinct ligand scaffolds, diverse linker types as well as three ligands for different druggable E3 ligases. A set of eighteen PROTACs with the WDR5 small molecule antagonist OICR-9429 was successfully synthesized in this thesis, as well as nine PROTACs bearing a pyrroloimidazole scaffold supervised in two master projects. This study evaluated the different steps of PROTAC-mediated target protein degradation. First, the in vitro affinity of the PROTACs to WDR5 was proven in two orthogonal biophysical assays, while the herein developed BRET assay showed cellular permeability and target engagement of the PROTACs. The degradation efficacy was evaluated in the context of a structure-activity-relationship study and revealed that degradation efficacy is affected by minimal changes in linker length and composition. Further biological experiments examined the proteasomal mechanism of action in MLL-rearragend cancer cells. The successful formation of the ternary complex was obtained by protein crystallography and completed the mechanism of action studies. The outcome of this study led to potent PROTACs and demonstrated that PROTAC-induced protein degradation can be achieved with different exit vectors and linker types. The best degrader was published as chemical probe Homer (https://www.thesgc.org/chemical-probes/homer) by the chemical probe program of the Structural Genomics Consortium. The obtained results reinforce WDR5 as potential cancer target and PROTAC technique as suitable method to explore proteins with manifold cellular functions like WDR5. ${ }^{174}$ 


\section{Conclusion and Outlook}

Since 2015, the biological relevance of the WD40-repeat containing protein 5 has continuously increased due to investigative research that aims to illuminate biological mechanisms and find new therapeutic approaches for severe diseases. ${ }^{82,} 102$ WDR5 has been rising as promising therapeutic target since its various interaction partners drive major oncogenic processes. ${ }^{114}$ These oncogenic drivers are e.g., the transcription factor family $\mathrm{MYC}^{103}$ that play a crucial role in neuroblastoma amplification ${ }^{114}$, and the MLL/ SET HMT complexes where rearrangements drive acute leukemia progression. ${ }^{79}, 83$ Both proteins rely on WDR5s' scaffolding role that recruit MYC to chromatin or ensure the stability and function of HMT complexes. Targeting WDR5 function was shown to be a promising approach for inhibiting leukemia (MLL-rearranged) and neuroblastoma (MYC-dependent) cell growth by inducing p53-dependent apoptosis. ${ }^{114}$ In addition to these wellestablished functions, WDR5 has additionally been shown to influence the expression of ribosomal subunits, making the moonlighting protein interesting for other cancer types, as alterations of ribosome biogenesis are a common feature of cancers. ${ }^{82,188}$

This thesis focused on the development to temper both oncogenic functions by degrading the scaffolding protein WDR5 using the PROTAC technique. The herein designed and developed heterobifunctional molecules relied on diverse WDR5 ligand scaffolds, that created different exit vectors from the WDR5 Win binding site, and comprised different E3 ligase ligands targeting Cereblon (7a-e), VHL (8a-j) and MDM2 (9a-c). The degrader molecules have been evaluated in various assays, starting from in vitro assays such as DSF and ITC which showed the high affinity of the PROTACs towards WDR5, to cellular assays that showed good cell permeability and on-target activity (BRET assay) or monitored degradation of WDR5 for a cluster of PROTACS (HiBiT). Further experiments showed the proteasome-mediated mechanism of action as well as the selectivity of WDR5 depletion.

However, only VHL-based PROTACs (8e-j) led to successful degradation and small changes in linker length and linker type resulted in significant changes in degradation efficacy. The herein used leukemia cell lines attested an incomplete degradation profile of the degraders and will need further investigation. Experiments from B. Adhikari from the University of Würzburg showed that cells could be sensitized to a better degradation profile by overexpressing VHL. This resulted in an increased degradation efficacy and suggested that using the heterobifunctional molecules in cell lines with higher VHL levels will also increase WDR5 degradation. The ongoing proliferation experiments showed that a more efficient depletion is required to see stronger attenuation of 
cancer cell growth. This is limited with the high amount of required concentration, and thus future medicinal chemists should focus on the chemical improvement of PROTAC (8g) lead structure.

Chemical modifications on the PROTAC scaffold could either be achieved by increasing solubility of the molecule or by enforcing ternary complex formation. Increasing solubility for the OICR-9429 derived degrader series would suggest implementing the original morpholine system, which was used in the OICR-9429 scaffold to generate solubility, while maintaining the exit vector of PROTAC (8g). ADME and PK/ PD studies could provide a more detailed feedback for cellular permeability. Regarding the BRET measurements of PROTACs (20a-g), the development of a pyrroloimidazole based tracer could likely determine the binding affinity of the PROTACs. Looking at the data from the HiBiT assay and combining them with the obtained crystal structure of the ternary complex, the OICR-9429 degrader series showed some space for chemical improvement, e.g. implementing alkine functions. While the HiBiT assay indicated that small changes in linker length - shortening/ prolonging of the butyl chain or substituting the alkyl chain into a [PEG] linker - effected the degradation profile extensively, the crystal structure showed a less favorable positioning of both proteins towards each other. This suggests that the cooperativity of ternary complex formation might not be favored, but additional biophysical experiments like SPR or ITC measurements and biological point mutation studies would be required to prove this hypothesis. As this work focused on the synthetic development of functional WDR5 degraders, the resulting additional biophysical questions have been upstaged.

A medicinal chemistry approach to improve the degradation profile beside improving the OICR9429 based degrader series would focus also on the pyrroloimidazole based scaffold of the PROTAC series (20a-g). An attempt to improve solubility could be the substitution of one [PEG] linker unit by a piperazine moiety. A better solubility might lower the required concentration in an aqueous environment, and proliferative effects of could be observed at lower concentrations. Another effect of the insertion of a piperazine moiety would be that the resulting PROTAC might be more rigid. Speculating about the outcome, this chemical change could either result in a more stable and long-lasting ternary complex (resulting in enhanced degradation efficacy) or the degradation could be completely abolished if the pyrroloimidazole based PROTAC contains a twisted exit vector and thus requires flexibility. To investigate this issue, a second ternary complex crystal structure would be beneficial.

In terms of biological experiments, additional qPCRs and Immunoblots of downregulated proteins in the proteomic experiments would potentialy give an insight on WDR5s' role in transcriptional processes. Therefore, compounds $(\mathbf{8 g})$ or $(\mathbf{2 0 b})$ presented here would already be suitable to 
address this question, as the effects have already been observed in the global proteomic change in Figure 19 and Figure 20. Otherwise, knock-down studies on WDR5 could be informative to clearify WDR5 interaction partners. ChIP- and RNA-Sequencing experiments of PROTACs would show commonalities and differences between the inhibitory versus the degradational approach. For in vivo studies, PROTACs with an improved degradation profile and solubility would definitely be required.

Although the herein investigated system has limitations and needs further characterization and optimization, it demonstrates the potential of the PROTAC approach. The heterobifunctional molecules presented here are suitable chemical tools that will allow comprehensive evaluation of WDR5 degradation in diverse cancer types and the potential of this strategy for drug discovery. 
70 Conclusion and Outlook 


\section{Deutsche Zusammenfassung}

Für jeden Betroffenen ist die Diagnose Krebs ein schwerwiegender Einschnitt in der Lebensqualität und -führung, da die Behandlung oftmals mit langen Chemotherapien einhergeht. Die enormen Kosten der Krebsbehandlungen stellen für das Gesundheitssystem eine hohe Belastung dar, die den Bedarf an neuer, günstiger und vor allem effizienter therapeutischer Ansätze verdeutlicht. Moderne Durchbrüche in der Krebsbehandlung stammen aus dem Forschungsbereich der zielgerichteten Molekulartherapie, eine Methode um spezifische Funktionen von Onkoproteinen zu inhibieren, oder aus dem Gebiet der Immuntherapien, die auf eine Stärkung des Immunsystems abzielt und damit Krebszellen effektiver vernichtet. Abgesehen von den beachtlichen Erfolgen beider Ansätze bei der Behandlung von Krebspatienten, bleiben auf dem Gebiet der Onkologie weiterhin Fragen zu den grundlegenden biologischen Prozessen unbeantwortet. Das Verständnis dieser Prozesse könnte Wege zur Überwindung, der im Verlauf der Therapie häufig auftretenden Medikamentenresistenzen, aufzeigen und Möglichkeiten für neue Therapieansätze eröffnen.

Zu den Onkoproteinen, die das Tumorwachstum in Leukemiezellen stark beeinflussen, gehören die Proteine der Klasse der mixed lineage leukemia (MLL) Histonmethyltransferasen. 50, 59, 62 Die großen, aus vielen Domänen bestehenden Proteinkomplexe markieren Histonenden der Chromatinstruktur mit Methylgruppen, um diese für die Gentranskription zugänglich zu machen. Genetische Fusionen des mll Gens, sogenannte Rearragments, führen zu MLL-fusion Produkten, die erheblich zum Verlauf der aggressiven akuten myeloischen Leukämie (AML) beitragen. ${ }^{60,182}$

Ein weiteres Onkoprotein ist für den Krankheitsverlauf vieler Krebsarten relevant, unter anderem weil dessen Überexprimierung in einem Drittel aller humanen Tumore beobachtet wurde: die Transkriptionsfaktorfamilie MYC. ${ }^{109-110}$ Auf molekularer Ebene sorgt MYC für die Transkription einer Vielzahl von Genen, deren Expression in direkter Abhängigkeit zu jeweiligen MYC Konzentration stehen. Studien belegen, dass hohe MYC Level die Expression von Genen regulieren, die essentiell für den Transformationsprozess und somit das Tumorwachstum sind. ${ }^{109-110,114,183-184}$ So zeigte sich unter anderem einen Zusammenhang zwischen MYC und der Amplifikation von Neuroblastomazellen auf. ${ }^{114}$ Da der Transkriptionsfaktor weder eine sabile tertiäre Proteinstruktur noch eine für Inhibitoren adressierbare Bindetasche aufweist, gilt MYC bis heute als undruggable. Neuere Forschungsansätze basieren auf der Inhibierung von Interaktionspartnern des Onkoproteins.

Sowohl die Histonmethyltransferase MLL1, als auch der Transkriptionsfaktor MYC interagieren mit einem ca. $37 \mathrm{kDa}$ Protein namens WD40-repeat containing Protein 5 (WDR5), das durch seine propellerförmige Struktur eine Oberfläche mit insgesamt zwei Bindestellen aufweist. Mehrere Studien 
zeigten, dass WDR5 die Stabilität und somit die Funktion epigenetischer Proteinkomplexe wie SET/ MLL und NSL gewährleistet. ${ }^{57,82}$ In diesem Kontext wurde WDR5 als relevantes Target für die MLLrearragend akute lymphatische Leukämie (ALL) postuliert. ${ }^{50,83,93-95, ~ 185-186 ~ W e i t e r e ~ S t u d i e n ~ z e i g t e n ~}$ zusätzliche Rollen von WDR5, wie die Interaktion zwischen WDR5 und dem Onkoprotein MYC sowie dessen Rekrutierung zum Chromatin. ${ }^{81,84,102,168,177}$ Dabei nutzt WDR5 seine zwei Bindestellen, um einerseits die Interaktion mit dem Transkriptionsfaktor und andererseits mit den Bindemotiven der Chromatinstruktur zu ermöglichen. ${ }^{81,84,86,102,168,177}$

Seit 2015 wurden erfolgreich mehrere niedermolekulare Wirkstoffe für die Inhibierung von WDR5 entwickelt. Dabei zielten die meisten der literaturbekannten Inhibitoren auf die Argininmotiverkennende WDR5-interacting (Win) Bindestelle, eine große, hydrophobe Bindetasche im Zentrum des WDR5-Propellers. ${ }^{81,83,93-95,167,185}$ Für das weniger zugängliche WDR5-binding Motiv (WBM), das eher einem kleinen Zwischenraum, denn einer Bindetasche auf der WDR5 Oberfläche ähnelt, konnten jedoch ebenfalls in den letzten Jahren erfolgreich Inhibitoren entwickelt werden. ${ }^{168}$ Die Resultate der besser erforschten Win Inhibitoren zeigten, dass WDR5 ein erfolgsversprechendes Target zur Inhibierung von leukämischen (MLL-r-abhängigen) und neuroblastomatischen (MYC-abhängigen) Zellwachstum ist. ${ }^{82-83,102}$

Häufig beschriebene Nachteile in Krebstherapien stellen entwickelte Wirkstoffresistenzen sowie eine erhöhte Wirkstofftoleranz dar. ${ }^{189}$ Eine dadurch bedingte Erhöhung der Dosis führt zu unerwünschten zellulären Effekten und Toxizitäten. Die PROTAC (Proteolysis targeting chimera) Technik beruht auf einem katalytischen Effekt und minimiert somit mögliche off-target Effekte. ${ }^{122}$ Die heterobifunktionellen Moleküle induzieren den Abbau des Zielproteins über das zelleigene UbiquitinProteasom-System anstatt dessen Enzymfunktion zu inhibieren. ${ }^{116,118,122}$ Der PROTAC induziert einen stabilen ternären Komplex zwischen dem Zielprotein und einer E3 Ubiquitin Ligase, was die Übertragung von Ubiquitin und eine anschließende Erkennung durch das Proteasom ermöglicht. Nach dem zelleigenen Abbau des Zielproteins wird der PROTAC freigesetzt und kann einen neuen Zyklus der Proteindegradation einleiten, was die erforderliche Menge an Wirkstoff verringert. Zahlreiche onkologisch relevante Zielproteine wurden bereits erfolgreich über das Ubiquitin-Proteasom-System (UPS) abgebaut und erste PROTACs befinden sich bereits in klinischen Studien. ${ }^{187}$

Da beide Bindestellen des WDR5 Proteins Interaktionen mit onkologisch bedeutsamen Faktoren eingehen, würde eine einseitige Inhibierung nur die Effekte der jeweiligen Bindestelle aufzeigen. Diese Limitierung könnte jedoch durch die Entwicklung von WDR5 PROTACs aufgehoben werden, da alle Gerüstfunktionen des Proteins und Protein-Protein-Interaktionen durch die Degradierung von WDR5 entfernt werden würden. 
Diese Dissertation beschäftigte sich mit dem Design, der Synthese sowie der biophysikalischen und biologischen Evaluierung von WDR5 PROTACs. Ausgehend von literaturbekannten WDR5 Liganden, dem OICR-9429 ${ }^{83},{ }^{93}$, DDO-2117 ${ }^{94}$ sowie einem pyrroloimidazol-basiertem Liganden ${ }^{167}$, wurden zwei verschiedene PROTAC Typen entworfen. Diese beiden Molekültypen besitzen einen unterschiedlichen geometrischen Austrittswinkel, wodurch die Chance auf eine erfolgreiche Komplexbildung zwischen WDR5, PROTAC und E3 Ligase erhöht wird. Da die Synthese des pyrroloimidazol-basierten Liganden in zwei Masterarbeiten diskutiert und evaluiert wurde, liegt der synthetische Fokus dieser Dissertation auf dem Piperazin-basierten WDR5 Liganden. Als Leitstruktur fungierten die Verbindungen OICR-9429 sowie DDO-2117. In einem ersten Syntheseplan wurden vier verschiedene WDR5 Liganden (6a-d), beruhend auf dem Arginin-Mimetika Methylpiperazin, aufgebaut. Im letzten Syntheseschritt wurden verschieden dekorierte Benzoesäuren verwendet, um den Liganden mit der höchsten Affinität zu generieren. In anschließenden biochemischen und biophysikalischen Evaluierungen der verschiedenen Liganden durch Thermofluor (DSF) und Biolumineszenz-Resonanzenergietransfer (BRET) Messungen zeigte sich Ligand (6d) als am geeignetsten für die Weiterentwicklung von PROTACs. Ausgehend vom WDR5 Ligand (6d) wurden heterobifunktionelle Moleküle mit verschiedenen Linkersystemen ([PEG]und alkyl-basiert, sowie aromatisch verbrückt) und verschiedenen E3 Ligase Liganden (Cereblon, VHL und MDM2) synthetisiert.

Die Affinität der OICR-9429-basierten PROTACs (7a-e), (8a-j) und (9a-c) wurde anschließend in in vitro Messungen validiert. Die DSF Messungen der Cereblon- (7a-e) und VHL-adressierenden PROTACs (8aj) deuteten auf eine hohe Affinität hin, während die MDM2-adressierenden PROTACs (9a-c) beinahe komplett ihre Affinität verloren. Orthogonale ITC Messungen ausgewählter Cereblon- und VHLaddressierenden PROTACs zeigten zweistellig nanomolare Affinitäten der heterobifunktionellen Moleküle. Die zelluläre Permeabilität der großen Moleküle wurde in einem hier etablierten BRET Assay untersucht. Zur Assay-Etablierung wurden drei Tracer (21a-c), basierend auf BODIPY Konjugaten, synthetisiert und getestet, bevor die PROTACs in intakten und lysierten Zellen vermessen wurden. Während die zellulären Affinitäten von Cereblon- und VHL-addressierenden PROTACs sich im niedrigen $\mu \mathrm{M}$ Bereich bewegten, wurden die nicht zellgänigen MDM2 PROTACs von weiteren Experimenten ausgeschlossen. Die hierin erzielten Resultate aus den durchgeführten Assays zeigen somit das Potenzial der Cereblon- und VHL-addressierenden PROTACs.

Die Degradierungeffizienz der WDR5 PROTACs (7a-e) und (8a-j) wurden in der Leukämie Zellinie MV411 untersucht, da diese die am meisten auftretende MLL-fusion Mutation AF4 birgt. Dabei wurde in zwei orthogonalen Assaysystemen der Proteinabbau von WDR5 nachgewiesen: sowohl der für das WDR5 Protein neu etablierte HiBiT Assay als auch die Western Blots quantifizierten den Abbau des WDR5 Proteins. Der beobachtete Abbau war bei einem PROTAC mit dem Pyrroloimidazol Grundgerüst (20b), sowie bei sechs der OICR-9429-basierten PROTACs (8e-j) erfolgreich. Bei beiden PROTAC Typen 
konnten die heterobifunktionellen Moleküle, die die VHL E3 Ligase addressierten, einen endogenen WDR5 Abbau hervorrufen. Die chemische Struktur des Linkers der verwendeten PROTACs steuerte die Effizienz des Abbaus: das über eine Butyl-Kette verknüpfte Molekül (8g) erzielte dabei die höchste WDR5 Degradierungseffizient von insgesamt 58\%. Zwei Negativkontrollen (22 und 23) wurden zusätzlich synthetisiert und anschließend vermessen, um die Effektivität von (8g) zu bestätigen.

Weitere Kontrollexperimente zeigten, dass der Abbau des WDR5 Proteins durch die heterobifunktionellen Moleküle stammte. Der Cycloheximide chase assay zeigte einen signifkanten Unterschied in der Proteinstabilität in den mit PROTAC (8g) behandelten Zellen.. Um einen Effekt auf die WDR5 Transkription auszuschließen, wurden die WDR5 mRNA Level in einer quantitative Polymerase-Kettenreaktion (qPCR) bestimmt. In einem dritten Experiment wurde über Westernblots gezeigt, dass der PROTAC (8g) verantwortlich für den proteasomalen Abbau des WDR5 Proteins ist: während Zellen, die mit dem VHL- oder dem WDR5-Liganden keinerlei Änderung der WDR5 Level aufwiesen, konnte bei PROTAC-behandelten Zellen eine verminderte Proteinkonzentration detektiert werden. Weiterhin blieben die zellulären WDR5 Level erhalten, wenn neben PROTAC-Behandlung ein Überschuss an WDR5 Ligand (6d) hinzugegeben wurde. Die durchgeführten Experimente belegen, dass die hier synthesierten PROTACs verantwortlich für den WDR5 Abbau sind.

Die Selektivität des effizientesten WDR5 PROTACs (8g) wurde in proteomische Messungen verifiziert. Die beobachteten Effekte sind durch den unvollständigen WDR5 Abbau schwach ausgeprägt, jedoch ist aus den Experimenten ersichtlich, dass nur das WDR5 Protein selektiv und in signifikanten Mengen abgebaut wird.

Da die hier generierten PROTACs das WDR5 Protein unvollständig abbauten, wurden Modelling- sowie Kristallisationsstudien zur möglichen Verbsserung des Moleküls (8g) durchgeführt. Die Bildung und Stabilität des ternären Komplexes zwischen Zielprotein, PROTAC und E3 Ligase ist hierbei essentiell für den Erfolg des Proteinabbaus, sodass vor allem nach Möglichkeiten gesucht wurden, den ternären Komplex zwischen WDR5, VHL und (8g) zu stabilisieren. Die Daten des Modellings stützten sich auf die Annahme der Kooperativität, die von einer Stabilisierung des Komplex durch zusätzliche ProteinProtein-Interaktionen ausgeht, während die erhaltene Komplexstruktur nur geringe Interaktionen zwischen beiden Proteinen zeigte. Die geringen PPI beschränken die Möglichkeiten für eine chemische Optimierung des PROTACs und künftige Arbeiten zielen vermehrt auf eine Verbesserung der Löslichkeit und Affinität der heterobifunktionellen Moleküle ab.

In weiterführenden biologischen Experimenten konnte gezeigt werden, dass die Degradierung aufgrund der VHL E3 Ligase Expressionslevel in den AF4 Krebszellen limitiert ist: eine Überexpression von VHL Protein steigerte hierbei den WDR5 Abbau. ${ }^{174}$ Dies bedeutet, dass für zukünftige 
Untersuchungen die VHL Level berücksichtigt werden müssen um den WDR5 Abbau und seine biologischen Auswirkungen noch genauere Erforschungen braucht.

Zusammenfassend wurden in dieser Arbeit eine Reihe von PROTACs für das WDR5 Protein entwickelt, die erst in biophysikalischen Assays und anschließend in biologischen Systemen charakterisiert wurden. Diese Studie zeigt die Abhängigkeit zwischen Linkerlänge und Degradierungeffizienz auf, sowie die Notwendigkeit, PROTACs mit verschiedenen Grundgerüsten, Linkern und E3 Ligase Liganden zu synthetisieren. Die umfassende Charakterisierung der PROTACs auf chemischen und biologischem Weg ermöglichte es, PROTAC (8g) unter dem Namen Homer (https://www.thesgc.org/chemicalprobes/homer) im Rahmen des donated chemical probes Portals zu veröffentlichen, um möglichst weitreichende Untersuchungen der unzähligen WDR5 Funktionen zu ermöglichen. 
76 Deutsche Zusammenfassung 


\section{Experimental part}

\subsection{General and measuring instruments}

\section{General}

Unless otherwise stated, all reactions in this work were carried out at $25^{\circ} \mathrm{C}$ under an argon atmosphere. Distillations marked "removed under reduced pressure/in vacuo" were carried out with a rotary evaporator under membrane pump vacuum at $40{ }^{\circ} \mathrm{C}$. Drying of the substances, for solids and high-boiling liquids, was performed in the high vacuum of an oil pump or in a vacuum drying oven at $40{ }^{\circ} \mathrm{C}$. The molecular weights in brackets () refer to the natural isotope distribution. The numerical values in square brackets [] refer to the masses of the most common isotopes ${ }^{1} \mathrm{H},{ }^{12} \mathrm{C},{ }^{14} \mathrm{~N},{ }^{16} \mathrm{O},{ }^{23} \mathrm{Na}$ and ${ }^{32} \mathrm{~S}$.

\section{Chemicals and solvents}

All chemicals and solvents used, were purchased from commercial suppliers as Abcr, Acros Organics, Alfa Aesar, ChemPur, Carl Roth, Fischer Scientific, Fluka, Fluorochem UK, Merck, Santa Cruz Biotechnology, Sigma Aldrich, TCI Europe and VWR International. The solvents were used in different degrees of purity: per analysi (p.a.), HPLC-grade and analytical grade. Unless otherwise indicated, all chemicals and solvents were used for synthesis without further purification. Deuterated solvents for NMR measurements were purchased from Eurisotop, France.

\section{Chromatographic methods}

\section{Thin-layer chromatography (TLC)}

For analytical thin-layer chromatography, silica gel coated aluminum plates with fluorescence indicator from Merck KGaA (TLC silica gel 60 F254 plates) were used. The chromatographic zones were detected with UV light of the wavelength $\lambda=254 \mathrm{~nm}$ (fluorescence quenching) and the wavelength $\lambda=365 \mathrm{~nm}$ (autofluorescence). In addition, $p$-anisaldehyde, cerium molybdate, 2,4-dinitrophenylhydrazine (DNP) and ninhydrin solution were used as staining reagents for the detection:

- $p$-Anisaldehyde solution: $3.7 \mathrm{~mL} p$-Anisaldehyde, $5 \mathrm{~mL}$ concentrated sulfuric acid, $1.5 \mathrm{~mL}$ glacial acetic acid, $135 \mathrm{~mL}$ absolute EtOH.

- Dinitrophenylhydrazine (DNP) solution: $12 \mathrm{~g}$ 2,4-Dinitrophenylhydrazine, $60 \mathrm{~mL}$ concentrated sulfuric acid and $80 \mathrm{~mL} \mathrm{H} \mathrm{H}_{2} \mathrm{O}$ in $200 \mathrm{~mL}$ of $95 \% \mathrm{EtOH}$.

- Ninhydrin solution: $1.5 \mathrm{~g}$ Ninhydrin, $15 \mathrm{~mL}$ glacial acetic acid, $500 \mathrm{~mL} \mathrm{MeOH}$. 
- Cerium molybdate solution: $12 \mathrm{~g}$ Ammonium molybdate, $0.5 \mathrm{~g}$ ceric ammonium molybdate, and $15 \mathrm{~mL}$ concentrated sulfuric acid in $235 \mathrm{~mL}$ of $\mathrm{H}_{2} \mathrm{O}$. TLC plates were immersed in the respective solution and then developed under the action of heat using a heat gun. The solvent mixtures specified correspond to the volume ratios.

\section{Preparative Column Chromatography}

Column chromatographic purifications were performed on silica gel with a particle size of 0.040-0.063 $\mathrm{mm}$ from Macherey-Nagel, unless otherwise specified. Flash chromatographic purifications were carried out with a PuriFlash XS system from Interchim and with silica columns of $15 \mu \mathrm{m}, 30 \mu \mathrm{m}$ and 50 $\mu \mathrm{m}$ particle size or RP C18 columns. Solvents were used in HPLC grade or technical grade purity. The noted solvent mixtures correspond to volume ratios.

Product purification was also performed on an Agilent 1260 Infinity II LC System [Eclipse XDB-C18 column $(7 \mu \mathrm{M}, 21.2 \times 250 \mathrm{~mm})]$ using a gradient of water/MeCN $+0.1 \%$ TFA (98:2-5:95) over $40 \mathrm{~min}$ with a flow rate of $21 \mathrm{~mL} / \mathrm{min}$.

\section{High Performance Liquid Chromatography (HPLC)}

The purity of the final compounds was determined to $>95 \%$ using an HPLC system an Agilent 1260 Infinity II LC System [Eclipse XDB-C18 column $(4.6 \times 250 \mathrm{~mm}, 5 \mu \mathrm{m})$ ] coupled to an Agilent InifinityLab LC/MSD using a gradient of water/MeCN $+0.1 \%$ TFA (98:2-5:95) over $25 \mathrm{~min}$ at a flow rate of $1 \mathrm{~mL} / \mathrm{min}$.

\section{NMR spectroscopy}

All samples were dissolved in deuterated solvent and measured on one of the following devices:

- Bruker DPX 250: $250 \mathrm{MHz}{ }^{1} \mathrm{H}-\mathrm{NMR}$

- Bruker Avance 300: $300 \mathrm{MHz}{ }^{1} \mathrm{H}-\mathrm{NMR}, 75 \mathrm{MHz}^{13} \mathrm{C}-\mathrm{NMR}$

- Bruker Avance 400: $400 \mathrm{MHz}{ }^{1} \mathrm{H}-\mathrm{NMR}, 101 \mathrm{MHz}{ }^{13} \mathrm{C}-\mathrm{NMR}$

- Bruker Avance 500: $500 \mathrm{MHz}{ }^{1} \mathrm{H}-\mathrm{NMR}, 126 \mathrm{MHz}{ }^{13} \mathrm{C}-\mathrm{NMR}$

- Bruker DRX 600: $600 \mathrm{MHz}{ }^{1} \mathrm{H}-\mathrm{NMR}, 150 \mathrm{MHz}{ }^{13} \mathrm{C}-\mathrm{NMR}$

- Bruker Avance 700: $700 \mathrm{MHz}{ }^{1} \mathrm{H}-\mathrm{NMR}, 176 \mathrm{MHz}{ }^{13} \mathrm{C}-\mathrm{NMR}$

- Bruker Avance 800: $800 \mathrm{MHz}{ }^{1} \mathrm{H}-\mathrm{NMR}, 201 \mathrm{MHz}{ }^{13} \mathrm{C}-\mathrm{NMR}$

The values given for the chemical shift $(\delta)$ refer to the signal of the particular deuterated solvent used in reference to the tetramethylsilane standard at $\delta=0 \mathrm{ppm}$.

- $\mathrm{CD}_{2} \mathrm{Cl}_{2}: \delta\left({ }^{1} \mathrm{H}\right)=5.32 \mathrm{ppm}, \delta\left({ }^{13} \mathrm{C}\right)=54.00 \mathrm{ppm}$

- $\mathrm{CDCl}_{3}: \delta\left({ }^{1} \mathrm{H}\right)=7.26 \mathrm{ppm}, \delta\left({ }^{13} \mathrm{C}\right)=77.16 \mathrm{ppm}$ 
- $\mathrm{CD}_{3} \mathrm{OD}: \delta\left({ }^{1} \mathrm{H}\right)=3.31 \mathrm{ppm}, \delta\left({ }^{13} \mathrm{C}\right)=49.00 \mathrm{ppm}$

- $\left(\mathrm{CD}_{3}\right)_{2} \mathrm{CO}: \delta\left({ }^{1} \mathrm{H}\right)=2.05 \mathrm{ppm}, \delta\left({ }^{13} \mathrm{C}\right)=29.84 \mathrm{ppm}$

- $\mathrm{D}_{2} \mathrm{O}: \delta\left({ }^{1} \mathrm{H}\right)=4.79 \mathrm{ppm}$

- DMSO-d6: $\delta\left({ }^{1} \mathrm{H}\right)=2.50 \mathrm{ppm}, \delta\left({ }^{13} \mathrm{C}\right)=39.52 \mathrm{ppm}$

${ }^{1} \mathrm{H}$-broadband decoupling was performed on the ${ }^{13} \mathrm{C}$-NMR-spectra. The obtained spectra were either evaluated with the software TopSpin 3.2 from Bruker or MestReNova from Mestrelab Research SL.

\section{Mass spectrometry}

Mass spectrometry was measured as a service at the Faculty of Biochemistry, Chemistry and Pharmacy at Goethe University, D-60438 Frankfurt am Main, Germany.

- High resolution mass spectrometry (FTMS + p MALDI-HRMS) was performed using a MALDI LTQ XL Orbitrap spectrometer from Thermo Scientific. The samples were measured without solvent in a HCCA matrix.

- Mass spectrometry (ESI) was measured in solution on a Surveyor MSQ spectrometer from ThermoFisher or directly from TLC using TLC-MS interface 2 from Camag.

- Mass spectrometry ( $p$ MALDI) was measured without solvent in a HCCA matrix on a VoyagerDE STR spectrometer from Perseptive Biosystems (today: Applied Biosystems).

\subsection{Biological methods}

\section{Protein Expression and purification}

Plasmids of WDR5A (aa 1-334 and aa 33-334) were a kind gift of M. Vedadi from the SGC Toronto. Expression and purification were performed in collaboration with A. Krämer from the Structural Genomics Consortium (SGC), Buchmann Institute for Life Sciences, Max-von-Laue-Str. 15, D60438 Frankfurt am Main, Germany. Briefly, WDR5 was overexpressed in E. coli BL21 using TB media. Protein expression was induced by addition of $0.5 \mathrm{mM} \mathrm{IPTG}$ and cells were grown overnight at $18^{\circ} \mathrm{C}$. Next morning, the cells were harvested and resuspended in Lysis buffer (50 mM HEPES buffer, pH 7.5, $500 \mathrm{mM} \mathrm{NaCl}, 20 \mathrm{mM}$ imidazole, $0.5 \mathrm{mM}$ TCEP and 5\% glycerol). For purification, the cells were lysed by sonication and centrifuged. The supernatant was loaded onto a Nickel-Sepharose column that had been equilibrated with $30 \mathrm{~mL}$ lysis buffer. The column was washed with $100 \mathrm{~mL}$ lysis buffer. WDR5 was eluted by an imidazole step gradient (50, 100, 200, 300 mM). Fractions containing WDR5 were pooled together, concentrated, and loaded onto a Superdex 200 16/60 HiLoad gel filtration column 
equilibrated with final buffer ( $25 \mathrm{mM}$ HEPES pH 7.5, $300 \mathrm{mM} \mathrm{NaCl}$ and $0.5 \mathrm{mM}$ TCEP). The protein was concentrated to approx. $400 \mu \mathrm{M}$. The buffer was kept and used for ITC experiments.

\section{DSF}

Ligand binding to WDR5 protein was detected on an MX3005P qPCR system from Agilent Technologies as described elsewhere. ${ }^{190}$ The measurements were assisted by A. Krämer from the Structural Genomics Consortium (SGC), Buchmann Institute for Life Sciences, Max-von-Laue-Str. 15, D-60438 Frankfurt am Main, Germany, who also provided the proteins. Briefly, protein was buffered in $25 \mathrm{mM}$ HEPES ( $\mathrm{pH}$ 7.5), $500 \mathrm{mM} \mathrm{NaCl}, 0.5 \mathrm{mM}$ TCEP and diluted to a final concentration of $2 \mu \mathrm{M}$, and the fluorescent dye SYPRO Orange was added at a dilution of 1:1000. Compounds were dissolved in DMSO $(10 \mathrm{mM})$ and added at a final concentration of $10 \mu \mathrm{M}$ to $20 \mu \mathrm{L}$ of protein-dye mix in a 96-well plate. Real-time melting curves were then recorded by heating the samples from 25 to $96^{\circ} \mathrm{C}$ in 71 cycles (heating rate of $270 \mathrm{~K} / \mathrm{h}$, excitation/emission filters $=492 / 610 \mathrm{~nm}$ ), and the melting point, $\mathrm{T}_{\mathrm{m}}$, was calculated using the Boltzmann equation.

\section{ITC}

Binding constant $\left(\mathrm{K}_{\mathrm{d}}\right)$, stoichiometry $(\mathrm{n})$ and thermodynamic binding parameters $(\Delta \mathrm{H}, \Delta \mathrm{S}$ and $\Delta \mathrm{G})$ of ligand-protein interactions were determined on a Nano-ITC from TA Instruments as described elsewhere. ${ }^{191}$ The measurements were assisted by A. Krämer from the Structural Genomics Consortium (SGC), Buchmann Institute for Life Sciences, Max-von-Laue-Str. 15, D-60438 Frankfurt am Main, Germany., who also provided the protein. Briefly, compounds were diluted to a final concentration of $10-25 \mu \mathrm{M}$ in buffer and placed into the sample cell. Proteins (80-120 $\mu \mathrm{M})$ were added using an initial injection of 3 or $4 \mu \mathrm{L}$, followed by $12-30$ injections of 6 or $8 \mu \mathrm{L}$ at $22^{\circ} \mathrm{C}$. Collected data were corrected by subtraction of pure DMSO injection heats. Data were analyzed by assuming a sigmoidal dose-response relationship (four parameters). Errors of fits were calculated using standard deviation and a confidence interval of $68 \%$ in GraphPad Prism.

\section{NanoBRET ${ }^{\mathrm{TM}}$}

The assay originally developed by Promega was performed in HEK293T cells by L. Berger from the Structural Genomics Consortium (SGC), Buchmann Institute for Life Sciences, Max-von-Laue-Str. 15, D60438 Frankfurt am Main, Germany, as described previously. ${ }^{174}$ Briefly, Full-length WDR5 was obtained as plasmids cloned in frame with a N- or C-terminal NanoLuc-fusion pNLF1 vector (Collaboration with SGC Toronto). Plasmids were transfected into HEK293T cells using FuGENE HD (Promega, E2312) and proteins were allowed to express for $20 \mathrm{~h}$. Serially diluted inhibitor and Tracer molecule (21c) at a concentration of $1 \mu \mathrm{M}$ determined previously as the Tracer (21c) $K_{D, \text { app }}$ were pipetted into white 384- 
well plates (Greiner 781 207) using an Echo acoustic dispenser (Labcyte). The corresponding proteintransfected cells were added and reseeded at a density of $2 \times 10^{5}$ cells $/ \mathrm{ml}$ after trypsinization and resuspending in Opti-MEM without phenol red (Life Technologies). The system was allowed to equilibrate for 2 hours at $37{ }^{\circ} \mathrm{C} / 5 \% \mathrm{CO}_{2}$ prior to BRET measurements. To measure BRET, NanoBRET ${ }^{\mathrm{TM}}$ NanoGlo Substrate and Extracellular NanoLuc Inhibitor (Promega, N2540) was added as per the manufacturer's protocol, and filtered luminescence was measured on a PHERAstar plate reader (BMG Labtech) equipped with a luminescence filter pair (450 nm BP filter (donor) and $610 \mathrm{~nm}$ LP filter (acceptor). Competitive displacement data were then graphed using GraphPad Prism 8 software using a normalized 3-parameter curve fit with the following equation: $y=\frac{100}{1+10^{x-\log \left(I C_{50}\right)}}$.

\section{Crystallization and structure determination}

Crystallization and structure determination of the compound synthesized in this work in complex with WDR5 and VHL were performed in collaboration with A. Krämer and M. Schwalm from the Structural Genomics Consortium (SGC), Buchmann Institute for Life Sciences, Max-von-Laue-Str. 15, D60438 Frankfurt am Main, Germany. The VHL plasmid was a kind gift of A. Ciulli from the University of Dundee. Protein expression and purification were performed by A. Krämer from the Structural Genomics Consortium (SGC), Buchmann Institute for Life Sciences, Max-von-Laue-Str. 15, D60438 Frankfurt am Main, Germany.

\section{Immunoblotting}

All procedures were performed by B. Adhikari from the University of Würzburg, Theodor-BoveriInstitut, Am Hubland, D-97974 Würzburg, Germany. ${ }^{174}$

Cell culture. Human MV4-11 (male) and human HL-60 (female) cells were cultured in RPMI-1640, whereas human HEK293 (female) cells were cultured in DMEM at $37{ }^{\circ} \mathrm{C}$ in $5 \% \mathrm{CO}_{2}$. Both media were supplemented with $10 \%$ FBS and $1 \%$ penicillin/streptomycin solution.

Immunoblotting. After compound treatment, cells were lysed in RIPA lysis buffer (50 mM HEPES pH 7.9, $140 \mathrm{mM} \mathrm{NaCl}, 1 \mathrm{mM}$ EDTA, 1\% Triton X-100, 0.1\% SDS, $0.1 \%$ sodium deoxycholate) and supplemented with protease and phosphatase inhibitors for $20 \mathrm{~min}$ at $4{ }^{\circ} \mathrm{C}$ head-over-tail. Supernatant had been collected after centrifugation. Bicinchoninic acid (BCA) assay was used for protein quantification. Equal amounts of protein were separated by Bis-Tris-PAGE and transferred to PVDF membranes (Millipore). The membranes were incubated with $5 \%(\mathrm{w} / \mathrm{v})$ non-fat dry milk in TBS-T (20 mM Tris- $\mathrm{HCl}, \mathrm{pH}$ 7.5, $150 \mathrm{mM} \mathrm{NaCl}, 0.1 \%(\mathrm{v} / \mathrm{v})$ Tween-20 for $1 \mathrm{~h}$ at room temperature for blocking and then incubated with the primary antibody overnight at $4{ }^{\circ} \mathrm{C}$. For visualization, horseradish peroxidase (HRP)-labelled secondary antibodies were used and detected using chemiluminescent HRP substrate (Millipore) in LAS4000 Mini (Fuji). The signal was quantified using ImageJ or Image Studio 
Lite (LI-COR Biosciences). Vinculin was used as a loading control. Antibodies used in this study were: WDR5 (Santa Cruz Biotechnology; sc-393080) and vinculin (Sigma; V9131).

\section{HiBit assay}

All procedures were performed by B. Adhikari from the University of Würzburg, Theodor-BoveriInstitut, Am Hubland, D-97974 Würzburg, Germany. ${ }^{174}$

Cloning. WDR5-HiBiT was cloned by PCR amplification of vector containing full length WDR5 using forward primer: CGCACCGGTATGGCGACGGAGGAGAAGAAGC and reverse primer: CGCGACGCGTTTAGCTAATCTTCTTGAACAGCCGCCAGCCGCTCACACCGGAGCTCCCGCAGTCACTCTTCCAC AGT. The PCR product was inserted into PRRL-PGK vector using Agel/Mlul restriction sites. HA-tagged VHL was cloned by PCR amplification of cDNA from MV4-11 cells as template (forward primer: CGCACCGGTATGTACCCTTACGACGTGCCCGACTACGCCGGGAGCTCCGGTCCCCGGAGGGCGGAGAAC and reverse primer: GGACTAGTTCAATCTCCCATCCGTTGATGTG) and inserted into pRRL-SFFV vector using Agel/Spel sites. The sequence of the cloned VHL was identified as isoform 1 by sanger sequencing.

MV4-11 ${ }^{\text {WDR5-HiBiT }}$ cell line generation. Lentiviral infection was used to generate stable MV4-11 ${ }^{\text {WDR5-HiBiT, }}$ MV4-11 ${ }^{\mathrm{VHL}}$ and MV4-11 $1^{\text {WDR5-HiBiT/NHL }}$ cells. Lentivirus was produced using plasmids psPAX2, PMD2.G and HiBiT-WDR5 or HA-VHL plasmid in HEK293 cells. MV4-11 cells were infected with filtered virus supernatant and selected after $72 \mathrm{~h}$ of infection for generation of MV4-11 $1^{\text {WDR5-HiBiT }}$ and MV4-11 ${ }^{\mathrm{VHL}}$ cells. MV4-11 ${ }^{\text {WDR5-HiBiT }}$ cells were used to prepare MV4-11 ${ }^{\text {WDR5-HiBiT/NHL }}$ cells.

HiBiT assay. HiBiT assay was performed as described previously. MV4-11 $1^{\text {WDR5-HiBiT }}$ or MV4-11 ${ }^{\text {WDR5-HiBiT/VHL }}$ cells were seeded and treated with serial dilutions of compounds for $6 \mathrm{~h}$ or $24 \mathrm{~h}$. Nano-Glo HiBiT Lytic Detection System (Promega) was used for the assay. Luminescence was measured on a GloMax 96 Microplate Luminometer (Promega). DC 50 was calculated using lower concentrations (showing sigmoidal behaviour) with the dose-response (four parameters) equation.

\section{Cycloheximide Chase Assay}

All procedures were performed by Bikash Adhikari from the University of Würzburg, Theodor-BoveriInstitut, Am Hubland, D-97974 Würzburg, Germany. ${ }^{174}$

Cycloheximide chase assay was performed as described previously. MV4-11 cells were treated with 50 $\mu \mathrm{g} / \mathrm{ml} \mathrm{CHX}$ with or without PROTACs for different time points. The cells were harvested in RIPA buffer and probed for immunoblotting. The intensity of WDR5 band at $0 \mathrm{~h}$ was set as 1 . The mean \pm SD from $\mathrm{n}=2$ biological experiments were plotted as $\log _{10}$ values. 


\section{Quantitative PCR (qPCR)}

All procedures were performed by B. Adhikari from the University of Würzburg, Theodor-BoveriInstitut, Am Hubland, D-97974 Würzburg, Germany. ${ }^{174}$

RT-qPCR. RT-qPCR was performed as described previously. Total RNA was extracted using peqGOLD TriFast (Peqlab). cDNA synthesis was carried out and cDNA were analysed by qPCR on a StepOnePlus Real-Time PCR System (Thermo Fisher Scientific) using the SYBR Green Master Mix (Thermo Fisher Scientific). Equal amounts of CDNA and SYBR Green Master Mix were added along with WDR5 primers (forward: CCAGTCTCGGCCGTTCATTT, reverse: CGTTCGGGGAGAACTTCACA). For analysis, expression was normalized to $\beta 2$-microglobulin expression. qPCR was done in technical triplicates.

\section{Quantitative Proteomics}

All procedures were performed by B. Adhikari from the University of Würzburg, Theodor-BoveriInstitut, Am Hubland, D-97974 Würzburg, Germany, and Nicola Berner from the Technical University of Munich, 85354 Freising, Germany. ${ }^{174}$

4 million MV4-11 cells (in $10 \mathrm{ml}$ ) were seeded at least in triplicates for each treatment evening before the treatment. Cells were treated for $9 \mathrm{~h}$ with either $1 \mu \mathrm{M}(\mathbf{6 d}), 1 \mu \mathrm{M}(\mathbf{8 g}), 5 \mu \mathrm{M}(\mathbf{2 0 b}), 5 \mu \mathrm{M}(\mathbf{1 7})$ or DMSO. After treatment, cells were washed twice with ice cold PBS, supplemented with protease and phosphatase inhibitor, and lysed in SDS lysis buffer (2\% SDS in $40 \mathrm{mM}$ Tris- $\mathrm{HCl}, \mathrm{pH} 7.6)$. In order to reduce viscosity, the sample was sonicated using a sonication water bath, boiled at $95{ }^{\circ} \mathrm{C}$ for $10 \mathrm{~min}$ and trifluoroacetic acid was added to a final concentration of $1 \%$. To neutralize the sample (final $\mathrm{pH}$ 7.6-8.0), $300 \mathrm{mM} \mathrm{N}$-methylmorpholin was added to a final concentration of $2 \%$. The protein concentration in cell lysate was determined using the Pierce ${ }^{\mathrm{TM}}$ BCA Protein Assay Kit (ThermoScientific) according to the protocol of the manufacturer. The beads suspension for sp3 sample workup was prepared by mixing magnetic SeraMag-A and SeraMag-B beads ( $10 \mu$ l per sample of each type; Cytiva) in a ratio of $1: 1$, washing them three times with $\mathrm{ddH}_{2} \mathrm{O}$ and resuspending them in $10 \mu \mathrm{L} d \mathrm{dH}_{2} \mathrm{O}$ per sample. A total of $200 \mu \mathrm{g}$ per sample was mixed with $10 \mu \mathrm{l}$ beads suspension. Acetonitrile was added to a final concentration of $70 \%$ and incubated at room temperature, $18 \mathrm{~min}, 800 \mathrm{rpm}$. After discarding the supernatant, beads were washed twice using $200 \mu \mathrm{l} 80 \%$ ethanol. For reduction and alkylation, beads were resuspended in $70 \mu \mathrm{l}$ of $2 \mathrm{mM} \mathrm{CaCl}_{2}$ in $40 \mathrm{mM}$ Tris pH 7.6. Proteins were reduced with $10 \mathrm{mM}$ dithiothreitol (DTT) for $45 \mathrm{~min}$ at $37{ }^{\circ} \mathrm{C}$ and $800 \mathrm{rpm}$, and alkylated with $55 \mathrm{mM}$ chloroacetamide (CAA) at room temperature in the dark for $30 \mathrm{~min}$. Proteins were digested (1:50 trypsin/substrate weight) overnight at $37^{\circ} \mathrm{C}$ and $1000 \mathrm{rpm}$. Samples were centrifuged (5 min, $20000 \mathrm{rcf}$ ), sonicated 3 times for $30 \mathrm{sec}$ and supernatant was collected. Beads were washed once with

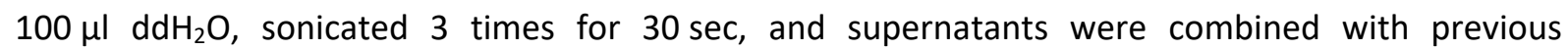
supernatants. Samples were acidified with formic acid (FA) to a final concentration of $1 \%$. Peptides 
were desalted using tC18 RP solid-phase extraction cartridges (Waters Corp.; wash solvent: 0.1\% FA; elution solvent: $0.1 \%$ FA in $50 \%$ acetonitrile (ACN). Samples were frozen at $-80^{\circ} \mathrm{C}$ freezer, dried in a SpeedVac, reconstituted in $0.1 \% \mathrm{FA}$ and peptide concentration was determined using a NanoDrop and stored at $-20{ }^{\circ} \mathrm{C}$ until LC-MS ${ }^{2}$ analysis.

A micro-flow LC-MSMS setup with a Q Exactive HF-X mass spectrometer (Thermo Fisher Scientific) was used as described in detail in previous publications. $50 \mu \mathrm{g}$ peptides dissolved in $0.1 \%$ FA were directly injected onto the microflow LC system. Online chromatography was performed using a commercially available Thermo Fisher Scientific Acclaim PepMap 100 C18 LC column ( $2 \mu \mathrm{m}$ particle size, $1 \mathrm{~mm}$ ID $\times 150 \mathrm{~mm}$; catalog number 164711). Column temperature was maintained at $55^{\circ} \mathrm{C}$ using the integrated column oven. Peptides were delivered at a flow rate of $50 \mu \mathrm{l} / \mathrm{min}$ and separated using a two-step linear gradient (120 $\mathrm{min}$ ) ranging from 1-24 \% (105 $\mathrm{min}$ ) and 24-35 \% (15 min) of LC solvent B (0.1 \% FA, 3 \% DMSO in ACN) in LC solvent A (0.1 \% FA, 3 \% DMSO. The Q Exactive HF-X was operated as follows: positive polarity; spray voltage $4 \mathrm{kV}$, capillary temperature $320^{\circ} \mathrm{C}$; vaporizer temperature $200{ }^{\circ} \mathrm{C}$. The flow rates of sheath gas, aux gas and sweep gas were set to 40,3 , and 0 , respectively. TopN was set to 50. Full MS was readout in the orbitrap, resolution was set to 120,000 and the mass range was set to 360-1300. Full MS AGC target value was $3 E 6$ with a maximum IT of $100 \mathrm{~ms}$ and RF lens value was set to 40 . Peptide match was set to preferred and default charge state was set to 2 . The dynamic exclusion duration was set to $40 \mathrm{~s}$ and exclude isotopes was switched on. For readout of MS2 spectra, orbitrap resolution was set to 15,000 and the mass range was set to 200-2000. The isolation width was set to $1.3 \mathrm{~m} / \mathrm{z}$, the first mass was fixed at $100 \mathrm{~m} / \mathrm{z}$, NCE was 28 . The AGC target value was set to $1 \mathrm{E} 5$ at a maximum IT of $22 \mathrm{~ms}$.

Protein and peptide identification and quantification was performed using MaxQuant (version 1.6.12.0) by searching the $\mathrm{MS}^{2}$ data against all canonical protein sequences as annotated in the UniProt reference database (human proteins only, downloaded 24.08.2020) using the search engine Andromeda. Carbamidomethylated cysteine was set as fixed modification; oxidation of methionine and $\mathrm{N}$-terminal protein acetylation were set as variable modification. Trypsin/P was specified as proteolytic enzyme and up to two missed cleavage sites were allowed. The minimum peptide length was set to seven and all data were adjusted to $1 \%$ peptide-spectrum-match (PSM) and $1 \%$ protein false discovery rate (FDR). LFQ based quantification was enabled including the match between runs option and without normalization.

Data analysis was performed using the Perseus software suite (version 1.6.14.0) and Microsoft Excel on identified and quantified protein groups as provided in the proteinGroups.txt file. Proteingroups.txt was filtered for contaminants and reverse hits, and median centric normalization and log2 transformation were performed. The pyrrolimidazole-based ligand (17) treated replicate 1 showed high differences to the other conditions and was not considered for further analysis. Entries were 
filtered for at least three valid values in one condition. Two-sample t-test were performed (s0:0.1, permutation-based FDR: 5\%, number of randomizations: 250). For principal component analysis (PCA) remaining missing values were replaced from normal distribution (width 0.3 , down shift: 1.8 ). The mass spectrometry proteomics data and complete MaxQuant search results have been deposited to the ProteomeXchange Consortium (http://www.proteomexchange.org/).

\subsection{Experimental part of chapter 3}

\subsubsection{Synthesis of WDR5 ligands}

Synthesis of 1-(4-bromo-2-nitrophenyl)-4-methylpiperazine (3)<smiles>CN1CCN(c2ccc(Br)cc2[N+](=O)[O-])CC1</smiles>

$5 \mathrm{~mL}$ (39.7 mmol, 1.00 eq) 5-Bromo-2-Fluoro-Nitrobenzene was dissolved in $20 \mathrm{~mL}$ EtOH. $4 \mathrm{~mL}$ (39.7 mmol, 1.00 eq) N-Methylpiperazine and $13.5 \mathrm{~mL}(79.4 \mathrm{mmol}, 2.00 \mathrm{eq})$ DIEA were added and the reaction mixture was stirred for $5 \mathrm{~h}$ at $80^{\circ} \mathrm{C}$. The reaction was cooled to $\mathrm{rt}$ and the solvent was removed under reduced pressure. The reaction mixture was diluted with water and extracted $8 \mathrm{x}$ with DCM. The combined organic phases were washed with $1 \mathrm{M} \mathrm{HCl}$, saturated $\mathrm{NaCl}$ solution, dried over $\mathrm{MgSO}_{4}$ and filtered. The crude product was purified via CC (gradient: $0 \%$ to $10 \% \mathrm{MeOH}$ in DCM). Other attempts of the synthesis of (3) are listed in Table 9.

Table 9: Synthesis of 1-(4-bromo-2-nitrophenyl)-4-methylpiperazine (3).

\begin{tabular}{|c|c|c|c|}
\hline $\begin{array}{c}\text { N- } \\
\text { Methylpiperazine }\end{array}$ & $\begin{array}{c}\text { 5-Bromo-2-Fluoro- } \\
\text { Nitrobenzene }\end{array}$ & DIEA & (3) \\
\hline $4 \mathrm{~mL}, 39.7 \mathrm{mmol}$, & $5 \mathrm{~mL}, 39.7 \mathrm{mmol}, 1.00$ & $13.5 \mathrm{~mL}, 79.4 \mathrm{mmol}$, & $11.1 \mathrm{~g}, 37 \mathrm{mmol}, 95$ \\
$1.00 \mathrm{eq}$ & eq & $2.00 \mathrm{eq}$ & $\%$ \\
\hline $4 \mathrm{~mL}, 39.7 \mathrm{mmol}$, & $5 \mathrm{~mL}, 39.7 \mathrm{mmol}, 1.00$ & $13.5 \mathrm{~mL}, 79.4 \mathrm{mmol}$, & $12.9 \mathrm{~g}, 38.7 \mathrm{mmol}$, \\
$1.00 \mathrm{eq}$ & eq & $2.00 \mathrm{eq}$ & $97 \%$. \\
\hline
\end{tabular}

Yield: $11.1 \mathrm{~g}, 37 \mathrm{mmol}, 95 \%$ of an orange powder. 
$\mathrm{R}_{\mathrm{f}}\left(5 \% \mathrm{MeOH} / \mathrm{CH}_{2} \mathrm{Cl}_{2}\right): 0.54$.

ESI: (calculated): $\left[\mathrm{M}+\mathrm{H}^{+}\right] 300.03 \mathrm{~g} / \mathrm{mol}$

(found): $\left[\mathrm{M}+\mathrm{H}^{+}\right] 299.98 \mathrm{~g} / \mathrm{mol}$.

${ }^{1} \mathrm{H} \mathrm{NMR}\left(250 \mathrm{MHz}, \mathrm{CDCl}_{3}\right) \delta=7.94\left(\mathrm{~d},{ }^{4} J=2.3 \mathrm{~Hz}, 1 \mathrm{H}\right), 7.65\left(\mathrm{dd},{ }^{3} J=8.7 \mathrm{~Hz},{ }^{4} J=2.4 \mathrm{~Hz}, 1 \mathrm{H}\right), 7.17\left(\mathrm{~d},{ }^{3} J=\right.$ $8.7 \mathrm{~Hz}, 1 \mathrm{H}), 3.43(\mathrm{~m}, 4 \mathrm{H}), 3.25(\mathrm{~m}, 4 \mathrm{H}), 2.80(\mathrm{~s}, 3 \mathrm{H}) \mathrm{ppm}$.

Synthesis of 5-bromo-2-(4-methylpiperazin-1-yl)aniline (4)<smiles>CN1CCN(c2ccc(Br)cc2N)CC1</smiles>

$2.00 \mathrm{~g}$ (7.40 mmol, $1.00 \mathrm{eq})$ 1-(4-bromo-2-nitrophenyl)-4-methylpiperazine $\mathbf{3}$ was suspended in a mixture of 1,4-Dioxane and water (3:1). $3.20 \mathrm{~g}$ (37 mmol, $7.50 \mathrm{eq)}$ Ammonium chloride was added, followed by a slow addition of $2.60 \mathrm{~g}(37 \mathrm{mmol}, 7.50 \mathrm{eq})$ Zinc dust. The reaction mixture was stirred until a colour change from orange to light pink was observed. The solvent was removed under reduced pressure. The reaction mixture was diluted with saturated $\mathrm{NaHCO}_{3}$ solution and extracted $6 \mathrm{x}$ with DCM. The combined organic phases were washed with saturated $\mathrm{NaCl}$ solution, dried over $\mathrm{MgSO}_{4}$, filtered and the solvent was removed under reduced pressure. Other attempts of the synthesis of (4) are listed in Table 10.

Table 10: Synthesis of 1-(4-bromo-2-nitrophenyl)-4-methylpiperazine (4).

\begin{tabular}{|c|c|c|c|}
\hline (3) & Ammonium chloride & Zinc dust & (4) \\
\hline $1.00 \mathrm{~g}, 3.34 \mathrm{mmol}$, & $1.40 \mathrm{~g}, 25.1 \mathrm{mmol}$, & $1.65 \mathrm{~g}, 25.1 \mathrm{mmol}$, & $733 \mathrm{mg}, 2.71 \mathrm{mmol}$, \\
$1.00 \mathrm{eq}$ & $7.50 \mathrm{eq}$ & $7.50 \mathrm{eq}$ & $81 \%$ \\
\hline $200 \mathrm{mg}, 0.67 \mathrm{mmol}$, & $269 \mathrm{mg}, 5.03 \mathrm{mmol}$, & $328 \mathrm{mg}, 5.03 \mathrm{mmol}$, & $145 \mathrm{mg}, 0.54 \mathrm{mmol}$, \\
$1.00 \mathrm{eq}$ & $7.50 \mathrm{eq}$ & $7.50 \mathrm{eq}$ & $80 \%$ \\
\hline $1.00 \mathrm{~g}, 3.34 \mathrm{mmol}$, & $1.40 \mathrm{~g}, 25.1 \mathrm{mmol}$, & $1.65 \mathrm{~g}, 25.1 \mathrm{mmol}$, & $841 \mathrm{mg}, 3.11 \mathrm{mmol}$, \\
$1.00 \mathrm{eq}$ & $7.50 \mathrm{eq}$ & $7.50 \mathrm{eq}$ & $93 \%$ \\
\hline $1.00 \mathrm{~g}, 3.34 \mathrm{mmol}$, & $1.40 \mathrm{~g}, 25.1 \mathrm{mmol}$, & $1.65 \mathrm{~g}, 25.1 \mathrm{mmol}$, & $752 \mathrm{mg}, 2.78 \mathrm{mmol}$, \\
$1.00 \mathrm{eq}$ & $7.50 \mathrm{eq}$ & $7.50 \mathrm{eq}$ & $83 \%$ \\
& & & \\
\hline
\end{tabular}


Yield: $1.57 \mathrm{mg}, 5.81 \mathrm{mmol}, 79 \%$ of a light pink solid.

$\mathrm{R}_{\mathrm{f}}\left(5 \% \mathrm{MeOH} / \mathrm{CH}_{2} \mathrm{Cl}_{2}\right): 0.3$.

ESI: (calculated): $\left[\mathrm{M}+\mathrm{H}^{+}\right] 270.06 \mathrm{~g} / \mathrm{mol}$

(found): $\left[\mathrm{M}+\mathrm{H}^{+}\right] 270.03 \mathrm{~g} / \mathrm{mol}$.

HPLC: RT = $10.9 \min (254 \mathrm{~nm}, 99 \%)$.

${ }^{1} \mathrm{H} \mathrm{NMR}\left(250 \mathrm{MHz}, \mathrm{CDCl}_{3}\right) \delta=6.96-6.70(\mathrm{~m}, 3 \mathrm{H}), 4.01(\mathrm{~s}, 2 \mathrm{H}), 2.92\left(\mathrm{t},{ }^{3} \mathrm{~J}=4.5 \mathrm{~Hz}, 4 \mathrm{H}\right), 2.60(\mathrm{~s}, 4 \mathrm{H}), 2.39$ $(\mathrm{s}, 3 \mathrm{H}) \mathrm{ppm}$.

Synthesis of tert-butyl 5-(3-amino-4-(4-methylpiperazin-1-yl)phenyl)-1H-indole-1-carboxylate (5a)<smiles>CN1CCN(c2ccc(-c3ccc4c(ccn4C(=O)OC(C)(C)C)c3)cc2N)CC1</smiles>

$1.58 \mathrm{~g}$ (4.42 mmol, $1.4 \mathrm{eq})$ 1-Boc-Indole-5-Boronic acid was dissolved in an Argon-purged solvent solution (1,4-Dioxane/ Water (3:1)) and $2.1 \mathrm{~g}(15.4 \mathrm{mmol}, 5 \mathrm{eq})$ potassium carbonate was added. The reaction mixture was stirred for $5 \mathrm{~min}$ at rt under Argon atmosphere, then $832 \mathrm{mg}$ ( $3.08 \mathrm{mmol}, 1 \mathrm{eq}$ ) 5-bromo-2-(4-methylpiperazin-1-yl)aniline and $262 \mathrm{mg}$ (0.31 mmol, $0.1 \mathrm{eq})$ XPhos Pd G3 were added. The reaction was stirred at $95{ }^{\circ} \mathrm{C}$ for $18 \mathrm{~h}$ under Argon atmosphere. The reaction mixture was cooled to rt, filtered over celite and washed with $\mathrm{MeOH}$. The solvent was removed under reduced pressure. The reaction mixture was diluted with water and extracted $3 x$ with DCM. The crude product was purified via FC (0\% to $10 \% \mathrm{MeOH} / \mathrm{DCM})$. Other attempts to synthesize (5b) are listed in Table $\mathbf{1 1 .}$ 
Table 11: Synthesis of tert-butyl 5-(3-amino-4-(4-methylpiperazin-1-yl)phenyl)-1H-indole-1-carboxylate (5b).

\begin{tabular}{|c|c|c|c|c|c|}
\hline $\begin{array}{c}\text { Boronic } \\
\text { acid }\end{array}$ & (4) & Base & $\begin{array}{c}\text { XPhos Pd } \\
\mathrm{G3}\end{array}$ & $\begin{array}{c}\text { Temperature/ } \\
\text { Heating time }\end{array}$ & \\
\hline $356 \mathrm{mg}$, & $200 \mathrm{mg}$, & $511 \mathrm{mg}$, & $62.6 \mathrm{mg}$, & $90{ }^{\circ} \mathrm{C} /$ & $160 \mathrm{mg}$, \\
$1.04 \mathrm{mmol}$, & $740 \mu \mathrm{mol}$, & $3.70 \mathrm{mmol}$, & $74 \mu \mathrm{mol}$, & $18 \mathrm{~h}$ & $393 \mu \mathrm{mol}$, \\
$1.4 \mathrm{eq}$ & $1 \mathrm{eq}$ & $5 \mathrm{eq} \mathrm{NaOH}$ & $0.1 \mathrm{eq}$ & & $53 \%$ \\
\hline
\end{tabular}

Yield: $814 \mathrm{mg}, 2.00 \mathrm{mmol}, 65 \%$ of a yellow solid.

$\mathrm{R}_{\mathrm{f}}\left(5 \% \mathrm{MeOH} / \mathrm{CH}_{2} \mathrm{Cl}_{2}\right): 0.13$.

ESI: (calculated): $\left[\mathrm{M}+\mathrm{H}^{+}\right] 407.24 \mathrm{~g} / \mathrm{mol}$

(found): $\left[\mathrm{M}+\mathrm{H}^{+}\right] 407.29 \mathrm{~g} / \mathrm{mol}$.

HPLC: RT = $12.5 \min (254 \mathrm{~nm}, 100 \%)$.

${ }^{1} \mathrm{H} \mathrm{NMR}\left(300 \mathrm{MHz}, \mathrm{CDCl}_{3}\right) \delta=8.16\left(\mathrm{~d},{ }^{3} \mathrm{~J}=8.6 \mathrm{~Hz}, 1 \mathrm{H}\right), 7.72(\mathrm{~s}, 1 \mathrm{H}), 7.61\left(\mathrm{~d},{ }^{3} \mathrm{~J}=3.5 \mathrm{~Hz}, 1 \mathrm{H}\right), 7.52\left(\mathrm{dd},{ }^{3} \mathrm{~J}\right.$ $\left.=8.7,{ }^{4} \mathrm{~J}=1.3 \mathrm{~Hz}, 1 \mathrm{H}\right), 7.14-6.98(\mathrm{~m}, 3 \mathrm{H}), 6.59\left(\mathrm{~d},{ }^{3} \mathrm{~J}=3.6 \mathrm{~Hz}, 1 \mathrm{H}\right), 4.07(\mathrm{~s}, 2 \mathrm{H}), 3.02(\mathrm{~s}, 4 \mathrm{H}), 2.62(\mathrm{~s}, 4 \mathrm{H})$, $2.39(\mathrm{~s}, 3 \mathrm{H}), 1.69(\mathrm{~s}, 9 \mathrm{H}) \mathrm{ppm}$.

\section{Synthesis of N-(5-bromo-2-(4-methylpiperazin-1-yl)phenyl)-3-methylbenzamide}<smiles>Cc1cccc(C(=O)Nc2cc(Br)ccc2N2CCN(C)CC2)c1</smiles>

$630 \mathrm{mg}$ (4.63 mmol, 1 eq) 2-Methylbenzoic acid and $2.64 \mathrm{mg}$ (6.94 mmol, $1.5 \mathrm{eq})$ HATU were dissolved in $1 \mathrm{~mL}$ DMF. $1.6 \mathrm{~mL}$ (9.25 mmol, 2 eq) DIEA was added. The mixture was stirred for $5 \mathrm{~min}$, then $1.50 \mathrm{~g}$ (5.55 mmol, 1 eq) 5-bromo-2-(4-methylpiperazin-1-yl)aniline were added. The solution was stirred at rt for $40 \mathrm{~h}$. The reaction was stopped with $1 \mathrm{~mL}$ water. Saturated $\mathrm{NaHCO}_{3}$ solution and saturated $\mathrm{NaCl}$ solution were added and the reaction mixture was extracted $4 \mathrm{x}$ with EA. The combined organic phases were washed with saturated $\mathrm{NaHCO}_{3}$ solution, dried over $\mathrm{MgSO}_{4}$ and filtered. The solvent of the 
organic phase was evapored under reduced pressure. The purification of the crude product was carried out on the $\mathrm{FC}$ system. The evapored fraction was dissolved in $2 \mathrm{M} \mathrm{HCl}$ in diethyl ether.

Yield: $687 \mathrm{mg}, 2.03 \mathrm{mmol}, 44 \%$ of a white solid.

$\mathrm{R}_{\mathrm{f}}\left(1 \% \mathrm{MeOH} / \mathrm{CH}_{2} \mathrm{Cl}_{2}\right): 0.12$.

ESI: (calculated): $\left[\mathrm{M}+\mathrm{H}^{+}\right] 388.10 \mathrm{~g} / \mathrm{mol}$ (found): $\left[\mathrm{M}+\mathrm{H}^{+}\right] 388.09 \mathrm{~g} / \mathrm{mol}$.

${ }^{1} \mathrm{H}$ NMR (500 MHz, DMSO) $\delta=10.46\left(\mathrm{~s}, 1 \mathrm{H}, \mathrm{H}(\mathrm{Cl})\right.$ salt), $9.49(\mathrm{~s}, 1 \mathrm{H}, \mathrm{NH}), 8.32\left(\mathrm{~d},{ }^{3} \mathrm{~J}=2.4 \mathrm{~Hz}, 1 \mathrm{H}\right), 7.75$ $(\mathrm{m}, 2 \mathrm{H}), 7.46(\mathrm{~m}, 2 \mathrm{H}), 7.37\left(\mathrm{dd},{ }^{3} \mathrm{~J}=8.5,{ }^{4} \mathrm{~J}=2.4 \mathrm{~Hz}, 1 \mathrm{H}\right), 7.24\left(\mathrm{~d},{ }^{3} \mathrm{~J}=8.6 \mathrm{~Hz}, 1 \mathrm{H}\right), 3.52(\mathrm{~m}, 2 \mathrm{H}), 3.28-$ $3.02(\mathrm{~m}, 6 \mathrm{H}), 2.85(\mathrm{~s}, 3 \mathrm{H}), 2.42(\mathrm{~s}, 3 \mathrm{H}) \mathrm{ppm}$.

${ }^{13} \mathrm{C}$ NMR (126 MHz, DMSO) $\delta=165.2,141.5,138.2,134.4,134.1,132.7,128.8,128.2,127.5,124.6$, $124.4,123.0,117.0,53.0,48.2,39.5,30.7,21.0 \mathrm{ppm}$.

\section{Synthesis of tert-butyl 5-(3-(3-methylbenzamido)-4-(piperazin-1-yl)phenyl)-1H-indole-1-carboxylate} (6a)

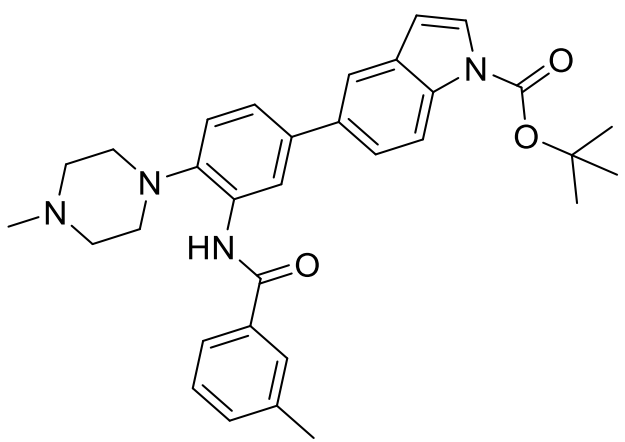

$318 \mathrm{mg}$, (0.82 mmol, 1 eq) of N-(5-bromo-2-(4-methylpiperazin-1-yl)phenyl)-3-methylbenzamide were suspended in $20 \mathrm{~mL}$ of an Argon purged Toluene/ Water mixture (3/1) and $364 \mathrm{mg}$ (1.06 mmol, $1.3 \mathrm{eq}$ ) tert-butyl 5-(4,4,5,5-tetramethyl-1,3,2-dioxaborolan-2-yl)-1H-indole-1-carboxylate, $165 \mathrm{mg}$ (4.01 mmol, 5 eq) sodium hydroxide and $69 \mathrm{mg}$ ( $80 \mu \mathrm{mol}, 0.1 \mathrm{eq}$ ) XPhos Pd G3 catalyst were added to the solution. The reaction mixture was heated at $95{ }^{\circ} \mathrm{C}$ for $20 \mathrm{~h}$. After cooling to $\mathrm{rt}$, the mixture was filtrated over celite, washed with $\mathrm{MeOH}$ and the excess solvent was evapored. The remaining solid was diluted with water and $\mathrm{CH}_{2} \mathrm{Cl}_{2}$, the aqueous phase extracted $3 x$ with $\mathrm{CH}_{2} \mathrm{Cl}_{2}$, dried over $\mathrm{MgSO}_{4}$ and the 
excess solvent was evapored. The crude product was purified via $\mathrm{FC}\left(\mathrm{MeOH} / \mathrm{CH}_{2} \mathrm{Cl}_{2}\right)$ to give a white foam.

Yield: $320 \mathrm{mg}, 611 \mu \mathrm{mol}, 74 \%$ of a white foam.

$\mathrm{R}_{\mathrm{f}}\left(1 \% \mathrm{MeOH} / \mathrm{CH}_{2} \mathrm{Cl}_{2}\right): 0.32$.

ESI: (calculated): $\left[\mathrm{M}+\mathrm{H}^{+}\right] 525.28 \mathrm{~g} / \mathrm{mol}$

(found): $\left[\mathrm{M}+\mathrm{H}^{+}\right] 525.18 \mathrm{~g} / \mathrm{mol}$.

HPLC: RT = $14.2 \min (254 \mathrm{~nm}, 96 \%)$.

${ }^{1} \mathrm{H}$ NMR $(250 \mathrm{MHz}, \mathrm{MeOD}) \delta=8.59(\mathrm{~s}, 1 \mathrm{H}), 8.17\left(\mathrm{~d},{ }^{3} \mathrm{~J}=8.8 \mathrm{~Hz}, 1 \mathrm{H}\right), 7.78(\mathrm{~m}, 3 \mathrm{H}), 7.64\left(\mathrm{~d},{ }^{3} \mathrm{~J}=3.7 \mathrm{~Hz}\right.$, $1 \mathrm{H}), 7.57\left(\mathrm{~d},{ }^{3} \mathrm{~J}=8.7 \mathrm{~Hz}, 1 \mathrm{H}\right), 7.46\left(\mathrm{~d},{ }^{3} \mathrm{~J}=4.8 \mathrm{~Hz}, 3 \mathrm{H}\right), 7.35\left(\mathrm{~d},{ }^{3} \mathrm{~J}=8.3 \mathrm{~Hz}, 1 \mathrm{H}\right), 6.67\left(\mathrm{~d},{ }^{3} \mathrm{~J}=3.6 \mathrm{~Hz}, 1 \mathrm{H}\right)$, $3.00\left(\mathrm{t},{ }^{3} \mathrm{~J}=4.3 \mathrm{~Hz}, 4 \mathrm{H}\right), 2.67(\mathrm{~s}, 4 \mathrm{H}), 2.47(\mathrm{~s}, 3 \mathrm{H}), 2.38(\mathrm{~s}, 3 \mathrm{H}), 1.70(\mathrm{~s}, 9 \mathrm{H}) \mathrm{ppm}$.

\section{Synthesis of $N$-(5-(1H-indol-5-yl)-2-(piperazin-1-yl)phenyl)-3-methylbenzamide}

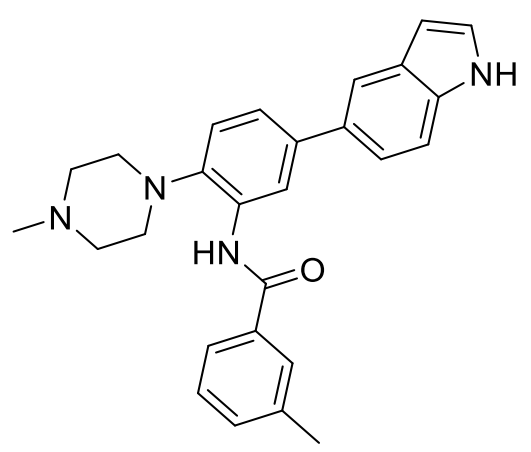

A mixture of $1 \mathrm{~mL}$ TFA and $1 \mathrm{~mL} \mathrm{CH} \mathrm{Cl}_{2}$ was added to $184 \mathrm{mg}$ (433 $\mu \mathrm{mol}, 1$ eq) tert-butyl 5-(3-(3methylbenzamido)-4-(piperazin-1-yl)phenyl)-1H-indole-1-carboxylate. The solution was stirred at $\mathrm{rt}$ for 30 min, then statured $\mathrm{NaHCO}_{3}$ was added. The mixture was extracted $4 \mathrm{x}$ with $\mathrm{CH}_{2} \mathrm{Cl}_{2}$, washed with brine, dried over $\mathrm{MgSO}_{4}$, filtered and excess solvent was evapored.

Yield: $164 \mathrm{mg}, 386 \mu \mathrm{mol}, 89 \%$ of a white solid.

$\mathrm{R}_{\mathrm{f}}\left(10 \% \mathrm{MeOH} / \mathrm{CH}_{2} \mathrm{Cl}_{2}\right): 0.62$.

ESI: (calculated): $\left[\mathrm{M}+\mathrm{H}^{+}\right] 425.23 \mathrm{~g} / \mathrm{mol}$ 
(found): $\left[\mathrm{M}+\mathrm{H}^{+}\right] 425.16 \mathrm{~g} / \mathrm{mol}$.

${ }^{1} \mathrm{H}$ NMR $\left(250 \mathrm{MHz}, \mathrm{CDCl}_{3}\right) \delta=11.06(\mathrm{~s}, 1 \mathrm{H}), 9.60(\mathrm{~s}, 1 \mathrm{H}), 8.83\left(\mathrm{~d},{ }^{3} \mathrm{~J}=2.0 \mathrm{~Hz}, 1 \mathrm{H}\right), 8.49(\mathrm{~s}, 1 \mathrm{H}), 7.90(\mathrm{~m}$, 1H), $7.78(s, 1 H), 7.74\left(d,{ }^{3} J=7.0 \mathrm{~Hz}, 1 \mathrm{H}\right), 7.50-7.42(\mathrm{~m}, 3 \mathrm{H}), 7.39(\mathrm{~s}, 2 \mathrm{H}), 7.27\left(\mathrm{~d},{ }^{3} \mathrm{~J}=8.2 \mathrm{~Hz}, 1 \mathrm{H}\right), 2.97$ $(\mathrm{m}, 4 \mathrm{H}), 2.62(\mathrm{~s}, 4 \mathrm{H}), 2.45(\mathrm{~s}, 3 \mathrm{H}), 2.36(\mathrm{~s}, 3 \mathrm{H}) \mathrm{ppm}$.

Synthesis of tert-butyl 5-(3-(2-chloro-4-fluoro-3-methyl-5-nitrobenzamido)-4-(4-methylpiperazin-1yl)phenyl)-1H-indole-1-carboxylate<smiles>Cc1c(F)c([N+](=O)[O-])cc(C(=O)Nc2cc(-c3ccc4c(ccn4C(=O)OC(C)(C)C)c3)ccc2N2CCN(C)CC2)c1Cl</smiles>

$37 \mathrm{mg}$, (160 $\mu \mathrm{mol}, 1 \mathrm{eq})$ of 2-chloro-4-fluoro-3-methyl-5-nitrobenzoic acid was dissolved in $1 \mathrm{~mL} \mathrm{DCM} /$ acetonitrile and $101 \mu \mathrm{L}(1.19 \mathrm{mmol}, 7.5 \mathrm{eq})$ thionyl chloride. The reaction mixture was stirred for $3 \mathrm{~h}$ at $50^{\circ} \mathrm{C}$ until a colour change from clear to yellow was observed. Excess thionyl chloride was removed under reduced pressure and the acyl chloride was evapored on a high vacuum line for $5 \mathrm{~min}$. The acyl chloride was diluted with $2 \mathrm{~mL}$ of DCM and a solution of $3 \mathrm{~mL}$ containing $65 \mathrm{mg}$ (160 $\mu \mathrm{mol}, 1 \mathrm{eq}$ ) tertbutyl 5-(3-amino-4-(4-methylpiperazin-1-yl)phenyl)-1H-indole-1-carboxylate and $26 \mu \mathrm{L}$ (320 $\mu \mathrm{mol}$, $2.00 \mathrm{eq}$ ) pyridine was added. The reaction mixture was stirred at $50{ }^{\circ} \mathrm{C}$ for $18 \mathrm{~h}$. The reaction mixture was cooled to rt, diluted with water and extracted $3 x$ with DCM. The crude product was purified by FC (0\% to $10 \% \mathrm{MeOH} / \mathrm{DCM}$ ). Other attempts to synthesize the product are listed in Table 12. 
Table 12: Synthesis of tert-butyl 5-(3-(2-chloro-4-fluoro-3-methyl-5-nitrobenzamido)-4-(4-methylpiperazin-1yl)phenyl)-1H-indole-1-carboxylate.

\begin{tabular}{|c|c|}
\hline (5b) & Product Yield \\
\hline $160 \mathrm{mg}, 0,39 \mu \mathrm{mol}$ & $198 \mathrm{mg}, 0.32 \mu \mathrm{mol}, 82 \%$. \\
\hline $112 \mathrm{mg}, 0.28 \mu \mathrm{mol}$ & $144 \mathrm{mg}, 0.23 \mu \mathrm{mol}, 83 \%$. \\
\hline $42 \mathrm{mg}, 0.11 \mu \mathrm{mol}$ & $47 \mathrm{mg}, 90 \mu \mathrm{mol}, 86 \%$. \\
\hline
\end{tabular}

Yield: $93 \mathrm{mg}, 149 \mu \mathrm{mol}, 93 \%$ of a red powder.

$\mathrm{R}_{\mathrm{f}}\left(5 \% \mathrm{MeOH} / \mathrm{CH}_{2} \mathrm{Cl}_{2}\right): 0.49$.

ESI: $\quad$ (calculated): $\left[\mathrm{M}+\mathrm{H}^{+}\right] 622.22 \mathrm{~g} / \mathrm{mol},\left[\mathrm{M}-\mathrm{Boc}^{+}\right] 522.17 \mathrm{~g} / \mathrm{mol}$

(found): $\left[\mathrm{M}+\mathrm{H}^{+}\right] 622.13 \mathrm{~g} / \mathrm{mol},\left[\mathrm{M}-\mathrm{Boc}^{+}\right] 522.29 \mathrm{~g} / \mathrm{mol}$.

HPLC: RT = $13.4 \min (254 \mathrm{~nm}, 95 \%)$.

${ }^{1} \mathrm{H}$ NMR $\left(500 \mathrm{MHz}, \mathrm{CD}_{2} \mathrm{Cl}_{2}\right) \delta=8.73\left(\mathrm{~d},{ }^{4} \mathrm{~J}=1 \mathrm{~Hz}, 1 \mathrm{H}\right), 8.19\left(\mathrm{~d},{ }^{3} \mathrm{~J}=7.7 \mathrm{~Hz}, 1 \mathrm{H}\right), 8.09\left(\mathrm{~d},{ }^{3} \mathrm{~J}=8.3 \mathrm{~Hz}, 1 \mathrm{H}\right)$, $7.74\left(\mathrm{~d},{ }^{4} \mathrm{~J}=1.1 \mathrm{~Hz}, 1 \mathrm{H}\right), 7.55\left(\mathrm{~d},{ }^{4} \mathrm{~J}=3.5 \mathrm{~Hz}, 1 \mathrm{H}\right), 7.51\left(\mathrm{dd},{ }^{3} \mathrm{~J}=8.6 \mathrm{~Hz},{ }^{4} \mathrm{~J}=, 1.5 \mathrm{~Hz}, 1 \mathrm{H}\right), 7.37\left(\mathrm{dd},{ }^{3} \mathrm{~J}=\right.$ $\left.8.2 \mathrm{~Hz},{ }^{4} \mathrm{~J}=2.0 \mathrm{~Hz}, 1 \mathrm{H}\right), 7.27\left(\mathrm{~d},{ }^{3} \mathrm{~J}=8.2 \mathrm{~Hz}, 1 \mathrm{H}\right), 6.56\left(\mathrm{~d},{ }^{3} \mathrm{~J}=3.6 \mathrm{~Hz}, 1 \mathrm{H}\right), 2.87\left(\mathrm{t},{ }^{3} \mathrm{~J}=4.3 \mathrm{~Hz}, 4 \mathrm{H}\right), 2.45-$ $2.40(\mathrm{~m}, 4 \mathrm{H}), 2.41(\mathrm{~s}, 3 \mathrm{H}), 2.21(\mathrm{~s}, 3 \mathrm{H}), 1.59(\mathrm{~s}, 9 \mathrm{H}) \mathrm{ppm}$. 
Synthesis of tert-butyl 5-(3-(5-amino-2-chloro-4-fluoro-3-methylbenzamido)-4-(4-methylpiperazin1-yl)phenyl)-1H-indole-1-carboxylate (6b)

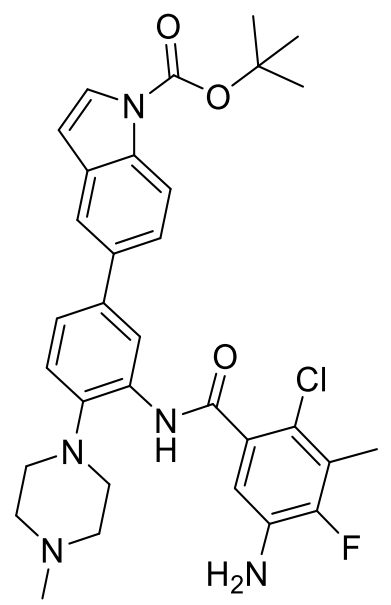

$100 \mathrm{mg}(160 \mu \mathrm{mol}, 1.0 \mathrm{eq})$ tert-butyl 5-(3-(2-chloro-4-fluoro-3-methyl-5-nitrobenzamido)-4-(4methylpiperazin-1-yl)phenyl)-1H-indole-1-carboxylate were dissolved in $6 \mathrm{~mL}$ dioxane/ water (3/1). $42 \mathrm{mg}$ ( $800 \mu \mathrm{mol}, 5.0 \mathrm{eq})$ ammonium chloride and $52 \mathrm{mg}(800 \mu \mathrm{mol}, 5.0$ eq) Zinc dust were added to the reaction solution and stirred for $1.5 \mathrm{~h}$. The reaction mixture was stirred until a colour change from orange to light pink was observed. The solvent was removed under reduced pressure. The reaction mixture was diluted with saturated $\mathrm{NaHCO}_{3}$ solution and extracted $6 \mathrm{x}$ with DCM. The combined organic phases were washed with saturated $\mathrm{NaCl}$ solution, dried over $\mathrm{MgSO}_{4}$, filtered and the solvent was removed under reduced pressure. The crude product was purified by FC.

Yield: $23 \mathrm{mg}, 40.5 \mu \mathrm{mol}, 25 \%$ of a white solid.

$\mathrm{R}_{\mathrm{f}}\left(5 \% \mathrm{MeOH} / \mathrm{CH}_{2} \mathrm{Cl}_{2}\right): 0.25$.

ESI: (calculated): $\left[\mathrm{M}+\mathrm{H}^{+}\right] 592.25 \mathrm{~g} / \mathrm{mol}$

(found): $\left[\mathrm{M}+\mathrm{H}^{+}\right] 592.28 \mathrm{~g} / \mathrm{mol}$.

${ }^{1} \mathrm{H} \mathrm{NMR}\left(400 \mathrm{MHz}, \mathrm{CD}_{2} \mathrm{Cl}_{2}\right) \delta=9.16(\mathrm{~s}, 1 \mathrm{H}), 8.85(\mathrm{~s}, 1 \mathrm{H}), 8.17\left(\mathrm{~d},{ }^{3} \mathrm{~J}=7.9 \mathrm{~Hz}, 1 \mathrm{H}\right), 7.83(\mathrm{~s}, 1 \mathrm{H}), 7.69-7.52$ $(\mathrm{m}, 2 \mathrm{H}), 7.40-7.30(\mathrm{~m}, 2 \mathrm{H}), 7.00\left(\mathrm{~d},{ }^{3} \mathrm{~J}=8.9 \mathrm{~Hz}, 1 \mathrm{H}\right), 6.64(\mathrm{~s}, 1 \mathrm{H}), 3.94(\mathrm{~s}, 2 \mathrm{H}, \mathrm{NH}), 2.93(\mathrm{~s}, 4 \mathrm{H}), 2.51(\mathrm{~s}$, $4 \mathrm{H}), 2.35(\mathrm{~s}, 3 \mathrm{H}), 2.28(\mathrm{~s}, 3 \mathrm{H}), 1.68(\mathrm{~s}, 9 \mathrm{H}) \mathrm{ppm}$. 
Synthesis of tert-butyl 5-(3-(6-hydroxy-4-(trifluoromethyl)nicotinamido)-4-(4-methylpiperazin-1yl)phenyl)-1H-indole-1-carboxylate (6c)

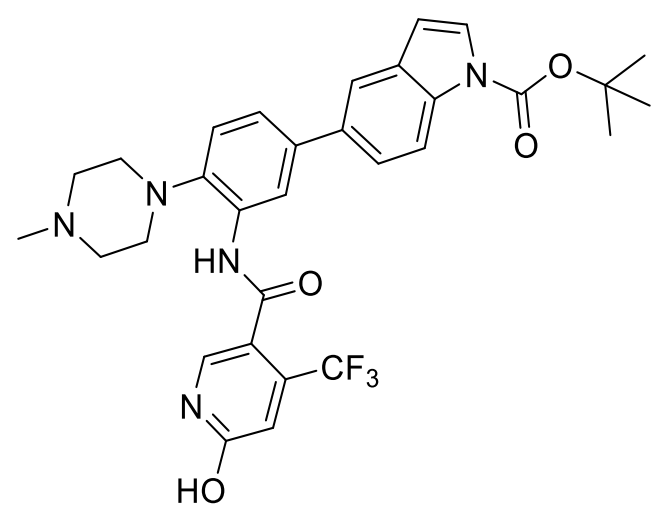

$33 \mathrm{mg}$, (159 $\mu \mathrm{mol}, 1 \mathrm{eq}$ ) of 6-Hydroxy-4-(trifluoromethyl)nicotinic acid was dissolved in $1 \mathrm{~mL}$ DCM/ acetonitrile and $58 \mu \mathrm{L}(797 \mu \mathrm{mol}, 5 \mathrm{eq})$ thionyl chloride. The reaction mixture was stirred for $3 \mathrm{~h}$ at $50^{\circ} \mathrm{C}$ until a colour change from clear to yellow was observed. Excess thionyl chloride was removed under reduced pressure and the acyl chloride was evapored on a high vacuum line for $5 \mathrm{~min}$. The acyl chloride was diluted with $2 \mathrm{~mL}$ of DCM and a solution of $3 \mathrm{~mL}$ containing $65 \mathrm{mg}$ (159 $\mu \mathrm{mol}, 1 \mathrm{eq}$ ) tertbutyl 5-(3-amino-4-(4-methylpiperazin-1-yl)phenyl)-1H-indole-1-carboxylate and $26 \mu \mathrm{L}$ (319 $\mu \mathrm{mol}$, 2.00 eq) pyridine was added. The reaction mixture was stirred at $50^{\circ} \mathrm{C}$ for $18 \mathrm{~h}$. The reaction mixture was cooled to rt, diluted with water and extracted $3 x$ with DCM. The crude product was purified via FC (0 \% to $10 \% \mathrm{MeOH} / \mathrm{DCM}$ ). Other attempts to synthesize (6b) are listed in Table 13.

Table 13: Synthesis of tert-butyl 5-(3-(6-hydroxy-4-(trifluoromethyl)nicotinamido)-4-(4-methylpiperazin-1yl)phenyl)-1H-indole-1-carboxylate (6c).

\begin{tabular}{|c|c|c|c|c|}
\hline Nicotinic acid & (5b) & Thionyl chloride & pyridine & (6b) \\
\hline $24 \mathrm{mg}, 118 \mu \mathrm{mol}$, & $48 \mathrm{mg}, 118 \mu \mathrm{mol}$, & $75 \mu \mathrm{L}, 886 \mu \mathrm{mol}$, & $19 \mu \mathrm{L}, 236 \mu \mathrm{mol}$, & $47 \mathrm{mg}, 79 \mu \mathrm{mol}$, \\
$1.0 \mathrm{eq}$ & $1.0 \mathrm{eq}$ & $7.5 \mathrm{eq}$ & $2.0 \mathrm{eq}$ & $67 \%$ \\
\hline
\end{tabular}

Yield: $45 \mathrm{mg}, 75.5 \mu \mathrm{mol}, 48 \%$ of a white powder.

$\mathrm{R}_{\mathrm{f}}\left(5 \% \mathrm{MeOH} / \mathrm{CH}_{2} \mathrm{Cl}_{2}\right): 0.1$.

ESI: (calculated): $\left[\mathrm{M}+\mathrm{H}^{+}\right] 596.25 \mathrm{~g} / \mathrm{mol}$

(found): [fragment $\left.{ }^{+}\right] 455.29 \mathrm{~g} / \mathrm{mol}(100) ;\left[\mathrm{M}+\mathrm{H}^{+}\right] 596.32 \mathrm{~g} / \mathrm{mol}(90)$. 
HPLC: RT = $12.3 \min (254 \mathrm{~nm}, 98 \%)$.

${ }^{1} \mathrm{H}$ NMR (600 MHz, MeOD) $\delta=8.26\left(\mathrm{~d},{ }^{4} J=1.7 \mathrm{~Hz}, 1 \mathrm{H}\right), 8.18\left(\mathrm{~d},{ }^{3} \mathrm{~J}=8.4 \mathrm{~Hz}, 1 \mathrm{H}\right), 8.03(\mathrm{~s}, 1 \mathrm{H}), 7.81\left(\mathrm{~d},{ }^{3} \mathrm{~J}\right.$ $=1.3 \mathrm{~Hz}, 1 \mathrm{H}), 7.64\left(\mathrm{~d},{ }^{3} \mathrm{~J}=3.7 \mathrm{~Hz}, 1 \mathrm{H}\right), 7.61-7.52(\mathrm{~m}, 2 \mathrm{H}), 7.37\left(\mathrm{~d},{ }^{3} \mathrm{~J}=8.3 \mathrm{~Hz}, 1 \mathrm{H}\right), 6.94(\mathrm{~s}, 1 \mathrm{H}), 6.67(\mathrm{~d}$, $\left.{ }^{3} \mathrm{~J}=3.6 \mathrm{~Hz}, 1 \mathrm{H}\right), 3.61(\mathrm{~m}, 2 \mathrm{H}), 3.28(\mathrm{~m}, 4 \mathrm{H}), 3.16(\mathrm{~m}, 2 \mathrm{H}), 2.96(\mathrm{~s}, 3 \mathrm{H}), 1.69(\mathrm{~s}, 9 \mathrm{H}) \mathrm{ppm}$.

Synthesis of tert-butyl 3'-amino-4'-(4-methylpiperazin-1-yl)-[1,1'-biphenyl]-4-carboxylate (5b)<smiles>CN1CCN(c2ccc(-c3ccc(C(=O)OC(C)(C)C)cc3)cc2N)CC1</smiles>

$395 \mathrm{mg}$ (1.78 mmol, $1.2 \mathrm{eq)} \mathrm{(4-(tert-butoxycarbonyl)phenyl)boronic} \mathrm{acid} \mathrm{was} \mathrm{dissolved} \mathrm{in} \mathrm{an} \mathrm{Argon-}$ purged solvent solution (1,4-Dioxane/ Water (3:1)) and $288 \mathrm{mg}$ (7.40 mmol, 5.0 eq) Sodium hydroxide was added. The reaction mixture was stirred for $5 \mathrm{~min}$ at $\mathrm{rt}$ under Argon atmosphere, then $400 \mathrm{mg}$ (1.48 mmol, 1.0 eq) 5-bromo-2-(4-methylpiperazin-1-yl)aniline and $171 \mathrm{mg}$ (148 $\mu \mathrm{mol}, 0.1 \mathrm{eq})$ $\mathrm{Pd}\left(\mathrm{PPh}_{3}\right)_{4}$ were added. The reaction was stirred at $90{ }^{\circ} \mathrm{C}$ for $18 \mathrm{~h}$ under Argon atmosphere. The reaction mixture was cooled to rt, filtered over celite and washed with $\mathrm{MeOH}$. The solvent was removed under reduced pressure. The reaction mixture was diluted with water and extracted $3 x$ with DCM. The crude product was purified via FC (0 \% to $10 \% \mathrm{MeOH} / \mathrm{DCM})$. Other attempts to synthesize 3'-amino-4'-(4methylpiperazin-1-yl)-[1,1'-biphenyl]-4-carboxylate (5b) are listed in Table 14.

Table 14: Synthesis 3'-amino-4'-(4-methylpiperazin-1-yl)-[1,1'-biphenyl]-4-carboxylate (5b).

\begin{tabular}{|c|c|c|c|c|c|}
\hline Boronic acid & (4) & $\mathrm{NaOH}$ & $\mathrm{Pd}\left(\mathrm{PPh}_{3}\right)_{4}$ & $\begin{array}{c}\text { Heating } \\
\text { temperature }\end{array}$ & (5b) \\
\hline $395 \mathrm{mg}$, & $400 \mathrm{mg}$, & $288 \mathrm{mg}$, & $171 \mathrm{mg}$, & $100^{\circ} \mathrm{C} / 17 \mathrm{~h}$ & $395 \mathrm{mg}$ \\
$1.78 \mathrm{mmol}$, & $1.48 \mathrm{mmol}$, & $7.40 \mathrm{mmol}$, & $148 \mu \mathrm{mol}$, & & $1.08 \mathrm{mmol}$, \\
$1.2 \mathrm{eq}$ & $1.0 \mathrm{eq}$ & $1.2 \mathrm{eq}$ & $0.1 \mathrm{eq}$ & & $73 \%$ \\
\hline $658 \mathrm{mg}$, & $495 \mathrm{mg}$, & $270 \mathrm{mg}$, & $210 \mathrm{mg}$, & $100^{\circ} \mathrm{C} / 17 \mathrm{~h}$ & $600 \mathrm{mg}$, \\
$2.96 \mathrm{mmol}$, & $1.83 \mathrm{mmol}$, & $6.75 \mathrm{mmol}$, & $180 \mu \mathrm{mol}$, & & $1.63 \mathrm{mmol}$, \\
$1.6 \mathrm{eq}$ & $1.0 \mathrm{eq}$ & $3.7 \mathrm{eq}$ & $0.1 \mathrm{eq}$ & & $89 \%$ \\
\hline
\end{tabular}


Yield: $395 \mathrm{mg}, 1.08 \mathrm{mmol}, 73 \%$ of a white solid.

$\mathrm{R}_{\mathrm{f}}\left(5 \% \mathrm{MeOH} / \mathrm{CH}_{2} \mathrm{Cl}_{2}\right): 0.28$.

ESI: (calculated): $\left[\mathrm{M}+\mathrm{H}^{+}\right] 368.23 \mathrm{~g} / \mathrm{mol}$ (found): $\left[\mathrm{M}+\mathrm{H}^{+}\right] 368.13 \mathrm{~g} / \mathrm{mol}$.

HPLC: $R T=11.9 \min (254 \mathrm{~nm}, 94 \%)$.

${ }^{1} \mathrm{H} \mathrm{NMR}\left(500 \mathrm{MHz}, \mathrm{CD}_{2} \mathrm{Cl}_{2}\right) \delta=7.98\left(\mathrm{~d},{ }^{3} \mathrm{~J}=8.5 \mathrm{~Hz}, 2 \mathrm{H}\right), 7.59\left(\mathrm{~d},{ }^{3} \mathrm{~J}=8.5 \mathrm{~Hz}, 2 \mathrm{H}\right), 7.06\left(\mathrm{~d},{ }^{3} \mathrm{~J}=8.7 \mathrm{~Hz}, 1 \mathrm{H}\right)$, $6.99(\mathrm{~m}, 2 \mathrm{H}), 3.97(\mathrm{~s}, 1 \mathrm{H}), 2.97(\mathrm{~s}, 4 \mathrm{H}), 2.61(\mathrm{~s}, 4 \mathrm{H}), 2.35(\mathrm{~s}, 3 \mathrm{H}), 1.58(\mathrm{~s}, 9 \mathrm{H}) \mathrm{ppm}$.

${ }^{13} \mathrm{C}$ NMR $\left(126 \mathrm{MHz}, \mathrm{CD}_{2} \mathrm{Cl}_{2}\right) \delta=165.9,145.5,142.4,139.8,136.5,130.8,130.1,126.8,120.4,117.6$, $113.8,81.1,56.0,51.0,46.1,28.3$ ppm. 
Synthesis of tert-butyl 3'-(6-hydroxy-4-(trifluoromethyl)nicotinamido)-4'-(4-methylpiperazin-1-yl)[1,1'-biphenyl]-4-carboxylate (6d)<smiles>CN1CCN(c2ccc(-c3ccc(C(=O)OC(C)(C)C)cc3)cc2NC(=O)c2cnc(O)cc2C(F)(F)F)CC1</smiles>

$56 \mathrm{mg},(272 \mu \mathrm{mol}, 1.00 \mathrm{eq})$ of 6-Hydroxy-4-(trifluoromethyl)nicotinic acid was dissolved in $1 \mathrm{~mL} \mathrm{DCM} /$ acetonitrile and $228 \mu \mathrm{L}(2.72 \mathrm{mmol}, 10 \mathrm{eq})$ thionyl chloride. The reaction mixture was stirred for $3 \mathrm{~h}$ at $50{ }^{\circ} \mathrm{C}$ until a colour change from clear to yellow was observed. Excess thionyl chloride was removed under reduced pressure and the acyl chloride was evapored on a high vacuum line for $5 \mathrm{~min}$. The acyl chloride was diluted with $2 \mathrm{~mL}$ of DCM and a solution of $3 \mathrm{~mL}$ containing $100 \mathrm{mg}$ ( $272 \mu \mathrm{mol}, 1.00 \mathrm{eq}$ ) 3'-amino-4'-(4-methylpiperazin-1-yl)-[1,1'-biphenyl]-4-carboxylate and $44 \mu \mathrm{L}$ (544 $\mu \mathrm{mol}, 2.00 \mathrm{eq}$ ) pyridine was added. The reaction mixture was stirred at $50{ }^{\circ} \mathrm{C}$ for $18 \mathrm{~h}$. The reaction mixture was cooled to $r$, diluted with water and extracted $3 x$ with DCM. The crude product was purified via FC $(0 \%$ to $10 \% \mathrm{MeOH} / \mathrm{DCM})$. Other attempts to synthesize (6d) are listed in Table 15.

Table 15: Synthesis of tert-butyl 3'-(6-hydroxy-4-(trifluoromethyl)nicotinamido)-4'-(4-methylpiperazin-1-yl)-

[1,1'-biphenyl]-4-carboxylate (6d).

\begin{tabular}{|c|c|c|c|c|}
\hline Nicotinic acid & (5a) & Thionyl chloride & pyridine & (6d) \\
\hline $49 \mathrm{mg}, 239 \mu \mathrm{mol}$, & $80 \mathrm{mg}, 217 \mu \mathrm{mol}$, & $183 \mu \mathrm{L}$, & $35 \mu \mathrm{L}, 435 \mu \mathrm{mol}$, & $64 \mathrm{mg}, 115 \mu \mathrm{mol}$, \\
$1.1 \mathrm{eq}$ & $1.0 \mathrm{eq}$ & $2.17 \mathrm{mmol}, 10 \mathrm{eq}$ & $2.0 \mathrm{eq}$ & $53 \%$ \\
\hline $192 \mathrm{mg}$, & $228 \mathrm{mg}$, & $522 \mu \mathrm{L}$, & $101 \mu \mathrm{L}$, & $167 \mathrm{mg}$, \\
$0.93 \mathrm{mmol}$, & $0.62 \mathrm{mmol}$, & $6.2 \mathrm{mmol}, 10 \mathrm{eq}$ & $1.24 \mathrm{mmol}$, & $0.3 \mathrm{mmol}, 48 \%$ \\
$1.5 \mathrm{eq}$ & $1.0 \mathrm{eq}$ & & $2.0 \mathrm{eq}$ & \\
& & & & \\
\hline
\end{tabular}

Yield: $109 \mathrm{mg}, 196 \mu \mathrm{mol}, 72 \%$ of a white solid.

$\mathrm{R}_{\mathrm{f}}\left(5 \% \mathrm{MeOH} / \mathrm{CH}_{2} \mathrm{Cl}_{2}\right): 0.21$. 
ESI: (calculated): $\left[\mathrm{M}+\mathrm{H}^{+}\right] 557.23 \mathrm{~g} / \mathrm{mol}$

(found): $\left[\mathrm{M}+\mathrm{H}^{+}\right] 557.08 \mathrm{~g} / \mathrm{mol}$.

HPLC: $R T=12.9 \min (254 \mathrm{~nm}, 93 \%)$.

${ }^{1} \mathrm{H} \mathrm{NMR}\left(500 \mathrm{MHz}, \mathrm{CDCl}_{3}\right) \delta=8.97(\mathrm{~s}, 1 \mathrm{H}), 8.69(\mathrm{~s}, 1 \mathrm{H}), 8.04\left(\mathrm{~d},{ }^{3} \mathrm{~J}=8.3 \mathrm{~Hz}, 2 \mathrm{H}\right), 7.89(\mathrm{~s}, 1 \mathrm{H}), 7.66\left(\mathrm{~d},{ }^{3} \mathrm{~J}\right.$ $=8.3 \mathrm{~Hz}, 2 \mathrm{H}), 7.48-7.30(\mathrm{~m}, 2 \mathrm{H}), 6.95(\mathrm{~s}, 1 \mathrm{H}), 3.05(\mathrm{~s}, 4 \mathrm{H}), 2.78(\mathrm{~s}, 4 \mathrm{H}), 2.51(\mathrm{~s}, 3 \mathrm{H}), 1.61(\mathrm{~s}, 9 \mathrm{H}) \mathrm{ppm}$.

${ }^{13} \mathrm{C}$ NMR (75 MHz, DMSO) $\delta=164.8,163.1,161.1,143.7,143.2,139.5(\mathrm{~m}), 138.6\left(\mathrm{q},{ }^{2} \mathrm{~J}=32 \mathrm{~Hz}\right), 135.0$, 134.1, 132.7, 130.1, 129.8, 126.4, 124.0, 122.1 (q, $\left.{ }^{1} \mathrm{~J}=273 \mathrm{~Hz}\right), 121.0,118.9(\mathrm{~m}), 111.6(\mathrm{~m}), 80.7,52.8$, 48.1, 42.3, $27.8 \mathrm{ppm}$.

Synthesis of 4-(4-bromo-2-nitrophenyl)morpholine<smiles>O=[N+]([O-])c1cc(Br)ccc1N1CCOCC1</smiles>

$1 \mathrm{~mL}$ (8.16 mmol, 1.00 eq) 5-Bromo-1-Flouro-2-Nitrobenzole and $704 \mu \mathrm{L}(8.16 \mathrm{mmol}, 1.00 \mathrm{eq})$ Morpholine were suspended in $5 \mathrm{~mL}$ EtOH and stirred at $80^{\circ} \mathrm{C}$ for $18 \mathrm{~h}$. The reaction was cooled to rt and the solvent was removed under reduced pressure. The reaction mixture was diluted with water and extracted $8 \mathrm{x}$ with DCM. The combined organic phases were washed with $1 \mathrm{M} \mathrm{HCl}$, saturated $\mathrm{NaCl}$ solution, dried over $\mathrm{MgSO}_{4}$ and filtered. The crude product was purified via CC (gradient: $25 \%$ to $50 \%$ EA in cHex).

Yield: $1.73 \mathrm{~g}, 6.03 \mathrm{mmol}, 74 \%$ of an orange powder.

$R_{f}(33 \% E A / c H e x): 0.47$.

${ }^{1} \mathrm{H}$ NMR $(250 \mathrm{MHz}, \mathrm{MeOD}) \delta=7.91\left(\mathrm{~d},{ }^{4} J=2.4 \mathrm{~Hz}, 1 \mathrm{H}\right), 7.67\left(\mathrm{dd},{ }^{3} J=8.8 \mathrm{~Hz},{ }^{4} J=2.4 \mathrm{~Hz}, 1 \mathrm{H}\right), 7.22\left(\mathrm{~d},{ }^{3} J\right.$ $=8.8 \mathrm{~Hz}, 1 \mathrm{H}), 3.85-3.66(\mathrm{~m}, 4 \mathrm{H}), 3.04-3.00(\mathrm{~m}, 4 \mathrm{H}) \mathrm{ppm}$. 
<smiles>Nc1cc(Br)ccc1N1CCOCC1</smiles>

$1.73 \mathrm{~g}$ (6.03 mmol, 1.00 eq) 4-(4-bromo-2-nitrophenyl)morpholine was suspended in a mixture of 1,4Dioxane and water (3:1). $2.96 \mathrm{~g}$ ( $45.2 \mathrm{mmol}, 7.50 \mathrm{eq}$ ) Ammonium chloride was added, followed by a slow addition of $2.42 \mathrm{~g}(45.2 \mathrm{mmol}, 7.50 \mathrm{eq})$ Zinc dust. The reaction mixture was stirred until a colour change from orange to light pink was observed. The solvent was removed under reduced pressure. The reaction mixture was diluted with saturated $\mathrm{NaHCO}_{3}$ solution and extracted $6 x$ with DCM. The combined organic phases were washed with saturated $\mathrm{NaCl}$ solution, dried over $\mathrm{MgSO}_{4}$, filtered and the solvent was removed under reduced pressure.

Yield: $603 \mathrm{mg}, 2.39 \mathrm{mmol}, 39 \%$ of a light pink solid

$\mathrm{R}_{\mathrm{f}}\left(10 \% \mathrm{MeOH} / \mathrm{CH}_{2} \mathrm{Cl}_{2}\right): 0.74$.

${ }^{1} \mathrm{H}$ NMR $(250 \mathrm{MHz}, \mathrm{MeOD}) \delta=6.89-6.84(\mathrm{~m}, 2 \mathrm{H}), 6.74\left(\mathrm{dd},{ }^{3} \mathrm{~J}=8.4 \mathrm{~Hz},{ }^{4} \mathrm{~J}=2.3 \mathrm{~Hz}, 1 \mathrm{H}\right), 3.88-3.77(\mathrm{~m}$, $4 \mathrm{H}), 2.91-2.74(\mathrm{~m}, 4 \mathrm{H}) \mathrm{ppm}$.

Synthesis of tert-butyl 4'-morpholino-3'-(6-oxo-4-(trifluoromethyl)-1,6-dihydropyridine-3carboxamido)-[1,1'-biphenyl]-4-carboxylate<smiles>CC(C)(C)OC(=O)c1ccc(-c2ccc(N3CCOCC3)c(NC(=O)c3c[nH]c(=O)cc3C(F)(F)F)c2)cc1</smiles>

$555 \mathrm{mg}$ (2.50 mmol, 1.6 eq) (4-(tert-butoxycarbonyl)phenyl)boronic acid was dissolved in an Argonpurged solvent solution (1,4-Dioxane/ Water (3:1)) and $187 \mathrm{mg}$ (4.68 mmol, 3.0 eq) Sodium hydroxide 
was added. The reaction mixture was stirred for $5 \mathrm{~min}$ at $\mathrm{rt}$ under Argon atmosphere, then $400 \mathrm{mg}$ (1.48 mmol, 1.0 eq) 5-bromo-2-morpholinoaniline and $132 \mathrm{mg}$ (156 $\mu \mathrm{mol}, 0.1 \mathrm{eq})$ Pd XPhos G3 were added. The reaction was stirred at $93^{\circ} \mathrm{C}$ for $24 \mathrm{~h}$ under Argon atmosphere. The reaction mixture was cooled to rt, filtered over celite and washed with $\mathrm{MeOH}$. The solvent was removed under reduced pressure. The reaction mixture was diluted with water and extracted $3 x$ with DCM. The crude product was purified via FC ( $0 \%$ to $10 \% \mathrm{MeOH} / \mathrm{DCM}$ ) to give $439 \mathrm{mg}$ (1.24 mmol, 79\%) of a white tert-butyl 3'-amino-4'-morpholino-[1,1'-biphenyl]-4-carboxylate.

$74 \mathrm{mg}$ (355 $\mathrm{mmol}, 1.20$ eq) 6-Hydroxy-4-(trifluoromethyl)nicotinic acid was dissolved in $1 \mathrm{~mL}$ DCM/ acetonitrile and $218 \mu \mathrm{L}(3 \mathrm{mmol}, 10 \mathrm{eq})$ thionyl chloride. The reaction mixture was stirred for $3 \mathrm{~h}$ at $50{ }^{\circ} \mathrm{C}$ until a colour change from clear to yellow was observed. Excess thionyl chloride was removed under reduced pressure and the acyl chloride was evapored on a high vacuum line for $5 \mathrm{~min}$. The acyl chloride was diluted with $2 \mathrm{~mL}$ of DCM and a solution of $3 \mathrm{~mL}$ containing $105 \mathrm{mg}$ (296 $\mu \mathrm{mol}, 1.00 \mathrm{eq}$ ) of tert-butyl 3'-amino-4'-morpholino-[1,1'-biphenyl]-4-carboxylate and $48 \mu \mathrm{L}$ (592 $\mu \mathrm{mol}, 2.00 \mathrm{eq}$ ) pyridine was added. The reaction mixture was stirred at $50{ }^{\circ} \mathrm{C}$ for $18 \mathrm{~h}$. The reaction mixture was cooled to $r$, diluted with water and extracted $3 \mathrm{x}$ with DCM. The crude product was purified via FC $(0 \%$ to $10 \% \mathrm{MeOH} / \mathrm{DCM})$.

Yield: $99 \mathrm{mg}, 182 \mu \mathrm{mol}, 51 \%$ of a white solid.

$\mathrm{R}_{\mathrm{f}}\left(10 \% \mathrm{MeOH} / \mathrm{CH}_{2} \mathrm{Cl}_{2}\right): 0.23$.

ESI: (calculated) $\left[\mathrm{M}+\mathrm{H}^{+}\right] 544.21 \mathrm{~g} / \mathrm{mol}$ (found) $\left[\mathrm{M}+\mathrm{H}^{+}\right] 544.21 \mathrm{~g} / \mathrm{mol}$.

${ }^{1} \mathrm{H} \mathrm{NMR}(250 \mathrm{MHz}, \mathrm{MeOD}) \delta=8.80(\mathrm{~s}, 1 \mathrm{H}), 8.65(\mathrm{~s}, 1 \mathrm{H}), 8.39\left(\mathrm{~d},{ }^{4} \mathrm{~J}=1.9 \mathrm{~Hz}, 1 \mathrm{H}\right), 8.00\left(\mathrm{~d},{ }^{3} \mathrm{~J}=8.3 \mathrm{~Hz}\right.$, $2 \mathrm{H}), 7.78(\mathrm{~s}, 1 \mathrm{H}), 7.70\left(\mathrm{~d},{ }^{3} \mathrm{~J}=8.3 \mathrm{~Hz}, 2 \mathrm{H}\right), 7.53\left(\mathrm{dd},{ }^{3} \mathrm{~J}=8.3 \mathrm{~Hz},{ }^{4} J=2.0 \mathrm{~Hz}, 1 \mathrm{H}\right), 7.33\left(\mathrm{~d},{ }^{3} J=8.4 \mathrm{~Hz}, 1 \mathrm{H}\right)$, $6.95(\mathrm{~s}, 1 \mathrm{H}), 3.85-3.78(\mathrm{~m}, 4 \mathrm{H}), 2.98-2.92(\mathrm{~m}, 4 \mathrm{H}), 1.60(\mathrm{~s}, 9 \mathrm{H}) \mathrm{ppm}$. 
<smiles>CN1CCN(c2ccc(-c3ccc(C(=O)NCCOCCNC(=O)OC(C)(C)C)cc3)cc2NC(=O)c2cnc(O)cc2C(F)(F)F)CC1</smiles>

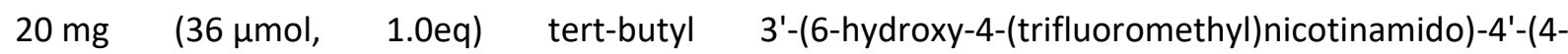
methylpiperazin-1-yl)-[1,1'-biphenyl]-4-carboxylate were dissolved in $0.5 \mathrm{~mL} \mathrm{DCM}$ and $0.5 \mathrm{~mL}$ TFA and stired at $\mathrm{rt}$ for $1 \mathrm{~h}$. Excess solvent was evapored. The solid was dissolved in $0.5 \mathrm{~mL} \mathrm{DMF}$, then $125 \mu \mathrm{L}$ (720 $\mu \mathrm{mol}, 20 \mathrm{eq}$ ) DIEA and $16.4 \mathrm{mg}$ ( $43 \mu \mathrm{mol}, 1.2 \mathrm{eq}$ ) HATU were added. After $15 \mathrm{~min}$, a solution of $7.7 \mathrm{mg}(38 \mu \mathrm{mol}, 1.05 \mathrm{eq}$ ) tert-butyl (2-(2-aminoethoxy)ethyl)carbamate in $0.5 \mathrm{~mL}$ DMF was added. The solution was stirred for $3 \mathrm{~h}$ at rt. The reaction mixture was quenched with $2 \mathrm{~mL}$ water and $2 \mathrm{~mL}$ saturated $\mathrm{NaHCO}_{3}$, then the reaction was extracted 3x with EA. The organic phase was dried over $\mathrm{MgSO}_{4}$, filtered and the solvent was removed under reduced pressure. The crude product was purified using by HPLC.

Yield: $18 \mathrm{mg}, 26.2 \mu \mathrm{mol}, 73 \%$ of a white solid.

ESI: (calculated) $\left[\mathrm{M}+\mathrm{H}^{+}\right] 687.31 \mathrm{~g} / \mathrm{mol}$

(found) $\left[\mathrm{M}+\mathrm{H}^{+}\right] 687.52 \mathrm{~g} / \mathrm{mol}$.

HPLC: RT = $11.6 \min (254 \mathrm{~nm}, 96 \%)$.

${ }^{1} \mathrm{H}$ NMR (400 MHz, DMSO) $\delta=9.47(\mathrm{~s}, 1 \mathrm{H}), 8.52\left(\mathrm{t},{ }^{3} \mathrm{~J}=5.4 \mathrm{~Hz}, 1 \mathrm{H}\right), 8.13\left(\mathrm{~d},{ }^{3} \mathrm{~J}=2.1 \mathrm{~Hz}\right), 7.97(\mathrm{~s}, 1 \mathrm{H})$, $7.93\left(d,{ }^{3} J=8.4 \mathrm{~Hz}, 2 \mathrm{H}\right), 7.68\left(\mathrm{~d},{ }^{3} \mathrm{~J}=8.5 \mathrm{~Hz}, 2 \mathrm{H}\right), 7.52\left(\mathrm{dd},{ }^{3} J=8.4 \mathrm{~Hz},{ }^{4} \mathrm{~J}=2.2 \mathrm{~Hz}\right), 7.27\left(\mathrm{~d},{ }^{3} J=8.4 \mathrm{~Hz}\right.$, $1 \mathrm{H}), 6.82(\mathrm{~s}, 1 \mathrm{H}), 6.76(\mathrm{~s}, 1 \mathrm{H}), 3.55-3.52(\mathrm{~m}, 2 \mathrm{H}), 3.47-3.38(\mathrm{~m}, 4 \mathrm{H}), 3.11-3.06(\mathrm{~m}, 2 \mathrm{H}), 2.99-2.85$ $(\mathrm{m}, 4 \mathrm{H}), 2.50(\mathrm{~s}, 4 \mathrm{H}) 2.23(\mathrm{~s}, 3 \mathrm{H}), 1.36(\mathrm{~s}, 9 \mathrm{H}) \mathrm{ppm}$. 
${ }^{13} \mathrm{C}$ NMR (101 MHz, DMSO) $\delta=168.0,166.0,162.8,161.2,144.9,142.2,140.0,139.5$ (q, ${ }^{2} \mathrm{~J}=35 \mathrm{~Hz}$ ), $134.2,133.00132 .3,132.2,128.3,127.9,126.0,123.9,122.6$ (q, ${ }^{1} \mathrm{~J}=240 \mathrm{~Hz}$ ), 122.3, 121.0, 120.4, 119.1 (m), $110.3(\mathrm{~m}), 77.59,68.99,68.7,54.7,51.0,45.7,39.1,38.9,28.2$ ppm.

Synthesis of tert-butyl (1-(3'-(6-hydroxy-4-(trifluoromethyl)nicotinamido)-4'-(4-methylpiperazin-1yl)-[1,1'-biphenyl]-4-yl)-1-oxo-5,8,11,14,17,20,23-heptaoxa-2-azapentacosan-25-yl)carbamate (6f)<smiles>CN1CCN(c2ccc(-c3ccc(C(=O)NCCOCCOCCOCCOCCOCCOCCOCCNC(=O)OC(C)(C)C)cc3)cc2NC(=O)c2cnc(O)cc2C(F)(F)F)CC1</smiles>

$20 \mathrm{mg} \quad(36 \mu \mathrm{mol}, \quad 1.00$ eq) tert-butyl 3'-(6-hydroxy-4-(trifluoromethyl)nicotinamido)-4'-(4methylpiperazin-1-yl)-[1,1'-biphenyl]-4-carboxylate were dissolved in $1 \mathrm{~mL}$ DCM and $1 \mathrm{~mL}$ TFA and stired at $\mathrm{rt}$ for $0.5 \mathrm{~h}$. Excess solvent was evapored. The solid was dissolved in $2 \mathrm{~mL} \mathrm{DMF}$, then $300 \mu \mathrm{L}$ ( $280 \mu \mathrm{mol}, 20 \mathrm{eq}$ ) DIEA and $16.4 \mathrm{mg}$ (43 $\mu \mathrm{mol}, 1.2 \mathrm{eq})$ HATU were added. After $15 \mathrm{~min}$, a solution of $17.7 \mathrm{mg}$ (38 $\mu \mathrm{mol}, 1.05 \mathrm{eq}$ ) tert-butyl (23-amino-3,6,9,12,15,18,21-heptaoxatricosyl)carbamate in $1 \mathrm{~mL}$ DMF was added. The solution was stirred for $3 \mathrm{~h}$ at $\mathrm{rt}$. The reaction mixture was quenched with $2 \mathrm{~mL}$ water and $2 \mathrm{~mL}$ saturated $\mathrm{NaHCO}_{3}$, then the reaction was extracted $3 x$ with EA. The organic phase was dried over $\mathrm{MgSO}_{4}$, filtered and the solvent was removed under reduced pressure. The crude product was purified using by RP-FC.

Yield: $10 \mathrm{mg}, 10.5 \mu \mathrm{mol}, 29 \%$ of a colourless oil.

$\mathrm{R}_{\mathrm{f}}\left(10 \% \mathrm{MeOH} / \mathrm{CH}_{2} \mathrm{Cl}_{2}\right): 0.25$.

ESI: (calculated) $\left[\mathrm{M}+\mathrm{H}^{+}\right] 951.47 \mathrm{~g} / \mathrm{mol}$ (found $\left[\mathrm{M}+\mathrm{H}^{+}\right] 951.95 \mathrm{~g} / \mathrm{mol}$.

HPLC: RT = $11.8 \min (254 \mathrm{~nm}, 94 \%)$.

${ }^{1} \mathrm{H} \mathrm{NMR}(400 \mathrm{MHz}, \mathrm{DMSO}) \delta=9.46(\mathrm{~s}, 1 \mathrm{H}), 8.53\left(\mathrm{t},{ }^{3} \mathrm{~J}=5.7 \mathrm{~Hz}, 1 \mathrm{H}\right), 8.12\left(\mathrm{~d},{ }^{4} \mathrm{~J}=2.2 \mathrm{~Hz}, 1 \mathrm{H}\right), 7.97(\mathrm{~s}, 1 \mathrm{H})$, $7.94\left(\mathrm{~d}, 3^{3} \mathrm{~J}=8.5 \mathrm{~Hz}, 2 \mathrm{H}\right), 7.68\left(\mathrm{~d},{ }^{3} J=8.5 \mathrm{~Hz}, 2 \mathrm{H}\right), 7.53\left(\mathrm{dd}, 3 J=8.4 \mathrm{~Hz},{ }^{4} J=2.2 \mathrm{~Hz}, 1 \mathrm{H}\right), 7.27\left(\mathrm{~d},{ }^{3} J=\right.$ 
$8.4 \mathrm{~Hz}, 1 \mathrm{H}), 6.82(\mathrm{~s}, 1 \mathrm{H}), 6.72\left(\mathrm{t},{ }^{3} \mathrm{~J}=5.3 \mathrm{~Hz}, 1 \mathrm{H}\right), 3.59-3.47(\mathrm{~m}, 28 \mathrm{H}), 3.44(\mathrm{~m}, 2 \mathrm{H}), 3.36\left(\mathrm{t},{ }^{3} \mathrm{~J}=6.1 \mathrm{~Hz}\right.$, $4 \mathrm{H}), 3.05\left(\mathrm{q},{ }^{3} \mathrm{~J}=6.0 \mathrm{~Hz}, 2 \mathrm{H}\right), 2.96-2.85(\mathrm{~m}, 4 \mathrm{H}), 2.24(\mathrm{~s}, 3 \mathrm{H}), 1.36(\mathrm{~s}, 9 \mathrm{H}) \mathrm{ppm}$.

Synthesis of tert-butyl (4-((3'-(6-hydroxy-4-(trifluoromethyl)nicotinamido)-4'-(4-methylpiperazin-1yl)-[1,1'-biphenyl]-4-carboxamido)methyl)benzyl)carbamate (6g)

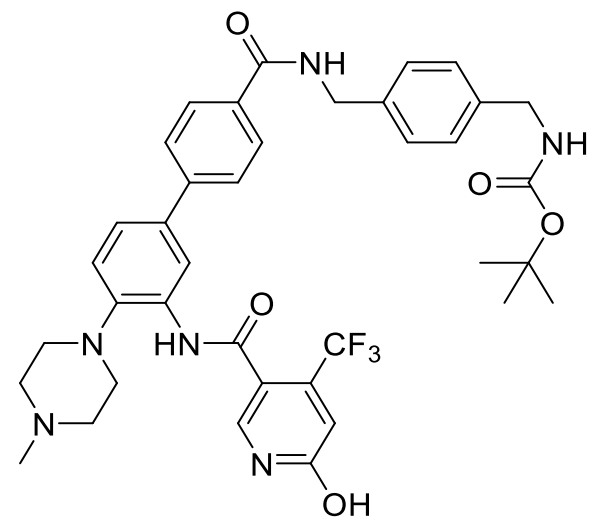

$20 \mathrm{mg} \quad$ (36 $\mathrm{\mu mol}, \quad 1.00 \mathrm{eq}) \quad$ tert-butyl $\quad 3$ '-(6-hydroxy-4-(trifluoromethyl)nicotinamido)-4'-(4methylpiperazin-1-yl)-[1,1'-biphenyl]-4-carboxylate were dissolved in $1 \mathrm{~mL}$ DCM and $1 \mathrm{~mL}$ TFA and stired at $\mathrm{rt}$ for $0.5 \mathrm{~h}$. Excess solvent was evapored. The solid was dissolved in $2 \mathrm{~mL} \mathrm{DMF}$, then $300 \mu \mathrm{L}$ ( $280 \mu \mathrm{mol}, 20 \mathrm{eq}$ ) DIEA and $16.4 \mathrm{mg}$ ( $43 \mu \mathrm{mol}, 1.2 \mathrm{eq}$ ) HATU were added. After $15 \mathrm{~min}$, a solution of $8.9 \mathrm{mg}$ ( $38 \mu \mathrm{mol}, 1.05 \mathrm{eq}$ ) tert-butyl (4-(aminomethyl)benzyl)carbamate in $1 \mathrm{~mL}$ DMF was added. The solution was stirred for $3 \mathrm{~h}$ at $\mathrm{rt}$. The reaction mixture was quenched with $2 \mathrm{~mL}$ water and $2 \mathrm{~mL}$ saturated $\mathrm{NaHCO}_{3}$, then the reaction was extracted 3x with EA. The organic phase was dried over $\mathrm{MgSO}_{4}$, filtered and the solvent was removed under reduced pressure. The crude product was purified using by RP-FC.

Yield: $14 \mathrm{mg}, 19.5 \mu \mathrm{mol}, 54 \%$ of a colourless oil.

$\mathrm{R}_{\mathrm{f}}\left(10 \% \mathrm{MeOH} / \mathrm{CH}_{2} \mathrm{Cl}_{2}\right): 0.11$.

ESI: (calculated) $\left[\mathrm{M}+\mathrm{H}^{+}\right] 719.32 \mathrm{~g} / \mathrm{mol}$

(found $\left[\mathrm{M}+\mathrm{H}^{+}\right] 719.54 \mathrm{~g} / \mathrm{mol}$.

HPLC: RT = $12.2 \min (254 \mathrm{~nm}, 91 \%)$. 
${ }^{1} \mathrm{H}$ NMR $(400 \mathrm{MHz}, \mathrm{MeOD}) \delta=8.29\left(\mathrm{~d},{ }^{4} \mathrm{~J}=1.8 \mathrm{~Hz}, 1 \mathrm{H}\right), 7.99(\mathrm{~s}, 1 \mathrm{H}), 7.92\left(\mathrm{~d},{ }^{3} \mathrm{~J}=8.4 \mathrm{~Hz}, 2 \mathrm{H}\right), 7.72\left(\mathrm{~d},{ }^{3} \mathrm{~J}\right.$ $=8.4 \mathrm{~Hz}, 2 \mathrm{H}), 7.52\left(\mathrm{dd},{ }^{3} J=8.3 \mathrm{~Hz},{ }^{4} J=2.1 \mathrm{~Hz}, 1 \mathrm{H}\right), 7.34(\mathrm{~m}, 3 \mathrm{H}), 7.25\left(\mathrm{~d},{ }^{3} J=8.1 \mathrm{~Hz}, 2 \mathrm{H}\right), 6.92(\mathrm{~s}, 1 \mathrm{H})$, $4.57(\mathrm{~s}, 2 \mathrm{H}), 4.21(\mathrm{~s}, 2 \mathrm{H}), 3.01(\mathrm{~s}, 4 \mathrm{H}), 2.69(\mathrm{~s}, 4 \mathrm{H}), 2.39(\mathrm{~s}, 3 \mathrm{H}), 1.44(\mathrm{~s}, 9 \mathrm{H}) \mathrm{ppm}$.

\subsubsection{Synthesis of E3 ligase linker}

Synthesis of 2-(2,6-dioxopiperidin-3-yl)-4-fluoroisoindoline-1,3-dione (L0)<smiles>O=C1CCC(N2C(=O)c3cccc(F)c3C2=O)C(=O)N1</smiles>

$2.00 \mathrm{~g}(12.0 \mathrm{mmol}, 1.00 \mathrm{eq})$ 4-Fluoroisobenzofuran-1,3-dione and $1.98 \mathrm{~g}(12.0 \mathrm{mmol}, 1.00 \mathrm{eq}) 3-$ Aminopiperidine-2,6-dione were dissolved in $15 \mathrm{~mL}$ pyridine and stirred for $18 \mathrm{~h}$ at $110^{\circ} \mathrm{C}$. The solution was cooled to $\mathrm{rt}$ and the pyridine was removed under reduced pressure. The crude solution was dissolved in water and DCM. The aqueous phase was separated and extracted 6x with DCM. The combined organic phases were washed with $1 \mathrm{M} \mathrm{HCl}$ and saturated $\mathrm{NaCl}$ solution. The combined organic phases were dried over $\mathrm{MgSO}_{4}$, filtered and excess solvent was removed under reduced pressure. The crude product was purified by column chromatography.

Yield: $1.13 \mathrm{~g}, 4.09 \mathrm{mmol}, 33 \%$ of a yellow solid.

$\mathrm{R}_{\mathrm{f}}\left(5 \% \mathrm{MeOH} / \mathrm{CH}_{2} \mathrm{Cl}_{2}\right): 0.53$.

ESI: (calculated): $\left[\mathrm{M}+2 \mathrm{H}^{+}\right] 278.06 \mathrm{~g} / \mathrm{mol}$ (found): $\left[\mathrm{M}+2 \mathrm{H}^{+}\right] 278.96 \mathrm{~g} / \mathrm{mol}$.

${ }^{1} \mathrm{H}$ NMR (250 MHz, DMSO) $\delta=11.13(\mathrm{~s}, 1 \mathrm{H}, 17-\mathrm{H}), 7.95$ (ddd, $\left.{ }^{3} \mathrm{~J}=8.3 \mathrm{~Hz},{ }^{3} \mathrm{~J}=7.5 \mathrm{~Hz},{ }^{4} \mathrm{~J}=4.6 \mathrm{~Hz}, 1 \mathrm{H}\right)$, $7.80-7.70(\mathrm{~m}, 2 \mathrm{H}), 5.18-5.10(\mathrm{~m}, 1 \mathrm{H}), 2.96-2.81(\mathrm{~m}, 1 \mathrm{H}), 2.65-2.43(\mathrm{~m}, 2 \mathrm{H}), 2.06(\mathrm{~m}, 1 \mathrm{H}) \mathrm{ppm}$. 
Synthesis

of

tert-butyl

(2-(2-((2-(2,6-dioxopiperidin-3-y))-1,3-dioxoisoindolin-4-

yl)amino)ethoxy)ethyl)carbamate (L1)

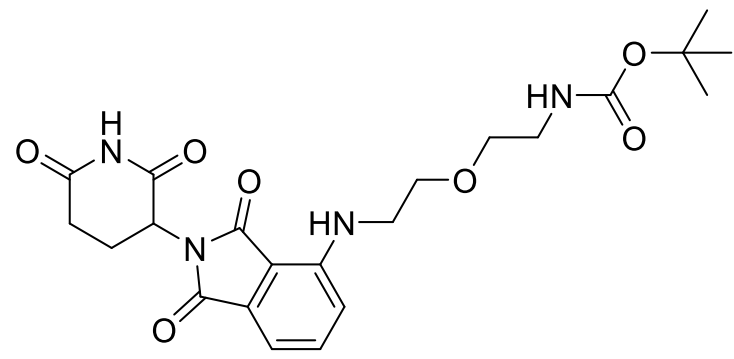

$150 \mathrm{mg}$ (543 $\mu \mathrm{mol}, 1.00$ eq) 2-(2,6-dioxopiperidin-3-yl)-4-fluoroisoindoline-1,3-dione, $111 \mathrm{mg}$ (543 $\mu \mathrm{mol}, 1.00 \mathrm{eq})$ tert-butyl (2-(2-aminoethoxy)ethyl)carbamate and $189 \mu \mathrm{L}(1.09 \mathrm{mmol}, 2.00 \mathrm{eq})$ DIEA were dissolved in $5 \mathrm{~mL} \mathrm{NMP}$ and stirred at $80^{\circ} \mathrm{C}$ for $16 \mathrm{~h}$. The solution was cooled to $\mathrm{rt}$ and diluted with $\mathrm{EA}$ and saturated $\mathrm{NaHCO}_{3}$ and saturated $\mathrm{NaCl}$ solution. The mixture was extracted $3 \mathrm{x}$ with $\mathrm{EA}$, dried over $\mathrm{MgSO}_{4}$, filtered and excess solvent was removed under reduced pressure. The crude product was purified by Flash Chromatography.

Yield: $102 \mathrm{mg}, 177 \mu \mathrm{mol} 33 \%$ of a yellow solid.

$R_{f}(30 \%$ Cyclohexane/EA): 0.46.

ESI: (calculated): $\left[\mathrm{M}+\mathrm{Na}^{+}\right] 483.19 \mathrm{~g} / \mathrm{mol}$

(found): [M+Na+] $483.23 \mathrm{~g} / \mathrm{mol}$.

HPLC: RT = $12.8 \min (254 \mathrm{~nm}, 100 \%)$.

1H NMR $(600 \mathrm{MHz}, \mathrm{MeOD}) \delta=7.52\left(\mathrm{t},{ }^{3} \mathrm{~J}=7.8 \mathrm{~Hz}, 1 \mathrm{H}\right), 7.06-7.02(\mathrm{~m}, 2 \mathrm{H}), 5.05\left(\mathrm{dd},{ }^{3} \mathrm{~J}=12.4 \mathrm{~Hz}, 4 \mathrm{~J}=\right.$ $5.3 \mathrm{~Hz}, 1 \mathrm{H}), 3.68\left(\mathrm{t},{ }^{3} \mathrm{~J}=5.2 \mathrm{~Hz}, 2 \mathrm{H}\right), 3.68-3.46(\mathrm{~m}, 4 \mathrm{H}), 3.23\left(\mathrm{t},{ }^{3} \mathrm{~J}=5.5 \mathrm{~Hz}, 2 \mathrm{H}\right), 2.85$ (ddd, ${ }^{2} \mathrm{~J}=19.2 \mathrm{~Hz}$, $\left.{ }^{3} \mathrm{~J}=14.3 \mathrm{~Hz},{ }^{4} \mathrm{~J}=5.2 \mathrm{~Hz}, 1 \mathrm{H}\right), 2.78-2.66(\mathrm{~m}, 2 \mathrm{H}), 2.16-2.06(\mathrm{~m}, 1 \mathrm{H}), 1.41(\mathrm{~s}, 9 \mathrm{H}) \mathrm{ppm}$.

13C NMR (126 MHz, MeOD) $\delta=174.6,171.5,170.7,169.2,158.4,148.2,137.2,133.8,118.3,112.1$, $111.2,80.1,70.9,70.4,50.2,49.0,43.2,41.3,32.2,28.7,23.8$ ppm. 
Synthesis of tert-butyl (2-(2-(2-(2-((2-(2,6-dioxopiperidin-3-yl)-1,3-dioxoisoindolin-4yl)amino)ethoxy)ethoxy)ethoxy)ethyl)carbamate (L2)<smiles>CC(C)(C)OC(=O)NCCCOCCOCCOCCCNc1cccc2c1C(=O)N(C1CCC(=O)NC1=O)C2=O</smiles>

$100 \mathrm{mg}$ (362 $\mu \mathrm{mol}, 1.00$ eq) 2-(2,6-dioxopiperidin-3-yl)-4-fluoroisoindoline-1,3-dione, $84.5 \mathrm{mg}$ (362 $\mu \mathrm{mol}, 1.00 \mathrm{eq})$ tert-butyl (3-(2-(2-(3-aminopropoxy)ethoxy)ethoxy)propyl)carbamate and $126 \mu \mathrm{L}$ (724 $\mathrm{mmol}, 2.00 \mathrm{eq}$ ) DIEA were dissolved in $5 \mathrm{~mL} \mathrm{NMP}$ and stirred at $80^{\circ} \mathrm{C}$ for $16 \mathrm{~h}$. The solution was cooled to rt and diluted with $\mathrm{EA}$ and saturated $\mathrm{NaHCO}_{3}$ and saturated $\mathrm{NaCl}$ solution. The mixture was extracted 3x with $\mathrm{EA}$, dried over $\mathrm{MgSO}_{4}$, filtered and excess solvent was removed under reduced pressure. The crude product was purified by Flash Chromatography.

Yield: $102 \mathrm{mg}, 177 \mu \mathrm{mol} 33 \%$ of a yellow solid.

$R_{f}(30 \%$ Cyclohexane/EA): 0.25 .

ESI: (calculated): $\left[\mathrm{M}+\mathrm{Na}^{+}\right] 599.27 \mathrm{~g} / \mathrm{mol}$

(found): $\left[\mathrm{M}+\mathrm{Na}^{+}\right] 599.36 \mathrm{~g} / \mathrm{mol}$.

HPLC: RT = $11.1 \mathrm{~min}(254 \mathrm{~nm}, 100 \%)$.

${ }^{1} \mathrm{H}$ NMR $(600 \mathrm{MHz}, \mathrm{MeOD}) \delta=7.55\left(\mathrm{dd},{ }^{3} J=8.5 \mathrm{~Hz},{ }^{3} \mathrm{~J}=7.2 \mathrm{~Hz}, 1 \mathrm{H}\right), 7.05\left(\mathrm{dd},{ }^{2} J=13.7,{ }^{3} J=7.8 \mathrm{~Hz}, 2 \mathrm{H}\right.$ ), $5.07-5.04(\mathrm{~m}, 1 \mathrm{H}), 3.69-3.68(\mathrm{~m}, 2 \mathrm{H}), 3.65-3.62(\mathrm{~m}, 10 \mathrm{H}), 3.45-3.42(\mathrm{~m}, 2 \mathrm{H}), 3.07\left(\mathrm{t},{ }^{3} \mathrm{~J}=6.4 \mathrm{~Hz}\right.$, $2 \mathrm{H}), 2.89-2.83(\mathrm{~m}, 1 \mathrm{H}), 2.76-2.70(\mathrm{~m}, 2 \mathrm{H}), 2.12-2.10(\mathrm{~m}, 1 \mathrm{H}), 1.94-1.89(\mathrm{~m}, 4 \mathrm{H}) \mathrm{ppm}$.

${ }^{13} \mathrm{C}$ NMR (126 MHz, MeOD) $\delta=174.6,171.7,170.7,169.3,148.2,137.3,133.9,118.0,111.8,111.0$, $71.4,71.2,71.1,70.4,70.2,50.2,41.4,39.95,32.2,30.1,28.1,23.8,18.5$ ppm. 
<smiles>CC(C)(C)OC(=O)NCCOCCOCCOCCOCCOCCNc1cccc2c1C(=O)N(C1CCC(=O)NC1=O)C2=O</smiles>

$200 \mathrm{mg}$ (720 $\mu \mathrm{mol}, 1.0$ eq) 2-(2,6-dioxopiperidin-3-yl)-4-fluoroisoindoline-1,3-dione, $84.5 \mathrm{mg}$ (720 $\mu \mathrm{mol}, 1.0$ eq) tert-butyl (17-amino-3,6,9,12,15-pentaoxaheptadecyl)carbamate and $242 \mu \mathrm{L}$ (1.45 mmol, 2.0 eq) DIEA were dissolved in $5 \mathrm{~mL} \mathrm{NMP}$ and stirred at $80^{\circ} \mathrm{C}$ for $16 \mathrm{~h}$. The solution was cooled to rt and diluted with $\mathrm{EA}$ and saturated $\mathrm{NaHCO}_{3}$ and saturated $\mathrm{NaCl}$ solution. The mixture was extracted 3x with $\mathrm{EA}$, dried over $\mathrm{MgSO}_{4}$, filtered and excess solvent was removed under reduced pressure. The crude product was purified by Flash Chromatography.

Yield: $210 \mathrm{mg}, 330 \mu \mathrm{mol}, 46 \%$ of a yellow oil.

$R_{f}(33 \%$ Cyclohexane/ 67\% EA): 0.13.

ESI: (calculated): $\left[\mathrm{M}+\mathrm{Na}^{+}\right] 659.29 \mathrm{~g} / \mathrm{mol}$ (found): $\left[\mathrm{M}+\mathrm{Na}^{+}\right] 659.30 \mathrm{~g} / \mathrm{mol}$.

HPLC: RT = $12.8 \min (254 \mathrm{~nm}, 93 \%)$.

${ }^{1} \mathrm{H}$ NMR $(250 \mathrm{MHz}, \mathrm{DMSO}) \delta=11.08(\mathrm{~s}, 1 \mathrm{H}), 7.58\left(\mathrm{dd},{ }^{3} \mathrm{~J}=8.4 \mathrm{~Hz},{ }^{3} \mathrm{~J}=7.2 \mathrm{~Hz}, 1 \mathrm{H}\right), 7.15\left(\mathrm{~d},{ }^{3} \mathrm{~J}=8.6 \mathrm{~Hz}\right.$, $1 \mathrm{H}), 7.04\left(\mathrm{~d},{ }^{3} \mathrm{~J}=7.0 \mathrm{~Hz}, 1 \mathrm{H}\right), 6.73\left(\mathrm{t},{ }^{3} \mathrm{~J}=4.7 \mathrm{~Hz}, 1 \mathrm{H}\right), 6.60\left(\mathrm{t},{ }^{3} \mathrm{~J}=5.6 \mathrm{~Hz}, 1 \mathrm{H}\right), 5.05\left(\mathrm{dd},{ }^{3} \mathrm{~J}=12.5 \mathrm{~Hz},{ }^{4} \mathrm{~J}=\right.$ $5.4 \mathrm{~Hz}, 1 \mathrm{H}), 3.62\left(\mathrm{t},{ }^{3} \mathrm{~J}=5.3 \mathrm{~Hz}, 2 \mathrm{H}\right), 3.58-3.43(\mathrm{~m}, 16 \mathrm{H}), 3.41-3.23(\mathrm{~m}, 4 \mathrm{H}), 3.05\left(\mathrm{q},{ }^{3} \mathrm{~J}=6.0 \mathrm{~Hz}, 2 \mathrm{H}\right)$, $2.99-2.77(\mathrm{~m}, 1 \mathrm{H}), 2.61-2.55(\mathrm{~m}, 2 \mathrm{H}), 2.04-2.02(\mathrm{~m}, 1 \mathrm{H}), 1.36(\mathrm{~s}, 9 \mathrm{H}) \mathrm{ppm}$. 
Synthesis of tert-butyl (23-((2-(2,6-dioxopiperidin-3-yl)-1,3-dioxoisoindolin-4-yl)amino)3,6,9,12,15,18,21-heptaoxatricosyl)carbamate (L4)<smiles>CC(C)(C)OC(=O)NCCOCCOCCOCCOCCOCCOCCOCCNc1cccc2c1C(=O)N(C1CCC(=O)NC1=O)C2=O</smiles>

$200 \mathrm{mg} \quad(720 \mu \mathrm{mol}, 1.0$ eq) 2-(2,6-dioxopiperidin-3-yl)-4-fluoroisoindoline-1,3-dione, $337 \mathrm{mg}$ (720 $\mu \mathrm{mol}, 1.0$ eq) tert-butyl (23-amino-3,6,9,12,15,18,21-heptaoxatricosyl)carbamate and $242 \mu \mathrm{L}$ (1.45 mmol, 2.0 eq) DIEA were dissolved in $5 \mathrm{~mL} \mathrm{NMP}$ and stirred at $80^{\circ} \mathrm{C}$ for $16 \mathrm{~h}$. The solution was cooled to rt and diluted with $\mathrm{EA}$ and saturated $\mathrm{NaHCO}_{3}$ and saturated $\mathrm{NaCl}$ solution. The mixture was extracted 3x with $\mathrm{EA}$, dried over $\mathrm{MgSO}_{4}$, filtered and excess solvent was removed under reduced pressure. The crude product was purified by Flash Chromatography.

Yield: $271 \mathrm{mg}, 374 \mu \mathrm{mol}, 52 \%$ of a yellow oil.

$R_{f}(50 \%$ Cyclohexane/ 50\% EA): 0.17.

ESI: (calculated): $\left[\mathrm{M}+\mathrm{Na}^{+}\right] 747.34 \mathrm{~g} / \mathrm{mol}$ (found): $\left[\mathrm{M}+\mathrm{Na}^{+}\right] 747.41 \mathrm{~g} / \mathrm{mol}$.

HPLC: RT $=12.7 \mathrm{~min}(254 \mathrm{~nm}, 100 \%)$.

${ }^{1} \mathrm{H}$ NMR $(600 \mathrm{MHz}, \mathrm{MeOD}) \delta=7.52\left(\mathrm{dd},{ }^{3} \mathrm{~J}=8.4,7.2 \mathrm{~Hz}, 1 \mathrm{H}\right), 7.06-7.02(\mathrm{~m}, 2 \mathrm{H}), 5.06-5.03(\mathrm{~m}, 1 \mathrm{H})$, $3.71\left(\mathrm{t}^{3}{ }^{3} \mathrm{~J}=5.3 \mathrm{~Hz}, 2 \mathrm{H}\right), 3.64-3.61(\mathrm{~m}, 4 \mathrm{H}), 3.62-3.57(\mathrm{~m}, 2 \mathrm{H}), 3.50-3.47(\mathrm{~m}, 4 \mathrm{H}), 3.21\left(\mathrm{t},{ }^{3} \mathrm{~J}=5.5 \mathrm{~Hz}\right.$, $2 \mathrm{H}), 2.93-2.81(\mathrm{~m}, 1 \mathrm{H}), 2.76-2.70(\mathrm{~m}, 2 \mathrm{H}), 2.13-2.11(\mathrm{~m}, 1 \mathrm{H}), 1.43(\mathrm{~s}, 9 \mathrm{H}) \mathrm{ppm}$.

${ }^{13} \mathrm{C}$ NMR (126 MHz, MeOD) $\delta=174.5,171.3,170.6,169.1,158.2,148.1,137.2,133.7,118.2,112.0$, $111.1,80.0,71.6,71.6,71.6,71.5,71.5,71.4,71.2$, 71.0, 70.5, 50.2, 43.2, 41.2, 32.2, 28.80, 23.77 ppm. 
Synthesis

of

tert-butyl

(4-((2-(2,6-dioxopiperidin-3-y))-1,3-dioxoisoindolin-4-

yl)amino)methyl)benzyl)carbamate (L5)<smiles>CC(C)(C)OC(=O)NCc1ccc(CNc2cccc3c2C(=O)N(C2CCC(=O)NC2=O)C3=O)cc1</smiles>

$150 \mathrm{mg} \quad(543 \mu \mathrm{mol}, 1.0$ eq) 2-(2,6-dioxopiperidin-3-yl)-4-fluoroisoindoline-1,3-dione, $128 \mathrm{mg}$ (543 $\mu \mathrm{mol}, 1.0 \mathrm{eq}$ ) tert-butyl (4-(aminomethyl)benzyl)carbamate and $284 \mu \mathrm{L}$ (1.63 mmol, 2.0 eq) DIEA were dissolved in $7 \mathrm{~mL}$ DMF and stirred at $80{ }^{\circ} \mathrm{C}$ for $16 \mathrm{~h}$. The solution was cooled to rt and diluted with EA and saturated $\mathrm{NaHCO}_{3}$ and saturated $\mathrm{NaCl}$ solution. The mixture was extracted $3 x$ with $\mathrm{EA}$, dried over $\mathrm{MgSO}_{4}$, filtered and excess solvent was removed under reduced pressure. The crude product was purified by Flash Chromatography.

Yield: $148 \mathrm{mg}, 301 \mu \mathrm{mol}, 55 \%$ of a yellow oil.

$\mathrm{R}_{\mathrm{f}}\left(5 \% \mathrm{MeOH} / \mathrm{CH}_{2} \mathrm{Cl}_{2}\right): 0.31$.

ESI: (calculated): $\left[\mathrm{M}+\mathrm{Na}^{+}\right] 515.19 \mathrm{~g} / \mathrm{mol}$

(found): [M+Na $] 515.27 \mathrm{~g} / \mathrm{mol}$.

HPLC: RT = $13.4 \min (254 \mathrm{~nm}, 76 \%)$.

${ }^{1} \mathrm{H}$ NMR $(250 \mathrm{MHz}, \mathrm{MeOD}) \delta=7.46\left(\mathrm{dd},{ }^{3} \mathrm{~J}=8.3 \mathrm{~Hz},{ }^{3} \mathrm{~J}=7.3 \mathrm{~Hz}, 1 \mathrm{H}\right), 7.30-7.27(\mathrm{~m}, 3 \mathrm{H}), 7.19-7.16(\mathrm{~m}$, 1H), $7.04\left(\mathrm{~d},{ }^{3} \mathrm{~J}=7.1 \mathrm{~Hz}, 1 \mathrm{H}\right), 6.93\left(\mathrm{~d},{ }^{3} \mathrm{~J}=8.5 \mathrm{~Hz}, 1 \mathrm{H}\right), 4.79-4.73(\mathrm{~m}, 1 \mathrm{H}), 4.55(\mathrm{~s}, 2 \mathrm{H}), 4.21(\mathrm{~s}, 2 \mathrm{H}), 2.89$ $-2.61(\mathrm{~m}, 1 \mathrm{H}), 2.49-2.47(\mathrm{~m}, 1 \mathrm{H}), 2.39-2.33(\mathrm{~m}, 1 \mathrm{H}), 2.12-2.01(\mathrm{~m}, 1 \mathrm{H}), 1.42(\mathrm{~s}, 9 \mathrm{H}) \mathrm{ppm}$. 
Synthesis

of

tert-butyl

(2-(3-)((S)-1-((2S,4R)-4-hydroxy-2-((4-(4-methylthiazol-5-

yl)benzyl)carbamoyl)pyrrolidin-1-yl)-3,3-dimethyl-1-oxobutan-2-yl)amino)-3-

oxopropoxy)ethyl)carbamate (L6)

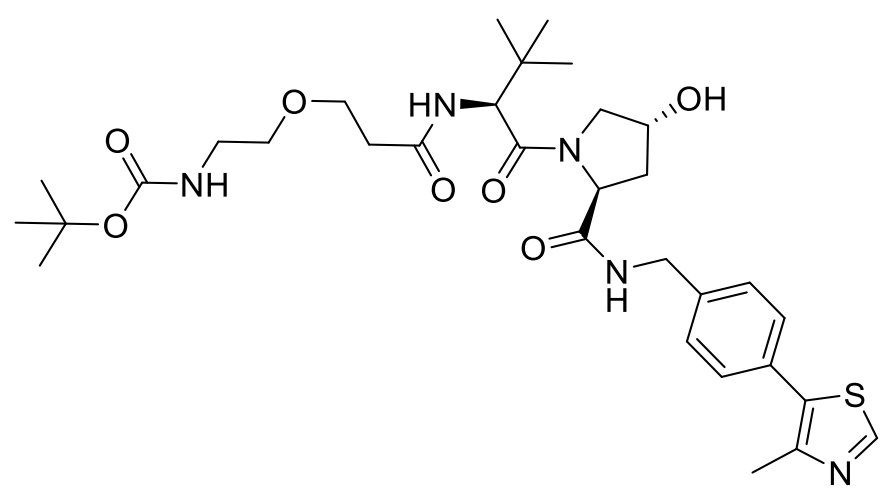

$71 \mathrm{mg}$ (307 $\mu \mathrm{mol}, 1.0$ eq) 3-(2-((tert-butoxycarbonyl)amino)ethoxy)propanoic acid were dissolved in

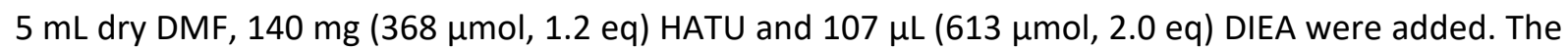

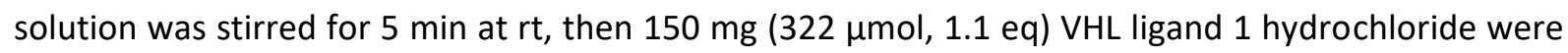
added to the solution and stirred under Argon atmosphere for $4 \mathrm{~h}$. The reaction mixture was quenched with $2 \mathrm{~mL}$ water and $2 \mathrm{~mL}$ saturated $\mathrm{NaHCO}_{3}$, then the reaction was extracted $5 \mathrm{x}$ with EA. The organic phase was dried over $\mathrm{MgSO}_{4}$, filtered and the solvent was removed under reduced pressure. The crude product was purified using an RP-FC system.

Yield: $80 \mathrm{mg}, 124 \mu \mathrm{mol}, 40 \%$ of a white solid.

$\mathrm{R}_{\mathrm{f}}\left(10 \% \mathrm{MeOH} / \mathrm{CH}_{2} \mathrm{Cl}_{2}\right): 0.15$.

ESI: (calculated): $\left[\mathrm{M}+\mathrm{H}^{+}\right] 646.31 \mathrm{~g} / \mathrm{mol}$

(found): $\left[\mathrm{M}+\mathrm{H}^{+}\right] 646.32 \mathrm{~g} / \mathrm{mol}$.

HPLC: RT = $12.1 \min (254 \mathrm{~nm}, 99 \%)$.

${ }^{1} \mathrm{H}$ NMR $(500 \mathrm{MHz}, \mathrm{DMSO}) \delta=8.98(\mathrm{~s}, 1 \mathrm{H}), 8.55\left(\mathrm{t},{ }^{3} \mathrm{~J}=6.0 \mathrm{~Hz}, 1 \mathrm{H}\right), 7.92\left(\mathrm{~d},{ }^{3} \mathrm{~J}=9.4 \mathrm{~Hz}, 1 \mathrm{H}\right), 7.42\left(\mathrm{~d},{ }^{3} \mathrm{~J}\right.$ $=8.4 \mathrm{~Hz}, 2 \mathrm{H}), 7.38\left(\mathrm{~d},{ }^{3} \mathrm{~J}=8.4 \mathrm{~Hz}, 2 \mathrm{H}\right), 6.69\left(\mathrm{t}, 3^{3} \mathrm{~J}=5.5 \mathrm{~Hz}, 1 \mathrm{H}\right), 5.12\left(\mathrm{~d}, 3^{3} \mathrm{~J}=3.6 \mathrm{~Hz}, 1 \mathrm{H}\right), 4.56\left(\mathrm{~d},{ }^{3} \mathrm{~J}=\right.$ $9.4 \mathrm{~Hz}, 1 \mathrm{H}), 4.49-4.39(\mathrm{~m}, 2 \mathrm{H}), 4.35(\mathrm{~s}, 1 \mathrm{H}), 4.22\left(\mathrm{dd},{ }^{3} \mathrm{~J}=15.8 \mathrm{~Hz},{ }^{4} \mathrm{~J}=5.4 \mathrm{~Hz}, 1 \mathrm{H}\right), 3.66\left(\mathrm{~d},{ }^{3} J=4.0 \mathrm{~Hz}\right.$, $1 \mathrm{H}), 3.63(\mathrm{~s}, 1 \mathrm{H}), 3.57\left(\mathrm{~d},{ }^{3} \mathrm{~J}=11.3 \mathrm{~Hz}, 2 \mathrm{H}\right), 3.40-3.33(\mathrm{~m}, 2 \mathrm{H}), 3.05\left(\mathrm{q},{ }^{3} \mathrm{~J}=6.0 \mathrm{~Hz}, 2 \mathrm{H}\right), 2.59-2.50(\mathrm{~m}$, $1 \mathrm{H}), 2.44(\mathrm{~s}, 3 \mathrm{H}), 2.40-2.29(\mathrm{~m}, 1 \mathrm{H}), 2.06-1.97(\mathrm{~m}, 1 \mathrm{H}), 1.92\left(\mathrm{dd},{ }^{3} J=8.5 \mathrm{~Hz},{ }^{4} \mathrm{~J}=4.5 \mathrm{~Hz}, 1 \mathrm{H}\right), 1.36(\mathrm{~s}$, 9H), $0.93(\mathrm{~s}, 9 \mathrm{H}) \mathrm{ppm}$. 
${ }^{13} \mathrm{C}$ NMR (126 MHz, DMSO) $\delta=171.9,170.0,169.5,155.5,151.4,147.7,139.5,131.2,129.6,128.6$, $127.4,77.6,68.87,66.6,58.7,56.4,56.3,41.67,39.9,35.4,28.2,26.3,15.9$ ppm.

Synthesis of tert-butyl (2-(2-(3-)((S)-1-((2S,4R)-4-hydroxy-2-((4-(4-methylthiazol-5yl)benzyl)carbamoyl)pyrrolidin-1-yl)-3,3-dimethyl-1-oxobutan-2-yl)amino)-3oxopropoxy)ethoxy)ethyl)carbamate (L7)

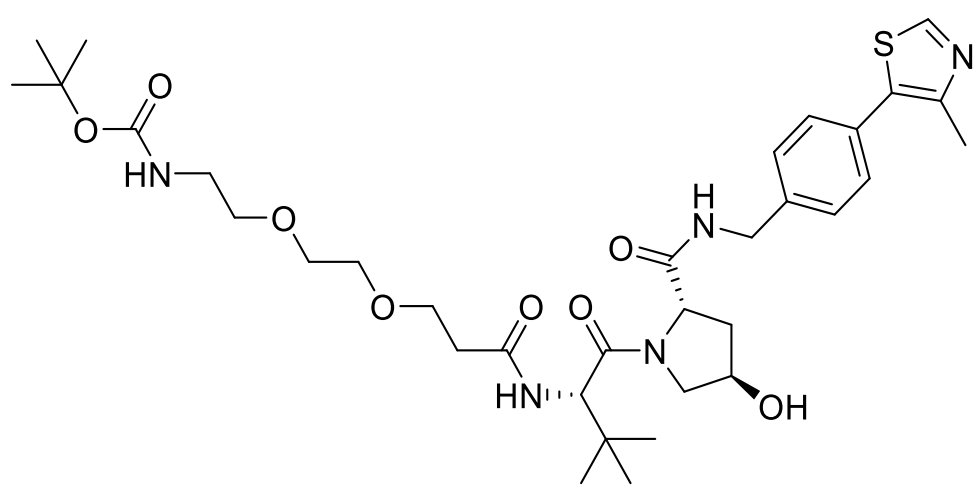

$49 \mathrm{mg}$ (178 $\mu \mathrm{mol}, 1.0$ eq) 2,2-dimethyl-4-oxo-3,8,11-trioxa-5-azatetradecan-14-oic acid were dissolved in $5 \mathrm{~mL}$ dry DMF, $82 \mathrm{mg}$ ( $214 \mu \mathrm{mol}, 1.2 \mathrm{eq})$ HATU and $62 \mu \mathrm{L}$ (356 $\mu \mathrm{mol}, 2.0$ eq) DIEA were added. The solution was stirred for $5 \mathrm{~min}$ at $\mathrm{rt}$, then $100 \mathrm{mg}$ ( $214 \mu \mathrm{mol}, 1.2 \mathrm{eq}$ ) VHL ligand 1 hydrochloride were added to the solution and stirred under Argon atmosphere for $4 \mathrm{~h}$. The reaction mixture was quenched with $2 \mathrm{~mL}$ water and $2 \mathrm{~mL}$ saturated $\mathrm{NaHCO}_{3}$, then the reaction was extracted $5 \mathrm{x}$ with EA. The organic phase was dried over $\mathrm{MgSO}_{4}$, filtered and the solvent was removed under reduced pressure. The crude product was purified using an RP-FC system.

Yield: $97 \mathrm{mg}, 141 \mu \mathrm{mol}, 80 \%$ of a white solid.

$\mathrm{R}_{\mathrm{f}}\left(10 \% \mathrm{MeOH} / \mathrm{CH}_{2} \mathrm{Cl}_{2}\right): 0.32$.

ESI: (calculated): $\left[\mathrm{M}+\mathrm{Na}^{+}\right] 712.33 \mathrm{~g} / \mathrm{mol}$ (found): $\left[\mathrm{M}+\mathrm{Na}^{+}\right] 712.32 \mathrm{~g} / \mathrm{mol}$.

HPLC: RT = $12.1 \mathrm{~min}(254 \mathrm{~nm}, 100 \%)$.

${ }^{1} \mathrm{H}$ NMR $(500 \mathrm{MHz}, \mathrm{MeOD}) \delta=8.87(\mathrm{~s}, 1 \mathrm{H}), 7.89\left(\mathrm{~d},{ }^{3} \mathrm{~J}=9.2 \mathrm{~Hz}, 1 \mathrm{H}\right), 7.47\left(\mathrm{~d},{ }^{3} \mathrm{~J}=8.3 \mathrm{~Hz}, 2 \mathrm{H}\right), 7.44-7.39$ $(\mathrm{m}, 2 \mathrm{H}), 4.66(\mathrm{~s}, 1 \mathrm{H}), 4.56\left(\mathrm{dd},{ }^{2} J=20.9 \mathrm{~Hz},{ }^{3} J=12.2 \mathrm{~Hz}, 2 \mathrm{H}\right), 4.50(\mathrm{~s}, 1 \mathrm{H}), 4.36\left(\mathrm{~d},{ }^{3} \mathrm{~J}=15.5 \mathrm{~Hz}, 1 \mathrm{H}\right), 3.89$ 
$\left(\mathrm{d},{ }^{3} \mathrm{~J}=11.0 \mathrm{~Hz}, 1 \mathrm{H}\right), 3.80\left(\mathrm{dd},{ }^{3} \mathrm{~J}=11.0 \mathrm{~Hz},{ }^{4} J=3.9 \mathrm{~Hz}, 1 \mathrm{H}\right), 3.74\left(\mathrm{dt},{ }^{3} J=10.9 \mathrm{~Hz},{ }^{4} J=5.3 \mathrm{~Hz}, 2 \mathrm{H}\right), 3.60$ $(\mathrm{s}, 4 \mathrm{H}), 3.49\left(\mathrm{t},{ }^{3} \mathrm{~J}=5.6 \mathrm{~Hz}, 2 \mathrm{H}\right), 3.20(\mathrm{~m}, 2 \mathrm{H}), 2.58(\mathrm{~m}, 1 \mathrm{H}), 2.52-2.48(\mathrm{~m}, 1 \mathrm{H}), 2.48(\mathrm{~s}, 3 \mathrm{H}), 2.21(\mathrm{~m}$, 1H), $2.09(\mathrm{~m}, 1 \mathrm{H}), 1.42(\mathrm{~s}, 9 \mathrm{H}), 1.04(\mathrm{~s}, 9 \mathrm{H}) \mathrm{ppm}$.

${ }^{13} \mathrm{C}$ NMR $(126 \mathrm{MHz}, \mathrm{MeOD}) \delta=174.4,173.7,172.1,158.4,152.8,149.0,140.3,133.4,131.5,130.4$, $129.0,80.1,71.4,71.2,71.1,68.3,60.8,58.9,58.0,43.7,41.3,38.9,37.3,36.8,28.8,27.0,15.8$ ppm.

Synthesis of tert-butyl ((S)-17-((2S,4R)-4-hydroxy-2-((4-(4-methylthiazol-5yl)benzyl)carbamoyl)pyrrolidine-1-carbonyl)-18,18-dimethyl-15-oxo-3,6,9,12-tetraoxa-16azanonadecyl)carbamate (L8)

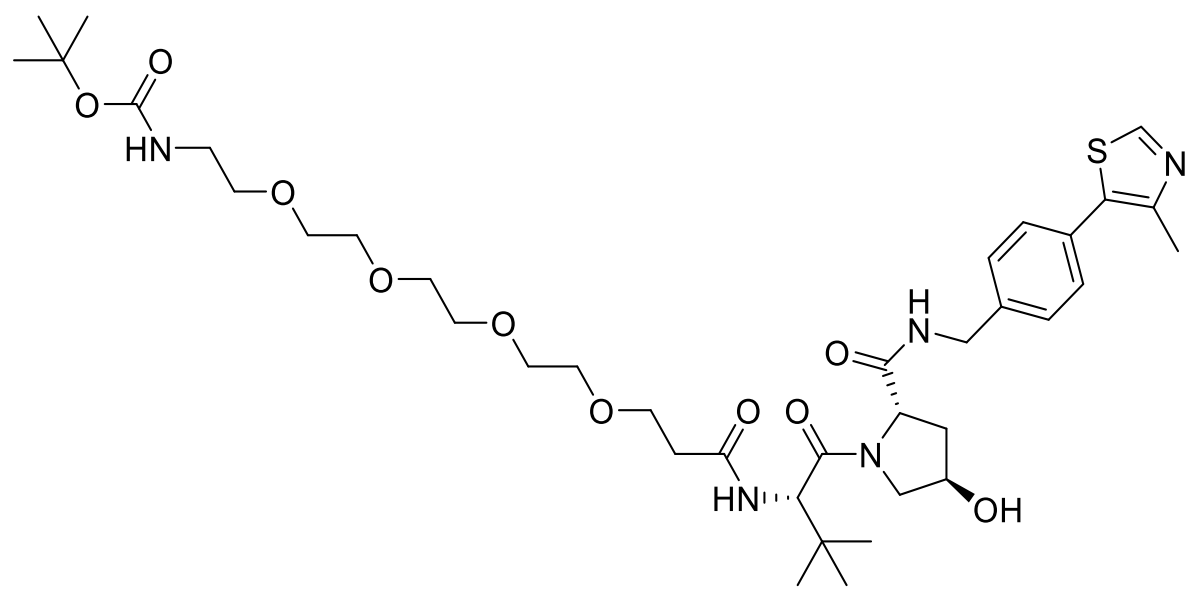

$65 \mathrm{mg}$ (178 $\mu \mathrm{mol}, 1.0$ eq) 2,2-dimethyl-4-oxo-3,8,11,14,17-pentaoxa-5-azaicosan-20-oic acid were dissolved in $5 \mathrm{~mL}$ dry DMF, $82 \mathrm{mg}$ ( $214 \mu \mathrm{mol}, 1.2 \mathrm{eq})$ HATU and $62 \mu \mathrm{L}$ (356 $\mu \mathrm{mol}, 2.0$ eq) DIEA were added. The solution was stirred for $5 \mathrm{~min}$ at $\mathrm{rt}$, then $100 \mathrm{mg}(214 \mu \mathrm{mol}, 1.2 \mathrm{eq}) \mathrm{VHL}$ ligand 1 hydrochloride were added to the solution and stirred under Argon atmosphere for $4 \mathrm{~h}$. The reaction mixture was quenched with $2 \mathrm{~mL}$ water and $2 \mathrm{~mL}$ saturated $\mathrm{NaHCO}_{3}$, then the reaction was extracted $5 \mathrm{x}$ with EA. The organic phase was dried over $\mathrm{MgSO}_{4}$, filtered and the solvent was removed under reduced pressure. The crude product was purified using an RP-FC system.

Yield: $42 \mathrm{mg}, 53.9 \mu \mathrm{mol}, 30 \%$ of a colourless oil.

$\mathrm{R}_{\mathrm{f}}\left(10 \% \mathrm{MeOH} / \mathrm{CH}_{2} \mathrm{Cl}_{2}\right): 0.22$.

ESI: (calculated): $\left[\mathrm{M}+\mathrm{Na}^{+}\right] 800.39 \mathrm{~g} / \mathrm{mol}$

(found): [M+Na $] 800.48 \mathrm{~g} / \mathrm{mol}$. 
HPLC: RT = $12.1 \min (254 \mathrm{~nm}, 99 \%)$.

${ }^{1} \mathrm{H}$ NMR $(500 \mathrm{MHz}, \mathrm{MeOD}) \delta=8.88(\mathrm{~s}, 1 \mathrm{H}), 7.47\left(\mathrm{~d},{ }^{3} \mathrm{~J}=8.3 \mathrm{~Hz}, 2 \mathrm{H}\right), 7.42\left(\mathrm{~d},{ }^{3} \mathrm{~J}=8.3 \mathrm{~Hz}, 2 \mathrm{H}\right), 4.65(\mathrm{~s}$, 1H), $4.60-4.52(\mathrm{~m}, 2 \mathrm{H}), 4.50(\mathrm{~s}, 1 \mathrm{H}), 4.36\left(\mathrm{~d},{ }^{3} \mathrm{~J}=15.5 \mathrm{~Hz}, 1 \mathrm{H}\right), 3.89\left(\mathrm{~d},{ }^{3} \mathrm{~J}=11.1 \mathrm{~Hz}, 1 \mathrm{H}\right), 3.80\left(\mathrm{dd},{ }^{3} J=\right.$ $\left.11.0 \mathrm{~Hz},{ }^{4} \mathrm{~J}=3.9 \mathrm{~Hz}, 1 \mathrm{H}\right), 3.76-3.70(\mathrm{~m}, 2 \mathrm{H}), 3.63-3.61(\mathrm{~m}, 10 \mathrm{H}), 3.60-3.58(\mathrm{~m}, 2 \mathrm{H}), 3.49\left(\mathrm{t},{ }^{3} \mathrm{~J}=\right.$ $5.6 \mathrm{~Hz}, 2 \mathrm{H}), 3.21\left(\mathrm{t},{ }^{3} \mathrm{~J}=5.6 \mathrm{~Hz}, 2 \mathrm{H}\right), 2.56\left(\mathrm{dd},{ }^{3} \mathrm{~J}=7.5 \mathrm{~Hz},{ }^{4} \mathrm{~J}=5.3 \mathrm{~Hz}, 1 \mathrm{H}\right), 2.48(\mathrm{~s}, 4 \mathrm{H}), 2.26-2.18(\mathrm{~m}$, $1 \mathrm{H}), 2.13-2.04(\mathrm{~m}, 1 \mathrm{H}), 1.43(\mathrm{~s}, 9 \mathrm{H}), 1.04(\mathrm{~s}, 9 \mathrm{H}) \mathrm{ppm}$.

${ }^{13} \mathrm{C}$ NMR (126 MHz, MeOD) $\delta=174.5,173.7,172.1,158.4,152.8,149.0,140.3,133.4,131.5,130.4$, $129.0,80.1,71.6,71.5,71.4,71.3,71.1,68.3,60.8,58.9,58.0,43.7,41.3,38.9,37.4,36.8,28.8,27.0$, $15.8 \mathrm{ppm}$.

Synthesis of tert-butyl (3-)((S)-1-((2S,4R)-4-hydroxy-2-((4-(4-methylthiazol-5yl)benzyl)carbamoyl)pyrrolidin-1-yl)-3,3-dimethyl-1-oxobutan-2-yl)amino)-3oxopropyl)carbamate (L9)<smiles>Cc1ncsc1-c1ccc(CNC(=O)[C@@H]2C[C@@H](O)CN2C(=O)[C@@H](NC(=O)CCNC(=O)OC(C)(C)C)C(C)(C)C)cc1</smiles>

$40 \mathrm{mg}(214 \mu \mathrm{mol}, 1.0$ eq) 3-((tert-butoxycarbonyl)amino)propanoic acid were dissolved in $2 \mathrm{~mL}$ dry DMF, $98 \mathrm{mg}$ ( $257 \mu \mathrm{mol}, 1.2 \mathrm{eq}) \mathrm{HATU}$ and $75 \mu \mathrm{L}$ (428 $\mu \mathrm{mol}, 2.0$ eq) DIEA were added. The solution was stirred for $5 \mathrm{~min}$ at $\mathrm{rt}$, then $100 \mathrm{mg}(214 \mu \mathrm{mol}, 1.0 \mathrm{eq}) \mathrm{VHL}$ ligand 1 hydrochloride were added to the solution and stirred under Argon atmosphere for $4 \mathrm{~h}$. The reaction mixture was quenched with $2 \mathrm{~mL}$ water and $2 \mathrm{~mL}$ saturated $\mathrm{NaHCO}_{3}$, then the reaction was extracted $5 \mathrm{x}$ with $\mathrm{EA}$. The organic phase was dried over $\mathrm{MgSO}_{4}$, filtered and the solvent was removed under reduced pressure. The crude product was purified using an RP-FC system.

Yield: $62 \mathrm{mg}, 103 \mu \mathrm{mol}, 48 \%$ of a white solid.

$\mathrm{R}_{\mathrm{f}}\left(10 \% \mathrm{MeOH} / \mathrm{CH}_{2} \mathrm{Cl}_{2}\right): 0.42$.

ESI: (calculated): $\left[\mathrm{M}+2 \mathrm{H}^{+}\right] 603.31 \mathrm{~g} / \mathrm{mol}$ 
(found): $\left[\mathrm{M}+2 \mathrm{H}^{+}\right] 603.26 \mathrm{~g} / \mathrm{mol}$.

${ }^{1} \mathrm{H}$ NMR $(300 \mathrm{MHz}, \mathrm{MeOD}) \delta=9.01(\mathrm{~s}, 1 \mathrm{H}), 7.48\left(\mathrm{~d},{ }^{3} \mathrm{~J}=8.0 \mathrm{~Hz}, 2 \mathrm{H}\right), 7.42\left(\mathrm{~d},{ }^{3} \mathrm{~J}=7.4 \mathrm{~Hz}, 2 \mathrm{H}\right), 4.59\left(\mathrm{~d},{ }^{3} \mathrm{~J}\right.$ $=12.9 \mathrm{~Hz}, 2 \mathrm{H}), 4.53-4.50(\mathrm{~m}, 2 \mathrm{H}), 4.36\left(\mathrm{~d},{ }^{3} \mathrm{~J}=15.2 \mathrm{~Hz}, 1 \mathrm{H}\right), 3.92\left(\mathrm{~d},{ }^{3} \mathrm{~J}=11.4 \mathrm{~Hz}, 1 \mathrm{H}\right), 3.80\left(\mathrm{~d},{ }^{3} J=\right.$ $10.2 \mathrm{~Hz}, 1 \mathrm{H}), 3.38-3.24(\mathrm{~m}, 2 \mathrm{H}), 2.53-2.43(\mathrm{~m}, 2 \mathrm{H}), 2.49(\mathrm{~s}, 3 \mathrm{H}), 2.31-2.15(\mathrm{~m}, 1 \mathrm{H}), 2.10(\mathrm{~m}, 1 \mathrm{H})$, $1.42(\mathrm{~s}, 9 \mathrm{H}), 1.04(\mathrm{~s}, 9 \mathrm{H}) \mathrm{ppm}$.

${ }^{13} \mathrm{C}$ NMR $(75 \mathrm{MHz}$, MeOD) $\delta=174.5,173.7,172.3,153.3,148.2,140.6,131.1,130.5,130.4,129.6$, $129.0,80.2,71.1,60.8,59.1,58.0,49.0,43.7,38.9,38.6,38.1,36.8,36.5,28.7,27.0,15.4$ ppm.

Synthesis of tert-butyl (4-)((S)-1-((2S,4R)-4-hydroxy-2-((4-(4-methylthiazol-5yl)benzyl)carbamoyl)pyrrolidin-1-yl)-3,3-dimethyl-1-oxobutan-2-yl)amino)-4oxobutyl)carbamate (L10)<smiles>Cc1ncsc1-c1ccc(CNC(=O)[C@@H]2C[C@@H](O)CN2C(=O)[C@@H](NC(=O)CCCNC(=O)OC(C)(C)C)C(C)(C)C)cc1</smiles>

$44 \mathrm{mg}$ (214 $\mu \mathrm{mol}, 1.0$ eq) 4-((tert-butoxycarbonyl)amino)butanoic acid were dissolved in $2 \mathrm{~mL}$ dry DMF, $98 \mathrm{mg}$ ( $257 \mu \mathrm{mol}, 1.2 \mathrm{eq})$ HATU and $75 \mu \mathrm{L}$ (428 $\mu \mathrm{mol}, 2.0$ eq) DIEA were added. The solution was stirred for $5 \mathrm{~min}$ at rt, then $100 \mathrm{mg}(214 \mu \mathrm{mol}, 1.0 \mathrm{eq}) \mathrm{VHL}$ ligand 1 hydrochloride were added to the solution and stirred under Argon atmosphere for $4 \mathrm{~h}$. The reaction mixture was quenched with $2 \mathrm{~mL}$ water and $2 \mathrm{~mL}$ saturated $\mathrm{NaHCO}_{3}$, then the reaction was extracted $5 \mathrm{x}$ with EA. The organic phase was dried over $\mathrm{MgSO}_{4}$, filtered and the solvent was removed under reduced pressure. The crude product was purified using an RP-FC system.

Yield: $61 \mathrm{mg}, 99.2 \mu \mathrm{mol}, 46 \%$ of a white solid.

$\mathrm{R}_{\mathrm{f}}\left(10 \% \mathrm{MeOH} / \mathrm{CH}_{2} \mathrm{Cl}_{2}\right): 0.45$.

ESI: (calculated): $\left[\mathrm{M}+\mathrm{Na}^{+}\right] 639.30 \mathrm{~g} / \mathrm{mol}$ (found): [M+Na+] $639.43 \mathrm{~g} / \mathrm{mol}$. 
${ }^{1} \mathrm{H}$ NMR (300 MHz, MeOD) $\delta=8.95(\mathrm{~s}, 1 \mathrm{H}), 7.47\left(\mathrm{~d},{ }^{3} \mathrm{~J}=8.4 \mathrm{~Hz}, 2 \mathrm{H}\right), 7.44-7.38(\mathrm{~m}, 2 \mathrm{H}), 4.55(\mathrm{~m}, 4 \mathrm{H})$, $4.36\left(\mathrm{~d},{ }^{3} \mathrm{~J}=15.5 \mathrm{~Hz}, 1 \mathrm{H}\right), 3.91(\mathrm{~m}, 1 \mathrm{H}), 3.80(\mathrm{~m}, 1 \mathrm{H}), 3.05\left(\mathrm{t},{ }^{3} \mathrm{~J}=6.8 \mathrm{~Hz}, 2 \mathrm{H}\right), 2.48(\mathrm{~s}, 3 \mathrm{H}), 2.29(\mathrm{~m}, 2 \mathrm{H})$, $2.24-2.15(\mathrm{~m}, 1 \mathrm{H}), 2.08(\mathrm{~m}, 1 \mathrm{H}), 1.85-1.65(\mathrm{~m}, 2 \mathrm{H}), 1.43(\mathrm{~s}, 9 \mathrm{H}), 1.04(\mathrm{~s}, 9 \mathrm{H}) \mathrm{ppm}$.

${ }^{13} \mathrm{C}$ NMR $(75 \mathrm{MHz}, \mathrm{MeOD}) \delta=175.4,174.5,172.4,153.1,148.6,140.4,133.7,131.2,130.4,129.4$, $129.0,79.9,71.1,60.8,59.2,58.0,43.7,40.7,38.9,36.5,33.9,28.8,27.4,27.0,15.6$ ppm.

Synthesis of tert-butyl (5-)((S)-1-((2S,4R)-4-hydroxy-2-((4-(4-methylthiazol-5yl)benzyl)carbamoyl)pyrrolidin-1-yl)-3,3-dimethyl-1-oxobutan-2-yl)amino)-5oxopentyl)carbamate (L11)

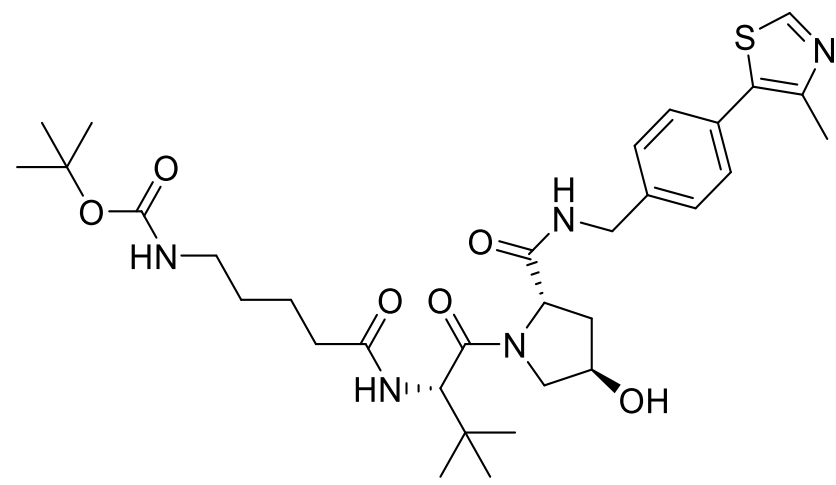

$39 \mathrm{mg}(178 \mu \mathrm{mol}, 1.0$ eq) 5-((tert-butoxycarbonyl)amino)pentanoic acid were dissolved in $5 \mathrm{~mL}$ dry DMF, $82 \mathrm{mg}$ (214 $\mu \mathrm{mol}, 1.2 \mathrm{eq})$ HATU and $62 \mu \mathrm{L}$ (356 $\mu \mathrm{mol}, 2.0$ eq) DIEA were added. The solution was stirred for $5 \mathrm{~min}$ at rt, then $100 \mathrm{mg}(214 \mu \mathrm{mol}, 1.2 \mathrm{eq}) \mathrm{VHL}$ ligand 1 hydrochloride were added to the solution and stirred under Argon atmosphere for $4 \mathrm{~h}$. The reaction mixture was quenched with $2 \mathrm{~mL}$ water and $2 \mathrm{~mL}$ saturated $\mathrm{NaHCO}_{3}$, then the reaction was extracted $5 \mathrm{x}$ with $\mathrm{EA}$. The organic phase was dried over $\mathrm{MgSO}_{4}$, filtered and the solvent was removed under reduced pressure. The crude product was purified using an RP-FC system.

Yield: $59 \mathrm{mg}, 93.8 \mu \mathrm{mol}, 53 \%$ of a white solid.

$\mathrm{R}_{\mathrm{f}}\left(10 \% \mathrm{MeOH} / 90 \% \mathrm{CH}_{2} \mathrm{Cl}_{2}\right): 0.37$.

ESI: (calculated): $\left[\mathrm{M}+\mathrm{Na}^{+}\right] 652.31 \mathrm{~g} / \mathrm{mol}$

(found): $\left[\mathrm{M}+\mathrm{Na}^{+}\right] 652.27 \mathrm{~g} / \mathrm{mol}$.

HPLC: RT = $12.1 \min (254 \mathrm{~nm}, 100 \%)$. 
${ }^{1} \mathrm{H}$ NMR $(500 \mathrm{MHz}, \mathrm{MeOD}) \delta=8.87(\mathrm{~s}, 1 \mathrm{H}), 7.46\left(\mathrm{~d},{ }^{3} \mathrm{~J}=8.3 \mathrm{~Hz}, 2 \mathrm{H}\right), 7.41\left(\mathrm{~d},{ }^{3} \mathrm{~J}=8.3 \mathrm{~Hz}, 2 \mathrm{H}\right), 4.63(\mathrm{~s}$, $1 \mathrm{H}), 4.56\left(\mathrm{dd},{ }^{2} J=19.3 \mathrm{~Hz},{ }^{3} \mathrm{~J}=11.8 \mathrm{~Hz}, 2 \mathrm{H}\right), 4.50(\mathrm{~s}, 1 \mathrm{H}), 4.36\left(\mathrm{~d},{ }^{2} \mathrm{~J}=15.5 \mathrm{~Hz}, 1 \mathrm{H}\right), 3.90\left(\mathrm{~d},{ }^{3} J=11.1 \mathrm{~Hz}\right.$, $1 \mathrm{H}), 3.80\left(\mathrm{dd},{ }^{3} \mathrm{~J}=10.9 \mathrm{~Hz},{ }^{4} \mathrm{~J}=3.9 \mathrm{~Hz}, 1 \mathrm{H}\right), 3.04\left(\mathrm{t},{ }^{3} \mathrm{~J}=6.9 \mathrm{~Hz}, 2 \mathrm{H}\right), 2.47(\mathrm{~s}, 3 \mathrm{H}), 2.30(\mathrm{~m}, 2 \mathrm{H}), 2.22(\mathrm{~m}$, $1 \mathrm{H}), 2.09(\mathrm{~m}, 1 \mathrm{H}), 1.68-1.58(\mathrm{~m}, 2 \mathrm{H}), 1.49(\mathrm{~m}, 2 \mathrm{H}), 1.42(\mathrm{~s}, 9 \mathrm{H}), 1.04(\mathrm{~s}, 9 \mathrm{H}) \mathrm{ppm}$.

${ }^{13} \mathrm{C}$ NMR (126 MHz, MeOD) $\delta=166.3,165.0,162.8,149.0,143.3,139.5,130.8,123.9,122.0,120.9$, $119.5,70.3,61.6,51.3,49.5,48.5,34.2,31.4,29.4,27.0,26.7,21.0,19.3,17.6,14.7,6.3$ ppm.

Synthesis of tert-butyl (6-)((S)-1-((2S,4R)-4-hydroxy-2-((4-(4-methylthiazol-5yl)benzyl)carbamoyl)pyrrolidin-1-yl)-3,3-dimethyl-1-oxobutan-2-yl)amino)-6oxohexyl)carbamate (L12)

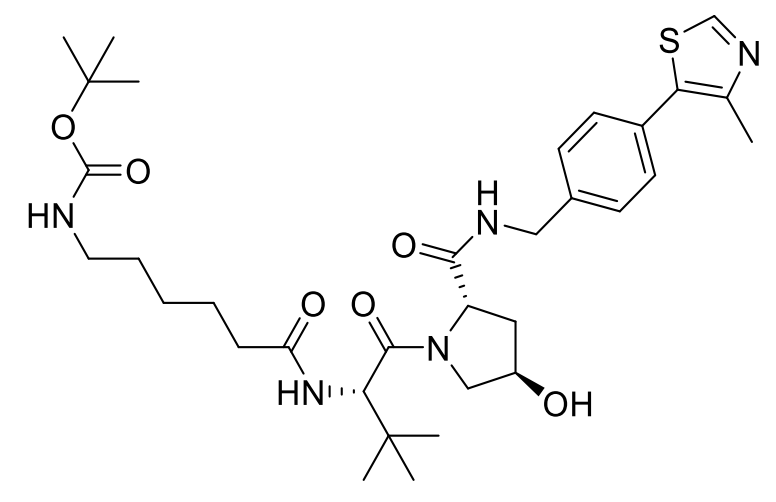

$41 \mathrm{mg}(178 \mu \mathrm{mol}, 1.0$ eq) 6-((tert-butoxycarbonyl)amino)hexanoic acid were dissolved in $5 \mathrm{~mL}$ dry DMF, $82 \mathrm{mg}$ ( $214 \mu \mathrm{mol}, 1.2 \mathrm{eq}) \mathrm{HATU}$ and $62 \mu \mathrm{L}$ (356 $\mu \mathrm{mol}, 2.0$ eq) DIEA were added. The solution was stirred for $5 \mathrm{~min}$ at rt, then $100 \mathrm{mg}(214 \mu \mathrm{mol}, 1.2 \mathrm{eq}) \mathrm{VHL}$ ligand 1 hydrochloride were added to the solution and stirred under Argon atmosphere for $4 \mathrm{~h}$. The reaction mixture was quenched with $2 \mathrm{~mL}$ water and $2 \mathrm{~mL}$ saturated $\mathrm{NaHCO}_{3}$, then the reaction was extracted $5 \mathrm{x}$ with EA. The organic phase was dried over $\mathrm{MgSO}_{4}$, filtered and the solvent was removed under reduced pressure. The crude product was purified using an RP-FC system.

Yield: $100 \mathrm{mg}, 155 \mu \mathrm{mol}, 87 \%$ of a white solid.

$\mathrm{R}_{\mathrm{f}}\left(5 \% \mathrm{MeOH} / \mathrm{CH}_{2} \mathrm{Cl}_{2}\right): 0.24$.

ESI: (calculated): $\left[\mathrm{M}+\mathrm{Na}^{+}\right] 666.32 \mathrm{~g} / \mathrm{mol}$ (found): $\left[\mathrm{M}+\mathrm{Na}^{+}\right] 666.30 \mathrm{~g} / \mathrm{mol}$. 
HPLC: RT = $12.3 \min (254 \mathrm{~nm}, 100 \%)$

${ }^{1} \mathrm{H}$ NMR (500 MHz, MeOD) $\delta=8.88(\mathrm{~s}, 1 \mathrm{H}), 7.47\left(\mathrm{~d},{ }^{3} \mathrm{~J}=8.4 \mathrm{~Hz}, 2 \mathrm{H}\right), 7.41\left(\mathrm{~d},{ }^{3} \mathrm{~J}=8.3 \mathrm{~Hz}, 2 \mathrm{H}\right), 4.63(\mathrm{~s}$, $1 \mathrm{H}), 4.58(\mathrm{~m}, 1 \mathrm{H}), 4.53(\mathrm{~m}, 1 \mathrm{H}) 4.50(\mathrm{~s}, 1 \mathrm{H}), 4.36(\mathrm{~m}, 1 \mathrm{H}), 3.91\left(\mathrm{~d},{ }^{3} \mathrm{~J}=11.0 \mathrm{~Hz}, 1 \mathrm{H}\right), 3.80\left(\mathrm{dd},{ }^{3} \mathrm{~J}=10.9 \mathrm{~Hz}\right.$, $\left.{ }^{4} J=3.9 \mathrm{~Hz}, 1 \mathrm{H}\right), 3.01(\mathrm{~m}, 2 \mathrm{H}), 2.47(\mathrm{~s}, 3 \mathrm{H}), 2.28(\mathrm{~m}, 2 \mathrm{H}), 2.21(\mathrm{~m}, 1 \mathrm{H}), 2.13-2.05(\mathrm{~m}, 1 \mathrm{H}), 1.66-1.59$ $(\mathrm{m}, 2 \mathrm{H}), 1.51-1.44(\mathrm{~m}, 2 \mathrm{H}), 1.42(\mathrm{~s}, 9 \mathrm{H}), 1.34(\mathrm{~m}, 2 \mathrm{H}), 1.04(\mathrm{~s}, 9 \mathrm{H}) \mathrm{ppm}$.

${ }^{13} \mathrm{C}$ NMR $(126 \mathrm{MHz}, \mathrm{MeOD}) \delta=175.9,174.5,172.3,158.5,152.8,149.0,140.3,133.4,131.5,130.4$, $129.0,79.8,71.1,60.8,59.0,58.0,43.7,41.2,38.9,36.5,30.6,28.8,27.5,27.0,26.7,15.8$ ppm.

Synthesis of tert-butyl (7-)((S)-1-((2S,4R)-4-hydroxy-2-((4-(4-methylthiazol-5yl)benzyl)carbamoyl)pyrrolidin-1-yl)-3,3-dimethyl-1-oxobutan-2-yl)amino)-7-oxoheptyl)carbamate (L13)

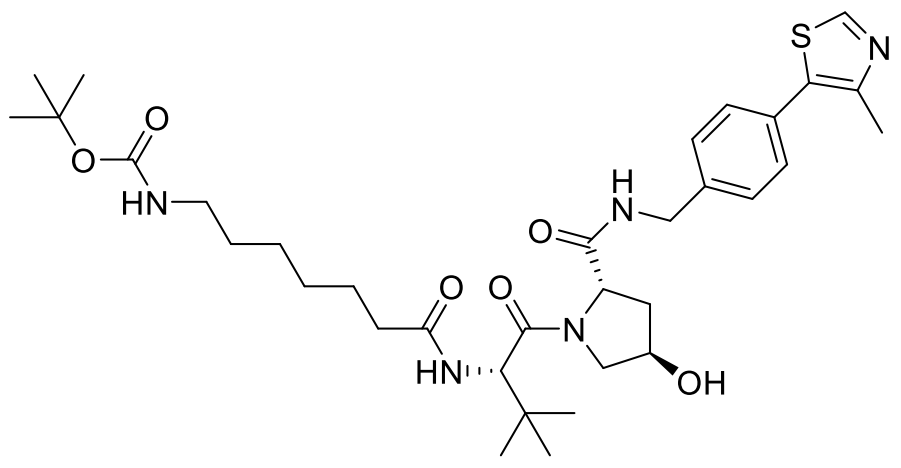

$44 \mathrm{mg}(178 \mu \mathrm{mol}, 1.0$ eq) 7-((tert-butoxycarbonyl)amino)heptanoic acid were dissolved in $5 \mathrm{~mL}$ dry DMF, $82 \mathrm{mg}$ ( $214 \mu \mathrm{mol}, 1.2 \mathrm{eq})$ HATU and $62 \mu \mathrm{L}$ (356 $\mu \mathrm{mol}, 2.0$ eq) DIEA were added. The solution was stirred for $5 \mathrm{~min}$ at rt, then $100 \mathrm{mg}(214 \mu \mathrm{mol}, 1.2 \mathrm{eq}) \mathrm{VHL}$ ligand 1 hydrochloride were added to the solution and stirred under Argon atmosphere for $4 \mathrm{~h}$. The reaction mixture was quenched with $2 \mathrm{~mL}$ water and $2 \mathrm{~mL}$ saturated $\mathrm{NaHCO}_{3}$, then the reaction was extracted $5 \mathrm{x}$ with $\mathrm{EA}$. The organic phase was dried over $\mathrm{MgSO}_{4}$, filtered and the solvent was removed under reduced pressure. The crude product was purified using an RP-FC system.

Yield: $76 \mathrm{mg}, 115 \mu \mathrm{mol}, 65 \%$ of a white solid.

$\mathrm{R}_{\mathrm{f}}\left(5 \% \mathrm{MeOH} / 95 \% \mathrm{CH}_{2} \mathrm{Cl}_{2}\right): 0.28$. 
ESI: (calculated): $\left[\mathrm{M}+\mathrm{Na}^{+}\right] 680.34 \mathrm{~g} / \mathrm{mol}$

(found): $\left[\mathrm{M}+\mathrm{Na}^{+}\right] 680.31 \mathrm{~g} / \mathrm{mol}$.

HPLC: RT = $12.5 \min (254 \mathrm{~nm}, 94 \%)$.

${ }^{1} \mathrm{H}$ NMR $(500 \mathrm{MHz}, \mathrm{MeOD}) \delta=8.87(\mathrm{~s}, 1 \mathrm{H}), 8.64\left(\mathrm{t},{ }^{3} \mathrm{~J}=6.0 \mathrm{~Hz}, 1 \mathrm{H}\right), 7.80\left(\mathrm{~d},{ }^{3} \mathrm{~J}=9.0 \mathrm{~Hz}, 1 \mathrm{H}, \mathrm{NH}\right), 7.46$ $\left(\mathrm{d},{ }^{3} \mathrm{~J}=8.3 \mathrm{~Hz}, 2 \mathrm{H}\right), 7.41\left(\mathrm{~d},{ }^{3} \mathrm{~J}=8.3 \mathrm{~Hz}, 2 \mathrm{H}\right), 4.66-4.62(\mathrm{~m}, 1 \mathrm{H}), 4.61-4.56(\mathrm{~m}, 1 \mathrm{H}), 4.54\left(\mathrm{~d},{ }^{3} \mathrm{~J}=15.5 \mathrm{~Hz}\right.$, $1 \mathrm{H}), 4.50(\mathrm{~s}, 1 \mathrm{H}), 4.36\left(\mathrm{~d},{ }^{3} \mathrm{~J}=15.5 \mathrm{~Hz}, 1 \mathrm{H}\right), 3.91\left(\mathrm{~d},{ }^{3} \mathrm{~J}=11.1 \mathrm{~Hz}, 1 \mathrm{H}\right), 3.80\left(\mathrm{dd},{ }^{3} \mathrm{~J}=10.9 \mathrm{~Hz},{ }^{4} \mathrm{~J}=3.9 \mathrm{~Hz}\right.$, $1 \mathrm{H}), 3.01\left(\mathrm{t},{ }^{3} \mathrm{~J}=7.0 \mathrm{~Hz}, 2 \mathrm{H}\right), 2.47(\mathrm{~s}, 3 \mathrm{H}), 2.37-2.23(\mathrm{~m}, 2 \mathrm{H}), 2.22-2.18(\mathrm{~m}, 1 \mathrm{H}), 2.08(\mathrm{~m}, 1 \mathrm{H}), 1.61$ $\left(\mathrm{dt},{ }^{3} \mathrm{~J}=14.2 \mathrm{~Hz},{ }^{4} \mathrm{~J}=7.2 \mathrm{~Hz}, 2 \mathrm{H}\right), 1.49-1.43(\mathrm{~m}, 2 \mathrm{H}), 1.42(\mathrm{~s}, 9 \mathrm{H}), 1.35-1.31(\mathrm{~m}, 4 \mathrm{H}), 1.04(\mathrm{~s}, 9 \mathrm{H}) \mathrm{ppm}$.

${ }^{13} \mathrm{C}$ NMR (126 MHz, MeOD) $\delta=176.0,174.4,172.3,158.5,152.8,149.0,140.3,133.4,131.5,130.3$, $129.0,79.7,71.1,60.8,59.0,58.0,43.7,41.3,38.9,36.5,30.8,29.9,28.8,27.5,27.0,26.9,15.8$ ppm.

Synthesis of tert-butyl (4-(2-)((S)-1-((2S,4R)-4-hydroxy-2-((4-(4-methylthiazol-5yl)benzyl)carbamoyl)pyrrolidin-1-yl)-3,3-dimethyl-1-oxobutan-2-yl)amino)-2oxoethyl)benzyl)carbamate (L14)

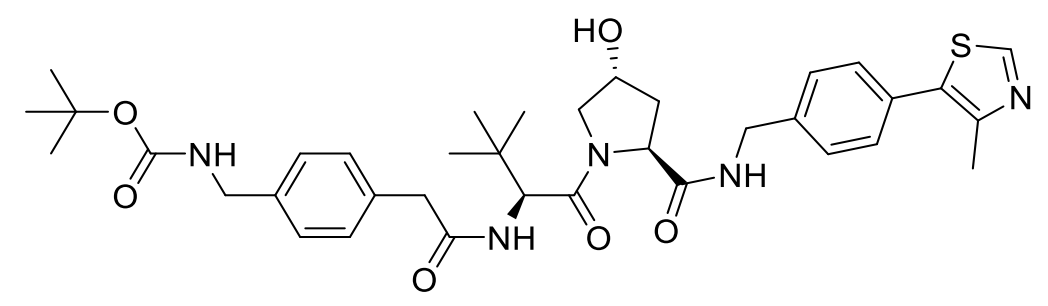

$81 \mathrm{mg}$ (307 $\mu \mathrm{mol}, 1.0$ eq) 2-(4-(((tert-butoxycarbonyl)amino)methyl)phenyl)acetic acid were dissolved

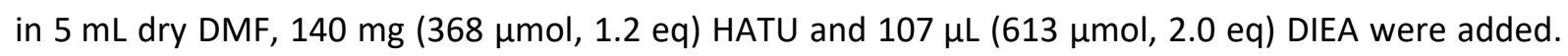
The solution was stirred for $5 \mathrm{~min}$ at $\mathrm{rt}$, then $150 \mathrm{mg}$ (322 $\mu \mathrm{mol}, 1.1 \mathrm{eq}) \mathrm{VHL}$ ligand 1 hydrochloride were added to the solution and stirred under Argon atmosphere for $4 \mathrm{~h}$. The reaction mixture was quenched with $2 \mathrm{~mL}$ water and $2 \mathrm{~mL}$ saturated $\mathrm{NaHCO}_{3}$, then the reaction was extracted $5 \mathrm{x}$ with $\mathrm{EA}$. The organic phase was dried over $\mathrm{MgSO}_{4}$, filtered and the solvent was removed under reduced pressure. The crude product was purified using an RP-FC system.

Yield: $65 \mathrm{mg}, 95.9 \mu \mathrm{mol}, 31 \%$ of a yellow solid.

$\mathrm{R}_{\mathrm{f}}\left(10 \% \mathrm{MeOH} / \mathrm{CH}_{2} \mathrm{Cl}_{2}\right): 0.11$. 
ESI: (calculated): $\left[\mathrm{M}+\mathrm{H}^{+}\right] 678.33 \mathrm{~g} / \mathrm{mol}$

(found): $\left[\mathrm{M}+\mathrm{H}^{+}\right] 678.34 \mathrm{~g} / \mathrm{mol}$.

HPLC: RT = $12.5 \min (254 \mathrm{~nm}, 100 \%)$.

${ }^{1} \mathrm{H}$ NMR (500 MHz, DMSO) $\delta=8.98(\mathrm{~s}, 1 \mathrm{H}), 8.57\left(\mathrm{t},{ }^{3} \mathrm{~J}=6.1 \mathrm{~Hz}, 1 \mathrm{H}\right), 8.07\left(\mathrm{~d},{ }^{3} \mathrm{~J}=9.3 \mathrm{~Hz}, 1 \mathrm{H}\right), 7.42\left(\mathrm{~d},{ }^{3} \mathrm{~J}\right.$ $=8.4 \mathrm{~Hz}, 2 \mathrm{H}), 7.39\left(\mathrm{~d}, 3^{3} \mathrm{~J}=8.4 \mathrm{~Hz}, 2 \mathrm{H}\right), 7.33\left(\mathrm{t},{ }^{3} \mathrm{~J}=6.3 \mathrm{~Hz}, 1 \mathrm{H}\right), 7.20\left(\mathrm{~d},{ }^{3} \mathrm{~J}=8.0 \mathrm{~Hz}, 2 \mathrm{H}\right), 7.13\left(\mathrm{~d}, 3^{3} \mathrm{~J}=\right.$ $8.0 \mathrm{~Hz}, 2 \mathrm{H}), 5.11\left(\mathrm{~d},{ }^{3} \mathrm{~J}=3.6 \mathrm{~Hz}, 1 \mathrm{H}\right), 4.51\left(\mathrm{~d},{ }^{3} \mathrm{~J}=9.4 \mathrm{~Hz}, 1 \mathrm{H}\right), 4.47-4.40(\mathrm{~m}, 2 \mathrm{H}), 4.34(\mathrm{~s}, 1 \mathrm{H}), 4.22(\mathrm{dd}$, $\left.{ }^{3} J=15.8 \mathrm{~Hz},{ }^{4} J=5.5 \mathrm{~Hz}, 1 \mathrm{H}\right), 4.08\left(\mathrm{~d},{ }^{3} J=6.1 \mathrm{~Hz}, 2 \mathrm{H}\right), 3.66(\mathrm{~m}, 1 \mathrm{H}), 3.62\left(\mathrm{~d},{ }^{3} J=13.2 \mathrm{~Hz}, 2 \mathrm{H}\right), 3.41\left(\mathrm{~d},{ }^{3} J\right.$ $=13.8 \mathrm{~Hz}, 1 \mathrm{H}), 2.45(\mathrm{~s}, 3 \mathrm{H}), 2.06-1.98(\mathrm{~m}, 1 \mathrm{H}), 1.90(\mathrm{~m}, 1 \mathrm{H}), 1.38(\mathrm{~s}, 9 \mathrm{H}), 0.91(\mathrm{~s}, 9 \mathrm{H}) \mathrm{ppm}$.

${ }^{13} \mathrm{C}$ NMR $(126 \mathrm{MHz}, \mathrm{DMSO}) \delta=171.9,170.0,169.5,155.7,151.4,147.7,139.5,138.1,134.9,131.1$, 129.6, 128.9, 128.6, 127.4, 126.7, 77.7, 68.8, 58.7, 56.4, 56.4, 43.1, 41.6, 40.0, 35.4, 28.2, 26.3, $15.9 \mathrm{ppm}$.

Synthesis of tert-butyl (5-) ((S)-1-((2S,4S)-4-hydroxy-2-((4-(4-methylthiazol-5yl)benzyl)carbamoyl)pyrrolidin-1-yl)-3,3-dimethyl-1-oxobutan-2-yl)amino)-5oxopentyl)carbamate (L15)<smiles>Cc1ncsc1-c1ccc(CNC(=O)[C@@H]2CC(O)CN2C(=O)[C@@H](NC(=O)CCCCNC(=O)OC(C)(C)C)C(C)(C)C)cc1</smiles>

$5.63 \mathrm{~g}$ ( $30.9 \mathrm{mmol}, 1.00 \mathrm{eq})$ 4-Bromobenzonitrile and $4.5 \mathrm{~mL}$ (30.9 mmol, $1.00 \mathrm{eq})$ 4-Methylthiazole were dissolved in $25 \mathrm{~mL}$ Dimethylacetamide and $2 \mathrm{~mL}$ water. $6.00 \mathrm{~g}(61.8 \mathrm{mmol}, 2.00 \mathrm{eq})$ potassium acetate and $32 \mathrm{mg}$ ( $3.09 \mathrm{mmol}, 0.10 \mathrm{eq}$ ) palladium(II)acetate were added. The mixture was stirred for $24 \mathrm{~h}$ at $115^{\circ} \mathrm{C}$. Excess solvent was removed under reduced pressure. The reaction mixture was dissolved with water and ethyl acetate. The phases were separated and the aqueous phase was extracted four times with ethylacetate. The combined organic phases were dried over MgSO4, filtered and excess solvent was removed under reduced pressure. The purification of the crude product was done by column chromatography to obtain $2.36 \mathrm{~g}(11.6 \mathrm{mmol}, 38 \%)$ of 4 -(4-methylthiazol-5yl)benzonitrile. 
This product was dissolved in THF and added slowly to $24 \mathrm{~mL}(23.1 \mathrm{mmol}, 2.00 \mathrm{eq})$ of a cold solution of $1 \mathrm{M} \mathrm{LiAlH}$ in THF. The reaction mixture was allowed to reach $r t$ and then heated at $40{ }^{\circ} \mathrm{C}$ for $18 \mathrm{~h}$. The reaction mixture was cooled to $\mathrm{rt}$ and water was added slowly. The solution was filtered and washed with $10 \% \mathrm{MeOH} / \mathrm{CH}_{2} \mathrm{Cl}_{2}$. The solvent was removed under reduced pressure, then the crude mixture was diluted with $\mathrm{CH}_{2} \mathrm{Cl}_{2}$. The phases were separated, the aqueous phase was extracted four times with $\mathrm{CH}_{2} \mathrm{Cl}_{2}$. The combined organic phases were dried over MgSO4, filtered and excess solvent was removed under reduced pressure to obtain $2.00 \mathrm{~g}(9.79 \mathrm{mmol}, 84 \%)$ of crude (4-(4-methylthiazol5-yl)phenyl)methanamine.

$351 \mathrm{mg}$ (1.52 mmol, 1.05 eq) (2S,4S)-1-(tert-butoxycarbonyl)-4-hydroxypyrrolidine-2-carboxylic acid and $661 \mathrm{mg}$ (1.74 mmol, 1.20 eq) HATU were dissolved in $7 \mathrm{~mL} \mathrm{DMF.} 505 \mu \mathrm{L}$ (2.89 mmol, 2.00 eq) DIEA were added and the mixture was stirred for $15 \mathrm{~min}$ at rt. $296 \mathrm{mg}(1.45 \mathrm{mmol}, 1.00 \mathrm{eq})$ (4-(4methylthiazol-5-yl)phenyl)methanamine was added to the mixture and stirred for $3 \mathrm{~h}$ at $\mathrm{rt}$. The reaction was stopped with $1 \mathrm{~mL}$ water. Saturated $\mathrm{NaHCO}_{3}$ solution and saturated $\mathrm{NaCl}$ solution were added and the reaction mixture was extracted $4 \mathrm{x}$ with EA. The combined organic phases were washed with saturated $\mathrm{NaHCO}_{3}$ solution, dried over $\mathrm{MgSO}_{4}$ and filtered. The solvent of the organic phase was evapored under reduced pressure. The purification of the crude product was done by Flash Chromatography to obtain $286 \mathrm{mg}(685 \mu \mathrm{mol}, 47 \%)$ of tert-butyl $(2 \mathrm{~S}, 4 \mathrm{~S})-4$-hydroxy-2-((4-)(4methylthiazol-5-yl)benzyl)carbamoyl)pyrrolidine-1-carboxylate.

The obtained product was dissolved in $1 \mathrm{~mL}$ TFA and $1 \mathrm{~mL} \mathrm{CH}_{2} \mathrm{Cl}_{2}$ and stirred for $1 \mathrm{~h}$ at $\mathrm{rt}$. Excess solvent was removed under reduced pressure. $158 \mathrm{mg}$ (685 $\mu \mathrm{mol}, 1.00$ eq) (S)-2-((tert-butoxycarbonyl)amino)3,3-dimethylbutanoic acid and $312 \mathrm{mg}$ (822 $\mu \mathrm{mol}, 1.20$ eq) HATU were dissolved in $5 \mathrm{~mL}$ DMF. $238 \mu \mathrm{L}$ ( $1.37 \mathrm{mmol}, 2.00 \mathrm{eq}$ ) DIEA were added and the mixture was stirred for $15 \mathrm{~min}$ at rt. $286 \mathrm{mg}$ (685 $\mu \mathrm{mol}$, 1.00 eq) of deprotected tert-butyl (2S,4S)-4-hydroxy-2-((4-(4-methylthiazol-5yl)benzyl)carbamoyl)pyrrolidine-1-carboxylate was dissolved in $1 \mathrm{~mL}$ DMF and DIEA was added until the $\mathrm{pH}$ was basic. The mixture was added to the active ester species and stirred for $3 \mathrm{~h}$ at $\mathrm{rt}$. The reaction was stopped with $1 \mathrm{~mL}$ water. Saturated $\mathrm{NaHCO}_{3}$ solution and saturated $\mathrm{NaCl}$ solution were added and the reaction mixture was extracted $4 x$ with EA. The combined organic phases were washed with saturated $\mathrm{NaHCO}_{3}$ solution, dried over $\mathrm{MgSO}_{4}$ and filtered. The solvent of the organic phase was evapored under reduced pressure. The purification of the crude product was done by Flash Chromatography to obtain $242 \mathrm{mg}(456 \mu \mathrm{mol}, 67 \%)$ tert-butyl ((S)-1-((2S,4S)-4-hydroxy-2-((4-(4methylthiazol-5-yl)benzyl)carbamoyl)pyrrolidin-1-yl)-3,3-dimethyl-1-oxobutan-2-yl)carbamate.

$114 \mathrm{mg} \quad(216 \mu \mathrm{mol}, 1.00$ eq) tert-butyl ((S)-1-((2S,4S)-4-hydroxy-2-((4-(4-methylthiazol-5yl)benzyl)carbamoyl)pyrrolidin-1-yl)-3,3-dimethyl-1-oxobutan-2-yl)carbamate were dissolved in $1 \mathrm{~mL}$

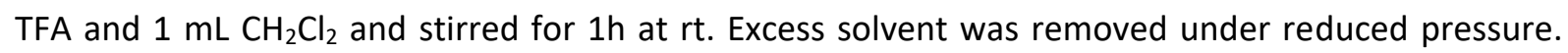

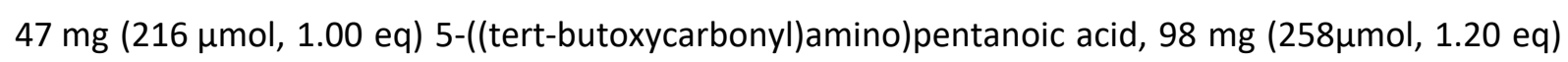


HATU and $75 \mu \mathrm{L}(429 \mu \mathrm{mol}, 2.00$ eq) DIPEA were dissolved in $1 \mathrm{~mL}$ DMF and stirred for $15 \mathrm{~min}$ at $\mathrm{rt}$. The deprotected amine was dissolved in $1 \mathrm{~mL}$ DMF and DIEA was added until the $\mathrm{pH}$ was basic. The mixture was added to the active ester species and stirred for $3 \mathrm{~h}$ at $\mathrm{rt}$. The reaction was stopped with $1 \mathrm{~mL}$ water. Saturated $\mathrm{NaHCO}_{3}$ solution and saturated $\mathrm{NaCl}$ solution were added and the reaction mixture was extracted $4 x$ with EA. The combined organic phases were washed with saturated $\mathrm{NaHCO}_{3}$ solution, dried over $\mathrm{MgSO}_{4}$ and filtered. The solvent of the organic phase was evapored under reduced pressure. The purification of the crude product was carried out on a preparative HPLC system.

Yield: $60 \mathrm{mg}, 95.3 \mu \mathrm{mol}, 44 \%$ of a white solid.

ESI: (calculated) $\left[\mathrm{M}+\mathrm{Na}^{+}\right] 652.31 \mathrm{~g} / \mathrm{mol}$

(found) $\left[\mathrm{M}+\mathrm{Na}^{+}\right] 652.31 \mathrm{~g} / \mathrm{mol}$.

HPLC: RT = $12.0 \mathrm{~min}(254 \mathrm{~nm}, 100 \%)$.

${ }^{1} \mathrm{H}$ NMR $(500 \mathrm{MHz}, \mathrm{MeOD}) \delta=8.92(\mathrm{~s}, 1 \mathrm{H}), 7.53-7.35(\mathrm{~m}, 4 \mathrm{H}), 4.63(\mathrm{~s}, 1 \mathrm{H}), 4.59-4.52(\mathrm{~m}, 2 \mathrm{H}), 4.51-$ $4.48(\mathrm{~m}, 1 \mathrm{H}), 4.36\left(\mathrm{~d},{ }^{3} \mathrm{~J}=15.5 \mathrm{~Hz}, 1 \mathrm{H}\right), 3.91-3.89(\mathrm{~m}, 1 \mathrm{H}), 3.80\left(\mathrm{dd},{ }^{3} J=11.0 \mathrm{~Hz},{ }^{4} J=3.9 \mathrm{~Hz}, 1 \mathrm{H}\right), 3.04$ $\left(t,{ }^{3} J=6.9 \mathrm{~Hz}, 2 \mathrm{H}\right), 2.48(\mathrm{~s}, 3 \mathrm{H}), 2.38-2.17(\mathrm{~m}, 3 \mathrm{H}), 2.11-2.06(\mathrm{~m}, 1 \mathrm{H}), 1.68-1.56(\mathrm{~m}, 2 \mathrm{H}), 1.51-1.46$ $(\mathrm{m}, 2 \mathrm{H}), 1.42(\mathrm{~s}, 9 \mathrm{H}), 1.04(\mathrm{~s}, 9 \mathrm{H}) \mathrm{ppm}$.

${ }^{13} \mathrm{C}$ NMR (126 MHz, MeOD) $\delta=175.7,174.5,172.3,158.5,153.0,148.7,140.4,133.6,131.3,130.4$, $129.0,79.8,71.1,60.8,59.0,58.0,49.0,43.7,40.9,38.9,36.5,36.2,30.5,28.8,27.0,24.2,15.7$ ppm. 
122 Experimental part 


\subsubsection{Synthesis of heterobifunctional molecules}

Synthesis

of

$N-(4 '-((2-$ (2-)(2-(2,6-dioxopiperidin-3-yl)-1,3-dioxoisoindolin-4-

yl)amino)ethoxy)ethyl)carbamoyl)-4-(4-methylpiperazin-1-yl)-[1,1'-biphenyl]-3-yl)-6-hydroxy-4-

(trifluoromethyl)nicotinamide (7a)

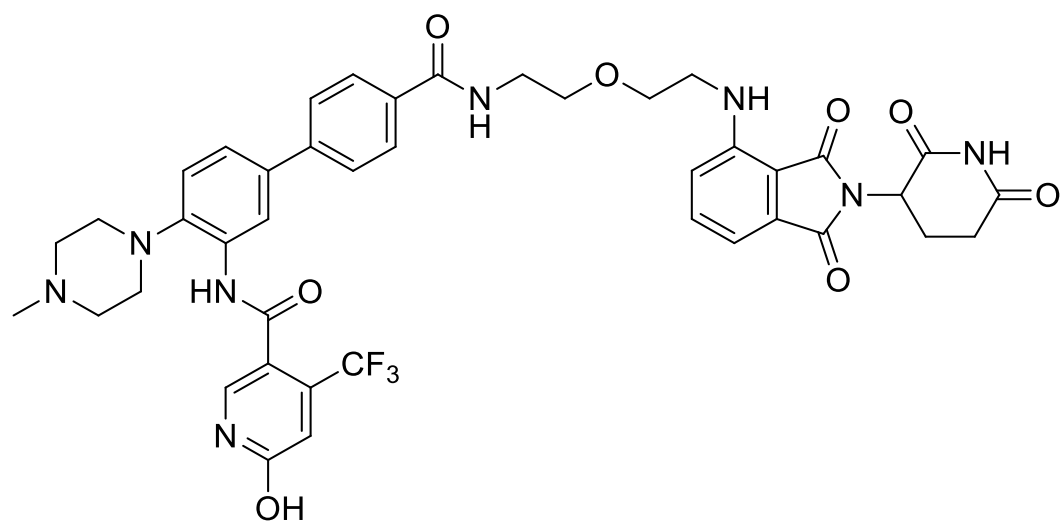

$16.0 \mathrm{mg}(28.7 \mu \mathrm{mol}, 1.00 \mathrm{eq})$ of tert-butyl 3'-(6-hydroxy-4-(trifluoromethyl)nicotinamido)-4'-(4methylpiperazin-1-yl)-[1,1'-biphenyl]-4-carboxylate was diluted in $1 \mathrm{~mL} \mathrm{DCM}$ and $1 \mathrm{~mL}$ TFA was added. In a second vial, $14.0 \mathrm{mg}(30.1 \mu \mathrm{mol}, 1.05 \mathrm{eq})$ of tert-butyl (2-(2-((2-(2,6-dioxopiperidin-3-yl)-1,3dioxoisoindolin-4-yl)amino)ethoxy)ethyl)carbamate was diluted in $1 \mathrm{~mL}$ DCM and $1 \mathrm{~mL}$ TFA was added. The two reaction mixtures were stirred for $1 \mathrm{~h}$ at $\mathrm{rt}$. Excess solvent and TFA was removed under reduced pressure.

The deprotected species of tert-butyl 3'-(6-hydroxy-4-(trifluoromethyl)nicotinamido)-4'-(4methylpiperazin-1-yl)-[1,1'-biphenyl]-4-carboxylate was dissolved in $0.5 \mathrm{~mL}$ DMF and $49 \mu \mathrm{L}$ $(0.29 \mathrm{mmol}, 10 \mathrm{eq})$ DIEA was added until the $\mathrm{pH}$ of the solution was basic. $13.0 \mathrm{mg}$ (34.4 $\mu \mathrm{mol}, 1.20$ eq) HATU was added. The reaction mixture was stirred for $20 \mathrm{~min}$ at rt. The TFA salt of tert-butyl (2-(2((2-(2,6-dioxopiperidin-3-yl)-1,3-dioxoisoindolin-4-yl)amino)ethoxy)ethyl)carbamate was dissolved in $0.5 \mathrm{~mL}$ DMF and $11 \mu \mathrm{L}(65 \mu \mathrm{mol}, 2.00 \mathrm{eq})$ DIEA were added to the solution. The mixture was added to the active ester of tert-butyl 3'-(6-hydroxy-4-(trifluoromethyl)nicotinamido)-4'-(4-methylpiperazin-1yl)-[1,1'-biphenyl]-4-carboxylate and stirred at rt for $4.5 \mathrm{~h}$. The reaction was stopped with $1 \mathrm{~mL}$ water. Saturated $\mathrm{NaHCO}_{3}$ solution and saturated $\mathrm{NaCl}$ solution were added and the reaction mixture was extracted $4 \mathrm{x}$ with EA. The combined organic phases were washed with saturated $\mathrm{NaHCO}_{3}$ solution, dried over $\mathrm{MgSO}_{4}$ and filtered. The solvent of the organic phase was evapored under reduced pressure. The purification of the crude product was done via a HPLC system.

Yield: $17.2 \mathrm{mg}, 20.4 \mu \mathrm{mol}, 71 \%$ of a yellow solid. 
MALDI: (calculated): $\left[\mathrm{M}+\mathrm{H}^{+}\right] 843.31 \mathrm{~g} / \mathrm{mol}$

(found): $\left[\mathrm{M}+\mathrm{H}^{+}\right] 843.41 \mathrm{~g} / \mathrm{mol}$.

HPLC: RT = $11.6 \min (254 \mathrm{~nm}, 100 \%)$.

HRMS: (calculated) $\left[\mathrm{M}+\mathrm{H}^{+}\right] 843.3072 \mathrm{~g} / \mathrm{mol}$

(found) $\left[\mathrm{M}+\mathrm{H}^{+}\right] 843.3067 \mathrm{~g} / \mathrm{mol}$.

${ }^{1} \mathrm{H}$ NMR $(500 \mathrm{MHz}, \mathrm{DMSO}) \delta=12.54(\mathrm{~s}, 1 \mathrm{H}), 11.08(\mathrm{~s}, 1 \mathrm{H}), 9.52(\mathrm{~s}, 1 \mathrm{H}), 8.53\left(\mathrm{t},{ }^{3} \mathrm{~J}=5.6 \mathrm{~Hz}, 1 \mathrm{H}\right), 8.14(\mathrm{~d}$, $\left.{ }^{4} J=1.8 \mathrm{~Hz}, 1 \mathrm{H}\right), 8.00(\mathrm{~s}, 1 \mathrm{H}), 7.91\left(\mathrm{~d},{ }^{3} J=8.4 \mathrm{~Hz}, 2 \mathrm{H}\right), 7.66\left(\mathrm{~d},{ }^{3} J=8.4 \mathrm{~Hz}, 2 \mathrm{H}\right), 7.60-7.48(\mathrm{~m}, 2 \mathrm{H}), 7.28$ $\left(d,{ }^{3} J=8.4 \mathrm{~Hz}, 1 \mathrm{H}\right), 7.15\left(\mathrm{~d},{ }^{3} \mathrm{~J}=8.6 \mathrm{~Hz}, 1 \mathrm{H}\right), 7.02\left(\mathrm{~d},{ }^{3} \mathrm{~J}=7.0 \mathrm{~Hz}, 1 \mathrm{H}\right), 6.83(\mathrm{~s}, 1 \mathrm{H}), 6.63\left(\mathrm{t},{ }^{3} \mathrm{~J}=5.7 \mathrm{~Hz}, 1 \mathrm{H}\right)$, $5.03\left(\mathrm{dd},{ }^{3} \mathrm{~J}=12.8 \mathrm{~Hz},{ }^{4} \mathrm{~J}=5.4 \mathrm{~Hz}, 1 \mathrm{H}\right), 3.66\left(\mathrm{t},{ }^{3} \mathrm{~J}=5.4 \mathrm{~Hz}, 2 \mathrm{H}\right), 3.61\left(\mathrm{t},{ }^{3} \mathrm{~J}=6.0 \mathrm{~Hz}, 2 \mathrm{H}\right), 3.48\left(\mathrm{dd},{ }^{3} \mathrm{~J}=\right.$ $\left.13.3 \mathrm{~Hz},{ }^{4} \mathrm{~J}=4.9 \mathrm{~Hz}, 4 \mathrm{H}\right), 2.99(\mathrm{~s}, 4 \mathrm{H}), 2.91-2.80(\mathrm{~m}, 1 \mathrm{H}), 2.75(\mathrm{~s}, 4 \mathrm{H}), 2.58-2.57(\mathrm{~m}, 1 \mathrm{H}), 2.52(\mathrm{~s}, 1 \mathrm{H})$, $2.42(\mathrm{~s}, 3 \mathrm{H}), 2.01-1.98(\mathrm{~m}, 1 \mathrm{H}) \mathrm{ppm}$.

${ }^{13} \mathrm{C}$ NMR (126 MHz, DMSO) $\delta=172.7,170.1,168.9,167.3,166.0,162.9,161.1,146.4,144.3,142.1$, $139.3,138.5\left(q,{ }^{2} J=33 \mathrm{~Hz}\right), 136.2,134.5,133.0,132.3,132.1,127.9,126.0,123.9,122.3,122.0\left(q,{ }^{1} J=\right.$ $275 \mathrm{~Hz}), 120.6,119.0,117.5,111.7,110.7,109.2,68.8,68.8,54.1,50.1,48.5,44.8,41.7,31.0,25.5$, $22.1 \mathrm{ppm}$. 
Synthesis of $\mathrm{N}$-(4'-((2-(2-(2-(2-)(2-(2,6-dioxopiperidin-3-y))-1,3-dioxoisoindolin-4yl)amino)ethoxy)ethoxy)ethoxy)ethyl)carbamoyl)-4-(4-methylpiperazin-1-yl)-[1,1'-biphenyl]-3-yl)6-hydroxy-4-(trifluoromethyl)nicotinamide (7b)

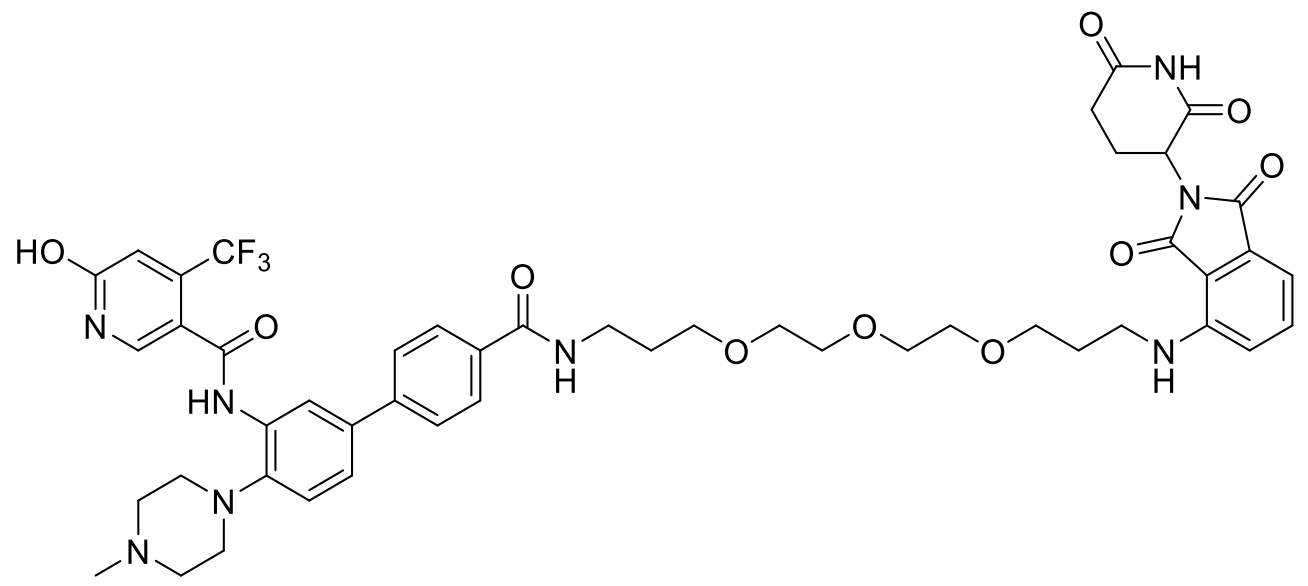

$15.0 \mathrm{mg}(27.0 \mu \mathrm{mol}, 1.00 \mathrm{eq})$ of tert-butyl 3'-(6-hydroxy-4-(trifluoromethyl)nicotinamido)-4'-(4methylpiperazin-1-yl)-[1,1'-biphenyl]-4-carboxylate was diluted in $1 \mathrm{~mL} \mathrm{DCM}$ and $1 \mathrm{~mL}$ TFA was added. In a second vial, $16.0 \mathrm{mg}(28.0 \mu \mathrm{mol}, 1.05 \mathrm{eq})$ of tert-butyl (2-(2-(2-(2-((2-(2,6-dioxopiperidin-3-yl)-1,3dioxoisoindolin-4-yl)amino)ethoxy)ethoxy)ethoxy)ethyl)carbamate was diluted in $1 \mathrm{~mL} D C M$ and $1 \mathrm{~mL}$ TFA was added. The two reaction mixtures were stirred for $1 \mathrm{~h}$ at $\mathrm{rt}$. Excess solvent and TFA was removed under reduced pressure.

The deprotected species of tert-butyl 3'-(6-hydroxy-4-(trifluoromethyl)nicotinamido)-4'-(4methylpiperazin-1-yl)-[1,1'-biphenyl]-4-carboxylate was dissolved in $0.5 \mathrm{~mL}$ DMF and $40 \mu \mathrm{L}$ (459 $\mu \mathrm{mol}$, 9.00 eq) of DIEA was added until the pH of the solution was basic. $15 \mathrm{mg}(40.5 \mu \mathrm{mol}, 1.50 \mathrm{eq})$ HATU was added. The reaction mixture was stirred for $20 \mathrm{~min}$ at $\mathrm{rt}$. The deprotected species of tert-butyl (2(2-(2-(2-((2-(2,6-dioxopiperidin-3-yl)-1,3-dioxoisoindolin-4-

yl)amino)ethoxy)ethoxy)ethoxy)ethyl)carbamate was dissolved in $0.5 \mathrm{~mL} \mathrm{DMF}$ and $10 \mu \mathrm{L}(54 \mu \mathrm{mol}$, $2.00 \mathrm{eq})$ DIEA were added to the solution. The mixture was added to the active ester of AD100 and stirred at $\mathrm{rt}$ for $2.5 \mathrm{~h}$. The reaction was stopped with $1 \mathrm{~mL}$ water. Saturated $\mathrm{NaHCO}_{3}$ solution and saturated $\mathrm{NaCl}$ solution were added and the reaction mixture was extracted $4 \mathrm{x}$ with $\mathrm{EA}$. The combined organic phases were washed with saturated $\mathrm{NaHCO}_{3}$ solution, dried over $\mathrm{MgSO}_{4}$ and filtered. The solvent of the organic phase was evapored under reduced pressure. The purification of the crude product was carried out on the HPLC system.

Yield: $8.58 \mathrm{mg}, 8.95 \mu \mathrm{mol}, 33 \%$ of a light yellow solid. 
(found) $\left[\mathrm{M}+\mathrm{H}^{+}\right] 959.47 \mathrm{~g} / \mathrm{mol}$.

HPLC: RT = $11.8 \min (254 \mathrm{~nm}, 98 \%)$

HRMS: (calculated) $\left[\mathrm{M}+\mathrm{H}^{+}\right] 959.3909 \mathrm{~g} / \mathrm{mol}$

(found) $\left[\mathrm{M}+\mathrm{H}^{+}\right] 959.3905 \mathrm{~g} / \mathrm{mol}$.

${ }^{1} \mathrm{H}$ NMR $(500 \mathrm{MHz}, \mathrm{DMSO}) \delta=11.08(\mathrm{~s}, 1 \mathrm{H}), 9.45(\mathrm{~s}, 1 \mathrm{H}), 8.44\left(\mathrm{t},{ }^{3} \mathrm{~J}=5.5 \mathrm{~Hz}, 1 \mathrm{H}\right), 8.12\left(\mathrm{~d},{ }^{4} \mathrm{~J}=1.7 \mathrm{~Hz}\right.$, $1 \mathrm{H}), 7.98(\mathrm{~s}, 1 \mathrm{H}), 7.91\left(\mathrm{~d},{ }^{3} \mathrm{~J}=8.4 \mathrm{~Hz}, 2 \mathrm{H}\right), 7.67\left(\mathrm{~d},{ }^{3} \mathrm{~J}=8.4 \mathrm{~Hz}, 2 \mathrm{H}\right), 7.61-7.54(\mathrm{~m}, 1 \mathrm{H}), 7.51\left(\mathrm{dd},{ }^{3} J=\right.$ $\left.8.3 \mathrm{~Hz},{ }^{4} \mathrm{~J}=2.2 \mathrm{~Hz}, 1 \mathrm{H}\right), 7.26\left(\mathrm{~d},{ }^{3} \mathrm{~J}=8.4 \mathrm{~Hz}, 1 \mathrm{H}\right), 7.08\left(\mathrm{~d},{ }^{3} \mathrm{~J}=8.6 \mathrm{~Hz}, 1 \mathrm{H}\right), 7.01\left(\mathrm{~d},{ }^{3} \mathrm{~J}=7.0 \mathrm{~Hz}, 1 \mathrm{H}\right), 6.81$ $(\mathrm{s}, 1 \mathrm{H}), 6.65\left(\mathrm{t},{ }^{3} \mathrm{~J}=5.9 \mathrm{~Hz}, 1 \mathrm{H}\right), 5.04\left(\mathrm{dd},{ }^{3} \mathrm{~J}=12.7 \mathrm{~Hz},{ }^{4} \mathrm{~J}=5.4 \mathrm{~Hz}, 1 \mathrm{H}\right), 3.61-3.35(\mathrm{~m}, 21 \mathrm{H}), 2.95-2.89$ $(\mathrm{m}, 3 \mathrm{H}), 2.91-2.84(\mathrm{~m}, 1 \mathrm{H}), 2.62-2.55(\mathrm{~m}, 1 \mathrm{H}), 2.23(\mathrm{~s}, 3 \mathrm{H}), 2.07-1.95(\mathrm{~m}, 1 \mathrm{H}), 1.85-1.72(\mathrm{~m}$, 5H) ppm.

${ }^{13} \mathrm{C}$ NMR (126 MHz, DMSO) $\delta=172.8,170.1,168.8,167.3,165.8,162.8,162.3,146.4,144.8,142.1$, $139.0,138.5\left(q,{ }^{2} J=32 \mathrm{~Hz}\right), 136.2,134.2,133.2,132.2,132.2,127.8,126.0,123.9,122.2,122.0\left(q,{ }^{1} J=\right.$ $275 \mathrm{~Hz}), 120.4,118.8,117.1,111.7,110.3,109.0,69.8,69.7,69.7,69.6,68.3,68.2,54.7,51.0,48.5$, $45.8,40.4,36.7,35.8,31.0,29.4,28.9,22.1$ ppm. 
Synthesis of $N$-(4'-((17-((2-(2,6-dioxopiperidin-3-yl)-1,3-dioxoisoindolin-4-yl)amino)-3,6,9,12,15pentaoxaheptadecyl)carbamoyl)-4-(4-methylpiperazin-1-yl)-[1,1'-biphenyl]-3-yl)-6-hydroxy-4(trifluoromethyl)nicotinamide (7c)<smiles>CN1CCN(c2ccc(-c3ccc(C(=O)NCCOCCOCCOCCOCCOCCNc4cccc5c4C(=O)N(C4CCC(=O)NC4=O)C5=O)cc3)cc2NC(=O)c2cnc(O)cc2C(F)(F)F)CC1</smiles>

$15.0 \mathrm{mg}(27.0 \mu \mathrm{mol}, 1.00 \mathrm{eq})$ of tert-butyl 3'-(6-hydroxy-4-(trifluoromethyl)nicotinamido)-4'-(4methylpiperazin-1-yl)-[1,1'-biphenyl]-4-carboxylate was diluted in $1 \mathrm{~mL} \mathrm{DCM}$ and $1 \mathrm{~mL}$ TFA was added. In a second vial, $18.0 \mathrm{mg}(28.0 \mu \mathrm{mol}, 1.05 \mathrm{eq})$ of tert-butyl (17-((2-(2,6-dioxopiperidin-3-yl)-1,3dioxoisoindolin-4-yl)amino)-3,6,9,12,15-pentaoxaheptadecyl)carbamate was diluted in $1 \mathrm{~mL}$ DCM and $1 \mathrm{~mL}$ TFA was added. The two reaction mixtures were stirred for $1 \mathrm{~h}$ at rt. Excess solvent and TFA was removed under reduced pressure.

The deprotected species of tert-butyl 3'-(6-hydroxy-4-(trifluoromethyl)nicotinamido)-4'-(4methylpiperazin-1-yl)-[1,1'-biphenyl]-4-carboxylate was dissolved in $0.5 \mathrm{~mL}$ DMF and $47 \mu \mathrm{L}$ ( $0.27 \mathrm{mmol}, 10 \mathrm{eq})$ DIEA was added until the $\mathrm{pH}$ of the solution was basic. $12.0 \mathrm{mg}(32.0 \mu \mathrm{mol}, 1.20 \mathrm{eq})$ HATU was added. The reaction mixture was stirred for $20 \mathrm{~min}$ at rt. The TFA salt of tert-butyl (17-((2(2,6-dioxopiperidin-3-yl)-1,3-dioxoisoindolin-4-yl)amino)-3,6,9,12,15-pentaoxaheptadecyl)carbamate was dissolved in $0.5 \mathrm{~mL}$ DMF and $11 \mu \mathrm{L}(65 \mu \mathrm{mol}, 2.00 \mathrm{eq})$ DIEA were added to the solution. The mixture was added to the active ester of tert-butyl 3'-(6-hydroxy-4-(trifluoromethyl)nicotinamido)-4'-(4methylpiperazin-1-yl)-[1,1'-biphenyl]-4-carboxylate and stirred at $\mathrm{rt}$ for $4.5 \mathrm{~h}$. The reaction was stopped with $1 \mathrm{~mL}$ water. Saturated $\mathrm{NaHCO}_{3}$ solution and saturated $\mathrm{NaCl}$ solution were added and the reaction mixture was extracted $4 \mathrm{x}$ with $\mathrm{EA}$. The combined organic phases were washed with saturated $\mathrm{NaHCO}_{3}$ solution, dried over $\mathrm{MgSO}_{4}$ and filtered. The solvent of the organic phase was evapored under reduced pressure. The purification of the crude product was carried out on the HPLC system.

Yield: $23.6 \mathrm{mg}, 23.1 \mu \mathrm{mol}, 86 \%$ of a yellow oil.

MALDI: (calculated): $\left[\mathrm{M}+\mathrm{H}^{+}\right] 1019.4121 \mathrm{~g} / \mathrm{mol}$

(found): $\left[\mathrm{M}+\mathrm{H}^{+}\right] 1019.52 \mathrm{~g} / \mathrm{mol}$. 
HPLC: RT = $11.6 \min (254 \mathrm{~nm}, 100 \%)$.

HRMS: (calculated) $\left[\mathrm{M}+\mathrm{H}^{+}\right] 1019.4121 \mathrm{~g} / \mathrm{mol}$

(found) $\left[\mathrm{M}+\mathrm{H}^{+}\right] 1019.4117 \mathrm{~g} / \mathrm{mol}$.

${ }^{1} \mathrm{H}$ NMR $(500 \mathrm{MHz}, \mathrm{DMSO}) \delta=11.10(\mathrm{~s}, 1 \mathrm{H}), 9.20(\mathrm{~s}, 1 \mathrm{H}), 8.59\left(\mathrm{~d},{ }^{3} \mathrm{~J}=5.2 \mathrm{~Hz}, 1 \mathrm{H}\right), 8.32\left(\mathrm{~d},{ }^{4} \mathrm{~J}=1.7 \mathrm{~Hz}\right.$, $1 \mathrm{H}), 8.14(\mathrm{~s}, 1 \mathrm{H}), 7.94\left(\mathrm{~d},{ }^{3} \mathrm{~J}=8.3 \mathrm{~Hz}, 6 \mathrm{H}\right), 7.68\left(\mathrm{~d},{ }^{3} \mathrm{~J}=8.4 \mathrm{~Hz}, 6 \mathrm{H}\right), 7.57\left(\mathrm{dd},{ }^{3} J=8.4 \mathrm{~Hz},{ }^{3} J=7.3 \mathrm{~Hz}, 1 \mathrm{H}\right)$, $7.46\left(\mathrm{dd},{ }^{3} \mathrm{~J}=8.3 \mathrm{~Hz},{ }^{4} \mathrm{~J}=2.0 \mathrm{~Hz}, 1 \mathrm{H}\right), 7.29\left(\mathrm{~d},{ }^{3} J=8.4 \mathrm{~Hz}, 1 \mathrm{H}\right), 7.13\left(\mathrm{t},{ }^{3} J=8.4 \mathrm{~Hz}, 1 \mathrm{H}\right), 7.06-6.94(\mathrm{~m}$, $1 \mathrm{H}), 6.58\left(\mathrm{dd},{ }^{2} \mathrm{~J}=12.2 \mathrm{~Hz},{ }^{3} \mathrm{~J}=6.4 \mathrm{~Hz}, 1 \mathrm{H}\right), 6.46(\mathrm{~s}, 1 \mathrm{H}), 5.05\left(\mathrm{dd},{ }^{3} \mathrm{~J}=12.7 \mathrm{~Hz},{ }^{4} \mathrm{~J}=5.4 \mathrm{~Hz}, 1 \mathrm{H}\right), 3.67-$ $3.32(\mathrm{~m}, 28 \mathrm{H}), 2.89\left(\mathrm{t},{ }^{3} \mathrm{~J}=4.4 \mathrm{~Hz}, 4 \mathrm{H}\right), 2.60-2.56(\mathrm{~m}, 1 \mathrm{H}), 2.48(\mathrm{~s}, 1 \mathrm{H}), 2.28-2.25(\mathrm{~m}, 1 \mathrm{H}), 2.22(\mathrm{~s}$, $3 \mathrm{H}), 2.08-1.95(\mathrm{~m}, 1 \mathrm{H}) \mathrm{ppm}$.

${ }^{13} \mathrm{C}$ NMR (126 MHz, DMSO) $\delta=172.8,170.1,168.9,167.3,165.9,163.1,161.1,146.4,142.9,142.0$, $139.5,138.6\left(q,{ }^{2} J=32 \mathrm{~Hz}\right), 136.2,135.3,133.2,132.6,132.1,128.0,126.1,123.9,122.3,122.1\left(q,{ }^{1} J=\right.$ $275 \mathrm{~Hz}), 121.0,119.0,117.4,111.7,110.7,109.2,69.8,69.8,69.7,69.6,68.9,68.9,52.8,48.6,48.1$, 42.4, 41.7, 38.9, 31.0, $22.1 \mathrm{ppm}$. 
<smiles>CN1CCN(c2ccc(-c3ccc(C(=O)NCCOCCOCCOCCOCCOCCOCCOCCNc4cccc5c4C(=O)N(C4CCC(=O)NC4=O)C5=O)cc3)cc2NC(=O)c2cnc(O)cc2C(F)(F)F)CC1</smiles>

$15.0 \mathrm{mg}(27.0 \mu \mathrm{mol}, 1.00 \mathrm{eq})$ of tert-butyl 3'-(6-hydroxy-4-(trifluoromethyl)nicotinamido)-4'-(4methylpiperazin-1-yl)-[1,1'-biphenyl]-4-carboxylate was diluted in $1 \mathrm{~mL} \mathrm{DCM}$ and $1 \mathrm{~mL}$ TFA was added. In a second vial, $20.0 \mathrm{mg}(28.0 \mu \mathrm{mol}, 1.05 \mathrm{eq})$ of tert-butyl (23-((2-(2,6-dioxopiperidin-3-yl)-1,3dioxoisoindolin-4-yl)amino)-3,6,9,12,15,18,21-heptaoxatricosyl)carbamate was diluted in $1 \mathrm{~mL}$ DCM and $1 \mathrm{~mL}$ TFA was added. The two reaction mixtures were stirred for $1 \mathrm{~h}$ at $\mathrm{rt}$. Excess solvent and TFA was removed under reduced pressure.

The deprotected species of tert-butyl 3'-(6-hydroxy-4-(trifluoromethyl)nicotinamido)-4'-(4methylpiperazin-1-yl)-[1,1'-biphenyl]-4-carboxylate was dissolved in $0.5 \mathrm{~mL}$ DMF and $47 \mu \mathrm{L}$ ( $0.27 \mathrm{mmol}, 10 \mathrm{eq})$ DIEA was added until the $\mathrm{pH}$ of the solution was basic. $12.0 \mathrm{mg}$ ( $32.0 \mu \mathrm{mol}, 1.20 \mathrm{eq})$ HATU was added. The reaction mixture was stirred for 20 min at rt. The TFA salt of tert-butyl (23-((2(2,6-dioxopiperidin-3-yl)-1,3-dioxoisoindolin-4-yl)amino)-3,6,9,12,15,18,21-

heptaoxatricosyl)carbamate was dissolved in $0.5 \mathrm{~mL} \mathrm{DMF}$ and DIEA was added until the $\mathrm{pH}$ of the solution was basic. The mixture was added to the active ester of tert-butyl 3'-(6-hydroxy-4(trifluoromethyl)nicotinamido)-4'-(4-methylpiperazin-1-yl)-[1,1'-biphenyl]-4-carboxylate and stirred at rt for $4.5 \mathrm{~h}$. The reaction was stopped with $1 \mathrm{~mL}$ water. Saturated $\mathrm{NaHCO}_{3}$ solution and saturated $\mathrm{NaCl}$ solution were added and the reaction mixture was extracted $4 x$ with EA. The combined organic phases were washed with saturated $\mathrm{NaHCO}_{3}$ solution, dried over $\mathrm{MgSO}_{4}$ and filtered. The solvent of the organic phase was evapored under reduced pressure. The purification of the crude product was carried out on the HPLC system.

Yield: $20.5 \mathrm{mg}, 18.5 \mu \mathrm{mol}, 69 \%$ of a yellow oil.

MALDI: (calculated) $\left[\mathrm{M}+\mathrm{H}^{+}\right] 1107.46 \mathrm{~g} / \mathrm{mol}$

(found) $\left[\mathrm{M}+\mathrm{H}^{+}\right] 1107.52 \mathrm{~g} / \mathrm{mol}$. 
HPLC: RT = $11.7 \min (254 \mathrm{~nm}, 97 \%)$.

HRMS: (calculated) $\left[\mathrm{M}+\mathrm{H}^{+}\right] 1107.4645 \mathrm{~g} / \mathrm{mol}$ (found) $\left[\mathrm{M}+\mathrm{H}^{+}\right] 1107.4638 \mathrm{~g} / \mathrm{mol}$.

${ }^{1} \mathrm{H}$ NMR (500 MHz, DMSO) $\delta=12.51(\mathrm{~s}, 1 \mathrm{H}), 11.08(\mathrm{~s}, 1 \mathrm{H}), 9.47(\mathrm{~s}, 1 \mathrm{H}), 8.54\left(\mathrm{t},{ }^{3} \mathrm{~J}=5.6 \mathrm{~Hz}, 1 \mathrm{H}\right), 8.12(\mathrm{~d}$, $\left.{ }^{4} J=2.0 \mathrm{~Hz}, 1 \mathrm{H}\right), 7.98(\mathrm{~s}, 1 \mathrm{H}), 7.94\left(\mathrm{~d},{ }^{3} J=8.4 \mathrm{~Hz}, 2 \mathrm{H}\right), 7.68\left(\mathrm{~d},{ }^{3} \mathrm{~J}=8.4 \mathrm{~Hz}, 2 \mathrm{H}\right), 7.60-7.55(\mathrm{~m}, 1 \mathrm{H}), 7.52$ $\left(\mathrm{dd},{ }^{3} J=8.3 \mathrm{~Hz},{ }^{4} J=2.2 \mathrm{~Hz}, 1 \mathrm{H}\right), 7.26\left(\mathrm{~d},{ }^{3} J=8.4 \mathrm{~Hz}, 1 \mathrm{H}\right), 7.13\left(\mathrm{~d},{ }^{3} J=8.6 \mathrm{~Hz}, 1 \mathrm{H}\right), 7.03\left(\mathrm{~d},{ }^{3} J=7.0 \mathrm{~Hz}\right.$, $1 \mathrm{H}), 6.82(\mathrm{~s}, 1 \mathrm{H}), 6.59\left(\mathrm{t},{ }^{3} \mathrm{~J}=5.7 \mathrm{~Hz}, 1 \mathrm{H}\right), 5.05\left(\mathrm{dd},{ }^{3} \mathrm{~J}=12.7 \mathrm{~Hz},{ }^{4} \mathrm{~J}=5.4 \mathrm{~Hz}, 1 \mathrm{H}\right), 3.61\left(\mathrm{t},{ }^{3} \mathrm{~J}=5.4 \mathrm{~Hz}, 2 \mathrm{H}\right)$, $3.58-3.41(\mathrm{~m}, 32 \mathrm{H}), 3.33-3.25(\mathrm{~m}, 2 \mathrm{H}), 2.95-2.89(\mathrm{~m}, 4 \mathrm{H}), 2.89-2.83(\mathrm{~m}, 1 \mathrm{H}), 2.61-2.51(\mathrm{~m}, 2 \mathrm{H})$, $2.23(\mathrm{~s}, 3 \mathrm{H}), 2.06-1.96(\mathrm{~m}, 1 \mathrm{H}) \mathrm{ppm}$.

${ }^{13} \mathrm{C}$ NMR (126 MHz, DMSO) $\delta=172.7,170.0,168.9,167.3,165.9,162.8,161.1,146.4,144.9,142.2$, $139.2,138.5\left(q,{ }^{2} J=32 \mathrm{~Hz}\right), 136.2,134.2,132.9,132.2,132.1,127.9,126.0,123.9,122.3,122.0\left(q,{ }^{1} J=\right.$ $275 \mathrm{~Hz}), 120.4,119.0,117.4,111.7,110.64109 .2,69.8,69.8,69.7,69.6,68.9,68.9,54.7,51.0,48.6$, 45.7, 41.7, 31.0, 22.1 ppm. 
Synthesis

of

N-(4'-((4-(((2-(2,6-dioxopiperidin-3-yl)-1,3-dioxoisoindolin-4-

yl)amino)methyl)benzyl)carbamoyl)-4-(4-methylpiperazin-1-yl)-[1,1'-biphenyl]-3-yl)-6-hydroxy-4-

(trifluoromethyl)nicotinamide (7e)

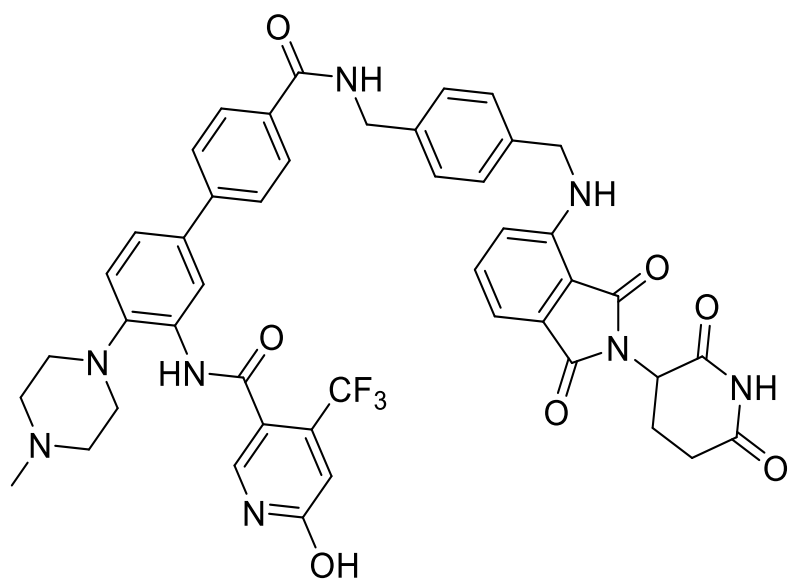

$13.0 \mathrm{mg}(23.0 \mu \mathrm{mol}, 1.00 \mathrm{eq})$ of tert-butyl 3'-(6-hydroxy-4-(trifluoromethyl)nicotinamido)-4'-(4methylpiperazin-1-yl)-[1,1'-biphenyl]-4-carboxylate was diluted in $1 \mathrm{~mL}$ DCM and $1 \mathrm{~mL}$ TFA was added. In a second vial, $12.0 \mathrm{mg}(25.0 \mu \mathrm{mol}, 1.05 \mathrm{eq})$ of AD130 was diluted in $1 \mathrm{~mL}$ DCM and $1 \mathrm{~mL}$ TFA was added. The two reaction mixtures were stirred for $1 \mathrm{~h}$ at $\mathrm{rt}$. Excess solvent and TFA was removed under reduced pressure.

The deprotected species of tert-butyl 3'-(6-hydroxy-4-(trifluoromethyl)nicotinamido)-4'-(4methylpiperazin-1-yl)-[1,1'-biphenyl]-4-carboxylate was dissolved in $0.5 \mathrm{~mL}$ DMF and DIEA was added until the $\mathrm{pH}$ of the solution was basic. $10.0 \mathrm{mg}(28.0 \mu \mathrm{mol}, 1.20 \mathrm{eq})$ HATU was added. The reaction mixture was stirred for $20 \mathrm{~min}$ at rt. The deprotected species of tert-butyl (4-(((2-(2,6-dioxopiperidin3-yl)-1,3-dioxoisoindolin-4-yl)amino)methyl)benzyl)carbamate was dissolved in $0.5 \mathrm{~mL}$ DMF and DIEA were added until the $\mathrm{pH}$ of the solution was basic. The mixture was added to the active ester of tertbutyl 3'-(6-hydroxy-4-(trifluoromethyl)nicotinamido)-4'-(4-methylpiperazin-1-yl)-[1,1'-biphenyl]-4carboxylate and stirred at $\mathrm{rt}$ for $3 \mathrm{~h}$. The reaction was stopped with $1 \mathrm{~mL}$ water. Saturated $\mathrm{NaHCO}_{3}$ solution and saturated $\mathrm{NaCl}$ solution were added, and the reaction mixture was extracted $4 \mathrm{x}$ with $\mathrm{EA}$. The combined organic phases were washed with saturated $\mathrm{NaHCO}_{3}$ solution, dried over $\mathrm{MgSO}_{4}$ and filtered. The solvent of the organic phase was evapored under reduced pressure. The purification of the crude product was carried out on the HPLC system.

Yield: $7.6 \mathrm{mg}, 6.9 \mu \mathrm{mol}, 30 \%$ of a yellow solid as TFA salt (1:2/ product: TFA.)

MALDI: (calculated): [M+Na+] $897.29 \mathrm{~g} / \mathrm{mol} ;\left[\mathrm{M}+\mathrm{H}^{+}\right] 875.31 \mathrm{~g} / \mathrm{mol}$

(found): [M+Na+ $897.20 \mathrm{~g} / \mathrm{mol}(100) ;\left[\mathrm{M}+\mathrm{H}^{+}\right] 875.22 \mathrm{~g} / \mathrm{mol}(70)$. 
HRMS: (calculated): $\left[\mathrm{M}+\mathrm{H}^{+}\right] 875.3123 \mathrm{~g} / \mathrm{mol}$ (found): $\left[\mathrm{M}+\mathrm{H}^{+}\right] 875.3120 \mathrm{~g} / \mathrm{mol}$.

HPLC: RT = $12.1 \mathrm{~min}(254 \mathrm{~nm}, 95 \%)$.

${ }^{1} \mathrm{H}$ NMR (500 MHz, DMSO) $\delta=11.09(\mathrm{~s}, 1 \mathrm{H}), 10.11(\mathrm{~s}, 1 \mathrm{H}), 9.61(\mathrm{~s}, 1 \mathrm{H}), 9.08\left(\mathrm{t},{ }^{3} \mathrm{~J}=5.9 \mathrm{~Hz}, 1 \mathrm{H}\right), 8.25-8$. $22(\mathrm{~m}, 1 \mathrm{H}), 8.04(\mathrm{~s}, 1 \mathrm{H}), 7.95\left(\mathrm{~d},{ }^{3} \mathrm{~J}=8.4 \mathrm{~Hz}, 2 \mathrm{H}\right), 7.69\left(\mathrm{~d},{ }^{3} \mathrm{~J}=8.4 \mathrm{~Hz}, 2 \mathrm{H}\right), 7.56\left(\mathrm{dd},{ }^{3} J=8.3 \mathrm{~Hz},{ }^{4} \mathrm{~J}=2.1 \mathrm{~Hz}\right.$, 1H), $7.450-7.47(\mathrm{~m}, 1 \mathrm{H}), 7.38-7.28(\mathrm{~m}, 3 \mathrm{H}), 7.28-7.15(\mathrm{~m}, 3 \mathrm{H}), 7.00\left(\mathrm{~d},{ }^{3} J=7.0 \mathrm{~Hz}, 1 \mathrm{H}\right), 6.95\left(\mathrm{~d},{ }^{3} \mathrm{~J}=\right.$ $8.6 \mathrm{~Hz}, 1 \mathrm{H}), 6.84(\mathrm{~s}, 1 \mathrm{H}), 5.08-5.04(\mathrm{~m}, 1 \mathrm{H}), 4.56\left(\mathrm{~d},{ }^{3} \mathrm{~J}=4.4 \mathrm{~Hz}, 2 \mathrm{H}\right), 4.49\left(\mathrm{~d},{ }^{3} \mathrm{~J}=4.8 \mathrm{~Hz}, 2 \mathrm{H}\right), 3.55-3.52$ $(\mathrm{m}, 2 \mathrm{H}), 3.33-3.19(\mathrm{~m}, 4 \mathrm{H}), 3.08-3.04(\mathrm{~m}, 2 \mathrm{H}), 2.92-2.82(\mathrm{~m}, 4 \mathrm{H}), 2.59-2.54(\mathrm{~m}, 2 \mathrm{H}), 2.04-2.00(\mathrm{~m}$, 1H) ppm.

${ }^{13} \mathrm{C}$ NMR $(126 \mathrm{MHz}$, DMSO) $\delta=173.3,170.5,169.2,167.7,166.3,163.6,161.6,146.6,143.4,142.5$, 140.6, 139.5, 139.0, 138.6, 136.6, 135.8, 133.6, 133.1, 132.6, 129.0, 128.5, 126.6, 126.4, 126.1, 125.9, 124.4, 122.9, 122.6 (d, ${ }^{1} J=275 \mathrm{~Hz}$ ), 121.5, 119.0, 118.1, 111.3, 111.2, 110.0, 53.3, 49.0, 48.6, 45.9, 43.0, 42.9, 31.4, $22.6 \mathrm{ppm}$. 
Synthesis

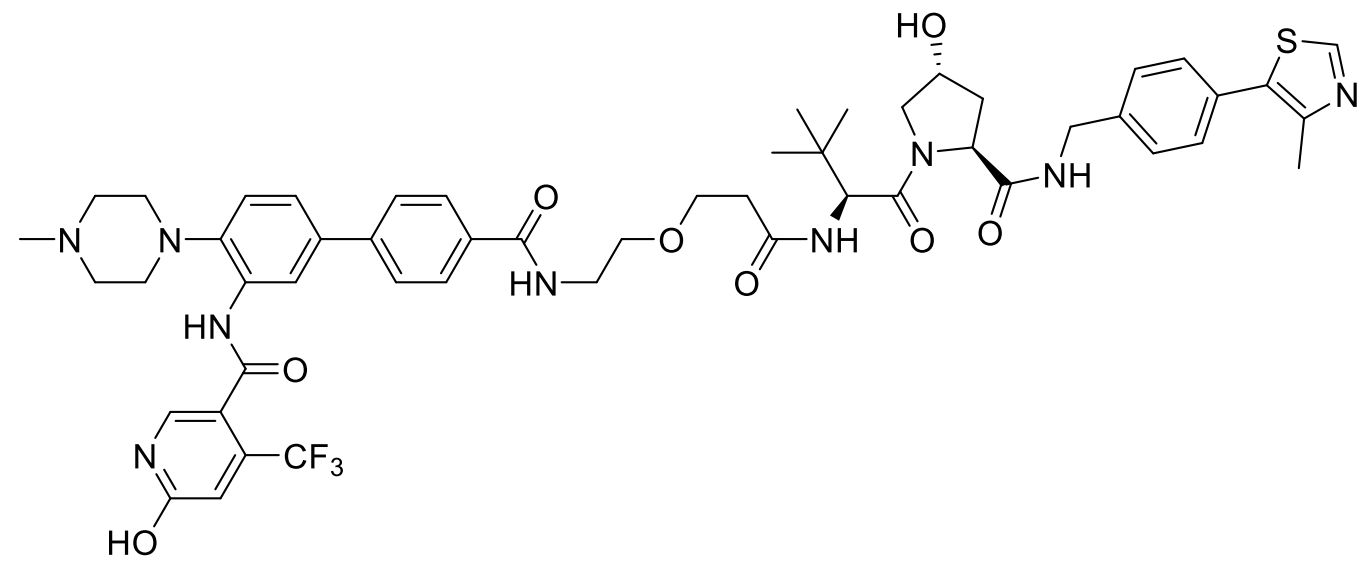

$30 \mathrm{mg} \quad(47 \mu \mathrm{mol}, 1.05$ eq) tert-butyl (2-(3-(((S)-1-((2S,4R)-4-hydroxy-2-((4-(4-methylthiazol-5yl)benzyl)carbamoyl)pyrrolidin-1-yl)-3,3-dimethyl-1-oxobutan-2-yl)amino)-3oxopropoxy)ethyl)carbamate were dissolved in $1 \mathrm{~mL} \mathrm{CH}{ }_{2} \mathrm{Cl}_{2}$ and $1 \mathrm{~mL}$ TFA was added. The solution was stirred for $1 \mathrm{~h}$ at rt. Excess solvent was removed under reduced pressure.

$25 \mathrm{mg}$ (45 $\mu \mathrm{mol}, 1.00$ eq) tert-butyl 3'-(6-hydroxy-4-(trifluoromethyl)nicotinamido)-4'-(4methylpiperazin-1-yl)-[1,1'-biphenyl]-4-carboxylate were dissolved in $1 \mathrm{~mL} \mathrm{CH}_{2} \mathrm{Cl}_{2}$ and $1 \mathrm{~mL}$ TFA was added. The solution was stirred for $1 \mathrm{~h}$ at $\mathrm{rt}$. Excess solvent was removed under reduced pressure. The crude species was dissolved in $0.5 \mathrm{~mL}$ DMF and DIEA was added until the $\mathrm{pH}$ of the solution was basic. $20.5 \mathrm{mg}(54 \mu \mathrm{mol}, 1.20 \mathrm{eq})$ HATU was added. The reaction mixture was stirred for $20 \mathrm{~min}$ at rt. The crude species of tert-butyl (2-(3-)((S)-1-((2S,4R)-4-hydroxy-2-((4-(4-methylthiazol-5yl)benzyl)carbamoyl)pyrrolidin-1-yl)-3,3-dimethyl-1-oxobutan-2-yl)amino)-3-

oxopropoxy)ethyl)carbamate were dissolved in $0.5 \mathrm{~mL} \mathrm{DMF}$ and DIEA were added until the $\mathrm{pH}$ of the solution was basic. The mixture was added to the active ester of tert-butyl 3'-(6-hydroxy-4(trifluoromethyl)nicotinamido)-4'-(4-methylpiperazin-1-yl)-[1,1'-biphenyl]-4-carboxylate and stirred at rt for $4 \mathrm{~h}$. The reaction was stopped with $1 \mathrm{~mL}$ water. Saturated $\mathrm{NaHCO}_{3}$ solution and saturated $\mathrm{NaCl}$ solution were added and the reaction mixture was extracted $4 x$ with EA. The combined organic phases were washed with saturated $\mathrm{NaHCO}_{3}$ solution, dried over $\mathrm{MgSO}_{4}$ and filtered. The solvent of the organic phase was evapored under reduced pressure. The purification of the crude product was carried out on the preparative HPLC system.

Yield: $27.4 \mathrm{mg}, 26.6 \mu \mathrm{mol}, 59 \%$ of a white solid. 
MALDI: (calculated): [M+Na+] $1050.41 \mathrm{~g} / \mathrm{mol}$ (found): $\left[\mathrm{M}+\mathrm{Na}^{+}\right] 1050.20 \mathrm{~g} / \mathrm{mol}$.

HRMS: (calculated): [M+Na+] $1050.4129 \mathrm{~g} / \mathrm{mol}$ (found): $\left[\mathrm{M}+\mathrm{Na}^{+}\right] 1050.4121 \mathrm{~g} / \mathrm{mol}$.

HPLC: RT = $11.3 \min (254 \mathrm{~nm}, 97 \%)$.

${ }^{1} \mathrm{H}$ NMR (500 MHz, DMSO) $\delta=9.47(\mathrm{~s}, 1 \mathrm{H}), 8.97(\mathrm{~s}, 1 \mathrm{H}), 8.56\left(\mathrm{t},{ }^{3} \mathrm{~J}=6.1 \mathrm{~Hz}, 1 \mathrm{H}\right), 8.50\left(\mathrm{t},{ }^{3} \mathrm{~J}=5.5 \mathrm{~Hz}, 1 \mathrm{H}\right)$, $8.12\left(\mathrm{~d},{ }^{3} \mathrm{~J}=2.1 \mathrm{~Hz}, 1 \mathrm{H}\right), 8.02-7.91(\mathrm{~m}, 4 \mathrm{H}), 7.68\left(\mathrm{~d},{ }^{3} \mathrm{~J}=8.5 \mathrm{~Hz}, 2 \mathrm{H}\right), 7.52\left(\mathrm{dd},{ }^{3} \mathrm{~J}=8.4 \mathrm{~Hz},{ }^{4} \mathrm{~J}=2.2 \mathrm{~Hz}\right.$, $1 \mathrm{H}), 7.45-7.33(\mathrm{~m}, 4 \mathrm{H}), 7.26\left(\mathrm{~d},{ }^{3} \mathrm{~J}=8.4 \mathrm{~Hz}, 1 \mathrm{H}\right), 6.82(\mathrm{~s}, 1 \mathrm{H}), 5.13(\mathrm{~s}, 1 \mathrm{H}), 4.56\left(\mathrm{~d},{ }^{3} \mathrm{~J}=9.4 \mathrm{~Hz}, 1 \mathrm{H}\right), 4.46$ $-4.38(\mathrm{~m}, 2 \mathrm{H}), 4.35(\mathrm{~s}, 1 \mathrm{H}), 4.24-4.20(\mathrm{~m}, 1 \mathrm{H}), 3.71-3.58(\mathrm{~m}, 4 \mathrm{H}), 3.58-3.48(\mathrm{~m}, 2 \mathrm{H}), 3.44-3.41(\mathrm{~m}$, $4 \mathrm{H}), 2.98-2.85(\mathrm{~m}, 4 \mathrm{H}), 2.61-2.52(\mathrm{~m}, 1 \mathrm{H}), 2.44-2.34(\mathrm{~m}, 2 \mathrm{H}), 2.23(\mathrm{~s}, 3 \mathrm{H}), 2.07-1.99(\mathrm{~m}, 1 \mathrm{H}), 1.96$ $-1.84(\mathrm{~m}, 1 \mathrm{H}), 0.92(\mathrm{~s}, 9 \mathrm{H}) \mathrm{ppm}$.

${ }^{13} \mathrm{C}$ NMR (126 MHz, DMSO) $\delta=171.9,170.0,169.6,165.9,162.8,161.2,151.4,147.7,144.9,142.2$, $139.5,139.2,138.5\left(q,{ }^{2} \mathrm{~J}=32 \mathrm{~Hz}\right), 134.2,132.9,132.2,131.2,129.6,128.6,127.9,127.4,126.0,123.9$, $122.3,122.0\left(\mathrm{~d},{ }^{1} \mathrm{~J}=275 \mathrm{~Hz}\right.$ ), 120.4, 119.0, 111.7, 68.9, 68.6, 66.6, 58.7, 56.4, 56.3, 54.7, 51.0, 45.7, $41.7,39.0,37.9,35.6,35.4,26.3,15.9$ ppm. 
Synthesis of 6-hydroxy-N-(4'-((2--(2-(3-)((S)-1-((2S,4R)-4-hydroxy-2-((4-(4-methylthiazol-5-

yl)benzyl)carbamoyl)pyrrolidin-1-yl)-3,3-dimethyl-1-oxobutan-2-yl)amino)-3-

oxopropoxy)ethoxy)ethyl)carbamoyl)-4-(4-methylpiperazin-1-yl)-[1,1'-biphenyl]-3-yl)-4-

(trifluoromethyl)nicotinamide (8b)

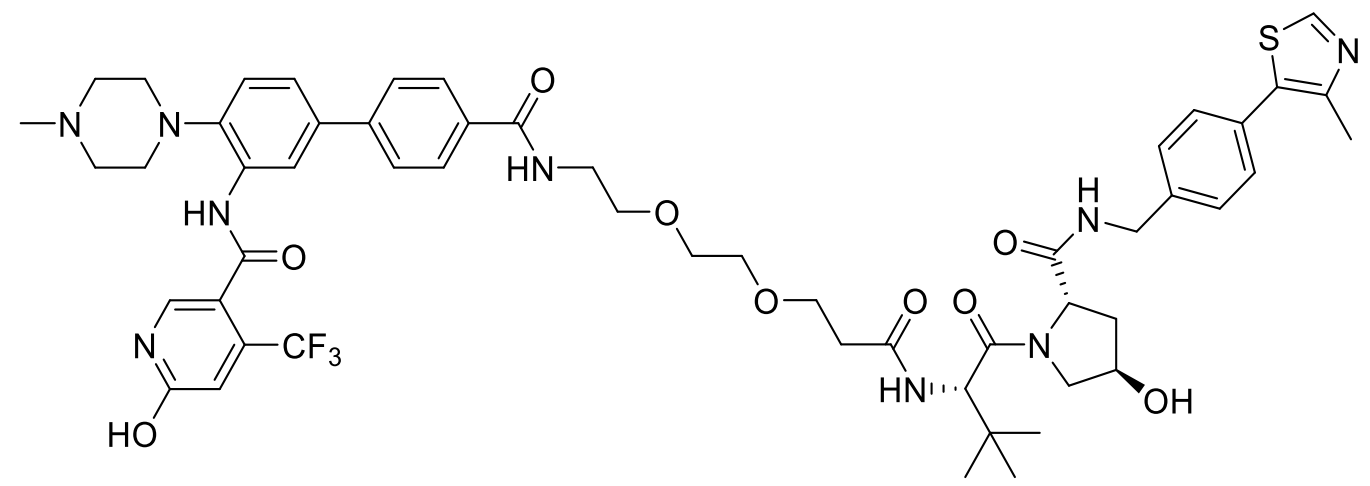

$18.0 \mathrm{mg}(32.0 \mu \mathrm{mol}, 1.00 \mathrm{eq})$ of tert-butyl 3'-(6-hydroxy-4-(trifluoromethyl)nicotinamido)-4'-(4methylpiperazin-1-yl)-[1,1'-biphenyl]-4-carboxylate was diluted in $1 \mathrm{~mL} \mathrm{DCM}$ and $1 \mathrm{~mL}$ TFA was added. In a second vial, $23.4 \mathrm{mg}(34.0 \mu \mathrm{mol}, 1.05 \mathrm{eq})$ of tert-butyl (2-(2-(3-(((S)-1-((2S,4R)-4-hydroxy-2-((4-(4methylthiazol-5-yl)benzyl)carbamoyl)pyrrolidin-1-yl)-3,3-dimethyl-1-oxobutan-2-yl)amino)-3oxopropoxy)ethoxy)ethyl)carbamate was diluted in $1 \mathrm{~mL}$ DCM and $1 \mathrm{~mL}$ TFA was added. The two reaction mixtures were stirred for $1 \mathrm{~h}$ at $\mathrm{rt}$. Excess solvent and TFA was removed under reduced pressure.

The deprotected species of tert-butyl 3'-(6-hydroxy-4-(trifluoromethyl)nicotinamido)-4'-(4methylpiperazin-1-yl)-[1,1'-biphenyl]-4-carboxylate was dissolved in $0.5 \mathrm{~mL} \mathrm{DMF}$ and DIEA was added until the $\mathrm{pH}$ of the solution was basic. $18.4 \mathrm{mg}(49.0 \mu \mathrm{mol}, 1.50 \mathrm{eq})$ HATU was added. The reaction mixture was stirred for $20 \mathrm{~min}$ at rt. The TFA salt of tert-butyl (2-(2-(3-)((S)-1-((2S,4R)-4-hydroxy-2-((4(4-methylthiazol-5-yl)benzyl)carbamoyl)pyrrolidin-1-yl)-3,3-dimethyl-1-oxobutan-2-yl)amino)-3oxopropoxy)ethoxy)ethyl)carbamate was dissolved in $0.5 \mathrm{~mL} \mathrm{DMF}$ and $11 \mu \mathrm{L}$ (65 $\mu \mathrm{mol}, 2.00$ eq) DIEA were added to the solution. The mixture was added to the active ester of tert-butyl 3'-(6-hydroxy-4(trifluoromethyl)nicotinamido)-4'-(4-methylpiperazin-1-yl)-[1,1'-biphenyl]-4-carboxylate and stirred at rt for $2.5 \mathrm{~h}$. The reaction was stopped with $1 \mathrm{~mL}$ water. Saturated $\mathrm{NaHCO}_{3}$ solution and saturated $\mathrm{NaCl}$ solution were added and the reaction mixture was extracted $4 x$ with EA. The combined organic phases were washed with saturated $\mathrm{NaHCO}_{3}$ solution, dried over $\mathrm{MgSO}_{4}$ and filtered. The solvent of the organic phase was evapored under reduced pressure. The purification of the crude product was carried out on the HPLC system.

Yield: $11.1 \mathrm{mg}, 10.4 \mu \mathrm{mol}, 33 \%$ of a white solid. 
MALDI: (calculated): [M+Na+] $1094.44 \mathrm{~g} / \mathrm{mol}$ (found): [M+Na+] $1094.44 \mathrm{~g} / \mathrm{mol}$.

HPLC: RT = $11.3 \min (254 \mathrm{~nm}, 100 \%)$.

HRMS: (calculated): [M+Na+] $1094.4392 \mathrm{~g} / \mathrm{mol}$

(found): $\left[\mathrm{M}+\mathrm{Na}^{+}\right] 1094.4384 \mathrm{~g} / \mathrm{mol}$.

${ }^{1} \mathrm{H}$ NMR $(600 \mathrm{MHz}, \mathrm{DMSO}) \delta=9.92(\mathrm{~s}, 1 \mathrm{H}), 9.59(\mathrm{~s}, 1 \mathrm{H}), 8.97(\mathrm{~s}, 1 \mathrm{H}), 8.56\left(\mathrm{t},{ }^{3} \mathrm{~J}=5.6 \mathrm{~Hz}, 2 \mathrm{H}\right), 8.22(\mathrm{~s}$, $1 \mathrm{H}), 8.04(\mathrm{~s}, 1 \mathrm{H}), 7.95-7.90(\mathrm{~m}, 3 \mathrm{H}), 7.69\left(\mathrm{~d},{ }^{3} \mathrm{~J}=8.2 \mathrm{~Hz}, 2 \mathrm{H}\right), 7.55\left(\mathrm{dd},{ }^{3} \mathrm{~J}=8.3 \mathrm{~Hz},{ }^{4} J=1.4 \mathrm{~Hz}, 1 \mathrm{H}\right), 7.42$ - $7.37(\mathrm{~m}, 4 \mathrm{H}), 7.31\left(\mathrm{~d},{ }^{3} \mathrm{~J}=8.3 \mathrm{~Hz}, 1 \mathrm{H}\right), 6.83(\mathrm{~s}, 1 \mathrm{H}), 4.55\left(\mathrm{~d},{ }^{3} \mathrm{~J}=9.4 \mathrm{~Hz}, 1 \mathrm{H}\right), 4.49-4.38(\mathrm{~m}, 2 \mathrm{H}), 4.35$ $(\mathrm{s}, 1 \mathrm{H}), 4.22\left(\mathrm{dd},{ }^{3} \mathrm{~J}=15.8 \mathrm{~Hz},{ }^{4} \mathrm{~J}=5.5 \mathrm{~Hz}, 2 \mathrm{H}\right), 3.68-3.65(\mathrm{~m}, 1 \mathrm{H}), 3.64-3.56(\mathrm{~m}, 3 \mathrm{H}), 3.56-3.47(\mathrm{~m}$, $8 \mathrm{H}), 3.46-4.42(\mathrm{~m}, 2 \mathrm{H}), 3.28-3.25(\mathrm{~m}, 2 \mathrm{H}), 3.24-3.19(\mathrm{~m}, 2 \mathrm{H}), 3.06-3.02(\mathrm{~m}, 2 \mathrm{H}), 2.87(\mathrm{~s}, 3 \mathrm{H}), 2.53$ $(\mathrm{s}, 1 \mathrm{H}), 2.44(\mathrm{~s}, 3 \mathrm{H}), 2.40-2.29(\mathrm{~m}, 1 \mathrm{H}), 2.08-2.00(\mathrm{~m}, 1 \mathrm{H}), 1.96-1.86(\mathrm{~m}, 1 \mathrm{H}), 0.93(\mathrm{~s}, 9 \mathrm{H}) \mathrm{ppm}$.

${ }^{13} \mathrm{C}$ NMR (126 MHz, DMSO) $\delta=171.9,170.0,169.6,165.9,163.1,161.1,151.5,147.7,142.9,142.0$, $139.5,139.1,138.5$ (q, ${ }^{2} J=32 \mathrm{~Hz}$ ), 135.3, 133.2, 132.64, 131.2, 129.6, 128.6, 128.0, 127.4, 126.1, 123.9, $122.32,122.1$ (q, $\left.{ }^{1} J=275 \mathrm{~Hz}\right), 121.0,119.0,118.8,111.6,69.6,69.5,69.0,68.9,66.9,58.7,56.4,56.3$, $52.85,48.1,42.4,41.7,38.0,35.7,35.4,26.3,15.9$ ppm. 
Synthesis

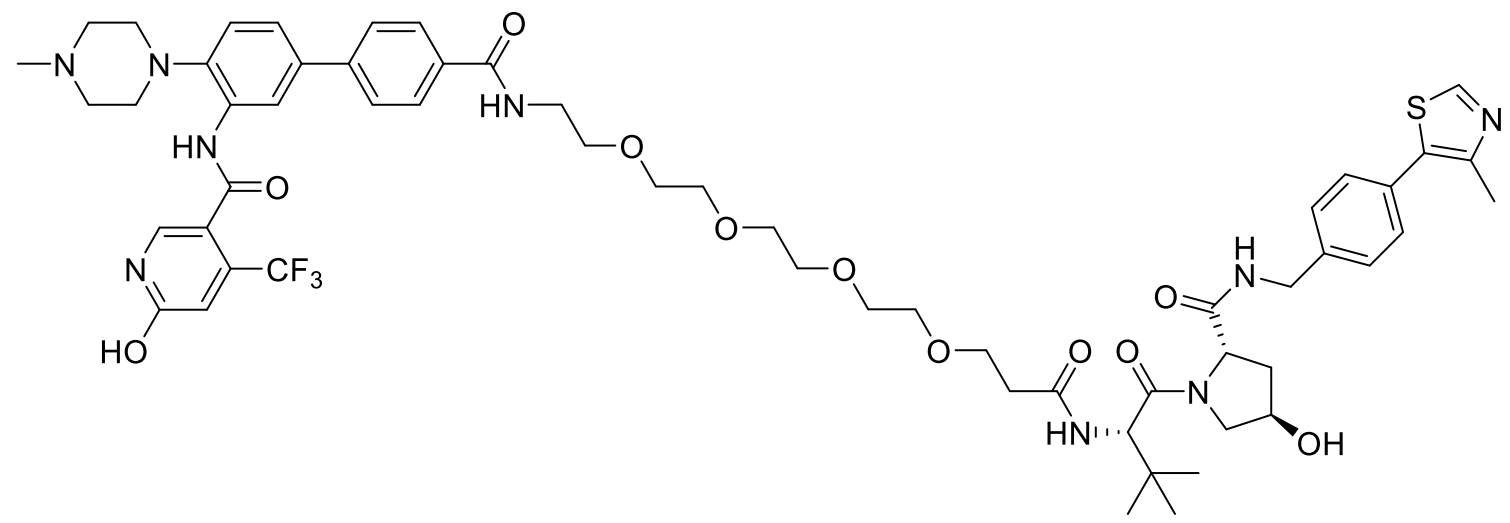

$18.0 \mathrm{mg}(32.0 \mu \mathrm{mol}, 1.00 \mathrm{eq})$ of tert-butyl 3'-(6-hydroxy-4-(trifluoromethyl)nicotinamido)-4'-(4methylpiperazin-1-yl)-[1,1'-biphenyl]-4-carboxylate was diluted in $1 \mathrm{~mL} \mathrm{DCM}$ and $1 \mathrm{~mL}$ TFA was added. In a second vial, $26.4 \mathrm{mg}(34.0 \mu \mathrm{mol}, 1.05 \mathrm{eq})$ of tert-butyl ((S)-17-((2S,4R)-4-hydroxy-2-((4-(4methylthiazol-5-yl)benzyl)carbamoyl)pyrrolidine-1-carbonyl)-18,18-dimethyl-15-oxo-3,6,9,12tetraoxa-16-azanonadecyl)carbamate was diluted in $1 \mathrm{~mL}$ DCM and $1 \mathrm{~mL}$ TFA was added. The two reaction mixtures were stirred for $1 \mathrm{~h}$ at $\mathrm{rt}$. Excess solvent and TFA was removed under reduced pressure.

The deprotected species of tert-butyl 3'-(6-hydroxy-4-(trifluoromethyl)nicotinamido)-4'-(4methylpiperazin-1-yl)-[1,1'-biphenyl]-4-carboxylate was dissolved in $0.5 \mathrm{~mL}$ DMF and DIEA was added until the $\mathrm{pH}$ of the solution was basic. $18.4 \mathrm{mg}(49.0 \mu \mathrm{mol}, 1.50 \mathrm{eq})$ HATU was added. The reaction mixture was stirred for $20 \mathrm{~min}$ at rt. The deprotected species of tert-butyl ((S)-17-((2S,4R)-4-hydroxy2-((4-(4-methylthiazol-5-yl)benzyl)carbamoyl)pyrrolidine-1-carbonyl)-18,18-dimethyl-15-oxo-

3,6,9,12-tetraoxa-16-azanonadecyl)carbamate was dissolved in $0.5 \mathrm{~mL} \mathrm{DMF}$ and $11 \mu \mathrm{L}$ (65 $\mu \mathrm{mol}, 2.00$ eq) DIEA were added to the solution. The mixture was added to the active ester of tert-butyl 3'-(6hydroxy-4-(trifluoromethyl)nicotinamido)-4'-(4-methylpiperazin-1-yl)-[1,1'-biphenyl]-4-carboxylate and stirred at $\mathrm{rt}$ for $2.5 \mathrm{~h}$. The reaction was stopped with $1 \mathrm{~mL}$ water. Saturated $\mathrm{NaHCO}_{3}$ solution and saturated $\mathrm{NaCl}$ solution were added and the reaction mixture was extracted $4 \mathrm{x}$ with $\mathrm{EA}$. The combined organic phases were washed with saturated $\mathrm{NaHCO}_{3}$ solution, dried over $\mathrm{MgSO}_{4}$ and filtered. The solvent of the organic phase was evapored under reduced pressure. The purification of the crude product was carried out on the HPLC system.

Yield: $2.34 \mathrm{mg}, 2.02 \mu \mathrm{mol}, 6 \%$ of a white solid. 
MALDI: (calculated) $\left[\mathrm{M}+\mathrm{H}^{+}\right] 1160.51 \mathrm{~g} / \mathrm{mol}$

(found) $\left[\mathrm{M}+\mathrm{H}^{+}\right] 1160.58 \mathrm{~g} / \mathrm{mol}$.

HPLC: RT = $11.4 \min (254 \mathrm{~nm}, 99 \%)$.

HRMS: (calculated) $\left[\mathrm{M}+\mathrm{Na}^{+}\right] 1182.4916 \mathrm{~g} / \mathrm{mol}$

(found) $\left[\mathrm{M}+\mathrm{Na}^{+}\right] 1182.4913 \mathrm{~g} / \mathrm{mol}$

${ }^{1} \mathrm{H}$ NMR $(500 \mathrm{MHz}, \mathrm{DMSO}) \delta=9.76(\mathrm{~s}, 1 \mathrm{H}), 9.57(\mathrm{~s}, 1 \mathrm{H}), 8.97(\mathrm{~s}, 1 \mathrm{H}), 8.56\left(\mathrm{t},{ }^{3} \mathrm{~J}=5.4 \mathrm{~Hz}, 2 \mathrm{H}\right), 8.21\left(\mathrm{~d},{ }^{4} \mathrm{~J}\right.$ $=1.8 \mathrm{~Hz}, 1 \mathrm{H}), 8.03(\mathrm{~s}, 1 \mathrm{H}), 7.95\left(\mathrm{~d},{ }^{3} \mathrm{~J}=8.4 \mathrm{~Hz}, 2 \mathrm{H}\right), 7.90\left(\mathrm{~d},{ }^{3} \mathrm{~J}=9.4 \mathrm{~Hz}, 1 \mathrm{H}\right), 7.69\left(\mathrm{~d},{ }^{3} \mathrm{~J}=8.4 \mathrm{~Hz}, 2 \mathrm{H}\right), 7.56$ $\left(\mathrm{dd},{ }^{3} J=8.3 \mathrm{~Hz},{ }^{4} \mathrm{~J}=2.1 \mathrm{~Hz}, 1 \mathrm{H}\right), 7.40\left(\mathrm{q},{ }^{3} \mathrm{~J}=8.3 \mathrm{~Hz}, 5 \mathrm{H}\right), 7.31\left(\mathrm{~d},{ }^{3} \mathrm{~J}=8.4 \mathrm{~Hz}, 1 \mathrm{H}\right), 6.84(\mathrm{~s}, 1 \mathrm{H}), 5.12(\mathrm{~s}$, $1 \mathrm{H}), 4.54\left(\mathrm{~d},{ }^{3} \mathrm{~J}=9.4 \mathrm{~Hz}, 1 \mathrm{H}\right), 4.48-4.39(\mathrm{~m}, 2 \mathrm{H}), 4.35(\mathrm{~s}, 1 \mathrm{H}), 4.21\left(\mathrm{dd}, 3 \mathrm{~J}=15.9 \mathrm{~Hz},{ }^{4} J=5.5 \mathrm{~Hz}, 1 \mathrm{H}\right)$, $3.68-3.64(\mathrm{~m}, 1 \mathrm{H}), 3.64-3.57(\mathrm{~m}, 4 \mathrm{H}), 3.56-3.41(\mathrm{~m}, 2 \mathrm{H}), 3.25-3.15(\mathrm{~m}, 2 \mathrm{H}), 3.05-3.00(\mathrm{~m}, 2 \mathrm{H})$, $2.86(\mathrm{~s}, 3 \mathrm{H}), 2.44(\mathrm{~s}, 3 \mathrm{H}), 2.37-2.30(\mathrm{~m}, 1 \mathrm{H}), 2.07-1.99(\mathrm{~m}, 1 \mathrm{H}), 1.95-1.85(\mathrm{~m}, 1 \mathrm{H}), 0.93(\mathrm{~s}, 9 \mathrm{H}) \mathrm{ppm}$.

${ }^{13} \mathrm{C}$ NMR (126 MHz, DMSO) $\delta=171.9,170.0,169.5,165.9,163.1,161.1,151.5,147.6,142.9,142.0$, $139.5,139.1,138.5\left(q,{ }^{2} J=33 \mathrm{~Hz}\right), 135.4,133.2,132.7,131.2,129.6,128.6,128.0,127.4,126.1,123.9$, $122.3,122.1\left(q,{ }^{1} J=277 \mathrm{~Hz}\right), 121.0,118.9,111.7,69.8,69.7,69.7,69.6,69.5,68.9,68.9,66.9,58.7$, 56.4, 56.3, 52.8, 48.1, 42.4, 41.7, 38.0, 35.7, 35.4, 26.3, 15.9 ppm. 
Synthesis

of

6-hydroxy-N-(4'-(((S)-1-((2S,4R)-4-hydroxy-2-((4-(4-methylthiazol-5-

yl)benzyl)carbamoyl)pyrrolidin-1-yl)-3,3-dimethyl-1-oxobutan-2-yl)carbamoyl)-4-(4-

methylpiperazin-1-yl)-[1,1'-biphenyl]-3-yl)-4-(trifluoromethyl)nicotinamide (8d)<smiles>Cc1ncsc1-c1ccc(CNC(=O)[C@@H]2C[C@@H](O)CN2C(=O)[C@@H](NC(=O)c2ccc(-c3ccc(N4CCN(C)CC4)c(NC(=O)c4cnc(O)cc4C(F)(F)F)c3)cc2)C(C)(C)C)cc1</smiles>

$20 \mathrm{mg} \quad(36 \mu \mathrm{mol}, 1.00 \mathrm{eq})$ tert-butyl 3'-(6-hydroxy-4-(trifluoromethyl)nicotinamido)-4'-(4methylpiperazin-1-yl)-[1,1'-biphenyl]-4-carboxylate were dissolved in $1 \mathrm{~mL} \mathrm{CH}_{2} \mathrm{Cl}_{2}$ and $1 \mathrm{~mL}$ TFA was added. The solution was stirred for $1 \mathrm{~h}$ at $\mathrm{rt}$. Excess solvent was removed under reduced pressure. The crude species was dissolved in $0.5 \mathrm{~mL}$ DMF and DIEA was added until the $\mathrm{pH}$ of the solution was basic. $16.4 \mathrm{mg}$ ( $43 \mu \mathrm{mol}, 1.20 \mathrm{eq}$ ) HATU was added. The reaction mixture was stirred for $20 \mathrm{~min}$ at rt. $17.0 \mathrm{mg}$ (36 $\mu \mathrm{mol}, 1.00 \mathrm{eq}$ ) VHL ligand 1 was dissolved in $0.5 \mathrm{~mL}$ DMF and added to the active ester of tert-butyl 3'-(6-hydroxy-4-(trifluoromethyl)nicotinamido)-4'-(4-methylpiperazin-1-yl)-[1,1'-biphenyl]-4carboxylate and stirred at $\mathrm{rt}$ for $4 \mathrm{~h}$. The reaction was stopped with $1 \mathrm{~mL}$ water. Saturated $\mathrm{NaHCO}_{3}$ solution and saturated $\mathrm{NaCl}$ solution were added and the reaction mixture was extracted $4 \mathrm{x}$ with $\mathrm{EA}$. The combined organic phases were washed with saturated $\mathrm{NaHCO}_{3}$ solution, dried over $\mathrm{MgSO}_{4}$ and filtered. The solvent of the organic phase was evapored under reduced pressure. The purification of the crude product was carried out on the preparative HPLC system.

Yield: $22.2 \mathrm{mg}, 24.3 \mu \mathrm{mol}, 67 \%$ of a white solid.

ESI: (calculated): $\left[\mathrm{M}+\mathrm{H}^{+}\right] 913.37 \mathrm{~g} / \mathrm{mol}$

(found): $\left[\mathrm{M}+\mathrm{H}^{+}\right] 913.63 \mathrm{~g} / \mathrm{mol}$.

MADLI: (calculated): [M+Na+] $935.35 \mathrm{~g} / \mathrm{mol}$

(found): [M+Na+] $935.10 \mathrm{~g} / \mathrm{mol}$. 
HRMS: (calculated) $\left[\mathrm{M}+\mathrm{Na}^{+}\right] 935.3496 \mathrm{~g} / \mathrm{mol}$, (found) $\left[\mathrm{M}+\mathrm{Na}^{+}\right] 935.3486 \mathrm{~g} / \mathrm{mol}$.

HPLC: RT = $11.8 \min (254 \mathrm{~nm}, 96 \%)$.

${ }^{1} \mathrm{H}$ NMR $(600 \mathrm{MHz}, \mathrm{DMSO}) \delta=9.94(\mathrm{~s}, 1 \mathrm{H}), 9.59(\mathrm{~s}, 1 \mathrm{H}), 8.99(\mathrm{~s}, 1 \mathrm{H}), 8.59\left(\mathrm{t},{ }^{3} \mathrm{~J}=5.7 \mathrm{~Hz}, 1 \mathrm{H}\right), 8.23(\mathrm{~s}$, $1 \mathrm{H}), 8.03\left(\mathrm{~d},{ }^{3} \mathrm{~J}=10.0 \mathrm{~Hz}, 2 \mathrm{H}\right), 7.98\left(\mathrm{~d},{ }^{3} \mathrm{~J}=7.8 \mathrm{~Hz}, 2 \mathrm{H}\right), 7.69\left(\mathrm{~d},{ }^{3} \mathrm{~J}=7.8 \mathrm{~Hz}, 2 \mathrm{H}\right), 7.56\left(\mathrm{~d},{ }^{3} J=8.3 \mathrm{~Hz}, 1 \mathrm{H}\right)$, $7.41\left(\mathrm{q},{ }^{3} \mathrm{~J}=8.1 \mathrm{~Hz}, 4 \mathrm{H}\right), 7.33\left(\mathrm{~d},{ }^{3} \mathrm{~J}=8.3 \mathrm{~Hz}, 1 \mathrm{H}\right), 6.84(\mathrm{~s}, 1 \mathrm{H}), 4.80\left(\mathrm{~d},{ }^{3} \mathrm{~J}=9.0 \mathrm{~Hz}, 1 \mathrm{H}\right), 4.47\left(\mathrm{t},{ }^{3} \mathrm{~J}=8.1 \mathrm{~Hz}\right.$, $1 \mathrm{H}), 4.47-4.41(\mathrm{~m}, 2 \mathrm{H}), 4.39(\mathrm{~s}, 1 \mathrm{H}), 4.25\left(\mathrm{dd},{ }^{3} \mathrm{~J}=15.7 \mathrm{~Hz},{ }^{4} \mathrm{~J}=5.3 \mathrm{~Hz}, 1 \mathrm{H}\right), 3.75(\mathrm{~s}, 2 \mathrm{H}), 3.54-3.52(\mathrm{~m}$, $2 \mathrm{H}), 3.29-3.17(\mathrm{~m}, 2 \mathrm{H}), 3.23-3.19(\mathrm{~m}, 2 \mathrm{H}), 3.06-3.02(\mathrm{~m}, 2 \mathrm{H}), 2.87(\mathrm{~s}, 3 \mathrm{H}), 2.45(\mathrm{~s}, 3 \mathrm{H}), 2.10-2.03$ $(\mathrm{m}, 1 \mathrm{H}), 1.96-1.90(\mathrm{~m}, 1 \mathrm{H}), 1.05(\mathrm{~s}, 9 \mathrm{H}) \mathrm{ppm}$.

${ }^{13} \mathrm{C}$ NMR $(151 \mathrm{MHz}$, DMSO) $\delta=171.9,169.5,166.2,162.9,161.1,151.5,147.8,142.9,142.4,139.5$, $139.4,138.8$ (q, $\left.{ }^{2} J=33 \mathrm{~Hz}\right), 134.5,132.7,132.4,131.2,129.7,128.7,128.5,127.5,126.0,124.0,122.3$, $121.1\left(q,{ }^{1} J=275 \mathrm{~Hz}\right), 120.6,119.1,111.6,68.9,58.8,57.3,56.5,52.8,48.1,41.7,40.4,37.9,35.6,26.5$, 15.9 ppm. 
Synthesis of 6-hydroxy-N-(4'-((3-)((S)-1-((2S,4R)-4-hydroxy-2-((4-(4-methylthiazol-5yl)benzyl)carbamoyl)pyrrolidin-1-yl)-3,3-dimethyl-1-oxobutan-2-yl)amino)-3oxopropyl)carbamoyl)-4-(4-methylpiperazin-1-yl)-[1,1'-biphenyl]-3-yl)-4(trifluoromethyl)nicotinamide (8e)<smiles>Cc1ncsc1-c1ccc(CNC(=O)[C@@H]2C[C@@H](O)CN2C(=O)[C@@H](NC(=O)CCNC(=O)c2ccc(-c3ccc(N4CCN(C)CC4)c(NC(=O)c4cnc(O)cc4C(F)(F)F)c3)cc2)C(C)(C)C)cc1</smiles>

$21.6 \mathrm{mg}$ (36 $\mu \mathrm{mol}, 1.00$ eq) tert-butyl (3-(((S)-1-((2S,4R)-4-hydroxy-2-((4-(4-methylthiazol-5yl)benzyl)carbamoyl)pyrrolidin-1-yl)-3,3-dimethyl-1-oxobutan-2-yl)amino)-3-oxopropyl)carbamate were dissolved in $1 \mathrm{~mL} \mathrm{CH} \mathrm{Cl}_{2}$ and $1 \mathrm{~mL}$ TFA was added. The solution was stirred for $1 \mathrm{~h}$ at $\mathrm{rt}$. Excess solvent was removed under reduced pressure.

$20 \mathrm{mg}$ (36 $\mu \mathrm{mol}$, 1.00 eq) tert-butyl 3'-(6-hydroxy-4-(trifluoromethyl)nicotinamido)-4'-(4methylpiperazin-1-yl)-[1,1'-biphenyl]-4-carboxylate were dissolved in $1 \mathrm{~mL} \mathrm{CH}_{2} \mathrm{Cl}_{2}$ and $1 \mathrm{~mL}$ TFA was added. The solution was stirred for $1 \mathrm{~h}$ at $\mathrm{rt}$. Excess solvent was removed under reduced pressure. The crude species was dissolved in $0.5 \mathrm{~mL}$ DMF and DIEA was added until the $\mathrm{pH}$ of the solution was basic. $16.4 \mathrm{mg}$ ( $43 \mu \mathrm{mol}, 1.20 \mathrm{eq}$ ) HATU was added. The reaction mixture was stirred for $20 \mathrm{~min}$ at rt. The crude species of tert-butyl (3-)((S)-1-((2S,4R)-4-hydroxy-2-((4-(4-methylthiazol-5yl)benzyl)carbamoyl)pyrrolidin-1-yl)-3,3-dimethyl-1-oxobutan-2-yl)amino)-3-oxopropyl)carbamate was dissolved in $0.5 \mathrm{~mL}$ DMF and DIEA were added until the $\mathrm{pH}$ of the solution was basic. The mixture was added to the active ester of tert-butyl 3'-(6-hydroxy-4-(trifluoromethyl)nicotinamido)-4'-(4methylpiperazin-1-yl)-[1,1'-biphenyl]-4-carboxylate and stirred at $\mathrm{rt}$ for $4 \mathrm{~h}$. The reaction was stopped with $1 \mathrm{~mL}$ water. Saturated $\mathrm{NaHCO}_{3}$ solution and saturated $\mathrm{NaCl}$ solution were added and the reaction mixture was extracted $4 x$ with EA. The combined organic phases were washed with saturated $\mathrm{NaHCO}_{3}$ solution, dried over $\mathrm{MgSO}_{4}$ and filtered. The solvent of the organic phase was evapored under reduced pressure. The purification of the crude product was carried out on the preparative HPLC system. 
MALDI: (calculated): [M+Na+] $1006.39 \mathrm{~g} / \mathrm{mol}$ (found): [M+Na+] $1006.42 \mathrm{~g} / \mathrm{mol}$.

HRMS: (calculated): [M+Na $] 1006.3867 \mathrm{~g} / \mathrm{mol}$ (found): $\left[\mathrm{M}+\mathrm{Na}^{+}\right] 1006.3870 \mathrm{~g} / \mathrm{mol}$.

HPLC: RT = $11.2 \min (254 \mathrm{~nm}, 100 \%)$.

${ }^{1} \mathrm{H}$ NMR $(600 \mathrm{MHz}, \mathrm{DMSO}) \delta=10.24(\mathrm{~s}, 1 \mathrm{H}), 9.92(\mathrm{~s}, 1 \mathrm{H}), 8.97(\mathrm{~s}, 1 \mathrm{H}), 8.56\left(\mathrm{t},{ }^{3} \mathrm{~J}=5.5 \mathrm{~Hz}, 1 \mathrm{H}\right), 8.50-$ $8.48(\mathrm{~m}, 1 \mathrm{H}), 8.34(\mathrm{~s}, 1 \mathrm{H}), 8.16(\mathrm{~s}, 1 \mathrm{H}), 8.01\left(\mathrm{~d}, 3^{3} \mathrm{~J}=9.2 \mathrm{~Hz}, 1 \mathrm{H}\right), 7.94\left(\mathrm{~d},{ }^{3} \mathrm{~J}=8.3 \mathrm{~Hz}, 2 \mathrm{H}\right), 7.67\left(\mathrm{~d},{ }^{3} \mathrm{~J}=\right.$ $7.8 \mathrm{~Hz}, 2 \mathrm{H}), 7.59\left(\mathrm{~d},{ }^{3} \mathrm{~J}=8.5 \mathrm{~Hz}, 1 \mathrm{H}\right), 7.40\left(\mathrm{dd},{ }^{2} J=22.6 \mathrm{~Hz},{ }^{3} \mathrm{~J}=7.6 \mathrm{~Hz}, 4 \mathrm{H}\right), 7.34\left(\mathrm{~d},{ }^{3} J=8.3 \mathrm{~Hz}, 1 \mathrm{H}\right), 7.31$ $(\mathrm{s}, 1 \mathrm{H}), 4.57\left(\mathrm{~d},{ }^{3} \mathrm{~J}=9.2 \mathrm{~Hz}, 1 \mathrm{H}\right), 4.47-4.39(\mathrm{~m}, 2 \mathrm{H}), 4.36(\mathrm{~s}, 1 \mathrm{H}), 4.22\left(\mathrm{dd},{ }^{3} \mathrm{~J}=15.9 \mathrm{~Hz},{ }^{4} \mathrm{~J}=5.3 \mathrm{~Hz}, 1 \mathrm{H}\right)$, $3.72-3.64(\mathrm{~m}, 2 \mathrm{H}), 3.59-3.52(\mathrm{~m}, 2 \mathrm{H}), 3.49\left(\mathrm{dd},{ }^{3} J=15.9 \mathrm{~Hz},{ }^{4} J=7.6 \mathrm{~Hz}, 2 \mathrm{H}\right), 3.31\left(\mathrm{~d},{ }^{3} J=11.4 \mathrm{~Hz}, 2 \mathrm{H}\right)$, $3.06(\mathrm{~s}, 4 \mathrm{H}), 2.86(\mathrm{~s}, 3 \mathrm{H}), 2.62-2.54(\mathrm{~m}, 2 \mathrm{H}), 2.44(\mathrm{~s}, 3 \mathrm{H}), 2.09-1.99(\mathrm{~m}, 1 \mathrm{H}), 1.95-1.87(\mathrm{~m}, 1 \mathrm{H}), 0.93$ $(\mathrm{s}, 9 \mathrm{H}) \mathrm{ppm}$.

${ }^{13} \mathrm{C}$ NMR $(151 \mathrm{MHz}$, DMSO) $\delta=171.9,170.4,169.6,165.8,163.1,161.1,151.5,147.6,142.9,142.0$, $139.5,139.0,138.5$ (q, $\left.{ }^{2} J=32 \mathrm{~Hz}\right), 135.3,133.3,132.6,131.2,129.6,128.6,127.9,127.4,126.1,124.0$, $122.4,122.1\left(q,{ }^{1} \mathrm{~J}=279 \mathrm{~Hz}\right), 121.0,118.9,111.7,68.9,58.7,56.5,56.4,52.9,48.1,42.4,41.7,38.0$, $36.3,35.3,34.9,26.4,15.9 \mathrm{ppm}$. 
Synthesis of 6-hydroxy-N-(4'-((4-)((S)-1-((2S,4R)-4-hydroxy-2-((4-(4-methylthiazol-5yl)benzyl)carbamoyl)pyrrolidin-1-yl)-3,3-dimethyl-1-oxobutan-2-yl)amino)-4-oxobutyl)carbamoyl)4-(4-methylpiperazin-1-yl)-[1,1'-biphenyl]-3-yl)-4-(trifluoromethyl)nicotinamide (8f)

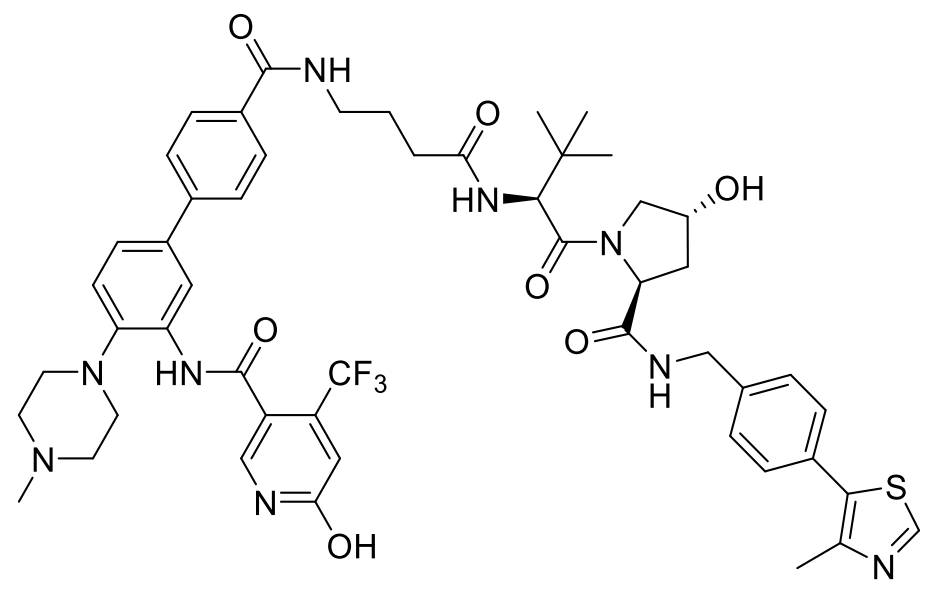

$22.1 \quad \mathrm{mg} \quad(36 \mu \mathrm{mol}, \quad 1.00 \mathrm{eq}) \quad(4-(((\mathrm{S})-1-((2 \mathrm{~S}, 4 \mathrm{R})-4-h y d r o x y-2-((4-(4-m e t h y l t h i a z o l-5-$ yl)benzyl)carbamoyl)pyrrolidin-1-yl)-3,3-dimethyl-1-oxobutan-2-yl)amino)-4-oxobutyl)carbamate were dissolved in $1 \mathrm{~mL} \mathrm{CH} \mathrm{Cl}_{2}$ and $1 \mathrm{~mL}$ TFA was added. The solution was stirred for $1 \mathrm{~h}$ at $\mathrm{rt}$. Excess solvent was removed under reduced pressure.

$20 \mathrm{mg}$ (36 $\mu \mathrm{mol}$, $1.00 \mathrm{eq}$ tert-butyl 3'-(6-hydroxy-4-(trifluoromethyl)nicotinamido)-4'-(4methylpiperazin-1-yl)-[1,1'-biphenyl]-4-carboxylate were dissolved in $1 \mathrm{~mL} \mathrm{CH}_{2} \mathrm{Cl}_{2}$ and $1 \mathrm{~mL}$ TFA was added. The solution was stirred for $1 \mathrm{~h}$ at rt. Excess solvent was removed under reduced pressure. The crude species was dissolved in $0.5 \mathrm{~mL}$ DMF and DIEA was added until the $\mathrm{pH}$ of the solution was basic. $16.4 \mathrm{mg}$ (43 $\mu \mathrm{mol}, 1.20 \mathrm{eq}$ ) HATU was added. The reaction mixture was stirred for $20 \mathrm{~min}$ at rt. The crude species of (4-(((S)-1-((2S,4R)-4-hydroxy-2-((4-(4-methylthiazol-5-yl)benzyl)carbamoyl)pyrrolidin1-yl)-3,3-dimethyl-1-oxobutan-2-yl)amino)-4-oxobutyl)carbamate was dissolved in 0.5 mL DMF and DIEA were added until the $\mathrm{pH}$ of the solution was basic. The mixture was added to the active ester of tert-butyl 3'-(6-hydroxy-4-(trifluoromethyl)nicotinamido)-4'-(4-methylpiperazin-1-yl)-[1,1'-biphenyl]4-carboxylate and stirred at $\mathrm{rt}$ for $4 \mathrm{~h}$. The reaction was stopped with $1 \mathrm{~mL}$ water. Saturated $\mathrm{NaHCO}_{3}$ solution and saturated $\mathrm{NaCl}$ solution were added and the reaction mixture was extracted $4 \mathrm{x}$ with $\mathrm{EA}$. The combined organic phases were washed with saturated $\mathrm{NaHCO}_{3}$ solution, dried over $\mathrm{MgSO}_{4}$ and filtered. The solvent of the organic phase was evapored under reduced pressure. The purification of the crude product was carried out on the preparative HPLC system.

Yield: $14.7 \mathrm{mg}, 14.7 \mu \mathrm{mol}, 41 \%$ of a white solid.

ESI: (calculated): $\left[\mathrm{M}+\mathrm{H}^{+}\right] 998.42 \mathrm{~g} / \mathrm{mol}$ 
(found): $\left[\mathrm{M}+\mathrm{H}^{+}\right](70) 998.35 \mathrm{~g} / \mathrm{mol}$.

MALDI: (calculated): [M+Na+] $1020.40 \mathrm{~g} / \mathrm{mol}$ (found): $\left[\mathrm{M}+\mathrm{Na}^{+}\right] 1020.34 \mathrm{~g} / \mathrm{mol}$.

HRMS: (calculated) $\left[\mathrm{M}+\mathrm{Na}^{+}\right] 1020.4024 \mathrm{~g} / \mathrm{mol}$, (found) $\left[\mathrm{M}+\mathrm{Na}^{+}\right] 1020.4010 \mathrm{~g} / \mathrm{mol}$.

HPLC: RT = $11.3 \min (254 \mathrm{~nm}, 100 \%)$.

${ }^{1} \mathrm{H} \mathrm{NMR}(600 \mathrm{MHz}, \mathrm{DMSO}) \delta=9.82(\mathrm{~s}, 1 \mathrm{H}), 9.58(\mathrm{~s}, 1 \mathrm{H}), 8.97(\mathrm{~s}, 1 \mathrm{H}), 8.55\left(\mathrm{t},{ }^{3} \mathrm{~J}=6.0 \mathrm{~Hz}, 1 \mathrm{H}\right), 8.52\left(\mathrm{t},{ }^{3} \mathrm{~J}\right.$ $=5.5 \mathrm{~Hz}, 1 \mathrm{H}), 8.21(\mathrm{~s}, 1 \mathrm{H}), 8.03(\mathrm{~s}, 1 \mathrm{H}), 7.95\left(\mathrm{~d},{ }^{3} \mathrm{~J}=8.2 \mathrm{~Hz}, 3 \mathrm{H}\right), 7.69\left(\mathrm{~d},{ }^{3} \mathrm{~J}=8.3 \mathrm{~Hz}, 2 \mathrm{H}\right), 7.56\left(\mathrm{dd},{ }^{3} \mathrm{~J}=\right.$ $\left.8.3 \mathrm{~Hz},{ }^{4} J=1.9 \mathrm{~Hz}, 1 \mathrm{H}\right), 7.40\left(\mathrm{dd},{ }^{2} J=22.3 \mathrm{~Hz},{ }^{3} J=8.1 \mathrm{~Hz}, 4 \mathrm{H}\right), 7.32\left(\mathrm{~d},{ }^{3} J=8.4 \mathrm{~Hz}, 1 \mathrm{H}\right), 6.84(\mathrm{~s}, 1 \mathrm{H}), 4.56$ $\left(d,{ }^{3} \mathrm{~J}=9.3 \mathrm{~Hz}, 1 \mathrm{H}\right), 4.43\left(\mathrm{dd}^{3} \mathrm{~J}=14.8 \mathrm{~Hz},{ }^{4} \mathrm{~J}=6.9 \mathrm{~Hz}, 2 \mathrm{H}\right), 4.36(\mathrm{~s}, 1 \mathrm{H}), 4.22\left(\mathrm{dd},{ }^{3} \mathrm{~J}=15.8 \mathrm{~Hz},{ }^{4} \mathrm{~J}=5.4 \mathrm{~Hz}\right.$, $1 \mathrm{H}), 3.71-3.63(\mathrm{~m}, 2 \mathrm{H}), 3.52\left(\mathrm{~d},{ }^{3} \mathrm{~J}=11.2 \mathrm{~Hz}, 2 \mathrm{H}\right), 3.28\left(\mathrm{~d},{ }^{3} \mathrm{~J}=7.4 \mathrm{~Hz}, 4 \mathrm{H}\right), 3.22-3.18(\mathrm{~m}, 2 \mathrm{H}), 3.05-$ $3.01(\mathrm{~m}, 2 \mathrm{H}), 2.87(\mathrm{~s}, 3 \mathrm{H}), 2.44(\mathrm{~s}, 3 \mathrm{H}), 2.37-2.30(\mathrm{~m}, 1 \mathrm{H}), 2.25-2.20(\mathrm{~m}, 1 \mathrm{H}), 2.05-2.00(\mathrm{~m}, 1 \mathrm{H}), 1.95$ $-1.87(\mathrm{~m}, 1 \mathrm{H}), 1.81-1.72(\mathrm{~m}, 2 \mathrm{H}), 0.95(\mathrm{~s}, 9 \mathrm{H}) \mathrm{ppm}$.

${ }^{13} \mathrm{C}$ NMR (201 MHz, DMSO) $\delta=171.9,171.8,169.6,165.8,162.8,161.0,151.4,147.7,144.8,142.0$, 139.5, 139.1, 138.7 (q, ${ }^{2} J=33 \mathrm{~Hz}$ ), 134.3, 133.2, 132.2, 131.1, 129.6, 128.6, 127.9, 127.4, 126.0, 124.0, $122.4,122.0\left(q,{ }^{1} J=276 \mathrm{~Hz}\right), 120.4,119.0,111.6,68.9,58.5,56.2,56.1,52.6,48.0,42.1,41.4,38.7$, $37.7,35.1,32.4,26.2,25.4,15.6 \mathrm{ppm}$. 
Synthesis

of

6-hydroxy-N-(4'-((5-)((S)-1-((2S,4R)-4-hydroxy-2-((4-(4-methylthiazol-5-

yl)benzyl)carbamoyl)pyrrolidin-1-yl)-3,3-dimethyl-1-oxobutan-2-yl)amino)-5-

oxopentyl)carbamoyl)-4-(4-methylpiperazin-1-yl)-[1,1'-biphenyl]-3-yl)-4-

(trifluoromethyl)nicotinamide (8g/ Homer)

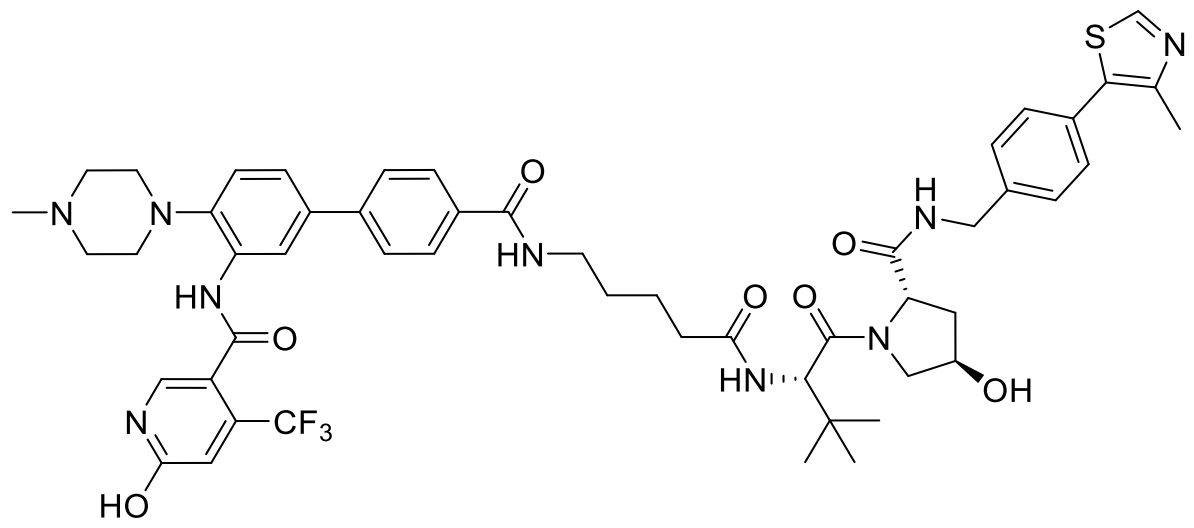

$18.0 \mathrm{mg}$ (32.0 $\mu \mathrm{mol}, 1.00 \mathrm{eq})$ of tert-butyl 3'-(6-hydroxy-4-(trifluoromethyl)nicotinamido)-4'-(4methylpiperazin-1-yl)-[1,1'-biphenyl]-4-carboxylate was diluted in $1 \mathrm{~mL}$ DCM and $1 \mathrm{~mL}$ TFA was added. In a second vial, $21.4 \mathrm{mg}(34.0 \mu \mathrm{mol}, 1.05 \mathrm{eq})$ of tert-butyl (5-(( $\mathrm{R})-1-((2 \mathrm{~S}, 4 \mathrm{R})-4$-hydroxy-2-((4-(4methylthiazol-5-yl)benzyl)carbamoyl)pyrrolidin-1-yl)-3,3-dimethyl-1-oxobutan-2-yl)amino)-5oxopentyl)carbamate was diluted in $1 \mathrm{~mL} \mathrm{DCM}$ and $1 \mathrm{~mL}$ TFA was added. The two reaction mixtures were stirred for $1 \mathrm{~h}$ at $\mathrm{rt}$. Excess solvent and TFA was removed under reduced pressure.

The deprotected species of tert-butyl 3'-(6-hydroxy-4-(trifluoromethyl)nicotinamido)-4'-(4methylpiperazin-1-yl)-[1,1'-biphenyl]-4-carboxylate was dissolved in $0.5 \mathrm{~mL}$ DMF and DIEA was added until the $\mathrm{pH}$ of the solution was basic. $18.4 \mathrm{mg}(49.0 \mu \mathrm{mol}, 1.50 \mathrm{eq})$ HATU was added. The reaction mixture was stirred for 20 min at rt. The deprotected species of tert-butyl (5-(( $R)-1-((2 S, 4 R)-4$-hydroxy2-((4-(4-methylthiazol-5-yl)benzyl)carbamoyl)pyrrolidin-1-yl)-3,3-dimethyl-1-oxobutan-2-yl)amino)-5oxopentyl)carbamate was dissolved in $0.5 \mathrm{~mL}$ DMF and $11 \mu \mathrm{L}$ (65 $\mu \mathrm{mol}, 2.00 \mathrm{eq}$ ) DIEA were added to the solution. The mixture was added to the active ester of tert-butyl 3'-(6-hydroxy-4(trifluoromethyl)nicotinamido)-4'-(4-methylpiperazin-1-yl)-[1,1'-biphenyl]-4-carboxylate and stirred at rt for $2.5 \mathrm{~h}$. The reaction was stopped with $1 \mathrm{~mL}$ water. Saturated $\mathrm{NaHCO}_{3}$ solution and saturated $\mathrm{NaCl}$ solution were added and the reaction mixture was extracted $4 x$ with EA. The combined organic phases were washed with saturated $\mathrm{NaHCO}_{3}$ solution, dried over $\mathrm{MgSO}_{4}$ and filtered. The solvent of the organic phase was evapored under reduced pressure. The purification of the crude product was carried out on the HPLC system.

Yield: $7.42 \mathrm{mg}, 7.33 \mu \mathrm{mol}, 23 \%$ of a white solid. 
MALDI: (calculated): $\left[\mathrm{M}+\mathrm{H}^{+}\right] 1012.43 \mathrm{~g} / \mathrm{mol}$ (found): $\left[\mathrm{M}+\mathrm{H}^{+}\right] 1012.53 \mathrm{~g} / \mathrm{mol}$.

HPLC: RT = $11.4 \min (254 \mathrm{~nm}, 100 \%)$.

HRMS: (calculated) $\left[\mathrm{M}+\mathrm{Na}^{+}\right] 1034.4180 \mathrm{~g} / \mathrm{mol}$ (found) $\left[\mathrm{M}+\mathrm{Na}^{+}\right] 1034.4171 \mathrm{~g} / \mathrm{mol}$.

${ }^{1} \mathrm{H}$ NMR $(500 \mathrm{MHz}, \mathrm{DMSO}) \delta=9.77(\mathrm{~s}, 1 \mathrm{H}), 9.57(\mathrm{~s}, 1 \mathrm{H}), 8.97(\mathrm{~s}, 1 \mathrm{H}), 8.55\left(\mathrm{t},{ }^{3} \mathrm{~J}=6.1 \mathrm{~Hz}, 1 \mathrm{H}\right), 8.50\left(\mathrm{t},{ }^{3} \mathrm{~J}\right.$ $=5.6 \mathrm{~Hz}, 1 \mathrm{H}), 8.21\left(\mathrm{~d},{ }^{4} \mathrm{~J}=1.8 \mathrm{~Hz}, 1 \mathrm{H}\right), 8.03(\mathrm{~s}, 1 \mathrm{H}), 7.94\left(\mathrm{~d},{ }^{3} \mathrm{~J}=8.4 \mathrm{~Hz}, 2 \mathrm{H}\right), 7.86\left(\mathrm{~d},{ }^{3} \mathrm{~J}=9.3 \mathrm{~Hz}, 1 \mathrm{H}\right), 7.68$ $\left(d,{ }^{3} J=8.4 \mathrm{~Hz}, 2 \mathrm{H}\right), 7.55\left(\mathrm{dd},{ }^{3} J=8.3 \mathrm{~Hz},{ }^{4} J=2.1 \mathrm{~Hz}, 1 \mathrm{H}\right), 7.42-7.39(\mathrm{~m}, 4 \mathrm{H}), 7.32\left(\mathrm{~d},{ }^{3} J=8.4 \mathrm{~Hz}, 1 \mathrm{H}\right)$, $6.84(\mathrm{~s}, 1 \mathrm{H}), 5.12(\mathrm{~s}, 1 \mathrm{H}), 4.54\left(\mathrm{~d},{ }^{3} \mathrm{~J}=9.4 \mathrm{~Hz}, 1 \mathrm{H}\right), 4.48-4.38(\mathrm{~m}, 2 \mathrm{H}), 4.35(\mathrm{~s}, 1 \mathrm{H}), 4.21\left(\mathrm{dd},{ }^{3} \mathrm{~J}=15.9 \mathrm{~Hz}\right.$, $\left.{ }^{4} J=5.5 \mathrm{~Hz}, 1 \mathrm{H}\right), 3.70-3.63(\mathrm{~m}, 2 \mathrm{H}), 3.55-3.46(\mathrm{~m}, 2 \mathrm{H}), 3.29-3.23(\mathrm{~m}, 4 \mathrm{H}), 3.07-3.02(\mathrm{~m}, 2 \mathrm{H}), 2.86$ $(\mathrm{s}, 3 \mathrm{H}), 2.53-2.50(\mathrm{~m}, 2 \mathrm{H}), 2.44(\mathrm{~s}, 3 \mathrm{H}), 2.35-2.26(\mathrm{~m}, 1 \mathrm{H}), 2.19-2.14(\mathrm{~m}, 1 \mathrm{H}), 2.09-1.99(\mathrm{~m}, 1 \mathrm{H})$, $1.93-1.88(\mathrm{~m}, 1 \mathrm{H}), 1.60-1.47(\mathrm{~m}, 4 \mathrm{H}), 0.94(\mathrm{~s}, 9 \mathrm{H}) \mathrm{ppm}$.

${ }^{13} \mathrm{C}$ NMR (126 MHz, DMSO) $\delta=172.0,172.0,169.7,165.8,163.2,151.4,147.7,144.6,142.1,139.5$, $139.1,138.1\left(\mathrm{~d},{ }^{2} \mathrm{~J}=32 \mathrm{~Hz}\right), 134.4,133.2,132.5,131.2,129.6,128.6,127.9,127.4,126.0,123.7,121.8$, $122.3\left(q,{ }^{1} J=276 \mathrm{~Hz}\right), 120.5,117.7,111.7,68.9,58.7,56.3,56.3,54.8,51.1,45.8,41.6,38.7,37.9,35.2$, $34.7,28.9,26.4,23.1,15.9 \mathrm{ppm}$. 
Synthesis

of

6-hydroxy-N-(4'-((6-)((S)-1-((2S,4R)-4-hydroxy-2-((4-(4-methylthiazol-5-

yl)benzyl)carbamoyl)pyrrolidin-1-yl)-3,3-dimethyl-1-oxobutan-2-yl)amino)-6-oxohexyl)carbamoyl)-

4-(4-methylpiperazin-1-yl)-[1,1'-biphenyl]-3-yl)-4-(trifluoromethyl)nicotinamide (8h)

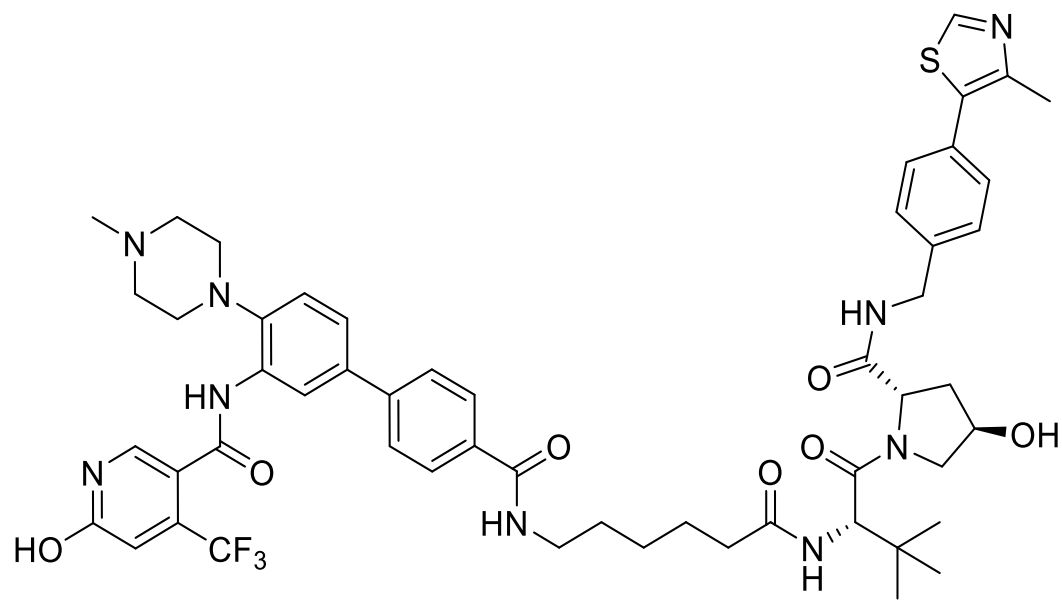

$18.0 \mathrm{mg}(32.0 \mu \mathrm{mol}, 1.00 \mathrm{eq})$ of tert-butyl 3'-(6-hydroxy-4-(trifluoromethyl)nicotinamido)-4'-(4methylpiperazin-1-yl)-[1,1'-biphenyl]-4-carboxylate was diluted in $1 \mathrm{~mL} \mathrm{DCM}$ and $1 \mathrm{~mL}$ TFA was added. In a second vial, $21.9 \mathrm{mg}(34.0 \mu \mathrm{mol}, 1.05 \mathrm{eq})$ of tert-butyl (6-(((S)-1-((2S,4R)-4-hydroxy-2-((4-(4methylthiazol-5-yl)benzyl)carbamoyl)pyrrolidin-1-yl)-3,3-dimethyl-1-oxobutan-2-yl)amino)-6oxohexyl)carbamate was diluted in $1 \mathrm{~mL}$ DCM and $1 \mathrm{~mL}$ TFA was added. The two reaction mixtures were stirred for $1 \mathrm{~h}$ at $\mathrm{rt}$. Excess solvent and TFA was removed under reduced pressure.

The deprotected species of tert-butyl 3'-(6-hydroxy-4-(trifluoromethyl)nicotinamido)-4'-(4methylpiperazin-1-yl)-[1,1'-biphenyl]-4-carboxylate was dissolved in $0.5 \mathrm{~mL}$ DMF and DIEA was added until the $\mathrm{pH}$ of the solution was basic. $18.4 \mathrm{mg}(49.0 \mu \mathrm{mol}, 1.50 \mathrm{eq})$ HATU was added. The reaction mixture was stirred for $20 \mathrm{~min}$ at rt. The deprotected species of tert-butyl (6-)((S)-1-((2S,4R)-4-hydroxy2-((4-(4-methylthiazol-5-yl)benzyl)carbamoyl)pyrrolidin-1-yl)-3,3-dimethyl-1-oxobutan-2-yl)amino)-6oxohexyl)carbamate was dissolved in $0.5 \mathrm{~mL}$ DMF and $11 \mu \mathrm{L}$ (65 $\mu \mathrm{mol}, 2.00$ eq) DIEA were added to the solution. The mixture was added to the active ester of tert-butyl 3'-(6-hydroxy-4(trifluoromethyl)nicotinamido)-4'-(4-methylpiperazin-1-yl)-[1,1'-biphenyl]-4-carboxylate and stirred at $\mathrm{rt}$ for $2.5 \mathrm{~h}$. The reaction was stopped with $1 \mathrm{~mL}$ water. Saturated $\mathrm{NaHCO}_{3}$ solution and saturated $\mathrm{NaCl}$ solution were added and the reaction mixture was extracted $4 x$ with EA. The combined organic phases were washed with saturated $\mathrm{NaHCO}_{3}$ solution, dried over $\mathrm{MgSO}_{4}$ and filtered. The solvent of the organic phase was evapored under reduced pressure. The purification of the crude product was carried out on the HPLC system.

Yield: $6.65 \mathrm{mg}, 6.48 \mu \mathrm{mol}, 20 \%$ of a white solid. 
MALDI: (calculated) $\left[\mathrm{M}+\mathrm{Na}^{+}\right] 1048.43 \mathrm{~g} / \mathrm{mol} ;\left[\mathrm{M}+\mathrm{H}^{+}\right] 1026.45 \mathrm{~g} / \mathrm{mol}$ (found) $\left[\mathrm{M}+\mathrm{Na}^{+}\right] 1048.43 \mathrm{~g} / \mathrm{mol}\left[\mathrm{M}+\mathrm{H}^{+}\right] 1026.44 \mathrm{~g} / \mathrm{mol}$.

HPLC: RT = $11.4 \min (254 \mathrm{~nm}, 100 \%)$.

HRMS: (calculated) $\left[\mathrm{M}+\mathrm{Na}^{+}\right] 1048.4337 \mathrm{~g} / \mathrm{mol}$

(found) $\left[\mathrm{M}+\mathrm{Na}^{+}\right] 1048.4338 \mathrm{~g} / \mathrm{mol}$.

${ }^{1} \mathrm{H}$ NMR $(500 \mathrm{MHz}, \mathrm{DMSO}) \delta=9.96(\mathrm{~s}, 1 \mathrm{H}), 9.59(\mathrm{~s}, 1 \mathrm{H}), 8.98(\mathrm{~s}, 1 \mathrm{H}), 8.56(\mathrm{t}, J=6.0 \mathrm{~Hz}, 1 \mathrm{H}), 8.48(\mathrm{t}, J=$ $5.6 \mathrm{~Hz}, 1 \mathrm{H}), 8.22(\mathrm{~d}, J=2.0 \mathrm{~Hz}, 1 \mathrm{H}), 8.04(\mathrm{~s}, 1 \mathrm{H}), 7.94(\mathrm{~d}, J=8.5 \mathrm{~Hz}, 2 \mathrm{H}), 7.84(\mathrm{~d}, J=9.3 \mathrm{~Hz}, 1 \mathrm{H}), 7.68(\mathrm{~d}$, $J=8.4 \mathrm{~Hz}, 2 \mathrm{H}), 7.55(\mathrm{dd}, J=8.3,2.2 \mathrm{~Hz}, 1 \mathrm{H}), 7.45-7.36(\mathrm{~m}, 4 \mathrm{H}), 7.32(\mathrm{~d}, J=8.4 \mathrm{~Hz}, 1 \mathrm{H}), 6.84(\mathrm{~s}, 1 \mathrm{H})$, $4.54(\mathrm{~d}, J=9.4 \mathrm{~Hz}, 1 \mathrm{H}), 4.47-4.38(\mathrm{~m}, 2 \mathrm{H}), 4.35(\mathrm{~s}, 1 \mathrm{H}), 4.22(\mathrm{dd}, J=15.9,5.4 \mathrm{~Hz}, 1 \mathrm{H}), 3.68-3.65(\mathrm{~m}$, $2 \mathrm{H}), 3.53(\mathrm{~d}, J=11.2 \mathrm{~Hz}, 2 \mathrm{H}), 3.28-3.20(\mathrm{~m}, 6 \mathrm{H}), 3.04(\mathrm{t}, J=11.3 \mathrm{~Hz}, 2 \mathrm{H}), 2.87(\mathrm{~s}, 3 \mathrm{H}), 2.44(\mathrm{~s}, 3 \mathrm{H}), 2.31$ $-2.22(\mathrm{~m}, 1 \mathrm{H}), 2.17-2.10(\mathrm{~m}, 1 \mathrm{H}), 2.05-2.01(\mathrm{~m}, 1 \mathrm{H}), 1.93-1.87(\mathrm{~m}, 1 \mathrm{H}), 1.56-1.50(\mathrm{~m}, 4 \mathrm{H}), 1.35-$ $1.25(\mathrm{~m}, 2 \mathrm{H}), 0.93(\mathrm{~s}, 9 \mathrm{H}) \mathrm{ppm}$.

${ }^{13} \mathrm{C}$ NMR (126 MHz, DMSO) $\delta=172.1,172.0,169.7,165.7,163.1,161.1,151.5,147.7,142.9,141.9$, $139.5,139.0,138.5$ (q, $\left.{ }^{2} J=33 \mathrm{~Hz}\right), 135.4,133.5,132.6,131.2,129.6,128.6,127.9,127.4,126.1,123.9$, $122.3,122.1\left(q,{ }^{1} J=276 \mathrm{~Hz}\right) 121.0,118.9,118.8,111.6,68.9,58.7,56.4,56.3,52.8,48.1,42.4,41.7$, $38.0,35.2,34.9,29.0,26.4,26.2,25.3,15.9$ ppm. 
Synthesis of 6-hydroxy-N-(4'-((7-)((S)-1-((2S,4R)-4-hydroxy-2-((4-(4-methylthiazol-5-

yl)benzyl)carbamoyl)pyrrolidin-1-yl)-3,3-dimethyl-1-oxobutan-2-yl)amino)-7oxoheptyl)carbamoyl)-4-(4-methylpiperazin-1-yl)-[1,1'-biphenyl]-3-yl)-4-

(trifluoromethyl)nicotinamide (8i)

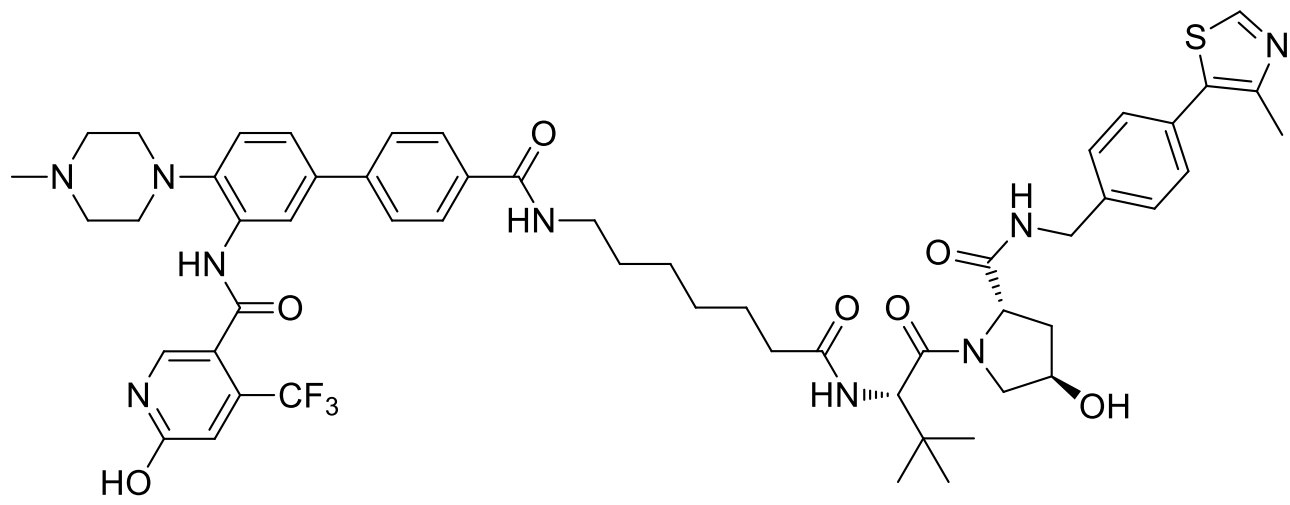

$18.0 \mathrm{mg}(32.0 \mu \mathrm{mol}, 1.00 \mathrm{eq})$ of tert-butyl 3'-(6-hydroxy-4-(trifluoromethyl)nicotinamido)-4'-(4methylpiperazin-1-yl)-[1,1'-biphenyl]-4-carboxylate was diluted in $1 \mathrm{~mL} \mathrm{DCM}$ and $1 \mathrm{~mL}$ TFA was added. In a second vial, $22.3 \mathrm{mg}$ (34.0 $\mathrm{mmol}, 1.05 \mathrm{eq})$ of (7-(((S)-1-((2S,4R)-4-hydroxy-2-((4-(4-methylthiazol-5yl)benzyl)carbamoyl)pyrrolidin-1-yl)-3,3-dimethyl-1-oxobutan-2-yl)amino)-7-oxoheptyl)carbamate was diluted in $1 \mathrm{~mL}$ DCM and $1 \mathrm{~mL}$ TFA was added. The two reaction mixtures were stirred for $1 \mathrm{~h}$ at rt. Excess solvent and TFA was removed under reduced pressure.

The deprotected species of tert-butyl 3'-(6-hydroxy-4-(trifluoromethyl)nicotinamido)-4'-(4methylpiperazin-1-yl)-[1,1'-biphenyl]-4-carboxylate was dissolved in $0.5 \mathrm{~mL} \mathrm{DMF}$ and DIEA was added until the $\mathrm{pH}$ of the solution was basic. $18.4 \mathrm{mg}(49.0 \mu \mathrm{mol}, 1.50 \mathrm{eq})$ HATU was added. The reaction mixture was stirred for $20 \mathrm{~min}$ at rt. The deprotected species of (7-()(S)-1-((2S,4R)-4-hydroxy-2-((4-(4methylthiazol-5-yl)benzyl)carbamoyl)pyrrolidin-1-yl)-3,3-dimethyl-1-oxobutan-2-yl)amino)-7oxoheptyl)carbamate was dissolved in $0.5 \mathrm{~mL} \mathrm{DMF}$ and $11 \mu \mathrm{L}$ (65 $\mu \mathrm{mol}, 2.00$ eq) DIEA were added to the solution. The mixture was added to the active ester of tert-butyl 3'-(6-hydroxy-4(trifluoromethyl)nicotinamido)-4'-(4-methylpiperazin-1-yl)-[1,1'-biphenyl]-4-carboxylate and stirred at rt for $2.5 \mathrm{~h}$. The reaction was stopped with $1 \mathrm{~mL}$ water. Saturated $\mathrm{NaHCO}_{3}$ solution and saturated $\mathrm{NaCl}$ solution were added and the reaction mixture was extracted $4 x$ with EA. The combined organic phases were washed with saturated $\mathrm{NaHCO}_{3}$ solution, dried over $\mathrm{MgSO}_{4}$ and filtered. The solvent of the organic phase was evapored under reduced pressure. The purification of the crude product was carried out on the HPLC system.

Yield: $4.67 \mathrm{mg}, 4.49 \mu \mathrm{mol}, 14 \%$ of a white solid. 
MALDI: (calculated): $\left[\mathrm{M}+\mathrm{H}^{+}\right] 1040.21 \mathrm{~g} / \mathrm{mol}$ (found): $\left[\mathrm{M}+\mathrm{H}^{+}\right] 1040.25 \mathrm{~g} / \mathrm{mol}$.

HPLC: RT = $11.6 \min (254 \mathrm{~nm}, 98 \%)$.

HRMS: (calculated) $\left[\mathrm{M}+\mathrm{Na}^{+}\right] 1062.45 \mathrm{~g} / \mathrm{mol}$ (found) $\left[\mathrm{M}+\mathrm{Na}^{+}\right] 1062.45 \mathrm{~g} / \mathrm{mol}$.

${ }^{1} \mathrm{H}$ NMR (500 MHz, DMSO) $\delta=12.55(\mathrm{~s}, 1 \mathrm{H}), 9.52(\mathrm{~s}, 1 \mathrm{H}), 8.97(\mathrm{~s}, 1 \mathrm{H}), 8.55\left(\mathrm{t},{ }^{3} \mathrm{~J}=6.0 \mathrm{~Hz}, 1 \mathrm{H}\right), 8.46\left(\mathrm{t},{ }^{3} \mathrm{~J}\right.$ $=5.6 \mathrm{~Hz}, 1 \mathrm{H}), 8.17(\mathrm{~s}, 1 \mathrm{H}), 8.01(\mathrm{~s}, 1 \mathrm{H}), 7.93\left(\mathrm{~d},{ }^{3} \mathrm{~J}=8.4 \mathrm{~Hz}, 2 \mathrm{H}\right), 7.84\left(\mathrm{~d},{ }^{3} \mathrm{~J}=9.4 \mathrm{~Hz}, 1 \mathrm{H}\right), 7.68\left(\mathrm{~d},{ }^{3} \mathrm{~J}=8.4\right.$ $\mathrm{Hz}, 2 \mathrm{H}), 7.54\left(\mathrm{dd},{ }^{3} \mathrm{~J}=8.3 \mathrm{~Hz},{ }^{4} \mathrm{~J}=2.1 \mathrm{~Hz}, 1 \mathrm{H}\right), 7.40\left(\mathrm{q},{ }^{3} \mathrm{~J}=8.3 \mathrm{~Hz}, 4 \mathrm{H}\right), 7.30\left(\mathrm{~d},{ }^{3} \mathrm{~J}=8.4 \mathrm{~Hz}, 1 \mathrm{H}\right), 6.83(\mathrm{~s}$, $1 \mathrm{H}), 5.11\left(\mathrm{~d},{ }^{3} \mathrm{~J}=3.6 \mathrm{~Hz}, 1 \mathrm{H}\right), 4.54\left(\mathrm{~d},{ }^{3} \mathrm{~J}=9.4 \mathrm{~Hz}, 1 \mathrm{H}\right), 4.49-4.38(\mathrm{~m}, 2 \mathrm{H}), 4.35(\mathrm{~s}, 1 \mathrm{H}), 4.21\left(\mathrm{dd},{ }^{2} J=\right.$ $\left.16.0 \mathrm{~Hz},{ }^{3} \mathrm{~J}=5.4 \mathrm{~Hz}, 1 \mathrm{H}\right), 3.72-3.58(\mathrm{~m}, 2 \mathrm{H}), 3.26\left(\mathrm{dd},{ }^{3} \mathrm{~J}=13.3 \mathrm{~Hz},{ }^{4} \mathrm{~J}=6.7 \mathrm{~Hz}, 4 \mathrm{H}\right), 3.05(\mathrm{~s}, 4 \mathrm{H}), 2.44(\mathrm{~s}$, $3 \mathrm{H}), 2.32-2.28(\mathrm{~m}, 1 \mathrm{H}), 2.19-2.07(\mathrm{~m}, 1 \mathrm{H}), 2.07-1.98(\mathrm{~m}, 1 \mathrm{H}), 1.93-1.88(\mathrm{~m}, 1 \mathrm{H}), 1.52$ - $1.47(\mathrm{~m}$, $4 \mathrm{H}), 1.31-1.23(\mathrm{~m}, 3 \mathrm{H}), 0.93(\mathrm{~s}, 9 \mathrm{H}) \mathrm{ppm}$.

${ }^{13} \mathrm{C}$ NMR (126 MHz, DMSO) $\delta=172.1,171.9,169.7,165.7,163.1,161.1,151.4,147.7,142.9,141.8$, $139.5,139.1,138.5\left(q,{ }^{2} J=32 \mathrm{~Hz}\right), 135.4,133.5,132.6,131.2,129.6,128.6,127.9,127.4,126.1,123.9$, $123.1,122.3,122.1\left(q,{ }^{1} J=273 \mathrm{~Hz}\right), 121.0,119.0,111.6,68.9,58.7,56.3,56.3,52.8,48.1,42.3,41.6$, $38.4,38.0,35.2,34.8,29.1,26.4,25.4,15.9$ ppm. 
Synthesis

of

6-hydroxy-N-(4'-((4-(2-)((S)-1-((2S,4R)-4-hydroxy-2-((4-(4-methylthiazol-5-

yl)benzyl)carbamoyl)pyrrolidin-1-yl)-3,3-dimethyl-1-oxobutan-2-yl)amino)-2-

oxoethyl)benzyl)carbamoyl)-4-(4-methylpiperazin-1-yl)-[1,1'-biphenyl]-3-yl)-4-

(trifluoromethyl)nicotinamide (8j)<smiles>Cc1ncsc1-c1ccc(CNC(=O)[C@@H]2C[C@@H](O)CN2C(=O)[C@@H](NC(=O)Cc2ccc(CNC(=O)c3ccc(-c4ccc(N5CCN(C)CC5)c(NC(=O)c5cnc(O)cc5C(F)(F)F)c4)cc3)cc2)C(C)(C)C)cc1</smiles>

$32 \mathrm{mg}(47 \mu \mathrm{mol}, 1.05$ eq) tert-butyl (4-(2-(((S)-1-((2S,4R)-4-hydroxy-2-((4-(4-methylthiazol-5yl)benzyl)carbamoyl)pyrrolidin-1-yl)-3,3-dimethyl-1-oxobutan-2-yl)amino)-2-

oxoethyl)benzyl)carbamate were dissolved in $1 \mathrm{~mL} \mathrm{CH}{ }_{2} \mathrm{Cl}_{2}$ and $1 \mathrm{~mL}$ TFA was added. The solution was stirred for $1 \mathrm{~h}$ at $\mathrm{rt}$. Excess solvent was removed under reduced pressure.

$25 \mathrm{mg}$ (45 $\mu \mathrm{mol}, 1.00$ eq) tert-butyl 3'-(6-hydroxy-4-(trifluoromethyl)nicotinamido)-4'-(4methylpiperazin-1-yl)-[1,1'-biphenyl]-4-carboxylate were dissolved in $1 \mathrm{~mL} \mathrm{CH}{ }_{2} \mathrm{Cl}_{2}$ and $1 \mathrm{~mL}$ TFA was added. The solution was stirred for $1 \mathrm{~h}$ at $\mathrm{rt}$. Excess solvent was removed under reduced pressure. The crude species was dissolved in $0.5 \mathrm{~mL}$ DMF and DIEA was added until the $\mathrm{pH}$ of the solution was basic. $20.5 \mathrm{mg}(54 \mu \mathrm{mol}, 1.20 \mathrm{eq}$ ) HATU was added. The reaction mixture was stirred for $20 \mathrm{~min}$ at $\mathrm{rt}$. The crude species of tert-butyl (4-(2-)((S)-1-((2S,4R)-4-hydroxy-2-((4-(4-methylthiazol-5yl)benzyl)carbamoyl)pyrrolidin-1-yl)-3,3-dimethyl-1-oxobutan-2-yl)amino)-2-

oxoethyl)benzyl)carbamate was dissolved in $0.5 \mathrm{~mL}$ DMF and DIEA were added until the $\mathrm{pH}$ of the solution was basic. The mixture was added to the active ester of tert-butyl 3'-(6-hydroxy-4(trifluoromethyl)nicotinamido)-4'-(4-methylpiperazin-1-yl)-[1,1'-biphenyl]-4-carboxylate and stirred at rt for $4 \mathrm{~h}$. The reaction was stopped with $1 \mathrm{~mL}$ water. Saturated $\mathrm{NaHCO}_{3}$ solution and saturated $\mathrm{NaCl}$ solution were added and the reaction mixture was extracted $4 x$ with EA. The combined organic phases were washed with saturated $\mathrm{NaHCO}_{3}$ solution, dried over $\mathrm{MgSO}_{4}$ and filtered. The solvent of the organic phase was evapored under reduced pressure. The purification of the crude product was carried out on the preparative HPLC system.

Yield: $14.4 \mathrm{mg}, 13.6 \mu \mathrm{mol}, 30 \%$ of a white solid. 
MALDI: (calculated): $\left[\mathrm{M}+\mathrm{H}^{+}\right] 1060.44 \mathrm{~g} / \mathrm{mol}$ (found): $\left[\mathrm{M}+\mathrm{H}^{+}\right] 1060.17 \mathrm{~g} / \mathrm{mol}$.

HRMS: (calculated): [M+Na+] $1082.4180 \mathrm{~g} / \mathrm{mol}$ (found): $\left[\mathrm{M}+\mathrm{Na}^{+}\right] 1082.4171 \mathrm{~g} / \mathrm{mol}$.

HPLC: RT = $11.6 \min (254 \mathrm{~nm}, 100 \%)$.

${ }^{1} \mathrm{H}$ NMR (500 MHz, DMSO) $\delta=9.47(\mathrm{~s}, 1 \mathrm{H}), 9.04\left(\mathrm{t},{ }^{3} \mathrm{~J}=6.0 \mathrm{~Hz}, 1 \mathrm{H}\right), 8.98(\mathrm{~s}, 1 \mathrm{H}), 8.56\left(\mathrm{t},{ }^{3} \mathrm{~J}=6.1 \mathrm{~Hz}, 1 \mathrm{H}\right)$, $8.12\left(d,{ }^{4} J=2.1 \mathrm{~Hz}, 1 \mathrm{H}\right), 8.09\left(\mathrm{~d},{ }^{3} \mathrm{~J}=9.3 \mathrm{~Hz}, 1 \mathrm{H}\right), 8.01-7.93(\mathrm{~m}, 3 \mathrm{H}), 7.69\left(\mathrm{~d},{ }^{3} \mathrm{~J}=8.5 \mathrm{~Hz}, 2 \mathrm{H}\right), 7.53(\mathrm{dd}$, $\left.{ }^{3} J=8.4 \mathrm{~Hz},{ }^{4} J=2.2 \mathrm{~Hz}, 1 \mathrm{H}\right), 7.40\left(\mathrm{q},{ }^{3} \mathrm{~J}=8.4 \mathrm{~Hz}, 4 \mathrm{H}\right), 7.27\left(\mathrm{~d},{ }^{3} \mathrm{~J}=8.4 \mathrm{~Hz}, 1 \mathrm{H}\right), 7.24(\mathrm{~s}, 4 \mathrm{H}), 6.82(\mathrm{~s}, 1 \mathrm{H})$, $5.11\left(\mathrm{~d},{ }^{3} J=3.1 \mathrm{~Hz}, 1 \mathrm{H}\right), 4.51\left(\mathrm{~d},{ }^{3} \mathrm{~J}=9.4 \mathrm{~Hz}, 1 \mathrm{H}\right), 4.47\left(\mathrm{~d},{ }^{3} \mathrm{~J}=5.8 \mathrm{~Hz}, 2 \mathrm{H}\right), 4.45-4.39(\mathrm{~m}, 2 \mathrm{H}), 4.33(\mathrm{~s}$, $1 \mathrm{H}), 4.22\left(\mathrm{dd},{ }^{3} \mathrm{~J}=15.9 \mathrm{~Hz},{ }^{4} \mathrm{~J}=5.5 \mathrm{~Hz}, 1 \mathrm{H}\right), 3.71-3.58(\mathrm{~m}, 3 \mathrm{H}), 3.44\left(\mathrm{~d},{ }^{3} \mathrm{~J}=13.9 \mathrm{~Hz}, 2 \mathrm{H}\right), 2.91\left(\mathrm{t},{ }^{3} \mathrm{~J}=\right.$ $4.3 \mathrm{~Hz}, 4 \mathrm{H}), 2.50(\mathrm{~s}, 4 \mathrm{H}), 2.44(\mathrm{~s}, 3 \mathrm{H}), 2.24(\mathrm{~s}, 3 \mathrm{H}), 2.07-1.97(\mathrm{~m}, 1 \mathrm{H}), 1.92-1.86(\mathrm{~m}, 1 \mathrm{H}), 0.92(\mathrm{~s}, 9 \mathrm{H})$ ppm.

${ }^{13} \mathrm{C}$ NMR (126 MHz, DMSO) $\delta=171.9,170.0,169.5,165.8,162.8,161.1,151.4,147.72,145.0,142.3$, $139.5,139.2,138.5\left(q,{ }^{2} J=32 \mathrm{~Hz}\right), 137.6,135.1,134.2,132.9,132.2,131.2,129.6,129.0,128.6,128.0$, 127.4, 127.0, 126.1, 124.0, 122.4, 122.0 (d, ${ }^{1} J=275 \mathrm{~Hz}$ ), 120.4, 119.0, 111.6, 68.9, 58.7, 56.5, 56.4, 54.7, $51.0,45.7,42.4,41.7,41.5,37.9,35.4,26.3,15.9$ ppm. 


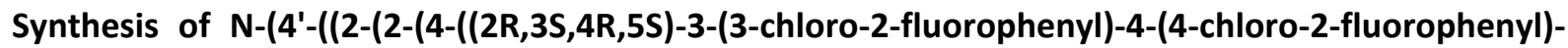
4-cyano-5-neopentylpyrrolidine-2-carboxamido)-3-methoxybenzamido)ethoxy)ethyl)carbamoyl)-4(4-methylpiperazin-1-yl)-[1,1'-biphenyl]-3-yl)-6-hydroxy-4-(trifluoromethyl)nicotinamide (9a)<smiles>COc1cc(C(=O)NCCOCCNC(=O)c2ccc(-c3ccc(N4CCN(C)CC4)c(NC(=O)c4cnc(O)cc4C(F)(F)F)c3)cc2)ccc1NC(=O)[C@@H]1N[C@@H](CC(C)(C)C)[C@](C#N)(c2ccc(Cl)cc2F)[C@H]1c1cccc(Cl)c1F</smiles>

$15.2 \mathrm{mg}$ ( $21 \mu \mathrm{mol}, 1.05 \mathrm{eq}$ ) Intermediate (6e) was dissolved in $2 \mathrm{~mL} \mathrm{TFA} / \mathrm{CH}_{2} \mathrm{Cl}_{2}$ and stirred for $1 \mathrm{~h}$ at

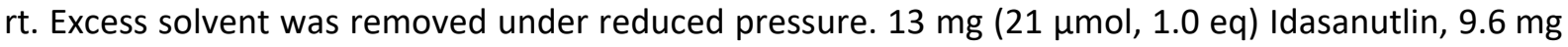
(25 $\mu \mathrm{mol}, 1.2 \mathrm{eq}) \mathrm{HATU}$ and $7 \mu \mathrm{L}(42 \mu \mathrm{mol}, 2.0$ eq) DIEA were dissolved in $1 \mathrm{~mL}$ DMF and stirred for 15 min at rt. Then, a solution of deprotected (6e) and $73 \mu \mathrm{L}(420 \mu \mathrm{mol}, 10$ eq) DIEA in $1 \mathrm{~mL}$ DMF were added to the solution and stirred for $12 \mathrm{~h}$ at $\mathrm{rt}$. The reaction mixture was quenched with $2 \mathrm{~mL}$ water and $2 \mathrm{~mL}$ saturated $\mathrm{NaHCO}_{3}$, then the reaction was extracted $3 x$ with EA. The organic phase was dried over $\mathrm{MgSO}_{4}$, filtered and the solvent was removed under reduced pressure. The crude product was purified by HPLC. The gained product was then dissolved in ethyl acetate and a solution of saturated $\mathrm{NaHCO}_{3}$ and saturated $\mathrm{NaCl}$ solution and extracted with ethyl acetate. The combined organic phases were dried over $\mathrm{MgSO}_{4}$ and the solvent was removed under reduced pressure.

Yield: $3.0 \mathrm{mg}, 2.5 \mu \mathrm{mol}, 12 \%$ of a clear oil.

MALDI: (calculated) $\left[\mathrm{M}+\mathrm{H}^{+}\right] 1184.49 \mathrm{~g} / \mathrm{mol}$, (found) $\left[\mathrm{M}+\mathrm{H}^{+}\right] 1184.40 \mathrm{~g} / \mathrm{mol}$.

HRMS: (calculated) $\left[\mathrm{M}+\mathrm{Na}^{+}\right] 1206.3805 \mathrm{~g} / \mathrm{mol}$, (found) $\left[\mathrm{M}+\mathrm{Na}^{+}\right] 1206.3821 \mathrm{~g} / \mathrm{mol}$.

HPLC: RT = $14.4 \min (254 \mathrm{~nm}, 98 \%)$. 
${ }^{1} \mathrm{H}$ NMR $(400 \mathrm{MHz}, \mathrm{DMSO}) \delta=10.45(\mathrm{~s}, 1 \mathrm{H}), 10.40(\mathrm{~s}, 1 \mathrm{H}), 9.45(\mathrm{~s}, 1 \mathrm{H}), 8.53\left(\mathrm{t},{ }^{3} \mathrm{~J}=5.5 \mathrm{~Hz}, 1 \mathrm{H}\right), 8.48(\mathrm{t}$, $\left.{ }^{3} J=5.5 \mathrm{~Hz}, 1 \mathrm{H}\right), 8.35(\mathrm{~s}, 1 \mathrm{H}), 8.32\left(\mathrm{~d},{ }^{3} \mathrm{~J}=8.5 \mathrm{~Hz}, 2 \mathrm{H}\right), 8.13\left(\mathrm{~d},{ }^{4} \mathrm{~J}=1.9 \mathrm{~Hz}, 1 \mathrm{H}\right), 7.92\left(\mathrm{~d},{ }^{3} J=8.4 \mathrm{~Hz}, 2 \mathrm{H}\right)$, $7.73\left(\mathrm{t},{ }^{3} \mathrm{~J}=7.3 \mathrm{~Hz}, 2 \mathrm{H}\right), 7.67\left(\mathrm{~d},{ }^{3} \mathrm{~J}=8.4 \mathrm{~Hz}, 2 \mathrm{H}\right), 7.60-7.56(\mathrm{~m}, 4 \mathrm{H}), 7.53-7.50(\mathrm{~m}, 3 \mathrm{H}), 7.44-7.38(\mathrm{~m}$, $1 \mathrm{H}), 7.38-7.31(\mathrm{~m}, 4 \mathrm{H}), 7.25\left(\mathrm{~d},{ }^{3} \mathrm{~J}=8.4 \mathrm{~Hz}, 1 \mathrm{H}\right), 6.81(\mathrm{~s}, 1 \mathrm{H}), 4.65-4.54(\mathrm{~m}, 4 \mathrm{H}), 4.40-4.33(\mathrm{~m}, 2 \mathrm{H})$, $3.99-3.95(\mathrm{~m}, 2 \mathrm{H}), 3.92(\mathrm{~s}, 3 \mathrm{H}), 3.90(\mathrm{~s}, 3 \mathrm{H}), 3.60-3.57(\mathrm{~m}, 5 \mathrm{H}), 3.48-3.43(\mathrm{~m}, 8 \mathrm{H}), 2.92-2.88(\mathrm{~m}$, $4 \mathrm{H}), 1.69-1.60(\mathrm{~m}, 2 \mathrm{H}), 1.39-1.36(\mathrm{~m}, 1 \mathrm{H}), 1.28-1.24(\mathrm{~m}, 2 \mathrm{H}), 0.96(\mathrm{~s}, 9 \mathrm{H}) \mathrm{ppm}$. Contains rotameres. 
Synthesis of $\mathrm{N}$-(4'-((1-(4-((2R,3S,4R,5S)-3-(3-chloro-2-fluorophenyl)-4-(4-chloro-2-fluorophenyl)-4cyano-5-neopentylpyrrolidine-2-carboxamido)-3-methoxyphenyl)-1-oxo-5,8,11,14,17,20,23heptaoxa-2-azapentacosan-25-yl)carbamoyl)-4-(4-methylpiperazin-1-yl)-[1,1'-biphenyl]-3-yl)-6hydroxy-4-(trifluoromethyl)nicotinamide (9b)

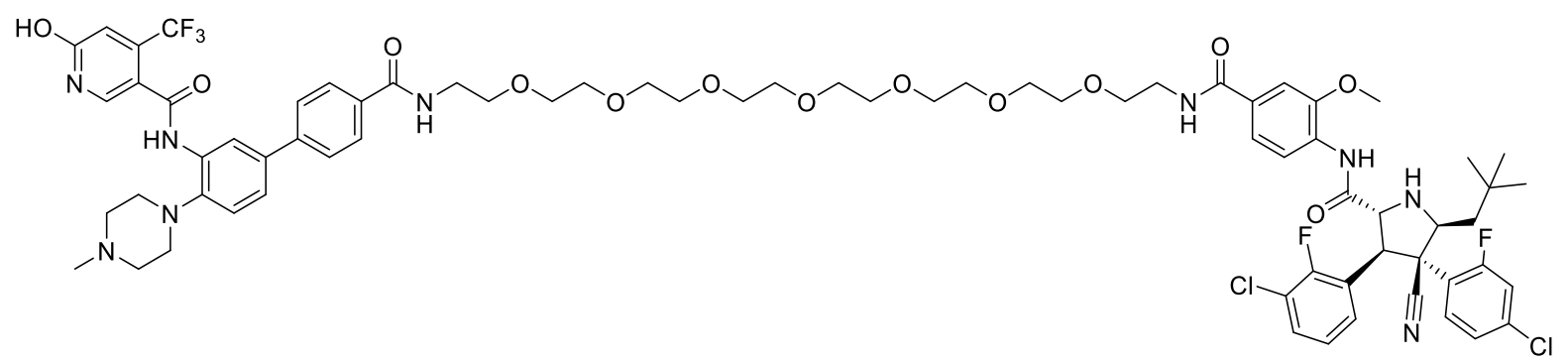

$8.9 \mathrm{mg}\left(9 \mu \mathrm{mol}, 1.0 \mathrm{eq}\right.$ ) Intermediate (6f) was dissolved in $2 \mathrm{~mL} \mathrm{TFA} / \mathrm{CH}_{2} \mathrm{Cl}_{2}$ and stirred for $1 \mathrm{~h}$ at $\mathrm{rt}$. Excess solvent was removed under reduced pressure. $5.5 \mathrm{mg}(9 \mu \mathrm{mol}, 1.0 \mathrm{eq})$ Idasanutlin, $4 \mathrm{mg}$ (11 $\mu \mathrm{mol}, 1.2 \mathrm{eq})$ HATU and $3 \mu \mathrm{L}(18 \mu \mathrm{mol}, 2.0 \mathrm{eq})$ DIEA were dissolved in $1 \mathrm{~mL}$ DMF and stirred for 15 min at rt. Then, a solution of deprotected (6f) and $31 \mu \mathrm{L}(180 \mu \mathrm{mol}, 10$ eq) DIEA in $1 \mathrm{~mL}$ DMF were added to the solution and stirred for $12 \mathrm{~h}$ at $\mathrm{rt}$. The reaction mixture was quenched with $2 \mathrm{~mL}$ water and $2 \mathrm{~mL}$ saturated $\mathrm{NaHCO}_{3}$, then the reaction was extracted $3 x$ with EA. The organic phase was dried over $\mathrm{MgSO}_{4}$, filtered and the solvent was removed under reduced pressure. The crude product was purified by HPLC. The gained product was then dissolved in ethyl acetate and a solution of saturated $\mathrm{NaHCO}_{3}$ and saturated $\mathrm{NaCl}$ solution and extracted with ethyl acetate. The combined organic phases were dried over $\mathrm{MgSO}_{4}$ and the solvent was removed under reduced pressure.

Yield: $3.3 \mathrm{mg}, 2.2 \mu \mathrm{mol}, 25 \%$ of a clear oil.

MALDI: (calculated) $\left[\mathrm{M}+\mathrm{H}^{+}\right] 1448.56 \mathrm{~g} / \mathrm{mol}$, (found) $\left[\mathrm{M}+\mathrm{H}^{+}\right] 1448.53 \mathrm{~g} / \mathrm{mol}$.

HRMS: (calculated) $\left[\mathrm{M}+\mathrm{Na}^{+}\right] 1470.5378 \mathrm{~g} / \mathrm{mol}$, (found) $\left[\mathrm{M}+\mathrm{Na}^{+}\right] 1470.5392 \mathrm{~g} / \mathrm{mol}$.

HPLC: RT = $14.6 \min (254 \mathrm{~nm}, 96 \%)$.

${ }^{1} \mathrm{H}$ NMR $(400 \mathrm{MHz}, \mathrm{DMSO}) \delta=10.47(\mathrm{~s}, 1 \mathrm{H}), 10.40(\mathrm{~s}, 1 \mathrm{H}), 9.46(\mathrm{~s}, 1 \mathrm{H}), 8.53\left(\mathrm{t},{ }^{3} \mathrm{~J}=5.5 \mathrm{~Hz}, 1 \mathrm{H}\right), 8.48(\mathrm{t}$, $\left.{ }^{3} J=5.4 \mathrm{~Hz}, 1 \mathrm{H}\right), 8.36\left(\mathrm{~d},{ }^{3} J=8.8 \mathrm{~Hz}, 1 \mathrm{H}\right), 8.32\left(\mathrm{~d},{ }^{3} J=8.4 \mathrm{~Hz}, 2 \mathrm{H}\right), 8.12\left(\mathrm{~d},{ }^{4} J=2.1 \mathrm{~Hz}, 1 \mathrm{H}\right), 7.92\left(\mathrm{~d},{ }^{3} J=\right.$ 
$8.5 \mathrm{~Hz}, 2 \mathrm{H}), 7.73\left(\mathrm{t},{ }^{3} \mathrm{~J}=7.2 \mathrm{~Hz}, 2 \mathrm{H}\right), 7.67\left(\mathrm{~d},{ }^{3} \mathrm{~J}=8.5 \mathrm{~Hz}, 2 \mathrm{H}\right), 7.60-7.56(\mathrm{~m}, 5 \mathrm{H}), 7.54-7.51(\mathrm{~m}, 2 \mathrm{H})$, $7.50-7.48(\mathrm{~m}, 1 \mathrm{H}), 7.40\left(\mathrm{dd},{ }^{3} \mathrm{~J}=8.4 \mathrm{~Hz},{ }^{4} \mathrm{~J}=2.4 \mathrm{~Hz}, 1 \mathrm{H}\right), 7.38-7.34(\mathrm{~m}, 3 \mathrm{H}), 7.34-7.32(\mathrm{~m}, 1 \mathrm{H}), 7.25$ $\left(\mathrm{d},{ }^{3} \mathrm{~J}=8.4 \mathrm{~Hz}, 1 \mathrm{H}\right), 6.82(\mathrm{~s}, 1 \mathrm{H}), 4.65-4.54(\mathrm{~m}, 4 \mathrm{H}), 4.41-4.33(\mathrm{~m}, 2 \mathrm{H}), 3.99-3.95(\mathrm{~m}, 2 \mathrm{H}), 3.92(\mathrm{~s}$, $3 \mathrm{H}), 3.90(\mathrm{~s}, 3 \mathrm{H}), 3.60-3.57(\mathrm{~m}, 5 \mathrm{H}), 3.49-3.41(\mathrm{~m}, 8 \mathrm{H}), 2.91-2.90(\mathrm{~m}, 4 \mathrm{H}), 1.68-1.61(\mathrm{~m}, 2 \mathrm{H}), 1.44$ $-1.33(\mathrm{~m}, 2 \mathrm{H}), 0.96(\mathrm{~s}, 9 \mathrm{H}) \mathrm{ppm}$. Contains rotameres. 
Synthesis of N-(4'-((4-)(4-((2R,3S,4R,5S)-3-(3-chloro-2-fluorophenyl)-4-(4-chloro-2-fluorophenyl)-4cyano-5-neopentylpyrrolidine-2-carboxamido)-3-methoxybenzamido)methyl)benzyl)carbamoyl)-4(4-methylpiperazin-1-yl)-[1,1'-biphenyl]-3-yl)-6-hydroxy-4-(trifluoromethyl)nicotinamide (9c)

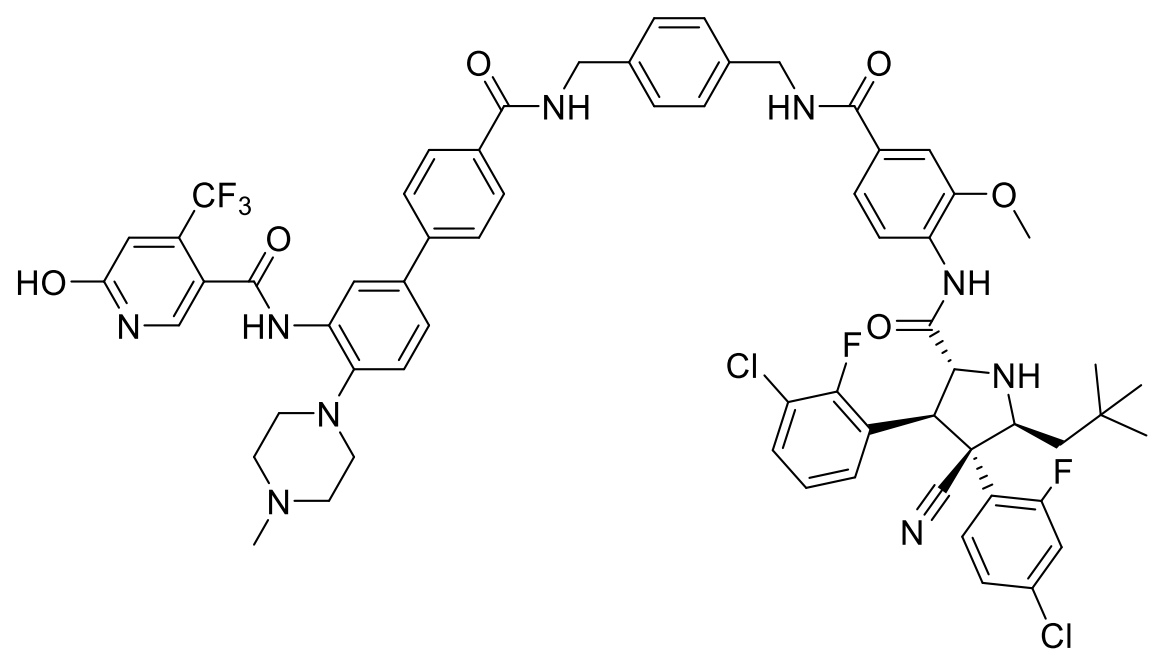

$13.5 \mathrm{mg}(24 \mu \mathrm{mol}, 1.0 \mathrm{eq})$ Intermediate $(6 \mathrm{~g})$ was dissolved in $2 \mathrm{~mL} \mathrm{TFA} / \mathrm{CH}_{2} \mathrm{Cl}_{2}$ and stirred for $1 \mathrm{~h}$ at $\mathrm{rt}$. Excess solvent was removed under reduced pressure. $15.7 \mathrm{mg}(25 \mu \mathrm{mol}, 1.1 \mathrm{eq})$ Idasanutlin, $11 \mathrm{mg}$ (29 $\mu \mathrm{mol}, 1.2 \mathrm{eq}) \mathrm{HATU}$ and $8 \mu \mathrm{L}$ (49 $\mu \mathrm{mol}, 2.0$ eq) DIEA were dissolved in $1 \mathrm{~mL}$ DMF and stirred for $15 \mathrm{~min}$ at rt. Then, a solution of deprotected $(6 \mathrm{~g})$ and $84 \mu \mathrm{L}(490 \mu \mathrm{mol}, 10 \mathrm{eq})$ DIEA in $1 \mathrm{~mL}$ DMF were added to the solution and stirred for $12 \mathrm{~h}$ at $\mathrm{rt}$. The reaction mixture was quenched with $2 \mathrm{~mL}$ water and 2 $\mathrm{mL}$ saturated $\mathrm{NaHCO}_{3}$, then the reaction was extracted $3 \mathrm{x}$ with $\mathrm{EA}$. The organic phase was dried over $\mathrm{MgSO}_{4}$, filtered and the solvent was removed under reduced pressure. The crude product was purified by HPLC. The gained product was then dissolved in ethyl acetate and a solution of saturated $\mathrm{NaHCO}_{3}$ and saturated $\mathrm{NaCl}$ solution and extracted with ethyl acetate. The combined organic phases were dried over $\mathrm{MgSO}_{4}$ and the solvent was removed under reduced pressure.

Yield: $3.7 \mathrm{mg}, 3.0 \mu \mathrm{mol}, 13 \%$ of a clear oil.

MALDI: (calculated) [M+K $] 1254.36 \mathrm{~g} / \mathrm{mol}$, (found) $\left[\mathrm{M}+\mathrm{K}^{+}\right] 1254.32 \mathrm{~g} / \mathrm{mol}$.

HRMS: (calculated) $\left[\mathrm{M}+\mathrm{Na}^{+}\right] 1238.3856 \mathrm{~g} / \mathrm{mol}$, (found) $\left[\mathrm{M}+\mathrm{Na}^{+}\right] 1238.3894 \mathrm{~g} / \mathrm{mol}$. 
${ }^{1} \mathrm{H}$ NMR $(250 \mathrm{MHz}, \mathrm{DMSO}) \delta=10.40(\mathrm{~s}, 1 \mathrm{H}), 9.13(\mathrm{~s}, 1 \mathrm{H}), 9.04(\mathrm{~s}, 1 \mathrm{H}), 8.96(\mathrm{~s}, 1 \mathrm{H}), 8.49(\mathrm{~s}, 3 \mathrm{H}), 8.44(\mathrm{~s}$, $1 \mathrm{H}), 8.31\left(\mathrm{~d},{ }^{3} \mathrm{~J}=9.1 \mathrm{~Hz}, 2 \mathrm{H}\right), 8.20\left(\mathrm{~d},{ }^{3} \mathrm{~J}=11.9 \mathrm{~Hz}, 1 \mathrm{H}\right), 7.98\left(\mathrm{~d},{ }^{3} \mathrm{~J}=7.9 \mathrm{~Hz}, 2 \mathrm{H}\right), 7.69\left(\mathrm{~d},{ }^{3} \mathrm{~J}=7.4 \mathrm{~Hz}, 2 \mathrm{H}\right)$, $7.61(\mathrm{~s}, 2 \mathrm{H}), 7.57-7.52(\mathrm{~m}, 2 \mathrm{H}), 7.52-7.47(\mathrm{~m}, 1 \mathrm{H}), 7.39(\mathrm{~s}, 1 \mathrm{H}), 7.36(\mathrm{~s}, 2 \mathrm{H}), 7.29(\mathrm{~s}, 4 \mathrm{H}), 6.52(\mathrm{~s}, 1 \mathrm{H})$, $6.13(\mathrm{~s}, 1 \mathrm{H}), 4.59-4.58(\mathrm{~m}, 2 \mathrm{H}), 4.51-4.41(\mathrm{~m}, 4 \mathrm{H}), 3.91(\mathrm{~s}, 4 \mathrm{H}), 3.57-3.50(\mathrm{~m}, 6 \mathrm{H}), 3.47\left(\mathrm{~d},{ }^{3} \mathrm{~J}=4.8 \mathrm{~Hz}\right.$, $2 \mathrm{H}), 3.11\left(\mathrm{~d},{ }^{3} \mathrm{~J}=5.1 \mathrm{~Hz}, 2 \mathrm{H}\right), 3.03-2.99(\mathrm{~m}, 2 \mathrm{H}), 2.44-2.37(\mathrm{~m}, 1 \mathrm{H}), 0.97(\mathrm{~s}, 9 \mathrm{H}) \mathrm{ppm}$. Contains rotameres. 
Synthesis

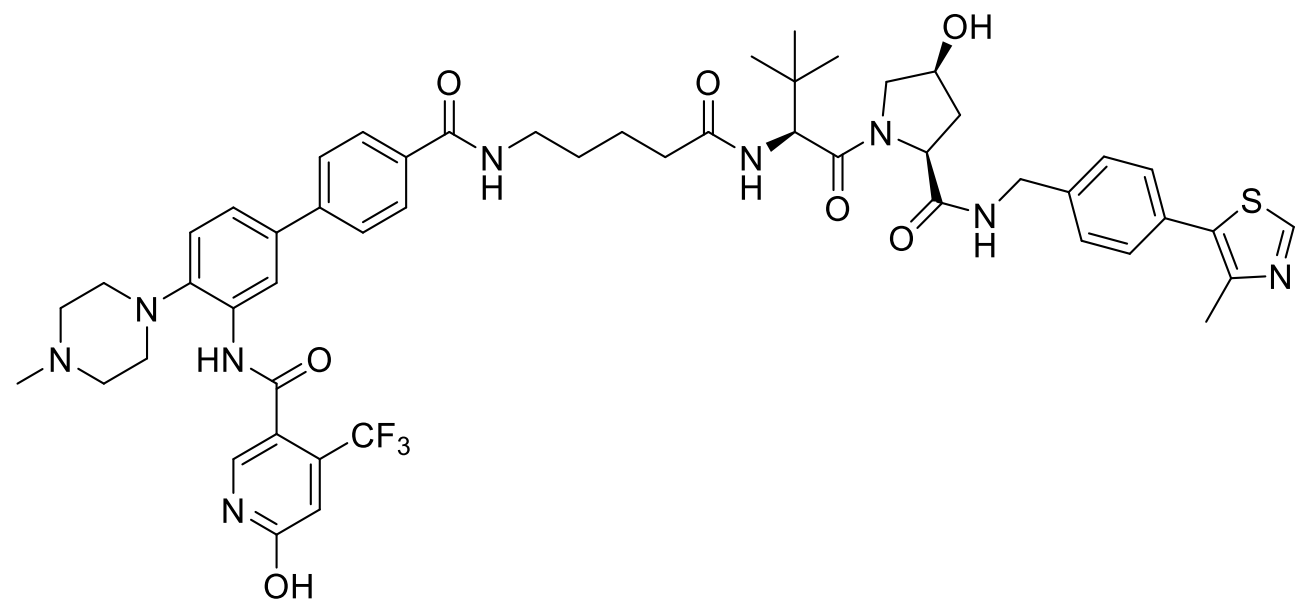

$20 \mathrm{mg} \quad(36 \mu \mathrm{mol}, 1.00$ eq) tert-butyl 3'-(6-hydroxy-4-(trifluoromethyl)nicotinamido)-4'-(4methylpiperazin-1-yl)-[1,1'-biphenyl]-4-carboxylate (6d) were dissolved in $1 \mathrm{~mL} \mathrm{CH}_{2} \mathrm{Cl}_{2}$ and $1 \mathrm{~mL}$ TFA was added. The solution was stirred for $1 \mathrm{~h}$ at rt. Excess solvent was removed under reduced pressure. The crude species was dissolved in $0.5 \mathrm{~mL}$ DMF and DIEA was added until the $\mathrm{pH}$ of the solution was basic. $16.4 \mathrm{mg}$ (43 $\mu \mathrm{mol}, 1.20 \mathrm{eq}$ ) HATU was added. The reaction mixture was stirred for $20 \mathrm{~min}$ at rt. $17.0 \mathrm{mg} \quad(36 \mu \mathrm{mol}, \quad 1.00 \mathrm{eq}) \quad$ tert-butyl $\quad(5-(((S)-1-((2 S, 4 S)-4-h y d r o x y-2-((4-(4-m e t h y l t h i a z o l-5-$ yl)benzyl)carbamoyl)pyrrolidin-1-yl)-3,3-dimethyl-1-oxobutan-2-yl)amino)-5-oxopentyl)carbamate (L15) was dissolved in $1 \mathrm{~mL} \mathrm{DCM}$ and $1 \mathrm{~mL}$ TFA and stirred for $1 \mathrm{~h}$ at $\mathrm{rt}$. Excess solvent was removed under reduced pressure. The crude species was dissolved in $0.5 \mathrm{~mL}$ DMF and DIEA was added until the $\mathrm{pH}$ of the solution was basic. The solution was added to the active ester of tert-butyl 3'-(6-hydroxy-4(trifluoromethyl)nicotinamido)-4'-(4-methylpiperazin-1-yl)-[1,1'-biphenyl]-4-carboxylate and stirred at $\mathrm{rt}$ for $4 \mathrm{~h}$. The reaction was stopped with $1 \mathrm{~mL}$ water. Saturated $\mathrm{NaHCO}_{3}$ solution and saturated $\mathrm{NaCl}$ solution were added and the reaction mixture was extracted $4 x$ with EA. The combined organic phases were washed with saturated $\mathrm{NaHCO}_{3}$ solution, dried over $\mathrm{MgSO}_{4}$ and filtered. The solvent of the organic phase was evapored under reduced pressure. The purification of the crude product was carried out on the preparative HPLC system. The gained product was then dissolved in ethyl acetate and a solution of saturated $\mathrm{NaHCO}_{3}$ and saturated $\mathrm{NaCl}$ solution and extracted with ethyl acetate. The combined organic phases were dried over $\mathrm{MgSO}_{4}$ and the solvent was removed under reduced pressure. 
Yield: $9 \mathrm{mg}, 8.9 \mu \mathrm{mol}, 18 \%$ of a white oil.

MALDI: (calculated) [M- $\left.\mathrm{CH}_{3}-\mathrm{tBu}+2 \mathrm{H}^{+}\right] 941.35 \mathrm{~g} / \mathrm{mol}$, (found) $\left[\mathrm{M}-\mathrm{CH}_{3}-\mathrm{tBu}+2 \mathrm{H}^{+}\right] 941.45 \mathrm{~g} / \mathrm{mol}$.

HRMS: (calculated) $\left[\mathrm{M}+\mathrm{Na}^{+}\right] 1034.4180 \mathrm{~g} / \mathrm{mol}$, (found) $\left[\mathrm{M}+\mathrm{Na}^{+}\right] 1034.4169 \mathrm{~g} / \mathrm{mol}$.

HPLC: RT = $11.4 \min (254 \mathrm{~nm}, 100 \%)$.

${ }^{1} \mathrm{H}$ NMR $(250 \mathrm{MHz}, \mathrm{MeOD}) \delta=9.03(\mathrm{~s}, 1 \mathrm{H}), 8.26\left(\mathrm{~d},{ }^{4} \mathrm{~J}=1.9 \mathrm{~Hz}, 1 \mathrm{H}\right), 8.03(\mathrm{~s}, 1 \mathrm{H}), 7.91\left(\mathrm{~d},{ }^{3} \mathrm{~J}=8.3 \mathrm{~Hz}\right.$, $2 \mathrm{H}), 7.71\left(\mathrm{~d},{ }^{3} J=8.3 \mathrm{~Hz}, 2 \mathrm{H}\right), 7.56\left(\mathrm{dd},{ }^{3} J=8.5 \mathrm{~Hz},{ }^{4} J=2.0 \mathrm{~Hz}, 1 \mathrm{H}\right), 7.44\left(\mathrm{dd},{ }^{2} J=15.1 \mathrm{~Hz},{ }^{3} J=7.8 \mathrm{~Hz}, 4 \mathrm{H}\right)$, $7.37\left(\mathrm{~d},{ }^{4} \mathrm{~J}=2.1 \mathrm{~Hz}, 1 \mathrm{H}\right), 6.94(\mathrm{~s}, 1 \mathrm{H}), 4.63(\mathrm{~s}, 1 \mathrm{H}), 4.56(\mathrm{~s}, 1 \mathrm{H}), 4.50(\mathrm{~s}, 2 \mathrm{H}), 4.35\left(\mathrm{~d},{ }^{3} \mathrm{~J}=15.7 \mathrm{~Hz}, 1 \mathrm{H}\right)$, $3.91\left(\mathrm{~d},{ }^{3} \mathrm{~J}=10.7 \mathrm{~Hz}, 1 \mathrm{H}\right), 3.80\left(\mathrm{dd},{ }^{3} \mathrm{~J}=10.9 \mathrm{~Hz},{ }^{4} \mathrm{~J}=3.6 \mathrm{~Hz}, 1 \mathrm{H}\right), 3.64-3.60(\mathrm{~m}, 2 \mathrm{H}), 3.44-3.39(\mathrm{~m}, 2 \mathrm{H})$, $3.36-3.24(\mathrm{~m}, 4 \mathrm{H}), 3.20-3.15(\mathrm{~m}, 2 \mathrm{H}), 2.97(\mathrm{~s}, 3 \mathrm{H}), 2.47(\mathrm{~s}, 3 \mathrm{H}), 2.39-2.33(\mathrm{~m}, 2 \mathrm{H}), 2.28-2.15(\mathrm{~m}$, $1 \mathrm{H}), 2.13-2.08(\mathrm{~m}, 1 \mathrm{H}), 1.79-1.59(\mathrm{~m}, 4 \mathrm{H}), 1.04(\mathrm{~s}, 9 \mathrm{H}) \mathrm{ppm}$. 
Synthesis

of

6-hydroxy-N-(4'-((5-)((S)-1-((2S,4R)-4-hydroxy-2-((4-(4-methylthiazol-5-

yl)benzyl)carbamoyl)pyrrolidin-1-yl)-3,3-dimethyl-1-oxobutan-2-yl)amino)-5-

oxopentyl)carbamoyl)-4-morpholino-[1,1'-biphenyl]-3-yl)-4-(trifluoromethyl)nicotinamide

(23) nc_WDR5)

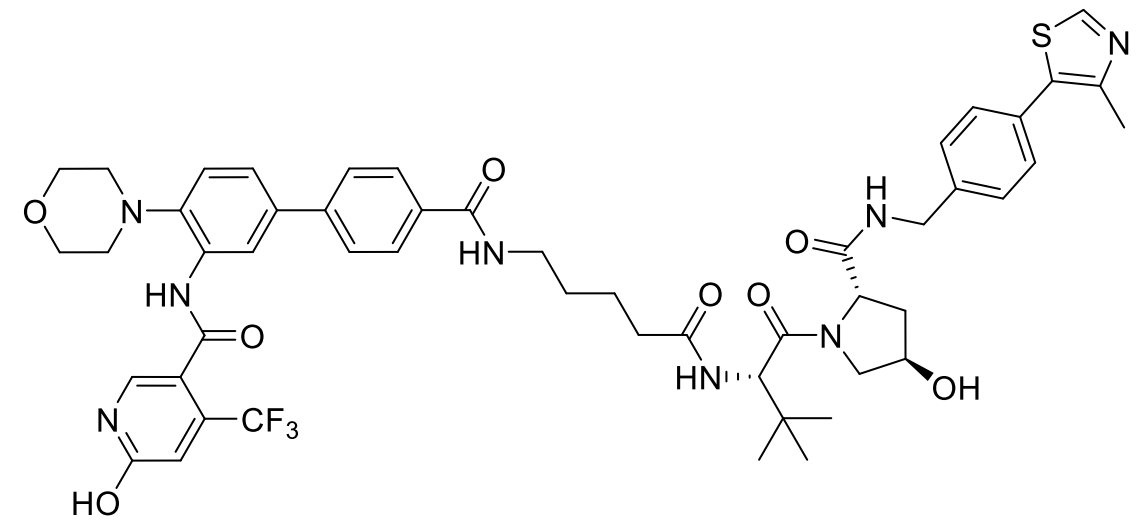

$21.0 \mathrm{mg} \quad(39.0 \mu \mathrm{mol}, \quad 1.00 \mathrm{eq})$ of tert-butyl 4'-morpholino-3'-(6-oxo-4-(trifluoromethyl)-1,6dihydropyridine-3-carboxamido)-[1,1'-biphenyl]-4-carboxylate was diluted in $1 \mathrm{~mL}$ DCM and $1 \mathrm{~mL}$ TFA was added. In a second vial, $24 \mathrm{mg}(39.0 \mu \mathrm{mol}, 1.00$ eq) of tert-butyl (5-(((R)-1-((2S,4R)-4-hydroxy-2((4-(4-methylthiazol-5-yl)benzyl)carbamoyl)pyrrolidin-1-yl)-3,3-dimethyl-1-oxobutan-2-yl)amino)-5oxopentyl)carbamate was diluted in $1 \mathrm{~mL}$ DCM and $1 \mathrm{~mL}$ TFA was added. The two reaction mixtures were stirred for $1 \mathrm{~h}$ at $\mathrm{rt}$. Excess solvent and TFA was removed under reduced pressure.

The deprotected species of tert-butyl 4'-morpholino-3'-(6-oxo-4-(trifluoromethyl)-1,6dihydropyridine-3-carboxamido)-[1,1'-biphenyl]-4-carboxylate was dissolved in $0.5 \mathrm{~mL} \mathrm{DMF}$ and DIEA was added until the $\mathrm{pH}$ of the solution was basic. $29.4 \mathrm{mg}(77.0 \mu \mathrm{mol}, 2.00 \mathrm{eq})$ HATU was added. The reaction mixture was stirred for $20 \mathrm{~min}$ at $\mathrm{rt}$. The deprotected species of tert-butyl (5-(((R)-1-((2S,4R)4-hydroxy-2-((4-(4-methylthiazol-5-yl)benzyl)carbamoyl)pyrrolidin-1-yl)-3,3-dimethyl-1-oxobutan-2yl)amino)-5-oxopentyl)carbamate was dissolved in $0.5 \mathrm{~mL}$ DMF and $67 \mu \mathrm{L}$ (387 $\mu \mathrm{mol}, 10.0 \mathrm{eq})$ DIEA were added to the solution. The mixture was added to the active ester of tert-butyl 4'-morpholino-3'(6-oxo-4-(trifluoromethyl)-1,6-dihydropyridine-3-carboxamido)-[1,1'-biphenyl]-4-carboxylate and stirred at rt for $2.5 \mathrm{~h}$. The reaction was stopped with $1 \mathrm{~mL}$ water. Saturated $\mathrm{NaHCO}_{3}$ solution and saturated $\mathrm{NaCl}$ solution were added and the reaction mixture was extracted $4 \mathrm{x}$ with $\mathrm{EA}$. The combined organic phases were washed with saturated $\mathrm{NaHCO}_{3}$ solution, dried over $\mathrm{MgSO}_{4}$ and filtered. The solvent of the organic phase was evapored under reduced pressure. The purification of the crude product was carried out on the HPLC system.

Yield: $19.5 \mathrm{mg}, 7.33 \mu \mathrm{mol}, 23 \%$ of a white solid as TFA salt (1:1/ product: TFA). 
ESI: (calculated): $[\mathrm{M}-\mathrm{H}]^{-} 998.11 \mathrm{~g} / \mathrm{mol}$ (found): [M-H] $998.53 \mathrm{~g} / \mathrm{mol}$.

MALDI: (calculated): $[\mathrm{M}+\mathrm{H}]^{+} 1000.13 \mathrm{~g} / \mathrm{mol}$ (found): $[\mathrm{M}+\mathrm{H}]^{+} 1000.19 \mathrm{~g} / \mathrm{mol}$.

HRMS: (calculated) $\left[\mathrm{M}+\mathrm{Na}^{+}\right] 1021.3864 \mathrm{~g} / \mathrm{mol}$

(found) $\left[\mathrm{M}+\mathrm{Na}^{+}\right] 1021.3902 \mathrm{~g} / \mathrm{mol}$.

${ }^{1} \mathrm{H}$ NMR (500 MHz, DMSO) $\delta=9.60(\mathrm{~s}, 1 \mathrm{H}), 8.98(\mathrm{~s}, 1 \mathrm{H}), 8.55\left(\mathrm{t},{ }^{3} \mathrm{~J}=6.1 \mathrm{~Hz}, 1 \mathrm{H}\right), 8.49\left(\mathrm{t},{ }^{3} \mathrm{~J}=5.6 \mathrm{~Hz}, 1 \mathrm{H}\right)$, $8.16\left(d,{ }^{4} J=2.1 \mathrm{~Hz}, 1 \mathrm{H}\right), 7.97(\mathrm{~s}, 1 \mathrm{H}), 7.93\left(\mathrm{~d},{ }^{3} \mathrm{~J}=8.5 \mathrm{~Hz}, 2 \mathrm{H}\right), 7.86\left(\mathrm{~d},{ }^{3} \mathrm{~J}=9.3 \mathrm{~Hz}, 1 \mathrm{H}\right), 7.68\left(\mathrm{~d},{ }^{3} \mathrm{~J}=8.5 \mathrm{~Hz}\right.$, 2H), $7.53\left(\mathrm{dd},{ }^{3} \mathrm{~J}=8.4 \mathrm{~Hz},{ }^{4} \mathrm{~J}=2.2 \mathrm{~Hz}, 1 \mathrm{H}\right), 7.43-7.37(\mathrm{~m}, 4 \mathrm{H}), 7.28\left(\mathrm{~d},{ }^{3} \mathrm{~J}=8.4 \mathrm{~Hz}, 1 \mathrm{H}\right), 6.82(\mathrm{~s}, 1 \mathrm{H}), 4.55$ $\left(\mathrm{d},{ }^{3} \mathrm{~J}=9.4 \mathrm{~Hz}, 1 \mathrm{H}\right), 4.47-4.40(\mathrm{~m}, 2 \mathrm{H}), 4.35(\mathrm{~s}, 1 \mathrm{H}), 4.24-4.20(\mathrm{~m}, 1 \mathrm{H}), 3.78-3.73(\mathrm{~m}, 4 \mathrm{H}), 3.69-3.65$ $(\mathrm{m}, 2 \mathrm{H}), 3.30-3.26(\mathrm{~m}, 2 \mathrm{H}), 2.92-2.88(\mathrm{~m}, 4 \mathrm{H}), 2.44(\mathrm{~s}, 3 \mathrm{H}), 2.34-2.28(\mathrm{~m}, 1 \mathrm{H}), 2.21-2.15(\mathrm{~m}, 1 \mathrm{H})$, $2.08-2.00(\mathrm{~m}, 1 \mathrm{H}), 1.93-1.88(\mathrm{~m}, 1 \mathrm{H}), 1.56-1.52(\mathrm{~m}, 4 \mathrm{H}), 0.94(\mathrm{~s}, 9 \mathrm{H}) \mathrm{ppm}$.

${ }^{13} \mathrm{C}$ NMR (126 MHz, DMSO) $\delta=172.0,171.9,169.7,165.7,162.9,161.1,151.5,147.7,144.6,142.5$, $139.5,139.2,138.6\left(q,{ }^{2} J=32 \mathrm{~Hz}\right), 134.6,133.3,132.4,131.2,129.6,128.6,127.9,127.4,126.0,124.0$, $123.1,122.3,122.1\left(q,{ }^{1} J=276 \mathrm{~Hz}\right), 120.6,119.0,111.7,68.9,66.2,58.7,56.4,56.3,53.6,51.6,41.8$, 41.6, 37.9, 35.2, 34.7, 28.9, 26.4, 23.1, 18.1, 16.7, 15.9 ppm. 
Synthesis of tert-butyl (1-(3'-(6-hydroxy-4-(trifluoromethyl)nicotinamido)-4'-(4-methylpiperazin-1yl)-[1,1'-biphenyl]-4-yl)-1-oxo-5,8,11,14,17,20,23,26,29-nonaoxa-2-azahentriacontan-31yl)carbamate (6h)<smiles>CN1CCN(c2ccc(-c3ccc(C(=O)NCCOCCOCCOCCOCCOCCOCCOCCOCCNC(=O)OC(C)(C)C)cc3)cc2NC(=O)c2c[nH]c(=O)cc2C(F)(F)F)CC1</smiles>

$22 \mathrm{mg} \quad(40 \mu \mathrm{mol}, \quad$ 1.0eq) tert-butyl 3'-(6-hydroxy-4-(trifluoromethyl)nicotinamido)-4'-(4methylpiperazin-1-yl)-[1,1'-biphenyl]-4-carboxylate were dissolved in $0.5 \mathrm{~mL} \mathrm{DCM}$ and $0.5 \mathrm{~mL}$ TFA and stired at $\mathrm{rt}$ for $1 \mathrm{~h}$. Excess solvent was evapored. The solid was dissolved in $0.5 \mathrm{~mL} \mathrm{DMF}$, then $137 \mu \mathrm{L}$ (720 $\mu \mathrm{mol}, 20 \mathrm{eq})$ DIEA and $18 \mathrm{mg}(47 \mu \mathrm{mol}, 1.2 \mathrm{eq})$ HATU were added. After $15 \mathrm{~min}$, a solution of $23.1 \mathrm{mg} \quad(42 \mu \mathrm{mol}, \quad 1.05$ eq) tert-butyl (29-amino-3,6,9,12,15,18,21,24,27nonaoxanonacosyl)carbamate in $0.5 \mathrm{~mL}$ DMF was added. The solution was stirred for $3 \mathrm{~h}$ at $\mathrm{rt}$. The reaction mixture was quenched with $2 \mathrm{~mL}$ water and $2 \mathrm{~mL}$ saturated $\mathrm{NaHCO}_{3}$, then the reaction was extracted 3x with EA. The organic phase was dried over $\mathrm{MgSO}_{4}$, filtered and the solvent was removed under reduced pressure. The crude product was purified using by HPLC.

Yield: $41 \mathrm{mg}, 35.6 \mu \mathrm{mol}, 89 \%$ of a colourless oil.

MALDI: (calculated) [M-Boc $\left.+\mathrm{H}^{+}\right] 939.47 \mathrm{~g} / \mathrm{mol}$

(found) [M-Boc+H'] $939.43 \mathrm{~g} / \mathrm{mol}$. 
${ }^{1} \mathrm{H}$ NMR $(400 \mathrm{MHz}, \mathrm{MeOD}) \delta=8.28\left(\mathrm{~d},{ }^{4} J=2.1 \mathrm{~Hz}, 1 \mathrm{H}\right), 8.04(\mathrm{~s}, 1 \mathrm{H}), 7.94\left(\mathrm{~d},{ }^{3} \mathrm{~J}=8.6 \mathrm{~Hz}, 2 \mathrm{H}\right), 7.78-7.71$ $(\mathrm{m}, 2 \mathrm{H}), 7.58\left(\mathrm{dd},{ }^{3} J=8.4 \mathrm{~Hz},{ }^{4} \mathrm{~J}=2.2 \mathrm{~Hz}, 1 \mathrm{H}\right), 7.40\left(\mathrm{~d},{ }^{3} \mathrm{~J}=8.4 \mathrm{~Hz}, 1 \mathrm{H}\right), 6.94(\mathrm{~s}, 1 \mathrm{H}), 3.72-3.56(\mathrm{~m}, 48 \mathrm{H})$, $2.97(\mathrm{~s}, 3 \mathrm{H}), 1.43(\mathrm{~s}, 9 \mathrm{H}) \mathrm{ppm}$.

Synthesis of tert-butyl (1-(3'-(6-hydroxy-4-(trifluoromethyl)nicotinamido)-4'-(4-methylpiperazin-1yl)-[1,1'-biphenyl]-4-yl)-1-oxo-6,9,12-trioxa-2-azapentadecan-15-yl)carbamate (6i)<smiles>CN1CCN(c2ccc(-c3ccc(C(=O)NCCCOCCOCCOCCCNC(=O)OC(C)(C)C)cc3)cc2NC(=O)c2c[nH]c(=O)cc2C(F)(F)F)CC1</smiles>

$84 \mathrm{mg} \quad(151 \mu \mathrm{mol}, \quad 1.0 \mathrm{eq}) \quad$ tert-butyl $\quad$ 3'-(6-hydroxy-4-(trifluoromethyl)nicotinamido)-4'-(4methylpiperazin-1-yl)-[1,1'-biphenyl]-4-carboxylate were dissolved in $1 \mathrm{~mL}$ DCM and $1 \mathrm{~mL}$ TFA and stired at $\mathrm{rt}$ for $1 \mathrm{~h}$. Excess solvent was evapored. The solid was dissolved in $2 \mathrm{~mL} \mathrm{DMF}$, then $526 \mu \mathrm{L}$ ( $3.02 \mathrm{mmol}, 20 \mathrm{eq}$ ) DIEA and $68.9 \mathrm{mg}(181 \mu \mathrm{mol}, 1.2 \mathrm{eq})$ HATU were added. After $15 \mathrm{~min}$, a solution of $50.8 \mathrm{mg}(158 \mu \mathrm{mol}, 1.05 \mathrm{eq})$ tert-butyl (3-(2-(2-(3-aminopropoxy)ethoxy)ethoxy)propyl)carbamate in $1 \mathrm{~mL}$ DMF was added. The solution was stirred for $3 \mathrm{~h}$ at $\mathrm{rt}$. The reaction mixture was quenched with $2 \mathrm{~mL}$ water and $2 \mathrm{~mL}$ saturated $\mathrm{NaHCO}_{3}$, then the reaction was extracted $3 x$ with EA. The organic phase was dried over $\mathrm{MgSO}_{4}$, filtered and the solvent was removed under reduced pressure. The crude product was purified using by HPLC.

Yield: $94.5 \mathrm{mg}, 118 \mu \mathrm{mol}, 78 \%$ of a colourless oil.

ESI: (calculated) $\left[\mathrm{M}+\mathrm{Na}^{+}\right] 825.37 \mathrm{~g} / \mathrm{mol}$

(found) $\left[\mathrm{M}+\mathrm{Na}^{+}\right] 825.05 \mathrm{~g} / \mathrm{mol}$.

HPLC: RT = $11.9 \min (254 \mathrm{~nm}, 100 \%)$

${ }^{1} \mathrm{H}$ NMR $(600 \mathrm{MHz}, \mathrm{MeOD}) \delta=8.29(\mathrm{~s}, 1 \mathrm{H}), 8.00(\mathrm{~s}, 1 \mathrm{H}), 7.89\left(\mathrm{~d},{ }^{3} \mathrm{~J}=8.4 \mathrm{~Hz}, 2 \mathrm{H}, 7.71\left(\mathrm{~d},{ }^{3} \mathrm{~J}=8.4 \mathrm{~Hz}, 2 \mathrm{H}\right)\right.$, $7.51\left(\mathrm{dd},{ }^{3} \mathrm{~J}=8.3 \mathrm{~Hz},{ }^{4} \mathrm{~J}=2.0 \mathrm{~Hz}\right), 7.33\left(\mathrm{~d},{ }^{3} \mathrm{~J}=8.3 \mathrm{~Hz}, 1 \mathrm{H}\right), 6.92(\mathrm{~s}, 1 \mathrm{H}), 3.68-3.64(\mathrm{~m}, 2 \mathrm{H}), 3.63-3.57$ 
$(\mathrm{m}, 6 \mathrm{H}), 3.55-3.51(\mathrm{~m}, 2 \mathrm{H}), 3.50\left(\mathrm{t},{ }^{3} \mathrm{~J}=6.7 \mathrm{~Hz}, 2 \mathrm{H}\right), 3.46\left(\mathrm{t},{ }^{3} \mathrm{~J}=6.2 \mathrm{~Hz}, 2 \mathrm{H}\right), 3.09\left(\mathrm{t},{ }^{3} \mathrm{~J}=6.8 \mathrm{~Hz}, 2 \mathrm{H}\right)$, $3.01\left(\mathrm{t},{ }^{3} \mathrm{~J}=4.2 \mathrm{~Hz}, 4 \mathrm{H}\right), 2.69(\mathrm{~s}, 4 \mathrm{H}), 2.39(\mathrm{~s}, 3 \mathrm{H}), 1.96-1.82(\mathrm{~m}, 2 \mathrm{H}), 1.76-1.62(\mathrm{~m}, 2 \mathrm{H}), 1.41(\mathrm{~s}$, 9H) ppm.

${ }^{13} \mathrm{C}$ NMR $(151 \mathrm{MHz}, \mathrm{MeOD}) \delta=169.7,165.0,164.3,164.3,145.3,144.7,141.41$ (d, J = 33.7 Hz), 140.5, 139.3 , 137.5, 134.4, 133.9, 129.5, 128.4, 127.3, 125.0, 123.4 (q, J = 275 Hz), 123.2, 122.7, 121.6, 119.8 (m), 114.6 (d, J = 8.2 Hz), 79.8, 72.5, 71.6, 71.3, 71.2, 70.6, 70.4, 56.2, 52.4, 45.5, 38.6, 37.8, 30.4, 30.0, 29.2, $28.3 \mathrm{ppm}$.

Synthesis of N-(4'-((2-(2-(3-(5,5-difluoro-7-(1H-pyrrol-2-yl)-5H-5l4,6l4-dipyrrolo[1,2-c:2',1'f][1,3,2]diazaborinin-3-yl)propanamido)ethoxy)ethyl)carbamoyl)-4-(4-methylpiperazin-1-yl)-[1,1'biphenyl]-3-yl)-6-hydroxy-4-(trifluoromethyl)nicotinamide (21a)

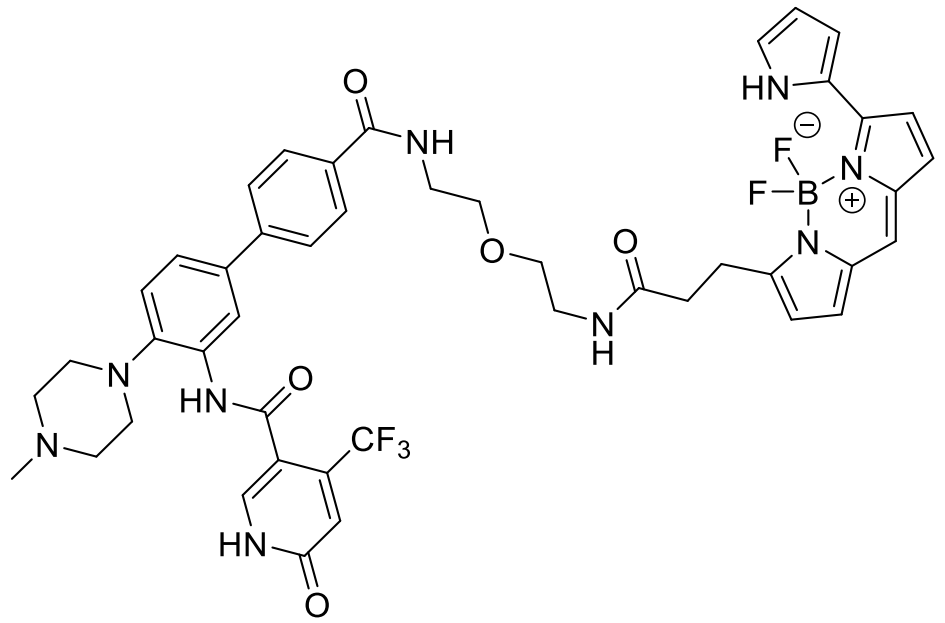

$12 \mathrm{mg} \quad(14 \mu \mathrm{mol}, \quad 1.0 \mathrm{eq})$ tert-butyl (2-(2-(3'-(6-hydroxy-4-(trifluoromethyl)nicotinamido)-4'-(4methylpiperazin-1-yl)-[1,1'-biphenyl]-4-carboxamido)ethoxy)ethyl)carbamate were dissolved in $0.5 \mathrm{~mL} \mathrm{DCM}$ and $0.5 \mathrm{~mL}$ TFA and stired at $\mathrm{rt}$ for $1 \mathrm{~h}$. Excess solvent was evapored. The solid was dissolved in $0.5 \mathrm{~mL}$ DMF, then $19 \mu \mathrm{L}(111 \mu \mathrm{mol}, 8.0$ eq) DIEA and $6.2 \mathrm{mg}$ (15 $\mu \mathrm{mol}, 1.1$ eq) 2,5dioxopyrrolidin-1-yl-3-(5,5-difluoro-7-(1H-pyrrol-2-yl)-5H-5|4,6|4-dipyrrolo[1,2-c:2',1'-

f] [1,3,2]diazaborinin-3-yl)propanoate in $0.5 \mathrm{~mL}$ DMF was added. The solution was stirred for $3 \mathrm{~h}$ at $\mathrm{rt}$. The reaction mixture was quenched with $2 \mathrm{~mL}$ water and $2 \mathrm{~mL}$ saturated $\mathrm{NaHCO}_{3}$, then the reaction was extracted 3x with EA. The organic phase was dried over $\mathrm{MgSO}_{4}$, filtered and the solvent was removed under reduced pressure. The crude product was purified using by HPLC.

Yield: $6.35 \mathrm{mg}, 7.08 \mu \mathrm{mol}, 77 \%$ of a purple solid. 
$\mathrm{R}_{\mathrm{f}}\left(20 \% \mathrm{MeOH} / \mathrm{CH}_{2} \mathrm{Cl}_{2}\right): 0.70$.

HPLC: RT = $12.1 \min (254 \mathrm{~nm}, 95 \%)$.

HRMS: (calculated) $\left[\mathrm{M}+\mathrm{Na}^{+}\right] 920.3449 \mathrm{~g} / \mathrm{mol}$

(found) $\left[\mathrm{M}+\mathrm{Na}^{+}\right] 920.3459 \mathrm{~g} / \mathrm{mol}$.

${ }^{1} \mathrm{H}$ NMR $(500 \mathrm{MHz}, \mathrm{MeOD}) \delta=8.21\left(\mathrm{~d},{ }^{4} \mathrm{~J}=2.1 \mathrm{~Hz}, 1 \mathrm{H}\right), 8.01(\mathrm{~s}, 1 \mathrm{H}), 7.85\left(\mathrm{~d},{ }^{3} \mathrm{~J}=8.4 \mathrm{~Hz}, 2 \mathrm{H}\right), 7.64\left(\mathrm{~d},{ }^{3} \mathrm{~J}\right.$ $=8.4 \mathrm{~Hz}, 2 \mathrm{H}), 7.46\left(\mathrm{dd},{ }^{3} \mathrm{~J}=8.4 \mathrm{~Hz},{ }^{4} \mathrm{~J}=2.1 \mathrm{~Hz}, 1 \mathrm{H}\right), 7.27\left(\mathrm{~d},{ }^{3} \mathrm{~J}=8.4 \mathrm{~Hz}, 1 \mathrm{H}\right), 7.21-7.07(\mathrm{~m}, 4 \mathrm{H}), 7.02-$ $6.90(\mathrm{~m}, 2 \mathrm{H}), 6.85\left(\mathrm{~d},{ }^{3} \mathrm{~J}=3.9 \mathrm{~Hz}, 1 \mathrm{H}\right), 6.32\left(\mathrm{dd},{ }^{3} \mathrm{~J}=3.8 \mathrm{~Hz},{ }^{4} \mathrm{~J}=2.6 \mathrm{~Hz}, 1 \mathrm{H}\right), 6.26\left(\mathrm{~d},{ }^{3} \mathrm{~J}=3.9 \mathrm{~Hz}, 1 \mathrm{H}\right), 3.61$ $(\mathrm{m}, 8 \mathrm{H}), 3.40\left(\mathrm{t},{ }^{3} \mathrm{~J}=5.2 \mathrm{~Hz}, 2 \mathrm{H}\right), 3.35-3.20(\mathrm{~m}, 6 \mathrm{H}), 3.13\left(\mathrm{t},{ }^{3} \mathrm{~J}=12.8 \mathrm{~Hz}, 2 \mathrm{H}\right), 2.96(\mathrm{~s}, 3 \mathrm{H}), 2.61\left(\mathrm{t},{ }^{3} \mathrm{~J}=\right.$ $7.6 \mathrm{~Hz}, 2 \mathrm{H}) \mathrm{ppm}$.

${ }^{13} \mathrm{C}$ NMR $(126 \mathrm{MHz}, \mathrm{MeOD}) \delta=174.97,169.9,165.3,163.9,156.2,152.1,144.3,143.9,141.4$ (q, J = $32.9 \mathrm{~Hz}), 139.7,138.9,138.6,134.9,134.4,133.8,133.1,129.0,127.9,127.4,126.0,124.9,124.6$, $123.8,123.4(q, J=273 \mathrm{~Hz}), 122.2,121.0,120.6,119.0,117.2,114.4,112.3,70.5,70.4,55.0,50.3,43.7$, $40.9,40.4,36.1,25.6 \mathrm{ppm}$. 


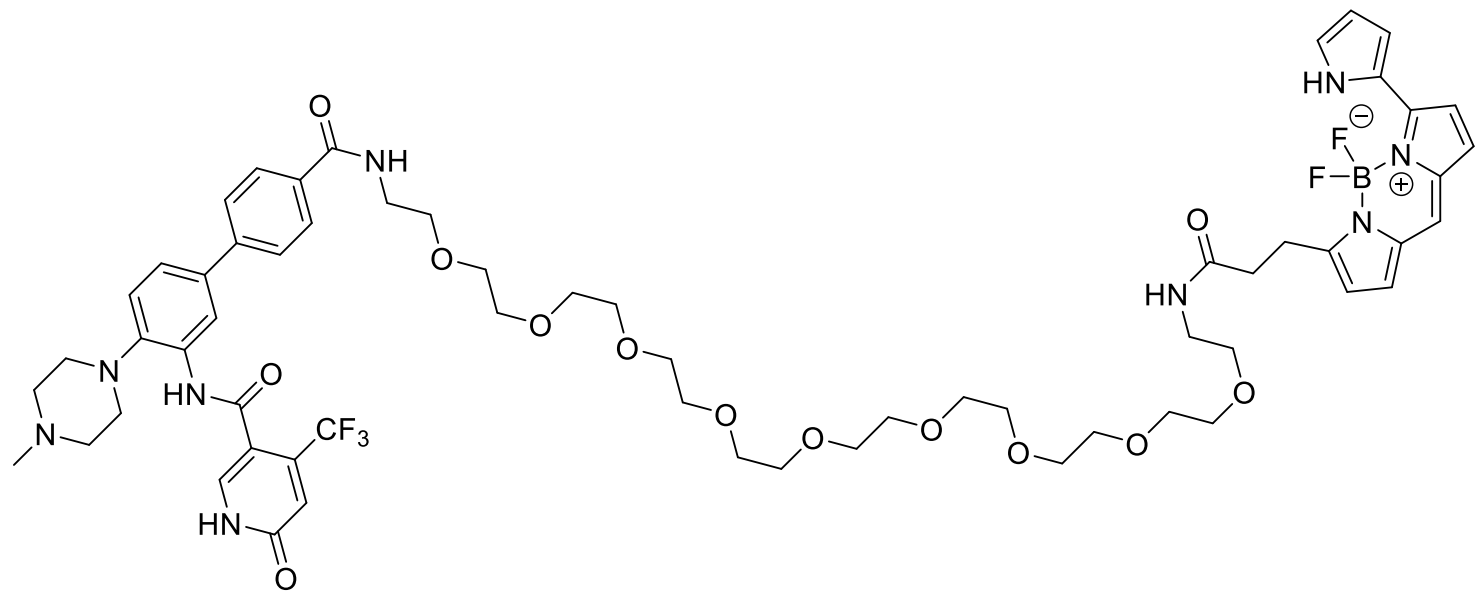

$12.5 \mathrm{mg} \quad(12 \mu \mathrm{mol}, \quad 1.0 \mathrm{eq}) \quad$ tert-butyl (1-(3'-(6-hydroxy-4-(trifluoromethyl)nicotinamido)-4'-(4methylpiperazin-1-yl)-[1,1'-biphenyl]-4-yl)-1-oxo-5,8,11,14,17,20,23,26,29-nonaoxa-2-

azahentriacontan-31-yl)carbamate were dissolved in $0.5 \mathrm{~mL} \mathrm{DCM}$ and $0.5 \mathrm{~mL}$ TFA and stired at rt for $1 \mathrm{~h}$. Excess solvent was evapored. The solid was dissolved in $0.5 \mathrm{~mL} \mathrm{DMF}$, then $17 \mu \mathrm{L}$ (10 $\mu \mathrm{mol}, 2.0 \mathrm{eq})$ DIEA and $5.8 \mathrm{mg}$ (14 $\mu \mathrm{mol}, 1.1 \mathrm{eq}$ ) 2,5-dioxopyrrolidin-1-yl-3-(5,5-difluoro-7-(1H-pyrrol-2-yl)-5H5|4,614-dipyrrolo[1,2-c:2',1'-f][1,3,2]diazaborinin-3-yl)propanoate in $0.5 \mathrm{~mL}$ DMF was added. The solution was stirred for $3 \mathrm{~h}$ at $\mathrm{rt}$. The reaction mixture was quenched with $2 \mathrm{~mL}$ water and $2 \mathrm{~mL}$ saturated $\mathrm{NaHCO}_{3}$, then the reaction was extracted 3x with EA. The organic phase was dried over $\mathrm{MgSO}_{4}$, filtered and the solvent was removed under reduced pressure. The crude product was purified using by HPLC.

Yield: $4.88 \mathrm{mg}, 3.9 \mu \mathrm{mol}, 33 \%$ of a purple solid.

HPLC: RT = $12.4 \min (254 \mathrm{~nm}, 100 \%)$.

HRMS: (calculated) $\left[\mathrm{M}+\mathrm{Na}^{+}\right] 1272.5546 \mathrm{~g} / \mathrm{mol}$

(found) $\left[\mathrm{M}+\mathrm{Na}^{+}\right] 1272.5583 \mathrm{~g} / \mathrm{mol}$.

${ }^{1} \mathrm{H}$ NMR $(400 \mathrm{MHz}, \mathrm{MeOD}) \delta=8.27\left(\mathrm{~d},{ }^{4} J=2.1 \mathrm{~Hz}, 1 \mathrm{H}\right), 8.02(\mathrm{~s}, 1 \mathrm{H}), 7.92\left(\mathrm{~d},{ }^{3} J=8.5 \mathrm{~Hz}, 2 \mathrm{H}\right), 7.72\left(\mathrm{~d}, 3^{3} J\right.$ $=8.5 \mathrm{~Hz}, 2 \mathrm{H}), 7.56\left(\mathrm{dd},{ }^{3} \mathrm{~J}=8.4 \mathrm{~Hz},{ }^{4} \mathrm{~J}=2.2 \mathrm{~Hz}, 1 \mathrm{H}\right), 7.36\left(\mathrm{~d},{ }^{3} \mathrm{~J}=8.4 \mathrm{~Hz}, 1 \mathrm{H}\right), 7.22(\mathrm{~s}, 1 \mathrm{H}), 7.21-7.13(\mathrm{~m}$, $3 \mathrm{H}), 7.00\left(\mathrm{~d},{ }^{4} \mathrm{~J}=4.6 \mathrm{~Hz}, 1 \mathrm{H}\right), 6.94(\mathrm{~s}, 1 \mathrm{H}), 6.91\left(\mathrm{~d},{ }^{4} \mathrm{~J}=3.9 \mathrm{~Hz}, 1 \mathrm{H}\right), 6.35-6.34(\mathrm{~m}, 1 \mathrm{H}), 6.32\left(\mathrm{~d},{ }^{4} \mathrm{~J}=4.0 \mathrm{~Hz}\right.$, 
168 Experimental part

$1 \mathrm{H}), 3.75-3.47(\mathrm{~m}, 44 \mathrm{H}), 3.36\left(\mathrm{t},{ }^{3} \mathrm{~J}=5.4 \mathrm{~Hz}, 2 \mathrm{H}\right), 3.27\left(\mathrm{t},{ }^{3} \mathrm{~J}=7.7 \mathrm{~Hz}, 4 \mathrm{H}\right), 2.95(\mathrm{~s}, 3 \mathrm{H}), 2.70-2.56(\mathrm{~m}$, 2H) ppm. 


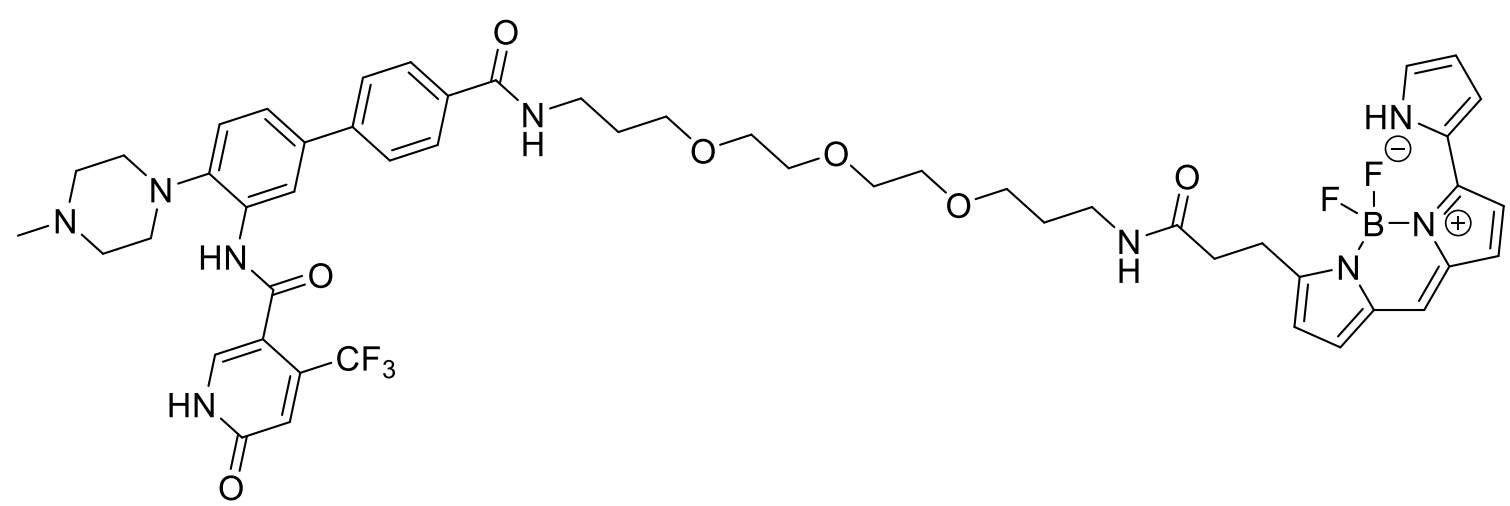

$10 \mathrm{mg} \quad(12 \mu \mathrm{mol}, \quad 1.0 \mathrm{eq})$ tert-butyl (1-(3'-(6-hydroxy-4-(trifluoromethyl)nicotinamido)-4'-(4methylpiperazin-1-yl)-[1,1'-biphenyl]-4-yl)-1-oxo-6,9,12-trioxa-2-azapentadecan-15-yl)carbamate were dissolved in $0.5 \mathrm{~mL}$ DCM and $0.5 \mathrm{~mL}$ TFA and stired at $\mathrm{rt}$ for $1 \mathrm{~h}$. Excess solvent was evapored.

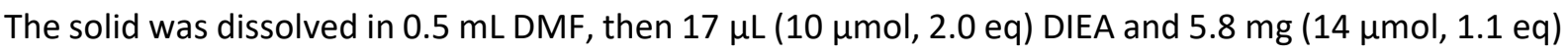
2,5-dioxopyrrolidin-1-yl-3-(5,5-difluoro-7-(1H-pyrrol-2-yl)-5H-5|4,6|4-dipyrrolo[1,2-c:2',1'-

f] $[1,3,2]$ diazaborinin-3-yl)propanoate in $0.5 \mathrm{~mL}$ DMF was added. The solution was stirred for $3 \mathrm{~h}$ at $\mathrm{rt}$. The reaction mixture was quenched with $2 \mathrm{~mL}$ water and $2 \mathrm{~mL}$ saturated $\mathrm{NaHCO}_{3}$, then the reaction was extracted 3x with EA. The organic phase was dried over $\mathrm{MgSO}_{4}$, filtered and the solvent was removed under reduced pressure. The crude product was purified using by HPLC.

Yield: $8.07 \mathrm{mg}, 7.96 \mu \mathrm{mol}, 66 \%$ of a purple solid.

$\mathrm{R}_{\mathrm{f}}\left(20 \% \mathrm{MeOH} / \mathrm{CH}_{2} \mathrm{Cl}_{2}\right): 0.76$.

HPLC: RT = $12.4 \min (254 \mathrm{~nm}, 100 \%)$.

HRMS: (calculated) $\left[\mathrm{M}+\mathrm{Na}^{+}\right] 1036.4286 \mathrm{~g} / \mathrm{mol}$

(found) [M+Na+] $1036.4303 \mathrm{~g} / \mathrm{mol}$.

${ }^{1} \mathrm{H} \mathrm{NMR}(600 \mathrm{MHz}, \mathrm{MeOD}) \delta=8.26\left(\mathrm{~d},{ }^{4} \mathrm{~J}=1.9 \mathrm{~Hz}, 1 \mathrm{H}\right), 8.03(\mathrm{~s}, 1 \mathrm{H}), 7.88\left(\mathrm{~d},{ }^{3} \mathrm{~J}=8.3 \mathrm{~Hz}, 2 \mathrm{H}\right), 7.70\left(\mathrm{~d},{ }^{3} \mathrm{~J}\right.$ $=8.4 \mathrm{~Hz}, 2 \mathrm{H}), 7.54\left(\mathrm{dd},{ }^{3} J=8.4 \mathrm{~Hz},{ }^{4} J=2.0 \mathrm{~Hz}, 1 \mathrm{H}\right), 7.35\left(\mathrm{~d},{ }^{3} J=8.4 \mathrm{~Hz}, 1 \mathrm{H}\right), 7.23-7.13(\mathrm{~m}, 4 \mathrm{H}), 6.99(\mathrm{~d}$, $\left.{ }^{4} J=4.6 \mathrm{~Hz}, 1 \mathrm{H}\right), 6.94(\mathrm{~s}, 1 \mathrm{H}), 6.89\left(\mathrm{~d},{ }^{4} \mathrm{~J}=3.9 \mathrm{~Hz}, 1 \mathrm{H}\right), 6.38-6.32(\mathrm{~m}, 1 \mathrm{H}), 6.28(\mathrm{~d}, 4 \mathrm{~J}=3.9 \mathrm{~Hz}, 1 \mathrm{H}), 3.62$ 
170 Experimental part

- $3.57(\mathrm{~m}, 10 \mathrm{H}), 3.50-3.47(\mathrm{~m}, 4 \mathrm{H}), 3.43\left(\mathrm{t},{ }^{3} \mathrm{~J}=6.1 \mathrm{~Hz}, 2 \mathrm{H}\right), 3.28-3.22(\mathrm{~m}, 8 \mathrm{H}), 3.13\left(\mathrm{t},{ }^{3} \mathrm{~J}=11.6 \mathrm{~Hz}\right.$, $2 \mathrm{H}), 2.94(\mathrm{~s}, 3 \mathrm{H}), 2.59\left(\mathrm{t},{ }^{3} \mathrm{~J}=7.7 \mathrm{~Hz}, 2 \mathrm{H}\right), 1.87\left(\mathrm{p},{ }^{3} \mathrm{~J}=6.3 \mathrm{~Hz}, 2 \mathrm{H}\right), 1.70\left(\mathrm{p},{ }^{3} \mathrm{~J}=6.4 \mathrm{~Hz}, 2 \mathrm{H}\right) \mathrm{ppm}$. 


\subsection{Differential Scanning Fluorimetrie}

Table 16: Thermal shift experiments of OICR-9429 derived molecules (6a-g), degraders (7a-e), (8a-j), (9a-c) and the negative controls (nc_VHL) and (nc_WDR5). $\Delta T_{m}$ : thermal shift change; SD: standard derivation; R1-R3: replicate 1-3. As negative controls, DMSO and Thalidomide, Idasanutlin and VH032 were used. As positive control OICR-9429 was used. The used concentrations for the experiments were $2 \mu \mathrm{M}$ WDR5 and $10 \mu \mathrm{M}$ compound. Table adapted with permission from "Design, Synthesis and Evaluation of WD40-repeat containing protein 5 (WDR5) Degraders by Dölle, Adhikari et al. ${ }^{174}$ Copyright 2021 American Chemical Society.

\begin{tabular}{|c|c|c|c|c|c|}
\hline ID & $\Delta T_{m}[\mathrm{~K}]$ & $\mathrm{SD}[\mathrm{K}]$ & $\mathrm{R} 1 \Delta T_{m}[\mathrm{~K}]$ & $\mathrm{R} 2 \Delta T_{m}[\mathrm{~K}]$ & $\mathrm{R} 3 \Delta T_{m}[\mathrm{~K}]$ \\
\hline $6 a$ & 1,2 & 0,1 & 1,2 & 1,3 & 1,1 \\
\hline $6 b$ & 3,5 & 0,2 & 3,6 & 3,3 & 3,4 \\
\hline $6 c$ & 12,7 & 0,3 & 13,0 & 12,7 & 12,3 \\
\hline $6 d$ & 20,8 & 0,6 & 20,3 & 21,5 & 20,7 \\
\hline $6 e$ & 5,7 & 0,4 & 6,1 & 5,2 & 5,7 \\
\hline $6 f$ & 4,1 & 0,6 & 5,0 & 3,4 & 4,0 \\
\hline $6 g$ & 4,2 & 0,7 & 3,4 & 4,2 & 5,1 \\
\hline $7 a$ & 13,6 & 0,2 & 13,4 & 13,7 & 13,6 \\
\hline $7 b$ & 12,7 & 0,3 & 13,0 & 12,6 & 12,3 \\
\hline $7 c$ & 9,0 & 0,5 & 8,9 & 9,5 & 8,6 \\
\hline $7 d$ & 11,9 & 0,3 & 11,8 & 12,3 & 11,7 \\
\hline $7 e$ & 12,5 & 0,6 & 12,8 & 11,8 & 12,8 \\
\hline $8 a$ & 15,3 & 0,2 & 15,2 & 15,3 & 15,6 \\
\hline $8 b$ & 7,7 & 0,5 & 7,6 & 7,4 & 8,3 \\
\hline $8 c$ & 12,5 & 0,2 & 12,4 & 12,3 & 12,7 \\
\hline $8 d$ & 15,6 & 0,0 & 15,6 & 15,6 & 15,5 \\
\hline $8 e$ & 11,0 & 0,3 & 10,9 & 11,5 & 10,7 \\
\hline $8 f$ & 10,4 & 0,7 & 10,8 & 11,0 & 9,4 \\
\hline $8 g$ & 13,2 & 0,1 & 13,2 & 13,1 & 13,1 \\
\hline $8 h$ & 9,7 & 2,8 & 8,2 & 7,9 & 12,9 \\
\hline $8 i$ & 3,5 & 0,4 & 3,8 & 3,0 & 3,7 \\
\hline $8 \mathbf{j}$ & 14,0 & 0,0 & 14,0 & 14,0 & n.d. \\
\hline $9 a$ & 0,9 & 0,2 & 1,2 & 0,8 & 0,8 \\
\hline $9 b$ & 0,3 & 0,4 & 0,7 & $-0,3$ & 0,5 \\
\hline $9 c$ & 0,7 & 0,2 & 0,6 & 0,6 & 1,0 \\
\hline OICR-9429 & 13,3 & 0,1 & 13,2 & 13,1 & 13,6 \\
\hline DMSO & 0,0 & 0,3 & $-0,1$ & $-0,4$ & 0,3 \\
\hline
\end{tabular}




\begin{tabular}{c|ccccc} 
22/nc_VHL & 12,2 & 0,1 & 12,2 & 12,4 & 12,1 \\
23/nc_WDR5 & 0,0 & 0,2 & 0,0 & $-0,1$ & 0,2 \\
VH032 & $-0,4$ & 0,1 & $-0,3$ & $-0,5$ & $-0,5$ \\
Thalidomide & 0,0 & $-0,1$ & 0,3 & 0,0 & $-0,5$ \\
Idasanutlin & $-0,2$ & 0,1 & $-0,1$ & $-0,1$ & $-0,4$
\end{tabular}

\subsection{Isothermal Titration Calorimetry}

Table 17: Thermodynamic properties of OICR-9429 derived molecule (6d), degraders (7a), (8a), (8e-j) and pyrroloimidazole-based inhibitor (17) and degrader (20b). $\mathrm{K}_{\mathrm{d}}$ : dissociation constant; SD: standard deviation; $\mathrm{n}$ : stoichiometry; $\Delta \mathrm{H}$ : enthalpy change $\Delta \mathrm{S}$ : entropy change; $\mathrm{T}$ : Temperature. Table adapted with permission from “Design, Synthesis and Evaluation of WD40-repeat containing protein 5 (WDR5) Degraders by Dölle, Adhikari et al. ${ }^{174}$ Copyright 2021 American Chemical Society.

\begin{tabular}{c|ccccc}
$\mathrm{ID}$ & $\mathrm{K}_{\mathrm{d}}[\mathrm{nM}]$ & $\mathrm{SD}[\mathrm{nM}]$ & $\mathrm{n}$ & $\Delta \mathrm{H}[\mathrm{kcal} / \mathrm{mol}]$ & $\mathrm{T} \Delta \mathrm{S}[\mathrm{kcal} / \mathrm{mol}]$ \\
\hline $\mathbf{6 d}$ & 25 & 6 & 1.0 & -9.4 & 0.8 \\
$\mathbf{7 a}$ & 12 & 4 & 1.0 & -8.1 & 2.6 \\
$\mathbf{8 a}$ & 41 & 9 & 1.0 & -10 & -0.2 \\
$\mathbf{8 e}$ & 9 & 2 & 1.1 & -6.3 & 4.5 \\
$\mathbf{8 f}$ & 6 & 2 & 1.0 & -10 & 1.1 \\
$\mathbf{8 g}$ & 18 & 5 & 1.1 & -7.4 & 3.2 \\
$\mathbf{8 h}$ & 12 & 4 & 1.0 & -9.3 & 1.4 \\
$\mathbf{8 i}$ & 11 & 3 & 1.1 & -10 & 0.7 \\
$\mathbf{8 j}$ & 33 & 5 & 1.0 & -7.9 & 2.2 \\
$\mathbf{1 4}$ & 125 & 34 & 1.0 & -4.9 & 4.4 \\
$\mathbf{1 7 b}$ & 97 & 31 & 1.0 & -6.6 & 2.8
\end{tabular}



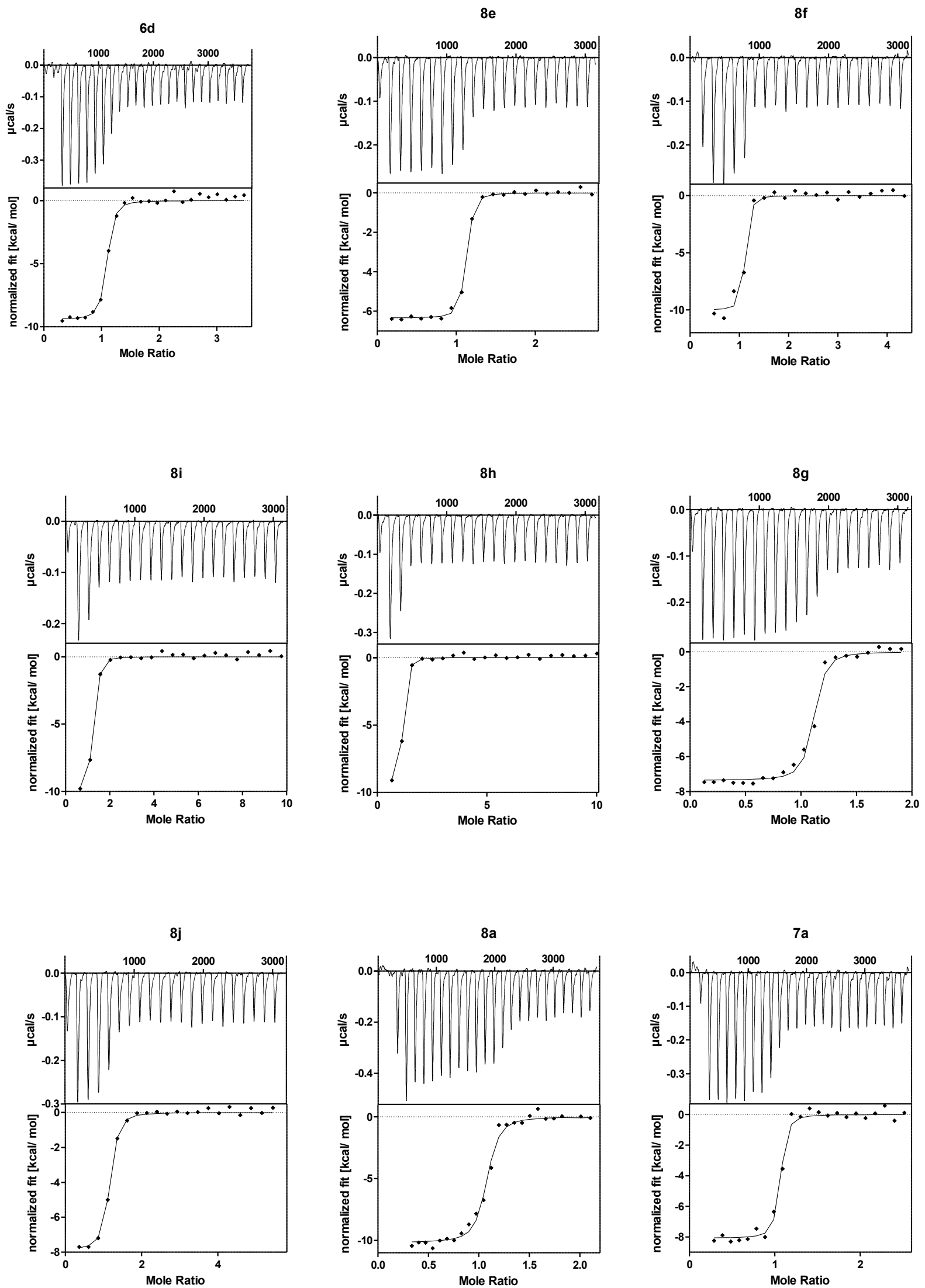

Figure 22: ITC curves of OICR-9429 derived molecule (6d) and degraders (7a), (8a), (8e-j). Figure reprinted 
174 Experimental part

(adapted) with permission from "Design, Synthesis and Evaluation of WD40-repeat containing protein 5 (WDR5) Degraders by Dölle, Adhikari et al. ${ }^{174}$ Copyright 2021 American Chemical Society. 


\section{Abbreviations}

\begin{tabular}{|c|c|}
\hline$\AA$ & Ångström \\
\hline $\mathrm{ACN}$ & acetonitrile \\
\hline ADI1 & 2-dihydroxy-3-keto-5-methylthiopentene dioxygenase \\
\hline ARID & AT-rich interactive domain-containing protein \\
\hline $\mathrm{ASH} 2 \mathrm{~L}$ & Absent-Small-Homeotic-2-Like protein \\
\hline ATAC & Ada2-containing \\
\hline ATRX & ATP-dependent helicase \\
\hline$\beta$ TRCP & beta-transducin repeat containing \\
\hline BAF & BRG1/BRM-associated factor \\
\hline BET & Bromodomain extra-terminal \\
\hline Boc & tertbutyloxycarbonyl \\
\hline BODIPY & 4,4-difluoro-4-bora-3a,4a-diaza-s-indacene \\
\hline BRCA1 & Breast cancer 1 \\
\hline BRD & Bromodomain \\
\hline BRET & Bioluminescence resonance energy transfer \\
\hline $\mathrm{BR}$ & Basic region \\
\hline C7orf26 & Chromosome 7 Open Reading Frame 26 \\
\hline C8orf & Human Ribosomal biogenesis factor \\
\hline CBP & CREB-binding protein \\
\hline CCDC117 & Coiled-coil domain-containing protein 117 \\
\hline $\mathrm{C} / \mathrm{EBPa}$ & ССАAT/enhancer-binding protein alpha \\
\hline CGGBP1 & CGG triplet repeat-binding protein 1 \\
\hline CHIP-Seq & Chromatin Immuno Precipitation DNA-Sequencing \\
\hline c-Hex & cyclohexane \\
\hline $\mathrm{CHX}$ & cycloheximide \\
\hline $\mathrm{c}-\mathrm{MYC}$ & Cellular MYC \\
\hline CRBN & Cereblon \\
\hline CUL4 & Cullin 4 \\
\hline DCAF16 & DDB1 And CUL4 Associated Factor 16 \\
\hline
\end{tabular}




\begin{tabular}{|c|c|}
\hline DCM & Dichloromethane \\
\hline DDB1 & DNA damage binding protein 1 \\
\hline DEAD & Diethyl azodicarboxylate \\
\hline DIEA & $\mathrm{N}, \mathrm{N}$-Diisoproylethylamine \\
\hline DLD & Dihydrolipoyl dehydrogenase \\
\hline DNA & Desoxyribonucleid acid \\
\hline DNMT & DNA (cytosine-5)-methyltransferase family \\
\hline DMF & $\mathrm{N}, \mathrm{N}$-Dimethylformamide \\
\hline DMSO & Dimethylsulfoxide \\
\hline DSF & differential scanning fluorimetrie \\
\hline dTAG & degradation tag \\
\hline DYP30 & Dumpy-30 protein \\
\hline EA & ethyl acetate \\
\hline EM & electron microscopy \\
\hline ESI & electronspray ionozation \\
\hline EtOH & ethanol \\
\hline eq & equivalent \\
\hline $\mathrm{EZH} 2$ & Enhancer of zeste homolog 2 \\
\hline FC & fold change \\
\hline G & free energy \\
\hline GO enrichment & Gene Ontology term enrichment \\
\hline $\mathrm{h}$ & hours \\
\hline $\mathrm{H}$ & enthalpy \\
\hline HAF & Histone acetylase \\
\hline HATU & $\begin{array}{l}\text { 1-[bis(dimethylamino)methylene]-1H-1,2,3-triazolo[4,5-b]pyridinium 3- } \\
\text { oxide hexafluorophosphate }\end{array}$ \\
\hline HDAC & Histone deacetylase \\
\hline $\mathrm{HLH}$ & Helix-loop-helix \\
\hline HMT & Histone Lysine Methyltransferase \\
\hline HOX & homeobox \\
\hline HPLC & high purity liquid chromatography \\
\hline HRMS & High resolution mass spectrometry \\
\hline
\end{tabular}




\begin{tabular}{|c|c|}
\hline HRNPU & Heterogeneous nuclear ribonucleoprotein U \\
\hline IAP & Inducer of Apoptosis \\
\hline IDH & isocitrate dehydrogenase \\
\hline INO80E & INO80 complex subunit E \\
\hline ITC & isothermal titration calorimetry \\
\hline JARID1C & Lysine-specific demethylase $5 \mathrm{C}$ \\
\hline K & Kelvin \\
\hline KANSL1 & KAT8 Regulatory NSL Complex Subunit 1 \\
\hline $\mathrm{kJ}$ & kilo Joule \\
\hline KMD & Lysine demethylase \\
\hline KMT & Lysine Methyltransferase \\
\hline LgBit & Large Bit \\
\hline IncRNA & Long non-coding RNA \\
\hline LSD1 & Lysine-specific histone demethylase $1 \mathrm{~A}$ \\
\hline LZ & leucine zipper \\
\hline M & molar \\
\hline MAX & myc-associated factor $X$ \\
\hline $\mathrm{Mb}$ & MYc boc \\
\hline MBDs & methylcytosine-binding proteins \\
\hline MDM2 & Mouse double minute 2 homolog \\
\hline $\mathrm{MeOH}$ & methanol \\
\hline $\min$ & minutes \\
\hline MLL & mixed lineage leukemia \\
\hline mRNA & messenger RNA \\
\hline MS & mass spectrometry \\
\hline MW & microwave \\
\hline$\mu \mathrm{M}$ & mircomolar \\
\hline $\mathrm{mM}$ & millimolar \\
\hline $\mathrm{n}$ & stochiometry \\
\hline NanoLuc & Nanoluciferase \\
\hline NCOA4 & Nuclear receptor coactivator 4 \\
\hline
\end{tabular}




\begin{tabular}{|c|c|}
\hline $\mathrm{nM}$ & nanomolar \\
\hline NMC & NUT midline carcinoma \\
\hline NMP & N-Methylpyrrolidone \\
\hline NMR & nulear magnetic resonance \\
\hline NLS & non-specific lethal \\
\hline NUT & Nuclear protein in testis \\
\hline Oct4 & octamer-binding transcription factor 4 \\
\hline $\mathrm{p}$ & significance \\
\hline Pax7 & Paired box protein 7 \\
\hline PBAF & polybromo-associated BAF factor \\
\hline PEG & Polyethyleneglycole \\
\hline PGD & 6-phosphogluconate dehydrogenase \\
\hline POI & Protein of interest \\
\hline PPI & Protein-protein interaction \\
\hline PROTAC & Proteolysis targeting chimera \\
\hline PSG & Protein synthesis genes \\
\hline qPCR & quantitative polymerase chain reation \\
\hline RbBP5 & Retinoblastoma Binding Protein 5 \\
\hline RING & Really interesting new gene \\
\hline RNF4 & RING finger protein 4 \\
\hline RPC10 & DNA-directed RNA polymerase III subunit \\
\hline RPG & Ribosomal protein genes \\
\hline RPL22L1 & 60S Ribosomal protein L22-like 1 \\
\hline RNA & Ribonucleid acid \\
\hline $\mathrm{rt}$ & room temperature \\
\hline S & entropy \\
\hline SAR & structure activity relationship \\
\hline SD & standard deviation \\
\hline SE & super enhancer \\
\hline SET1 & Suppressor of variegation 3-9, Enhancer of Zeste, Trithorax \\
\hline SMARCA2/4 & $\begin{array}{l}\text { SWI/SNF-related, matrix-associated actin-dependent regulator of } \\
\text { chromatin, subfamily A2/ } 4\end{array}$ \\
\hline
\end{tabular}




\begin{tabular}{|c|c|}
\hline $\mathrm{S}_{\mathrm{N}}$ & nucleophilic substitution \\
\hline$S_{N} A R$ & nucleophilic aromatic substitution \\
\hline SNF & SWI/SNF chromatin-remodeling complex subunit \\
\hline STOML2 & Stomatin-like protein 2 \\
\hline $\mathrm{T}$ & Temperature \\
\hline TET & ten-eleven translocation \\
\hline TFA & Triflouroacetic acid \\
\hline THF & Tetrahydrofuran \\
\hline TLC & thin layer chromatography \\
\hline TPD & Target protein degradation \\
\hline TRIM & tripartite motif family \\
\hline tRNA & Transfer RNA \\
\hline Ts & Tosylate \\
\hline TSA & thermal shift assay \\
\hline UPS & Ubiqutin Proteasome System \\
\hline UTX & Ubiquitously transcribed tetratricopeptide repeat, $\mathrm{X}$ chromosome \\
\hline VHL & Von Hippel Lindau \\
\hline WARS & Tryptophan-tRNA ligase \\
\hline WBM & WDR5 binding motif \\
\hline WDR5 & WD40- repeat containing protein 5 \\
\hline Win & WDR5 interacting \\
\hline XRCC3 & DNA repair protein \\
\hline
\end{tabular}


180 Abbreviations 


\section{References}

1. Luger, K.; Mäder, A. W.; Richmond, R. K.; Sargent, D. F.; Richmond, T. J., Crystal structure of the nucleosome core particle at $2.8 \AA$ A resolution. Nature 1997, 389 (6648), 251-260.

2. Bannister, A. J.; Kouzarides, T., Regulation of chromatin by histone modifications. Cell Research 2011, 21 (3), 381-395.

3. Jenuwein, T.; Allis, C. D., Translating the histone code. Science 2001, 293 (5532), 1074-80.

4. Park, S. H.; Ayoub, A.; Lee, Y.-T.; Xu, J.; Kim, H.; Zheng, W.; Zhang, B.; Sha, L.; An, S.; Zhang, Y.; Cianfrocco, M. A.; Su, M.; Dou, Y.; Cho, U.-S., Cryo-EM structure of the human MLL1 core complex bound to the nucleosome. Nature Communications 2019, 10 (1), 5540.

5. Rodríguez-Paredes, M.; Esteller, M., Cancer epigenetics reaches mainstream oncology. Nature Medicine 2011, 17 (3), 330-339.

6. Zhong, S.; Fei, Z.; Chen, Y. R.; Zheng, Y.; Huang, M.; Vrebalov, J.; McQuinn, R.; Gapper, N.; Liu, B.; Xiang, J.; Shao, Y.; Giovannoni, J. J., Single-base resolution methylomes of tomato fruit development reveal epigenome modifications associated with ripening. Nat Biotechnol 2013, 31 (2), 154-9.

7. Lang, Z.; Wang, Y.; Tang, K.; Tang, D.; Datsenka, T.; Cheng, J.; Zhang, Y.; Handa, A. K.; Zhu, J. K., Critical roles of DNA demethylation in the activation of ripening-induced genes and inhibition of ripening-repressed genes in tomato fruit. Proc Natl Acad Sci U S A 2017, 114 (22), E4511-e4519.

8. Dahlet, T.; Argüeso Lleida, A.; Al Adhami, H.; Dumas, M.; Bender, A.; Ngondo, R. P.; Tanguy, M.; Vallet, J.; Auclair, G.; Bardet, A. F.; Weber, M., Genome-wide analysis in the mouse embryo reveals the importance of DNA methylation for transcription integrity. Nature Communications 2020, 11 (1), 3153.

9. Etchegaray, J. P.; Mostoslavsky, R., Interplay between Metabolism and Epigenetics: A Nuclear Adaptation to Environmental Changes. Molecular cell 2016, 62 (5), 695-711.

10. Zhang, J.; Zhang, Y. Z.; Jiang, J.; Duan, C. G., The Crosstalk Between Epigenetic Mechanisms and Alternative RNA Processing Regulation. Front Genet 2020, 11, 998.

11. Hanahan, D.; Weinberg, R. A., Hallmarks of cancer: the next generation. Cell 2011, 144 (5), 64674.

12. Baylin, S. B.; Jones, P. A., Epigenetic Determinants of Cancer. Cold Spring Harbor perspectives in biology 2016, 8 (9).

13. Feinberg, A. P., Cancer epigenetics is no Mickey Mouse. Cancer Cell 2005, 8 (4), 267-8.

14. Jones, P. A.; Laird, P. W., Cancer epigenetics comes of age. Nat Genet 1999, 21 (2), 163-7.

15. Esteller, M., Epigenetics in cancer. The New England journal of medicine 2008, 358 (11), 1148-

59. 
16. Esteller, M., Cancer epigenomics: DNA methylomes and histone-modification maps. Nature reviews. Genetics 2007, 8 (4), 286-98.

17. Jones, P. A.; Taylor, S. M., Cellular differentiation, cytidine analogs and DNA methylation. Cell $1980,20(1), 85-93$

18. Plass, C.; Pfister, S. M.; Lindroth, A. M.; Bogatyrova, O.; Claus, R.; Lichter, P., Mutations in regulators of the epigenome and their connections to global chromatin patterns in cancer. Nature reviews. Genetics 2013, 14 (11), 765-80.

19. Shen, H.; Laird, P. W., Interplay between the cancer genome and epigenome. Cell 2013, 153 (1), 38-55.

20. You, J. S.; Jones, P. A., Cancer genetics and epigenetics: two sides of the same coin? Cancer Cell 2012, $22(1), 9-20$.

21. Garraway, L. A.; Lander, E. S., Lessons from the cancer genome. Cell 2013, 153 (1), 17-37.

22. Tarakhovsky, A., Tools and landscapes of epigenetics. Nature immunology 2010, 11 (7), 565-8.

23. O'Connor, O. A.; Bhagat, G.; Ganapathi, K.; Pedersen, M. B.; D'Amore, F.; Radeski, D.; Bates, S. E., Changing the paradigms of treatment in peripheral T-cell lymphoma: from biology to clinical practice. Clinical cancer research : an official journal of the American Association for Cancer Research 2014, 20 (20), 5240-54.

24. Biswas, S.; Rao, C. M., Epigenetic tools (The Writers, The Readers and The Erasers) and their implications in cancer therapy. European journal of pharmacology 2018, 837, 8-24.

25. Bushey, A. M.; Dorman, E. R.; Corces, V. G., Chromatin insulators: regulatory mechanisms and epigenetic inheritance. Molecular cell 2008, 32 (1), 1-9.

26. Filippakopoulos, P.; Knapp, S., Targeting bromodomains: epigenetic readers of lysine acetylation. Nature Reviews Drug Discovery 2014, 13 (5), 337-356.

27. Donati, B.; Lorenzini, E.; Ciarrocchi, A., BRD4 and Cancer: going beyond transcriptional regulation. Molecular cancer 2018, 17 (1), 164.

28. Filippakopoulos, P.; Qi, J.; Picaud, S.; Shen, Y.; Smith, W. B.; Fedorov, O.; Morse, E. M.; Keates, T.; Hickman, T. T.; Felletar, I.; Philpott, M.; Munro, S.; McKeown, M. R.; Wang, Y.; Christie, A. L.; West, N.; Cameron, M. J.; Schwartz, B.; Heightman, T. D.; La Thangue, N.; French, C. A.; Wiest, O.; Kung, A. L.; Knapp, S.; Bradner, J. E., Selective inhibition of BET bromodomains. Nature 2010, 468 (7327), 1067-73. 29. Stathis, A.; Bertoni, F., BET Proteins as Targets for Anticancer Treatment. Cancer Discov 2018, $8(1), 24-36$.

30. Kadoch, C.; Crabtree, G. R., Mammalian SWI/SNF chromatin remodeling complexes and cancer: Mechanistic insights gained from human genomics. Sci Adv 2015, 1 (5), e1500447.

31. Alfert, A.; Moreno, N.; Kerl, K., The BAF complex in development and disease. Epigenetics \& chromatin 2019, 12 (1), 19. 
32. Hodges, C.; Kirkland, J. G.; Crabtree, G. R., The Many Roles of BAF (mSWI/SNF) and PBAF Complexes in Cancer. Cold Spring Harbor perspectives in medicine 2016, 6 (8).

33. Bracken, A. P.; Brien, G. L.; Verrijzer, C. P., Dangerous liaisons: interplay between SWI/SNF, NuRD, and Polycomb in chromatin regulation and cancer. Genes \& development 2019, 33 (15-16), 936959.

34. Varela, I.; Tarpey, P.; Raine, K.; Huang, D.; Ong, C. K.; Stephens, P.; Davies, H.; Jones, D.; Lin, M. L.; Teague, J.; Bignell, G.; Butler, A.; Cho, J.; Dalgliesh, G. L.; Galappaththige, D.; Greenman, C.; Hardy, C.; Jia, M.; Latimer, C.; Lau, K. W.; Marshall, J.; McLaren, S.; Menzies, A.; Mudie, L.; Stebbings, L.; Largaespada, D. A.; Wessels, L. F.; Richard, S.; Kahnoski, R. J.; Anema, J.; Tuveson, D. A.; Perez-Mancera, P. A.; Mustonen, V.; Fischer, A.; Adams, D. J.; Rust, A.; Chan-on, W.; Subimerb, C.; Dykema, K.; Furge, K.; Campbell, P. J.; Teh, B. T.; Stratton, M. R.; Futreal, P. A., Exome sequencing identifies frequent mutation of the SWI/SNF complex gene PBRM1 in renal carcinoma. Nature 2011, 469 (7331), 539-42.

35. Shi, Y., Histone lysine demethylases: emerging roles in development, physiology and disease. Nature reviews. Genetics 2007, 8 (11), 829-33.

36. Chi, P.; Allis, C. D.; Wang, G. G., Covalent histone modifications--miswritten, misinterpreted and mis-erased in human cancers. Nat Rev Cancer 2010, 10 (7), 457-69.

37. Herz, H. M.; Garruss, A.; Shilatifard, A., SET for life: biochemical activities and biological functions of SET domain-containing proteins. Trends in biochemical sciences 2013, 38 (12), 621-39.

38. Glozak, M. A.; Seto, E., Histone deacetylases and cancer. Oncogene 2007, 26 (37), 5420-32.

39. Li, Y.; Seto, E., HDACs and HDAC Inhibitors in Cancer Development and Therapy. Cold Spring Harbor perspectives in medicine 2016, 6 (10).

40. Romero, D., HDAC inhibitors tested in phase III trial. Nature reviews. Clinical oncology 2019, 16 (8), 465 .

41. Hontecillas-Prieto, L.; Flores-Campos, R.; Silver, A.; de Álava, E.; Hajji, N.; García-Domínguez, D. J., Synergistic Enhancement of Cancer Therapy Using HDAC Inhibitors: Opportunity for Clinical Trials. Front Genet 2020, 11, 578011.

42. Manzo, F.; Tambaro, F. P.; Mai, A.; Altucci, L., Histone acetyltransferase inhibitors and preclinical studies. Expert Opin Ther Pat 2009, 19 (6), 761-74.

43. Bernstein, B. E.; Humphrey, E. L.; Erlich, R. L.; Schneider, R.; Bouman, P.; Liu, J. S.; Kouzarides, T.; Schreiber, S. L., Methylation of histone H3 Lys 4 in coding regions of active genes. Proc Natl Acad Sci U S A 2002, 99 (13), 8695-700.

44. Bannister, A. J.; Schneider, R.; Myers, F. A.; Thorne, A. W.; Crane-Robinson, C.; Kouzarides, T., Spatial distribution of di- and tri-methyl lysine 36 of histone H3 at active genes. J Biol Chem 2005, 280 (18), 17732-6. 
45. Mohan, M.; Herz, H. M.; Takahashi, Y. H.; Lin, C.; Lai, K. C.; Zhang, Y.; Washburn, M. P.; Florens, L.; Shilatifard, A., Linking H3K79 trimethylation to Wnt signaling through a novel Dot1-containing complex (DotCom). Genes \& development 2010, 24 (6), 574-89.

46. Greer, E. L.; Shi, Y., Histone methylation: a dynamic mark in health, disease and inheritance. Nature reviews. Genetics 2012, 13 (5), 343-57.

47. Song, Y.; Wu, F.; Wu, J., Targeting histone methylation for cancer therapy: enzymes, inhibitors, biological activity and perspectives. Journal of Hematology \& Oncology 2016, 9 (1), 49.

48. Shi, Y.; Lan, F.; Matson, C.; Mulligan, P.; Whetstine, J. R.; Cole, P. A.; Casero, R. A.; Shi, Y., Histone demethylation mediated by the nuclear amine oxidase homolog LSD1. Cell 2004, 119 (7), 94153.

49. Højfeldt, J. W.; Agger, K.; Helin, K., Histone lysine demethylases as targets for anticancer therapy. Nature reviews. Drug discovery 2013, 12 (12), 917-30.

50. Cao, F.; Townsend, E. C.; Karatas, H.; Xu, J.; Li, L.; Lee, S.; Liu, L.; Chen, Y.; Ouillette, P.; Zhu, J.; Hess, J. L.; Atadja, P.; Lei, M.; Qin, Z. S.; Malek, S.; Wang, S.; Dou, Y., Targeting MLL1 H3K4 methyltransferase activity in mixed-lineage leukemia. Molecular cell 2014, 53 (2), 247-61.

51. Shilatifard, A., The COMPASS family of histone H3K4 methylases: mechanisms of regulation in development and disease pathogenesis. Annu Rev Biochem 2012, 81, 65-95.

52. Sims, R. J., 3rd; Nishioka, K.; Reinberg, D., Histone lysine methylation: a signature for chromatin function. Trends in genetics : TIG 2003, 19 (11), 629-39.

53. Husmann, D.; Gozani, O., Histone lysine methyltransferases in biology and disease. Nat Struct Mol Biol 2019, 26 (10), 880-889.

54. Miller, J. L.; Grant, P. A., The role of DNA methylation and histone modifications in transcriptional regulation in humans. Sub-cellular biochemistry 2013, 61, 289-317.

55. Malik, S.; Bhaumik, S. R., Mixed lineage leukemia: histone H3 lysine 4 methyltransferases from yeast to human. Febs j 2010, 277 (8), 1805-21.

56. Taverna, S. D.; Li, H.; Ruthenburg, A. J.; Allis, C. D.; Patel, D. J., How chromatin-binding modules interpret histone modifications: lessons from professional pocket pickers. Nat Struct Mol Biol 2007, 14 (11), 1025-1040.

57. Vedadi, M.; Blazer, L.; Eram, M. S.; Barsyte-Lovejoy, D.; Arrowsmith, C. H.; Hajian, T., Targeting human SET1/MLL family of proteins. Protein Sci 2017, 26 (4), 662-676.

58. Ruthenburg, A. J.; Allis, C. D.; Wysocka, J., Methylation of lysine 4 on histone H3: intricacy of writing and reading a single epigenetic mark. Molecular cell 2007, 25 (1), 15-30.

59. Balgobind, B. V.; Raimondi, S. C.; Harbott, J.; Zimmermann, M.; Alonzo, T. A.; Auvrignon, A.; Beverloo, H. B.; Chang, M.; Creutzig, U.; Dworzak, M. N.; Forestier, E.; Gibson, B.; Hasle, H.; Harrison, C. J.; Heerema, N. A.; Kaspers, G. J.; Leszl, A.; Litvinko, N.; Nigro, L. L.; Morimoto, A.; Perot, C.; Pieters, 
R.; Reinhardt, D.; Rubnitz, J. E.; Smith, F. O.; Stary, J.; Stasevich, I.; Strehl, S.; Taga, T.; Tomizawa, D.; Webb, D.; Zemanova, Z.; Zwaan, C. M.; van den Heuvel-Eibrink, M. M., Novel prognostic subgroups in childhood 11q23/MLL-rearranged acute myeloid leukemia: results of an international retrospective study. Blood 2009, 114 (12), 2489-96.

60. Winters, A. C.; Bernt, K. M., MLL-Rearranged Leukemias-An Update on Science and Clinical Approaches. Frontiers in pediatrics 2017, 5, 4.

61. Tkachuk, D. C.; Kohler, S.; Cleary, M. L., Involvement of a homolog of Drosophila trithorax by 11q23 chromosomal translocations in acute leukemias. Cell 1992, 71 (4), 691-700.

62. Krivtsov, A. V.; Hoshii, T.; Armstrong, S. A., Mixed-Lineage Leukemia Fusions and Chromatin in Leukemia. Cold Spring Harbor perspectives in medicine 2017, 7 (11).

63. Guenther, M. G.; Jenner, R. G.; Chevalier, B.; Nakamura, T.; Croce, C. M.; Canaani, E.; Young, R. A., Global and Hox-specific roles for the MLL1 methyltransferase. Proc Natl Acad Sci U S A 2005, 102 (24), 8603-8.

64. Milne, T. A.; Briggs, S. D.; Brock, H. W.; Martin, M. E.; Gibbs, D.; Allis, C. D.; Hess, J. L., MLL targets SET domain methyltransferase activity to Hox gene promoters. Molecular cell 2002, 10 (5), 1107-17.

65. Qian, C.; Zhou, M. M., SET domain protein lysine methyltransferases: Structure, specificity and catalysis. Cell Mol Life Sci 2006, 63 (23), 2755-63.

66. Dou, Y.; Milne, T. A.; Ruthenburg, A. J.; Lee, S.; Lee, J. W.; Verdine, G. L.; Allis, C. D.; Roeder, R. G., Regulation of MLL1 H3K4 methyltransferase activity by its core components. Nat Struct Mol Biol 2006, 13 (8), 713-9.

67. Jiang, H.; Shukla, A.; Wang, X.; Chen, W. Y.; Bernstein, B. E.; Roeder, R. G., Role for Dpy-30 in ES cell-fate specification by regulation of H3K4 methylation within bivalent domains. Cell 2011, 144 (4), 513-25.

68. Shinsky, S. A.; Monteith, K. E.; Viggiano, S.; Cosgrove, M. S., Biochemical reconstitution and phylogenetic comparison of human SET1 family core complexes involved in histone methylation. $J$ Biol Chem 2015, 290 (10), 6361-75.

69. Patel, A.; Dharmarajan, V.; Vought, V. E.; Cosgrove, M. S., On the mechanism of multiple lysine methylation by the human mixed lineage leukemia protein-1 (MLL1) core complex. J Biol Chem 2009, 284 (36), 24242-56.

70. Steward, M. M.; Lee, J. S.; O'Donovan, A.; Wyatt, M.; Bernstein, B. E.; Shilatifard, A., Molecular regulation of H3K4 trimethylation by ASH2L, a shared subunit of MLL complexes. Nat Struct Mol Biol 2006, 13 (9), 852-4.

71. Haddad, J. F.; Yang, Y.; Takahashi, Y. H.; Joshi, M.; Chaudhary, N.; Woodfin, A. R.; Benyoucef, A.; Yeung, S.; Brunzelle, J. S.; Skiniotis, G.; Brand, M.; Shilatifard, A.; Couture, J. F., Structural Analysis 
of the Ash2L/Dpy-30 Complex Reveals a Heterogeneity in H3K4 Methylation. Structure 2018, 26 (12), 1594-1603.e4.

72. Hsu, P. L.; Li, H.; Lau, H. T.; Leonen, C.; Dhall, A.; Ong, S. E.; Chatterjee, C.; Zheng, N., Crystal Structure of the COMPASS H3K4 Methyltransferase Catalytic Module. Cell 2018, 174 (5), 1106-1116.e9. 73. Zhang, H.; Li, M.; Gao, Y.; Jia, C.; Pan, X.; Cao, P.; Zhao, X.; Zhang, J.; Chang, W., Structural implications of Dpy30 oligomerization for MLL/SET1 COMPASS H3K4 trimethylation. Protein \& cell 2015, 6 (2), 147-51.

74. Dong, X.; Peng, Y.; Peng, Y.; Xu, F.; He, X.; Wang, F.; Peng, X.; Qiang, B.; Yuan, J.; Rao, Z., Characterization and crystallization of human DPY-30-like protein, an essential component of dosage compensation complex. Biochim Biophys Acta 2005, 1753 (2), 257-62.

75. van Nuland, R.; Smits, A. H.; Pallaki, P.; Jansen, P. W.; Vermeulen, M.; Timmers, H. T., Quantitative dissection and stoichiometry determination of the human SET1/MLL histone methyltransferase complexes. Molecular and cellular biology 2013, 33 (10), 2067-77.

76. Xu, Z.; Gong, Q.; Xia, B.; Groves, B.; Zimmermann, M.; Mugler, C.; Mu, D.; Matsumoto, B.; Seaman, M.; Ma, D., A role of histone H3 lysine 4 methyltransferase components in endosomal trafficking. The Journal of cell biology 2009, 186 (3), 343-53.

77. Li, Y.; Han, J.; Zhang, Y.; Cao, F.; Liu, Z.; Li, S.; Wu, J.; Hu, C.; Wang, Y.; Shuai, J.; Chen, J.; Cao, L.; Li, D.; Shi, P.; Tian, C.; Zhang, J.; Dou, Y.; Li, G.; Chen, Y.; Lei, M., Structural basis for activity regulation of MLL family methyltransferases. Nature 2016, 530 (7591), 447-52.

78. Zhang, P.; Lee, H.; Brunzelle, J. S.; Couture, J. F., The plasticity of WDR5 peptide-binding cleft enables the binding of the SET1 family of histone methyltransferases. Nucleic Acids Res 2012, 40 (9), 4237-46.

79. Wysocka, J.; Swigut, T.; Milne, T. A.; Dou, Y.; Zhang, X.; Burlingame, A. L.; Roeder, R. G.; Brivanlou, A. H.; Allis, C. D., WDR5 associates with histone $\mathrm{H} 3$ methylated at $\mathrm{K} 4$ and is essential for H3 K4 methylation and vertebrate development. Cell 2005, 121 (6), 859-72.

80. Mishra, B. P.; Zaffuto, K. M.; Artinger, E. L.; Org, T.; Mikkola, H. K.; Cheng, C.; Djabali, M.; Ernst, P., The histone methyltransferase activity of MLL1 is dispensable for hematopoiesis and leukemogenesis. Cell Rep 2014, 7 (4), 1239-47.

81. Aho, E. R.; Wang, J.; Gogliotti, R. D.; Howard, G. C.; Phan, J.; Acharya, P.; Macdonald, J. D.; Cheng, K.; Lorey, S. L.; Lu, B.; Wenzel, S.; Foshage, A. M.; Alvarado, J.; Wang, F.; Shaw, J. G.; Zhao, B.; Weissmiller, A. M.; Thomas, L. R.; Vakoc, C. R.; Hall, M. D.; Hiebert, S. W.; Liu, Q.; Stauffer, S. R.; Fesik, S. W.; Tansey, W. P., Displacement of WDR5 from Chromatin by a WIN Site Inhibitor with Picomolar Affinity. Cell Rep 2019, 26 (11), 2916-2928.e13.

82. Aho, E. R.; Weissmiller, A. M.; Fesik, S. W.; Tansey, W. P., Targeting WDR5: A WINning AntiCancer Strategy? Epigenetics insights 2019, 12, 2516865719865282. 
83. Grebien, F.; Vedadi, M.; Getlik, M.; Giambruno, R.; Grover, A.; Avellino, R.; Skucha, A.; Vittori, S.; Kuznetsova, E.; Smil, D.; Barsyte-Lovejoy, D.; Li, F.; Poda, G.; Schapira, M.; Wu, H.; Dong, A.; Senisterra, G.; Stukalov, A.; Huber, K. V. M.; Schönegger, A.; Marcellus, R.; Bilban, M.; Bock, C.; Brown, P. J.; Zuber, J.; Bennett, K. L.; Al-awar, R.; Delwel, R.; Nerlov, C.; Arrowsmith, C. H.; Superti-Furga, G., Pharmacological targeting of the Wdr5-MLL interaction in C/EBP $\alpha$ N-terminal leukemia. Nature Chemical Biology 2015, 11 (8), 571-578.

84. Bryan, A. F.; Wang, J.; Howard, G. C.; Guarnaccia, A. D.; Woodley, C. M.; Aho, E. R.; Rellinger, E. J.; Matlock, B. K.; Flaherty, D. K.; Lorey, S. L.; Chung, D. H.; Fesik, S. W.; Liu, Q.; Weissmiller, A. M.; Tansey, W. P., WDR5 is a conserved regulator of protein synthesis gene expression. Nucleic Acids Res 2020, 48 (6), 2924-2941.

85. Punzi, S.; Balestrieri, C.; D'Alesio, C.; Bossi, D.; Dellino, G. I.; Gatti, E.; Pruneri, G.; Criscitiello, C.; Lovati, G.; Meliksetyan, M.; Carugo, A.; Curigliano, G.; Natoli, G.; Pelicci, P. G.; Lanfrancone, L., WDR5 inhibition halts metastasis dissemination by repressing the mesenchymal phenotype of breast cancer cells. Breast cancer research : BCR 2019, 21 (1), 123.

86. Zhu, J.; Sammons, M. A.; Donahue, G.; Dou, Z.; Vedadi, M.; Getlik, M.; Barsyte-Lovejoy, D.; Alawar, R.; Katona, B. W.; Shilatifard, A.; Huang, J.; Hua, X.; Arrowsmith, C. H.; Berger, S. L., Gain-offunction p53 mutants co-opt chromatin pathways to drive cancer growth. Nature 2015, 525 (7568), 206-11.

87. Terranova, R.; Agherbi, H.; Boned, A.; Meresse, S.; Djabali, M., Histone and DNA methylation defects at Hox genes in mice expressing a SET domain-truncated form of MII. Proc Natl Acad Sci U S A 2006, 103 (17), 6629-34.

88. Wang, P.; Lin, C.; Smith, E. R.; Guo, H.; Sanderson, B. W.; Wu, M.; Gogol, M.; Alexander, T.; Seidel, C.; Wiedemann, L. M.; Ge, K.; Krumlauf, R.; Shilatifard, A., Global analysis of H3K4 methylation defines MLL family member targets and points to a role for MLL1-mediated H3K4 methylation in the regulation of transcriptional initiation by RNA polymerase II. Molecular and cellular biology 2009, 29 (22), 6074-85.

89. Li, X.; Song, Y., Structure, function and inhibition of critical protein-protein interactions involving mixed lineage leukemia 1 and its fusion oncoproteins. J Hematol Oncol 2021, 14 (1), 56.

90. Borkin, D.; He, S.; Miao, H.; Kempinska, K.; Pollock, J.; Chase, J.; Purohit, T.; Malik, B.; Zhao, T.; Wang, J.; Wen, B.; Zong, H.; Jones, M.; Danet-Desnoyers, G.; Guzman, M. L.; Talpaz, M.; Bixby, D. L.; Sun, D.; Hess, J. L.; Muntean, A. G.; Maillard, I.; Cierpicki, T.; Grembecka, J., Pharmacologic inhibition of the Menin-MLL interaction blocks progression of MLL leukemia in vivo. Cancer Cell 2015, 27 (4), 589602. 
91. Grembecka, J.; He, S.; Shi, A.; Purohit, T.; Muntean, A. G.; Sorenson, R. J.; Showalter, H. D.; Murai, M. J.; Belcher, A. M.; Hartley, T.; Hess, J. L.; Cierpicki, T., Menin-MLL inhibitors reverse oncogenic activity of MLL fusion proteins in leukemia. Nat Chem Bio/ 2012, 8 (3), 277-84.

92. Shi, A.; Murai, M. J.; He, S.; Lund, G.; Hartley, T.; Purohit, T.; Reddy, G.; Chruszcz, M.; Grembecka, J.; Cierpicki, T., Structural insights into inhibition of the bivalent menin-MLL interaction by small molecules in leukemia. Blood 2012, 120 (23), 4461-9.

93. Getlik, M.; Smil, D.; Zepeda-Velázquez, C.; Bolshan, Y.; Poda, G.; Wu, H.; Dong, A.; Kuznetsova, E.; Marcellus, R.; Senisterra, G.; Dombrovski, L.; Hajian, T.; Kiyota, T.; Schapira, M.; Arrowsmith, C. H.; Brown, P. J.; Vedadi, M.; Al-Awar, R., Structure-Based Optimization of a Small Molecule Antagonist of the Interaction Between WD Repeat-Containing Protein 5 (WDR5) and Mixed-Lineage Leukemia 1 (MLL1). J Med Chem 2016, 59 (6), 2478-96.

94. Li, D. D.; Chen, W. L.; Wang, Z. H.; Xie, Y. Y.; Xu, X. L.; Jiang, Z. Y.; Zhang, X. J.; You, Q. D.; Guo, X. K., High-affinity small molecular blockers of mixed lineage leukemia 1 (MLL1)-WDR5 interaction inhibit MLL1 complex H3K4 methyltransferase activity. Eur J Med Chem 2016, 124, 480-489.

95. Li, D. D.; Wang, Z. H.; Chen, W. L.; Xie, Y. Y.; You, Q. D.; Guo, X. K., Structure-based design of ester compounds to inhibit MLL complex catalytic activity by targeting mixed lineage leukemia 1 (MLL1)-WDR5 interaction. Bioorg Med Chem 2016, 24 (22), 6109-6118.

96. Alicea-Velázquez, N. L.; Shinsky, S. A.; Loh, D. M.; Lee, J. H.; Skalnik, D. G.; Cosgrove, M. S., Targeted Disruption of the Interaction between WD-40 Repeat Protein 5 (WDR5) and Mixed Lineage Leukemia (MLL)/SET1 Family Proteins Specifically Inhibits MLL1 and SETd1A Methyltransferase Complexes. J Biol Chem 2016, 291 (43), 22357-22372.

97. Avdic, V.; Zhang, P.; Lanouette, S.; Groulx, A.; Tremblay, V.; Brunzelle, J.; Couture, J. F., Structural and biochemical insights into MLL1 core complex assembly. Structure 2011, 19 (1), 101-8.

98. Dias, J.; Van Nguyen, N.; Georgiev, P.; Gaub, A.; Brettschneider, J.; Cusack, S.; Kadlec, J.; Akhtar, A., Structural analysis of the KANSL1/WDR5/KANSL2 complex reveals that WDR5 is required for efficient assembly and chromatin targeting of the NSL complex. Genes \& development 2014, 28 (9), $929-42$.

99. Guelman, S.; Kozuka, K.; Mao, Y.; Pham, V.; Solloway, M. J.; Wang, J.; Wu, J.; Lill, J. R.; Zha, J., The double-histone-acetyltransferase complex ATAC is essential for mammalian development. Molecular and cellular biology 2009, 29 (5), 1176-88.

100. Wu, M. Z.; Tsai, Y. P.; Yang, M. H.; Huang, C. H.; Chang, S. Y.; Chang, C. C.; Teng, S. C.; Wu, K. J., Interplay between HDAC3 and WDR5 is essential for hypoxia-induced epithelial-mesenchymal transition. Molecular cell 2011, 43 (5), 811-22.

101. Yates, J. A.; Menon, T.; Thompson, B. A.; Bochar, D. A., Regulation of HOXA2 gene expression by the ATP-dependent chromatin remodeling enzyme CHD8. FEBS Lett 2010, 584 (4), 689-93. 
102. Guarnaccia, A. D.; Tansey, W. P., Moonlighting with WDR5: A Cellular Multitasker. Journal of clinical medicine 2018, 7 (2).

103. Thomas, L. R.; Wang, Q.; Grieb, B. C.; Phan, J.; Foshage, A. M.; Sun, Q.; Olejniczak, E. T.; Clark, T.; Dey, S.; Lorey, S.; Alicie, B.; Howard, G. C.; Cawthon, B.; Ess, K. C.; Eischen, C. M.; Zhao, Z.; Fesik, S. W.; Tansey, W. P., Interaction with WDR5 promotes target gene recognition and tumorigenesis by MYC. Molecular cell 2015, 58 (3), 440-52.

104. Ge, Z.; Song, E. J.; Kawasawa, Y. I.; Li, J.; Dovat, S.; Song, C., WDR5 high expression and its effect on tumorigenesis in leukemia. Oncotarget 2016, 7 (25), 37740-37754.

105. Chen, X.; Xie, W.; Gu, P.; Cai, Q.; Wang, B.; Xie, Y.; Dong, W.; He, W.; Zhong, G.; Lin, T.; Huang, J., Upregulated WDR5 promotes proliferation, self-renewal and chemoresistance in bladder cancer via mediating H3K4 trimethylation. Sci Rep 2015, 5, 8293.

106. Cui, Z.; Li, H.; Liang, F.; Mu, C.; Mu, Y.; Zhang, X.; Liu, J., Effect of high WDR5 expression on the hepatocellular carcinoma prognosis. Oncology letters 2018, 15 (5), 7864-7870.

107. Dai, X.; Guo, W.; Zhan, C.; Liu, X.; Bai, Z.; Yang, Y., WDR5 Expression Is Prognostic of Breast Cancer Outcome. PLoS One 2015, 10 (9), e0124964.

108. Vilhais-Neto, G. C.; Fournier, M.; Plassat, J.-L.; Sardiu, M. E.; Saraf, A.; Garnier, J.-M.; Maruhashi, M.; Florens, L.; Washburn, M. P.; Pourquié, O., The WHHERE coactivator complex is required for retinoic acid-dependent regulation of embryonic symmetry. Nature Communications 2017, 8 (1), 728.

109. Dang, C. V., MYC on the path to cancer. Cell 2012, 149 (1), 22-35.

110. Stine, Z. E.; Walton, Z. E.; Altman, B. J.; Hsieh, A. L.; Dang, C. V., MYC, Metabolism, and Cancer. Cancer Discovery 2015, 5 (10), 1024.

111. Baluapuri, A.; Wolf, E.; Eilers, M., Target gene-independent functions of MYC oncoproteins. Nature reviews. Molecular cell biology 2020, 21 (5), 255-267.

112. Adhikari, B.; Bozilovic, J.; Diebold, M.; Schwarz, J. D.; Hofstetter, J.; Schröder, M.; Wanior, M.; Narain, A.; Vogt, M.; Dudvarski Stankovic, N.; Baluapuri, A.; Schönemann, L.; Eing, L.; Bhandare, P.; Kuster, B.; Schlosser, A.; Heinzlmeir, S.; Sotriffer, C.; Knapp, S.; Wolf, E., PROTAC-mediated degradation reveals a non-catalytic function of AURORA-A kinase. Nature Chemical Biology 2020, 16 (11), 11791188.

113. Soucek, L.; Jucker, R.; Panacchia, L.; Ricordy, R.; Tatò, F.; Nasi, S., Omomyc, a potential Myc dominant negative, enhances Myc-induced apoptosis. Cancer Res 2002, 62 (12), 3507-10.

114. Sun, Y.; Bell, J. L.; Carter, D.; Gherardi, S.; Poulos, R. C.; Milazzo, G.; Wong, J. W.; Al-Awar, R.; Tee, A. E.; Liu, P. Y.; Liu, B.; Atmadibrata, B.; Wong, M.; Trahair, T.; Zhao, Q.; Shohet, J. M.; Haupt, Y.; Schulte, J. H.; Brown, P. J.; Arrowsmith, C. H.; Vedadi, M.; MacKenzie, K. L.; Hüttelmaier, S.; Perini, G.; 
Marshall, G. M.; Braithwaite, A.; Liu, T., WDR5 Supports an N-Myc Transcriptional Complex That Drives a Protumorigenic Gene Expression Signature in Neuroblastoma. Cancer Res 2015, 75 (23), 5143-54.

115. Marschalek, R., Mechanisms of leukemogenesis by MLL fusion proteins. British journal of haematology 2011, 152 (2), 141-54.

116. Sakamoto, K. M.; Kim, K. B.; Kumagai, A.; Mercurio, F.; Crews, C. M.; Deshaies, R. J., Protacs: chimeric molecules that target proteins to the Skp1-Cullin-F box complex for ubiquitination and degradation. Proc Natl Acad Sci U S A 2001, 98 (15), 8554-9.

117. Sakamoto, K. M.; Kim, K. B.; Verma, R.; Ransick, A.; Stein, B.; Crews, C. M.; Deshaies, R. J., Development of Protacs to target cancer-promoting proteins for ubiquitination and degradation. Molecular \& cellular proteomics : MCP 2003, 2 (12), 1350-8.

118. Gu, S.; Cui, D.; Chen, X.; Xiong, X.; Zhao, Y., PROTACs: An Emerging Targeting Technique for Protein Degradation in Drug Discovery. BioEssays : news and reviews in molecular, cellular and developmental biology 2018, 40 (4), e1700247.

119. Neklesa, T. K.; Winkler, J. D.; Crews, C. M., Targeted protein degradation by PROTACs. Pharmacol Ther 2017, 174, 138-144.

120. Liu, J.; Chen, H.; Kaniskan, H. Ü.; Xie, L.; Chen, X.; Jin, J.; Wei, W., TF-PROTACs Enable Targeted Degradation of Transcription Factors. Journal of the American Chemical Society 2021, 143 (23), $8902-$ 8910.

121. Doelle, A.; Adhikari, B.; Kraemer, A.; Weckesser, J.; Berner, N.; Berger, L.-M.; Diebold, M.; Szewczyk, M.; Barsyte-Lovejoy, D.; Arrowsmith, C.; Gerbel, J.; Loehr, F.; Doetsch, V.; Eilers, M.; HeinzImeir, S.; Kuester, B.; Sotriffer, C.; Wolf, E.; Knapp, S., Design, Synthesis and Evaluation of WDrepeat containing protein 5 (WDR5) degraders. bioRxiv 2021, 2021.04.12.439490.

122. Bondeson, D. P.; Mares, A.; Smith, I. E.; Ko, E.; Campos, S.; Miah, A. H.; Mulholland, K. E.; Routly, N.; Buckley, D. L.; Gustafson, J. L.; Zinn, N.; Grandi, P.; Shimamura, S.; Bergamini, G.; Faelth-Savitski, M.; Bantscheff, M.; Cox, C.; Gordon, D. A.; Willard, R. R.; Flanagan, J. J.; Casillas, L. N.; Votta, B. J.; den Besten, W.; Famm, K.; Kruidenier, L.; Carter, P. S.; Harling, J. D.; Churcher, I.; Crews, C. M., Catalytic in vivo protein knockdown by small-molecule PROTACs. Nat Chem Biol 2015, 11 (8), 611-7.

123. Burslem, G. M.; Smith, B. E.; Lai, A. C.; Jaime-Figueroa, S.; McQuaid, D. C.; Bondeson, D. P.; Toure, M.; Dong, H.; Qian, Y.; Wang, J.; Crew, A. P.; Hines, J.; Crews, C. M., The Advantages of Targeted Protein Degradation Over Inhibition: An RTK Case Study. Cell Chem Biol 2018, 25 (1), 67-77.e3.

124. Smith, B. E.; Wang, S. L.; Jaime-Figueroa, S.; Harbin, A.; Wang, J.; Hamman, B. D.; Crews, C. M., Differential PROTAC substrate specificity dictated by orientation of recruited E3 ligase. Nature Communications 2019, 10 (1), 131. 
125. Metzger, M. B.; Pruneda, J. N.; Klevit, R. E.; Weissman, A. M., RING-type E3 ligases: master manipulators of E2 ubiquitin-conjugating enzymes and ubiquitination. Biochim Biophys Acta 2014, 1843 (1), 47-60.

126. George, A. J.; Hoffiz, Y. C.; Charles, A. J.; Zhu, Y.; Mabb, A. M., A Comprehensive Atlas of E3 Ubiquitin Ligase Mutations in Neurological Disorders. Frontiers in Genetics 2018, 9 (29).

127. Lescouzères, L.; Bomont, P., E3 Ubiquitin Ligases in Neurological Diseases: Focus on Gigaxonin and Autophagy. Frontiers in physiology 2020, 11, 1022.

128. Zhao, Q.; Lan, T.; Su, S.; Rao, Y., Induction of apoptosis in MDA-MB-231 breast cancer cells by a PARP1-targeting PROTAC small molecule. Chem Commun (Camb) 2019, 55 (3), 369-372.

129. Tinworth, C. P.; Lithgow, H.; Dittus, L.; Bassi, Z. I.; Hughes, S. E.; Muelbaier, M.; Dai, H.; Smith, I. E. D.; Kerr, W. J.; Burley, G. A.; Bantscheff, M.; Harling, J. D., PROTAC-Mediated Degradation of Bruton's Tyrosine Kinase Is Inhibited by Covalent Binding. ACS Chem Biol 2019, 14 (3), 342-347.

130. Ward, C. C.; Kleinman, J. I.; Brittain, S. M.; Lee, P. S.; Chung, C. Y. S.; Kim, K.; Petri, Y.; Thomas, J. R.; Tallarico, J. A.; McKenna, J. M.; Schirle, M.; Nomura, D. K., Covalent Ligand Screening Uncovers a RNF4 E3 Ligase Recruiter for Targeted Protein Degradation Applications. ACS Chem Biol 2019, 14 (11), 2430-2440.

131. Ottis, P.; Toure, M.; Cromm, P. M.; Ko, E.; Gustafson, J. L.; Crews, C. M., Assessing Different E3 Ligases for Small Molecule Induced Protein Ubiquitination and Degradation. ACS Chem Biol 2017, 12 (10), 2570-2578.

132. Panicker, N.; Dawson, V. L.; Dawson, T. M., Activation mechanisms of the E3 ubiquitin ligase parkin. Biochem J 2017, 474 (18), 3075-3086.

133. Zhang, X.; Crowley, V. M.; Wucherpfennig, T. G.; Dix, M. M.; Cravatt, B. F., Electrophilic PROTACs that degrade nuclear proteins by engaging DCAF16. Nat Chem Biol 2019, 15 (7), 737-746.

134. Ito, T.; Ando, H.; Suzuki, T.; Ogura, T.; Hotta, K.; Imamura, Y.; Yamaguchi, Y.; Handa, H., Identification of a primary target of thalidomide teratogenicity. Science 2010, 327 (5971), 1345-50.

135. Donovan, K. A.; An, J.; Nowak, R. P.; Yuan, J. C.; Fink, E. C.; Berry, B. C.; Ebert, B. L.; Fischer, E. S., Thalidomide promotes degradation of SALL4, a transcription factor implicated in Duane Radial Ray syndrome. Elife 2018, 7.

136. Asatsuma-Okumura, T.; Ando, H.; De Simone, M.; Yamamoto, J.; Sato, T.; Shimizu, N.; Asakawa, K.; Yamaguchi, Y.; Ito, T.; Guerrini, L.; Handa, H., p63 is a cereblon substrate involved in thalidomide teratogenicity. Nat Chem Biol 2019, 15 (11), 1077-1084.

137. Fischer, E. S.; Böhm, K.; Lydeard, J. R.; Yang, H.; Stadler, M. B.; Cavadini, S.; Nagel, J.; Serluca, F.; Acker, V.; Lingaraju, G. M.; Tichkule, R. B.; Schebesta, M.; Forrester, W. C.; Schirle, M.; Hassiepen, U.; Ottl, J.; Hild, M.; Beckwith, R. E.; Harper, J. W.; Jenkins, J. L.; Thomä, N. H., Structure of the DDB1CRBN E3 ubiquitin ligase in complex with thalidomide. Nature 2014, 512 (7512), 49-53. 
138. Botting, J., The History of Thalidomide. Drug news \& perspectives 2002, 15 (9), 604-611.

139. Liu, Y.; Huang, X.; He, X.; Zhou, Y.; Jiang, X.; Chen-Kiang, S.; Jaffrey, S. R.; Xu, G., A novel effect of thalidomide and its analogs: suppression of cereblon ubiquitination enhances ubiquitin ligase function. Faseb j 2015, 29 (12), 4829-39.

140. Galdeano, C.; Gadd, M. S.; Soares, P.; Scaffidi, S.; Van Molle, I.; Birced, I.; Hewitt, S.; Dias, D. M.; Ciulli, A., Structure-Guided Design and Optimization of Small Molecules Targeting the ProteinProtein Interaction between the von Hippel-Lindau (VHL) E3 Ubiquitin Ligase and the Hypoxia Inducible Factor (HIF) Alpha Subunit with in Vitro Nanomolar Affinities. Journal of Medicinal Chemistry 2014, 57 (20), 8657-8663.

141. Cardote, T. A. F.; Gadd, M. S.; Ciulli, A., Crystal Structure of the Cul2-Rbx1-EloBC-VHL Ubiquitin Ligase Complex. Structure 2017, 25 (6), 901-911.e3.

142. Cyrus, K.; Wehenkel, M.; Choi, E. Y.; Lee, H.; Swanson, H.; Kim, K. B., Jostling for position: optimizing linker location in the design of estrogen receptor-targeting PROTACs. ChemMedChem 2010, 5 (7), 979-85.

143. Rodriguez-Gonzalez, A.; Cyrus, K.; Salcius, M.; Kim, K.; Crews, C. M.; Deshaies, R. J.; Sakamoto, K. M., Targeting steroid hormone receptors for ubiquitination and degradation in breast and prostate cancer. Oncogene 2008, 27 (57), 7201-11.

144. Sharma, L. K.; Lee, N. R.; Jang, E. R.; Lei, B.; Zhan, C. G.; Lee, W.; Kim, K. B., Activity-based nearinfrared fluorescent probe for LMP7: a chemical proteomics tool for the immunoproteasome in living cells. Chembiochem 2012, 13 (13), 1899-903.

145. Winter, G. E.; Buckley, D. L.; Paulk, J.; Roberts, J. M.; Souza, A.; Dhe-Paganon, S.; Bradner, J. E., DRUG DEVELOPMENT. Phthalimide conjugation as a strategy for in vivo target protein degradation. Science 2015, 348 (6241), 1376-81.

146. Lu, J.; Qian, Y.; Altieri, M.; Dong, H.; Wang, J.; Raina, K.; Hines, J.; Winkler, J. D.; Crew, A. P.; Coleman, K.; Crews, C. M., Hijacking the E3 Ubiquitin Ligase Cereblon to Efficiently Target BRD4. Chem Biol 2015, 22 (6), 755-63.

147. Zengerle, M.; Chan, K. H.; Ciulli, A., Selective Small Molecule Induced Degradation of the BET Bromodomain Protein BRD4. ACS Chem Bio/ 2015, 10 (8), 1770-7.

148. Lai, A. C.; Toure, M.; Hellerschmied, D.; Salami, J.; Jaime-Figueroa, S.; Ko, E.; Hines, J.; Crews, C. M., Modular PROTAC Design for the Degradation of Oncogenic BCR-ABL. Angew Chem Int Ed Engl 2016, 55 (2), 807-10.

149. Robb, C. M.; Contreras, J. I.; Kour, S.; Taylor, M. A.; Abid, M.; Sonawane, Y. A.; Zahid, M.; Murry, D. J.; Natarajan, A.; Rana, S., Chemically induced degradation of CDK9 by a proteolysis targeting chimera (PROTAC). Chem Commun (Camb) 2017, 53 (54), 7577-7580. 
150. Bondeson, D. P.; Smith, B. E.; Burslem, G. M.; Buhimschi, A. D.; Hines, J.; Jaime-Figueroa, S.; Wang, J.; Hamman, B. D.; Ishchenko, A.; Crews, C. M., Lessons in PROTAC Design from Selective Degradation with a Promiscuous Warhead. Cell Chem Biol 2018, 25 (1), 78-87.e5.

151. Zhang, C.; Han, X. R.; Yang, X.; Jiang, B.; Liu, J.; Xiong, Y.; Jin, J., Proteolysis Targeting Chimeras (PROTACs) of Anaplastic Lymphoma Kinase (ALK). Eur J Med Chem 2018, 151, 304-314.

152. Crew, A. P.; Raina, K.; Dong, H.; Qian, Y.; Wang, J.; Vigil, D.; Serebrenik, Y. V.; Hamman, B. D.; Morgan, A.; Ferraro, C.; Siu, K.; Neklesa, T. K.; Winkler, J. D.; Coleman, K. G.; Crews, C. M., Identification and Characterization of Von Hippel-Lindau-Recruiting Proteolysis Targeting Chimeras (PROTACs) of TANK-Binding Kinase 1. J Med Chem 2018, 61 (2), 583-598.

153. Huang, H. T.; Dobrovolsky, D.; Paulk, J.; Yang, G.; Weisberg, E. L.; Doctor, Z. M.; Buckley, D. L.; Cho, J. H.; Ko, E.; Jang, J.; Shi, K.; Choi, H. G.; Griffin, J. D.; Li, Y.; Treon, S. P.; Fischer, E. S.; Bradner, J. E.; Tan, L.; Gray, N. S., A Chemoproteomic Approach to Query the Degradable Kinome Using a Multikinase Degrader. Cell Chem Biol 2018, 25 (1), 88-99.e6.

154. Olson, C. M.; Jiang, B.; Erb, M. A.; Liang, Y.; Doctor, Z. M.; Zhang, Z.; Zhang, T.; Kwiatkowski, N.; Boukhali, M.; Green, J. L.; Haas, W.; Nomanbhoy, T.; Fischer, E. S.; Young, R. A.; Bradner, J. E.; Winter, G. E.; Gray, N. S., Pharmacological perturbation of CDK9 using selective CDK9 inhibition or degradation. Nat Chem Biol 2018, 14 (2), 163-170.

155. Powell, C. E.; Gao, Y.; Tan, L.; Donovan, K. A.; Nowak, R. P.; Loehr, A.; Bahcall, M.; Fischer, E. S.; Jänne, P. A.; George, R. E.; Gray, N. S., Chemically Induced Degradation of Anaplastic Lymphoma Kinase (ALK). J Med Chem 2018, 61 (9), 4249-4255.

156. Cromm, P. M.; Samarasinghe, K. T. G.; Hines, J.; Crews, C. M., Addressing Kinase-Independent Functions of Fak via PROTAC-Mediated Degradation. J Am Chem Soc 2018, 140 (49), 17019-17026.

157. Buhimschi, A. D.; Armstrong, H. A.; Toure, M.; Jaime-Figueroa, S.; Chen, T. L.; Lehman, A. M.; Woyach, J. A.; Johnson, A. J.; Byrd, J. C.; Crews, C. M., Targeting the C481S Ibrutinib-Resistance Mutation in Bruton's Tyrosine Kinase Using PROTAC-Mediated Degradation. Biochemistry 2018, 57 (26), 3564-3575.

158. Chen, H.; Chen, F.; Liu, N.; Wang, X.; Gou, S., Chemically induced degradation of CK2 by proteolysis targeting chimeras based on a ubiquitin-proteasome pathway. Bioorg Chem 2018, 81, 536544.

159. Sun, Y.; Zhao, X.; Ding, N.; Gao, H.; Wu, Y.; Yang, Y.; Zhao, M.; Hwang, J.; Song, Y.; Liu, W.; Rao, Y., PROTAC-induced BTK degradation as a novel therapy for mutated BTK C481S induced ibrutinibresistant B-cell malignancies. Cell Res 2018, 28 (7), 779-781.

160. Zorba, A.; Nguyen, C.; Xu, Y.; Starr, J.; Borzilleri, K.; Smith, J.; Zhu, H.; Farley, K. A.; Ding, W.; Schiemer, J.; Feng, X.; Chang, J. S.; Uccello, D. P.; Young, J. A.; Garcia-Irrizary, C. N.; Czabaniuk, L.; Schuff, B.; Oliver, R.; Montgomery, J.; Hayward, M. M.; Coe, J.; Chen, J.; Niosi, M.; Luthra, S.; Shah, J. C.; El- 
Kattan, A.; Qiu, X.; West, G. M.; Noe, M. C.; Shanmugasundaram, V.; Gilbert, A. M.; Brown, M. F.; Calabrese, M. F., Delineating the role of cooperativity in the design of potent PROTACs for BTK. Proc Natl Acad Sci U S A 2018, 115 (31), E7285-e7292.

161. Bian, J.; Ren, J.; Li, Y.; Wang, J.; Xu, X.; Feng, Y.; Tang, H.; Wang, Y.; Li, Z., Discovery of Wogoninbased PROTACs against CDK9 and capable of achieving antitumor activity. Bioorg Chem 2018, 81, 373381.

162. Kang, C. H.; Lee, D. H.; Lee, C. O.; Du Ha, J.; Park, C. H.; Hwang, J. Y., Induced protein degradation of anaplastic lymphoma kinase (ALK) by proteolysis targeting chimera (PROTAC). Biochem Biophys Res Commun 2018, 505 (2), 542-547.

163. Burslem, G. M.; Song, J.; Chen, X.; Hines, J.; Crews, C. M., Enhancing Antiproliferative Activity and Selectivity of a FLT-3 Inhibitor by Proteolysis Targeting Chimera Conversion. J Am Chem Soc 2018, 140 (48), 16428-16432.

164. Farnaby, W.; Koegl, M.; Roy, M. J.; Whitworth, C.; Diers, E.; Trainor, N.; Zollman, D.; Steurer, S.; Karolyi-Oezguer, J.; Riedmueller, C.; Gmaschitz, T.; Wachter, J.; Dank, C.; Galant, M.; Sharps, B.; Rumpel, K.; Traxler, E.; Gerstberger, T.; Schnitzer, R.; Petermann, O.; Greb, P.; Weinstabl, H.; Bader, G.; Zoephel, A.; Weiss-Puxbaum, A.; Ehrenhöfer-Wölfer, K.; Wöhrle, S.; Boehmelt, G.; Rinnenthal, J.; Arnhof, H.; Wiechens, N.; Wu, M. Y.; Owen-Hughes, T.; Ettmayer, P.; Pearson, M.; McConnell, D. B.; Ciulli, A., BAF complex vulnerabilities in cancer demonstrated via structure-based PROTAC design. Nat Chem Biol 2019, 15 (7), 672-680.

165. Gechijian, L. N.; Buckley, D. L.; Lawlor, M. A.; Reyes, J. M.; Paulk, J.; Ott, C. J.; Winter, G. E.; Erb, M. A.; Scott, T. G.; Xu, M.; Seo, H. S.; Dhe-Paganon, S.; Kwiatkowski, N. P.; Perry, J. A.; Qi, J.; Gray, N. S.; Bradner, J. E., Functional TRIM24 degrader via conjugation of ineffectual bromodomain and VHL ligands. Nat Chem Biol 2018, 14 (4), 405-412.

166. Chu, T. T.; Gao, N.; Li, Q. Q.; Chen, P. G.; Yang, X. F.; Chen, Y. X.; Zhao, Y. F.; Li, Y. M., Specific Knockdown of Endogenous Tau Protein by Peptide-Directed Ubiquitin-Proteasome Degradation. Cell Chem Biol 2016, 23 (4), 453-61.

167. Wang, F.; Jeon, K. O.; Salovich, J. M.; Macdonald, J. D.; Alvarado, J.; Gogliotti, R. D.; Phan, J.; Olejniczak, E. T.; Sun, Q.; Wang, S.; Camper, D.; Yuh, J. P.; Shaw, J. G.; Sai, J.; Rossanese, O. W.; Tansey, W. P.; Stauffer, S. R.; Fesik, S. W., Discovery of Potent 2-Aryl-6,7-dihydro-5 H-pyrrolo[1,2- a]imidazoles as WDR5-WIN-Site Inhibitors Using Fragment-Based Methods and Structure-Based Design. J Med Chem 2018, 61 (13), 5623-5642.

168. Chacón Simon, S.; Wang, F.; Thomas, L. R.; Phan, J.; Zhao, B.; Olejniczak, E. T.; Macdonald, J. D.; Shaw, J. G.; Schlund, C.; Payne, W.; Creighton, J.; Stauffer, S. R.; Waterson, A. G.; Tansey, W. P.; Fesik, S. W., Discovery of WD Repeat-Containing Protein 5 (WDR5)-MYC Inhibitors Using FragmentBased Methods and Structure-Based Design. J Med Chem 2020, 63 (8), 4315-4333. 
169. Carpino, L. A.; El-Faham, A.; Minor, C. A.; Albericio, F., Advantageous applications of azabenzotriazole (triazolopyridine)-based coupling reagents to solid-phase peptide synthesis. Journal of the Chemical Society, Chemical Communications 1994, (2), 201-203.

170. Valeur, E.; Bradley, M., Amide bond formation: beyond the myth of coupling reagents. Chem Soc Rev 2009, 38 (2), 606-31.

171. Carpino, L. A., 1-Hydroxy-7-azabenzotriazole. An efficient peptide coupling additive. Journal of the American Chemical Society 1993, 115 (10), 4397-4398.

172. Al-Warhi, T. I.; Al-Hazimi, H. M. A.; El-Faham, A., Recent development in peptide coupling reagents. Journal of Saudi Chemical Society 2012, 16 (2), 97-116.

173. Tang, X.; Tang, G.; Wang, H.; Luo, L.; Yang, D., A convenient and highly efficient synthesis of one kind of peptide nucleic acid monomer. Bulletin of the Chemical Society of Ethiopia 2012, 26.

174. Dölle, A.; Adhikari, B.; Krämer, A.; Weckesser, J.; Berner, N.; Berger, L.-M.; Diebold, M.; Szewczyk, M. M.; Barsyte-Lovejoy, D.; Arrowsmith, C. H.; Gebel, J.; Löhr, F.; Dötsch, V.; Eilers, M.; Heinzlmeir, S.; Kuster, B.; Sotriffer, C.; Wolf, E.; Knapp, S., Design, Synthesis, and Evaluation of WDRepeat-Containing Protein 5 (WDR5) Degraders. Journal of Medicinal Chemistry 2021.

175. Dale, N. C.; Johnstone, E. K. M.; White, C. W.; Pfleger, K. D. G., NanoBRET: The Bright Future of Proximity-Based Assays. Frontiers in Bioengineering and Biotechnology 2019, 7 (56).

176. Riching, K. M.; Mahan, S.; Corona, C. R.; McDougall, M.; Vasta, J. D.; Robers, M. B.; Urh, M.; Daniels, D. L., Quantitative Live-Cell Kinetic Degradation and Mechanistic Profiling of PROTAC Mode of Action. ACS Chemical Biology 2018, 13 (9), 2758-2770.

177. Guarnaccia, A. D.; Rose, K. L.; Wang, J.; Zhao, B.; Popay, T. M.; Wang, C. E.; Guerrazzi, K.; Hill, S.; Woodley, C. M.; Hansen, T. J.; Lorey, S. L.; Shaw, J. G.; Payne, W. G.; Weissmiller, A. M.; Olejniczak, E. T.; Fesik, S. W.; Liu, Q.; Tansey, W. P., Impact of WIN site inhibitor on the WDR5 interactome. Cell Rep 2021, 34 (3), 108636.

178. Fisher, S. L.; Phillips, A. J., Targeted protein degradation and the enzymology of degraders. Curr Opin Chem Biol 2018, 44, 47-55.

179. Roy, M. J.; Winkler, S.; Hughes, S. J.; Whitworth, C.; Galant, M.; Farnaby, W.; Rumpel, K.; Ciulli, A., SPR-Measured Dissociation Kinetics of PROTAC Ternary Complexes Influence Target Degradation Rate. ACS Chem Biol 2019, 14 (3), 361-368.

180. Gadd, M. S.; Testa, A.; Lucas, X.; Chan, K. H.; Chen, W.; Lamont, D. J.; Zengerle, M.; Ciulli, A., Structural basis of PROTAC cooperative recognition for selective protein degradation. Nat Chem Biol 2017, 13 (5), 514-521.

181. Testa, A.; Hughes, S. J.; Lucas, X.; Wright, J. E.; Ciulli, A., Structure-Based Design of a Macrocyclic PROTAC. Angew Chem Int Ed Eng/ 2020, 59 (4), 1727-1734. 
182. Meyer, C.; Burmeister, T.; Gröger, D.; Tsaur, G.; Fechina, L.; Renneville, A.; Sutton, R.; Venn, N. C.; Emerenciano, M.; Pombo-de-Oliveira, M. S.; Barbieri Blunck, C.; Almeida Lopes, B.; Zuna, J.; Trka, J.; Ballerini, P.; Lapillonne, H.; De Braekeleer, M.; Cazzaniga, G.; Corral Abascal, L.; van der Velden, V. H. J.; Delabesse, E.; Park, T. S.; Oh, S. H.; Silva, M. L. M.; Lund-Aho, T.; Juvonen, V.; Moore, A. S.; Heidenreich, O.; Vormoor, J.; Zerkalenkova, E.; Olshanskaya, Y.; Bueno, C.; Menendez, P.; TeiglerSchlegel, A.; zur Stadt, U.; Lentes, J.; Göhring, G.; Kustanovich, A.; Aleinikova, O.; Schäfer, B. W.; Kubetzko, S.; Madsen, H. O.; Gruhn, B.; Duarte, X.; Gameiro, P.; Lippert, E.; Bidet, A.; Cayuela, J. M.; Clappier, E.; Alonso, C. N.; Zwaan, C. M.; van den Heuvel-Eibrink, M. M.; Izraeli, S.; Trakhtenbrot, L.; Archer, P.; Hancock, J.; Möricke, A.; Alten, J.; Schrappe, M.; Stanulla, M.; Strehl, S.; Attarbaschi, A.; Dworzak, M.; Haas, O. A.; Panzer-Grümayer, R.; Sedék, L.; Szczepański, T.; Caye, A.; Suarez, L.; Cavé, H.; Marschalek, R., The MLL recombinome of acute leukemias in 2017. Leukemia 2018, 32 (2), 273-284.

183. Lorenzin, F.; Benary, U.; Baluapuri, A.; Walz, S.; Jung, L. A.; von Eyss, B.; Kisker, C.; Wolf, J.; Eilers, M.; Wolf, E., Different promoter affinities account for specificity in MYC-dependent gene regulation. Elife 2016, 5.

184. Walz, S.; Lorenzin, F.; Morton, J.; Wiese, K. E.; von Eyss, B.; Herold, S.; Rycak, L.; Dumay-Odelot, H.; Karim, S.; Bartkuhn, M.; Roels, F.; Wüstefeld, T.; Fischer, M.; Teichmann, M.; Zender, L.; Wei, C. L.; Sansom, O.; Wolf, E.; Eilers, M., Activation and repression by oncogenic MYC shape tumour-specific gene expression profiles. Nature 2014, 511 (7510), 483-7.

185. Chen, W.; Chen, X.; Li, D.; Wang, X.; Long, G.; Jiang, Z.; You, Q.; Guo, X., Discovery of a potent MLL1 and WDR5 protein-protein interaction inhibitor with in vivo antitumor activity. European Journal of Medicinal Chemistry 2021, 223, 113677.

186. Ye, X.; Chen, G.; Jin, J.; Zhang, B.; Wang, Y.; Cai, Z.; Ye, F., The Development of Inhibitors Targeting the Mixed Lineage Leukemia 1 (MLL1)-WD Repeat Domain 5 Protein (WDR5) Protein- Protein Interaction. Curr Med Chem 2020, 27 (33), 5530-5542.

187. Mullard, A., Targeted protein degraders crowd into the clinic. Nature reviews. Drug discovery 2021, 20 (4), 247-250.

188. Penzo, M.; Montanaro, L.; Treré, D.; Derenzini, M., The Ribosome Biogenesis-Cancer Connection. Cells 2019, 8 (1), 55.

189. Housman, G.; Byler, S.; Heerboth, S.; Lapinska, K.; Longacre, M.; Snyder, N.; Sarkar, S., Drug resistance in cancer: an overview. Cancers (Basel) 2014, 6 (3), 1769-1792.

190. Fedorov, O.; Niesen, F. H.; Knapp, S., Kinase inhibitor selectivity profiling using differential scanning fluorimetry. Methods Mol Biol 2012, 795, 109-18.

191. Wanior, M.; Preuss, F.; Ni, X.; Krämer, A.; Mathea, S.; Göbel, T.; Heidenreich, D.; Simonyi, S.; Kahnt, A. S.; Joerger, A. C.; Knapp, S., Pan-SMARCA/PB1 Bromodomain Inhibitors and Their Role in Regulating Adipogenesis. Journal of Medicinal Chemistry 2020. 


\section{Appendix}

ESI, ${ }^{1} \mathrm{H}-\mathrm{NMR},{ }^{13} \mathrm{C}-\mathrm{NMR}$ and HPLC of N-(5-bromo-2-(4-methylpiperazin-1-yl)phenyl)-3methylbenzamide

C:IXcaliburldataIAD22_3_foam 8/1/2018 10:45:08 AM

AD22_3_foam \#32-43 RT: 0.53-0.72 AV: 12 SB: 21 0.12-0.46 NL: 1.91E7

$\mathrm{T}:\{0,0\}+\mathrm{c}$ ESI !corona sid $=75.00$ det=1506.00 Full ms $[100.00-800.00]$

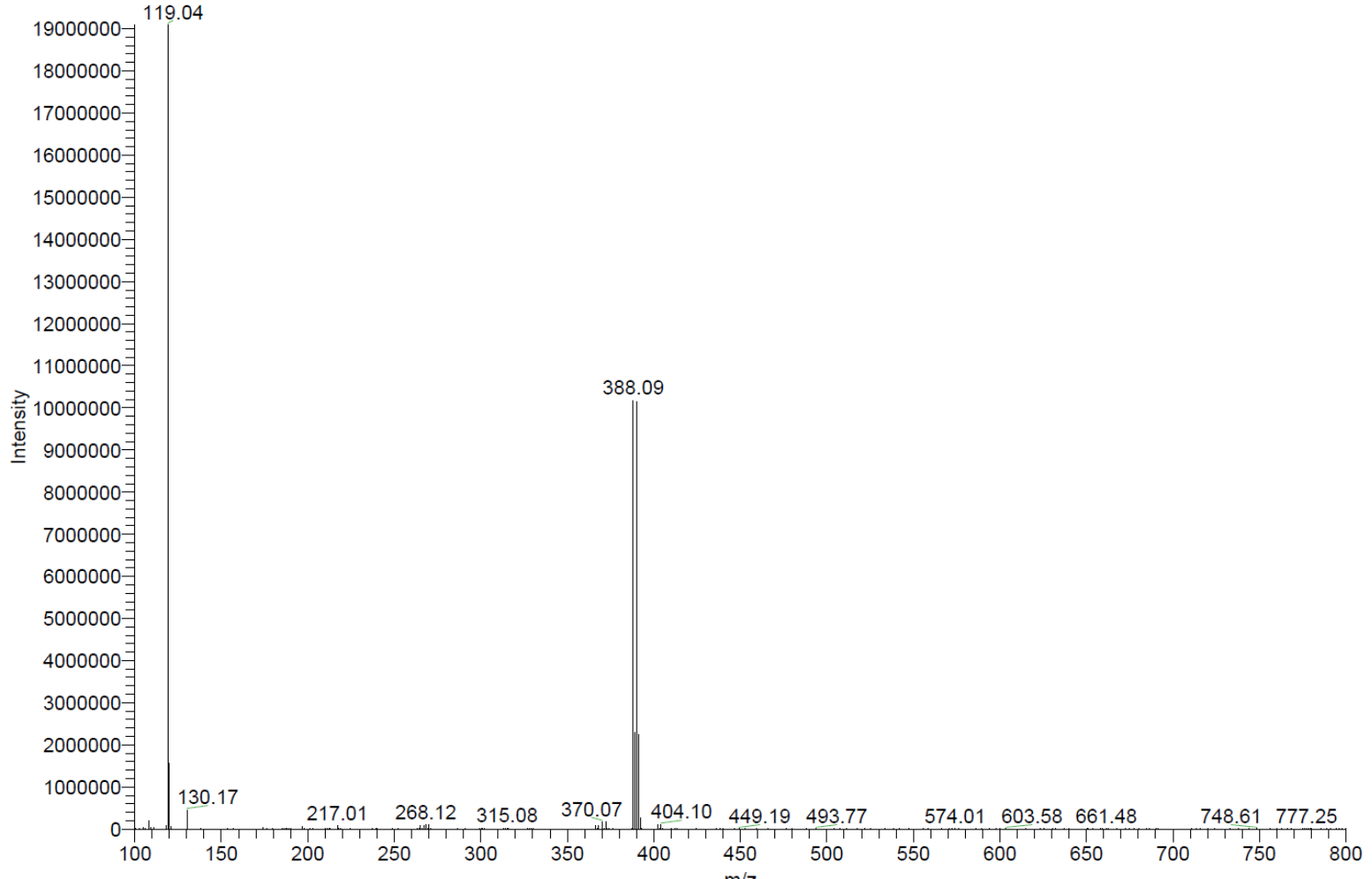

AV500-2018-08-28-adkn.25922

AD22.4
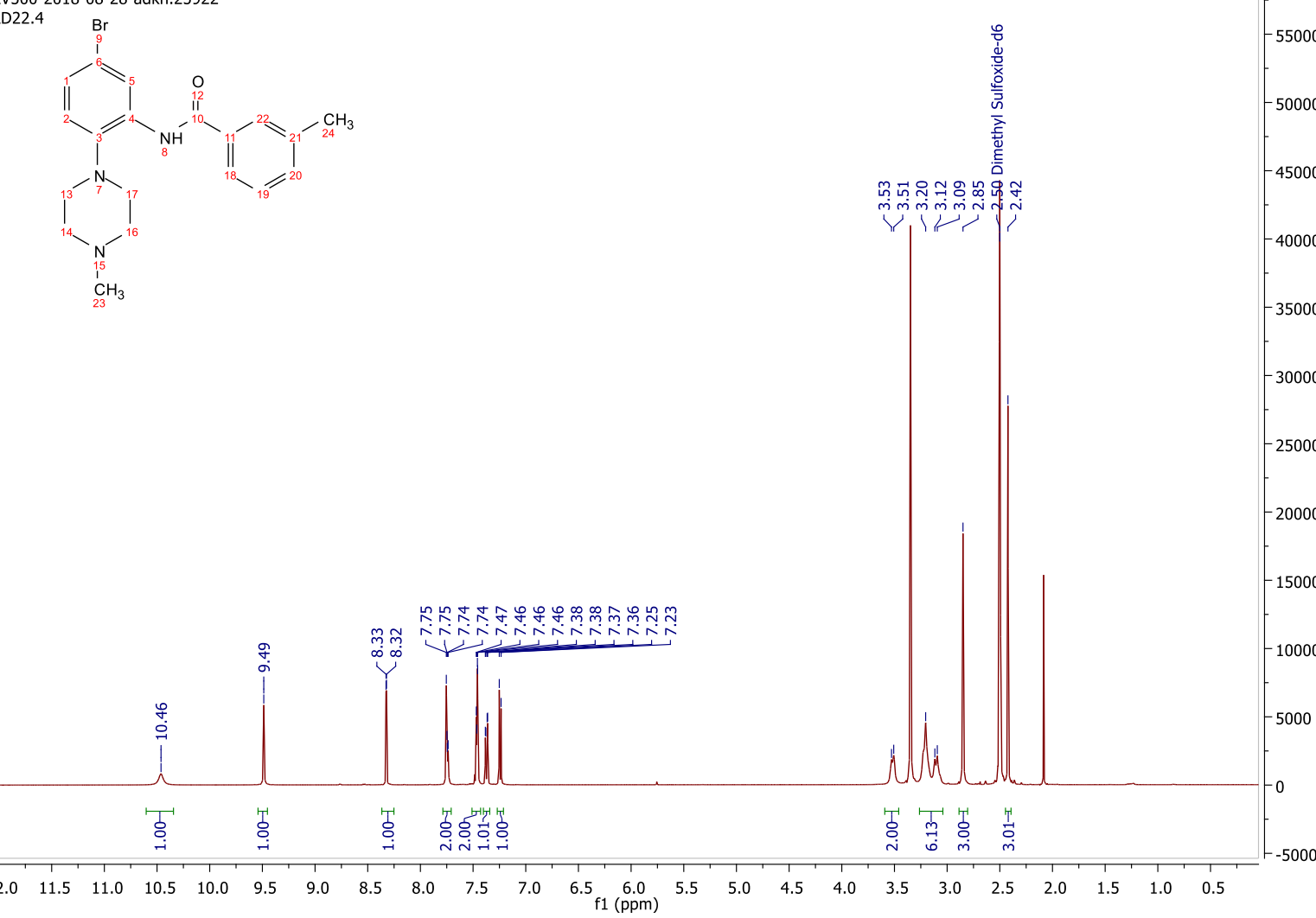

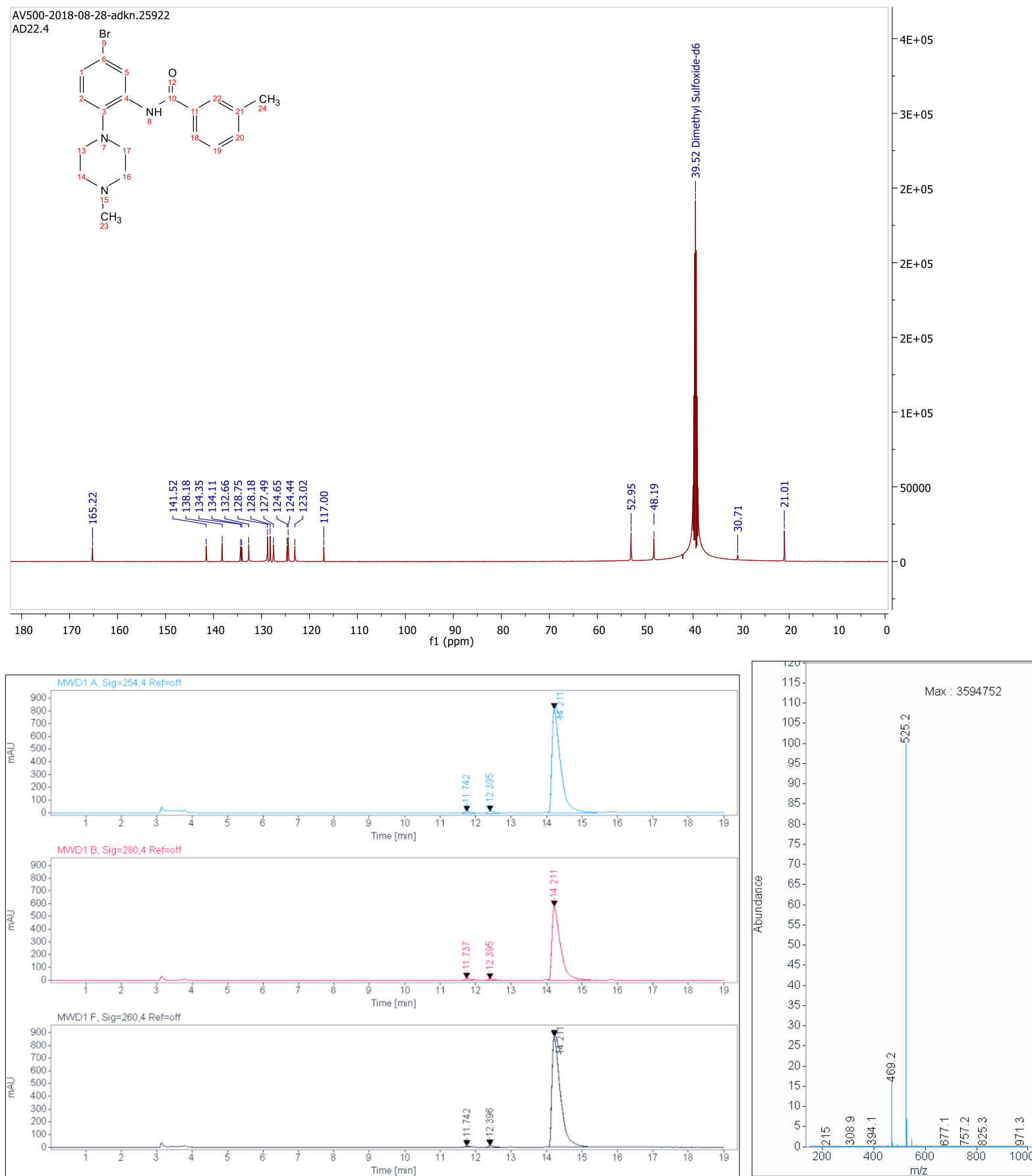

Signal: $\quad$ MWD1 A, Sig $=254,4$ Ref $=$ off

$\begin{array}{crrrr}\text { RT [min] Type } & \text { Width [min] } & \text { Area } & \text { Height } & \text { Area\% } \\ 11.742 \mathrm{MM} & 0.2481 & 242.9475 & 16.3228 & 1.6890 \\ 12.395 \mathrm{MM} & 0.2417 & 271.0590 & 18.6918 & 1.8844 \\ 14.211 \mathrm{VV} & 0.2550 & 13870.0791 & 817.4152 & 96.4266 \\ & \text { Sum } & 14384.0856 & & \end{array}$

Signal: $\quad$ MWD1 B, Sig $=280,4$ Ref $=$ off

$\begin{array}{crrrr}\text { RT [min] Type } & \text { Width [min] } & \text { Area } & \text { Height } & \text { Area\% } \\ 11.737 \mathrm{MM} & 0.2260 & 211.1424 & 15.5726 & 2.0895 \\ 12.395 \mathrm{MM} & 0.2529 & 184.8797 & 12.1860 & 1.8296 \\ 14.211 \mathrm{VV} & 0.2523 & 9708.7354 & 580.0815 & 96.0808\end{array}$




$$
\text { Sum } \quad 10104.7575
$$

Signal: $\quad$ MWD1 F, Sig=260,4 Ref=off

$\begin{array}{crrrr}\text { RT [min] Type } & \text { Width [min] } & \text { Area } & \text { Height } & \text { Area\% } \\ 11.742 \text { MM } & 0.1945 & 126.9072 & 10.8757 & 0.8444 \\ 12.396 \text { MM } & 0.2314 & 247.7028 & 17.8418 & 1.6481 \\ 14.211 \text { VV } & 0.2527 & 14654.5596 & 873.7964 & 97.5074\end{array}$

ESI and ${ }^{1} \mathrm{H}$-NMR of tert-butyl 5-(3-(3-methylbenzamido)-4-(piperazin-1-yl)phenyl)-1H-indole-1carboxylate (6a)

C:IXcaliburldatalAD40_3h

8/16/2018 12:21:42 PM

AD40_3h \#35-43 RT: 0.60-0.74 AV: 9 SB: 19 0.04-0.35 NL: 3.99E5

$\mathrm{T}:\{0, \overline{0}\}+\mathrm{c}$ ESI !corona sid $=75.00$ det $=1306.00$ Full ms $[100.00-1000.00]$

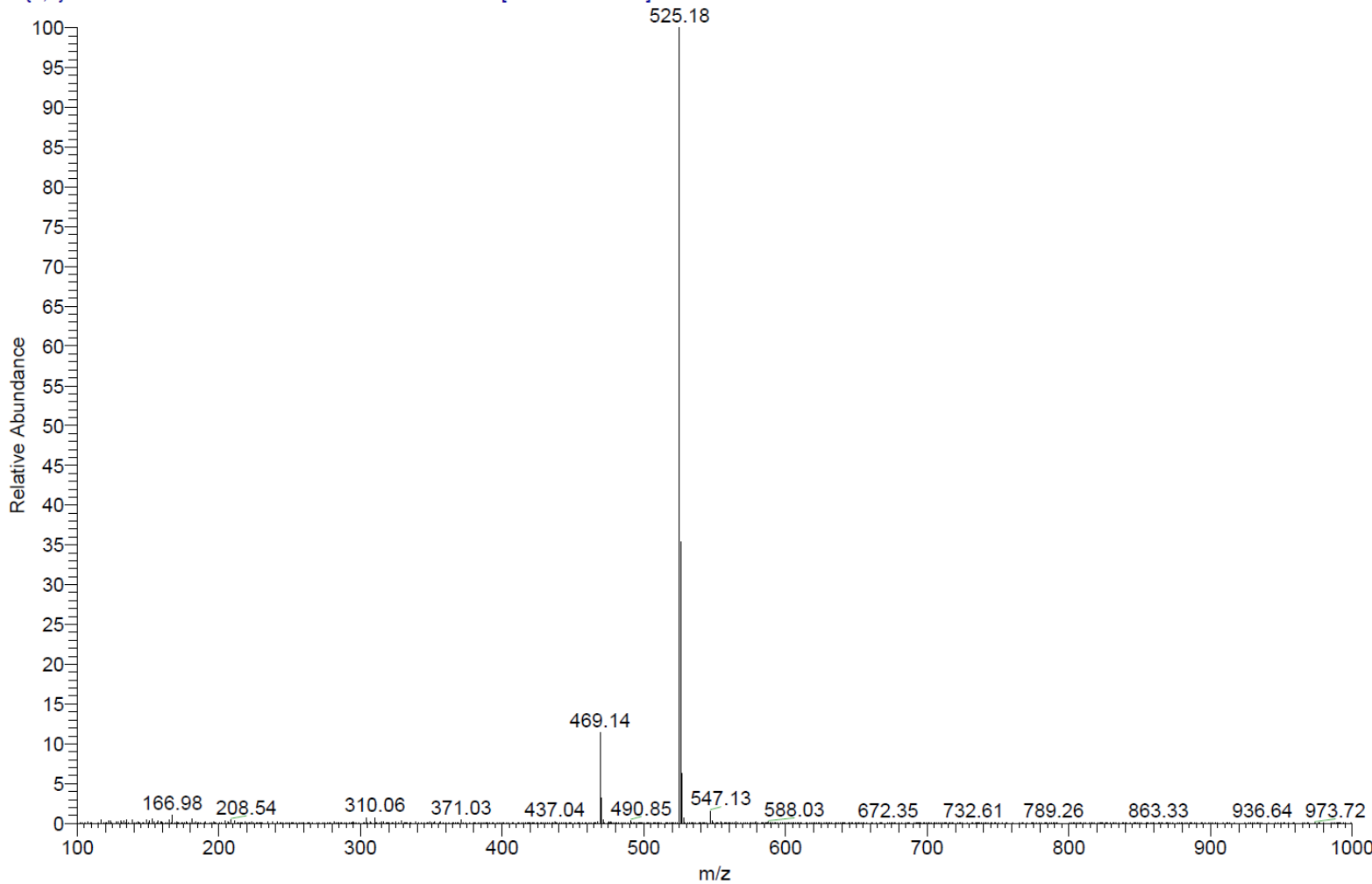




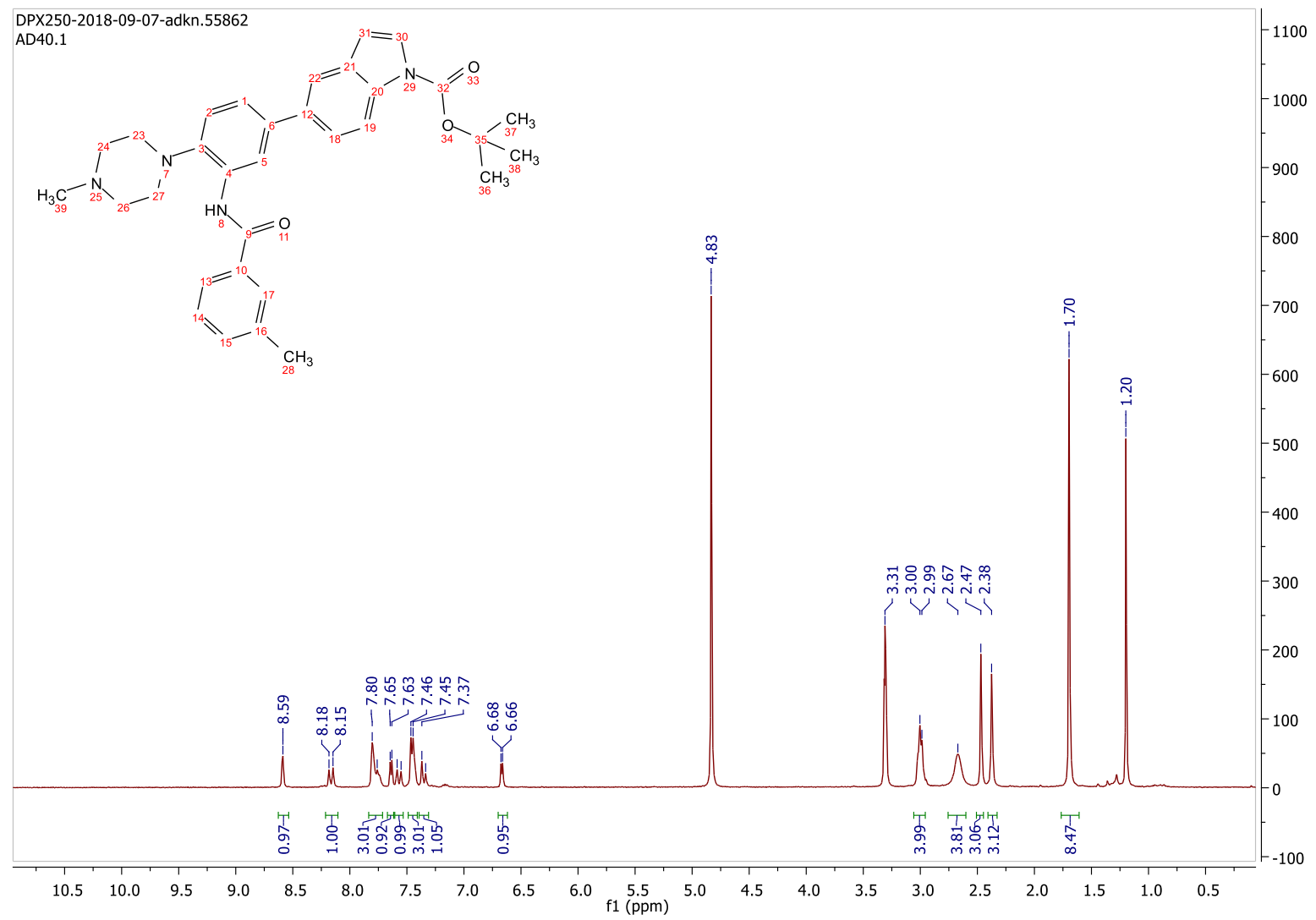




\section{ESI and ${ }^{1} \mathrm{H}-\mathrm{NMR}$ of $\mathrm{N}$-(5-(1H-indol-5-yl)-2-(piperazin-1-yl)phenyl)-3-methylbenzamide}

C:IXcaliburldataIAD37

8/13/2018 7:07:41 AM

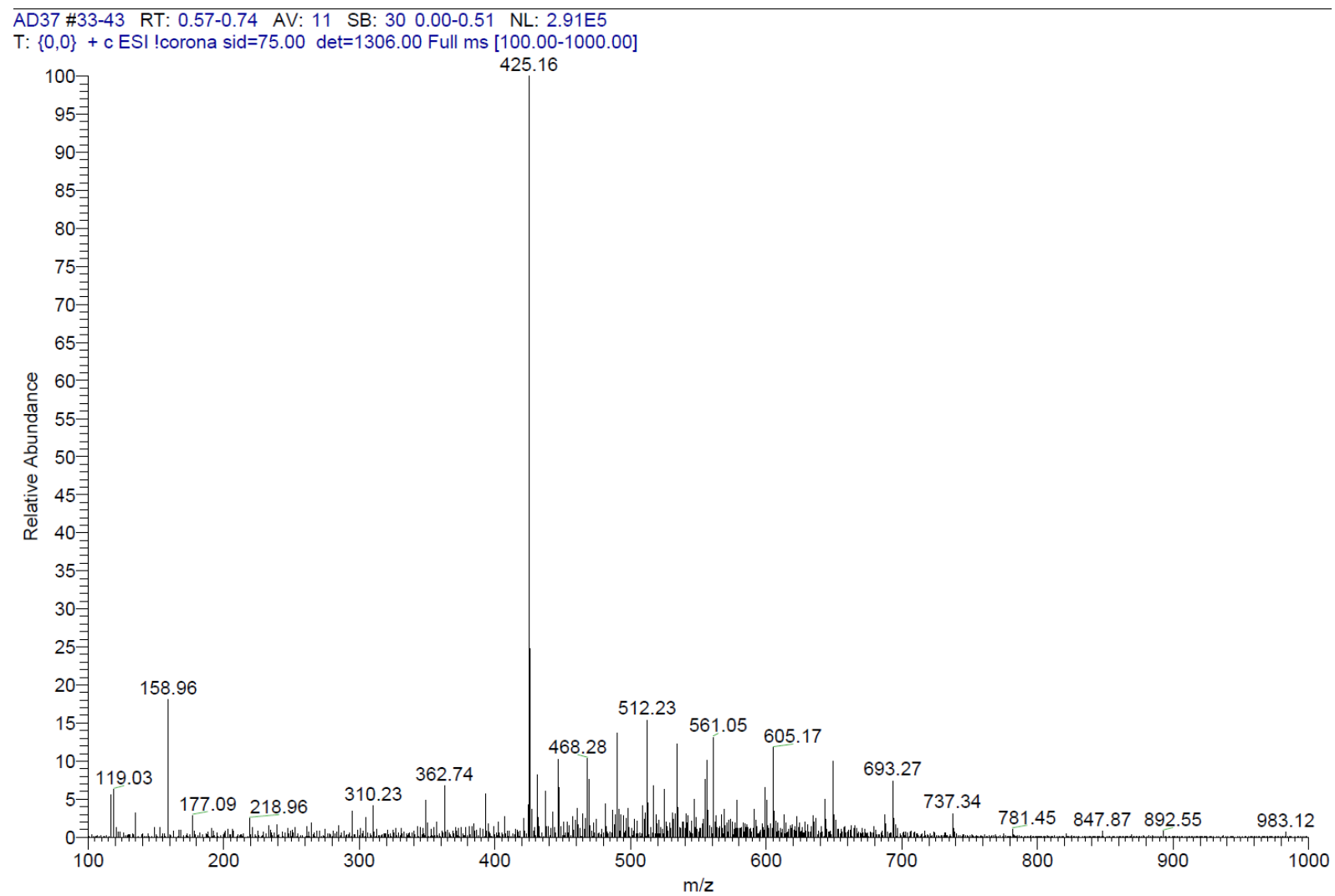

DPX250-2018-10-23-adkn.57058

AD37.1_bad resolved

1H_1D_ns CDCl3 /nmr kn 7

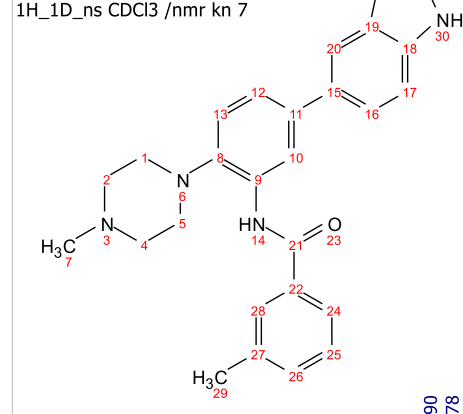
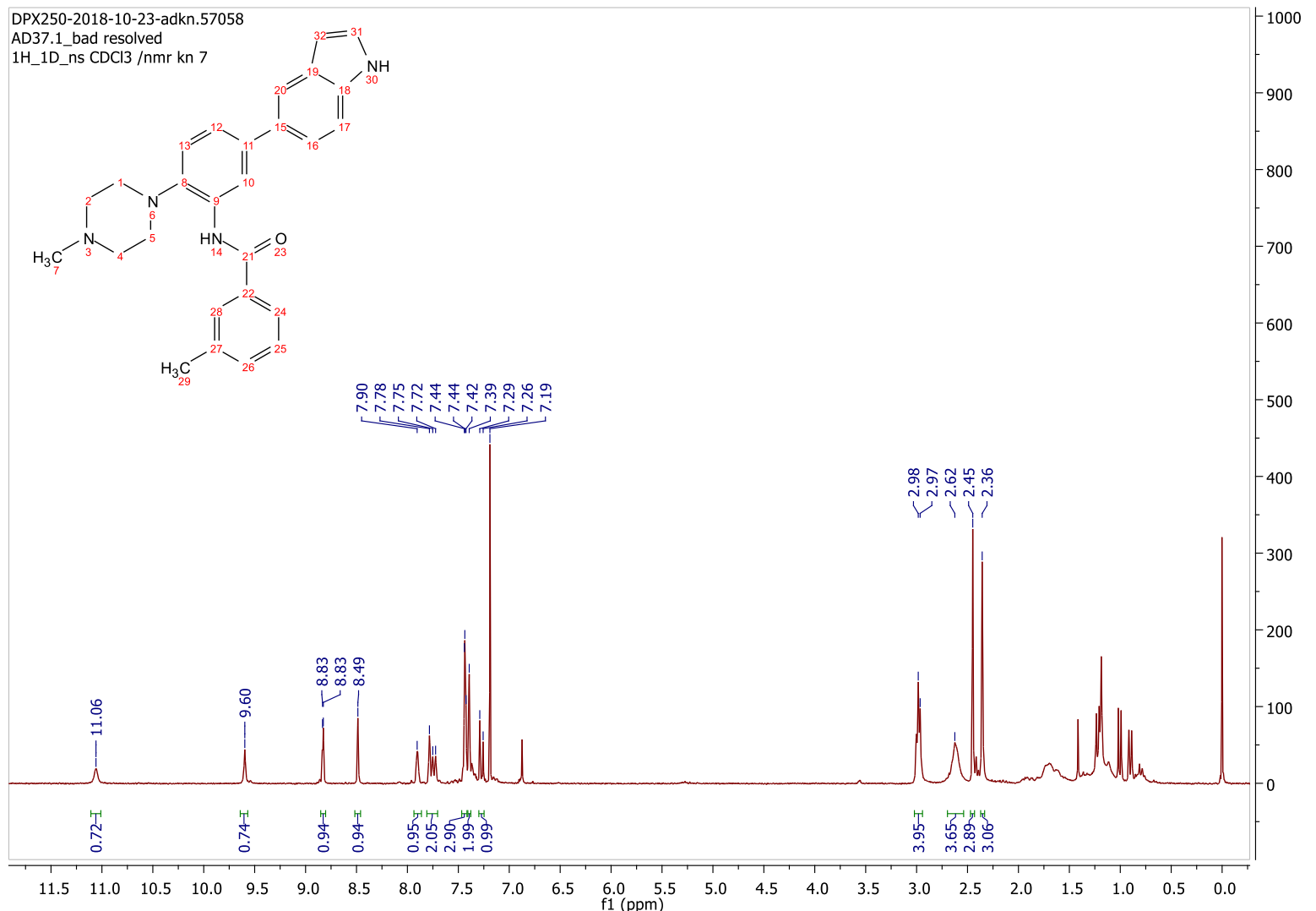
ESI, ${ }^{1} \mathrm{H}-\mathrm{NMR}$ and HPLC of tert-butyl 5-(3-(2-chloro-4-fluoro-3-methyl-5-nitrobenzamido)-4-(4methylpiperazin-1-yl)phenyl)-1H-indole-1-carboxylate

C:IXcaliburldataVAD64

12/3/2018 9:17:16 AM

AD64 \#39-44 RT: 0.68-0.77 AV: 6 SB: 26 0.07-0.52 NL: 3.49E6

$\mathrm{T}:\{0,0\}+\mathrm{c}$ ESI !corona sid $=75.00$ det $=1306.00$ Full ms [200.00-1200.00]
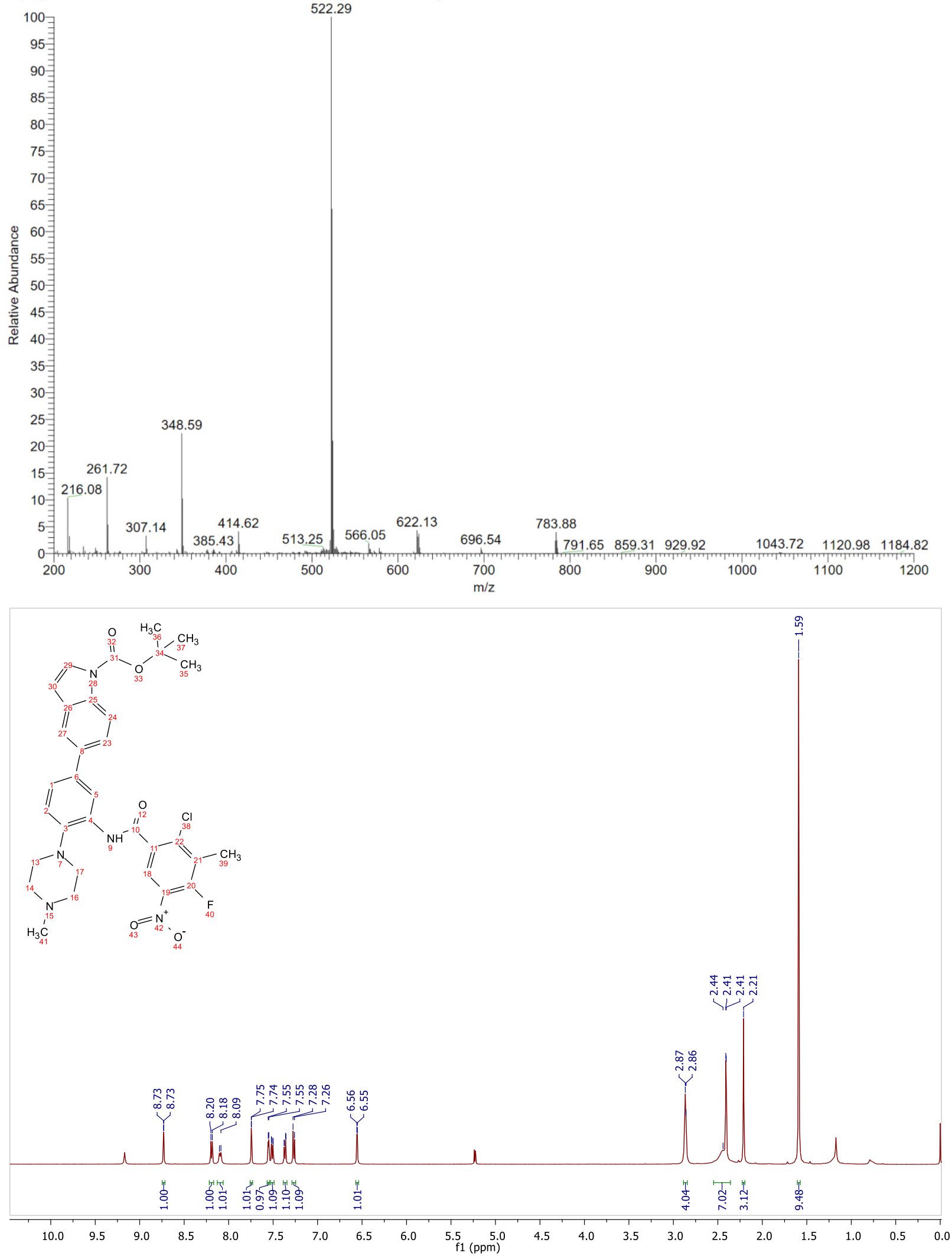
Signal: $\quad$ MSD1 TIC, MS File

$\begin{array}{crrrr}\text { RT [min] Type } & \text { Width [min] } & \text { Area } & \text { Height } & \text { Area\% } \\ 13.614 \text { BB } & 0.4825 & 440825536.0000 & 14593189.0000 & 100.0000 \\ & \text { Sum } & 440825536.0000 & & \end{array}$

Signal: $\quad$ MSD2 TIC, MS File

$\begin{array}{crrrr}\text { RT [min] Type } & \text { Width [min] } & \text { Area } & \text { Height } & \text { Area\% } \\ 12.838 \text { BV } & 0.5139 & 19723108.0000 & 546568.7500 & 19.1017 \\ 13.623 \text { VV } & 0.5145 & 21110724.0000 & 611107.0000 & 20.4456 \\ 15.941 \text { VV } & 1.0243 & 21339308.0000 & 267287.7188 & 20.6670 \\ 17.900 \mathrm{VV} & 1.7478 & 41079972.0000 & 292904.9375 & 39.7857 \\ & \text { Sum } & 103253112.0000 & & \end{array}$

Signal: $\quad$ MWD1 A, Sig=254,4 Ref=off

$\begin{array}{crrrr}\text { RT [min] Type } & \text { Width [min] } & \text { Area } & \text { Height } & \text { Area\% } \\ 12.661 \text { VV } & 0.1661 & 859.6909 & 81.4859 & 4.6480 \\ 13.414 \text { VV } & 0.2077 & 17636.1641 & 1356.2094 & 95.3520 \\ & \text { Sum } & 18495.8550 & & \end{array}$

Signal: $\quad$ MWD1 E, Sig $=280,4$ Ref=off

$\begin{array}{crrrr}\text { RT [min] Type } & \text { Width [min] } & \text { Area } & \text { Height } & \text { Area\% } \\ 12.661 \text { VV } & 0.1626 & 868.4492 & 84.6968 & 7.4586 \\ 13.414 \text { VV } & 0.2062 & 10775.1768 & 831.3713 & 92.5414 \\ & \text { Sum } & 11643.6260 & & \end{array}$

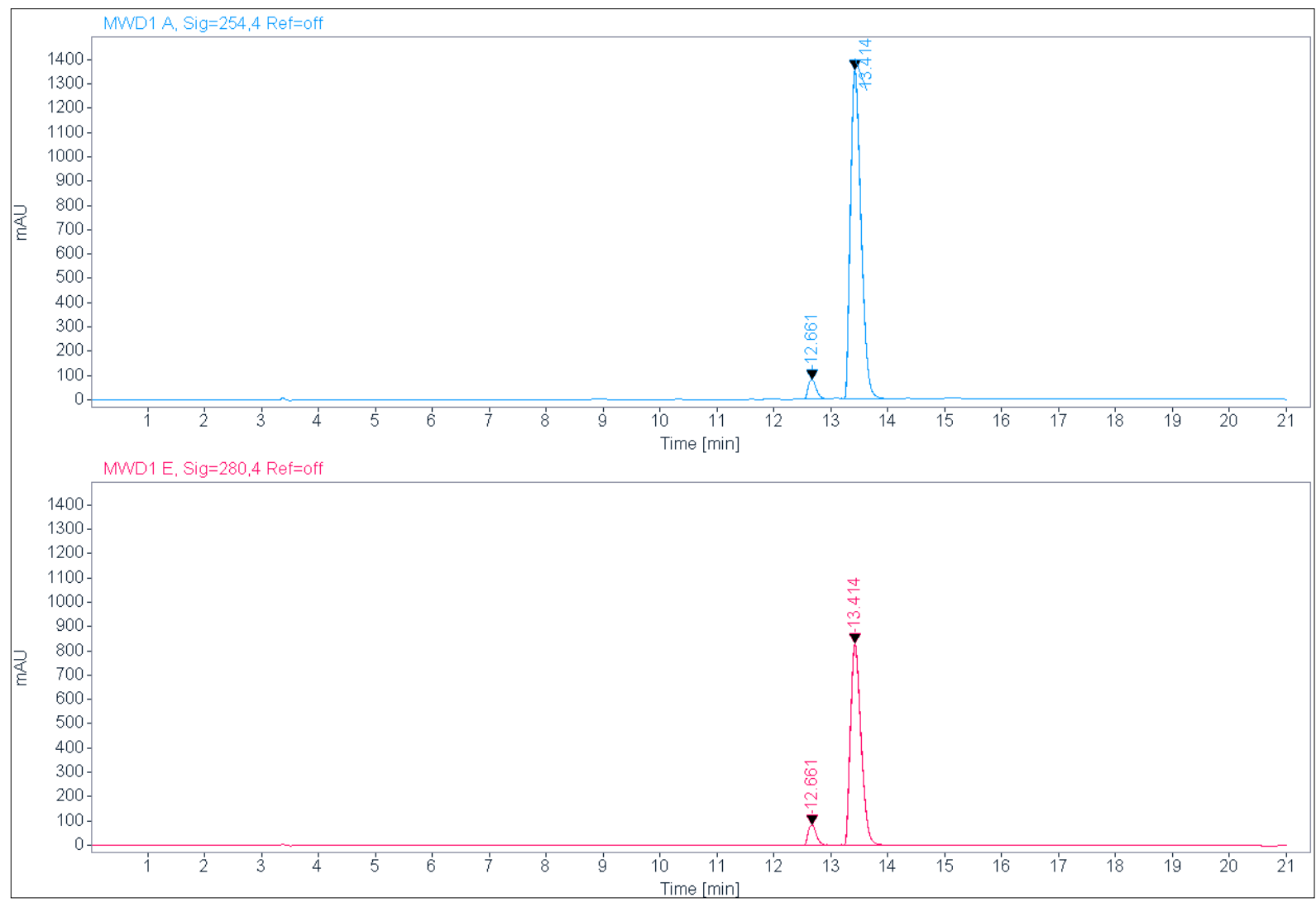



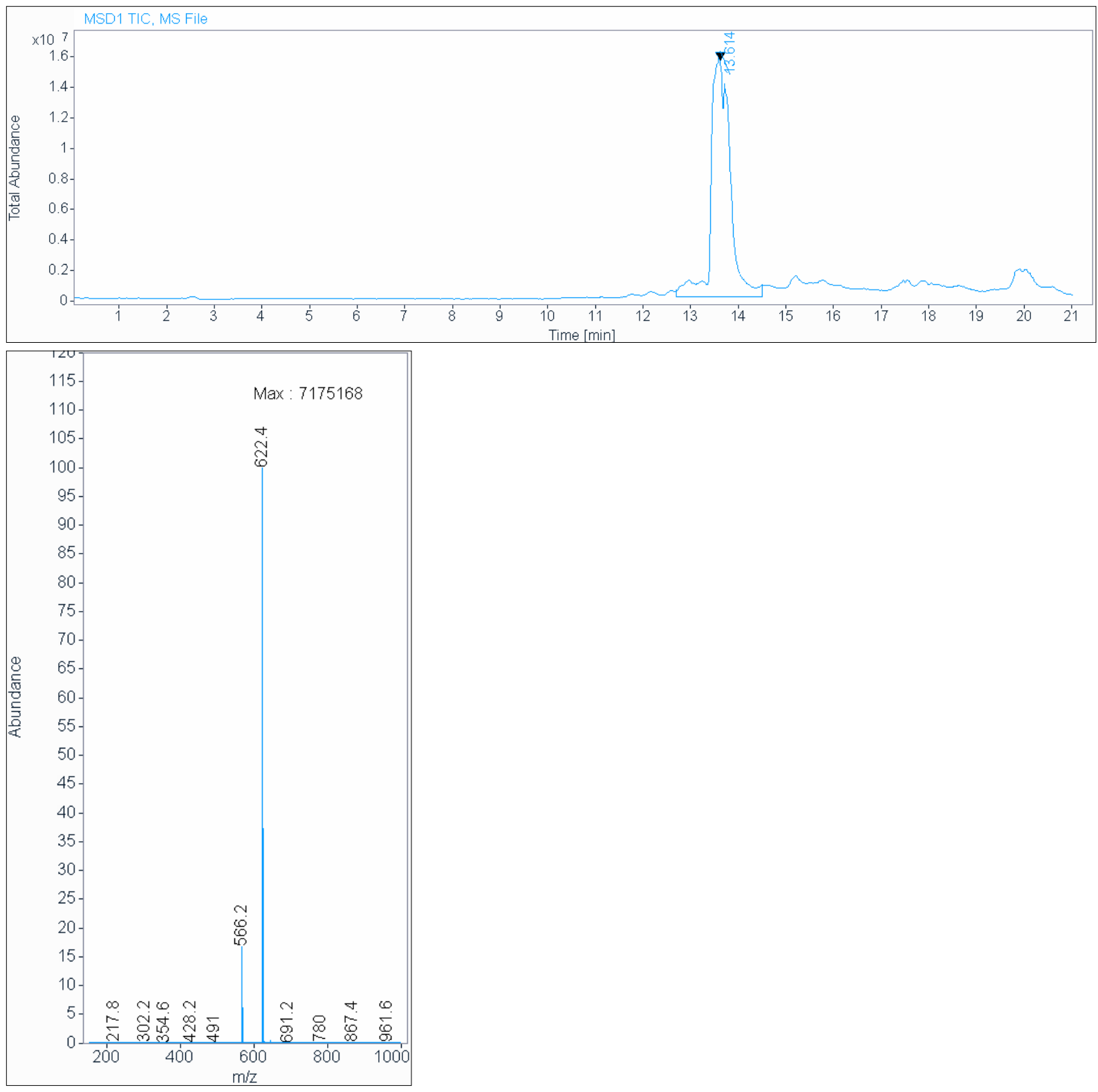
ESI and ${ }^{1} \mathrm{H}-\mathrm{NMR}$ of tert-butyl 5-(3-(5-amino-2-chloro-4-fluoro-3-methylbenzamido)-4-(4methylpiperazin-1-yl)phenyl)-1H-indole-1-carboxylate (6b)

C:IXcaliburldataVAD69

12/19/2018 2:43:50 PM

AD69 \#32-42 RT: 0.54-0.71 AV: 11 SB: 16 0.07-0.33 NL: 2.44E7

$\mathrm{T}:\{0,0\}+\mathrm{c}$ ESI !corona sid=75.00 det=1506.00 Full ms [105.00-1000.00]
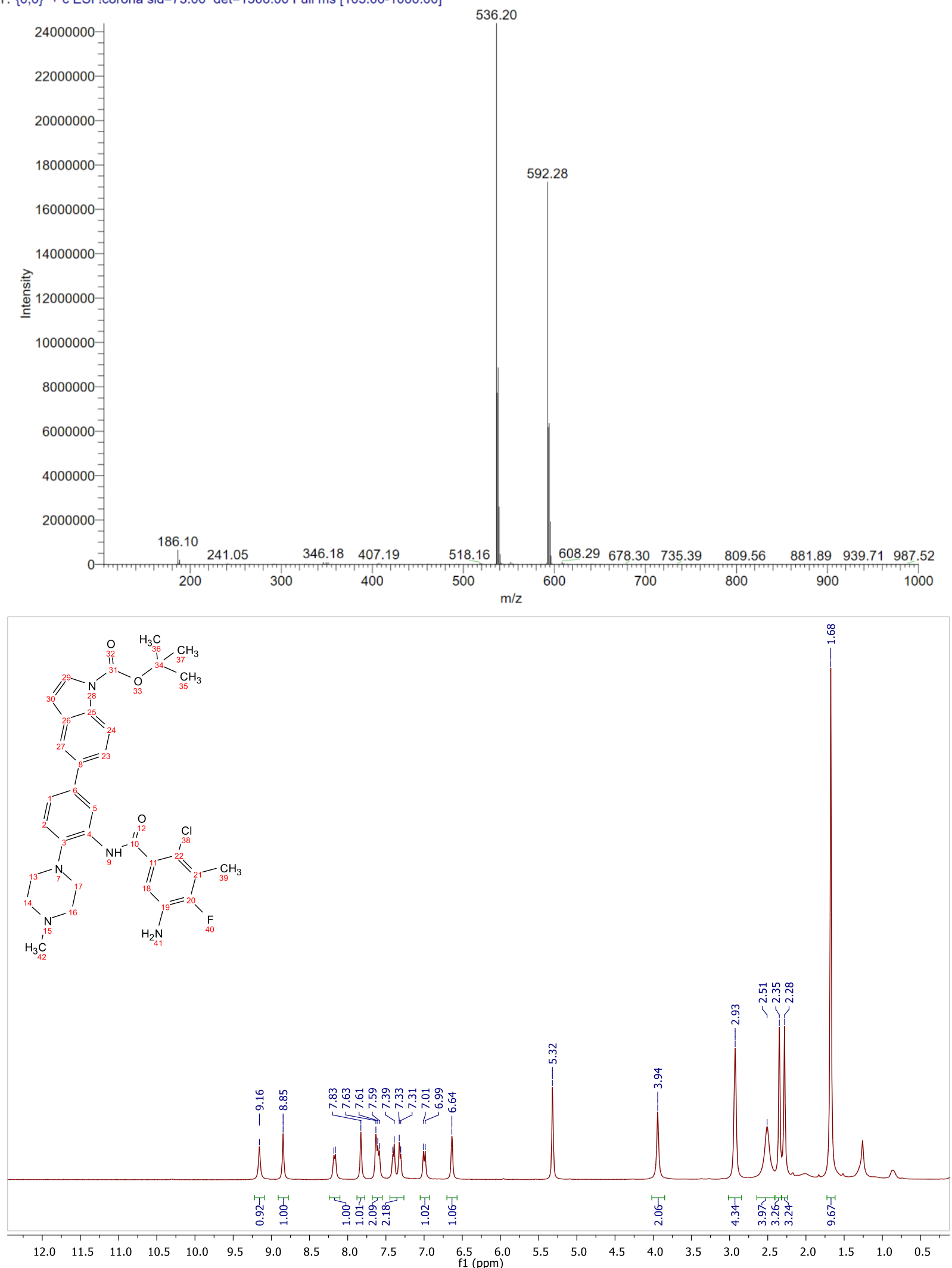
HPLC, ESI and ${ }^{1} \mathrm{H}-\mathrm{NMR}$ of tert-butyl 5-(3-amino-4-(4-methylpiperazin-1-yl)phenyl)-1H-indole-1carboxylate (5a)

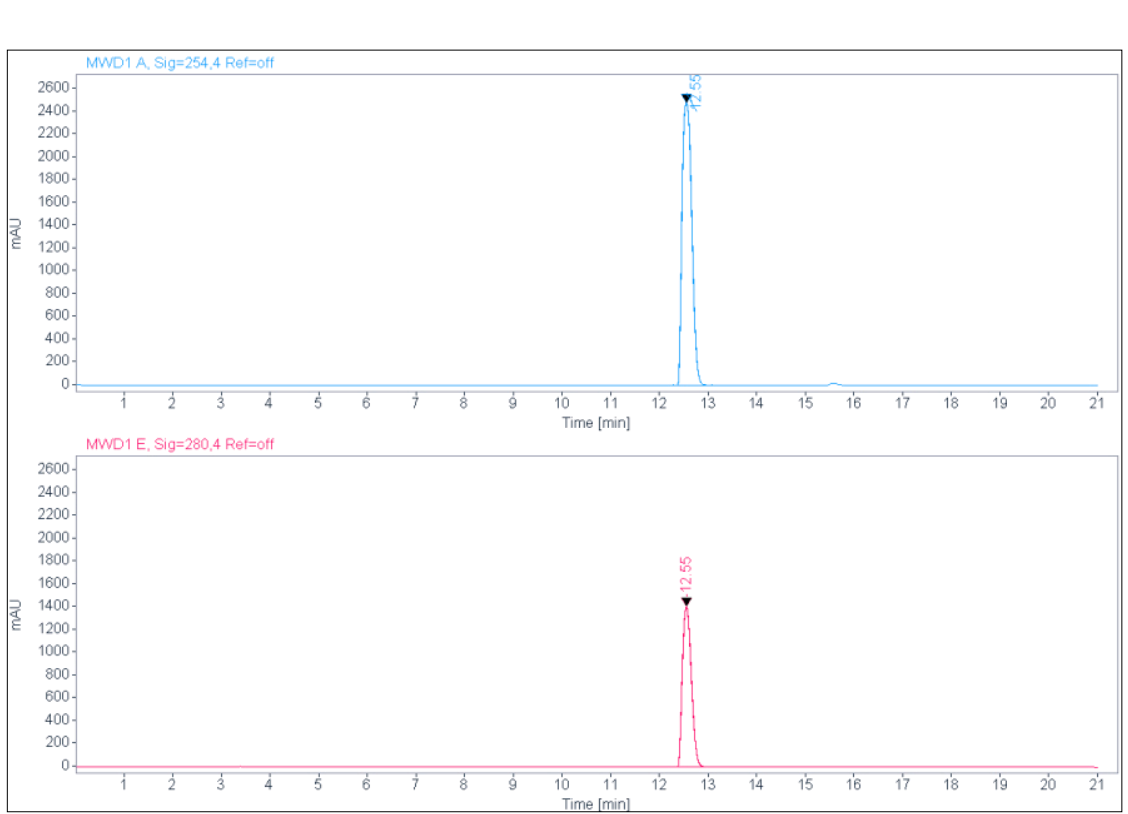

Peak RT $\quad 12.598$

Signal: $\quad$ MWD1 A, Sig=254,4 Ref=off

$\begin{array}{crrrr}\text { RT [min] Type } & \text { Width [min] } & \text { Area } & \text { Height } & \text { Area\% } \\ 12.550 \mathrm{VV} & 0.2245 & 34534.6094 & 2480.0020 & 100.0000 \\ & \text { Sum } & 34534.6094 & & \end{array}$

Signal: $\quad$ MWD1 E, Sig $=280,4$ Ref $=$ off

$\begin{array}{crrrr}\text { RT [min] Type } & \text { Width [min] } & \text { Area } & \text { Height } & \text { Area\% } \\ 12.550 \text { VV } & 0.2081 & 18136.9473 & 1409.5803 & 100.0000 \\ & \text { Sum } & 18136.9473 & & \end{array}$


C:IXcaliburldata|AD74-1

2/12/2019 9:03:14 AM

AD74-1 \#33-43 RT: 0.57-0.74 AV: 11 SB: 10 0.14-0.30 NL: 5.82E6

$\mathrm{T}:\{0,0\}+\mathrm{c}$ ESI !corona sid=75.00 det $=1306.00$ Full $\mathrm{ms}[100.00-1000.00]$

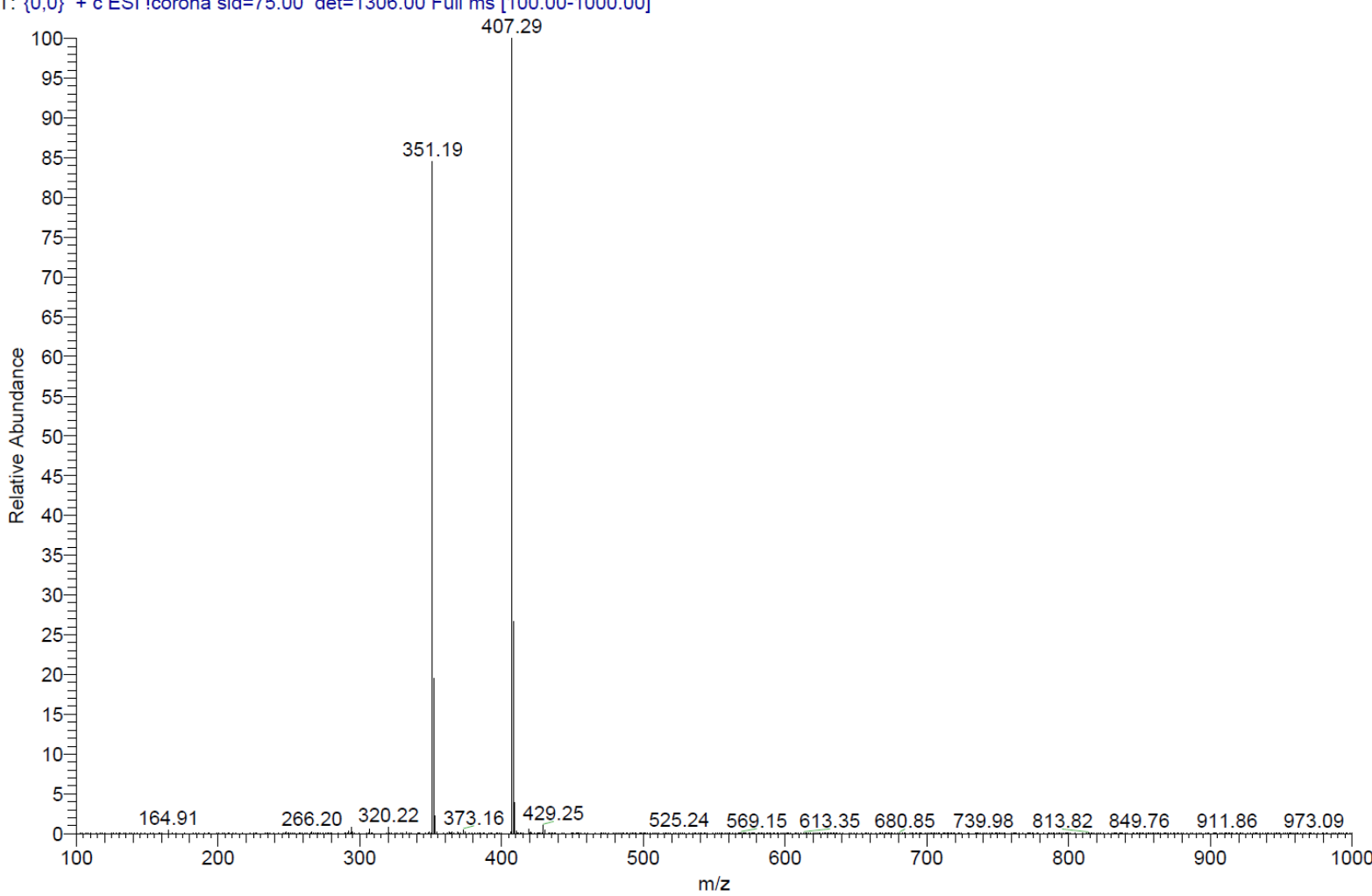

AV300-2019-01-23-adkn.26509

H1_1D CDCl3 /nmr kn 3 $\mathrm{m} / \mathrm{z}$

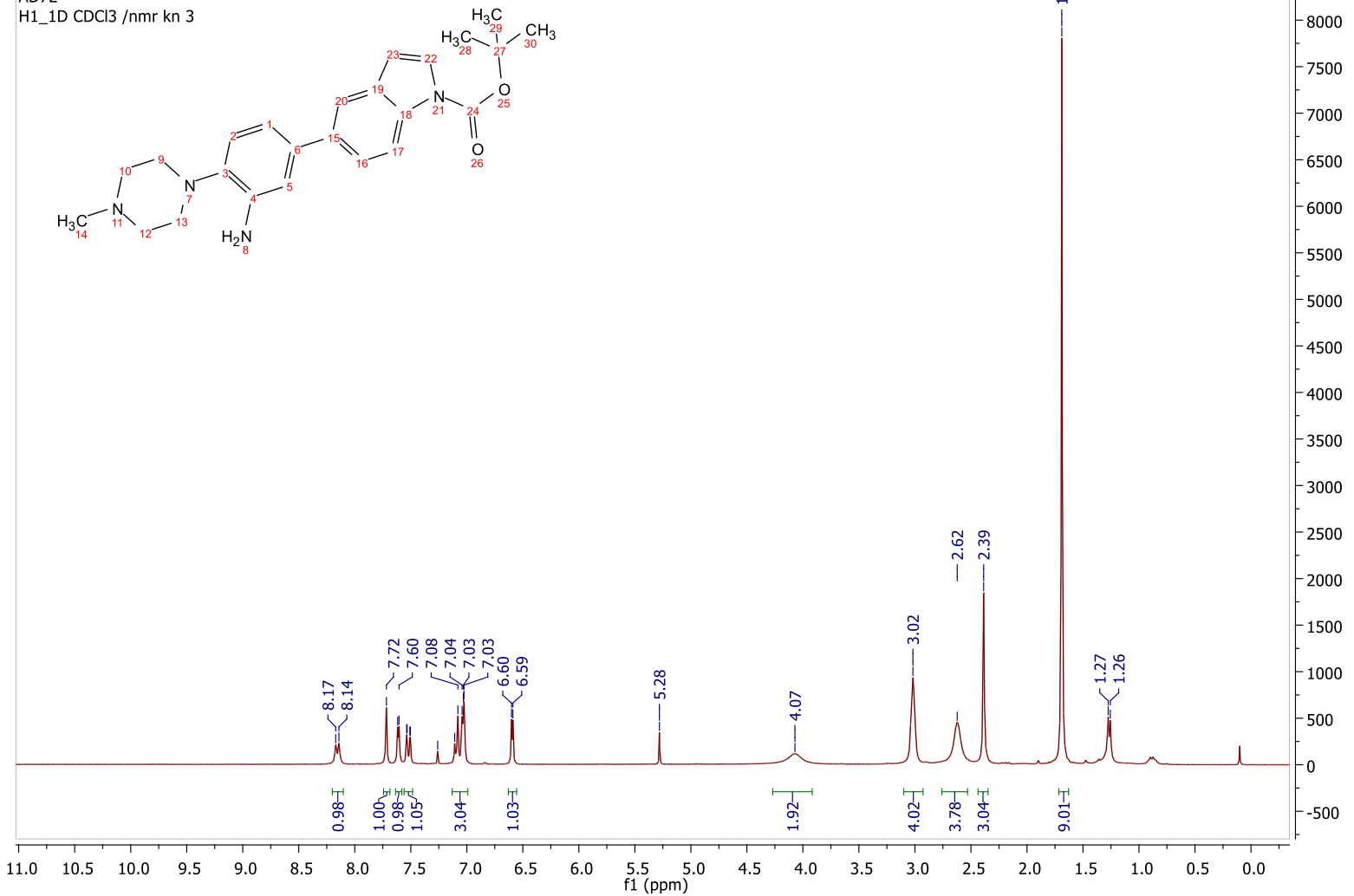


ESI, HPLC and ${ }^{1}$ H-NMR of tert-butyl 5-(3-(6-hydroxy-4-(trifluoromethyl)nicotinamido)-4-(4methylpiperazin-1-yl)phenyl)-1H-indole-1-carboxylate (6c)

C:IXcaliburldataIAD74-2

2/12/2019 9:04:37 AM

AD74-2 \#32-42 RT: 0.55-0.73 AV: 11 SB: 11 0.14-0.32 NL: 2.00E5

$\mathrm{T}:\{0,0\}+\mathrm{c}$ ESI !corona sid=75.00 det=1306.00 Full ms [100.00-1000.00]
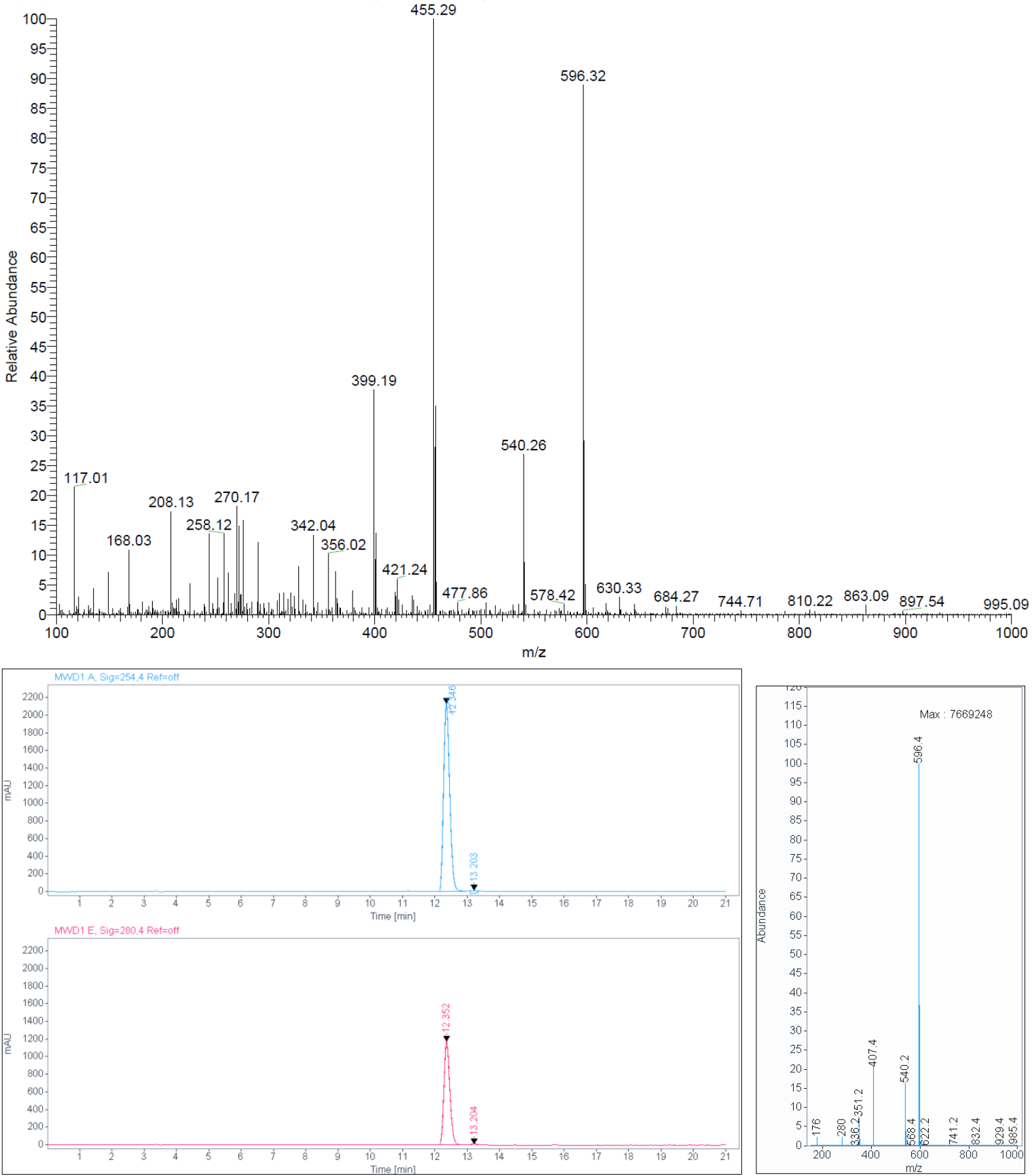

Signal: $\quad$ MWD1 A, Sig=254,4 Ref=off

$\begin{array}{crrrr}\text { RT [min] Type } & \text { Width [min] } & \text { Area } & \text { Height } & \text { Area\% } \\ 12.346 \text { VV } & 0.2079 & 27935.7637 & 2131.1035 & 98.3774 \\ 13.203 \text { MM } & 0.1934 & 460.7658 & 34.4330 & 1.6226 \\ & \text { Sum } & 28396.5295 & & \end{array}$

Signal: $\quad$ MWD1 E, Sig=280,4 Ref=off

$\begin{array}{crrrr}\text { RT [min] Type } & \text { Width [min] } & \text { Area } & \text { Height } & \text { Area\% } \\ 12.352 \text { VV } & 0.2061 & 14929.4092 & 1175.6801 & 99.8037\end{array}$


Sum

14958.7690

DRX600-2020-07-10-adkn.5061

Group AK_Knapp

AD74

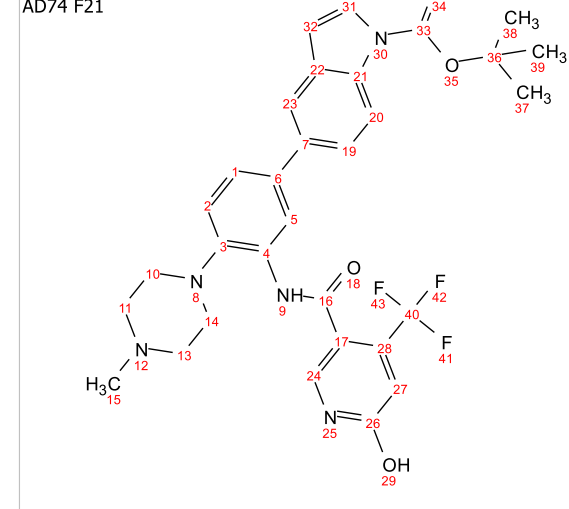

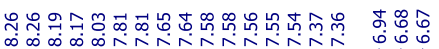

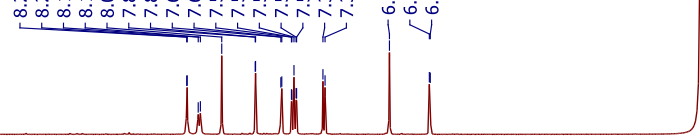

$\overbrace{}^{\circ}$

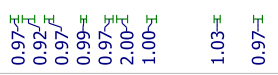

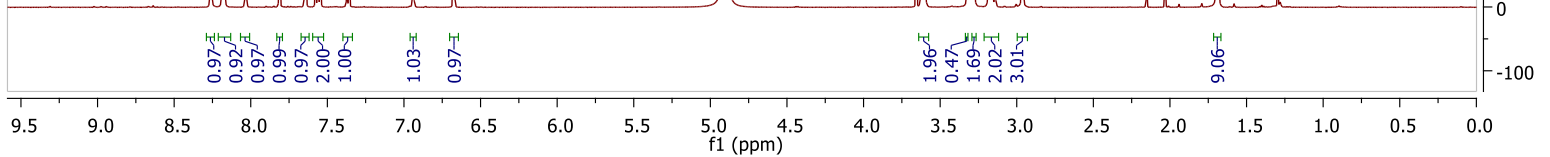


${ }^{1} \mathrm{H}-\mathrm{NMR}$ of 4-(4-bromo-2-nitrophenyl)morpholine

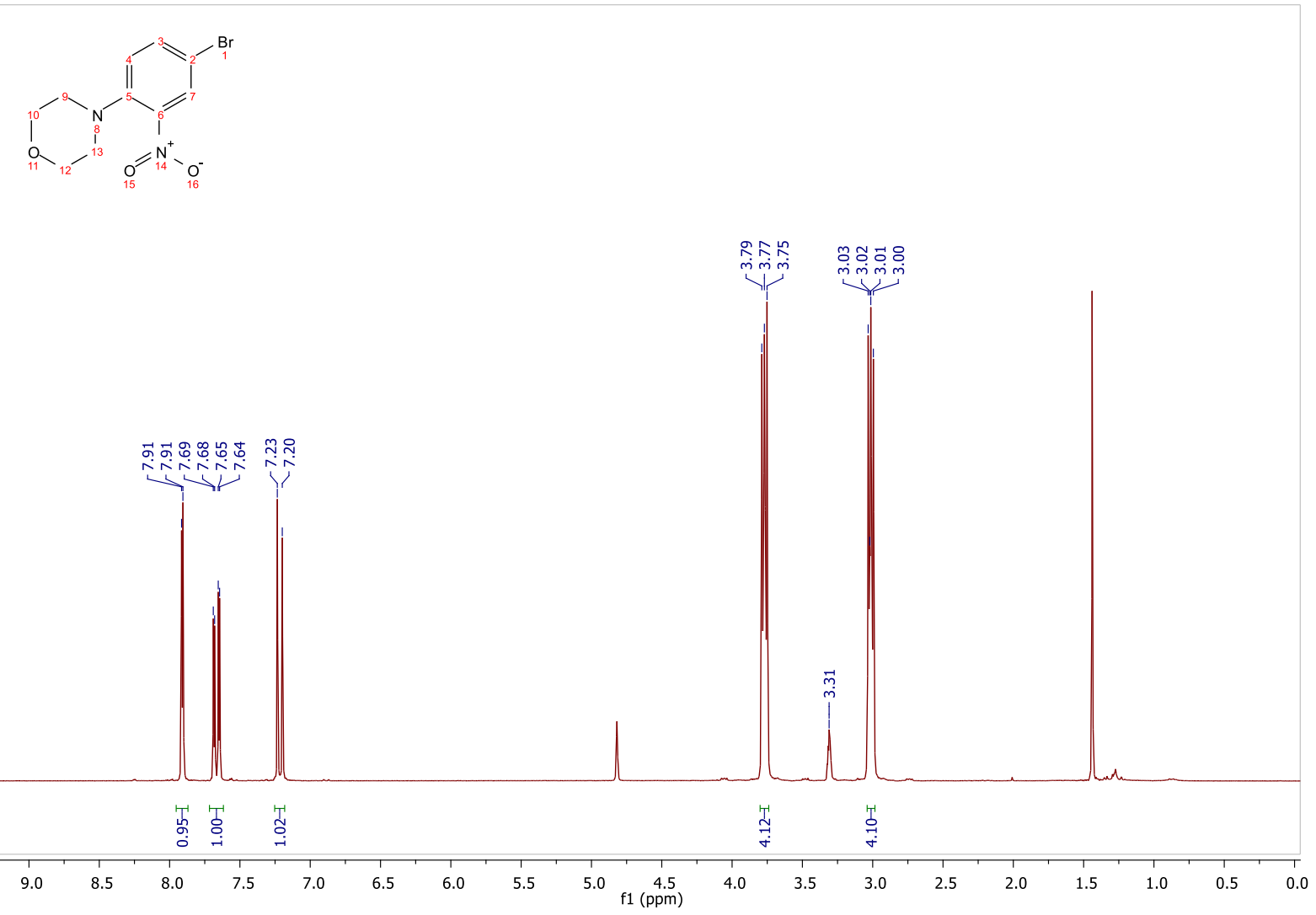

${ }^{1} \mathrm{H}$-NMR of 5-bromo-2-morpholinoaniline

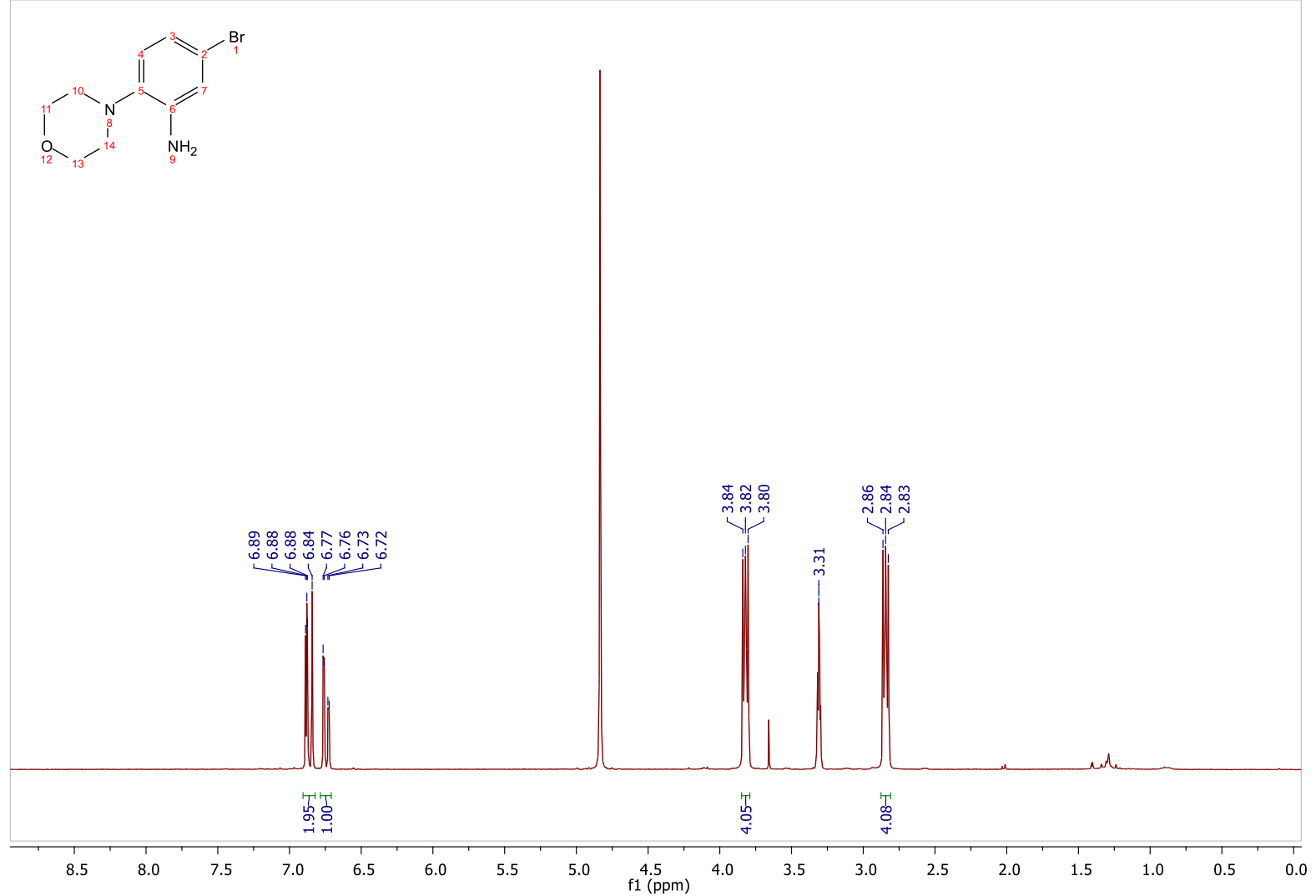


${ }^{1} \mathrm{H}-\mathrm{NMR}$ and ESI of tert-butyl 4'-morpholino-3'-(6-oxo-4-(trifluoromethyl)-1,6-dihydropyridine-3carboxamido)-[1,1'-biphenyl]-4-carboxylate

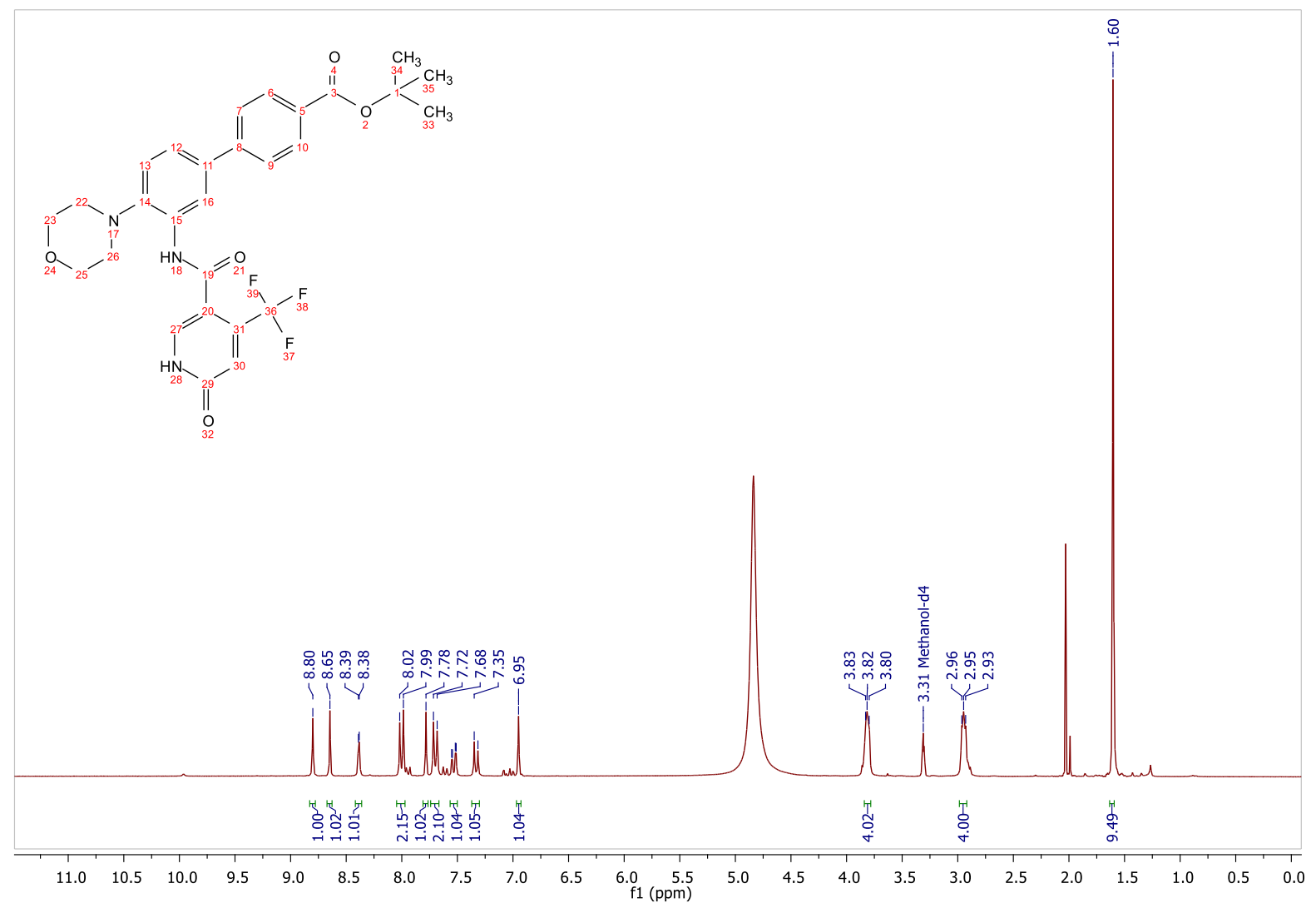

C:IXcaliburldatalAD165_weiß

2/15/2021 12:22:39 PM

AD165_weiß \#30-42 RT: 0.51-0.73 AV: 13 SB: 13 0.16-0.37 NL: 1.83E6

$\mathrm{T}:\{0,0\}+\mathrm{c}$ ESI !corona sid=75.00 det $=1600.00$ Full $\mathrm{ms}[105.00-1000.00]$

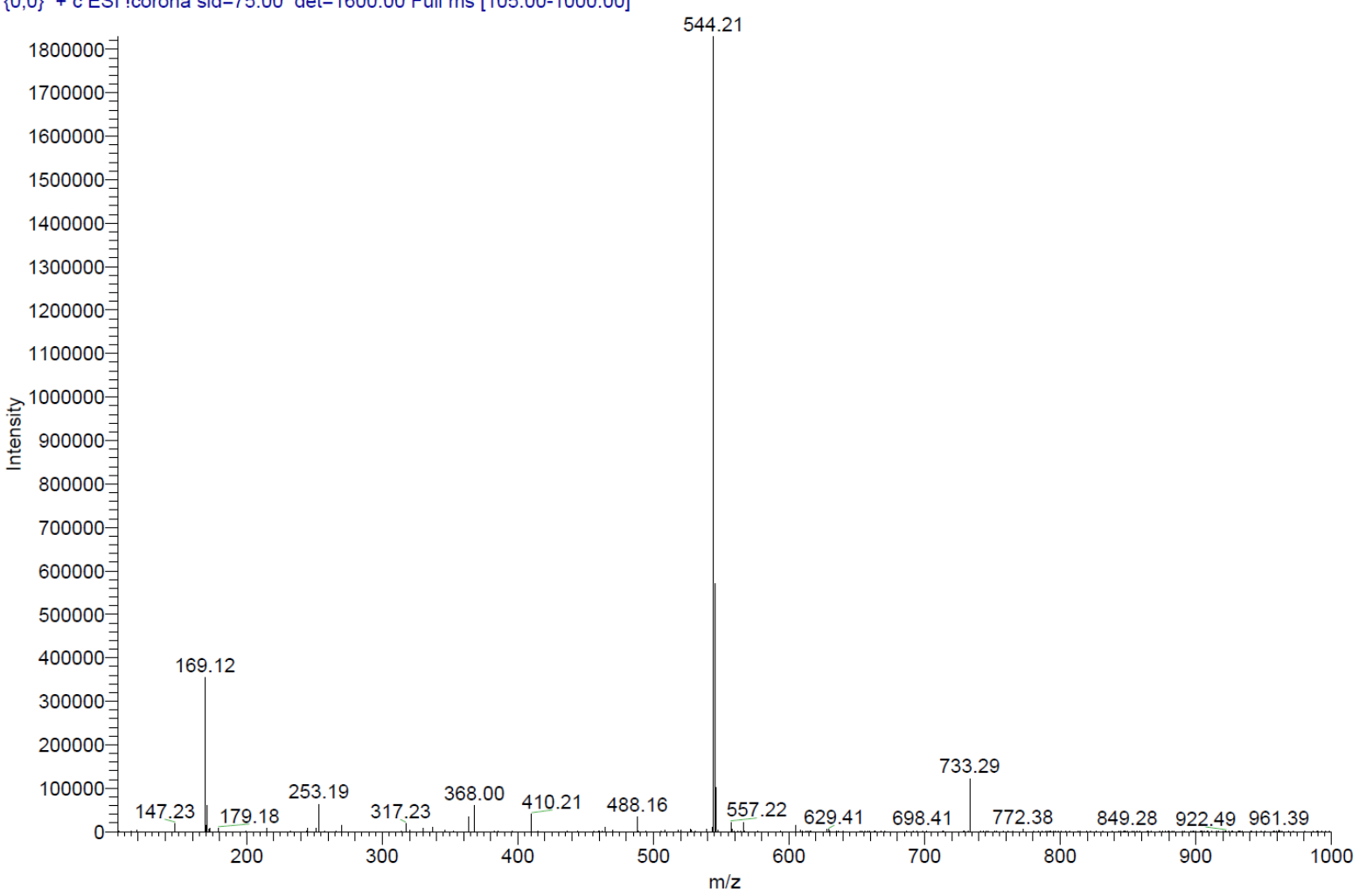




\section{ESI, ${ }^{1} \mathrm{H}-\mathrm{NMR}$ of 2-(2,6-dioxopiperidin-3-yl)-4-fluoroisoindoline-1,3-dione (L0)}

C:IXcaliburldata|NKO2

11/19/2019 9:56:33 AM

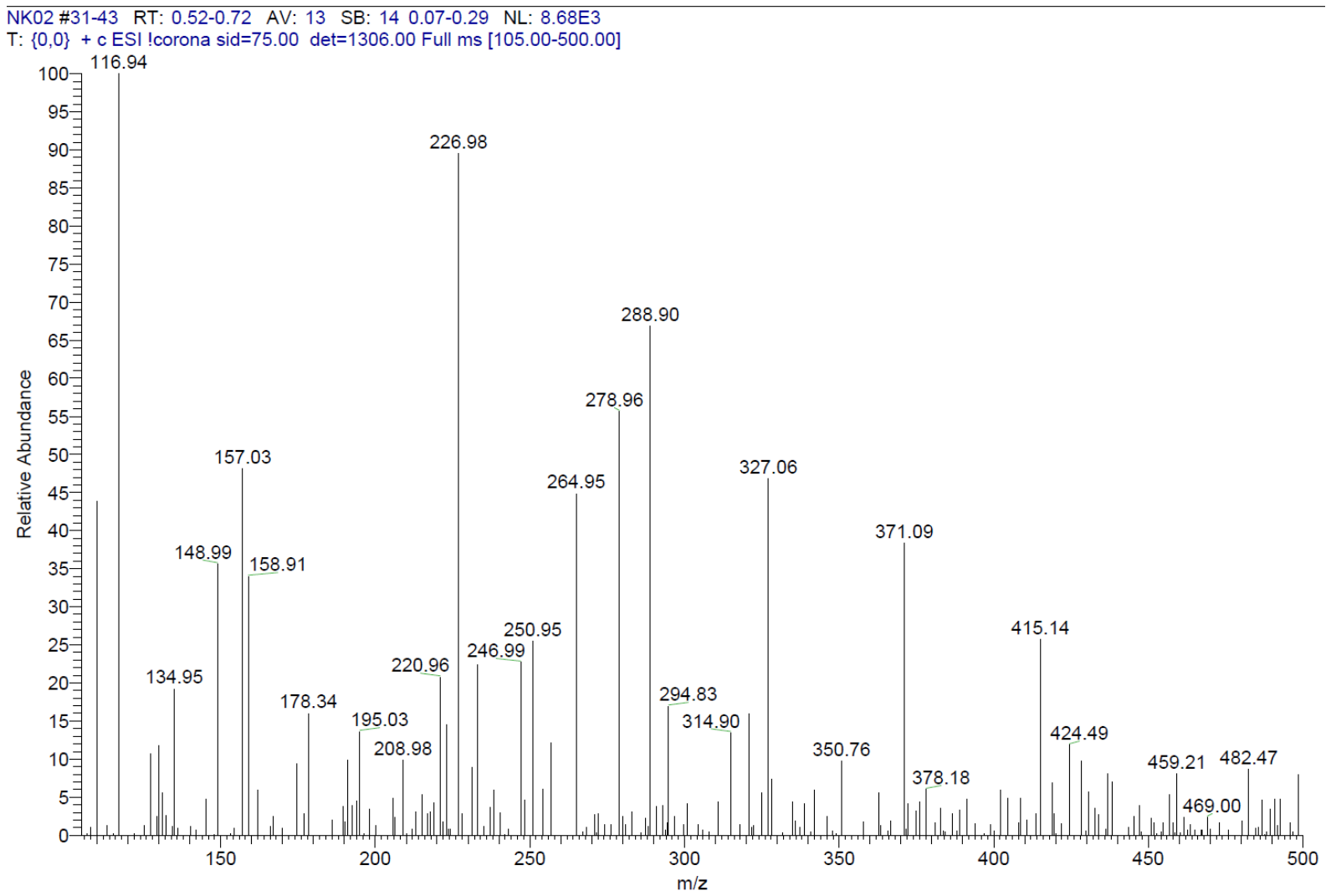

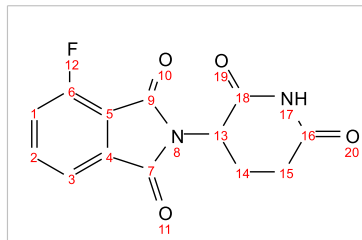
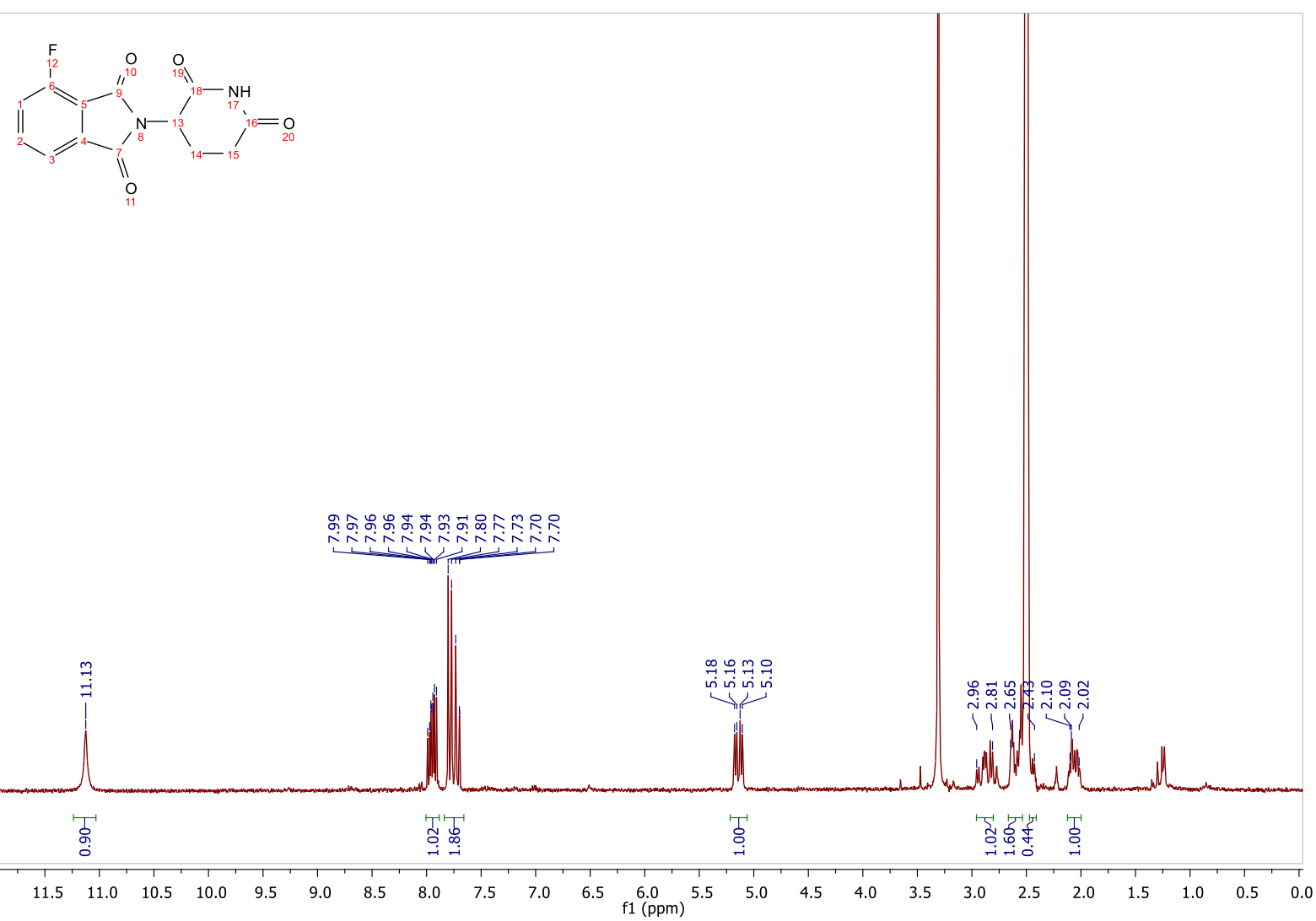
ESI, HPLC, ${ }^{1} \mathrm{H}-\mathrm{NMR}$ and ${ }^{13} \mathrm{C}-\mathrm{NMR}$ of tert-butyl (2-(2-((2-(2,6-dioxopiperidin-3-yl)-1,3-dioxoisoindolin4-yl)amino)ethoxy)ethyl)carbamate (L1)

C:IXcaliburldatalLLO6-1

3/13/2019 12:21:42 PM

L06-1 \#34-41 RT: 0.58-0.71 AV: 8 SB: 5 0.16-0.23 NL: 1.00E6

$\mathrm{T}:\{0,0\}+\mathrm{c}$ ESI !corona sid=75.00 det=1306.00 Full ms [100.00-900.00]

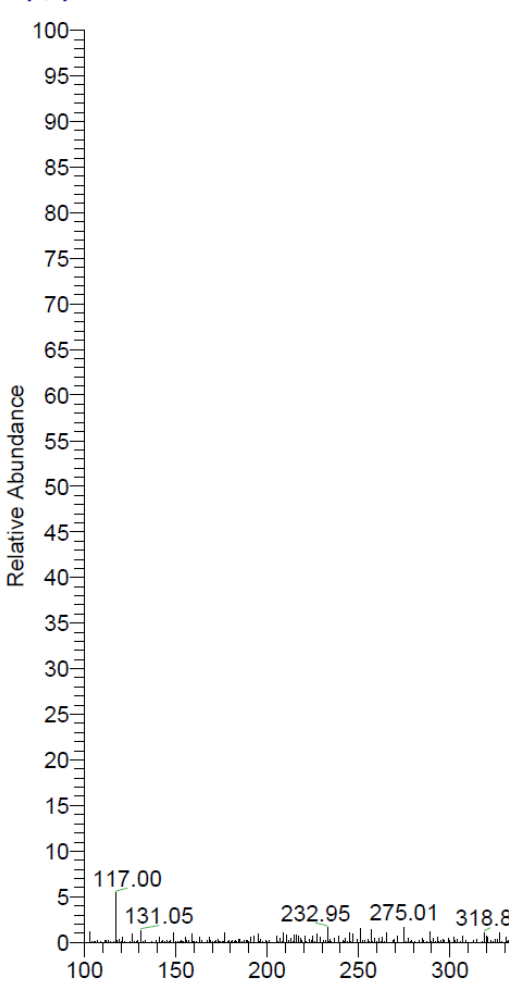

483.23

Signal: $\quad$ MWD1 A, Sig=254,4 Ref=off

$\begin{array}{rrrrr}\text { RT [min] Type } & \text { Width [min] } & \text { Area } & \text { Height } & \text { Area } \% \\ 3.313 \text { BV } & 0.0953 & 471.4035 & 74.0216 & 0.8010 \\ 12.868 \text { MM } & 0.2825 & 58377.2656 & 3443.8289 & 99.1990 \\ & \text { Sum } & 58848.6691 & & \end{array}$

Signal: $\quad$ MWD1 B, Sig $=280,4$ Ref=off

$\begin{array}{crrrr}\text { RT [min] Type } & \text { Width [min] } & \text { Area } & \text { Height } & \text { Area\% } \\ 12.805 \text { MM } & 0.2581 & 53369.2109 & 3445.7402 & 100.0000 \\ & \text { Sum } & 53369.2109 & & \end{array}$

Signal: $\quad$ MWD1 F, Sig $=260,4$ Ref $=$ off

$\begin{array}{crrrr}\text { RT [min] Type } & \text { Width [min] } & \text { Area } & \text { Height } & \text { Area\% } \\ 3.313 \mathrm{VV} & 0.0926 & 356.4311 & 58.9223 & 0.5828 \\ 12.774 \mathrm{MM} & 0.2846 & 60802.2852 & 3561.0684 & 99.4172\end{array}$



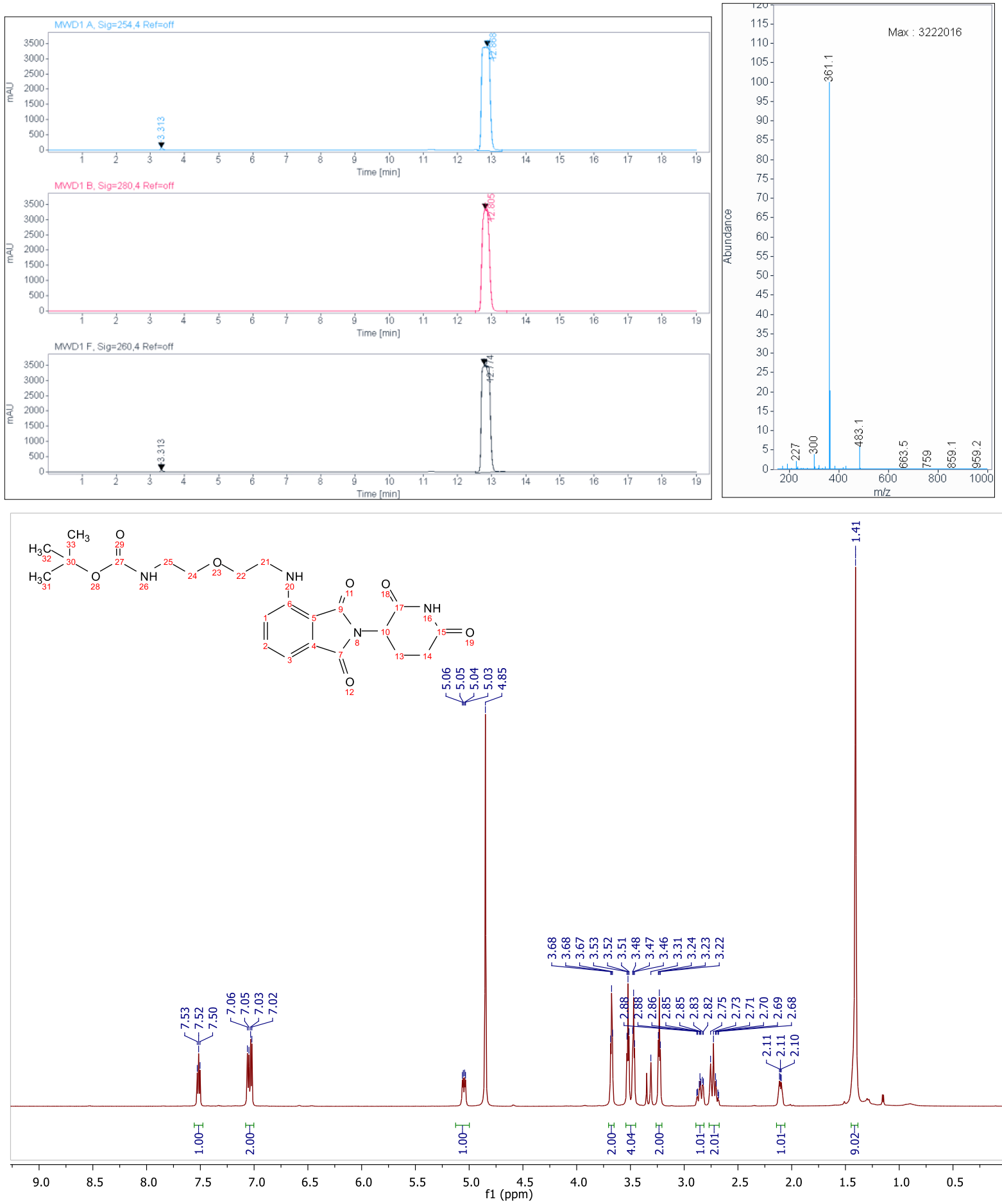


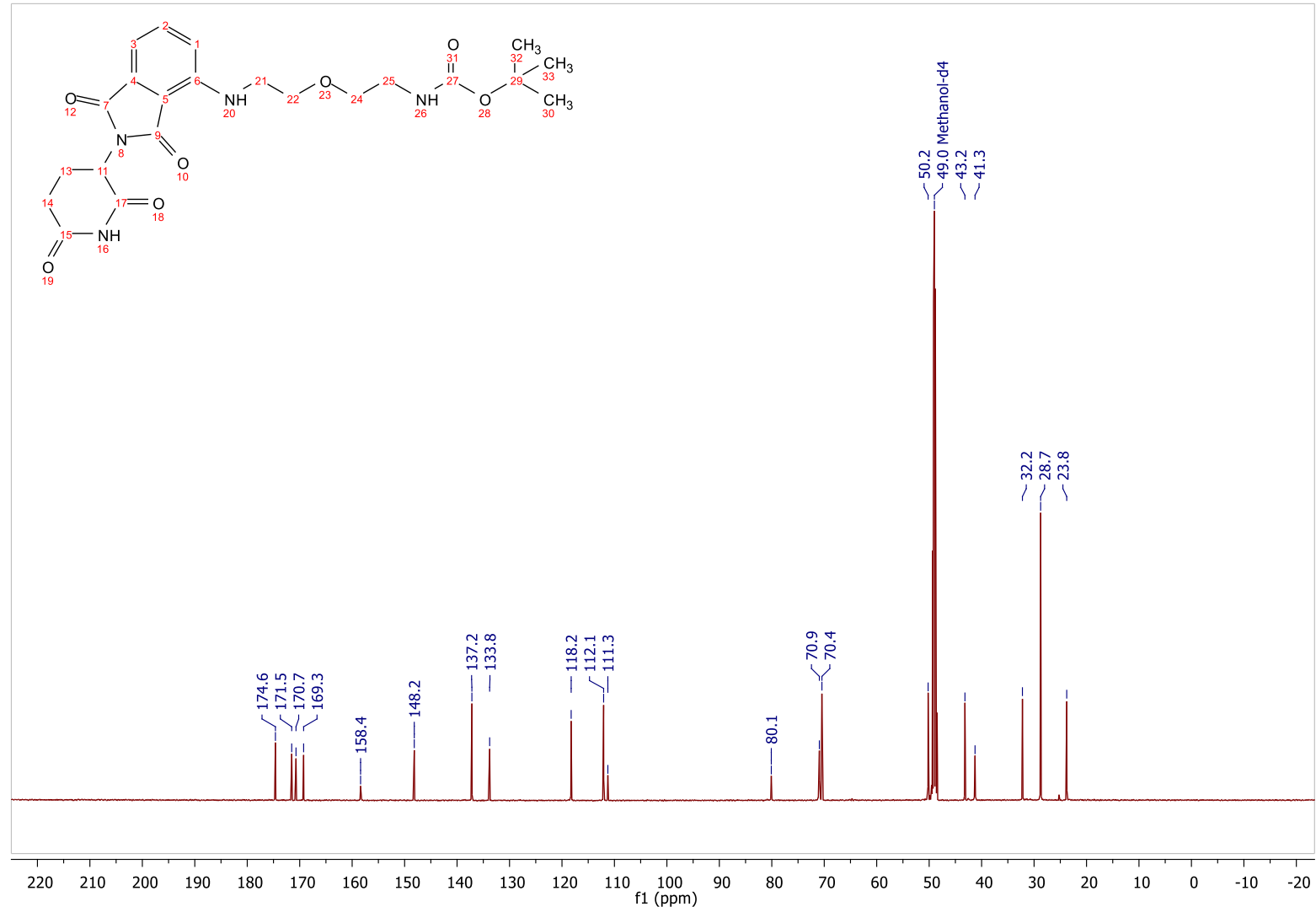


ESI, HPLC, ${ }^{1} \mathrm{H}-\mathrm{NMR}$ and ${ }^{13} \mathrm{C}-\mathrm{NMR}$ of tert-butyl (2-(2-(2-(2-((2-(2,6-dioxopiperidin-3-yl)-1,3dioxoisoindolin-4-yl)amino)ethoxy)ethoxy)ethoxy)ethyl)carbamate (L2)

C:IXcaliburldatalLLO7-1

3/13/2019 12.23.26 PM

LL07-1 \#36-43 RT: 0.62-0.74 AV: 8 SB: 6 0.11-0.20 NL: 7.75E5

$\mathrm{T}:\{0,0\}+\mathrm{c}$ ESI !corona sid $=75.00$ det $=1306.00$ Full $\mathrm{ms}[100.00-1000.00]$
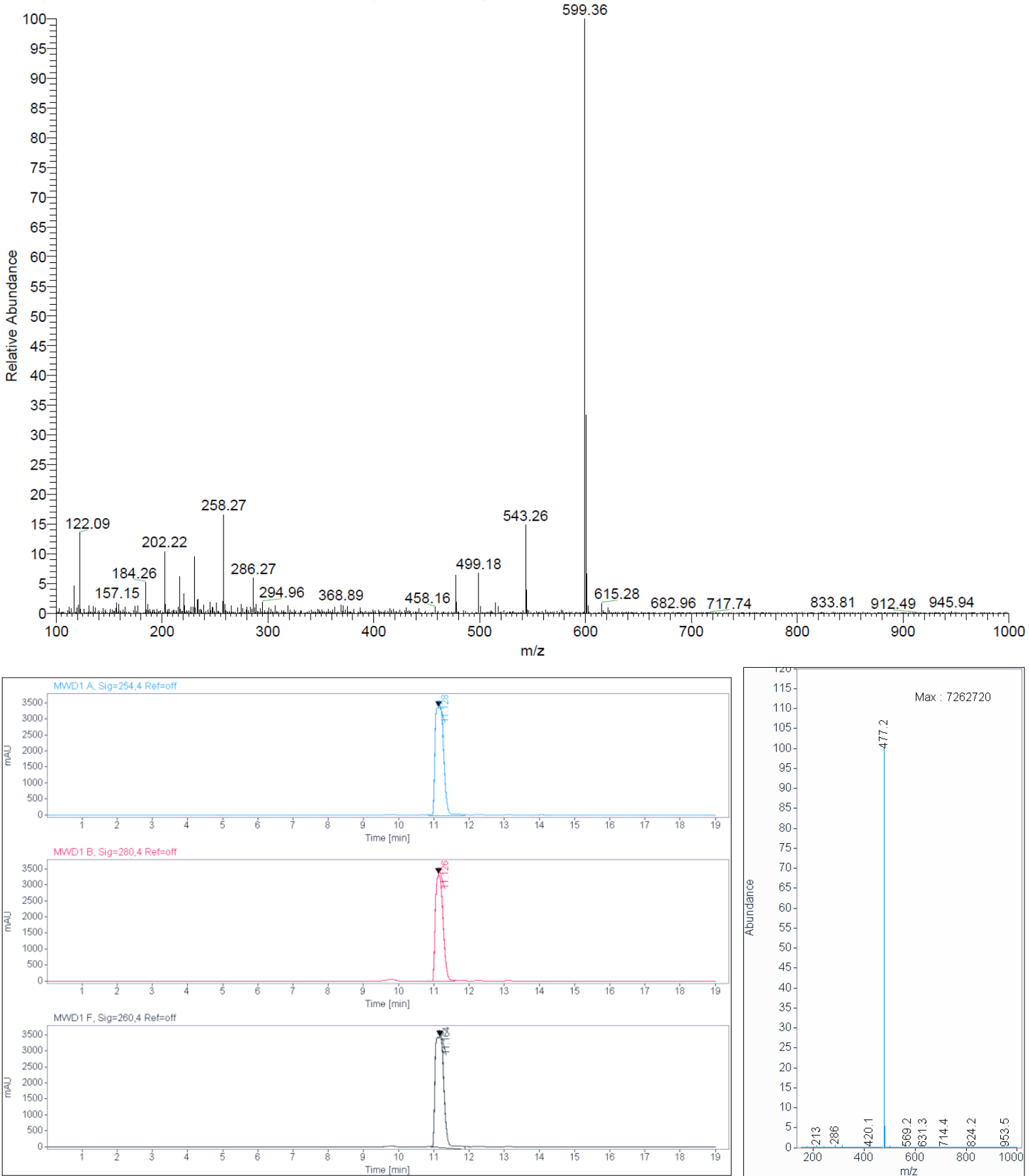

Signal: $\quad$ MWD1 A, Sig=254,4 Ref=off

$\begin{array}{crrrr}\text { RT [min] Type } & \text { Width [min] } & \text { Area } & \text { Height } & \text { Area\% } \\ 11.128 \text { MM } & 0.2843 & 58034.3398 & 3402.1462 & 100.0000 \\ & \text { Sum } & 58034.3398 & & \end{array}$

Signal: $\quad$ MWD1 B, Sig=280,4 Ref=off

$\begin{array}{crrrr}\text { RT [min] Type } & \text { Width [min] } & \text { Area } & \text { Height } & \text { Area\% } \\ 11.126 \text { VV } & 0.1779 & 50324.2734 & 3357.0464 & 100.0000\end{array}$


Sum $\quad 50324.2734$

Signal: $\quad$ MWD1 $F$, Sig=260,4 Ref=off

$\begin{array}{crrrr}\text { RT [min] Type } & \text { Width [min] } & \text { Area } & \text { Height } & \text { Area\% } \\ 11.164 \mathrm{MM} & 0.2940 & 61209.2969 & 3470.2668 & 100.0000\end{array}$

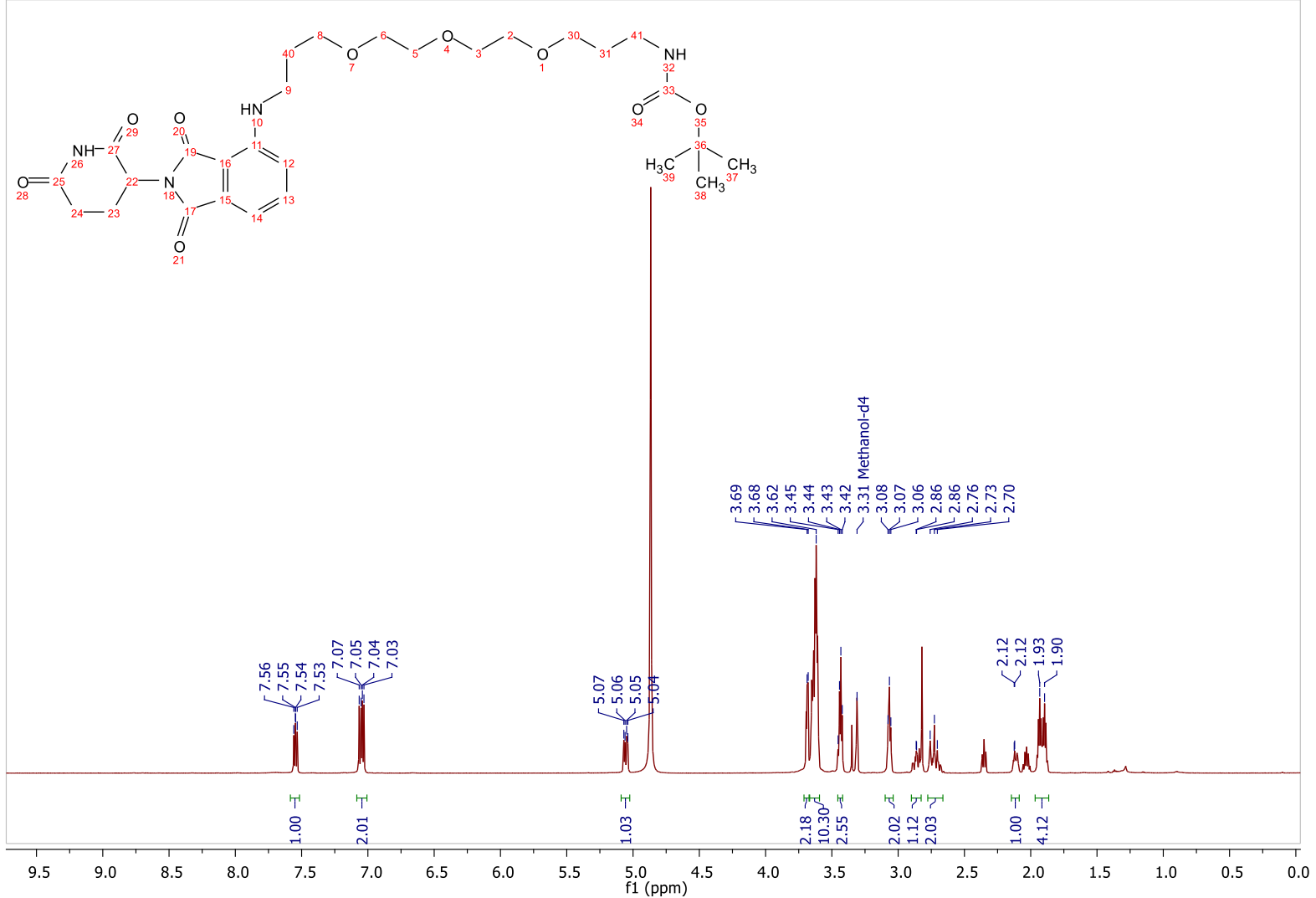




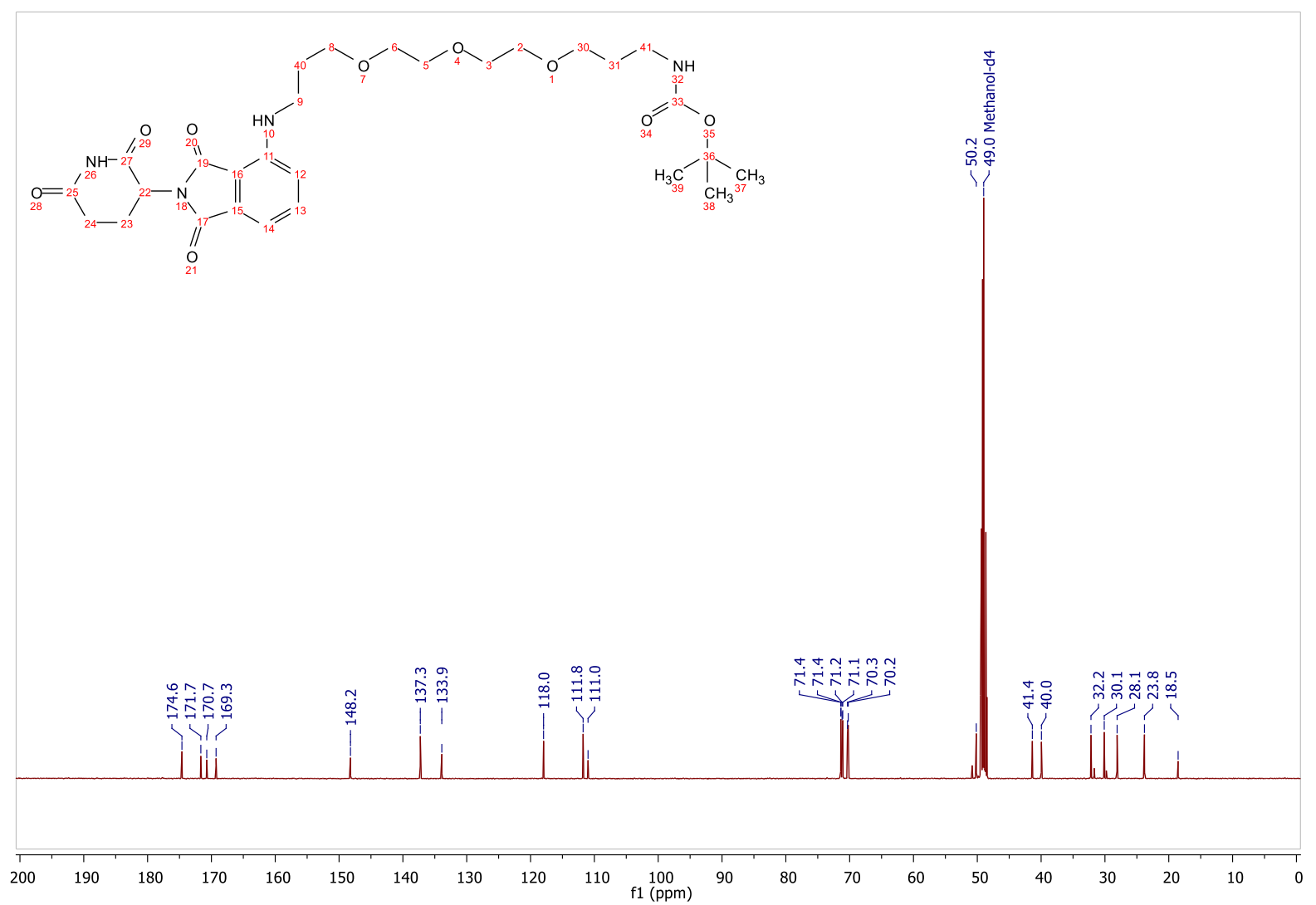


ESI, HPLC, ${ }^{1} \mathrm{H}-\mathrm{NMR}$ of tert-butyl (17-((2-(2,6-dioxopiperidin-3-yl)-1,3-dioxoisoindolin-4-yl)amino)3,6,9,12,15-pentaoxaheptadecyl)carbamate (L3)
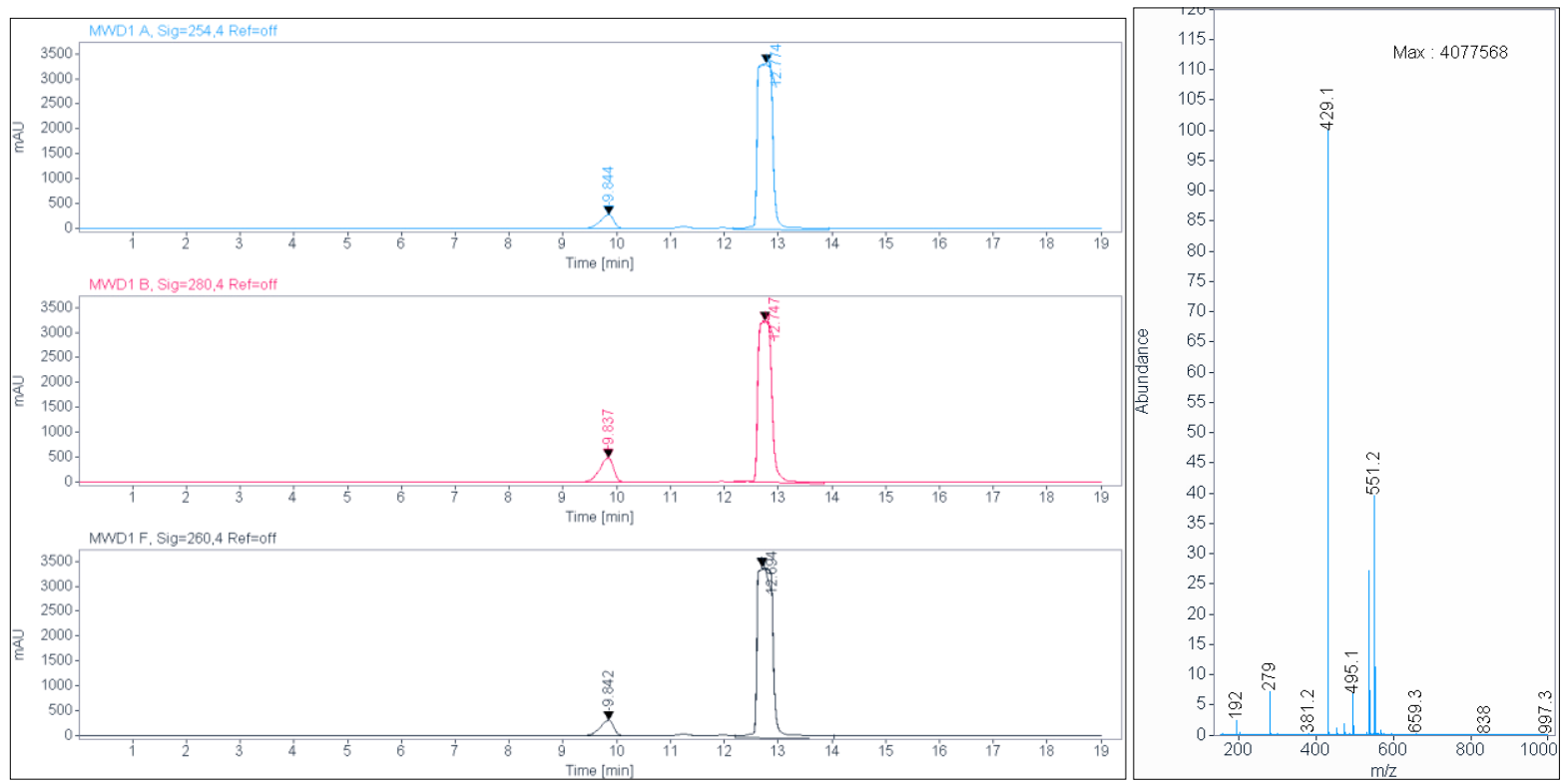

Signal: $\quad$ MWD1 A, Sig=254,4 Ref=off

$\begin{array}{crrrr}\text { RT [min] Type } & \text { Width [min] } & \text { Area } & \text { Height } & \text { Area\% } \\ 9.844 \text { VV } & 0.2442 & 4875.1143 & 277.2130 & 6.8073 \\ 12.774 \mathrm{MM} & 0.3324 & 66741.1328 & 3346.6414 & 93.1927 \\ & \text { Sum } & 71616.2471 & & \end{array}$

Signal: $\quad$ MWD1 B, Sig=280,4 Ref=off

$\begin{array}{crrrr}\text { RT [min] Type } & \text { Width [min] } & \text { Area } & \text { Height } & \text { Area\% } \\ 9.837 \text { VV } & 0.2515 & 8863.7100 & 489.0014 & 13.2680 \\ 12.747 \text { MM } & 0.2964 & 57941.5820 & 3258.0781 & 86.7320 \\ & \text { Sum } & 66805.2920 & & \end{array}$

Signal: $\quad$ MWD1 F, Sig=260,4 Ref=off

$\begin{array}{crrrr}\text { RT [min] Type } & \text { Width [min] } & \text { Area } & \text { Height } & \text { Area\% } \\ 9.842 \text { VV } & 0.2456 & 5633.9717 & 318.0999 & 7.2044 \\ 12.694 \mathrm{MM} & 0.3503 & 72567.7969 & 3452.9812 & 92.7956\end{array}$


C: XcaliburldataINK05-1

$11 / 28 / 2019$ 10:40:25 AM

NK05-1 \#37-43 RT: 0.64-0.75 AV: 7 SB: 17 0.09-0.37 NL: 6.15E5

$\mathrm{T}:\{0,0\}+\mathrm{c}$ ESI !corona sid=75.00 det=1306.00 Full ms [200.00-1200.00]
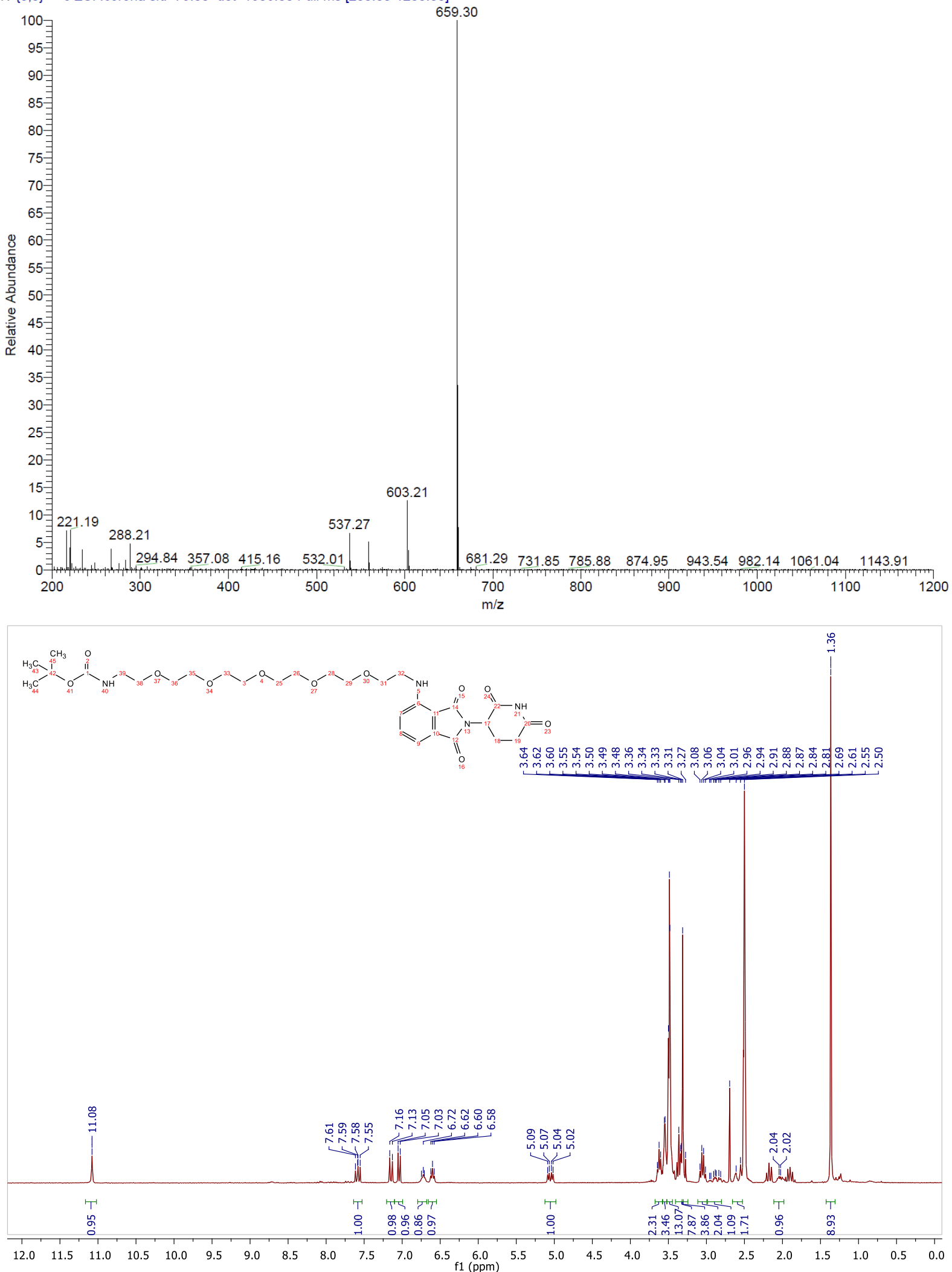
ESI, HPLC, ${ }^{1} \mathrm{H}-N M R$ and ${ }^{13} \mathrm{C}-\mathrm{NMR}$ of tert-butyl (23-((2-(2,6-dioxopiperidin-3-yl)-1,3-dioxoisoindolin-4yl)amino)-3,6,9,12,15,18,21-heptaoxatricosyl)carbamate (L4)

C:IXcaliburldataINK06-1

11/28/2019 10:41:49 AM

NK06-1 \#36-42 RT: 0.62-0.73 AV: 7 SB: 11 0.02-0.20 NL: 1.12E5

$\mathrm{T}:\{0,0\}+\mathrm{cESI}$ ! corona sid=75.00 det=1306.00 Full ms [200.00-1200.00]

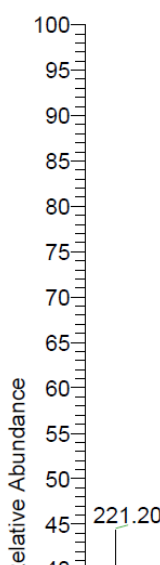

$\sum_{\frac{\pi}{0}}^{\pi} 45 \exists 221$

35

30沓

20 每 $234.16 \quad 332.13$

10 459.22 0 200

300 400 500

25.29
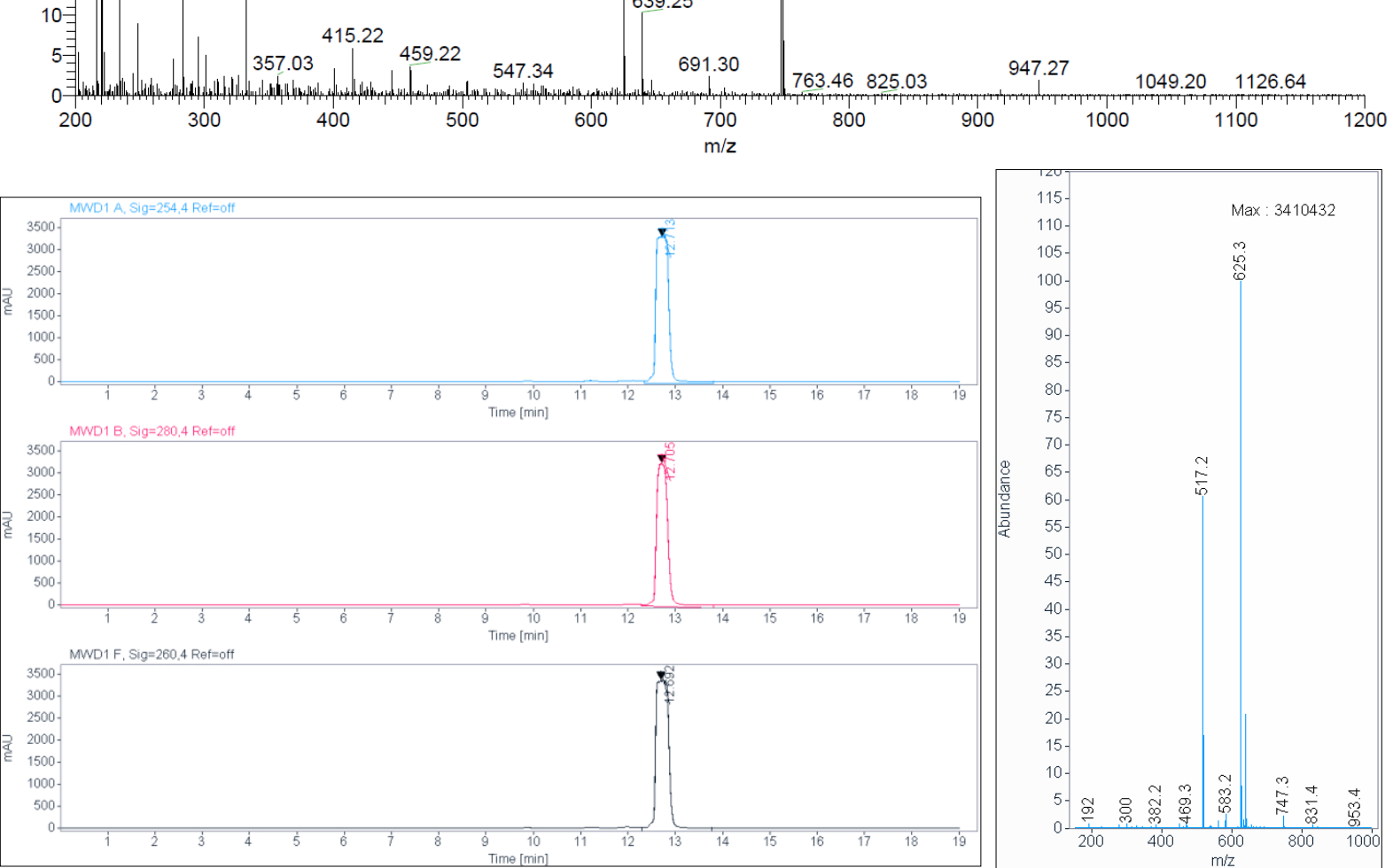

Signal: $\quad$ MWD1 A, Sig $=254,4$ Ref $=$ off

RT [min] Type Width [min]

12.713 MM

Area

Height

Area\%

64034.5742

3352.9675

100.0000

Signal: $\quad$ MWD1 B, Sig=280,4 Ref=off

RT [min] Type Width [min]

Area

Height

Area\% 
12.705 MM

$$
\begin{array}{rr}
0.2795 & 54819.2344 \\
\text { Sum } & 54819.2344
\end{array}
$$

3269.3167

100.0000

Signal: $\quad$ MWD1 $F$, Sig=260,4 Ref=off

$\begin{array}{ccrrr}\text { RT [min] Type } & \text { Width [min] } & \text { Area } & \text { Height } & \text { Area\% } \\ 12.692 \text { MM } & 0.3332 & 69116.1953 & 3456.9661 & 100.0000\end{array}$

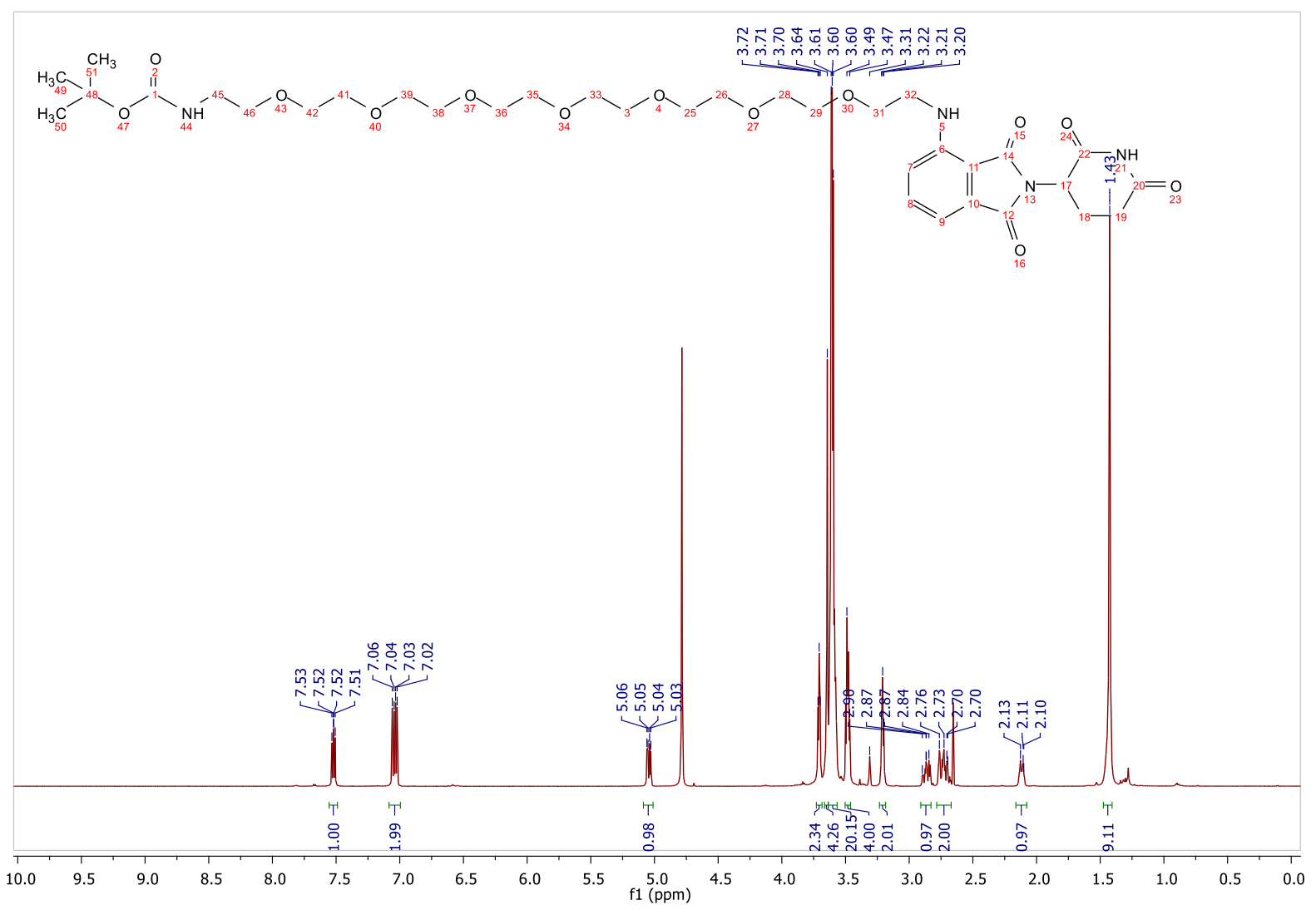




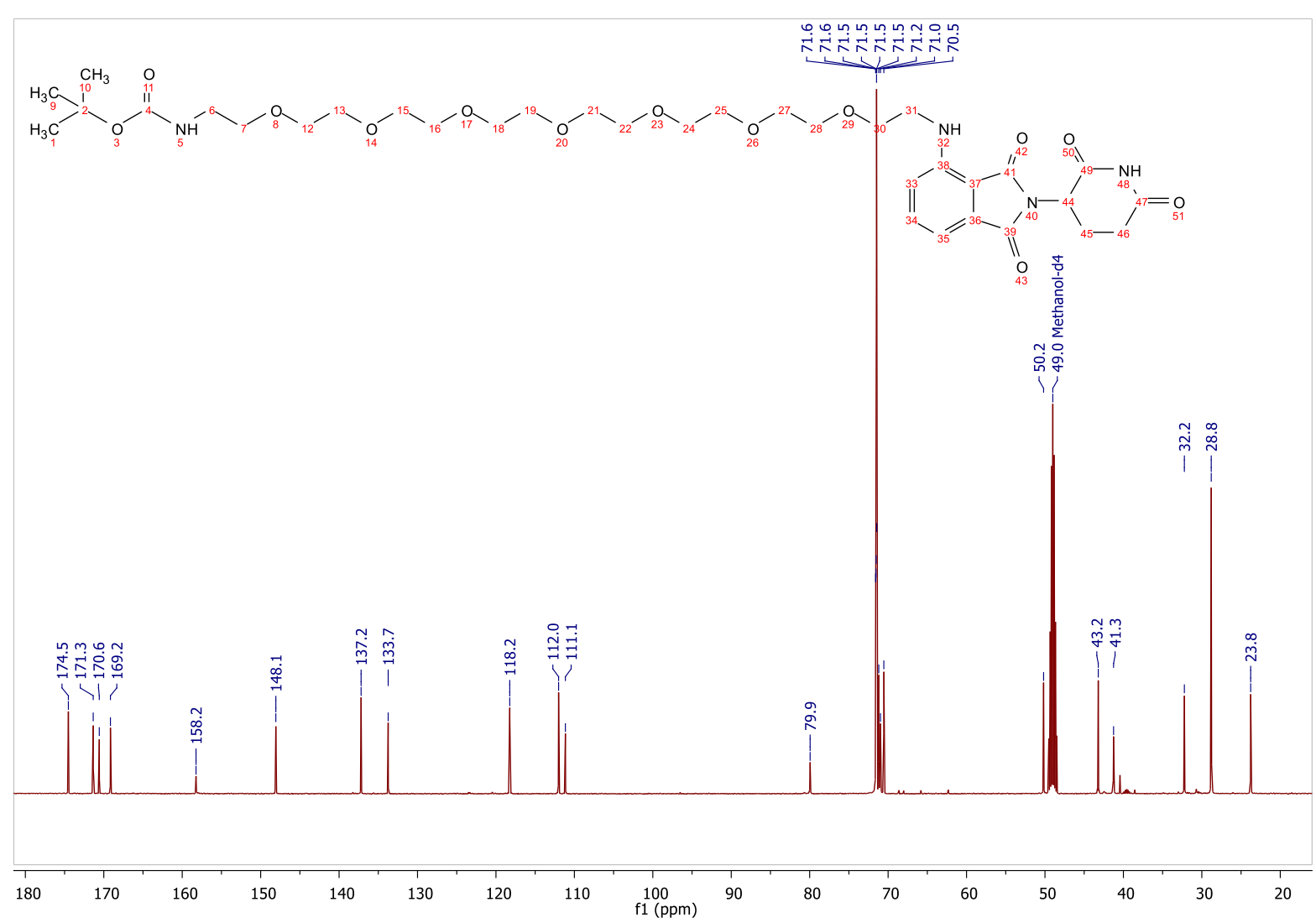


ESI, HPLC, ${ }^{1} \mathrm{H}-\mathrm{NMR}$ of tert-butyl (4-(((2-(2,6-dioxopiperidin-3-yl)-1,3-dioxoisoindolin-4yl)amino)methyl)benzyl)carbamate (L5)

C:IXcaliburldataIAD130-1

2/13/2020 9:55:03 AM
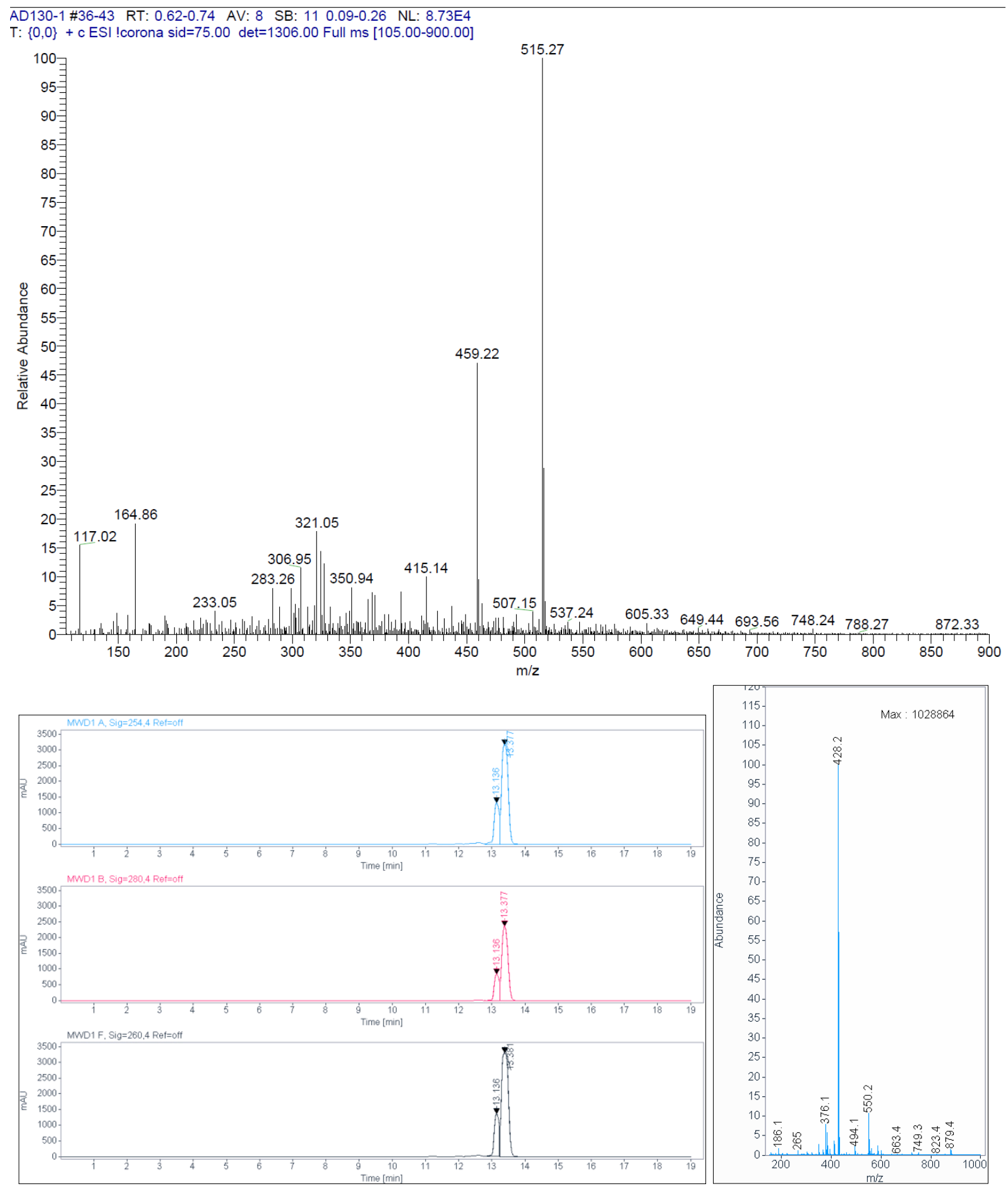

Signal: $\quad$ MWD1 A, Sig=254,4 Ref=off

RT [min] Type Width [min]

$13.136 \mathrm{VV}$

0.1695

0.1652

Sum

Area

14003.7656

43473.3477

57477.1133
Height Area\%

1322.683024 .3641

3166.834075 .6359

Signal: $\quad$ MWD1 B, Sig=280,4 Ref=off 


$\begin{array}{rrrr}13.136 \text { VV } & 0.1658 & 8677.5889 & 844.715322 .1242 \\ 13.377 \text { VV } & 0.1573 & 30544.4863 & 2370.890677 .8758 \\ & \text { Sum } & 39222.0752 & \end{array}$

Signal: $\quad$ MWD1 F, Sig=260,4 Ref=off

$\begin{array}{ccrc}\text { RT [min] Type } & \text { Width [min] } & \text { Area } & \text { Height Area\% } \\ 13.136 \mathrm{MM} & 0.1585 & 12919.3477 & 1358.925021 .3525 \\ 13.381 \mathrm{VV} & 0.1748 & 47585.8594 & 3308.088178 .6475\end{array}$

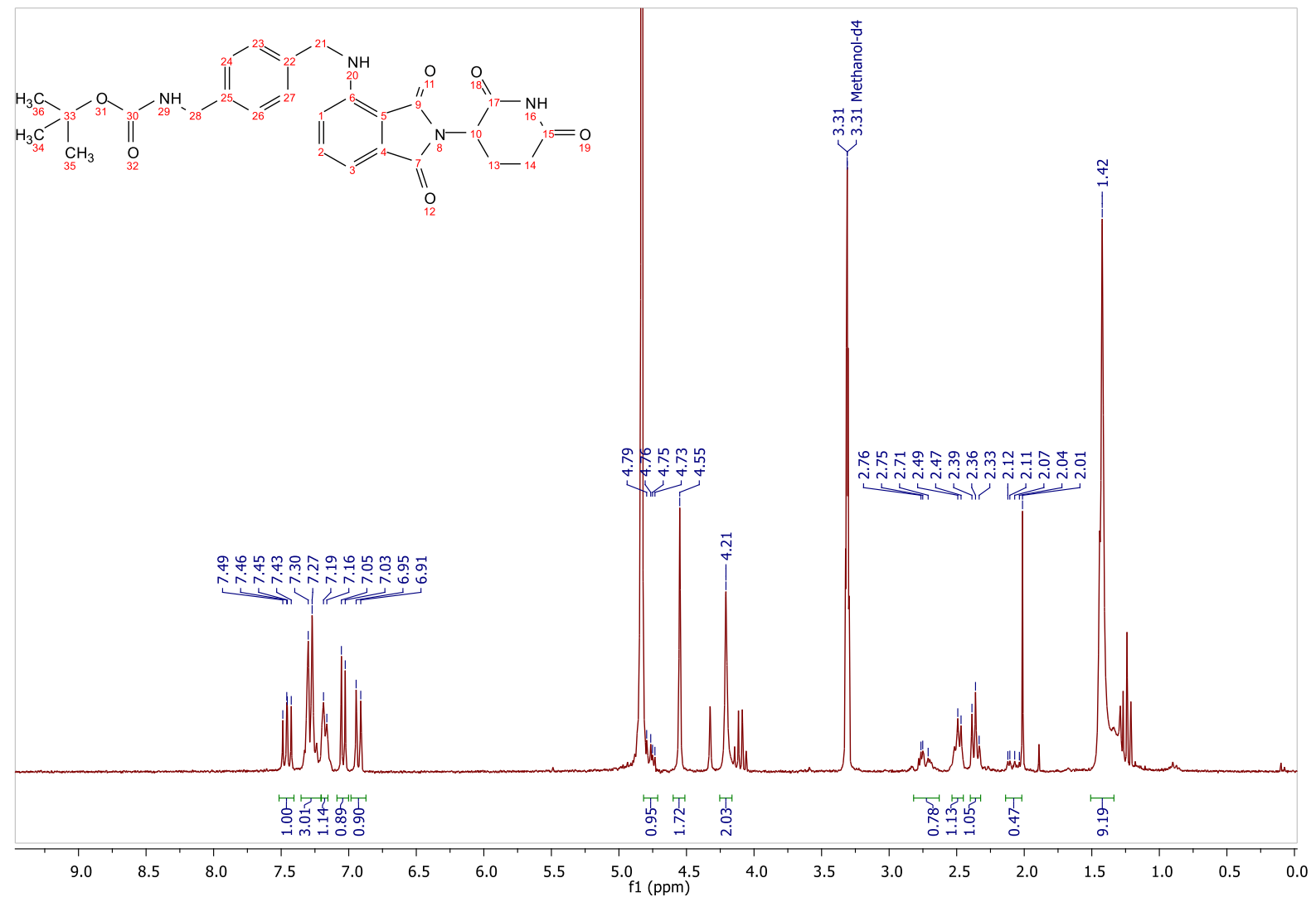


ESI, HPLC, ${ }^{1} \mathrm{H}-\mathrm{NMR}$ and ${ }^{13} \mathrm{C}-\mathrm{NMR}$ of tert-butyl (2-(3-(((S)-1-((2S,4R)-4-hydroxy-2-((4-(4-methylthiazol5-yl)benzyl)carbamoyl)pyrrolidin-1-yl)-3,3-dimethyl-1-oxobutan-2-yl)amino)-3oxopropoxy)ethyl)carbamate (L6)

C:IXcaliburldata\AD139

AD139 \#35-42 RT: 0.61-0.73 AV: 8 SB: 10 0.09-0.25 NL: $1.98 E 6$

T: $\{0,0\}+c$ ESI !corona sid=75.00 det=1306.00 Full ms [200.00-1200.00]
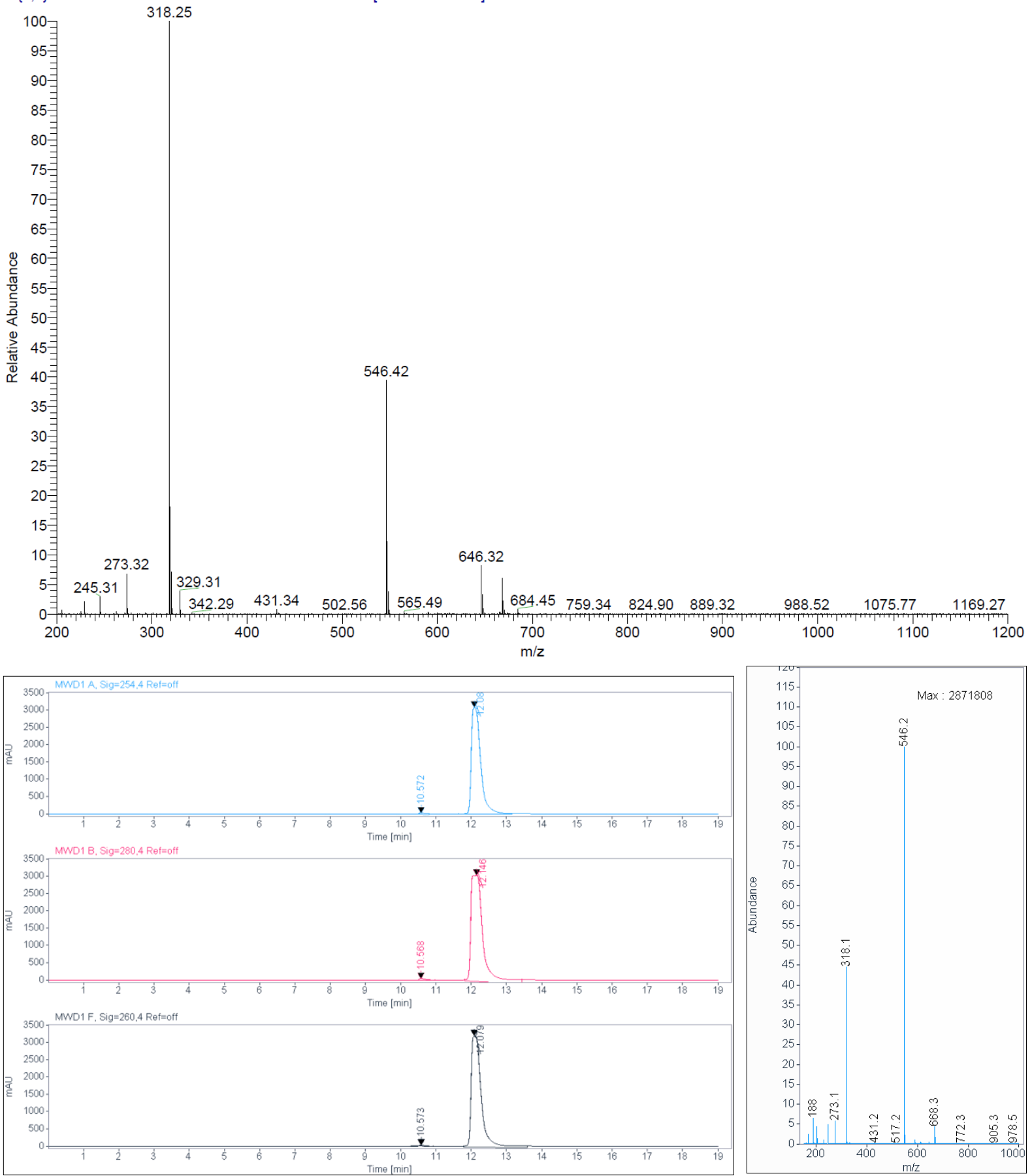

Signal: $\quad$ MWD1 A, Sig=254,4 Ref=off

$\begin{array}{crrr}\text { RT [min] Type } & \text { Width [min] } & \text { Area } & \text { Height Area\% } \\ 10.572 \mathrm{MM} & 0.2555 & 672.4956 & 43.86691 .1729 \\ 12.080 \mathrm{VV} & 0.2221 & 56661.4414 & 3088.263298 .8271 \\ & \text { Sum } & 57333.9370 & \end{array}$

Signal: $\quad$ MWD1 B, Sig=280,4 Ref=off 


$\begin{array}{lrrr}10.568 \mathrm{VV} & 0.2175 & 784.3678 & 51.84851 .0468 \\ 12.146 \mathrm{MM} & 0.4020 & 74143.3516 & 3073.821598 .9532 \\ & \text { Sum } & 74927.7194 & \end{array}$

Signal: $\quad$ MWD1 F, Sig=260,4 Ref=off

$\begin{array}{crrr}\text { RT [min] Type } & \text { Width [min] } & \text { Area } & \text { Height Area\% } \\ 10.573 \text { MM } & 0.2640 & 536.8670 & 33.89430 .8248 \\ 12.079 \text { MM } & 0.3343 & 64556.0977 & 3218.718899 .1752\end{array}$

AV500-2020-05-07-adkn.32688

Group AK_Knapp

AD139
$1 H$ DMS /nmr/Tag-Messung Tag-Messung 43

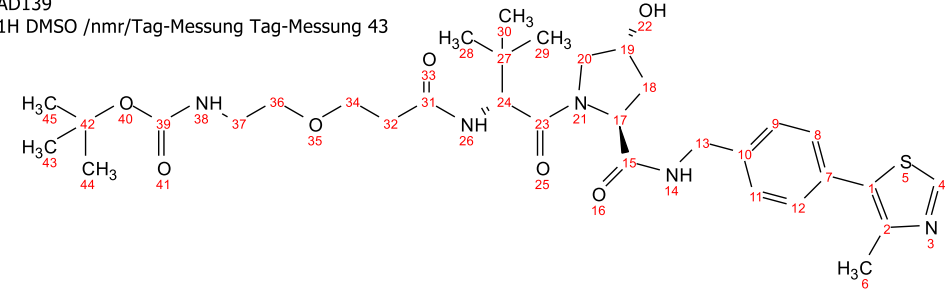

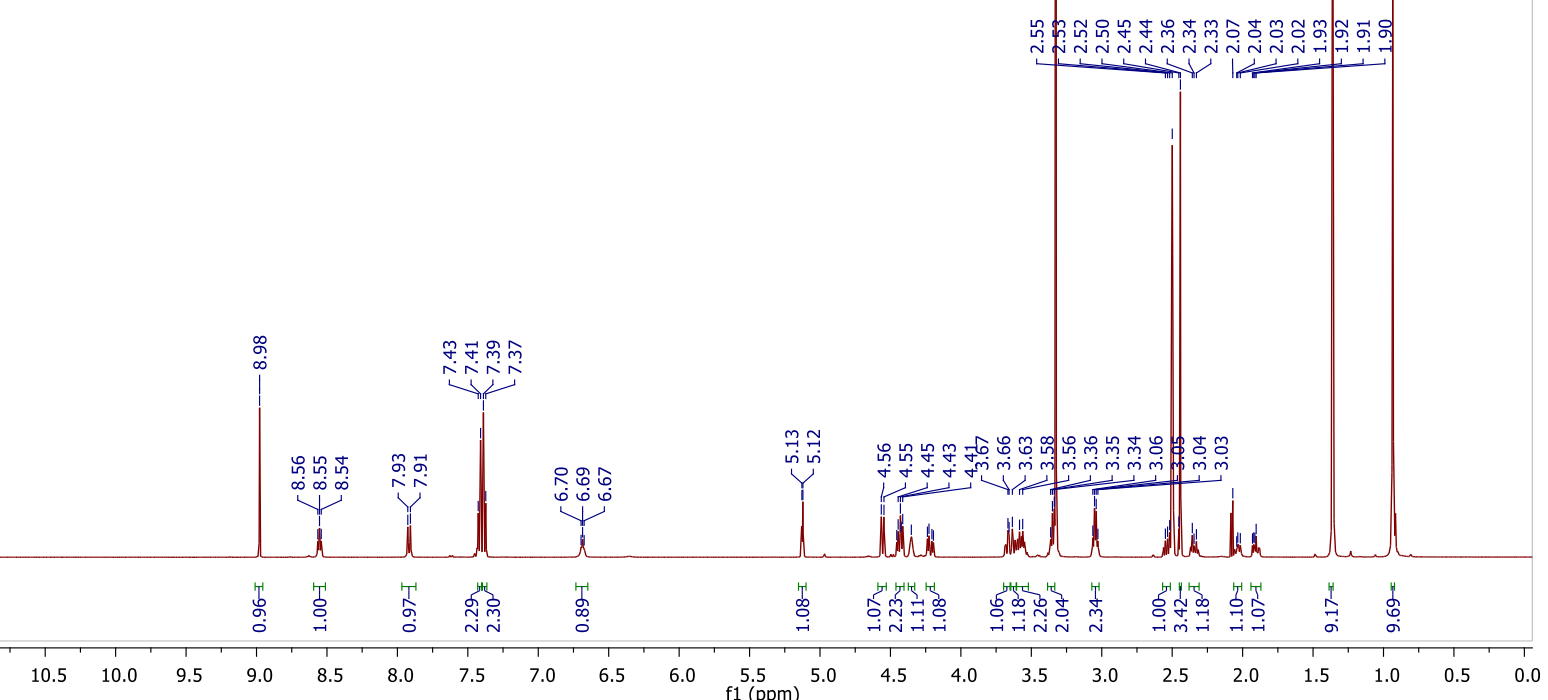




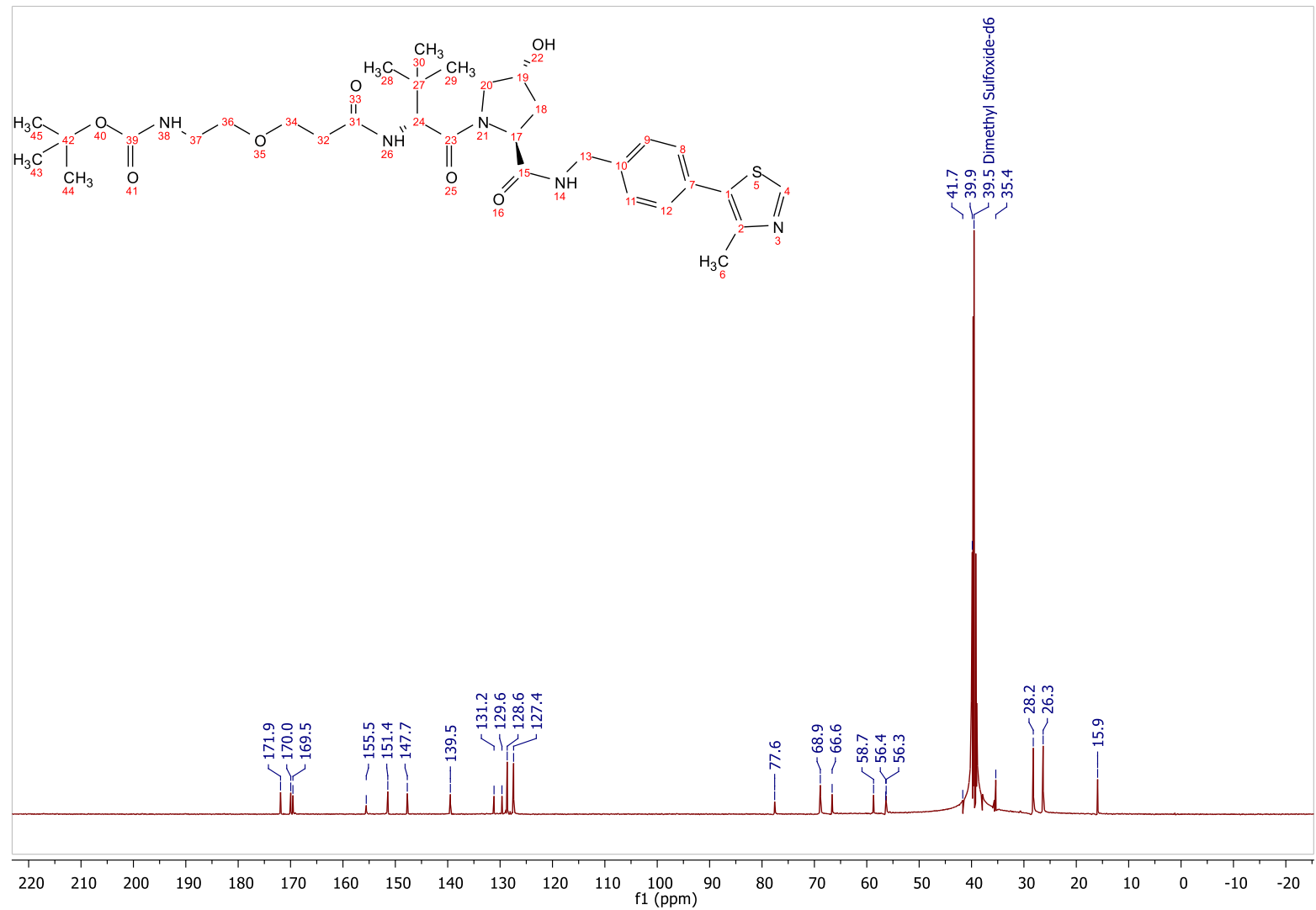


ESI, HPLC, ${ }^{1} \mathrm{H}-\mathrm{NMR}$ and ${ }^{13} \mathrm{C}-\mathrm{NMR}$ of tert-butyl (2-(2-(3-()(S)-1-((2S,4R)-4-hydroxy-2-((4-)(4methylthiazol-5-yl)benzyl)carbamoyl)pyrrolidin-1-yl)-3,3-dimethyl-1-oxobutan-2-yl)amino)-3oxopropoxy)ethoxy)ethyl)carbamate (L7)

C:IXcaliburldataIAD108-1

9/18/2019 7:35:17 AM

AD108-1 \#32-42 RT: 0.56-0.74 AV: 11 SB: 8 0.11-0.23 NL: $3.01 E 6$

$\mathrm{T}:\{0,0\}+\mathrm{c}$ ESI !corona sid=75.00 det=1306.00 Full ms [105.00-1200.00]
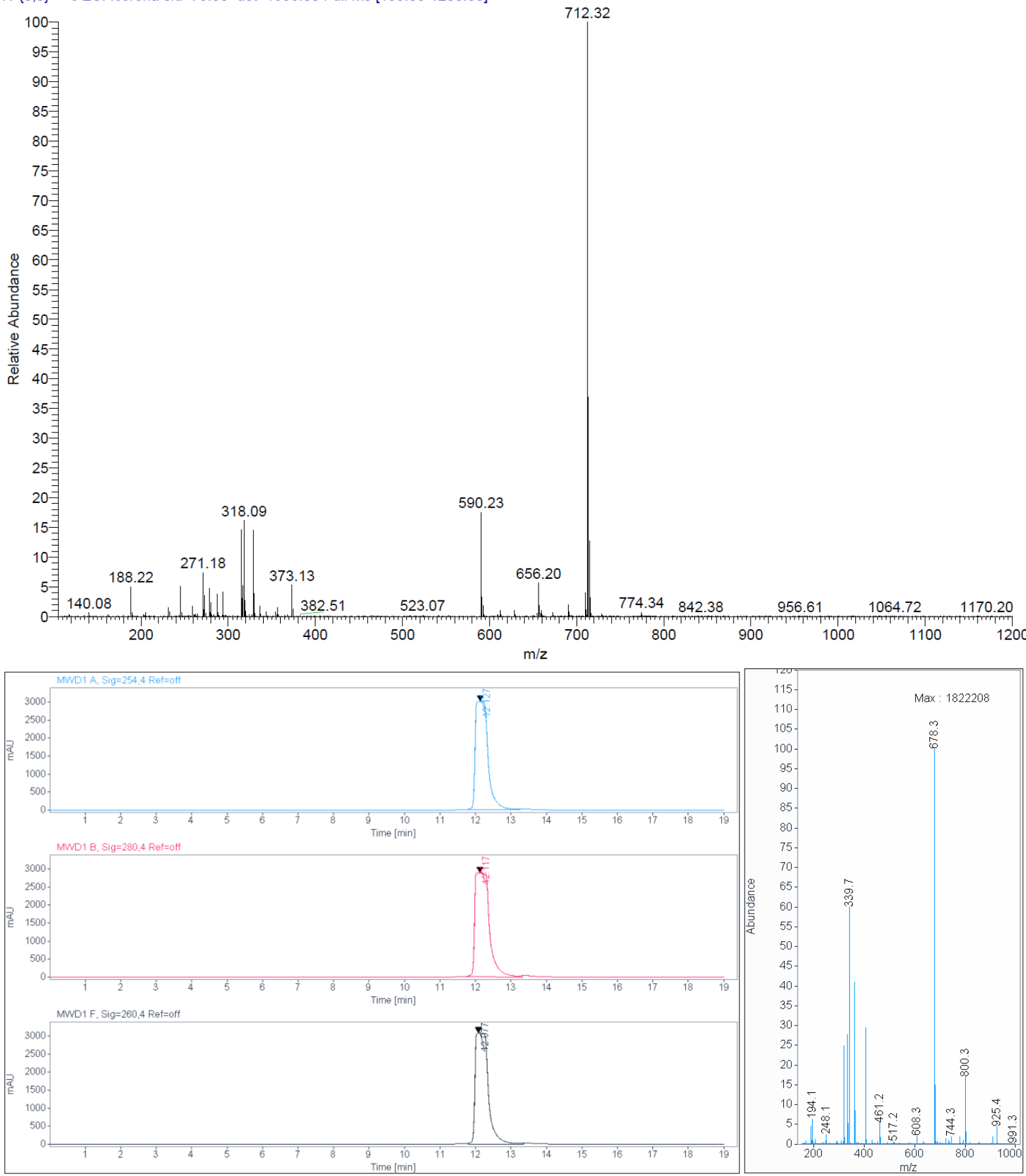

Signal: $\quad$ MWD1 A, Sig=254,4 Ref=off

$\begin{array}{crrrr}\text { RT [min] Type } & \text { Width [min] } & \text { Area } & \text { Height } & \text { Area\% } \\ 12.127 \mathrm{MM} & 0.4125 & 74880.8047 & 3025.3701 & 100.0000 \\ & \text { Sum } & 74880.8047 & & \end{array}$

Signal: $\quad$ MWD1 B, Sig=280,4 Ref=off 


$\begin{array}{rrrrr}12.117 \mathrm{MM} & 0.4749 & 82822.3672 & 2906.9238 & 100.0000 \\ & \text { Sum } & 82822.3672 & & \end{array}$

Signal: $\quad$ MWD1 $\mathrm{F}, \mathrm{Sig}=260,4 \mathrm{Ref}=\mathrm{off}$

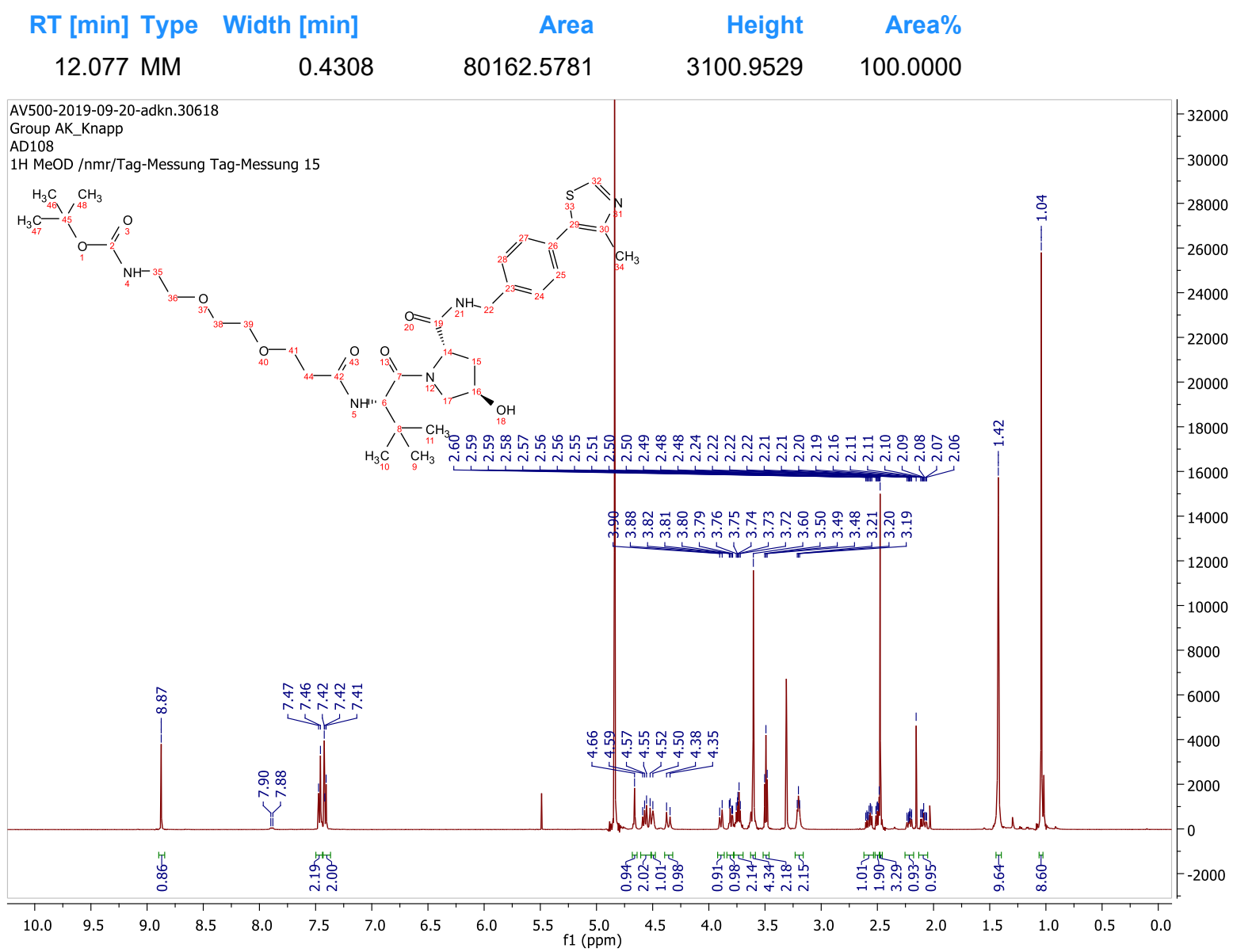




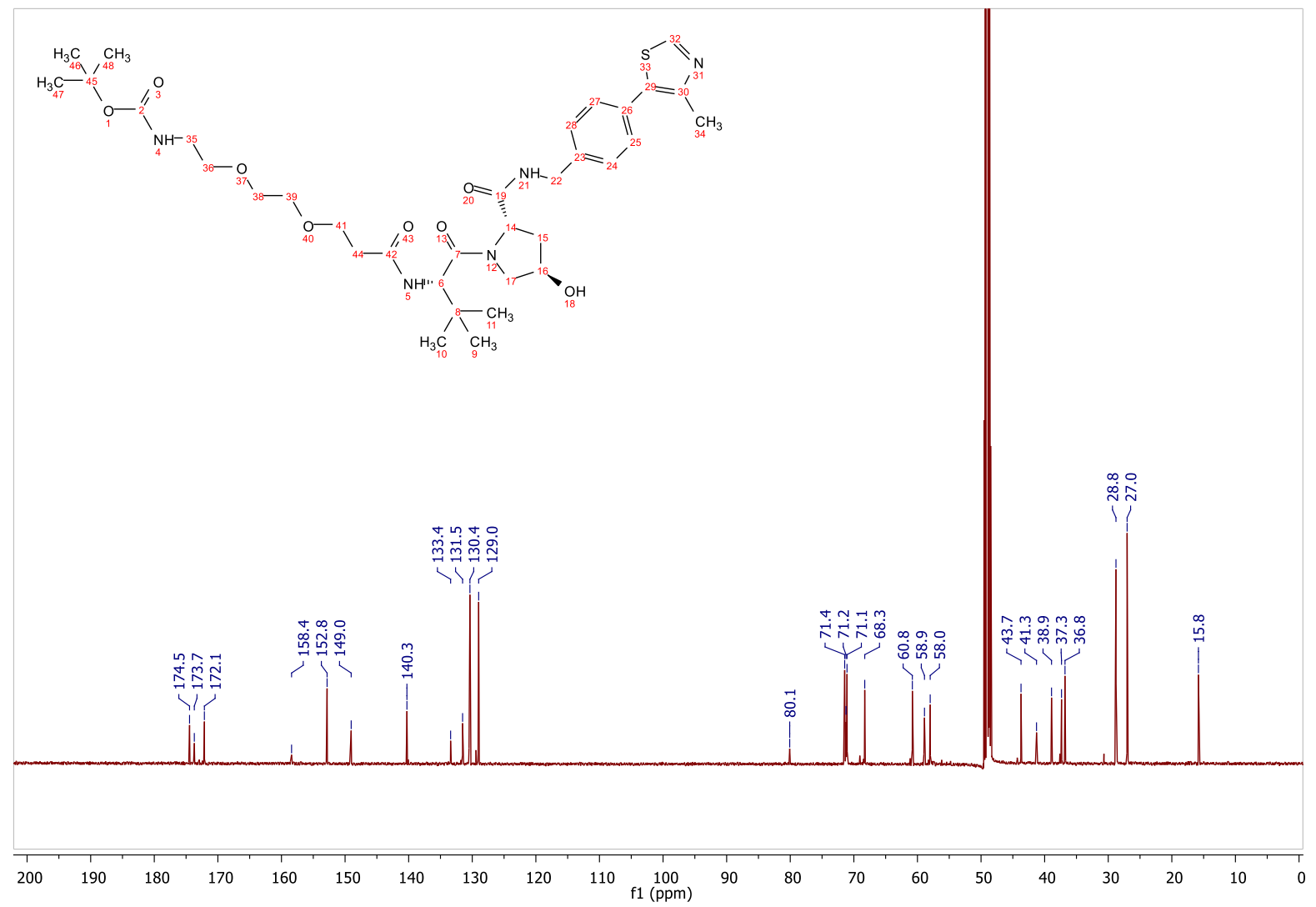


ESI, HPLC, ${ }^{1} \mathrm{H}-\mathrm{NMR}$ and ${ }^{13} \mathrm{C}-\mathrm{NMR}$ of tert-butyl ((S)-17-((2S,4R)-4-hydroxy-2-((4-(4-methylthiazol-5yl)benzyl)carbamoyl)pyrrolidine-1-carbonyl)-18,18-dimethyl-15-oxo-3,6,9,12-tetraoxa-16azanonadecyl)carbamate (L8)

C:IXcaliburldata|AD108-2

AD108-2 \#30-41 RT: 0.52-0.72 AV: 12 SB: 8 0.00-0.13 NL: 1.01E6

$\mathrm{T}:\{0,0\}+\mathrm{c}$ ESI !corona sid=75.00 det=1306.00 Full ms [105.00-1200.00]
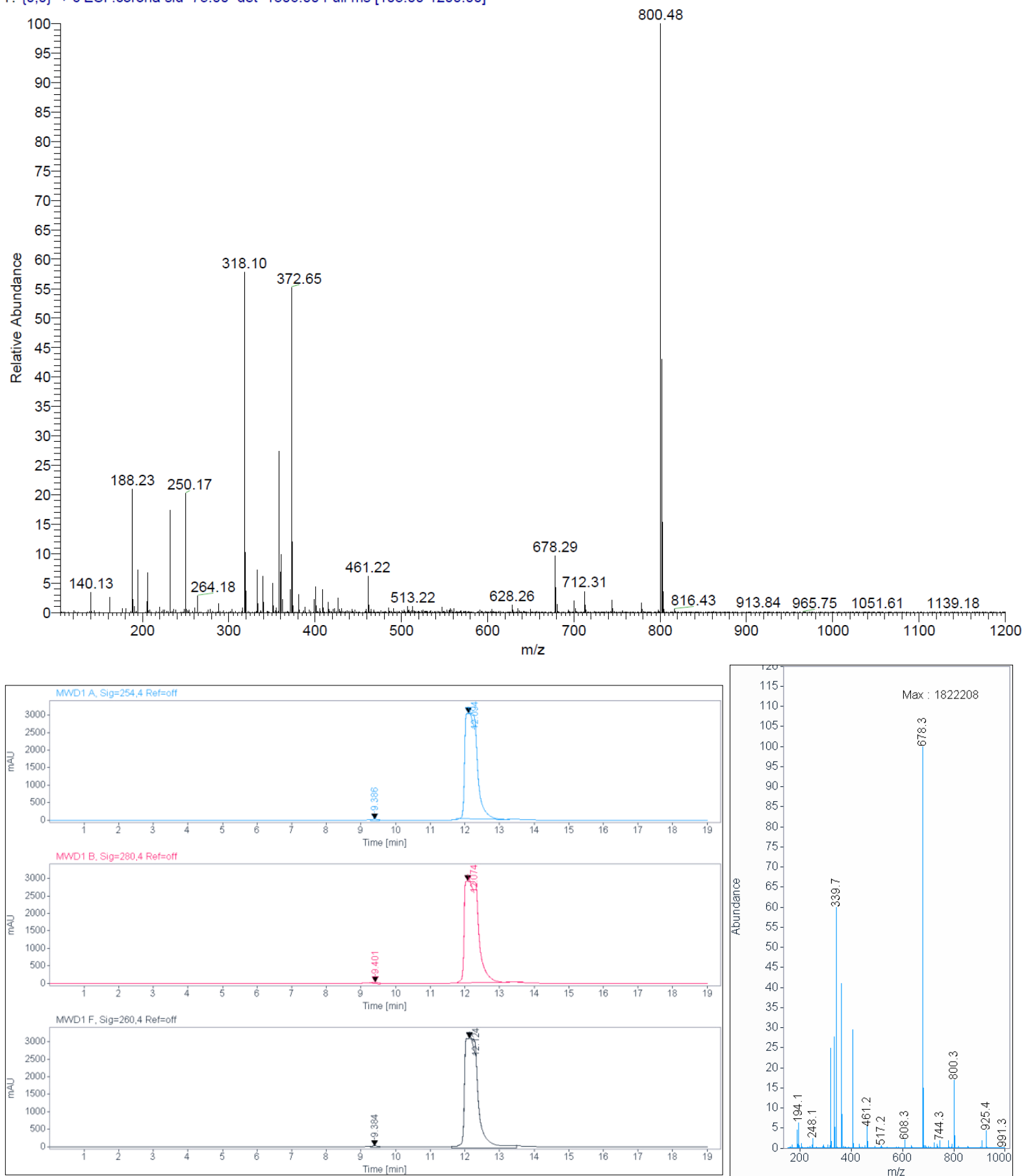

Signal: $\quad$ MWD1 A, Sig=254,4 Ref=off

$\begin{array}{rrr}\text { RT [min] Type } & \text { Width [min] } & \text { Area } \\ 9.386 \text { MM } & 0.2141 & 578.0328 \\ 12.094 \mathrm{MM} & 0.4188 & 76100.4609 \\ & \text { Sum } & 76678.4938\end{array}$

Height Area\%

44.98900 .7538

3028.603099 .2462

Signal: $\quad$ MWD1 B, Sig=280,4 Ref=off 


$\begin{array}{rrrr}\text { RT [min] Type } & \text { Width [min] } & \text { Area } & \text { Height Area\% } \\ 9.401 \mathrm{MM} & 0.2216 & 570.3035 & 42.88590 .6772 \\ 12.074 \mathrm{MM} & 0.4768 & 83642.8047 & 2923.658099 .3228 \\ & \text { Sum } & 84213.1082 & \end{array}$

Signal: $\quad$ MWD1 F, Sig=260,4 Ref=off

$\begin{array}{rrrr}\text { RT [min] Type } & \text { Width [min] } & \text { Area } & \text { Height Area\% } \\ 9.384 \mathrm{MM} & 0.2119 & 613.2241 & 48.22440 .6948 \\ 12.124 \mathrm{MM} & 0.4631 & 87650.6875 & 3154.525999 .3052 \\ & \text { Sum } & 88263.9116 & \end{array}$

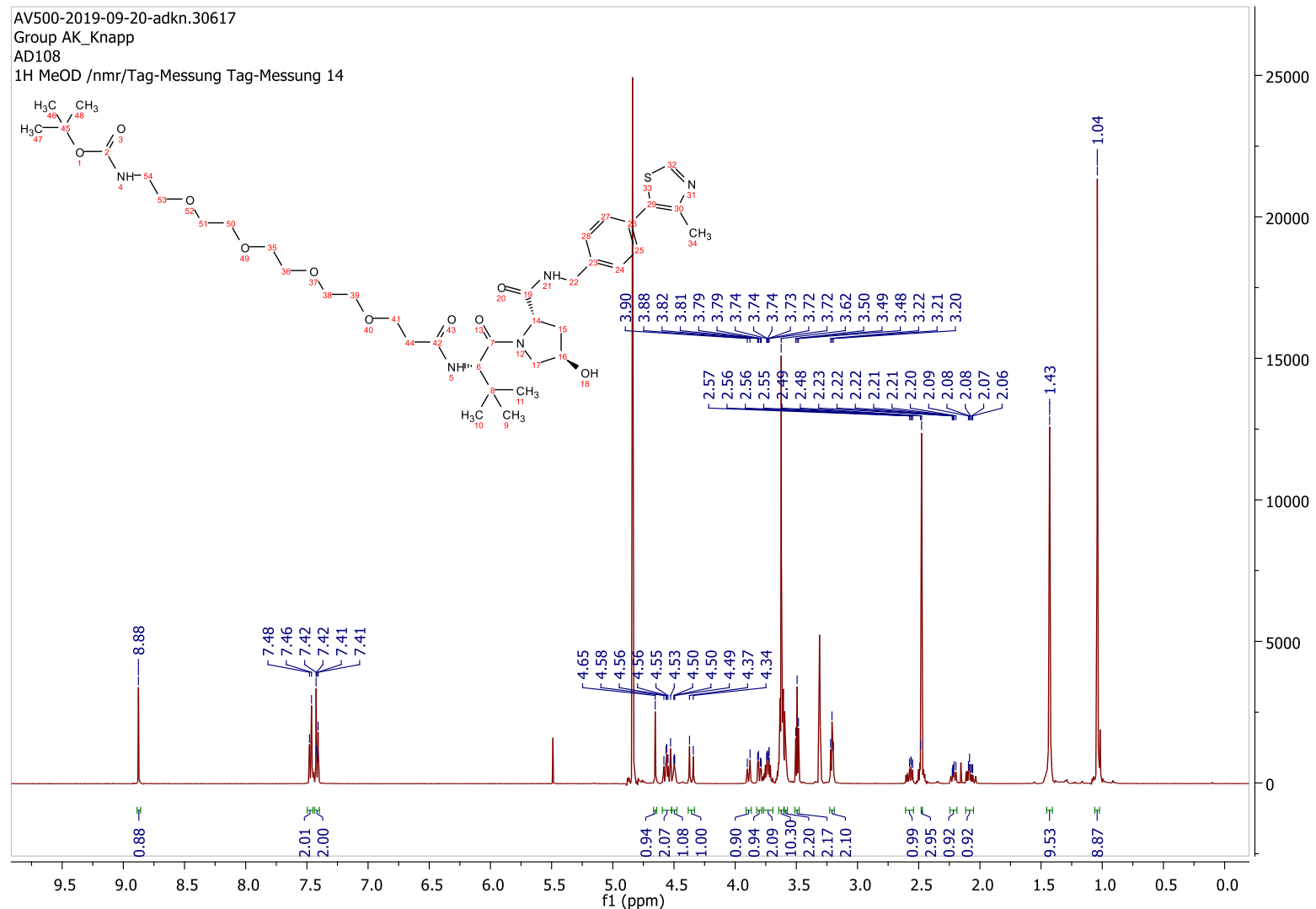


234

Appendix

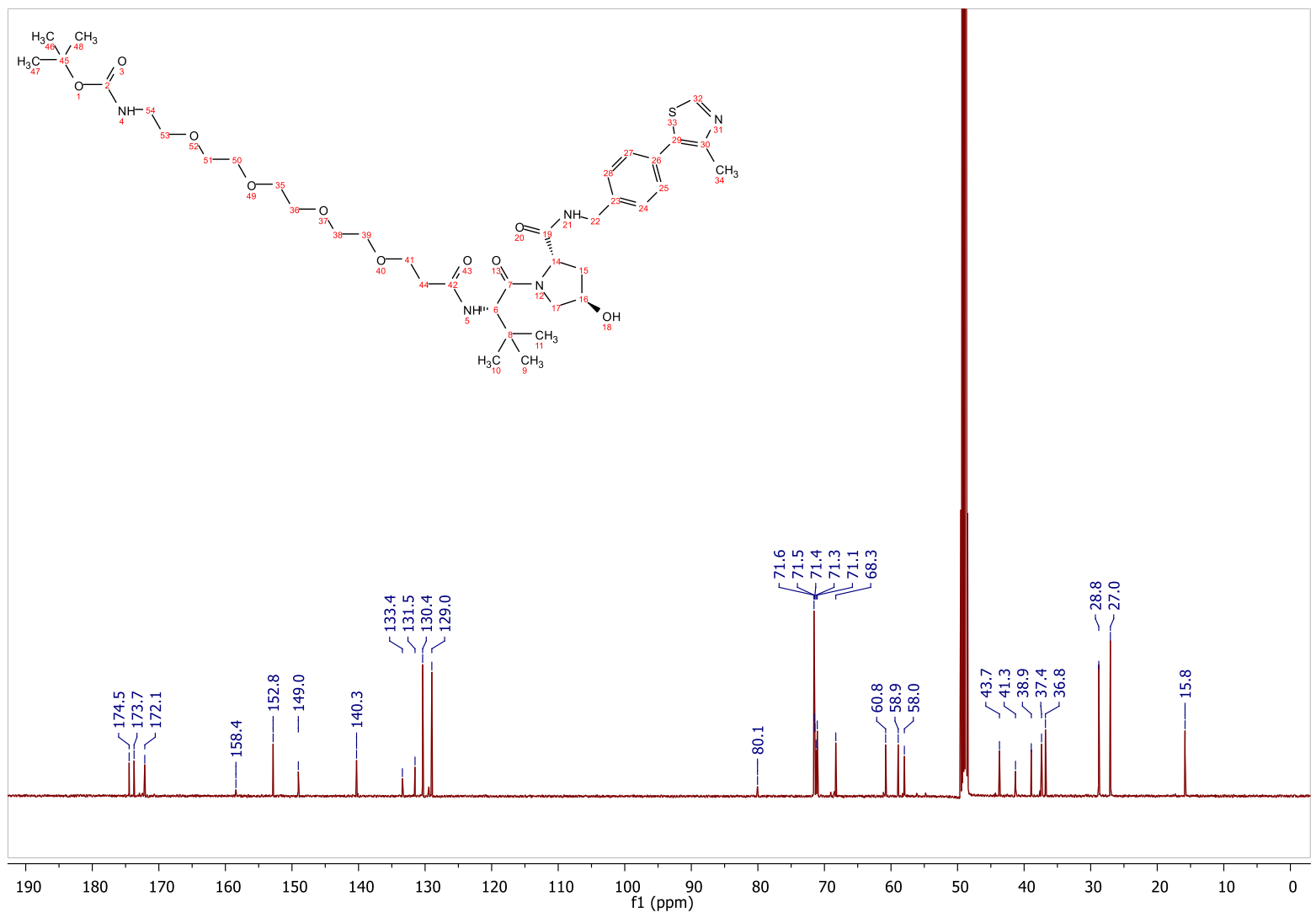


ESI, ${ }^{1} \mathrm{H}-\mathrm{NMR}$ and ${ }^{13} \mathrm{C}-\mathrm{NMR}$ of tert-butyl (3-)((S)-1-((2S,4R)-4-hydroxy-2-((4-(4-methylthiazol-5yl)benzyl)carbamoyl)pyrrolidin-1-yl)-3,3-dimethyl-1-oxobutan-2-yl)amino)-3-oxopropyl)carbamate (L9)

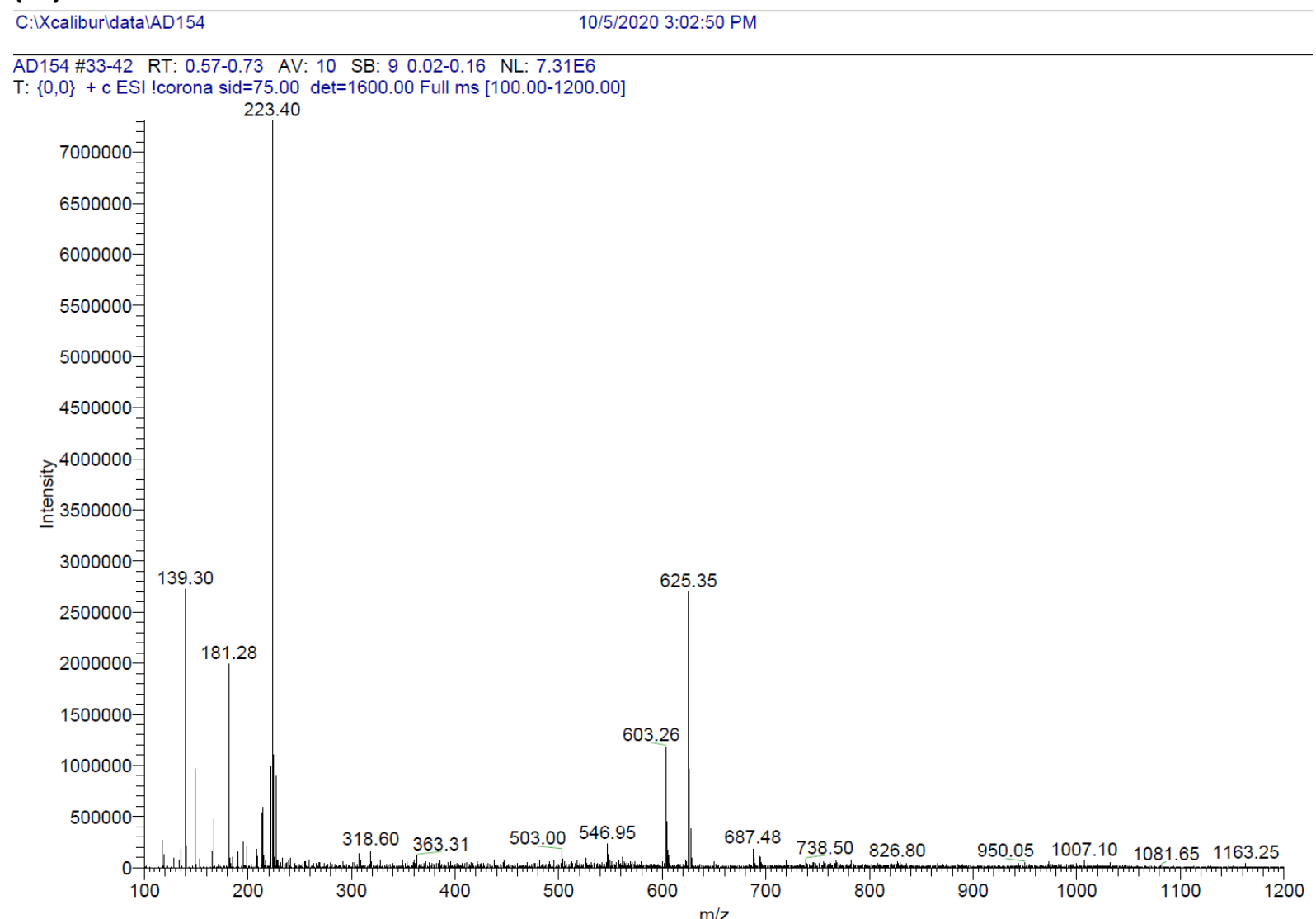

AV300-2020-10-12-adkn.37730

Group AK_Knapp

1H MeOD /nmr Tag-Messung 13

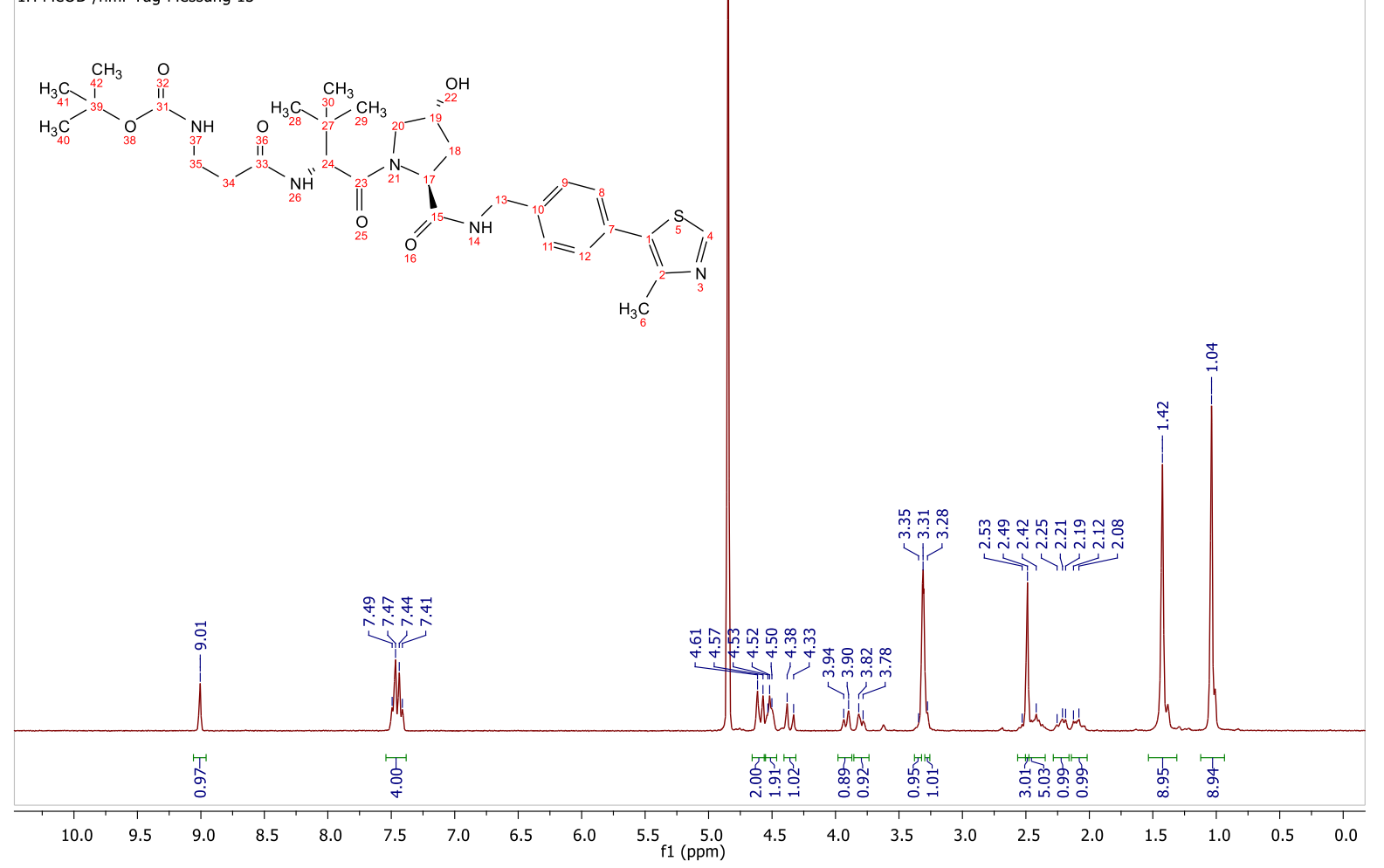



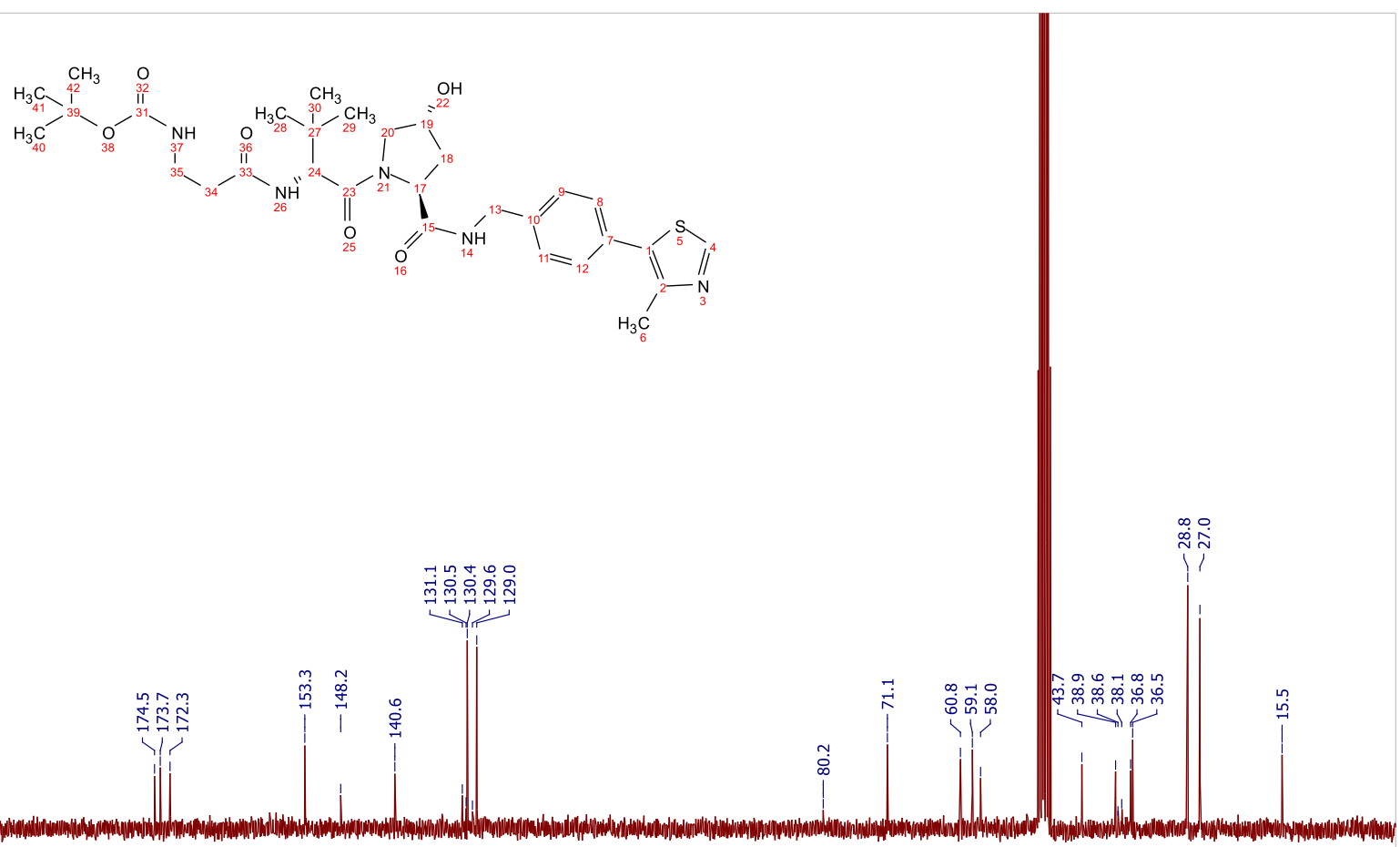

190

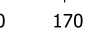

160

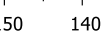

120 $\begin{array}{cc}100 & 90 \\ f 1(\mathrm{ppm})\end{array}$

$80 \quad 70$

$60 \quad 50 \quad 40$ 
ESI, ${ }^{1} \mathrm{H}-\mathrm{NMR}$ and ${ }^{13} \mathrm{C}-\mathrm{NMR}$ of tert-butyl (4-)((S)-1-((2S,4R)-4-hydroxy-2-((4-(4-methylthiazol-5yl)benzyl)carbamoyl)pyrrolidin-1-yl)-3,3-dimethyl-1-oxobutan-2-yl)amino)-4-oxobutyl)carbamate (L10)

C:IXcaliburldata|AD155

10/5/2020 3:04:33 PM

AD155 \#32-42 RT: 0.56-0.73 AV: 11 SB: 10 0.05-0.21 NL: 5.31E6

$\mathrm{T}:\{0,0\}+\mathrm{c}$ ESI !corona sid $=75.00$ det $=1600.00$ Full $\mathrm{ms}[100.00-1200.00]$

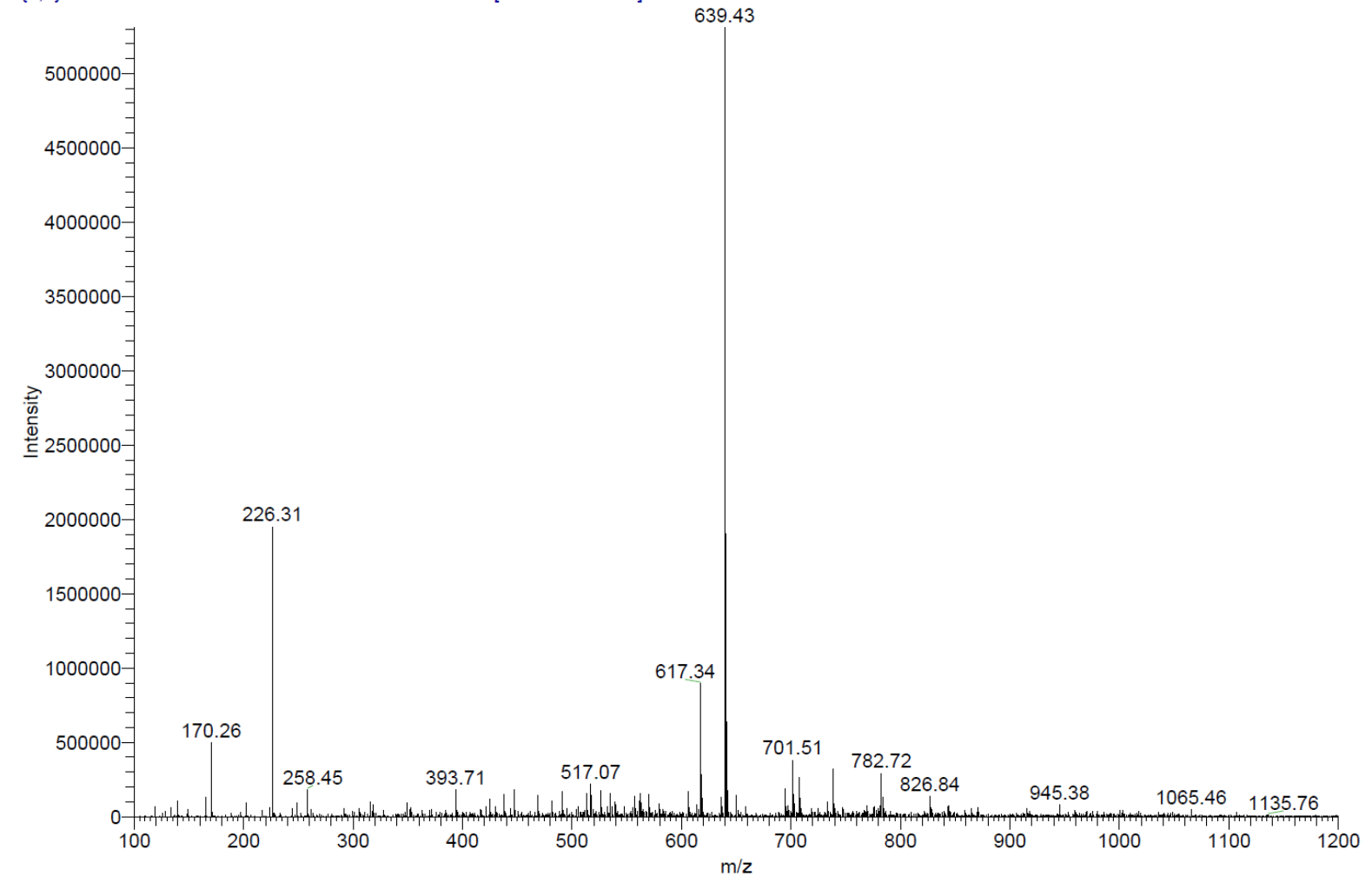

AV300-2020-10-12-adkn.37731

Group AK_Knapp

$1 \mathrm{H}$ MeOD /nmr Tag-Messung 14

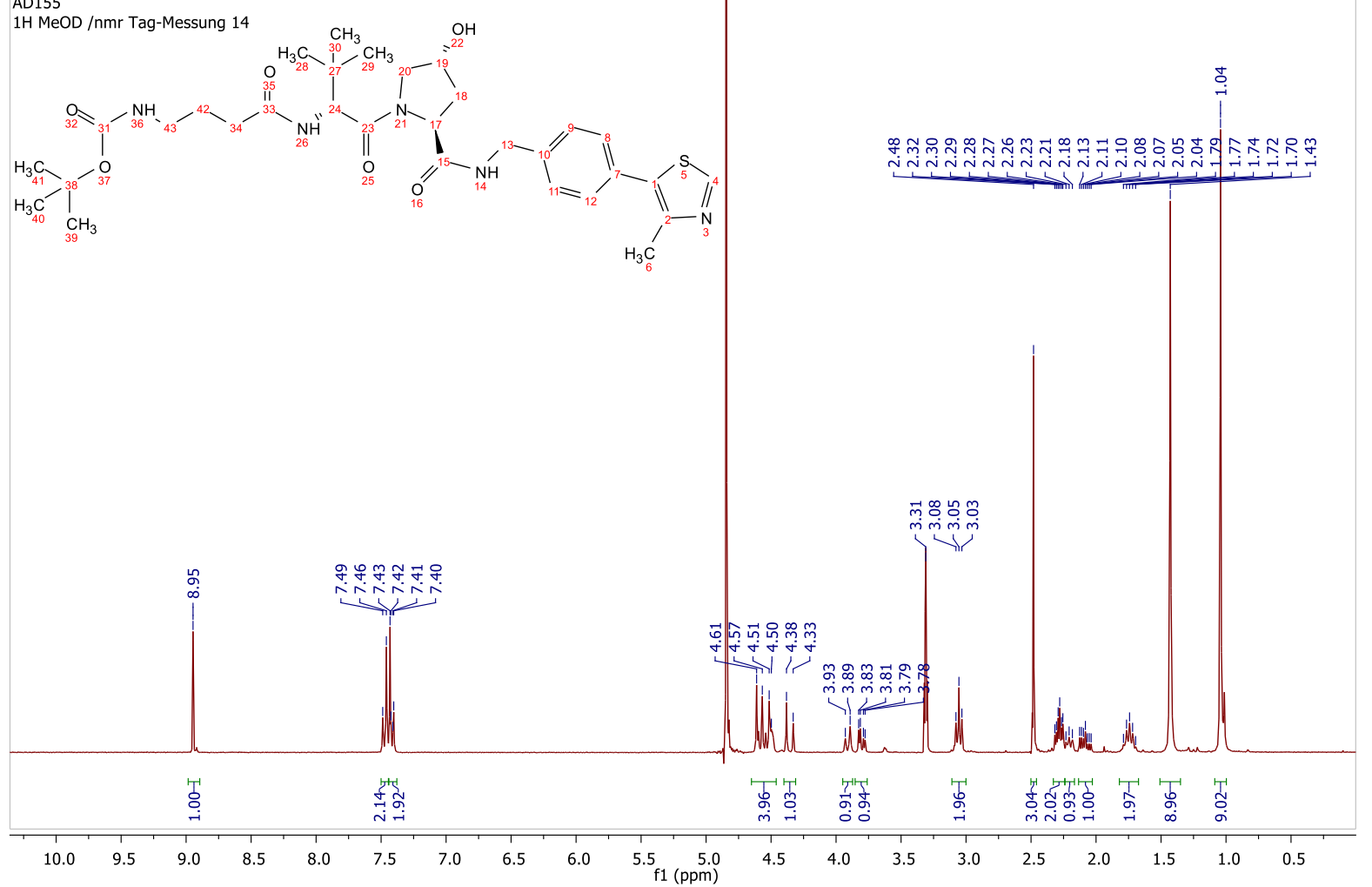




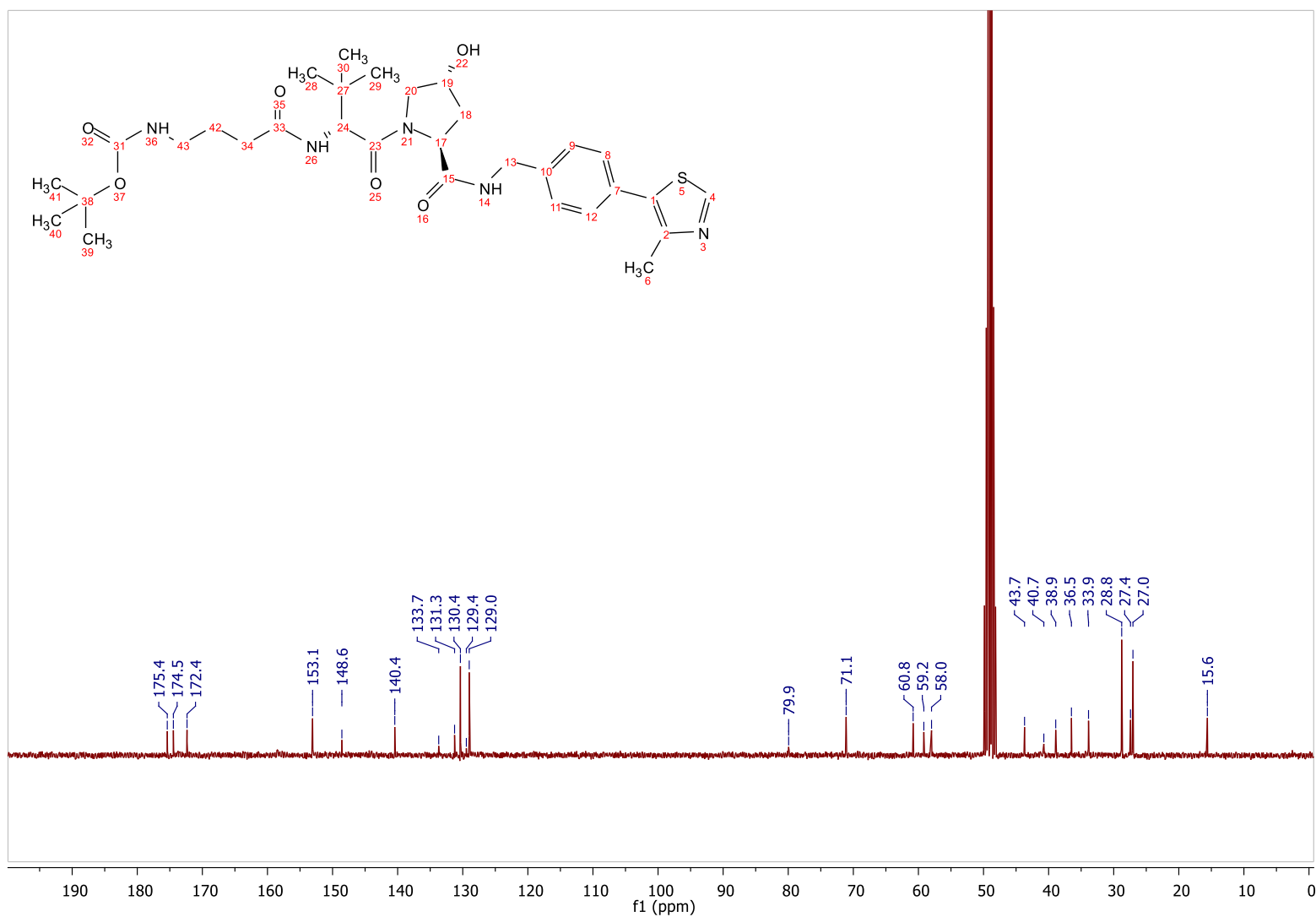


ESI, HPLC, ${ }^{1} \mathrm{H}-\mathrm{NMR}$ and ${ }^{13} \mathrm{C}-\mathrm{NMR}$ of tert-butyl (5-)((S)-1-((2S,4R)-4-hydroxy-2-((4-(4-methylthiazol-5yl)benzyl)carbamoyl)pyrrolidin-1-yl)-3,3-dimethyl-1-oxobutan-2-yl)amino)-5-oxopentyl)carbamate (L11)

C:Xcaliburldata|AD107

9/13/2019 6:56:17 AM

AD107 \#32-43 RT: 0.55-0.75 AV: 12 SB: 7 0.07-0.18 NL: 1.11E6

$\mathrm{T}:\{0,0\}+\mathrm{c}$ ESI !corona sid=75.00 det=1306.00 Full ms [200.00-1200.00]
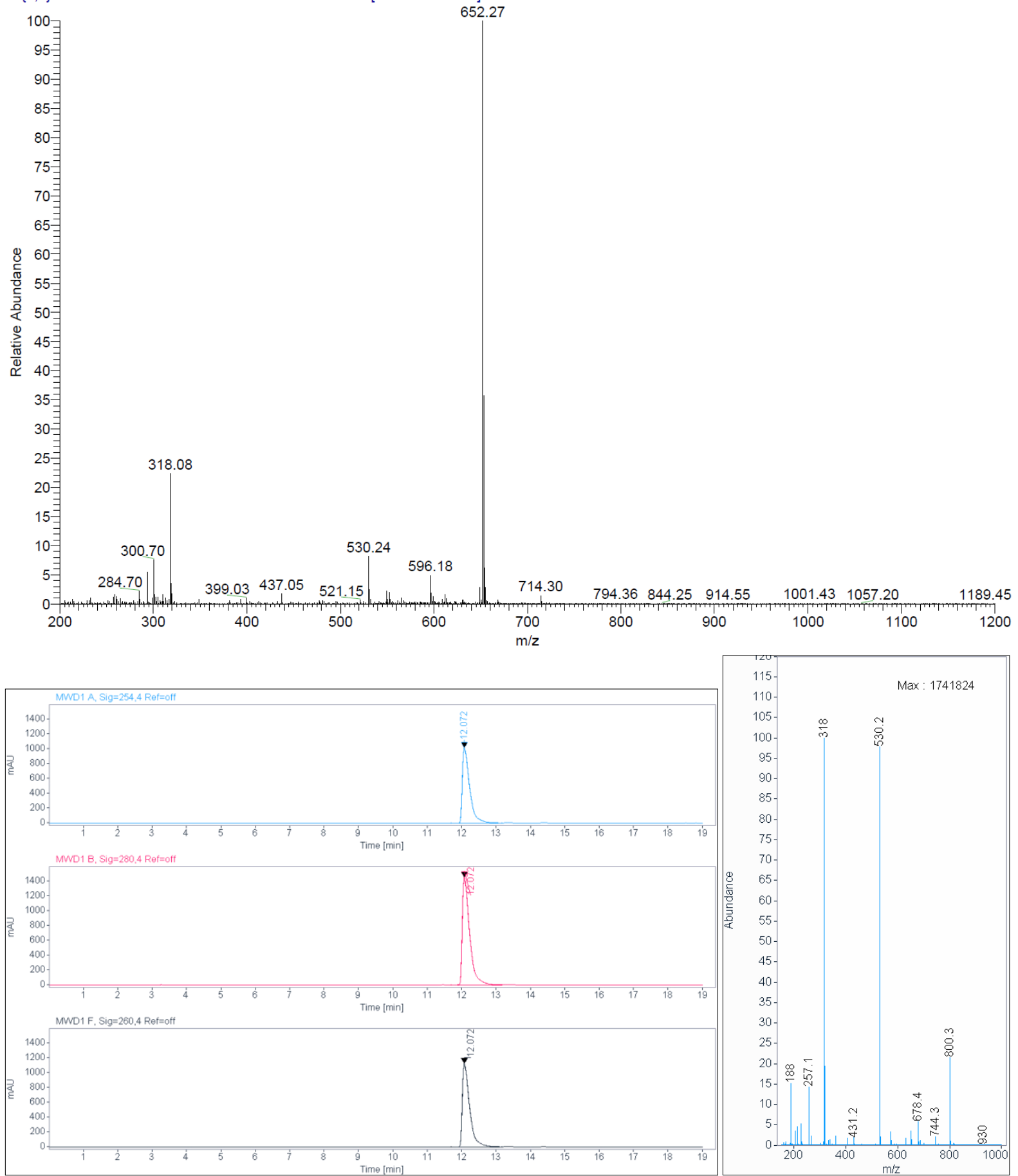

Signal: $\quad$ MWD1 A, Sig=254,4 Ref=off

RT [min] Type Width [min] $12.072 \mathrm{VV}$
0.2259

Sum
Area

15183.3105

15183.3105
Height Area\%

$1018.8142 \quad 100.0000$

Signal: $\quad$ MWD1 B, Sig=280,4 Ref=off 


$\begin{array}{crrrr}\text { RT [min] Type } & \text { Width [min] } & \text { Area } & \text { Height } & \text { Area\% } \\ 12.072 \text { VV } & 0.2262 & 21853.1191 & 1455.2832 & 100.0000 \\ & \text { Sum } & 21853.1191 & & \end{array}$

Signal: $\quad$ MWD1 F, Sig=260,4 Ref=off

$\begin{array}{ccrrr}\text { RT [min] Type } & \text { Width [min] } & \text { Area } & \text { Height } & \text { Area\% } \\ 12.072 \text { VV } & 0.2257 & 16775.8438 & 1126.5007 & 100.0000\end{array}$

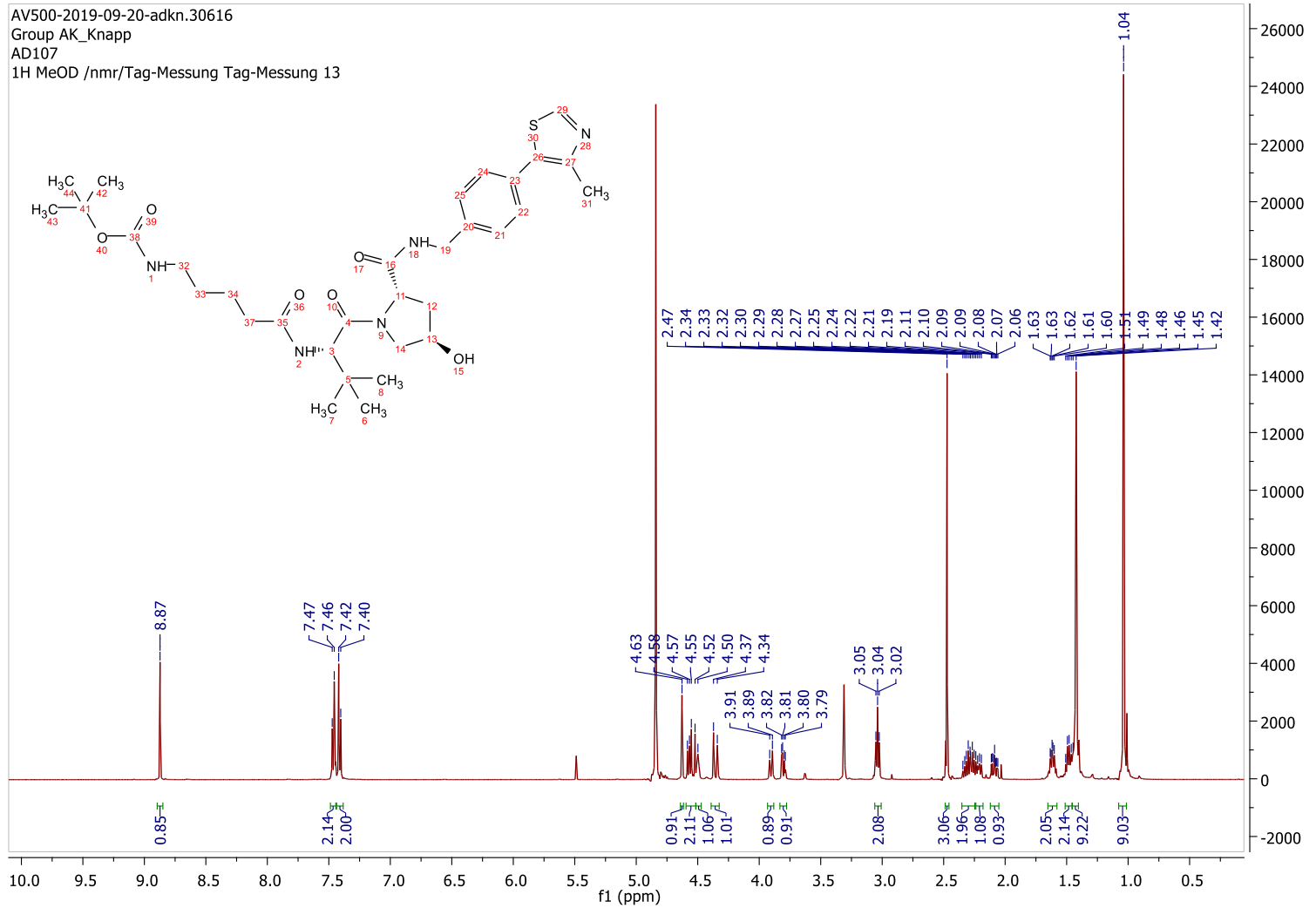




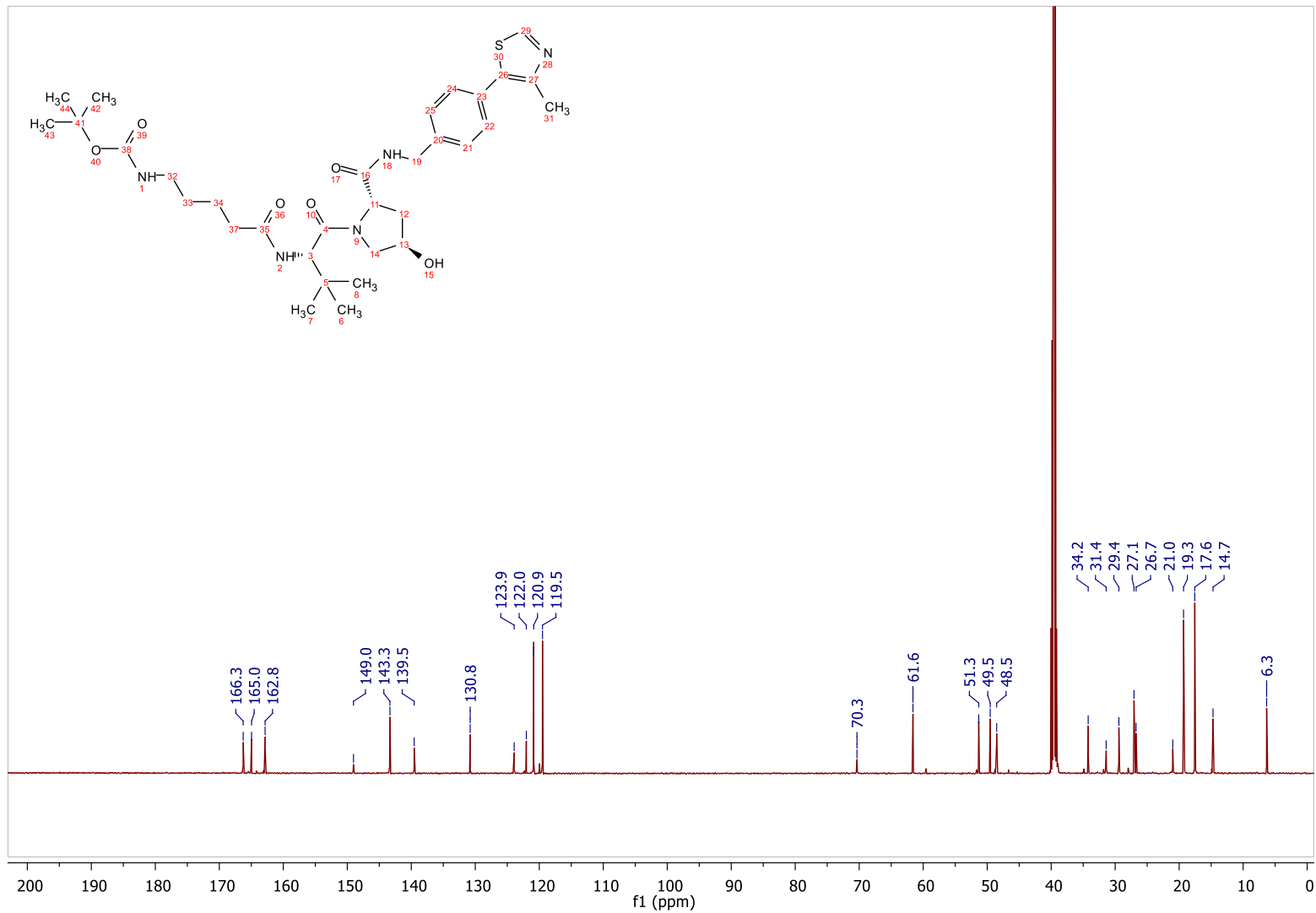


ESI, HPLC, ${ }^{1} \mathrm{H}-\mathrm{NMR}$ and ${ }^{13} \mathrm{C}-\mathrm{NMR}$ of tert-butyl (6-)((S)-1-((2S,4R)-4-hydroxy-2-((4-(4-methylthiazol-5yl)benzyl)carbamoyl)pyrrolidin-1-yl)-3,3-dimethyl-1-oxobutan-2-yl)amino)-6-oxohexyl)carbamate (L12)

C:IXcaliburldataIAD113-1

10/15/2019 3:46:17 PM

AD113-1\#36-42 RT: 0.62-0.73 AV: 7 SB: 9 0.07-0.21 NL: $1.64 E 6$

$\mathrm{T}:\{0,0\}+\mathrm{c} E \mathrm{SI}$ !corona sid=75.00 det=1306.00 Full ms [200.00-1200.00]
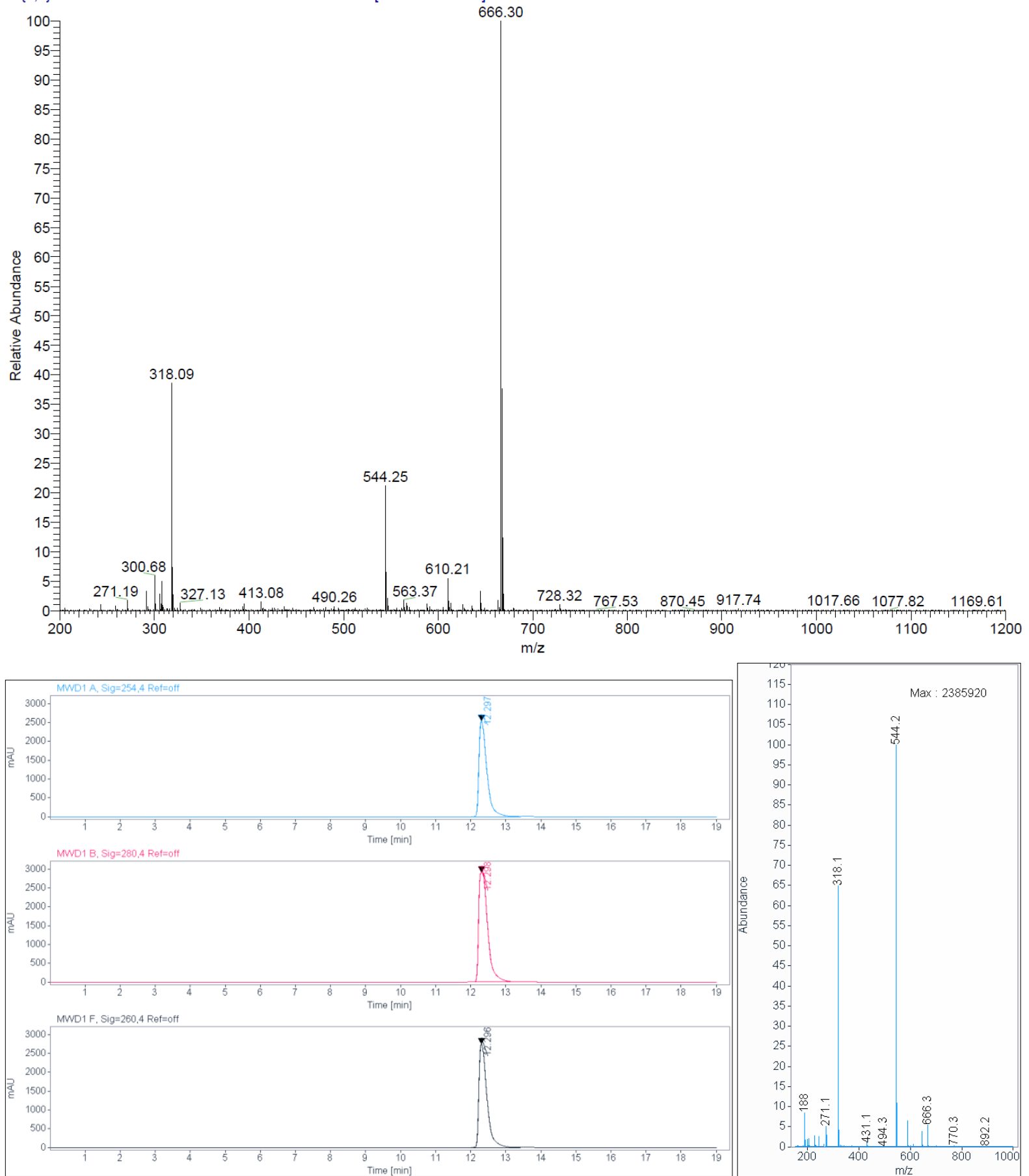

Signal: $\quad$ MWD1 A, Sig=254,4 Ref=off

$\begin{array}{rrrrr}\text { RT [min] Type } & \text { Width [min] } & \text { Area } & \text { Height } & \text { Area\% } \\ 12.297 \text { VV } & 0.2391 & 40065.7813 & 2553.0674 & 100.0000 \\ & \text { Sum } & 40065.7813 & & \end{array}$

Signal: $\quad$ MWD1 B, Sig=280,4 Ref=off 


$\begin{array}{crrrr}\text { RT [min] Type } & \text { Width [min] } & \text { Area } & \text { Height } & \text { Area\% } \\ 12.298 \text { MM } & 0.2941 & 51400.3750 & 2912.6912 & 100.0000 \\ & \text { Sum } & 51400.3750 & & \end{array}$

Signal: $\quad$ MWD1 F, Sig=260,4 Ref=off

$\begin{array}{ccrrr}\text { RT [min] Type } & \text { Width [min] } & \text { Area } & \text { Height } & \text { Area\% } \\ 12.296 \text { VV } & 0.2173 & 44116.5742 & 2763.2627 & 100.0000\end{array}$

AV500-2019-10-21-adkn.30870

Group AK_Knapp

1H MeOD /nmr/Tag-Messung Tag-Messung 19

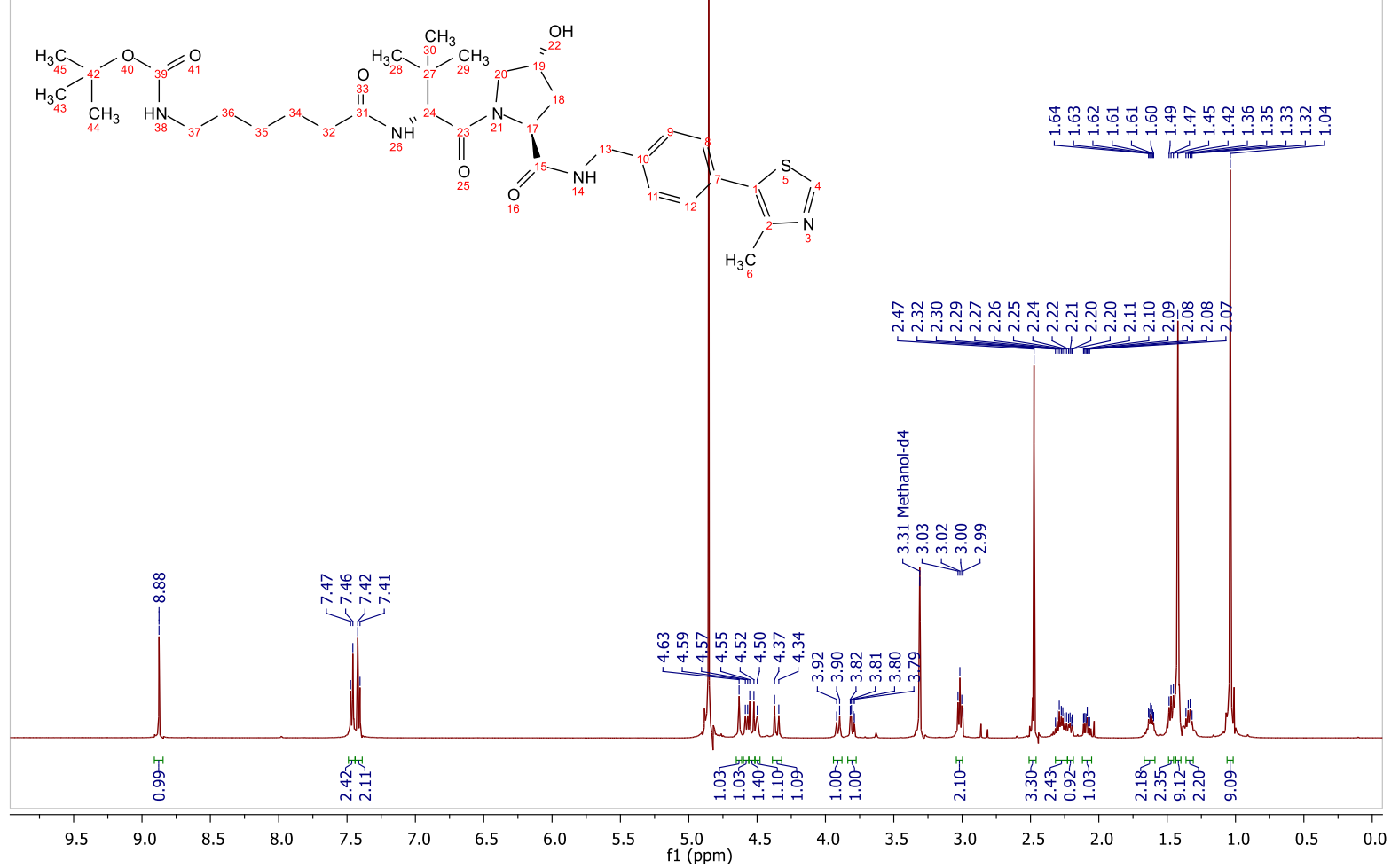




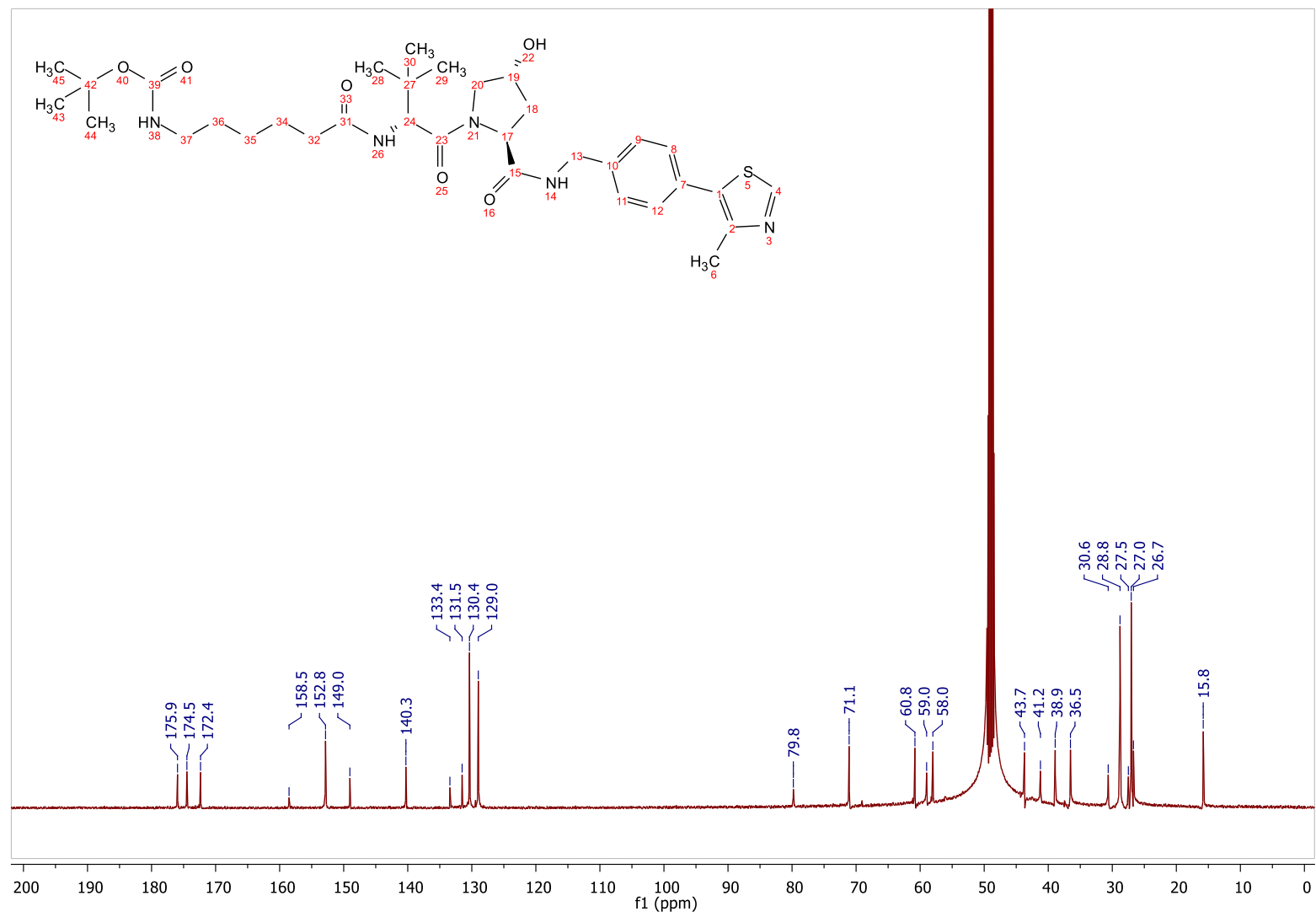


ESI, HPLC, ${ }^{1} \mathrm{H}-\mathrm{NMR}$ and ${ }^{13} \mathrm{C}-\mathrm{NMR}$ of tert-butyl (7-)((S)-1-((2S,4R)-4-hydroxy-2-((4-(4-methylthiazol-5yl)benzyl)carbamoyl)pyrrolidin-1-yl)-3,3-dimethyl-1-oxobutan-2-yl)amino)-7-oxoheptyl)carbamate (L13)

C:IXcaliburldata|AD106-2

9/16/2019 10:04:34 AM

AD106-2 \#34-42 RT: 0.59-0.73 AV: 9 SB: 11 0.04-0.22 NL: 1.04E6

$\mathrm{T}:\{0,0\}+\mathrm{c}$ ESI ! corona sid $=75.00$ det $=1306.00$ Full $\mathrm{ms}[105.00-1200.00]$
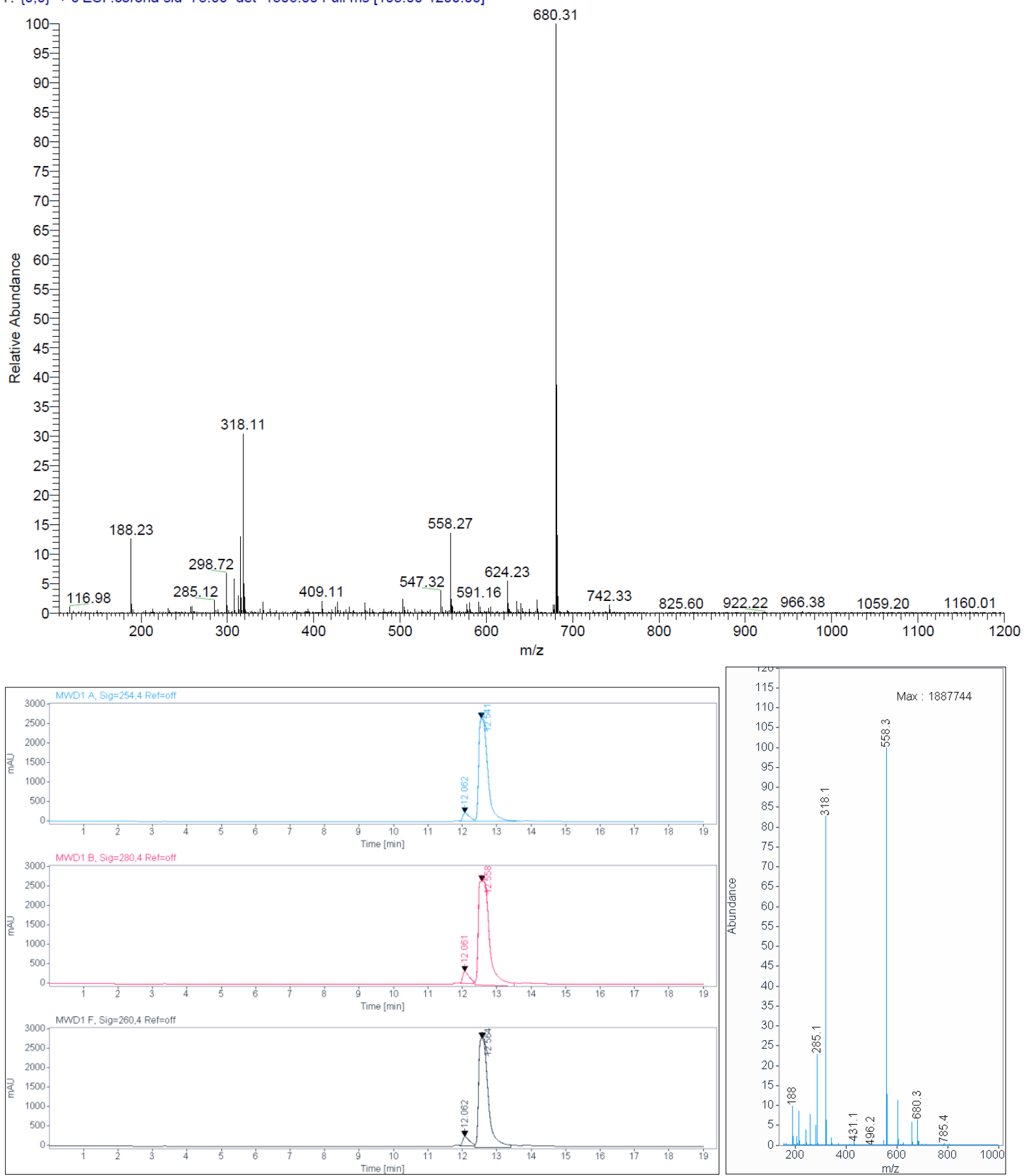

Signal: $\quad$ MWD1 $A$, Sig=254,4 Ref=off

$\begin{array}{rrrrr}\text { RT [min] Type } & \text { Width [min] } & \text { Area } & \text { Height } & \text { Area\% } \\ 12.062 \text { VV } & 0.2181 & 3252.9497 & 223.1664 & 6.2811 \\ 12.541 \text { VV } & 0.2368 & 48536.3945 & 2660.7231 & 93.7189 \\ & \text { Sum } & 51789.3442 & & \end{array}$

Signal: $\quad$ MWD1 B, Sig=280,4 Ref=off 


$\begin{array}{crrrr}\text { RT [min] Type } & \text { Width [min] } & \text { Area } & \text { Height } & \text { Area\% } \\ 12.061 \text { MM } & 0.2245 & 4209.9492 & 312.5555 & 6.5658 \\ 12.558 \text { MM } & 0.3742 & 59909.6172 & 2668.2478 & 93.4342 \\ & \text { Sum } & 64119.5664 & & \end{array}$

Signal: $\quad$ MWD1 F, Sig=260,4 Ref=off

$\begin{array}{ccrrr}\text { RT [min] Type } & \text { Width [min] } & \text { Area } & \text { Height } & \text { Area\% } \\ 12.062 \text { VV } & 0.2166 & 3560.8386 & 246.4372 & 6.1048 \\ 12.564 \text { MM } & 0.3241 & 54767.2188 & 2816.3435 & 93.8952\end{array}$

AV500-2019-09-20-adkn.30615

Group AK_Knapp

1H MeOD /nmr/Tag-Messung Tag-Messung 12
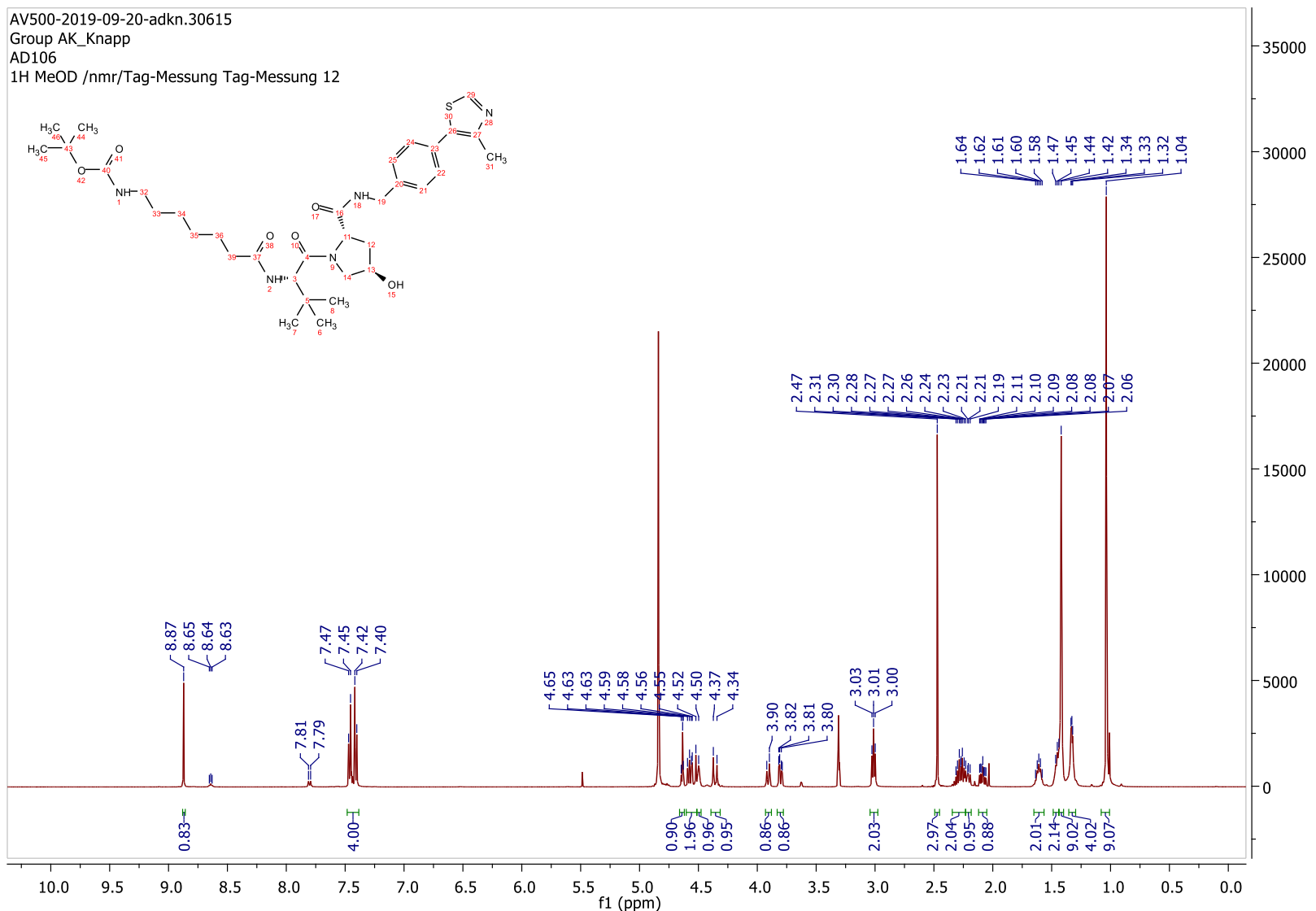


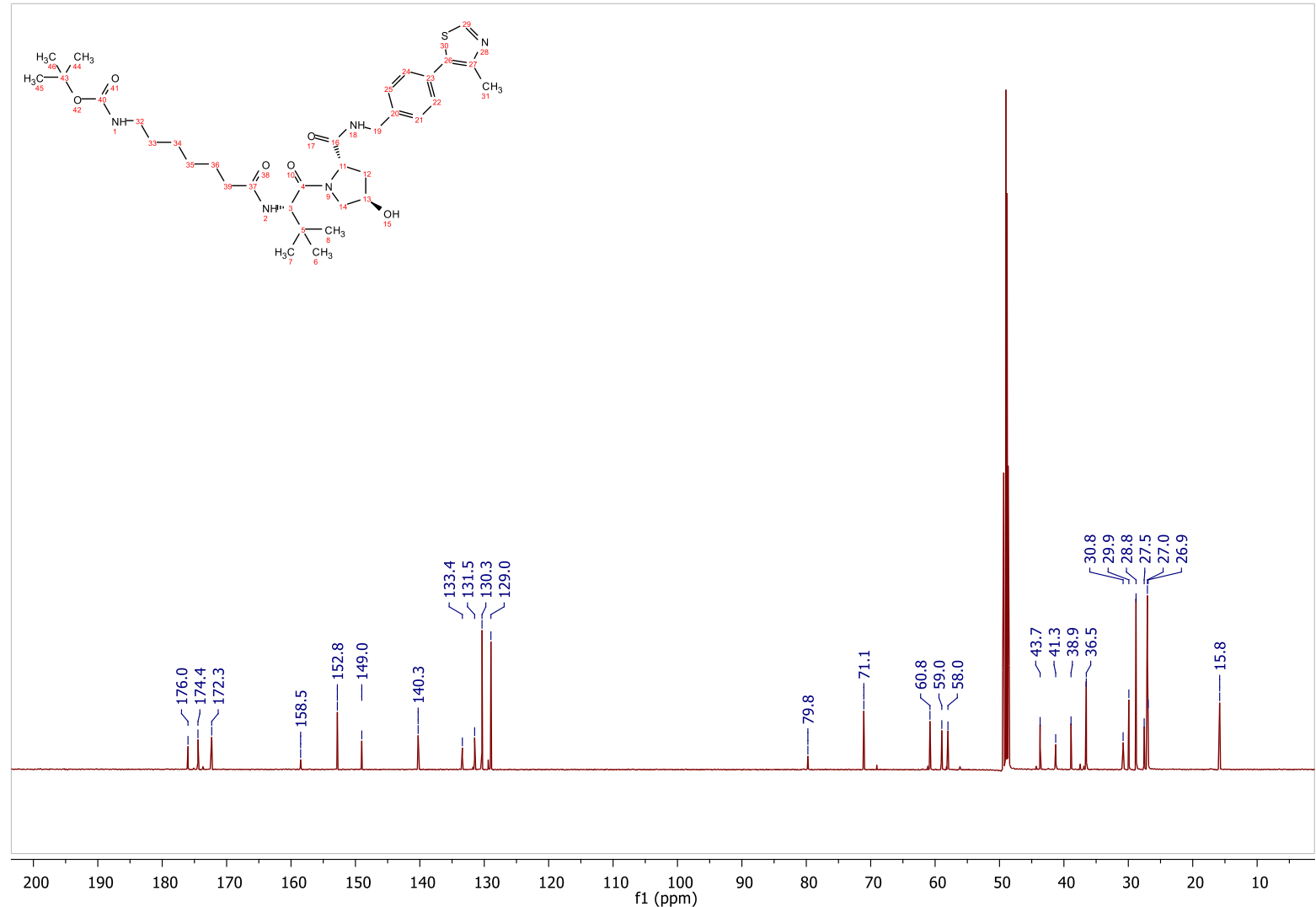


ESI, HPLC, ${ }^{1} \mathrm{H}-\mathrm{NMR}$ and ${ }^{13} \mathrm{C}-\mathrm{NMR}$ of tert-butyl (4-(2-(((S)-1-((2S,4R)-4-hydroxy-2-((4-(4-methylthiazol5-yl)benzyl)carbamoyl)pyrrolidin-1-yl)-3,3-dimethyl-1-oxobutan-2-yl)amino)-2oxoethyl)benzyl)carbamate (L14)

C:IXcaliburldataIAD140

AD140 \#35-42 RT: 0.61-0.73 AV: 8 SB: 7 0.09-0.20 NL: 3.27E6

$\mathrm{T}:\{0,0\}+\mathrm{c}$ ESI !corona sid=75.00 det=1306.00 Full ms [200.00-1200.00]
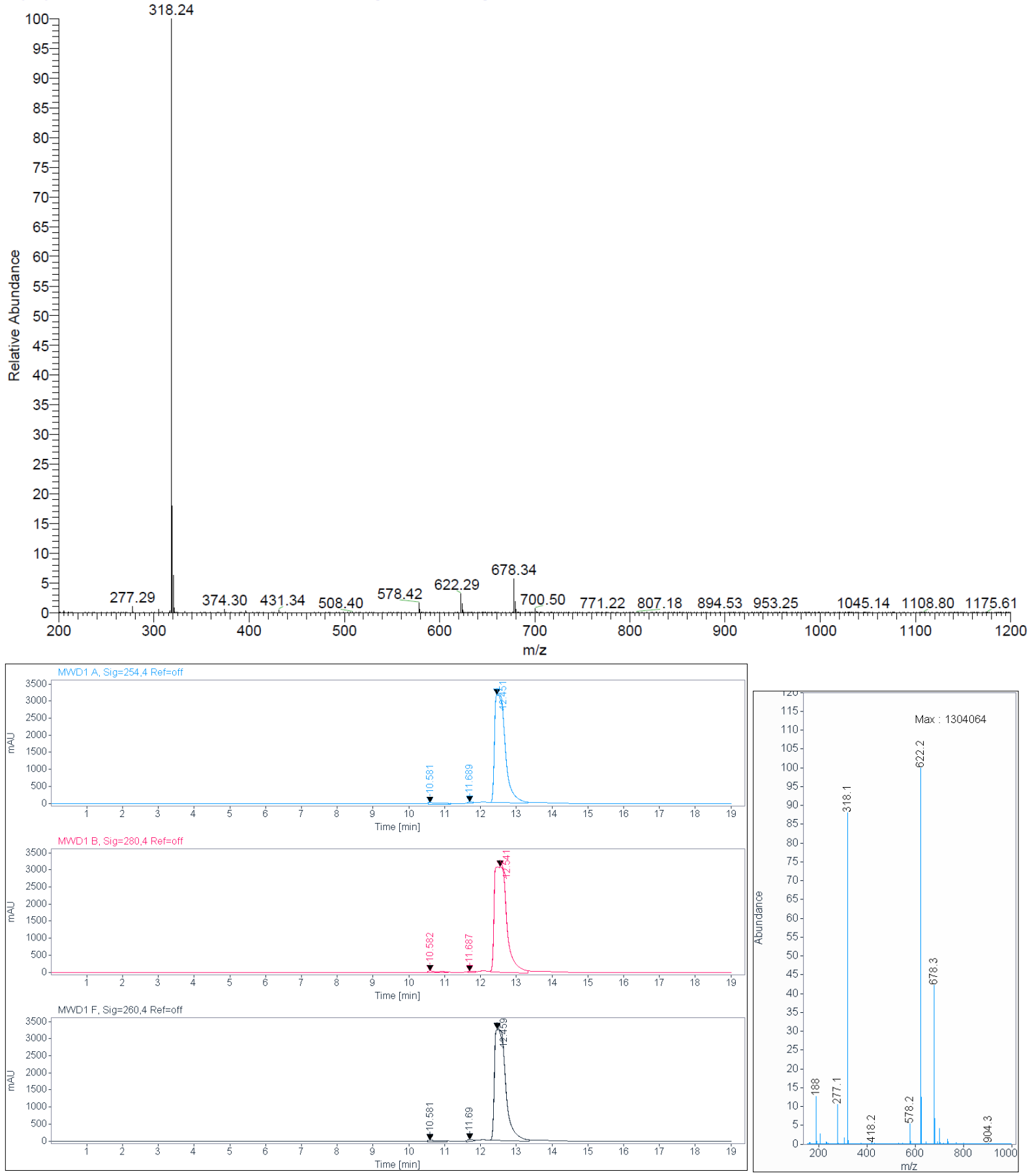

Signal: $\quad$ MWD1 A, Sig $=254,4$ Ref $=$ off

$\begin{array}{crrr}\text { RT [min] Type } & \text { Width [min] } & \text { Area } & \text { Height Area\% } \\ 10.581 \text { MM } & 0.4447 & 1334.5194 & 50.01071 .9565 \\ 11.689 \text { MM } & 0.1568 & 414.5808 & 44.07820 .6078 \\ 12.451 \mathrm{MM} & 0.3501 & 66460.5781 & 3164.229297 .4357 \\ & \text { Sum } & 68209.6783 & \end{array}$

Signal: $\quad$ MWD1 B, Sig=280,4 Ref=off 


$\begin{array}{crrr}\text { RT [min] Type } & \text { Width [min] } & \text { Area } & \text { Height Area\% } \\ 10.582 \text { MM } & 0.3997 & 1567.3302 & 65.35032 .0085 \\ 11.687 \text { MM } & 0.2019 & 685.3602 & 56.57470 .8783 \\ 12.541 \mathrm{MM} & 0.4061 & 75781.6641 & 3110.114597 .1132 \\ & \text { Sum } & 78034.3544 & \end{array}$

Signal: $\quad$ MWD1 F, Sig $=260,4$ Ref $=$ off

$\begin{array}{crrr}\text { RT [min] Type } & \text { Width [min] } & \text { Area } & \text { Height Area\% } \\ 10.581 \mathrm{MM} & 0.4067 & 1300.0765 & 53.27411 .7700 \\ 11.690 \mathrm{MM} & 0.1873 & 729.8845 & 64.95430 .9937 \\ 12.459 \mathrm{MM} & 0.3645 & 71421.4219 & 3265.356497 .2363\end{array}$

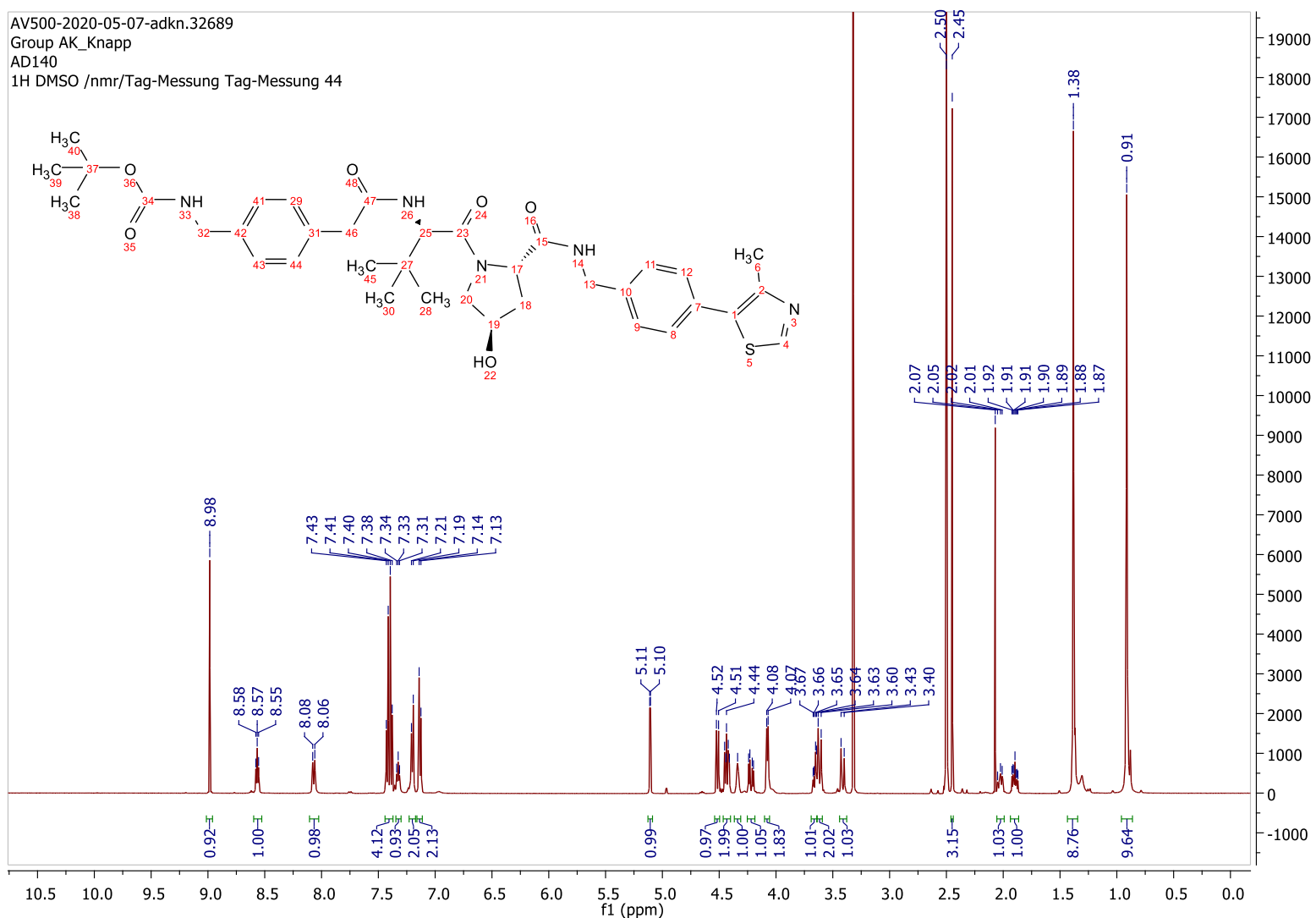



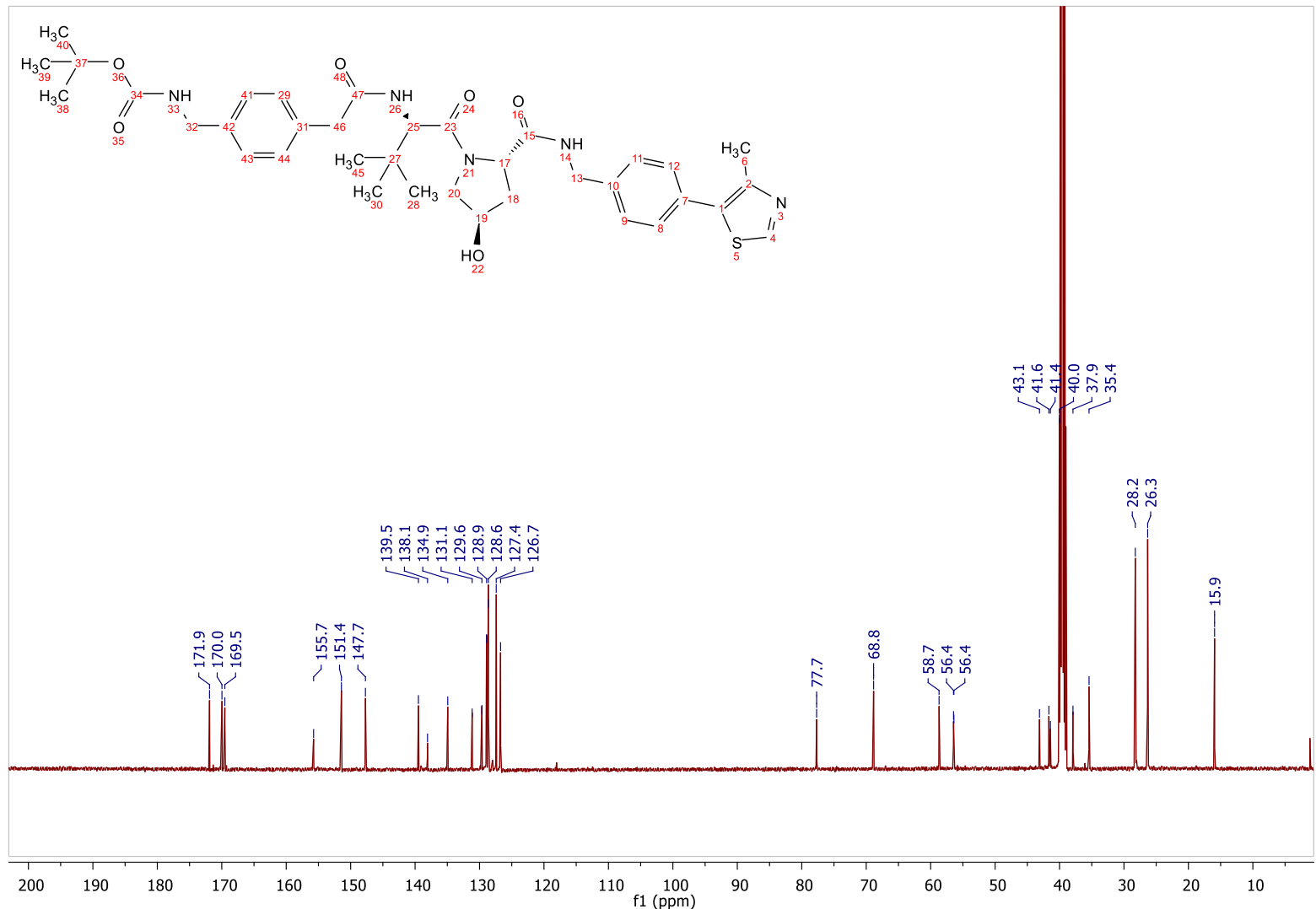
ESI, HPLC, ${ }^{1} \mathrm{H}-\mathrm{NMR}$ and ${ }^{13} \mathrm{C}-\mathrm{NMR}$ of tert-butyl (5-)((S)-1-((2S,4S)-4-hydroxy-2-((4-(4-methylthiazol-5yl)benzyl)carbamoyl)pyrrolidin-1-yl)-3,3-dimethyl-1-oxobutan-2-yl)amino)-5-oxopentyl)carbamate (L15)

C:IXcaliburldata|AD152-2

9/17/2020 8:08:35 AM

AD152-2 \#30-43 RT: 0.51-0.74 AV: 14 SB: 7 0.05-0.16 NL: $3.32 \mathrm{E} 4$

$\mathrm{T}:\{0,0\}+\mathrm{c} E S I$ !corona sid=75.00 det=1306.00 Full ms [105.00-1000.00]

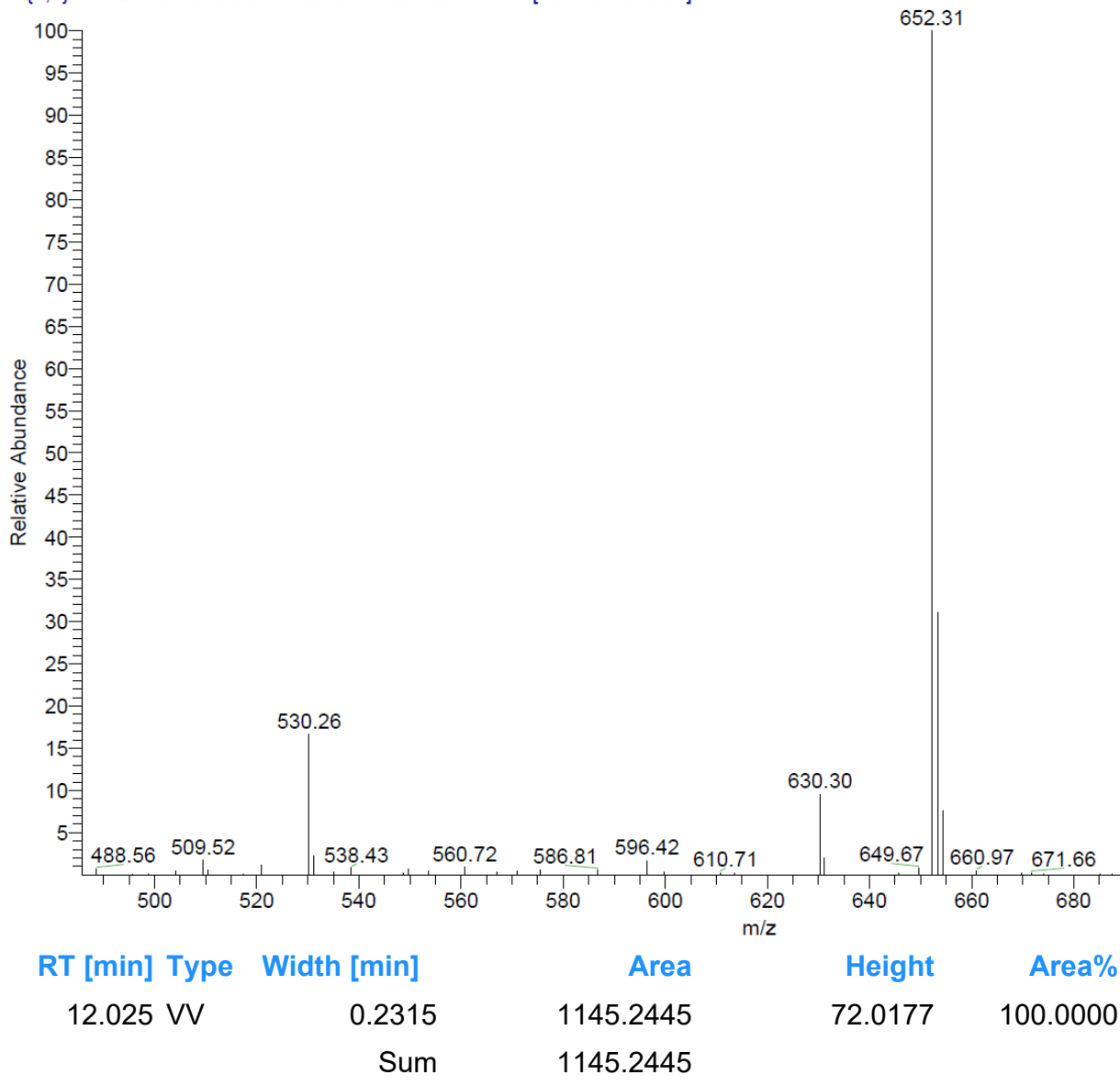

Signal: $\quad$ MWD1 B, Sig $=280,4$ Ref $=$ off

$\begin{array}{crrrr}\text { RT [min] Type } & \text { Width [min] } & \text { Area } & \text { Height } & \text { Area\% } \\ 12.025 \mathrm{VV} & 0.2326 & 1672.4189 & 104.5707 & 100.0000 \\ & \text { Sum } & 1672.4189 & & \end{array}$

Signal: $\quad$ MWD1 F, Sig $=260,4$ Ref $=$ off

$\begin{array}{crrrr}\text { RT [min] Type } & \text { Width [min] } & \text { Area } & \text { Height } & \text { Area\% } \\ 12.025 \text { VV } & 0.2292 & 1240.3011 & 78.9803 & 100.0000\end{array}$



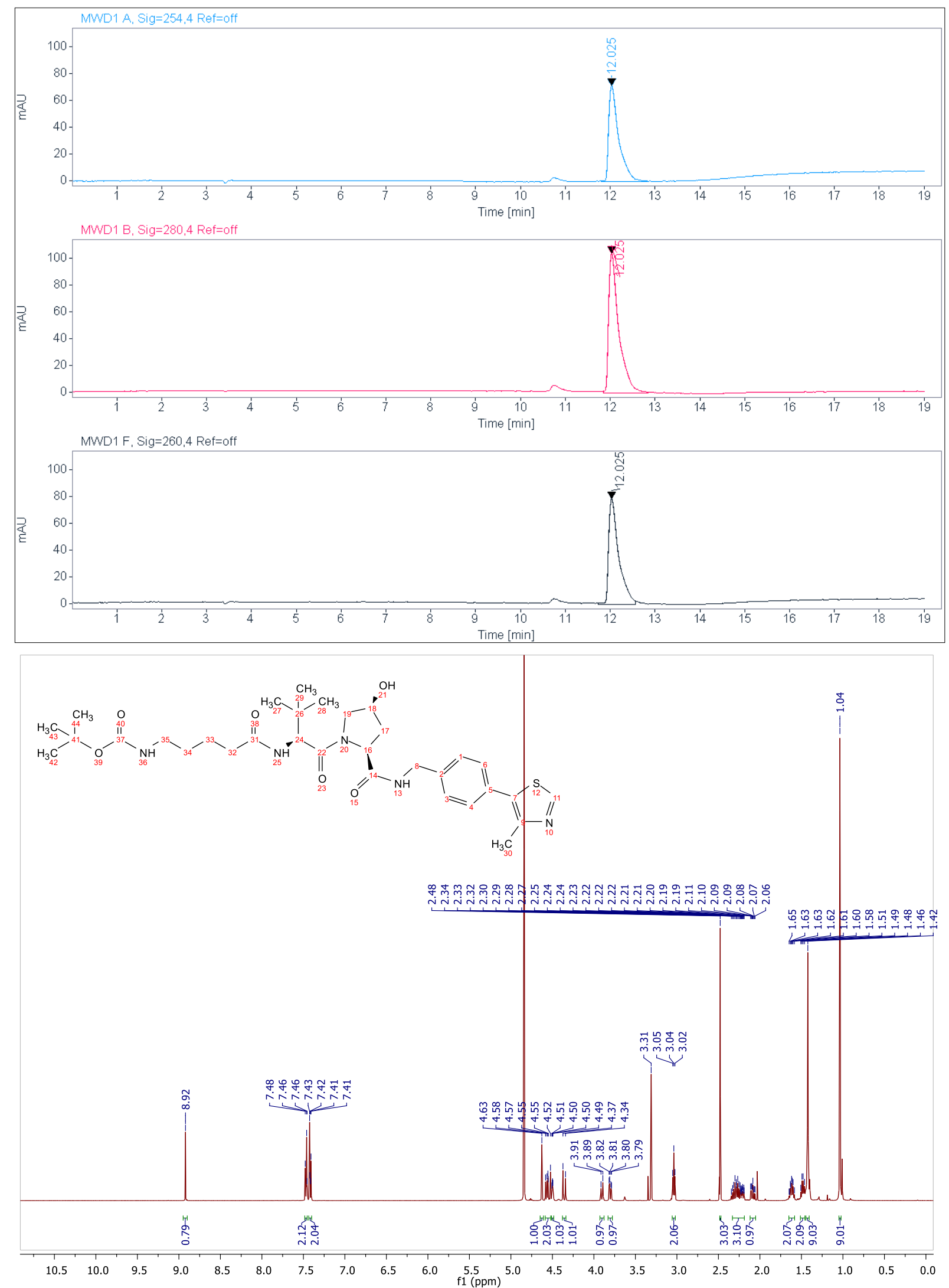


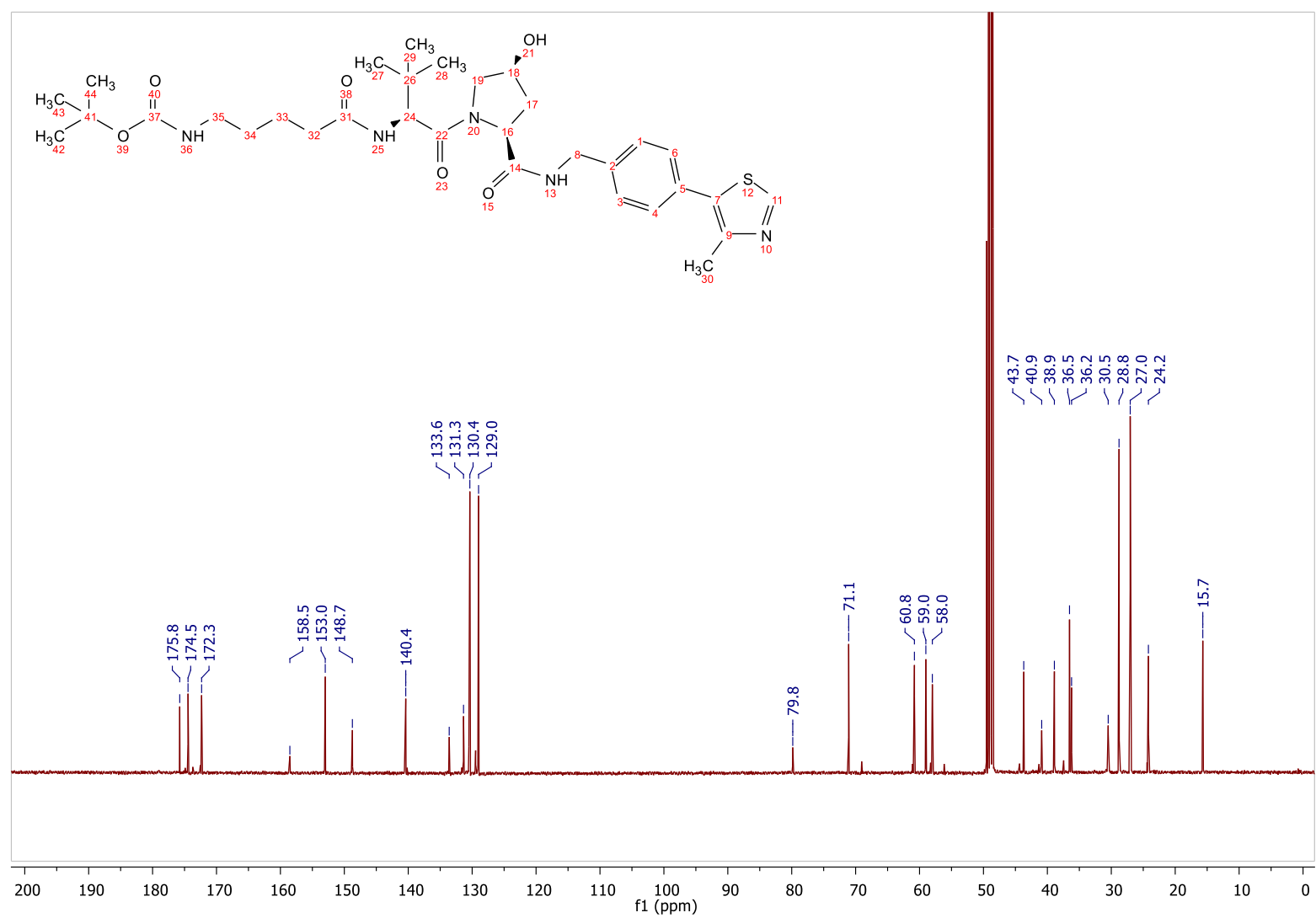


ESI, HPLC, ${ }^{1} \mathrm{H}-\mathrm{NMR}$ and ${ }^{13} \mathrm{C}-\mathrm{NMR}$ of tert-butyl (2-(2-(3'-(6-hydroxy-4-(trifluoromethyl)nicotinamido)4'-(4-methylpiperazin-1-yl)-[1,1'-biphenyl]-4-carboxamido)ethoxy)ethyl)carbamate (6e)

C:IXcaliburldata\AD133

5/4/2020 9:32:53 AM

AD133 \#36-42 RT: 0.63-0.74 AV: 7 SB: 15 0.04-0.29 NL: 2.44E5

$\mathrm{T}:\{0,0\}+\mathrm{c}$ ESI !corona sid=75.00 det=1306.00 Full ms [300.00-1500.00]
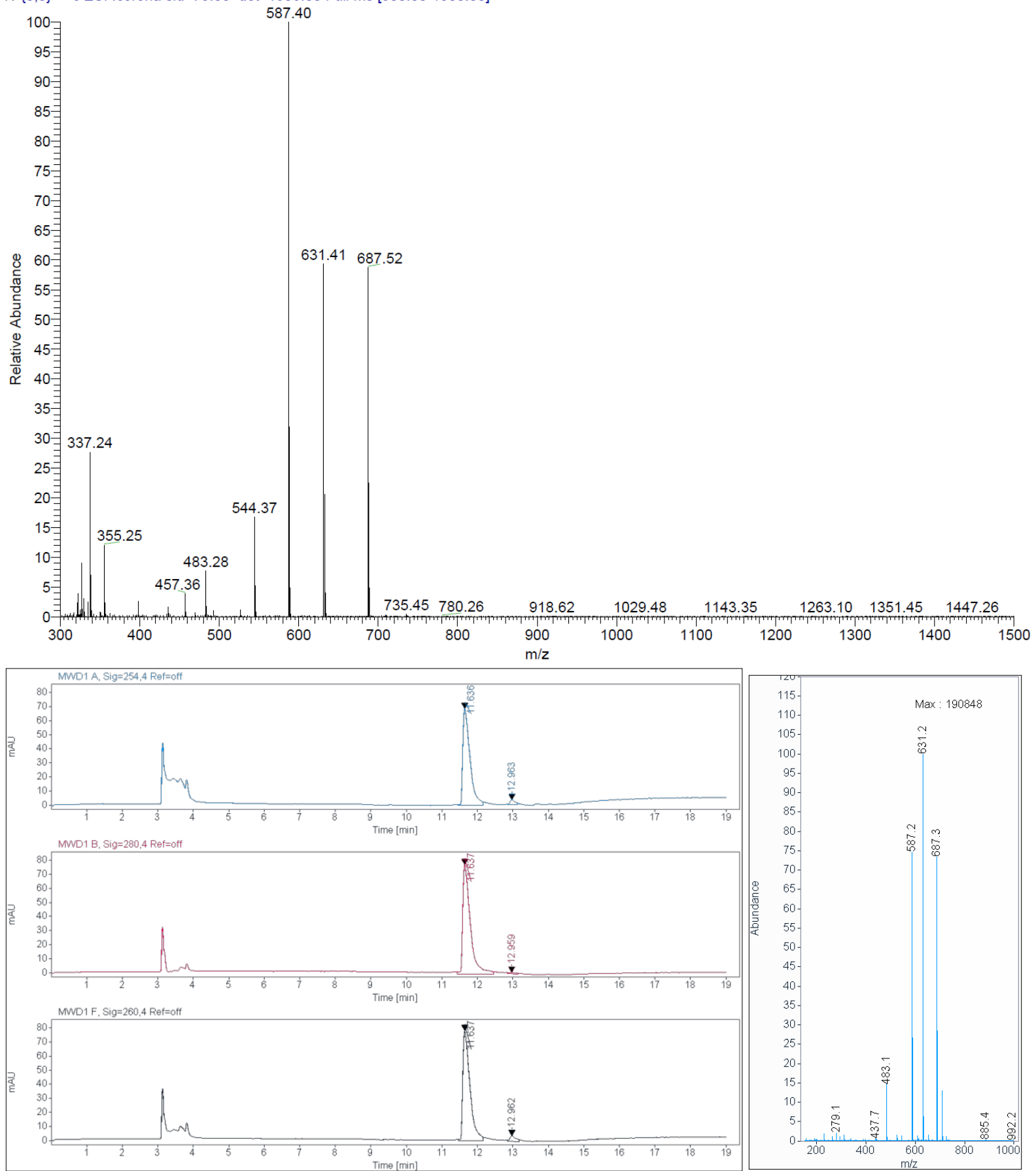

Signal: $\quad$ MWD1 A, Sig=254,4 Ref=off

$\begin{array}{crrr}\text { RT [min] Type } & \text { Width [min] } & \text { Area } & \text { Height Area\% } \\ 11.636 \text { VV } & 0.2088 & 960.0562 & 68.802596 .2284 \\ 12.963 \mathrm{MM} & 0.1685 & 37.6289 & 3.72203 .7716 \\ & \text { Sum } & 997.6851 & \end{array}$

Signal: $\quad$ MWD1 B, Sig=280,4 Ref=off 


$\begin{array}{lrrr}11.637 \mathrm{VV} & 0.2215 & 1175.1613 & 78.124598 .9523 \\ 12.959 \mathrm{MM} & 0.2024 & 12.4427 & 1.02471 .0477 \\ & \text { Sum } & 1187.6039 & \end{array}$

Signal: $\quad$ MWD1 F, Sig=260,4 Ref=off

$\begin{array}{crrr}\text { RT [min] Type } & \text { Width [min] } & \text { Area } & \text { Height Area\% } \\ 11.637 \text { VV } & 0.2084 & 1095.8492 & 78.236595 .5942 \\ 12.962 \mathrm{MM} & 0.2064 & 50.5057 & 4.07914 .4058\end{array}$

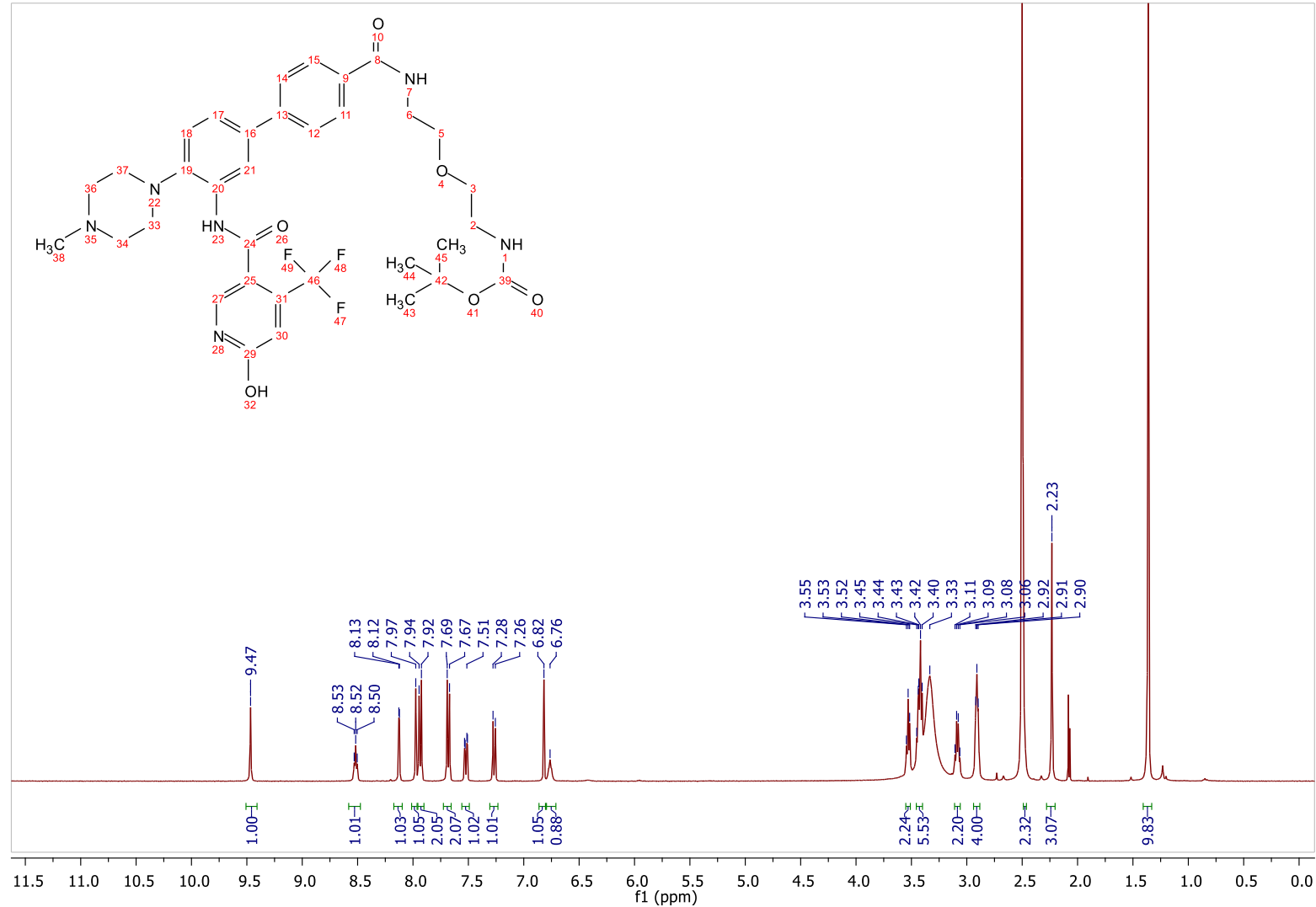




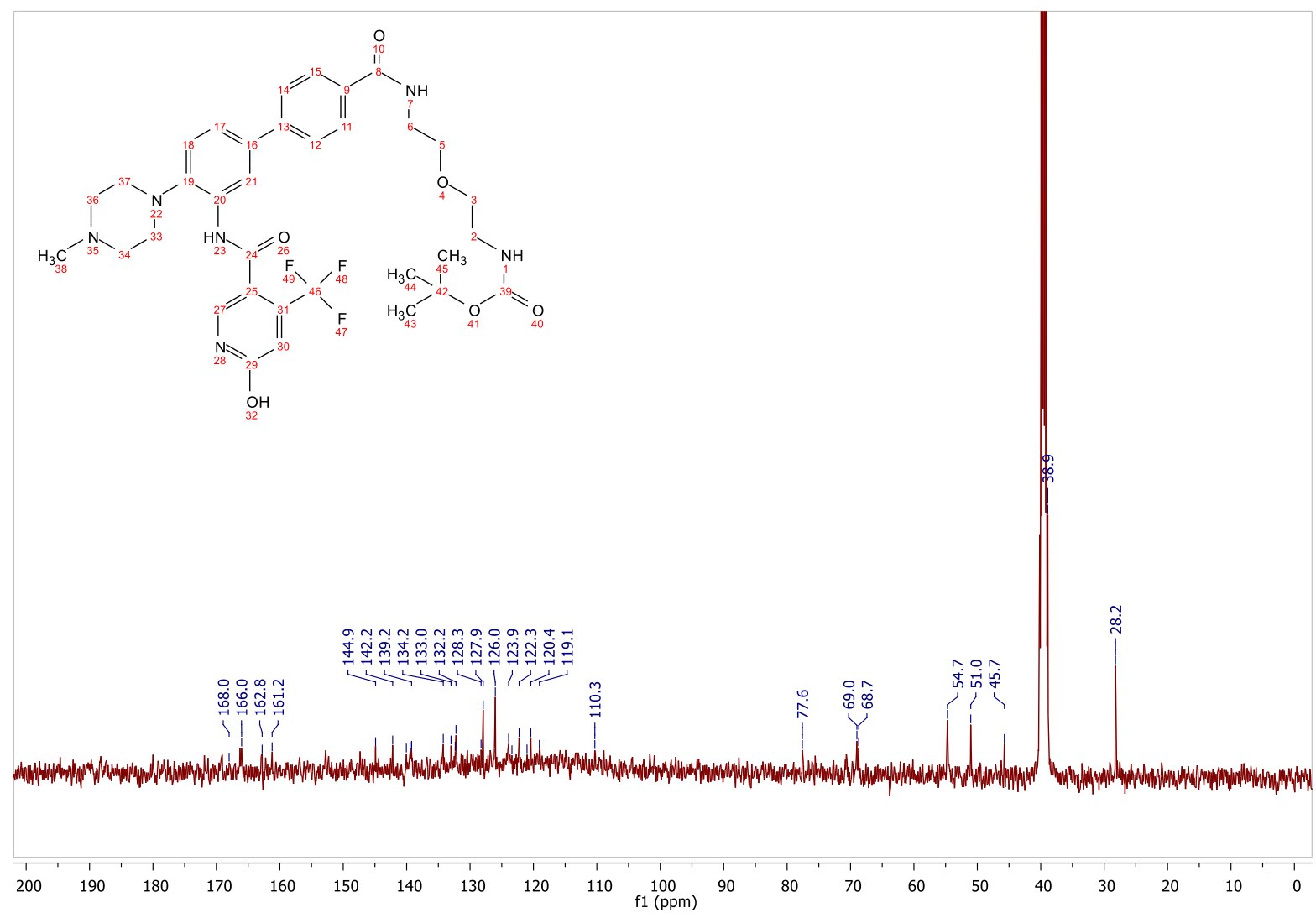


ESI, HPLC, ${ }^{1} \mathrm{H}-\mathrm{NMR}$ of tert-butyl (1-(3'-(6-hydroxy-4-(trifluoromethyl)nicotinamido)-4'-(4methylpiperazin-1-yl)-[1,1'-biphenyl]-4-yl)-1-oxo-5,8,11,14,17,20,23-heptaoxa-2-azapentacosan-25yl)carbamate (6f)

C:IXcaliburldata\AD134

AD134 \#38-42 RT: 0.67-0.74 AV: 5 SB: 9 0.04-0.18 NL: $5.92 E 5$

$\mathrm{T}:\{0,0\}+\mathrm{c}$ ESI !corona sid=75.00 det=1306.00 Full ms [300.00-1500.00]

$100 \exists \quad 426.42$

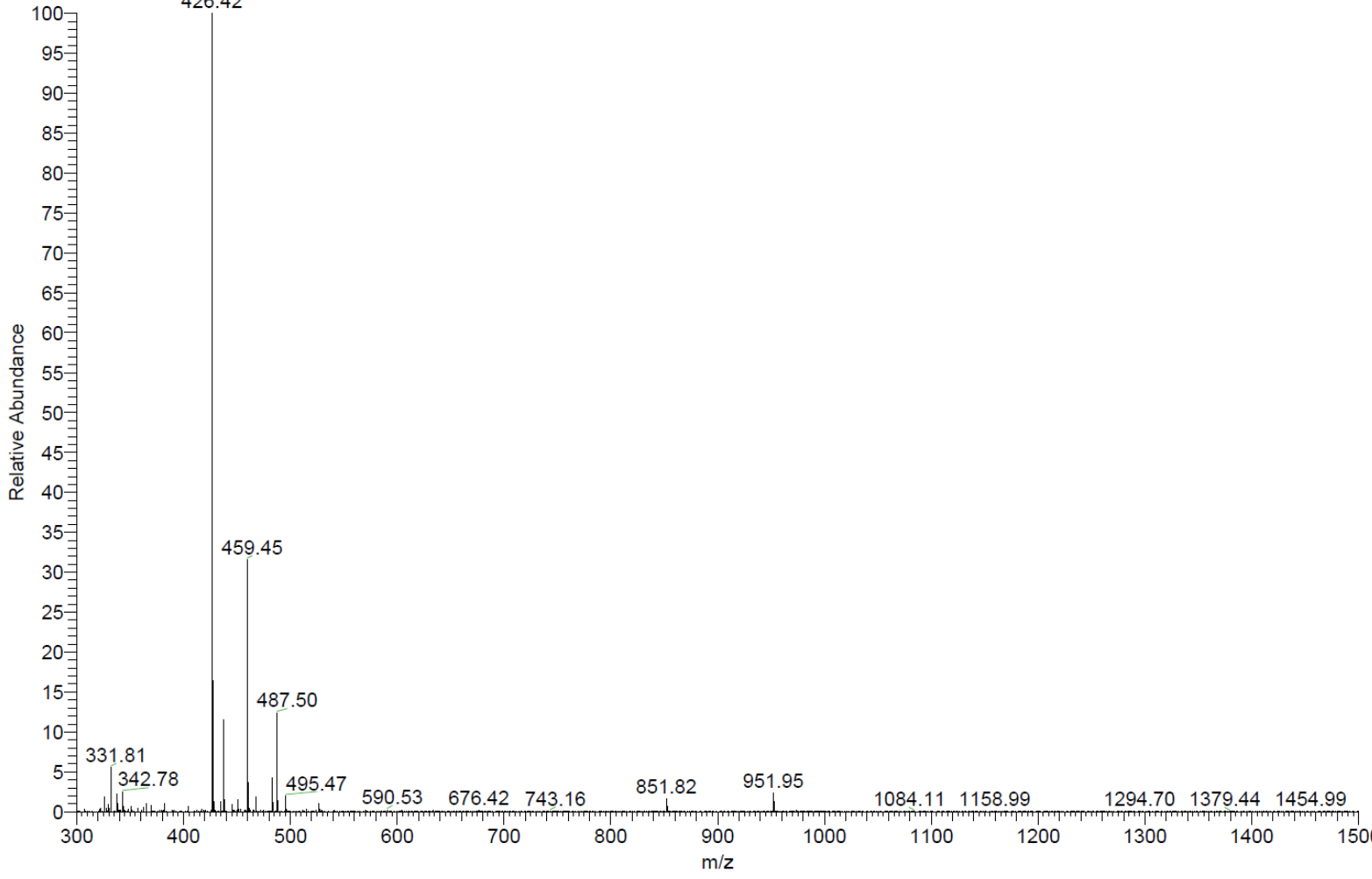

Sum $\quad 42114393.0000$

Signal: $\quad$ MWD1 A, Sig $=254,4$ Ref $=$ off

$\begin{array}{crrr}\text { RT [min] Type } & \text { Width [min] } & \text { Area } & \text { Height Area\% } \\ 11.751 \mathrm{VV} & 0.2234 & 917.7254 & 61.038694 .3249 \\ 12.958 \mathrm{MM} & 0.2044 & 55.2154 & 4.50215 .6751 \\ & \text { Sum } & 972.9408 & \end{array}$

Signal: $\quad$ MWD1 B, Sig=280,4 Ref=off

$\begin{array}{crrr}\text { RT [min] Type } & \text { Width [min] } & \text { Area } & \text { Height Area\% } \\ 11.751 \text { VV } & 0.2259 & 1091.4231 & 70.010498 .2189 \\ 12.954 \text { MM } & 0.2499 & 19.7914 & 1.32011 .7811 \\ & \text { Sum } & 1111.2145 & \end{array}$

Signal: $\quad$ MWD1 F, Sig $=260,4$ Ref $=$ off

$\begin{array}{ccrr}\text { RT [min] Type } & \text { Width [min] } & \text { Area } & \text { Height Area\% } \\ 11.751 \mathrm{VV} & 0.2199 & 1049.4673 & 69.590294 .7728 \\ 12.958 \mathrm{MM} & 0.2140 & 57.8829 & 4.50915 .2272\end{array}$



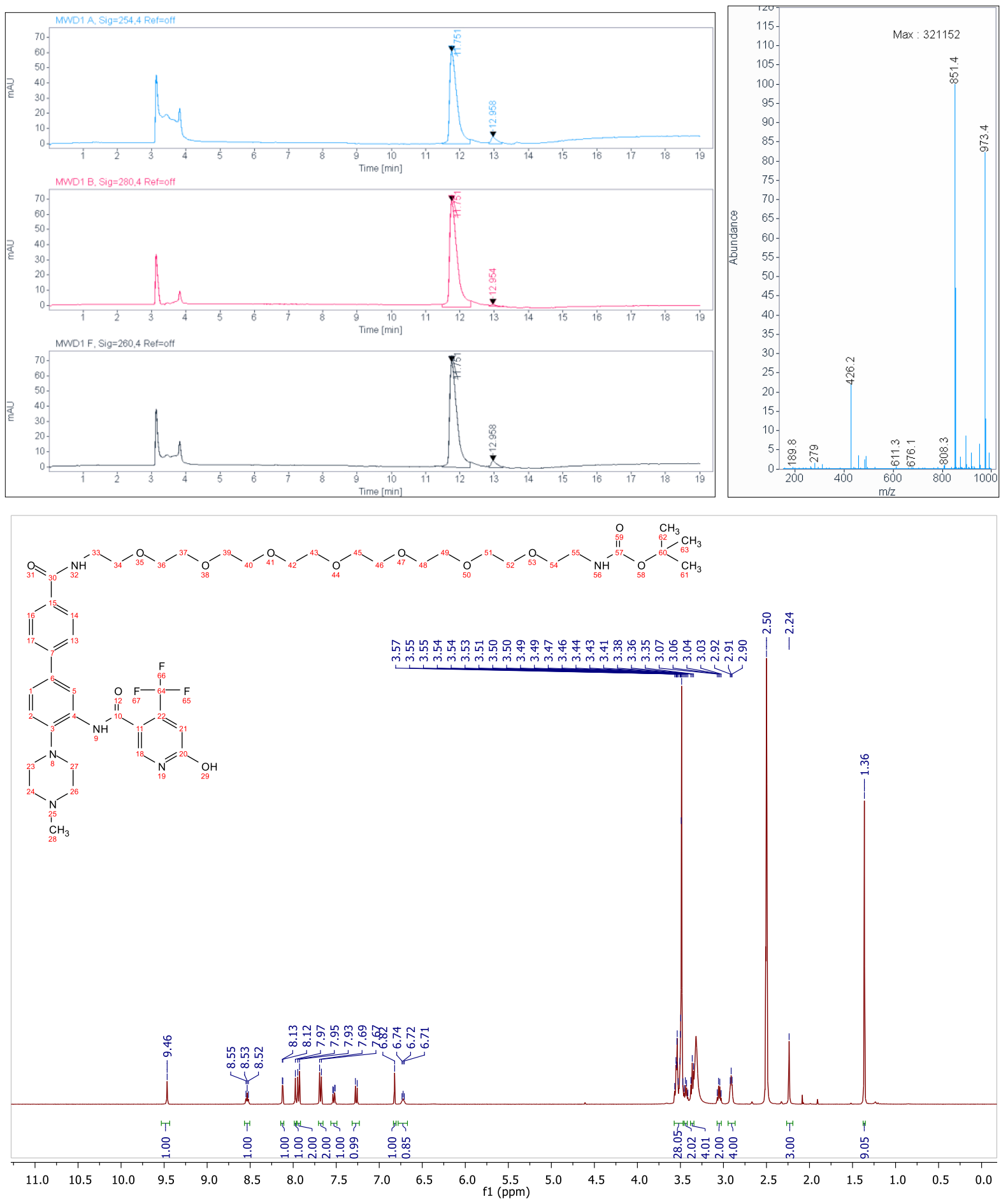
ESI, HPLC and ${ }^{1} \mathrm{H}-N M R$ of tert-butyl (4-((3'-(6-hydroxy-4-(trifluoromethyl)nicotinamido)-4'-(4methylpiperazin-1-yl)-[1,1'-biphenyl]-4-carboxamido)methyl)benzyl)carbamate (6g)

C:IXcaliburldata\AD135

5/4/2020 9:37:12 AM

AD135 \#33-43 RT: 0.58-0.76 AV: 11 SB: 10 0.07-0.23 NL: $2.49 E 5$

$\mathrm{T}:\{0,0\}+\mathrm{c}$ ESI !corona sid=75.00 det=1306.00 Full ms [300.00-1500.00]

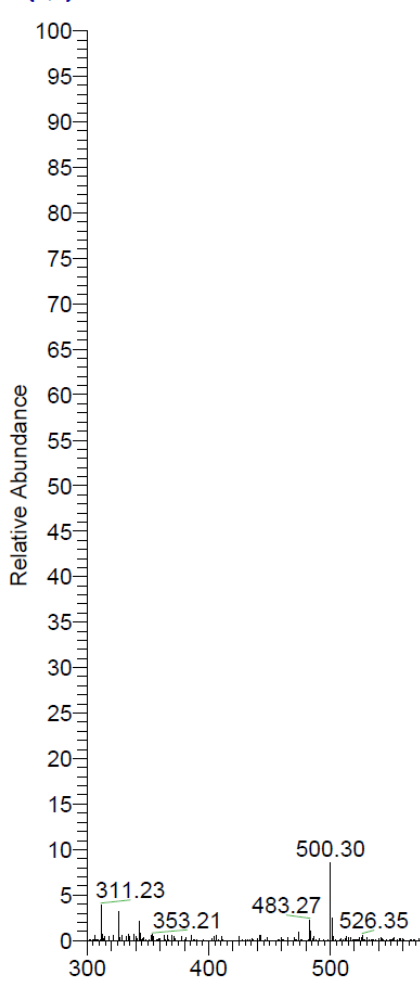
663.41

Signal: $\quad$ MWD1 A, Sig=254,4 Ref=off

$\begin{array}{crrr}\text { RT [min] Type } & \text { Width [min] } & \text { Area } & \text { Height Area\% } \\ 12.212 \mathrm{VV} & 0.2107 & 791.6523 & 56.420391 .4191 \\ 12.949 \mathrm{MM} & 0.2487 & 74.3068 & 4.98028 .5809 \\ & \text { Sum } & 865.9591 & \end{array}$

Signal: $\quad$ MWD1 B, Sig=280,4 Ref=off

$\begin{array}{crrr}\text { RT [min] Type } & \text { Width [min] } & \text { Area } & \text { Height Area\% } \\ 12.211 \text { VV } & 0.2149 & 943.0983 & 64.372696 .0057 \\ 12.766 \text { MM } & 0.2462 & 39.2378 & 2.65623 .9943 \\ & \text { Sum } & 982.3361 & \end{array}$

Signal: $\quad$ MWD1 F, Sig=260,4 Ref $=$ off

$\begin{array}{ccrc}\text { RT [min] Type } & \text { Width [min] } & \text { Area } & \text { Height Area\% } \\ 12.212 \mathrm{VV} & 0.2120 & 907.8795 & 64.207092 .2728 \\ 12.951 \mathrm{MM} & 0.2594 & 76.0286 & 4.88477 .7272\end{array}$



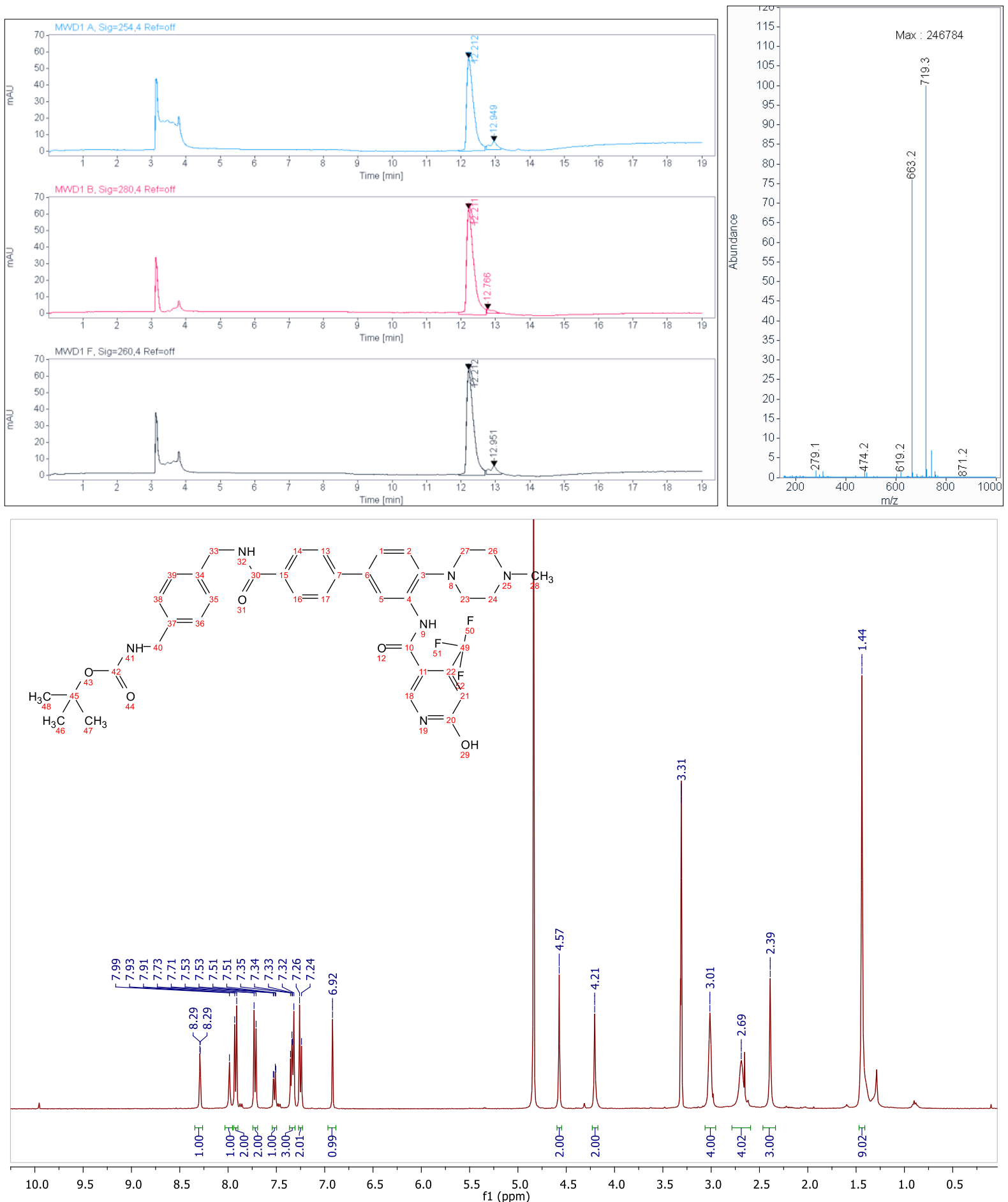
MALDI and ${ }^{1}$ H-NMR of tert-butyl (1-(3'-(6-hydroxy-4-(trifluoromethyl)nicotinamido)-4'-(4methylpiperazin-1-yl)-[1,1'-biphenyl]-4-yl)-1-oxo-5,8,11,14,17,20,23,26,29-nonaoxa-2azahentriacontan-31-yl)carbamate (6h)

Voyager Spec \#1[BP = 939.4, 31058]
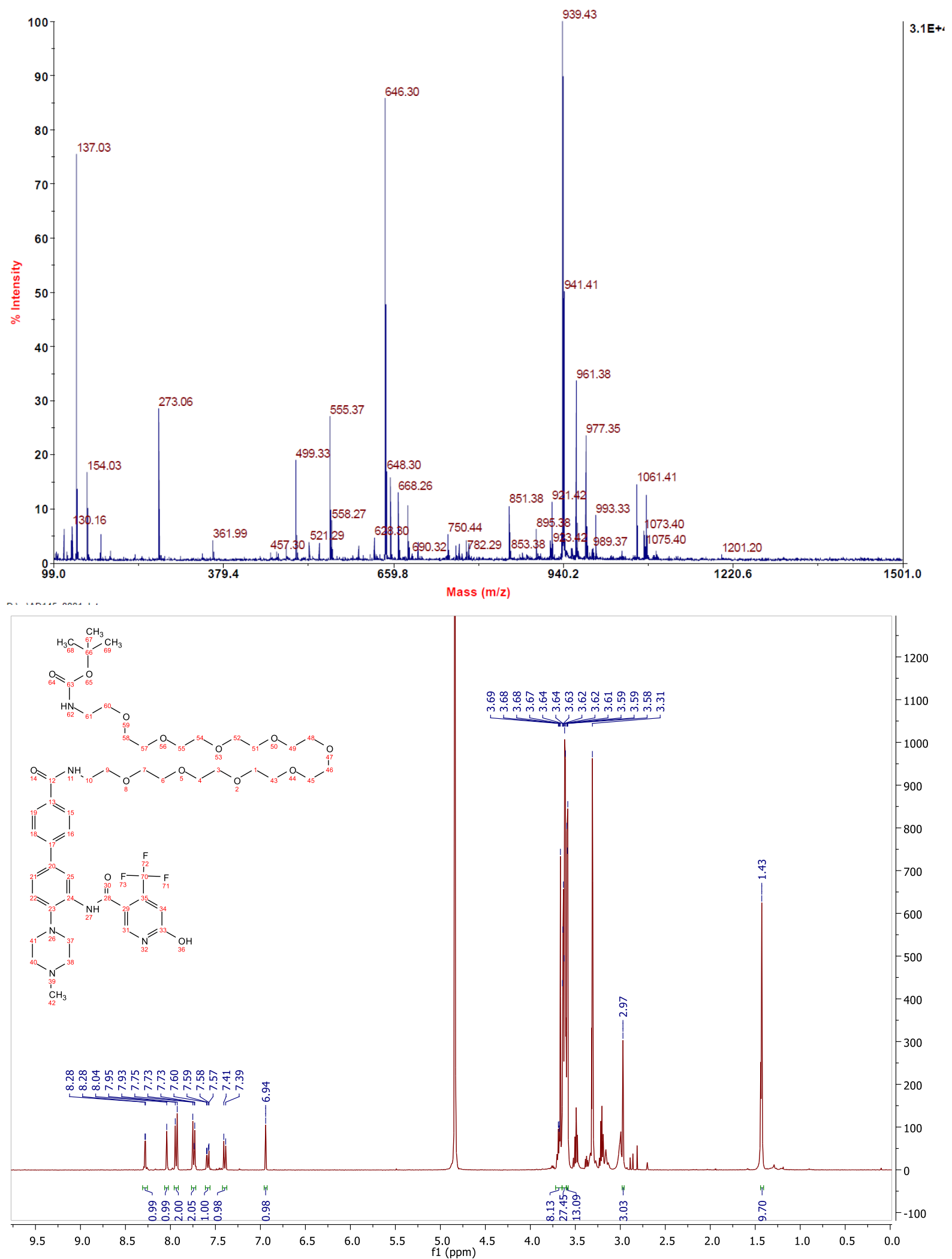
ESI, HPLC, ${ }^{1} \mathrm{H}-\mathrm{NMR}$ and ${ }^{13} \mathrm{C}-\mathrm{NMR}$ of tert-butyl (1-(3'-(6-hydroxy-4-(trifluoromethyl)nicotinamido)-4'(4-methylpiperazin-1-yl)-[1,1'-biphenyl]-4-yl)-1-oxo-6,9,12-trioxa-2-azapentadecan-15yl)carbamate (6i)

C:IXcaliburldataIAD143-9

5/26/2020 9:16:14 AM

AD143-9 \#38-42 RT: 0.67-0.74 AV: 5 SB: 13 0.07-0.29 NL: 4.26E5

T: $\{0,0\}+c$ ESI !corona sid=75.00 det=1506.00 Full ms [300.00-1500.00]
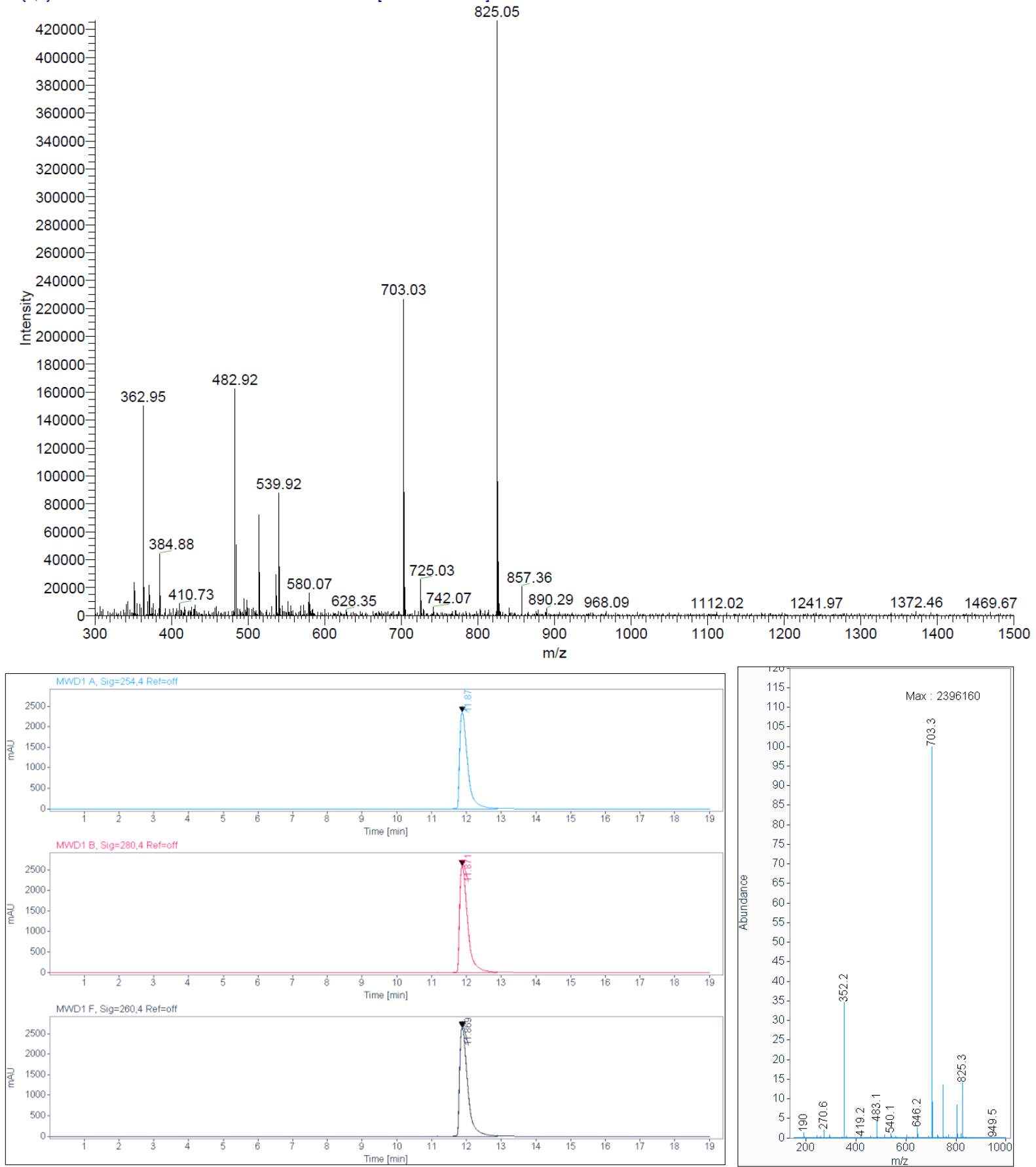

Signal: $\quad$ MWD1 A, Sig=254,4 Ref=off

RT [min] Type Width [min] 11.870 BV 100.0000

Signal: $\quad$ MWD1 B, Sig=280,4 Ref=off

RT [min] Type Width [min]

Area

Height

Area\% 
11.871 VV

$\begin{array}{rr}0.2203 & 42077.1367 \\ \text { Sum } & 42077.1367\end{array}$

$2606.3718 \quad 100.0000$

Signal: $\quad$ MWD1 F, Sig=260,4 Ref=off

$\begin{array}{ccrrr}\text { RT [min] Type } & \text { Width [min] } & \text { Area } & \text { Height } & \text { Area\% } \\ 11.869 \text { VV } & 0.2388 & 42766.1367 & 2656.3064 & 100.0000\end{array}$

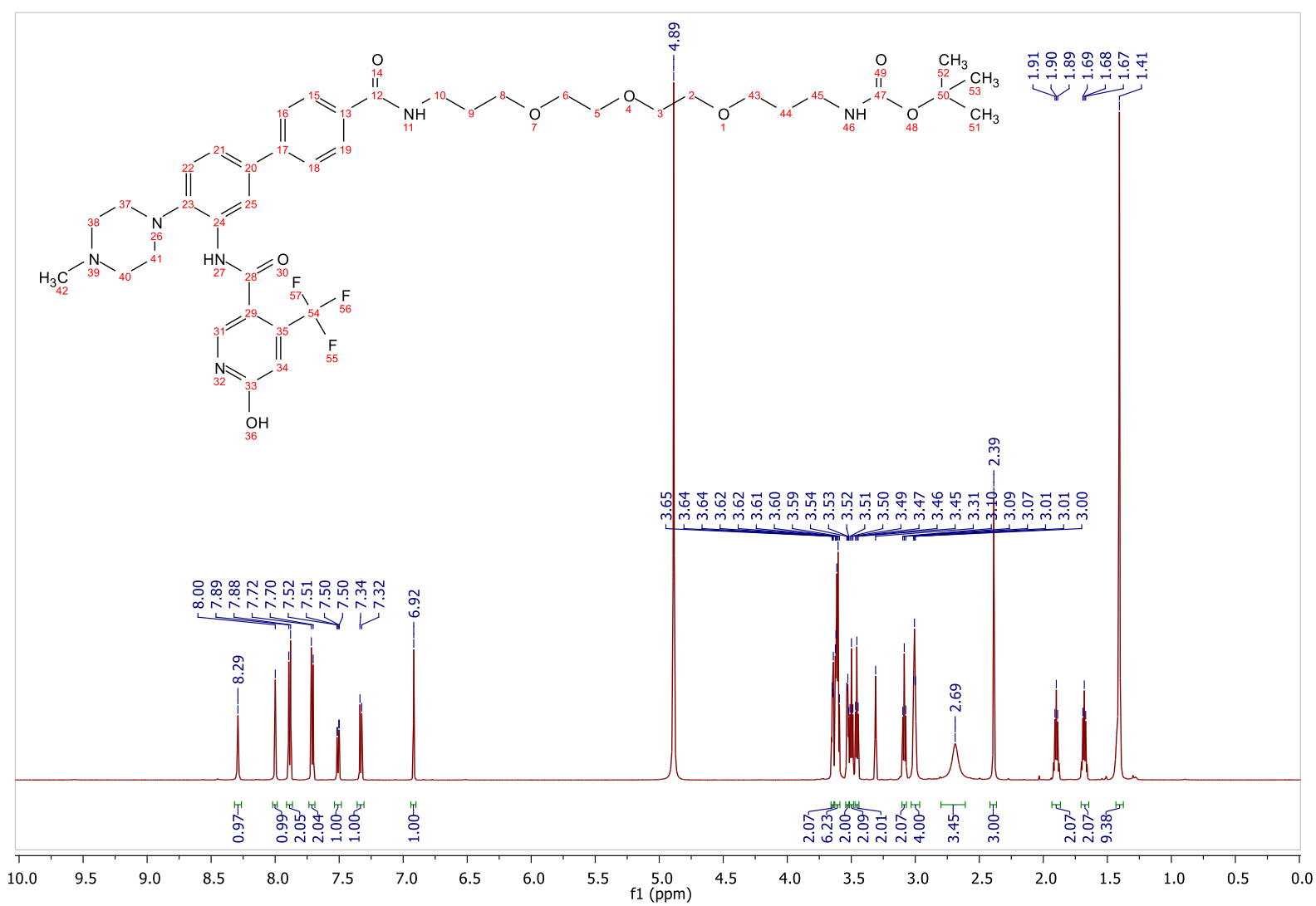




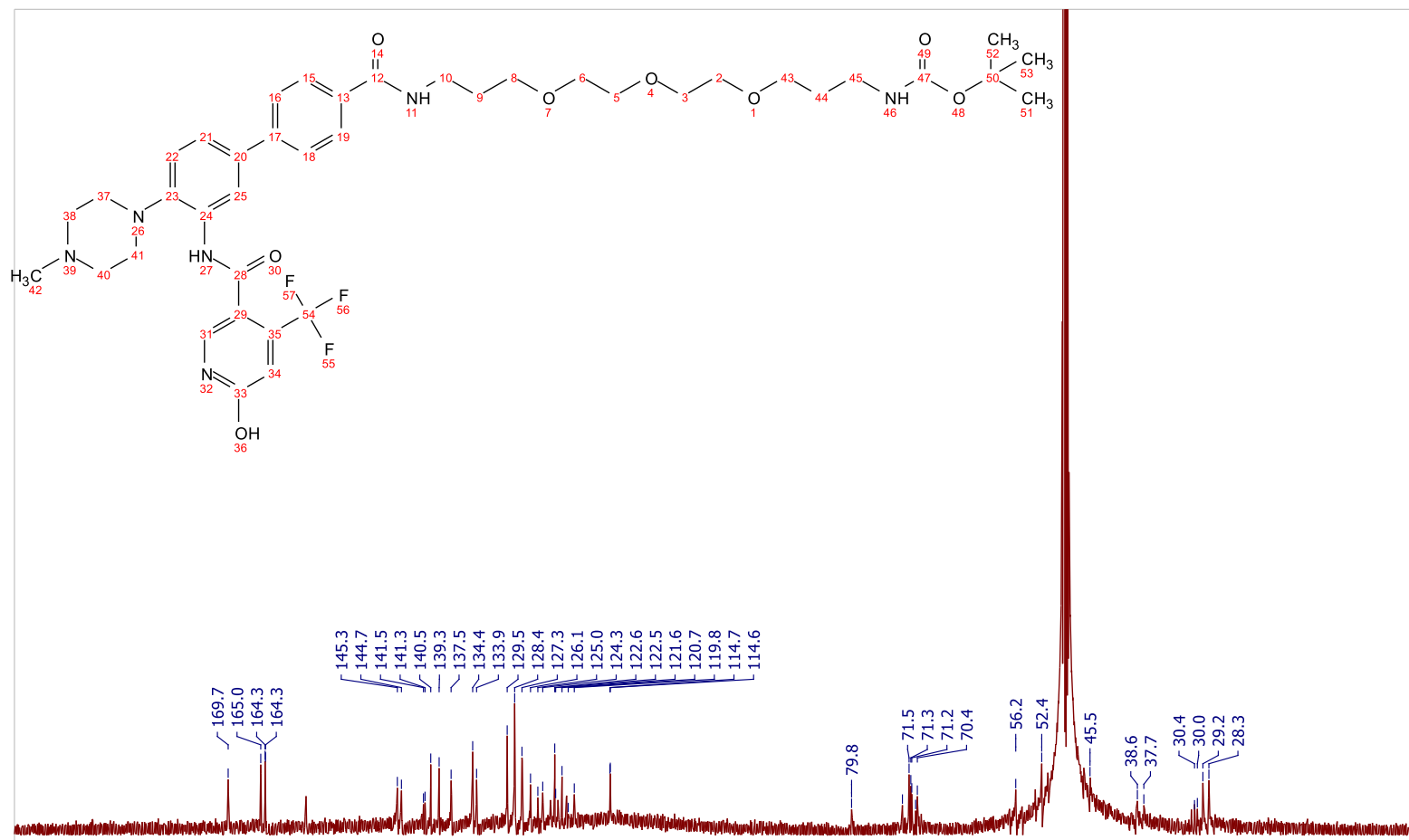

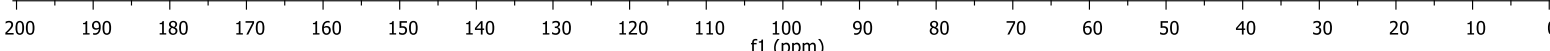


HPLC, HRMS, ${ }^{1} \mathrm{H}-\mathrm{NMR}$ and ${ }^{13} \mathrm{C}-\mathrm{NMR}$ of $\mathrm{N}$-(4'-((2-(2-(3-(5,5-difluoro-7-(1H-pyrrol-2-yl)-5H-5|4,614dipyrrolo[1,2-c:2',1'-f][1,3,2]diazaborinin-3-yl)propanamido)ethoxy)ethyl)carbamoyl)-4-(4methylpiperazin-1-yl)-[1,1'-biphenyl]-3-yl)-6-hydroxy-4-(trifluoromethyl)nicotinamide (19a)
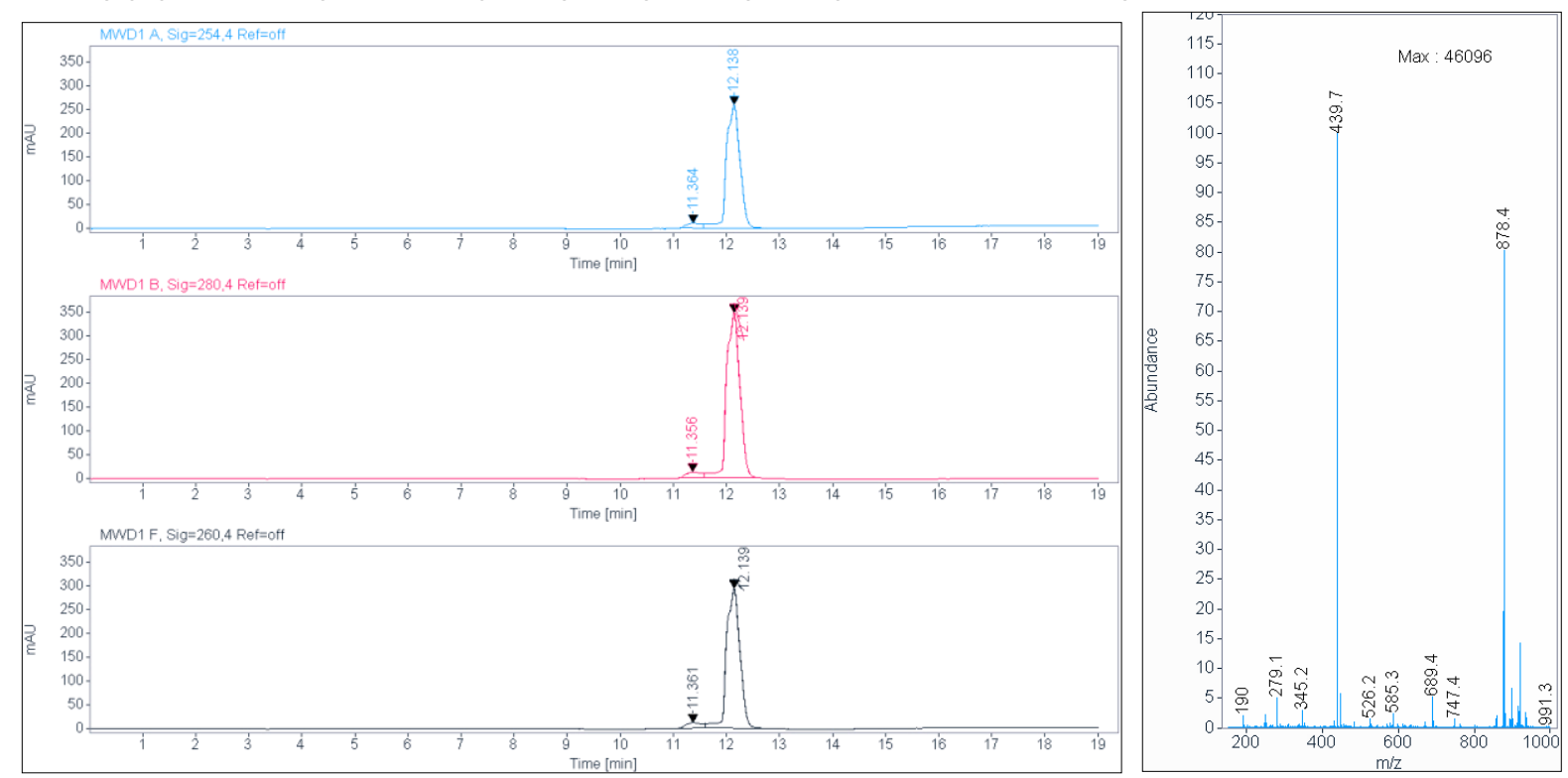

Signal: $\quad$ MWD1 A, Sig=254,4 Ref=off

$\begin{array}{crrr}\text { RT [min] Type } & \text { Width [min] } & \text { Area } & \text { Height Area\% } \\ 11.364 \mathrm{MM} & 0.3356 & 228.0416 & 11.32504 .6822 \\ 12.138 \mathrm{MM} & 0.2965 & 4642.3574 & 260.997195 .3178 \\ & \text { Sum } & 4870.3990 & \end{array}$

Signal: $\quad$ MWD1 B, Sig=280,4 Ref=off

$\begin{array}{crr}\text { RT [min] Type } & \text { Width [min] } & \text { Area } \\ 11.356 \text { MM } & 0.3379 & 265.3242 \\ 12.139 \text { MM } & 0.2911 & 6074.2964 \\ & \text { Sum } & 6339.6206\end{array}$

Signal: $\quad$ MWD1 F, Sig=260,4 Ref=off

$\begin{array}{ccrr}\text { RT [min] Type } & \text { Width [min] } & \text { Area } & \text { Height Area\% } \\ 11.361 \mathrm{MM} & 0.3556 & 252.2446 & 11.82154 .6842 \\ 12.139 \mathrm{MM} & 0.2909 & 5132.7949 & 294.102095 .3158\end{array}$


C:IUser|...|2020\10.08.2020\AD148_F8
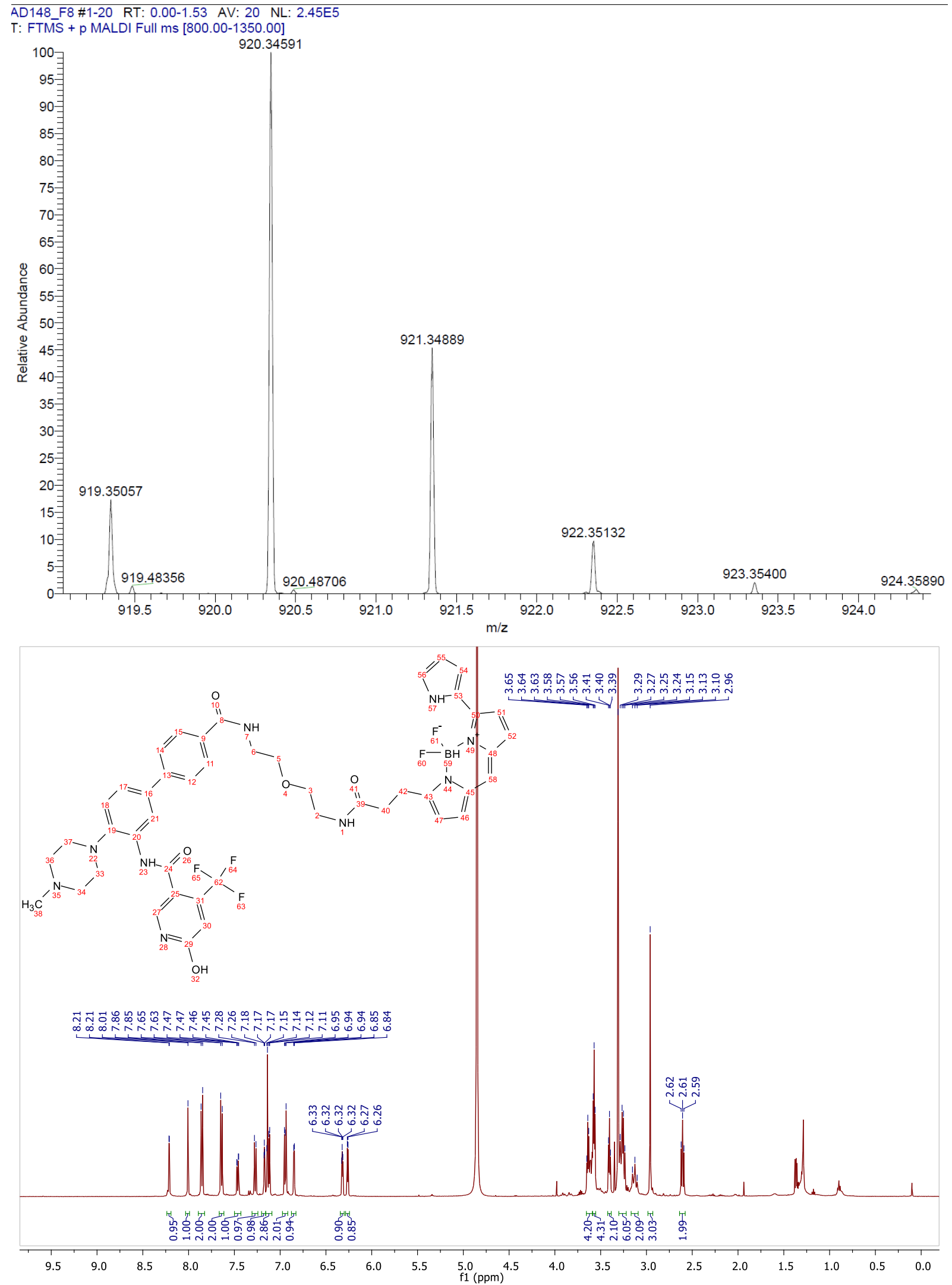
Appendix

267

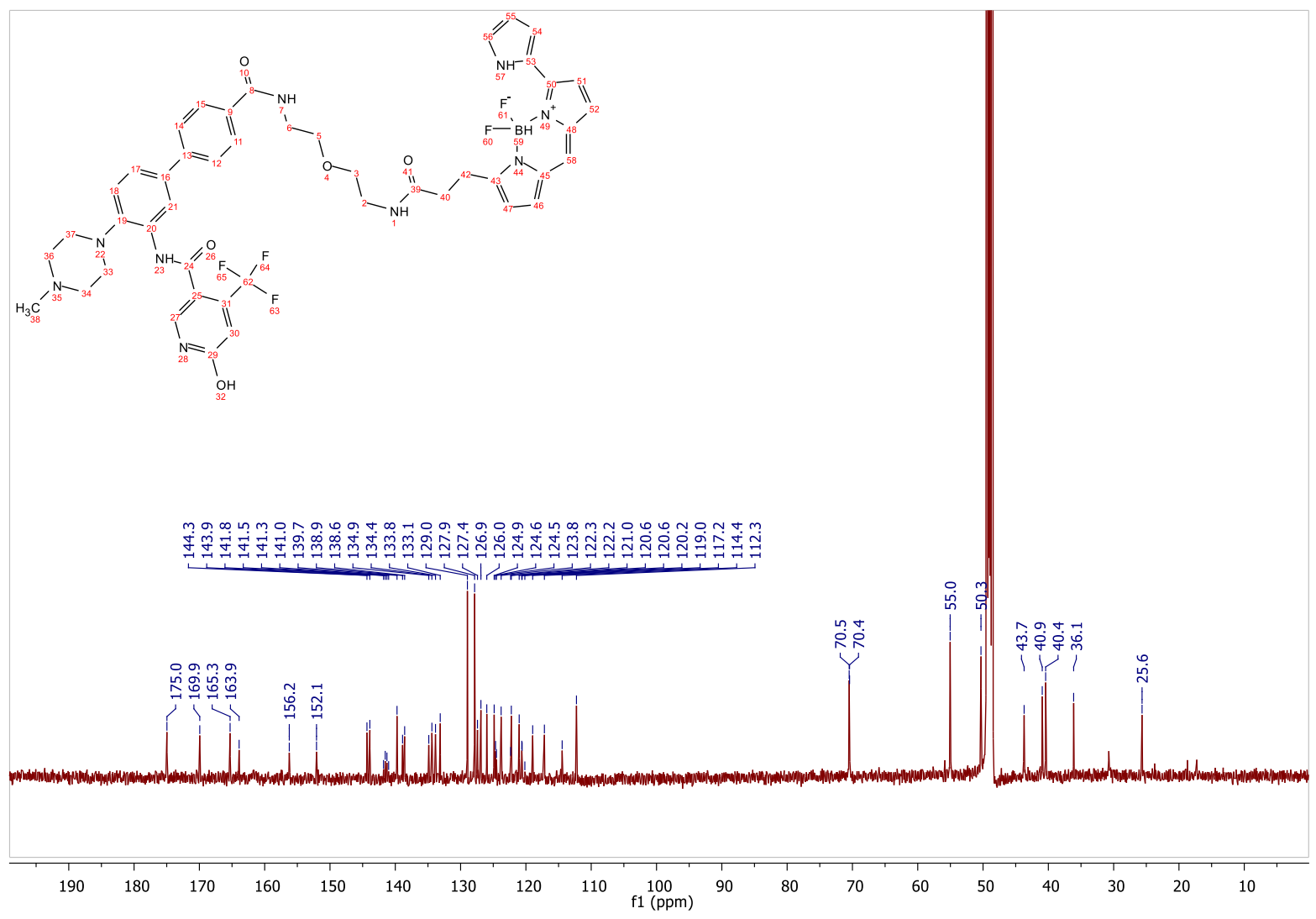


HPLC, HRMS, ${ }^{1} \mathrm{H}-\mathrm{NMR}$ of N-(4'-((33-(5,5-difluoro-7-(1H-pyrrol-2-yl)-5H-5|4,614-dipyrrolo[1,2-c:2',1'f][1,3,2]diazaborinin-3-yl)-31-oxo-3,6,9,12,15,18,21,24,27-nonaoxa-30-azatritriacontyl)carbamoyl)4-(4-methylpiperazin-1-yl)-[1,1'-biphenyl]-3-yl)-6-hydroxy-4-(trifluoromethyl)nicotinamide (19b)
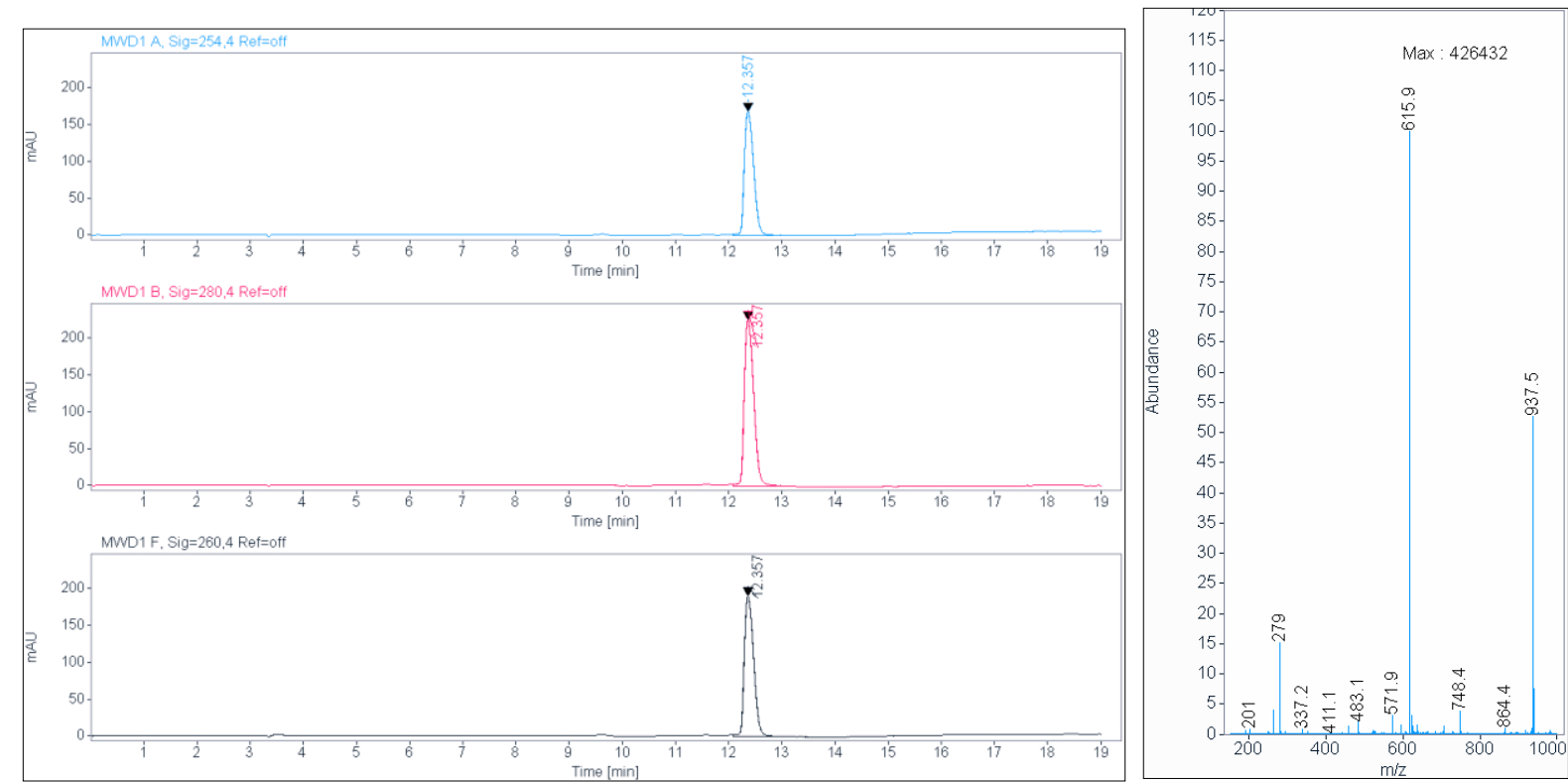

Signal: $\quad$ MWD1 A, Sig=254,4 Ref=off

RT [min] Type Width [min] 12.357 VV

Sum 2072.8545

Signal: $\quad$ MWD1 B, Sig $=280,4$ Ref $=$ off

$$
\begin{array}{cr}
\text { RT [min] Type } & \text { Width [min] } \\
12.357 \mathrm{VV} & 0.2005
\end{array}
$$

Sum

Area

Height

Area \%

2813.1299

225.5552

100.0000

Signal: $\quad$ MWD1 F, Sig=260,4 Ref=off

RT [min] Type Width [min] 12.357 VV
0.1988

Area 2353.3545
Height Area $\%$ $190.8938 \quad 100.0000$ 
C:IUserl...12020110.08.2020IAD147_F7

\section{AD147_F7 \#1-12 RT: 0.01-0.90 AV: 12 NL: 5.68E5}

T: FTMS + p MALDI Full ms [800.00-1350.00]
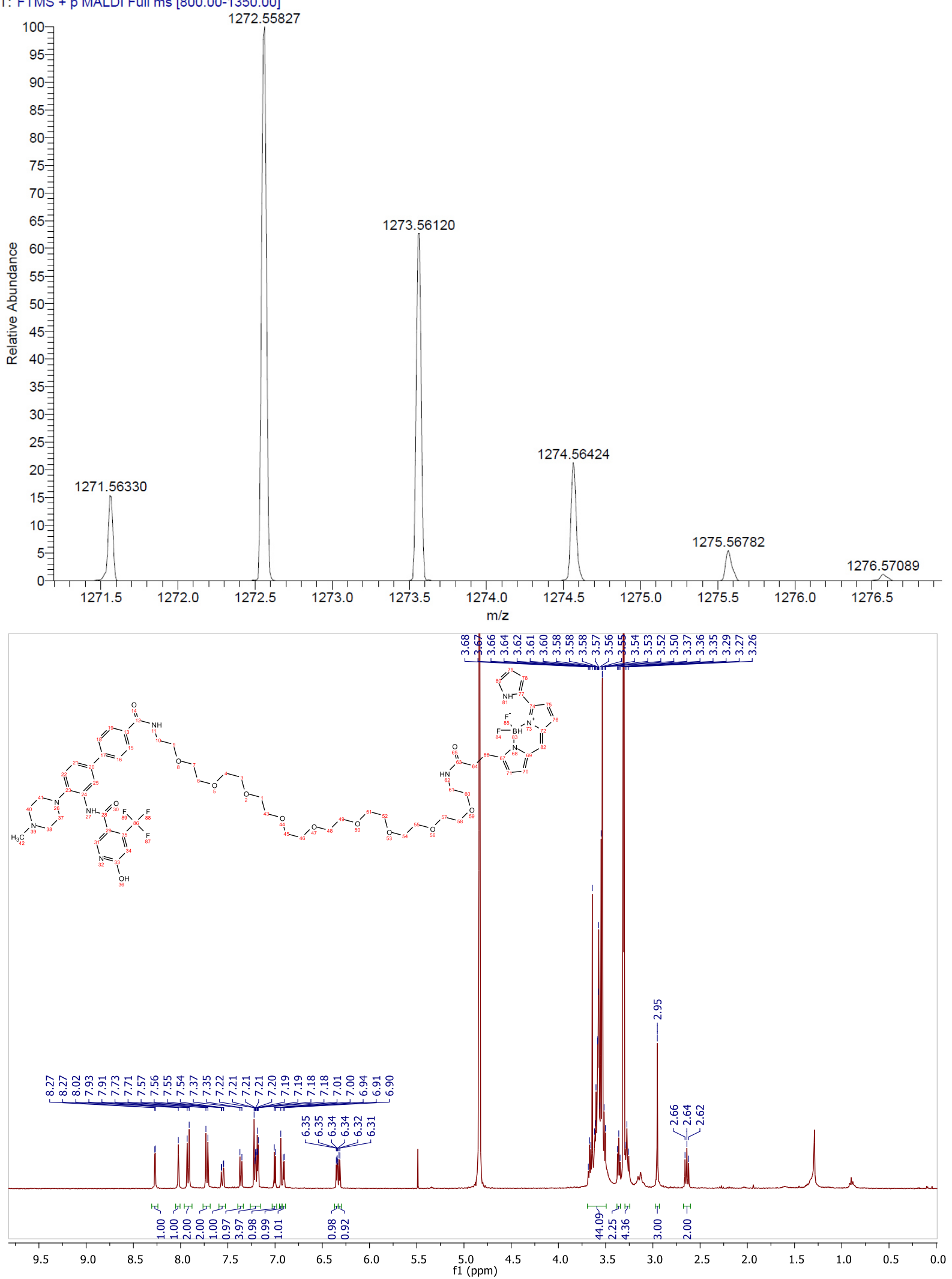
HPLC, HRMS and ${ }^{1} \mathrm{H}-\mathrm{NMR}$ of $\mathrm{N}$-(4'-((17-(5,5-difluoro-7-(1H-pyrrol-2-yl)-5H-5|4,6|4-dipyrrolo[1,2c:2',1'-f][1,3,2]diazaborinin-3-yl)-15-oxo-4,7,10-trioxa-14-azaheptadecyl)carbamoyl)-4-(4methylpiperazin-1-yl)-[1,1'-biphenyl]-3-yl)-6-hydroxy-4-(trifluoromethyl)nicotinamide (19c)
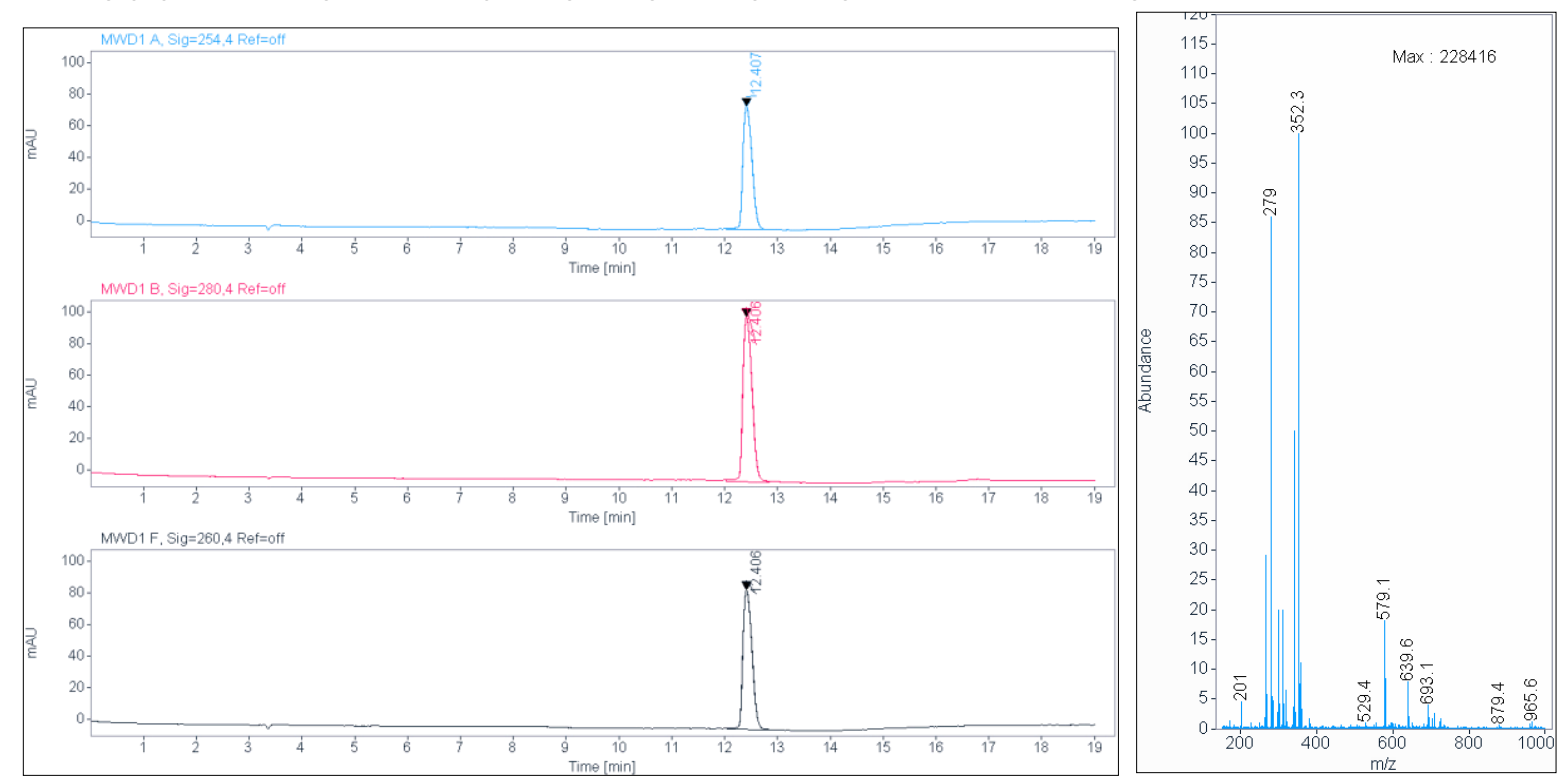

Signal: $\quad$ MWD1 A, Sig=254,4 Ref=off

$\begin{array}{crrrr}\text { RT [min] Type } & \text { Width [min] } & \text { Area } & \text { Height } & \text { Area\% } \\ 12.407 \text { VV } & 0.2010 & 936.6609 & 77.5274 & 100.0000 \\ & \text { Sum } & 936.6609 & & \end{array}$

Signal: $\quad$ MWD1 B, Sig=280,4 Ref=off

$\begin{array}{crrrr}\text { RT [min] Type } & \text { Width [min] } & \text { Area } & \text { Height } & \text { Area\% } \\ 12.406 \text { VV } & 0.2034 & 1279.1853 & 104.0625 & 100.0000 \\ & \text { Sum } & 1279.1853 & & \end{array}$

Signal: $\quad$ MWD1 F, Sig=260,4 Ref=off

$\begin{array}{crrrr}\text { RT [min] Type } & \text { Width [min] } & \text { Area } & \text { Height } & \text { Area\% } \\ 12.406 \text { VV } & 0.2009 & 1061.8375 & 87.9442 & 100.0000\end{array}$



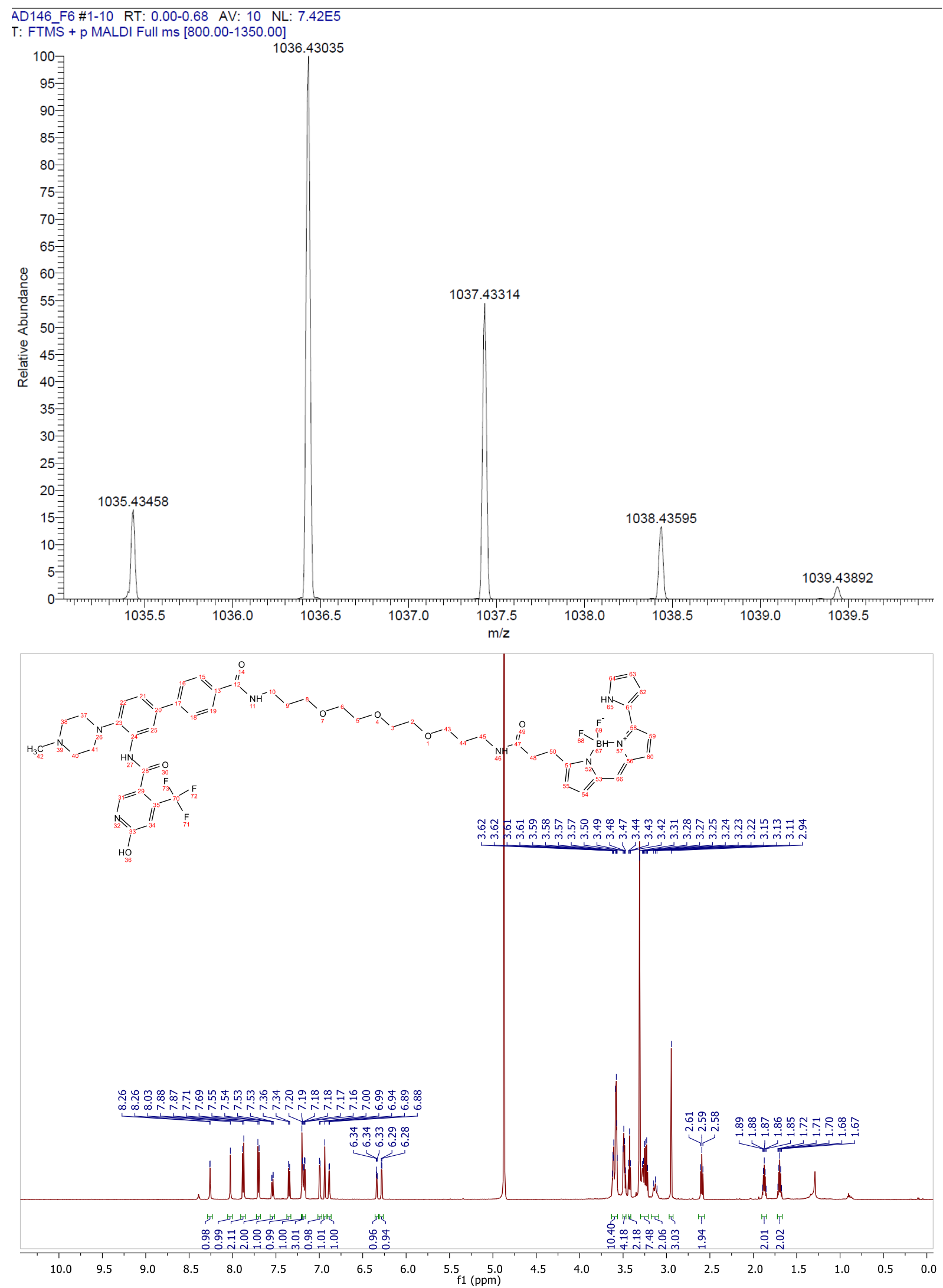
Appendix of intermediates 3-6

ESI and ${ }^{1} \mathrm{H}-\mathrm{NMR}$ of 1-(4-bromo-2-nitrophenyl)-4-methylpiperazine

(3) C:UcaliburldataVD14_2h 6/15/2018 12:18:41 PM

AD14_2h \#32-44 RT: 0.54-0.75 AV: 13 SB: 11 0.19-0.36 NL: 8.65E6

$\mathrm{T}:\{0, \overline{0}\}+\mathrm{c}$ ESI !corona sid $=75.00$ det $=1306.00$ Full ms $[105.00-600.00]$
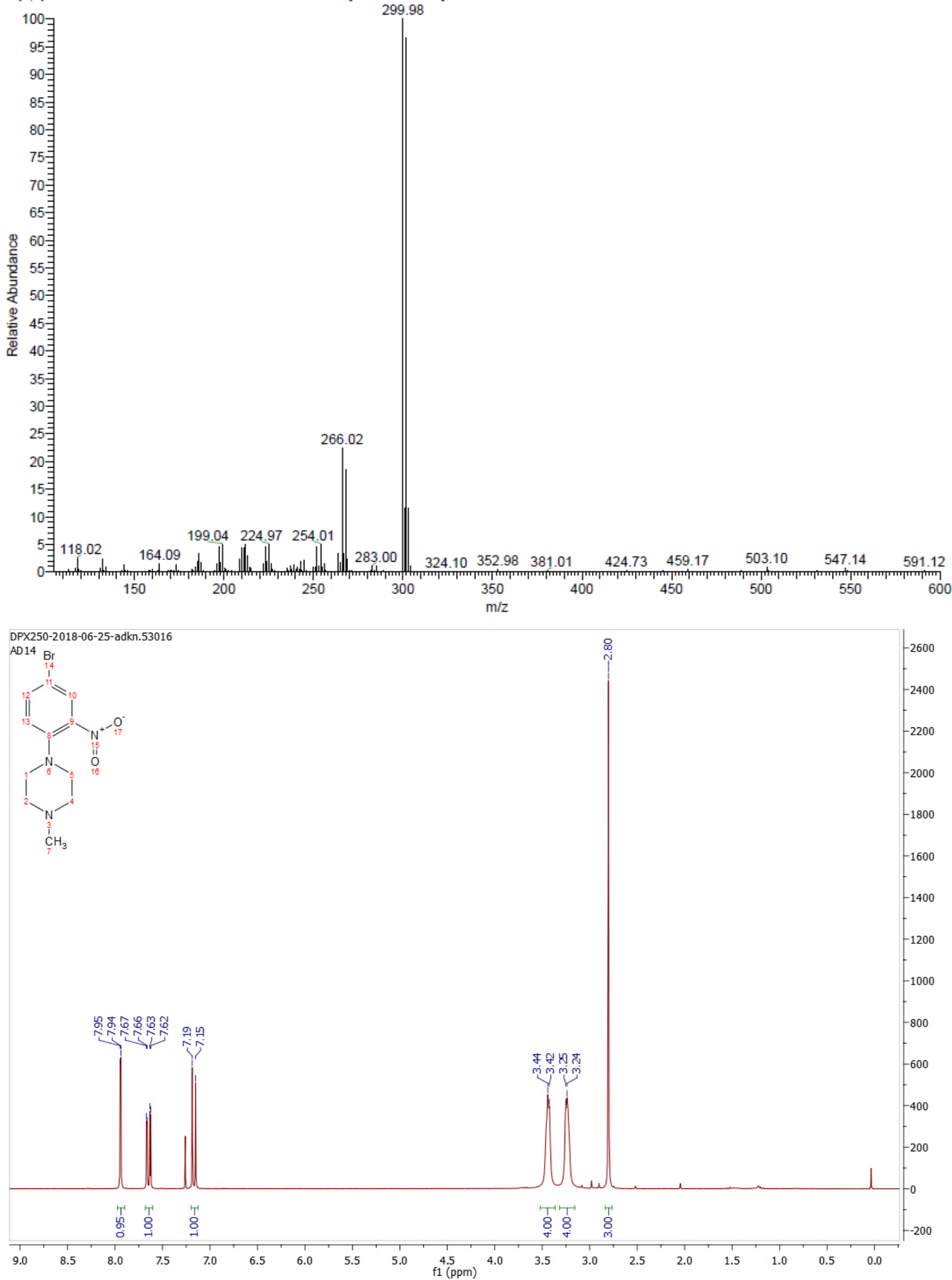
ESI, ${ }^{1} \mathrm{H}-\mathrm{NMR}$ and $\mathrm{HPLC}$ of 5-bromo-2-(4-methylpiperazin-1-yl)aniline C:XXaliburldatalAD21_1 7/2/2018 9:10:28 AM

(4)

AD21_1 \#36-43 RT: 0.61-0.73 AV: 8 SB: 10 0.07-0.23 NL: 5.44E6

$\mathrm{T}:\{0, \overline{0}\}+\mathrm{c}$ ESI $!$ corona sid $=75.00$ det $=1306.00$ Full $\mathrm{ms}[100.00-600.00]$

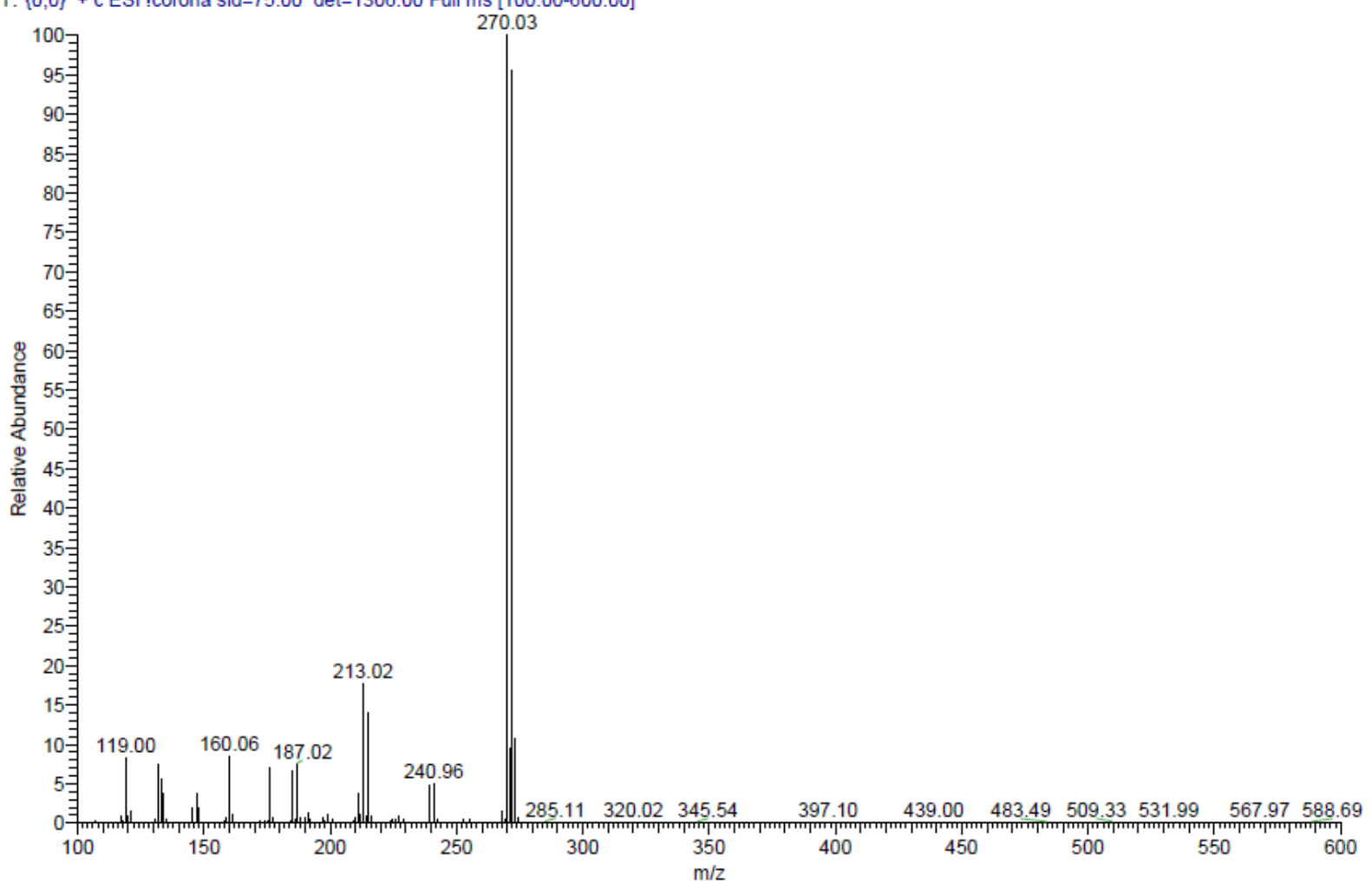

DPX250-2018-08-02-adkn.54465 AD21.3<smiles>CN1CNP(N2P=NP(Br)N=P2)N=P1</smiles>

(2)



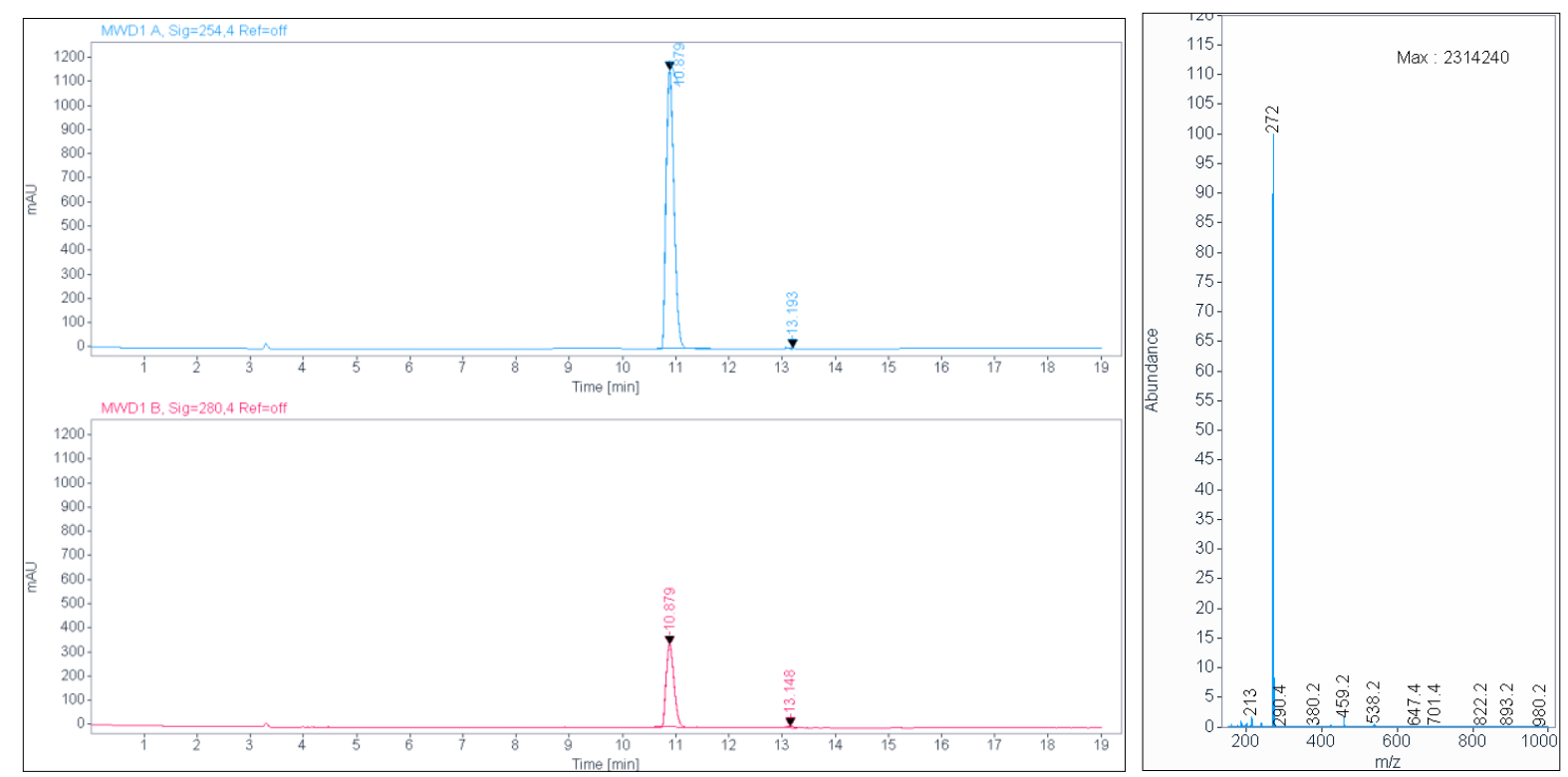

Signal: $\quad$ MWD1 A, Sig=254,4 Ref=off

$\begin{array}{ccrrr}\text { RT [min] Type } & \text { Width [min] } & \text { Area } & \text { Height } & \text { Area\% } \\ 10.879 \text { BV } & 0.1668 & 11862.8955 & 1155.4844 & 99.6042 \\ 13.193 \text { MM } & 0.0751 & 47.1392 & 10.4557 & 0.3958 \\ & \text { Sum } & 11910.0347 & & \end{array}$

Signal: $\quad$ MWD1 B, Sig=280,4 Ref=off

$\begin{array}{crrrr}\text { RT [min] Type } & \text { Width [min] } & \text { Area } & \text { Height } & \text { Area\% } \\ 10.879 \text { VV } & 0.1677 & 3558.1433 & 343.9120 & 98.2896 \\ 13.148 \mathrm{MM} & 0.1711 & 61.9159 & 6.0318 & 1.7104 \\ & \text { Sum } & 3620.0593 & & \end{array}$


ESI, HPLC, ${ }^{1} \mathrm{H}-\mathrm{NMR}$ and ${ }^{13} \mathrm{C}-\mathrm{NMR}$ of tert-butyl 3'-amino-4'-(4-methylpiperazin-1-yl)-[1,1'-biphenyl]-4carboxylate (5b)

C:IXcaliburldataIAD99-1 8/20/2019 8:21:08 AM

AD99-1 \#36-43 RT: 0.61-0.73 AV: 8 SB: 9 0.02-0.16 NL: 1.27E7

$\mathrm{T}:\{0,0\}+\mathrm{c}$ ESI !corona sid=75.00 det=1306.00 Full ms [100.00-700.00]
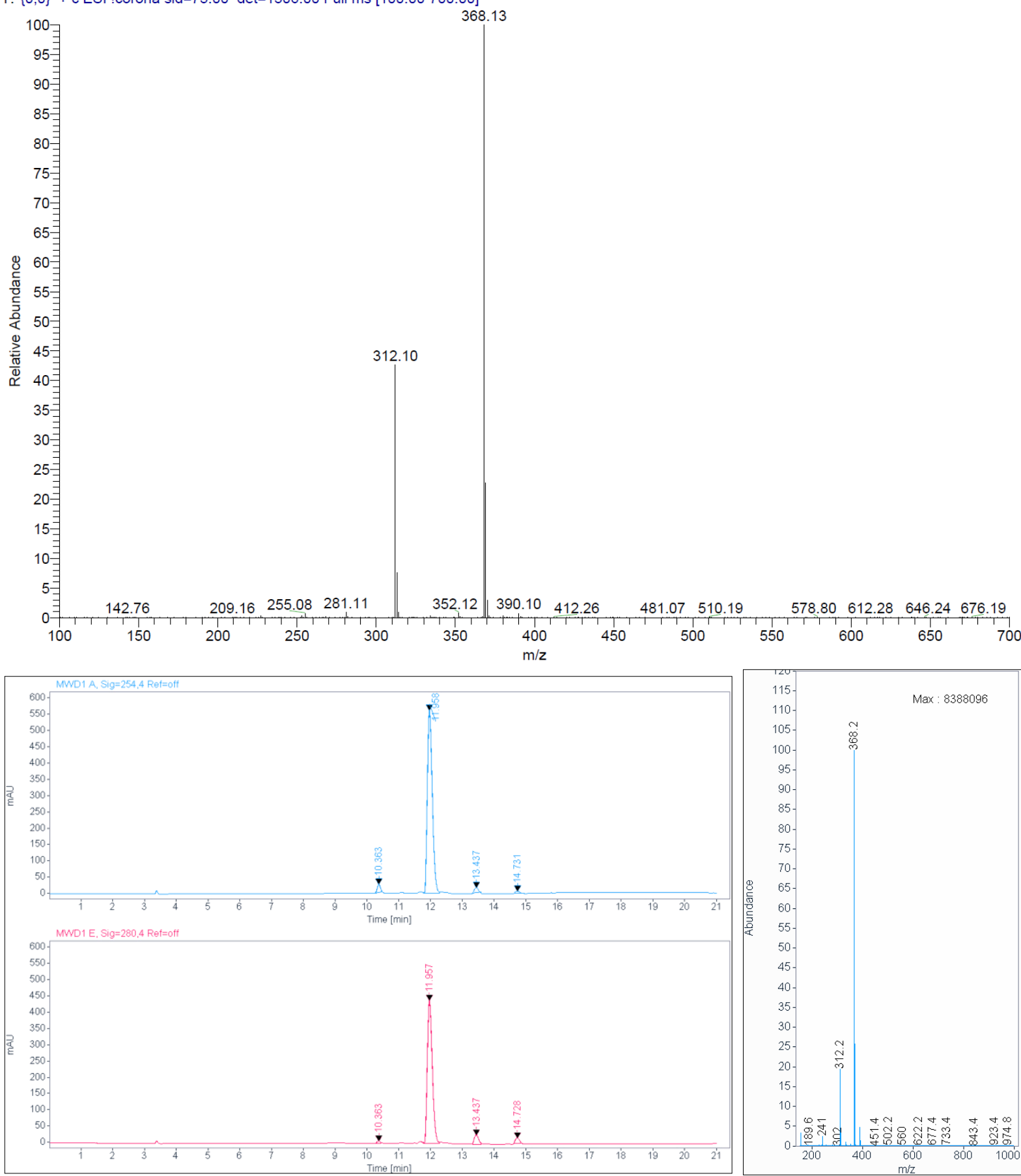

Signal: $\quad$ MWD1 A, Sig $=254,4$ Ref $=$ off

$\begin{array}{crrrr}\text { RT [min] Type } & \text { Width [min] } & \text { Area } & \text { Height } & \text { Area\% } \\ 10.363 \text { MM } & 0.1143 & 179.2153 & 26.1271 & 2.7597 \\ 11.958 \text { VV } & 0.1658 & 6113.2773 & 562.4454 & 94.1364 \\ 13.437 \text { MM } & 0.1422 & 136.3742 & 15.9810 & 2.1000 \\ 14.731 \mathrm{MM} & 0.1515 & 65.1996 & 7.1723 & 1.0040 \\ & \text { Sum } & 6494.0666 & & \end{array}$

Signal: $\quad$ MWD1 E, Sig=280,4 Ref=off 


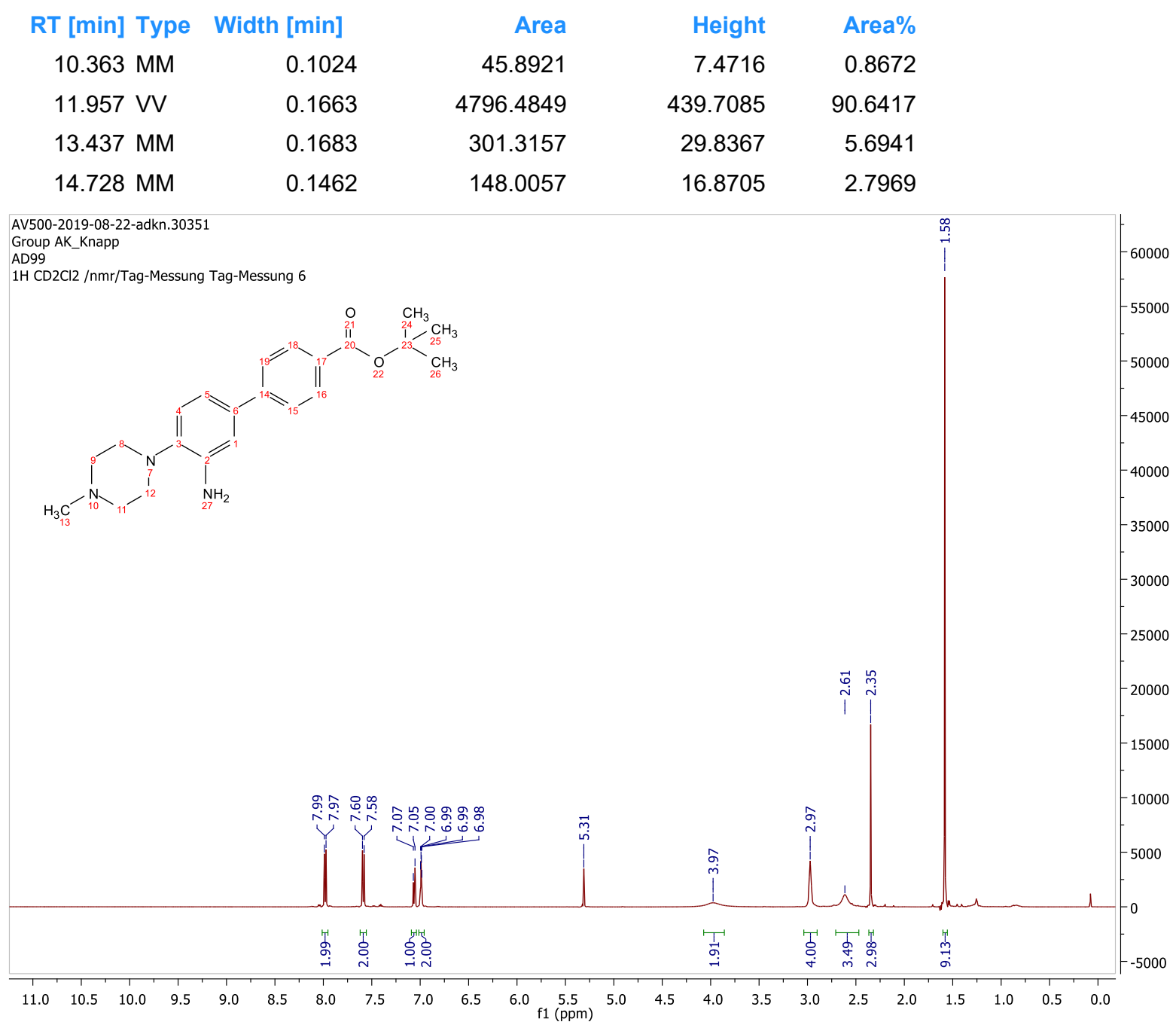




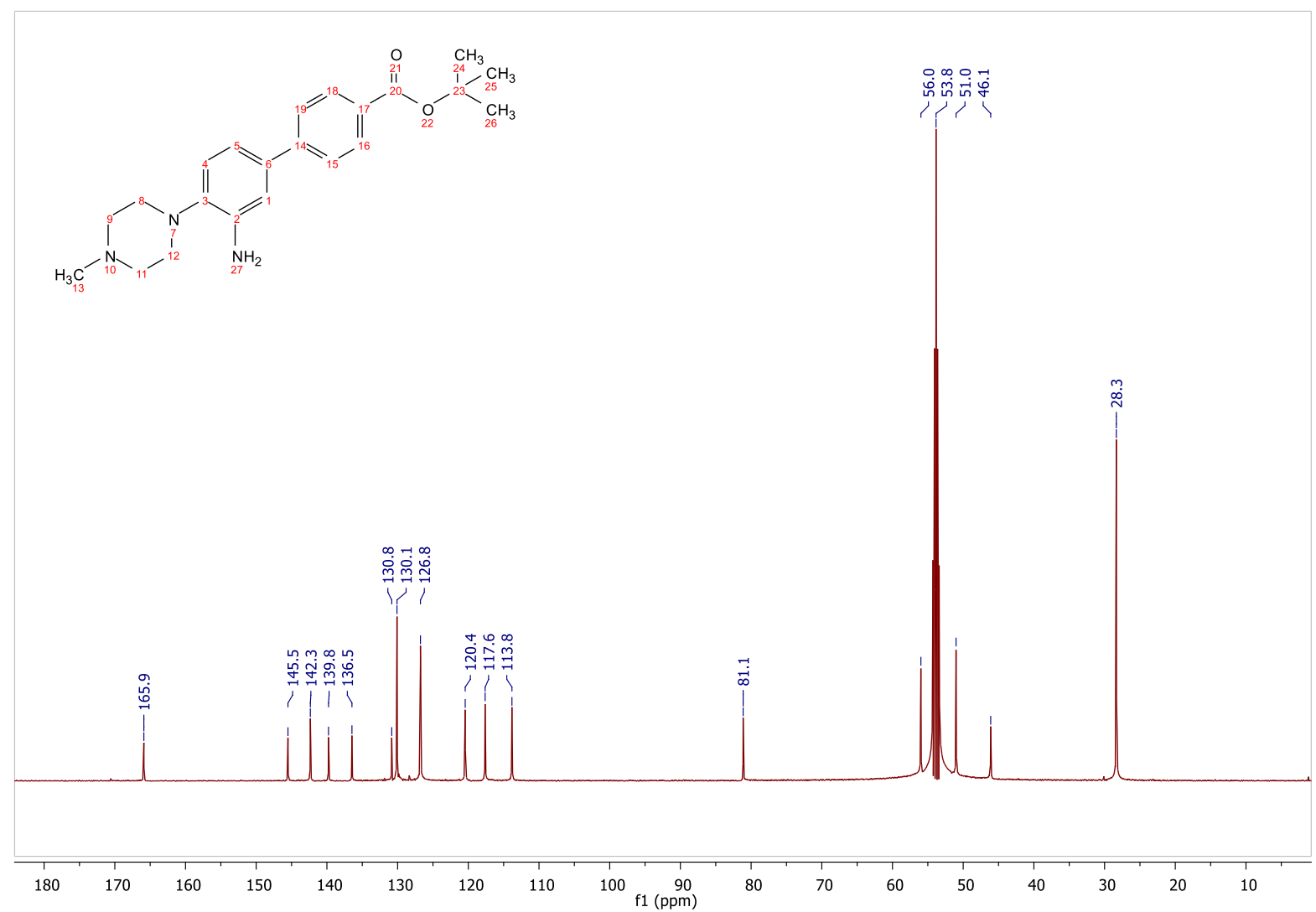


ESI, HPLC, ${ }^{1} \mathrm{H}-\mathrm{NMR}$ and ${ }^{13} \mathrm{C}-\mathrm{NMR}$ of tert-butyl 3'-(6-hydroxy-4-(trifluoromethyl)nicotinamido)-4'-(4methylpiperazin-1-yl)-[1,1'-biphenyl]-4-carboxylate (6d)

C:IXcaliburldata\AD100_1-F2

8/29/2019 9:34:41 AM
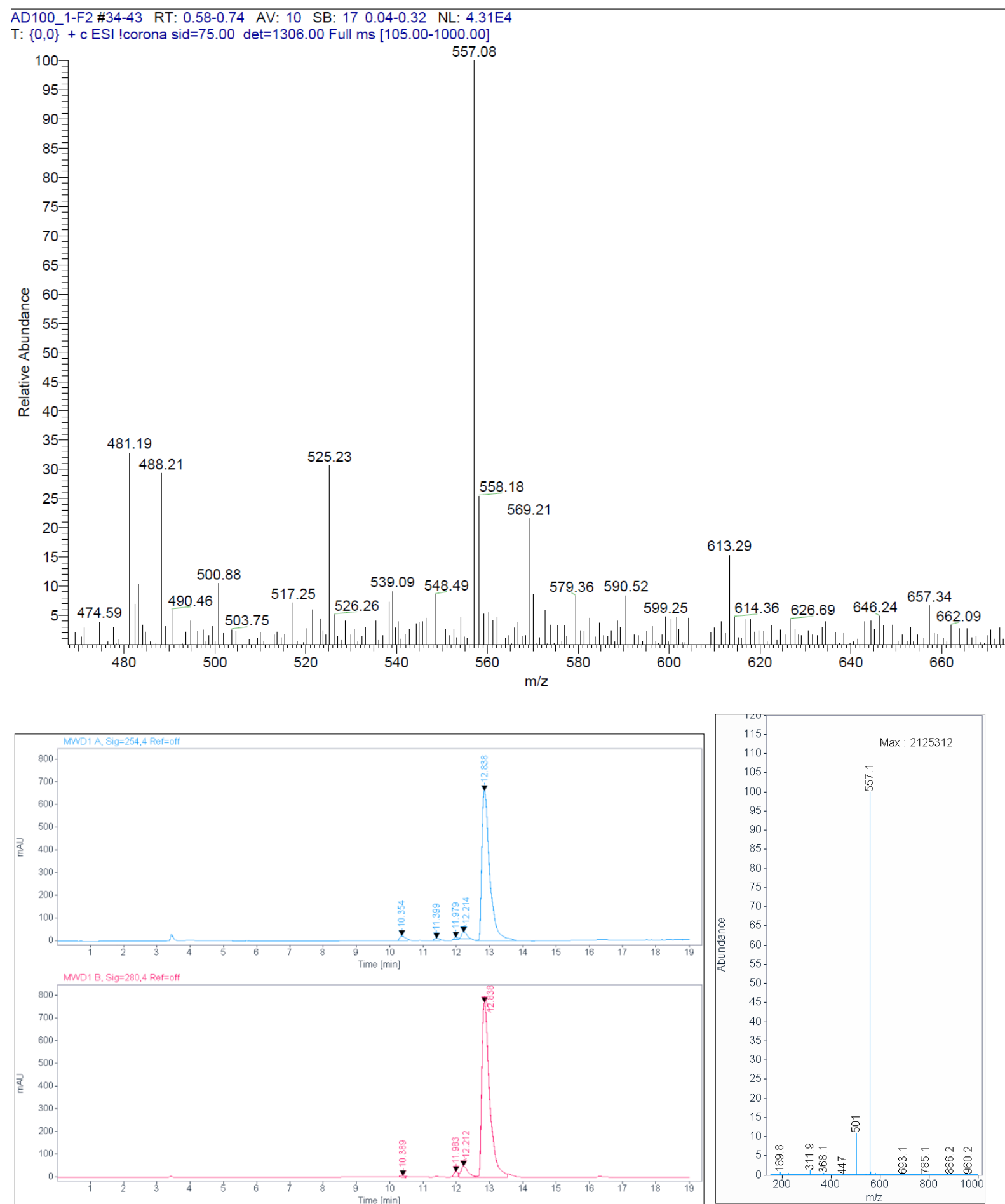

Signal: $\quad$ MWD1 A, Sig=254,4 Ref=off

$\begin{array}{crrrr}\text { RT [min] Type } & \text { Width [min] } & \text { Area } & \text { Height } & \text { Area\% } \\ 10.354 \text { MM } & 0.1955 & 264.3400 & 22.5354 & 2.2644 \\ 11.399 \mathrm{MM} & 0.1602 & 109.3309 & 11.3760 & 0.9366 \\ 11.979 \mathrm{MM} & 0.1438 & 97.5140 & 11.2982 & 0.8353 \\ 12.214 \mathrm{MM} & 0.1826 & 324.7970 & 29.6518 & 2.7823 \\ 12.838 \mathrm{VV} & 0.2499 & 10877.6895 & 661.4399 & 93.1814\end{array}$




\section{Sum 11673.6714}

Signal: $\quad$ MWD1 B, Sig=280,4 Ref=off

$\begin{array}{ccrrr}\text { RT [min] Type } & \text { Width [min] } & \text { Area } & \text { Height } & \text { Area\% } \\ 10.389 \text { MM } & 0.1813 & 84.4925 & 7.7665 & 0.6195 \\ 11.983 \text { MM } & 0.1406 & 191.5884 & 22.7102 & 1.4046 \\ 12.212 \text { VV } & 0.2312 & 748.0756 & 50.1011 & 5.4845 \\ 12.838 \text { VV } & 0.2487 & 12615.6523 & 771.6691 & 92.4914\end{array}$

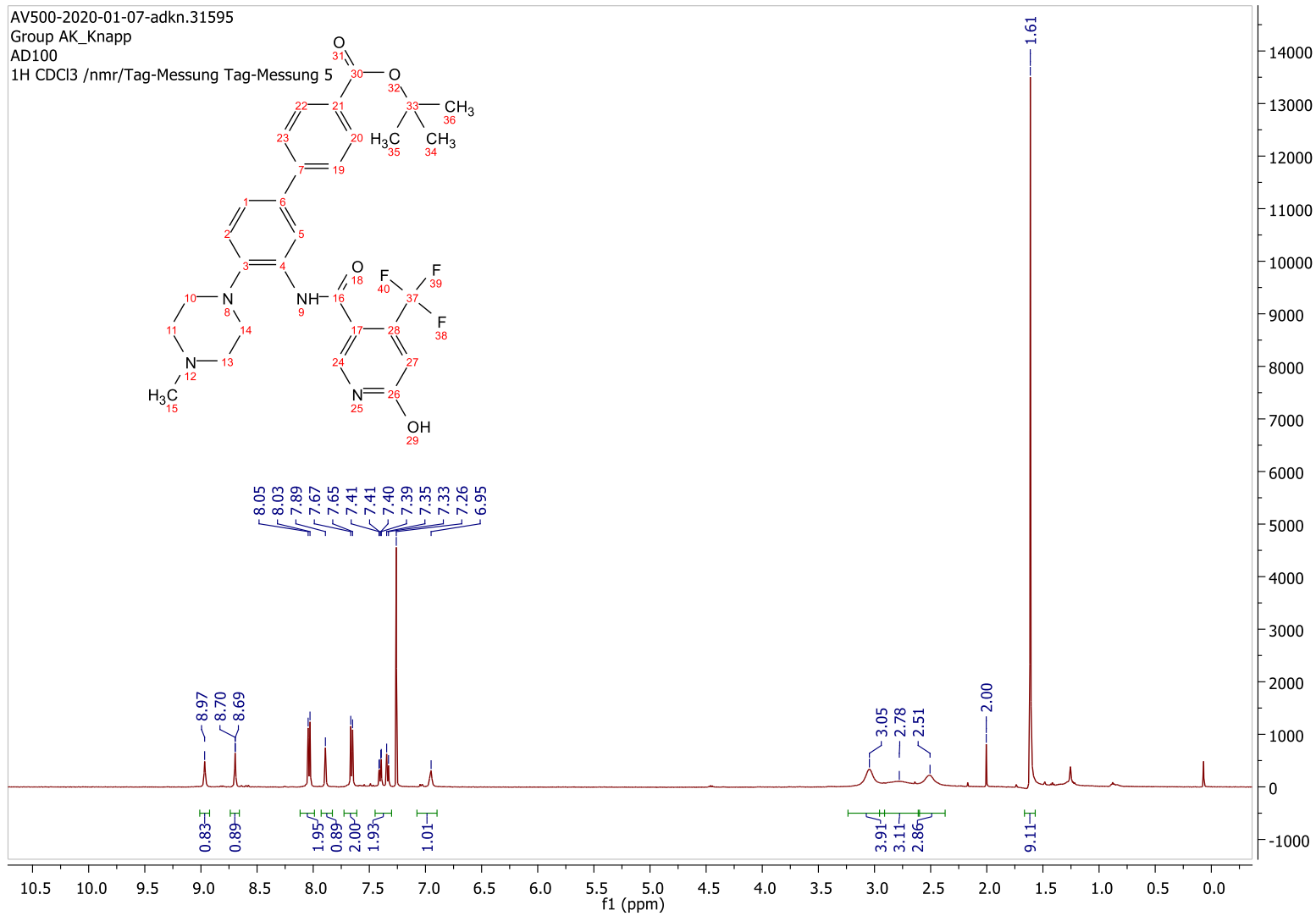




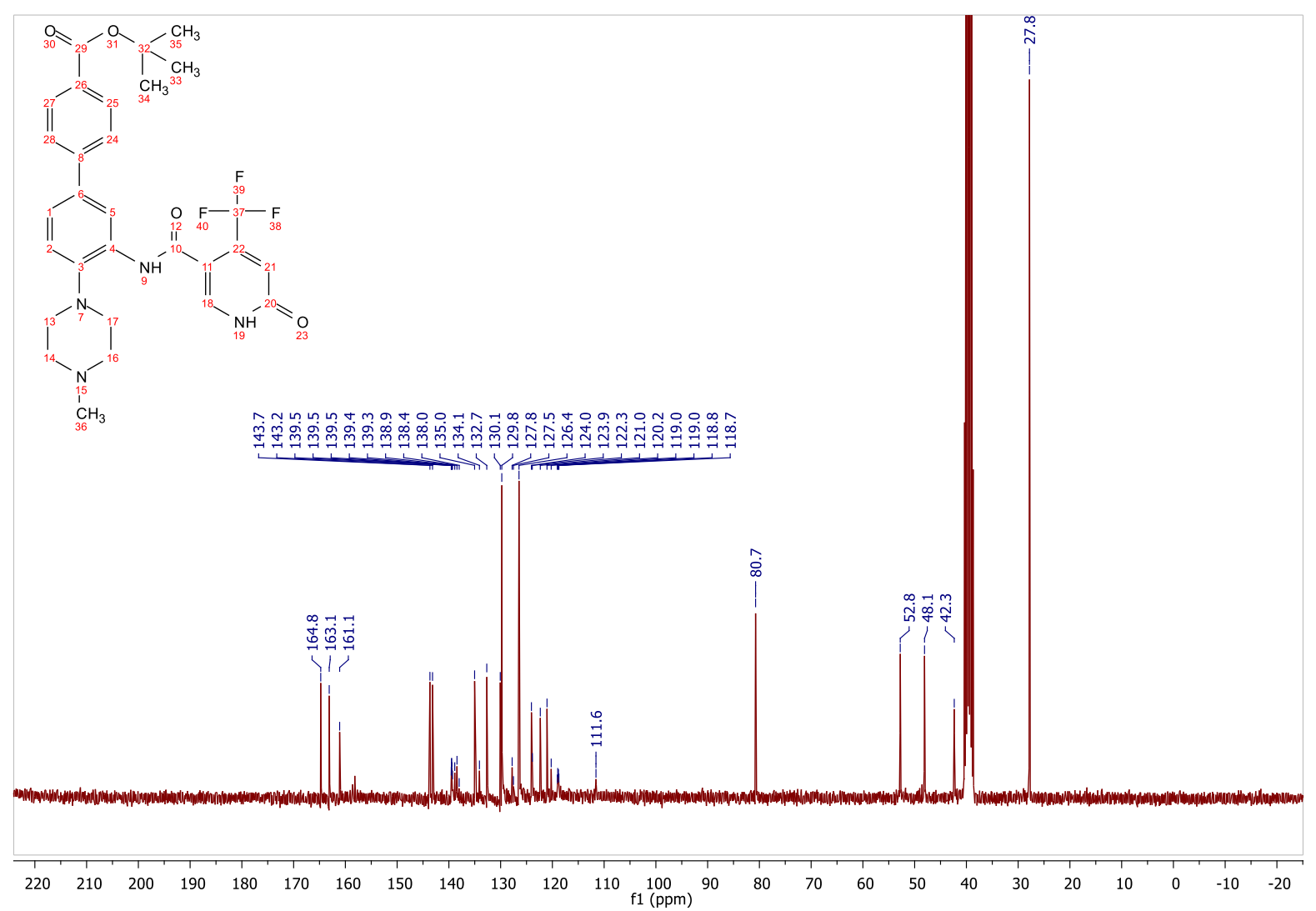


MALDI, HRMS, HPLC, ${ }^{1} \mathrm{H}-\mathrm{NMR},{ }^{13} \mathrm{C}-\mathrm{NMR},{ }^{13} \mathrm{C}-\mathrm{NMR},{ }^{13} \mathrm{C}$-DEPT9O, ${ }^{1} \mathrm{H}-{ }^{13} \mathrm{C}-\mathrm{HSQC},{ }^{1} \mathrm{H}-{ }^{13} \mathrm{C}-\mathrm{HMBC}$ of $\mathrm{N}-\left(4{ }^{-}-\right.$ ((2-(2-((2-(2,6-dioxopiperidin-3-yl)-1,3-dioxoisoindolin-4-yl)amino)ethoxy)ethyl)carbamoyl)-4-(4methylpiperazin-1-yl)-[1,1'-biphenyl]-3-yl)-6-hydroxy-4-(trifluoromethyl)nicotinamide (7a)

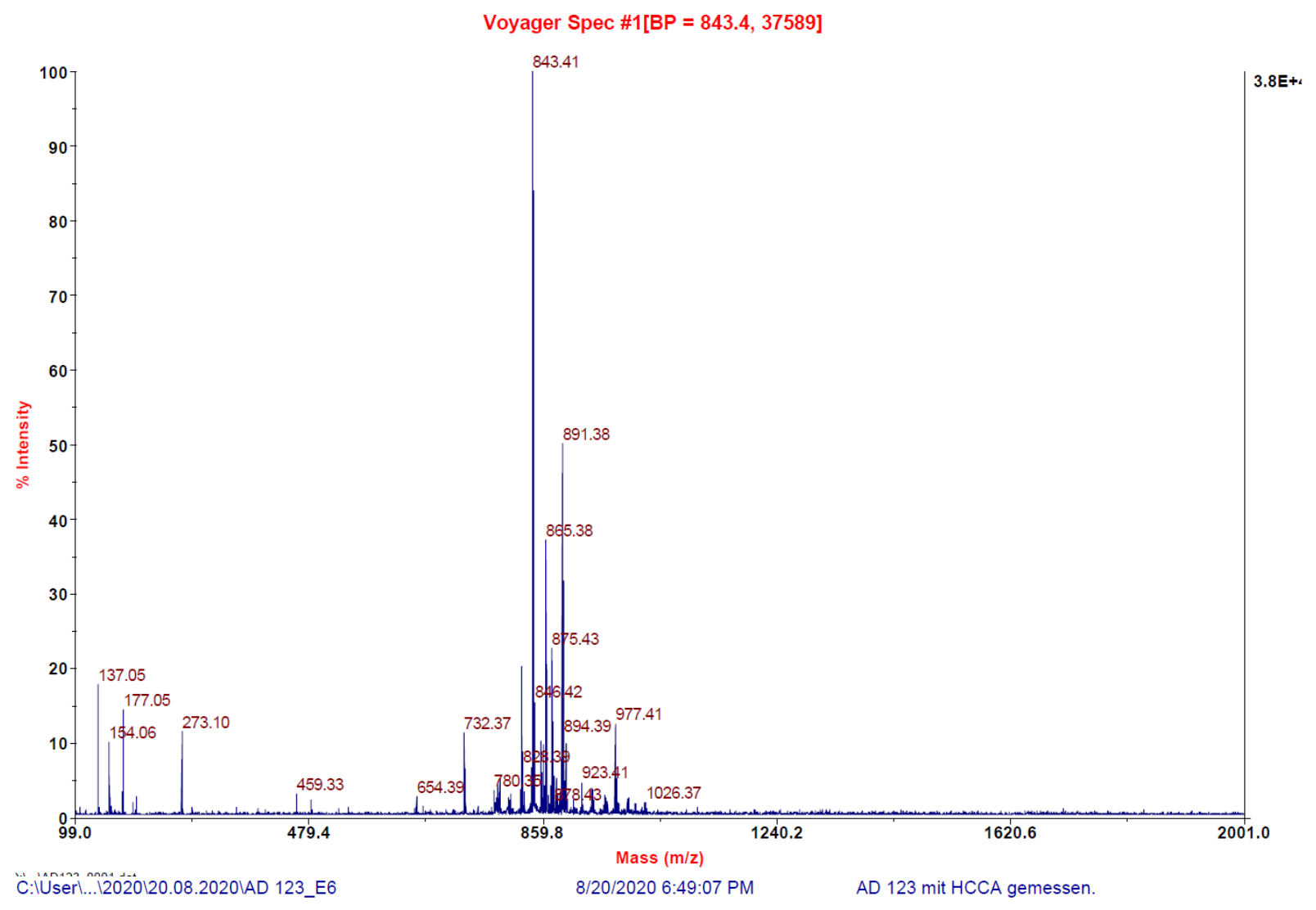

AD 123_E6 \#1-9 RT: 0.00-0.60 AV: 9 NL: 1.87E7

T: FTMS + p MALDI Full ms [700.00-1700.00]

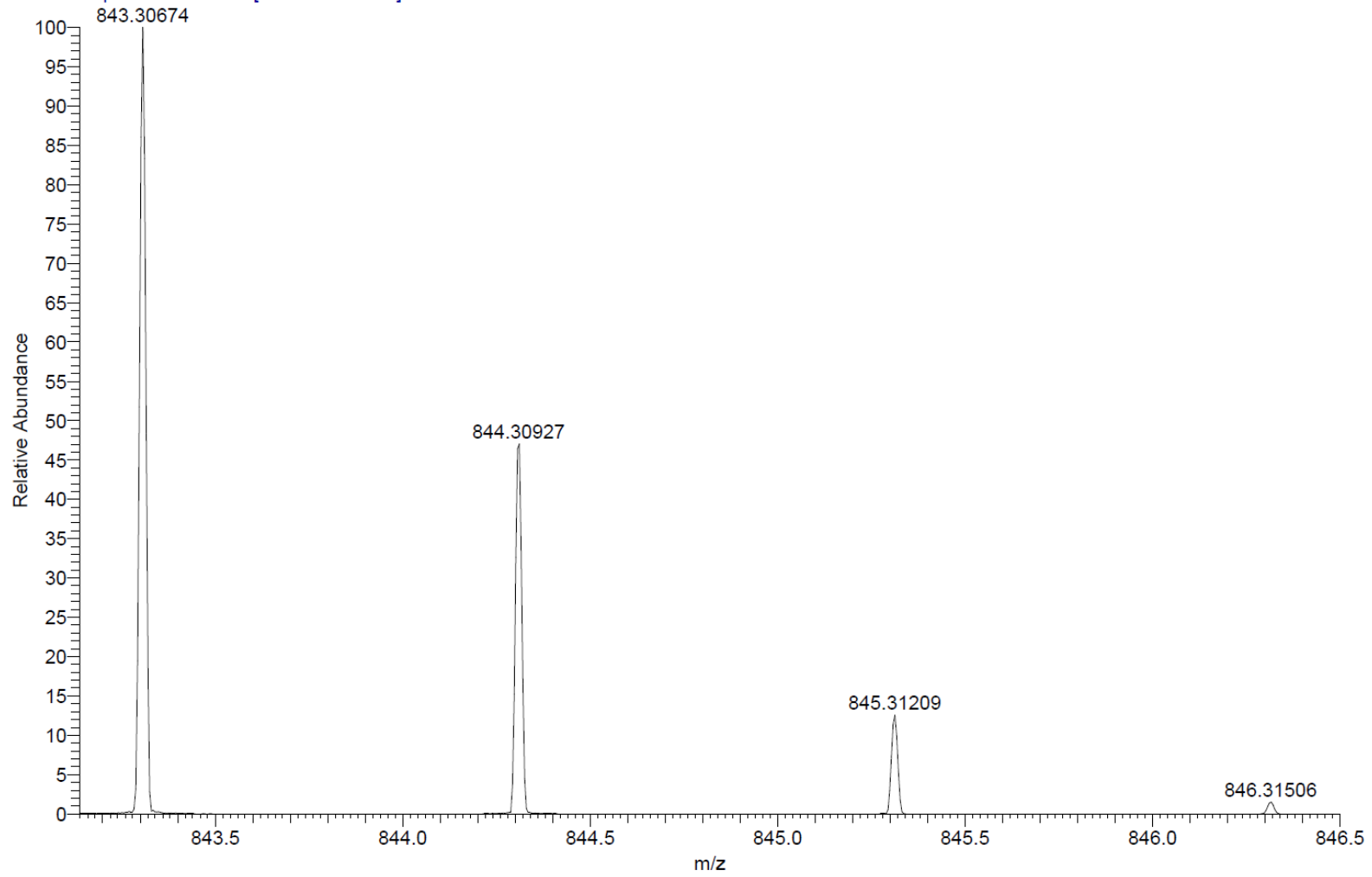




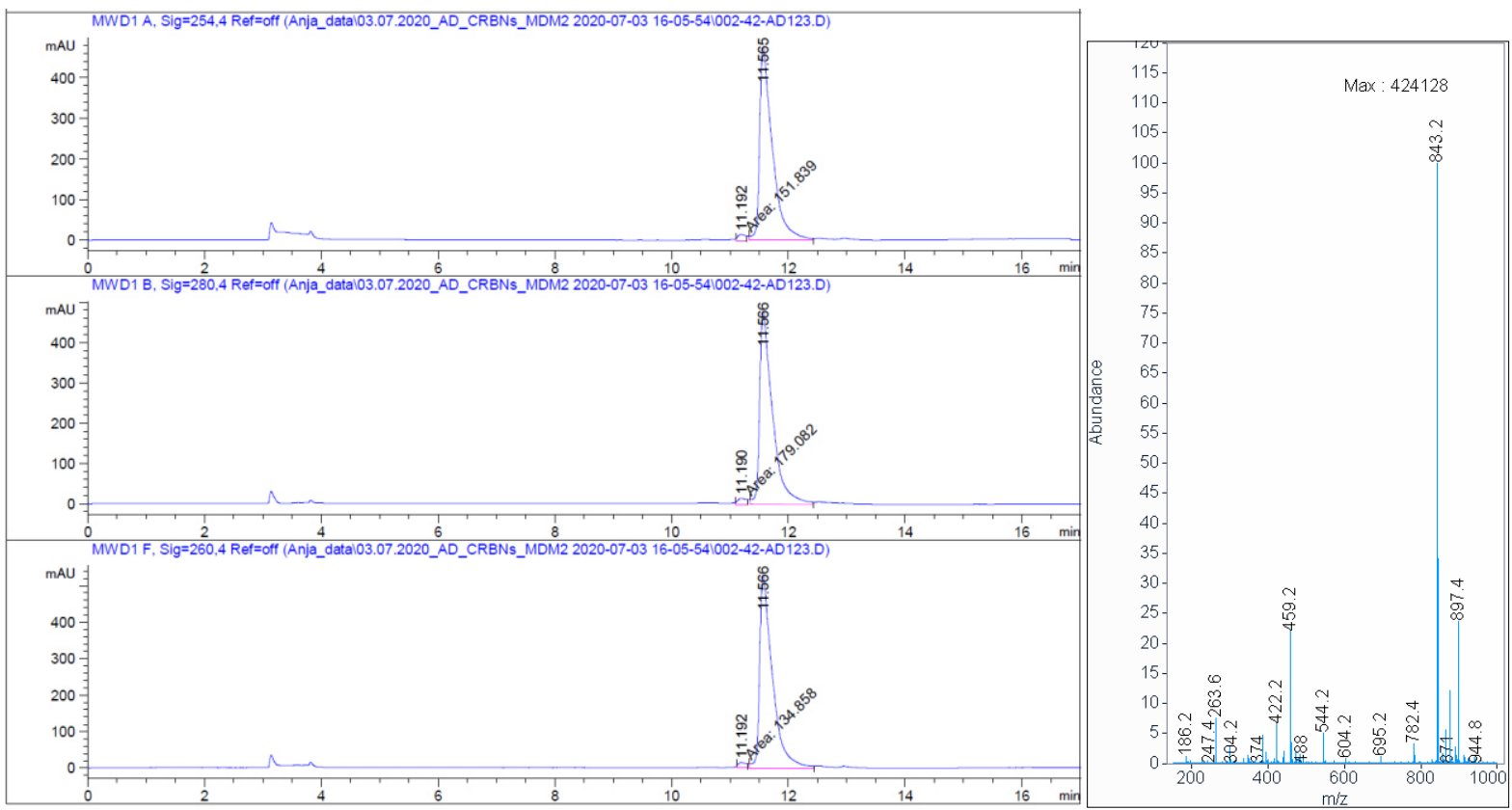

Signal: $\quad$ MWD1 A, Sig=254,4 Ref=off

$\begin{array}{crrrr}\text { RT [min] Type } & \text { Width [min] } & \text { Area } & \text { Height } & \text { Area\% } \\ 11.614 \text { MM } & 0.2199 & 202.6069 & 15.3539 & 100.0000 \\ & \text { Sum } & 202.6069 & & \end{array}$

Signal: $\quad$ MWD1 B, Sig=280,4 Ref=off

$\begin{array}{crrrr}\text { RT [min] Type } & \text { Width [min] } & \text { Area } & \text { Height } & \text { Area\% } \\ 11.612 \mathrm{MM} & 0.2239 & 212.1426 & 15.7890 & 100.0000 \\ & \text { Sum } & 212.1426 & & \end{array}$

Signal: $\quad$ MWD1 F, Sig=260,4 Ref=off

$\begin{array}{crrrr}\text { RT [min] Type } & \text { Width [min] } & \text { Area } & \text { Height } & \text { Area\% } \\ 11.612 \text { MM } & 0.2276 & 238.7010 & 17.4816 & 100.0000\end{array}$



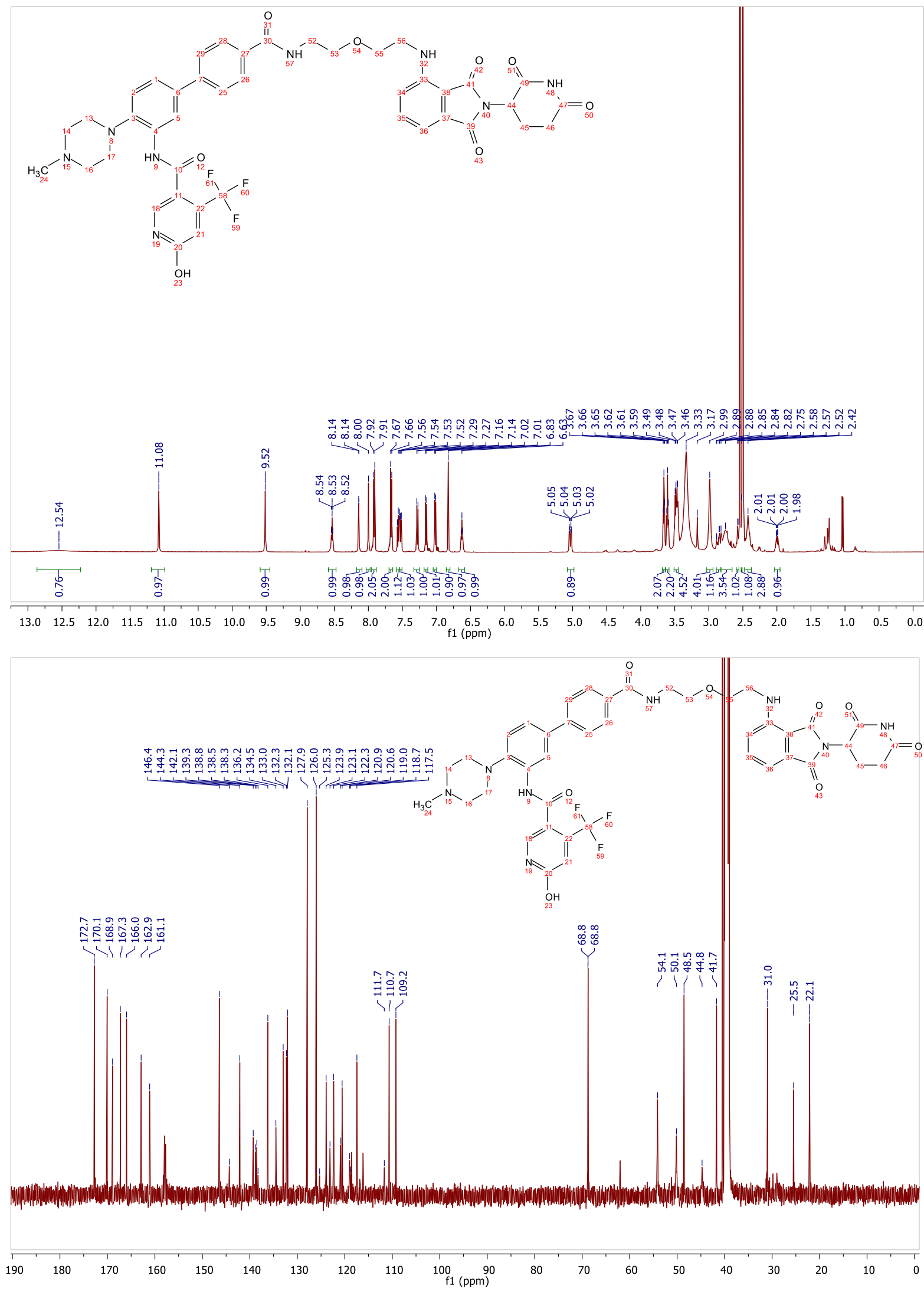


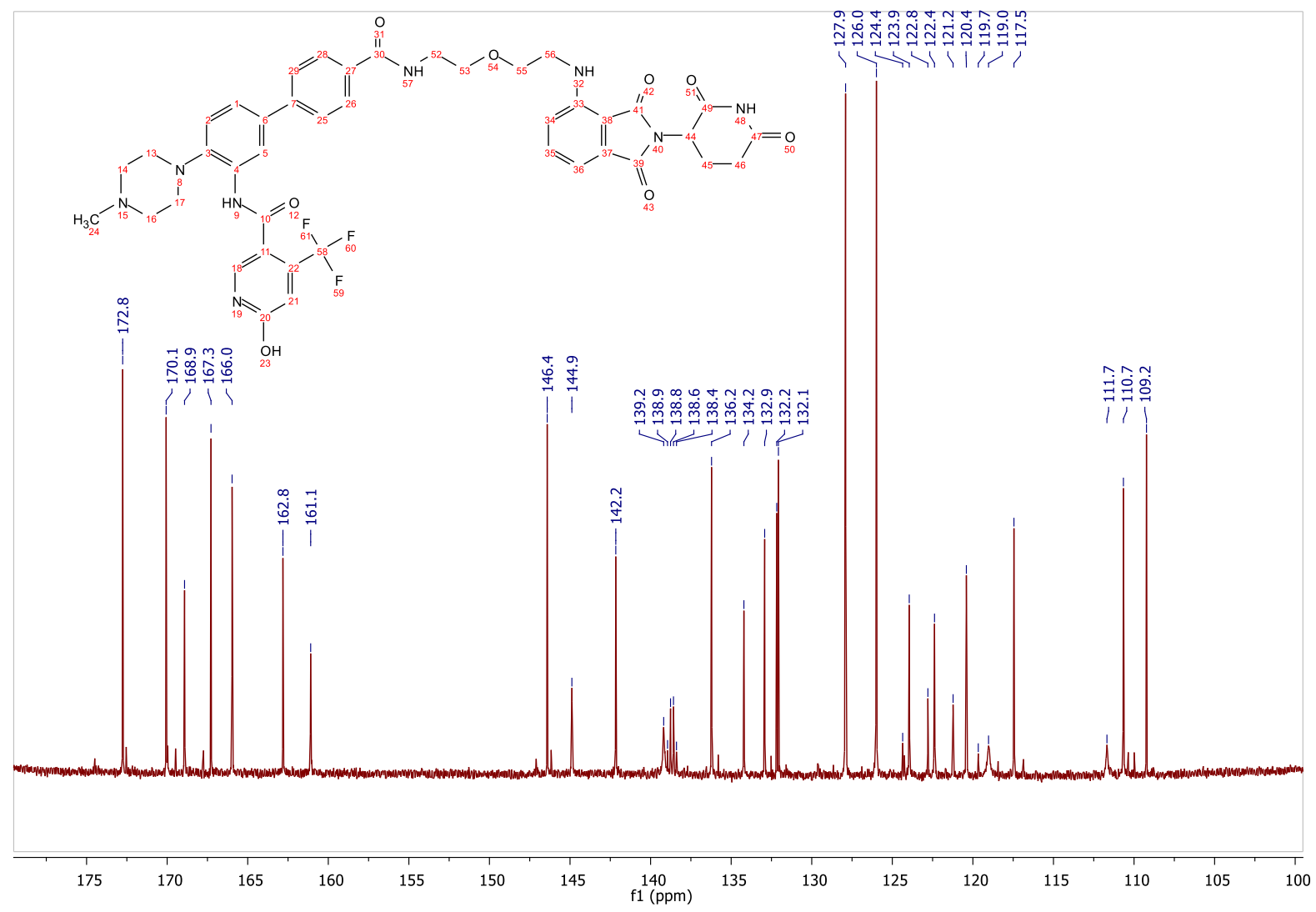

protac_700

compound \#123 ?mg in 500ul DMSO-d6 T=298K

$13 \mathrm{C}$ DEPT90 (only $\mathrm{CH}$ groups detected) NS=1k Aq=1.5s D1=1.5s protac_700/63 17.3.2021 $13 \mathrm{C}$ carrier at $140 \mathrm{ppm}, \mathrm{SW}=80 \mathrm{ppm}$, de $=50$ u

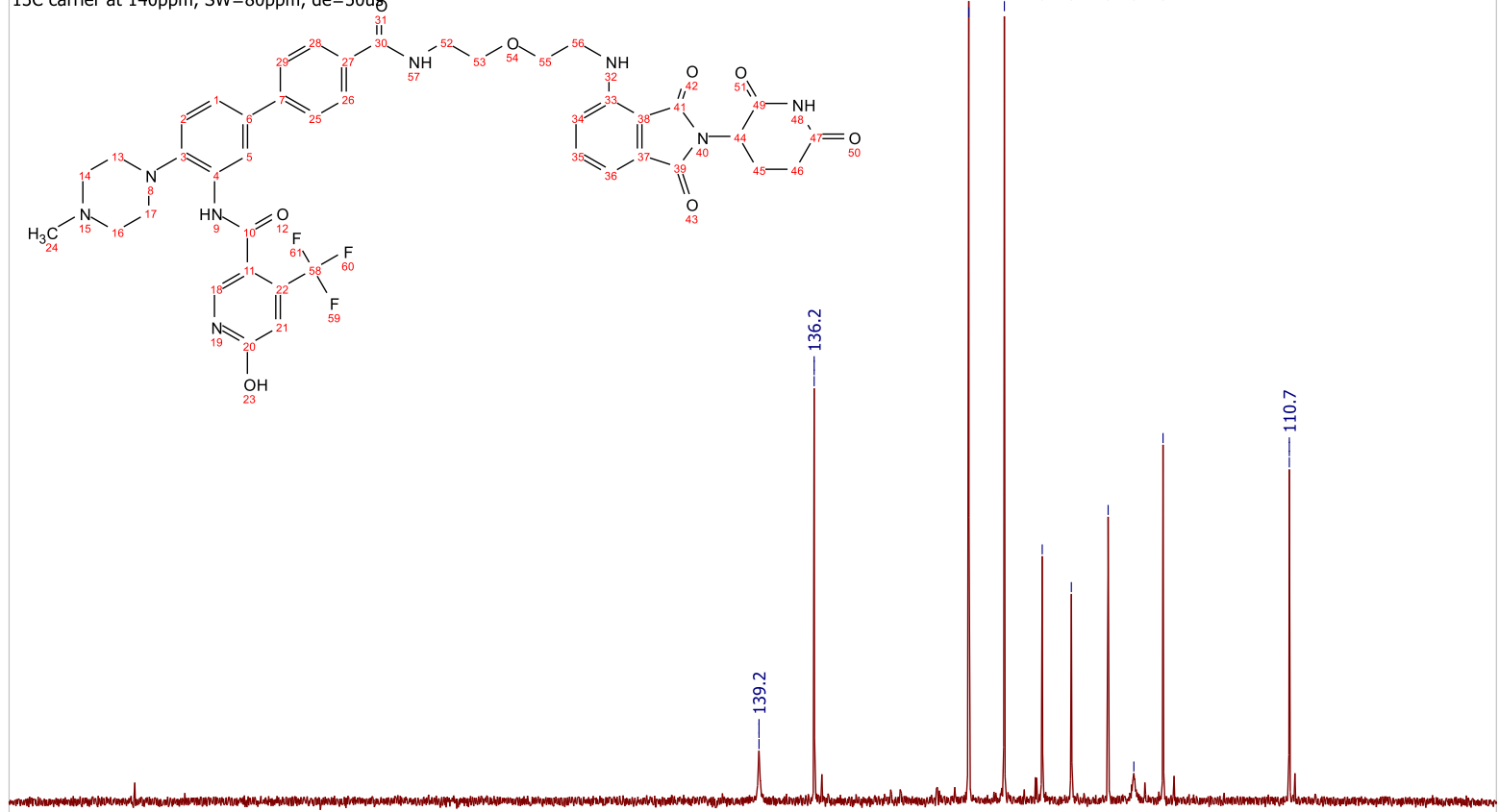

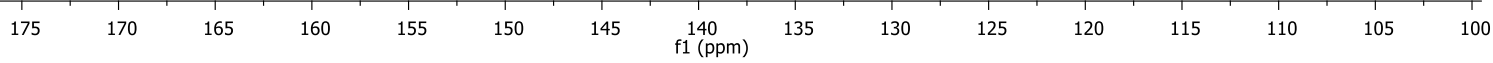



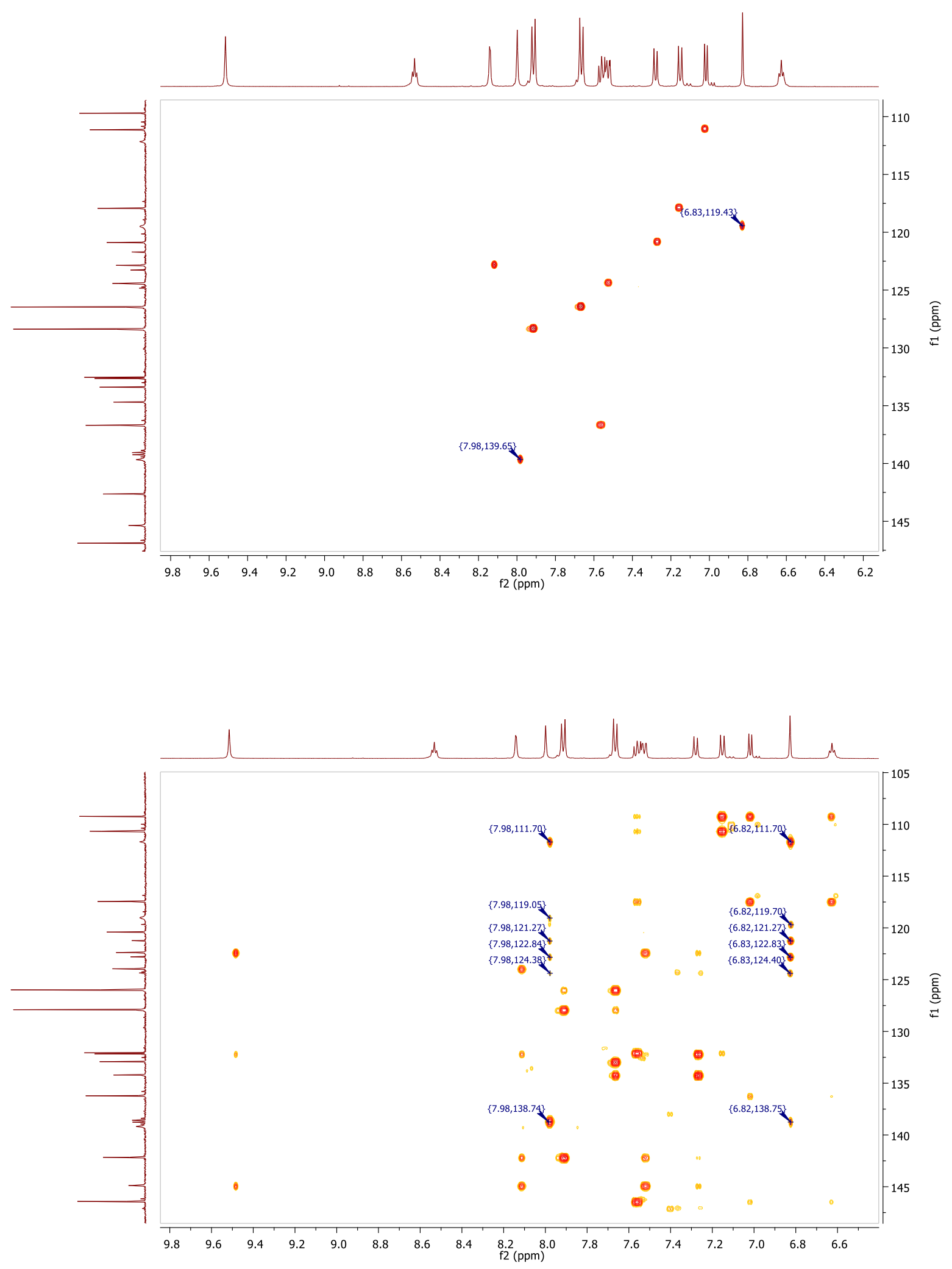
HPLC, MALDI, HRMS, ${ }^{1} \mathrm{H}-\mathrm{NMR}$ and ${ }^{13} \mathrm{C}-\mathrm{NMR},{ }^{13} \mathrm{C}-\mathrm{NMR},{ }^{1} \mathrm{H}-{ }^{13} \mathrm{C}-\mathrm{HSQC},{ }^{1} \mathrm{H}-{ }^{13} \mathrm{C}-\mathrm{HMBC}$ of $\mathrm{N}-\left(4{ }^{1}-((2-(2-(2-)\right.$ (2-((2-(2,6-dioxopiperidin-3-yl)-1,3-dioxoisoindolin-4-

yl)amino)ethoxy)ethoxy)ethoxy)ethyl)carbamoyl)-4-(4-methylpiperazin-1-yl)-[1,1'-biphenyl]-3-yl)6-hydroxy-4-(trifluoromethyl)nicotinamide (7b)
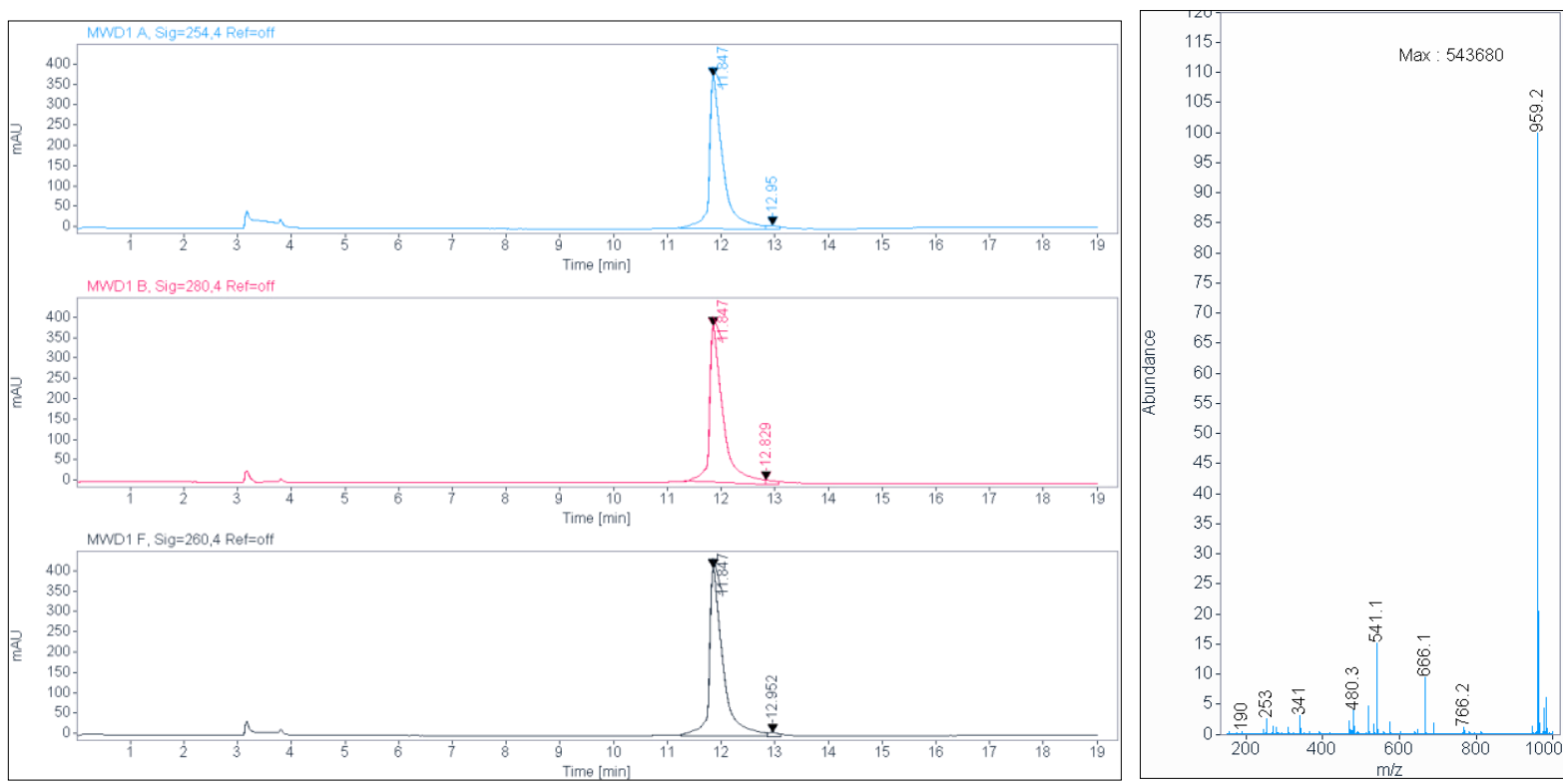

Signal: $\quad$ MWD1 A, Sig=254,4 Ref=off

$\begin{array}{crrr}\text { RT [min] Type } & \text { Width [min] } & \text { Area } & \text { Height Area\% } \\ 11.847 \mathrm{MM} & 0.2960 & 6658.2402 & 374.959097 .7984 \\ 12.950 \mathrm{MM} & 0.2326 & 149.8847 & 10.73862 .2016 \\ & \text { Sum } & 6808.1249 & \end{array}$

Signal: $\quad$ MWD1 B, Sig=280,4 Ref=off

$\begin{array}{crrr}\text { RT [min] Type } & \text { Width [min] } & \text { Area } & \text { Height Area\% } \\ 11.847 \mathrm{MM} & 0.2924 & 6744.6553 & 384.480198 .0727 \\ 12.829 \mathrm{MM} & 0.1510 & 132.5450 & 10.45351 .9273 \\ & \text { Sum } & 6877.2003 & \end{array}$

Signal: $\quad$ MWD1 F, Sig $=260,4$ Ref $=$ off

$\begin{array}{crrr}\text { RT [min] Type } & \text { Width [min] } & \text { Area } & \text { Height Area\% } \\ 11.847 \text { VV } & 0.2405 & 7302.5942 & 412.607698 .0674 \\ 12.952 \mathrm{MM} & 0.2115 & 143.9127 & 11.34331 .9326\end{array}$


Voyager Spec \#1[BP = 959.5, 37728]
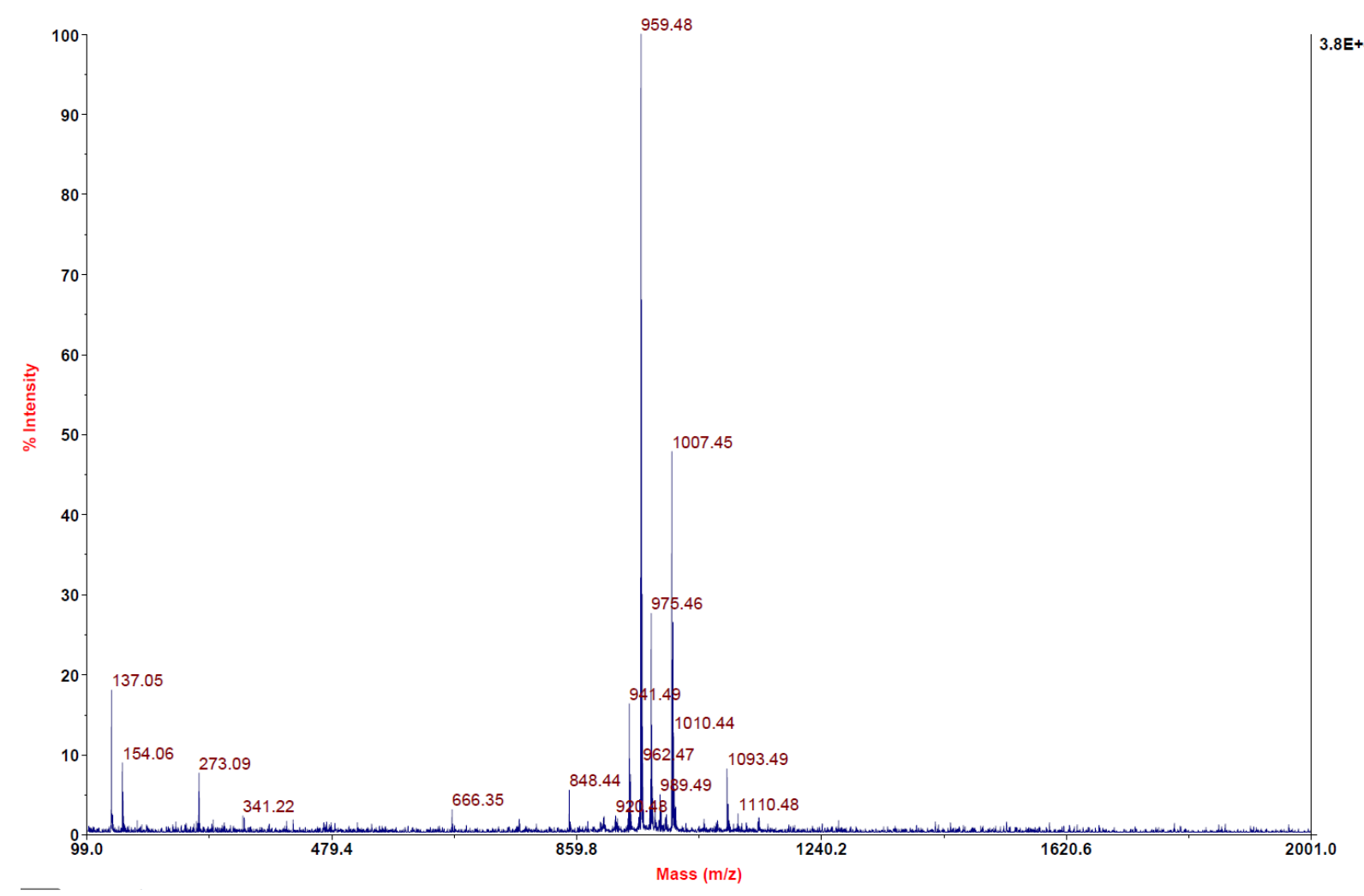

C:IUserl...I2020120.08.2020\AD 120_E4

8/20/2020 6:46:39 PM

AD 120 mit HCCA gemessen.

AD 120_E4 \#1-4 RT: 0.01-0.41 AV: 4 NL: 3.46E6

Г: FTMS + p MALDI Full ms [700.00-1700.00]

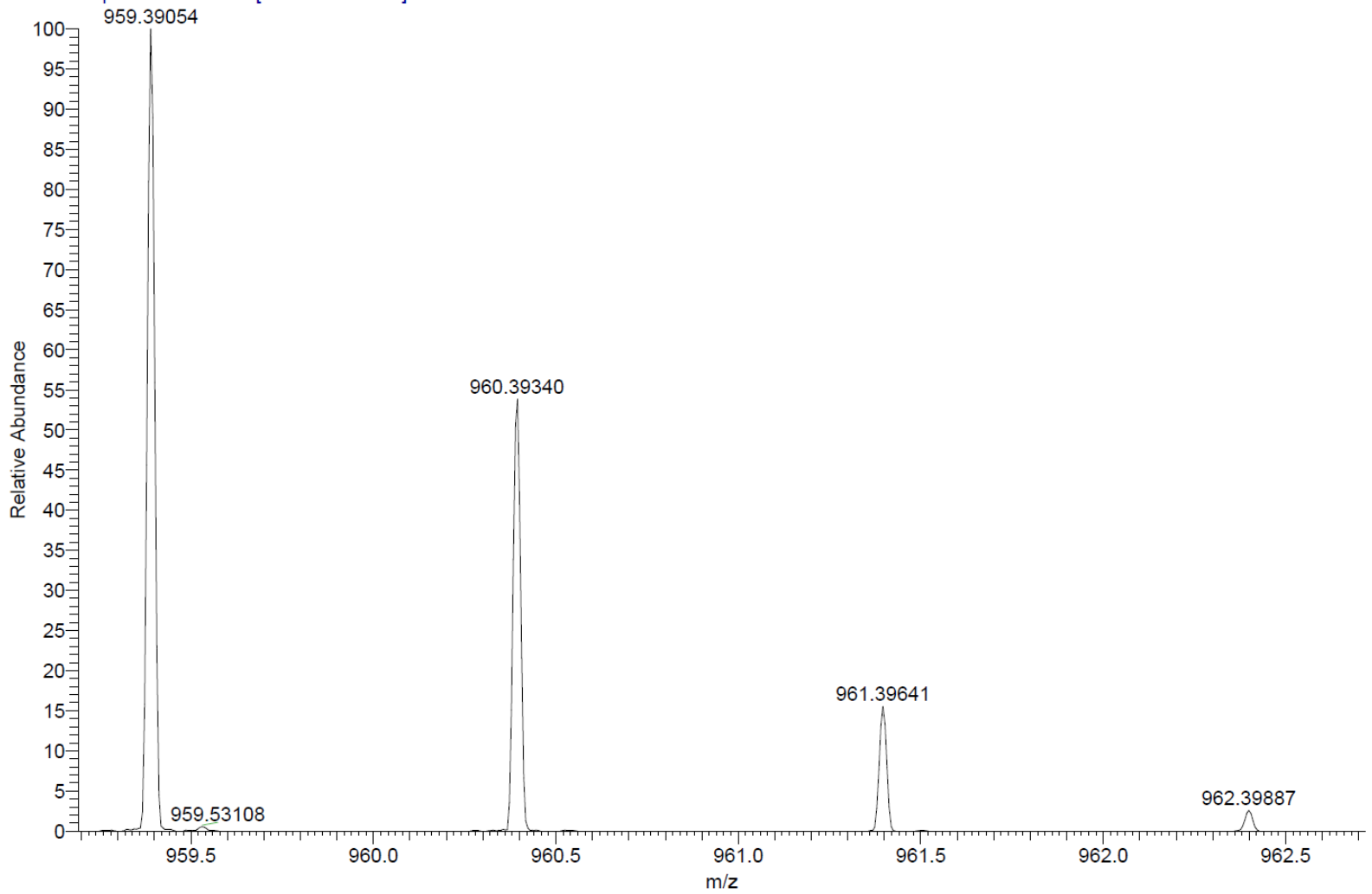



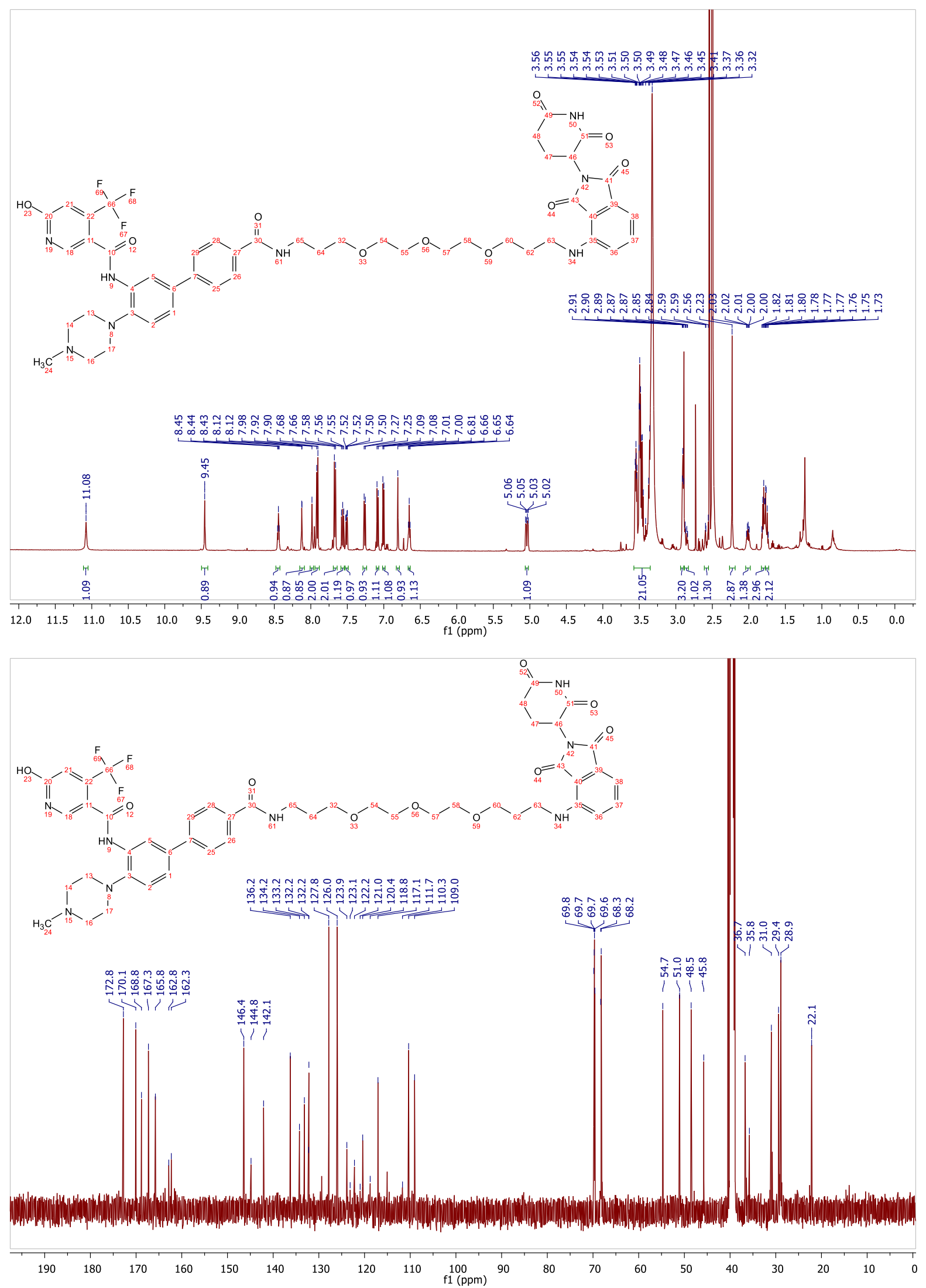

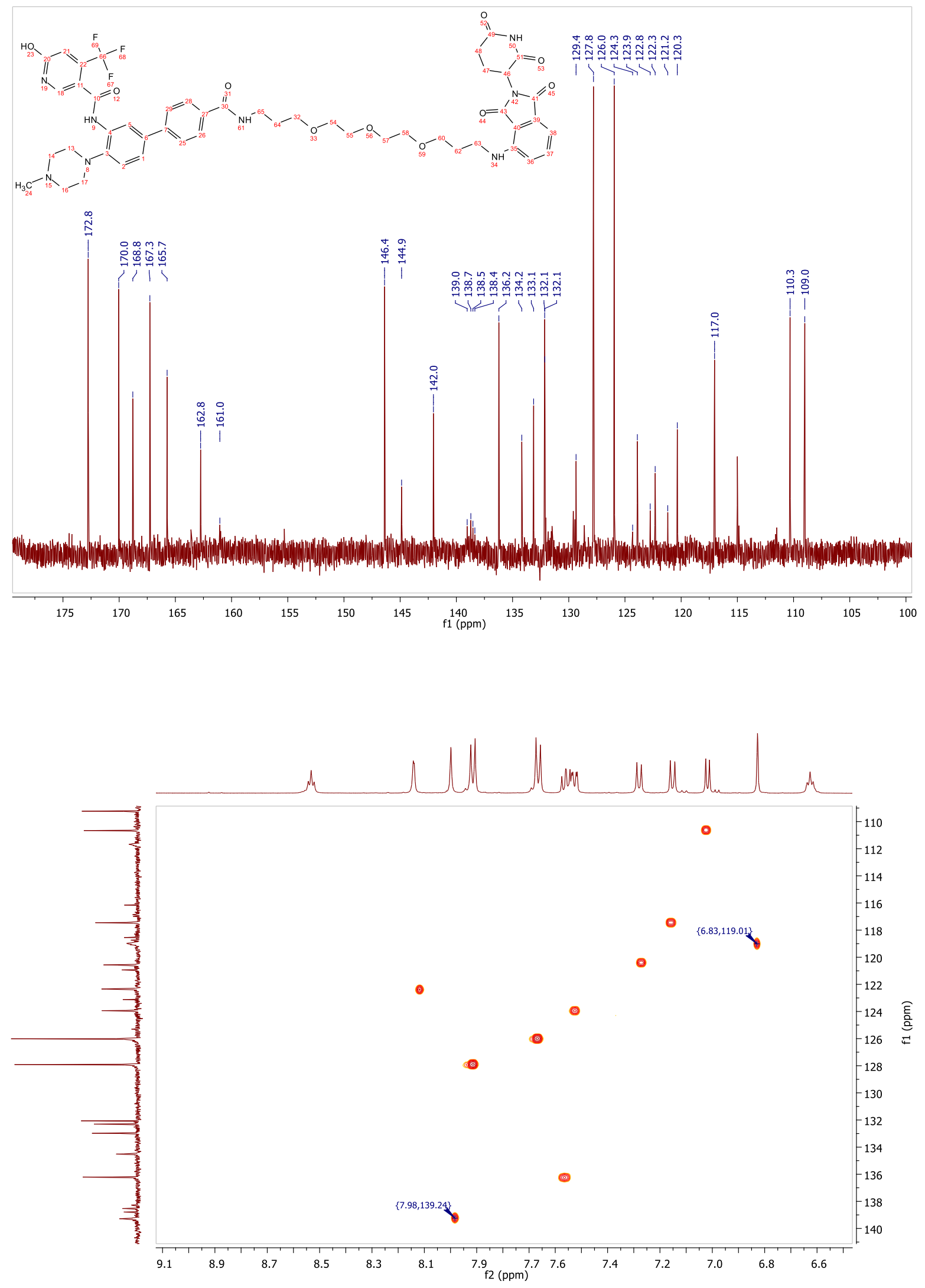


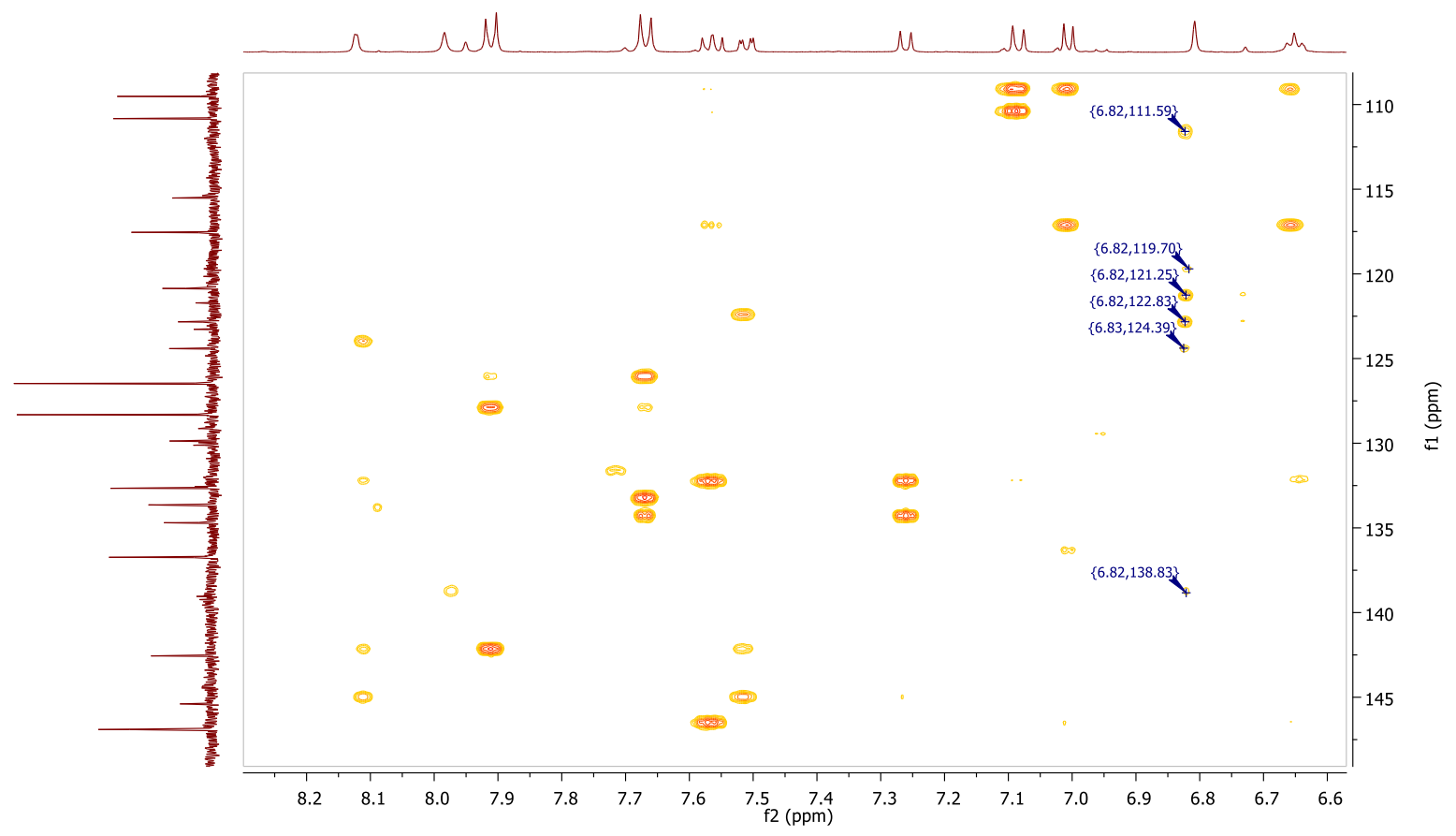


MALDI, HRMS, HPLC, ${ }^{1} \mathrm{H}-\mathrm{NMR},{ }^{13} \mathrm{C}-\mathrm{NMR},{ }^{1} \mathrm{H}-{ }^{13} \mathrm{C}-\mathrm{HSQC},{ }^{1} \mathrm{H}-{ }^{13} \mathrm{C}-\mathrm{HMBC}$ of $\mathrm{N}-(4 \mathrm{-}-((17-)((2-)(2,6-$ dioxopiperidin-3-yl)-1,3-dioxoisoindolin-4-yl)amino)-3,6,9,12,15-pentaoxaheptadecyl)carbamoyl)4-(4-methylpiperazin-1-yl)-[1,1'-biphenyl]-3-yl)-6-hydroxy-4-(trifluoromethyl)nicotinamide (7c)

Voyager Spec \#1 [BP = 635.4, 5083]

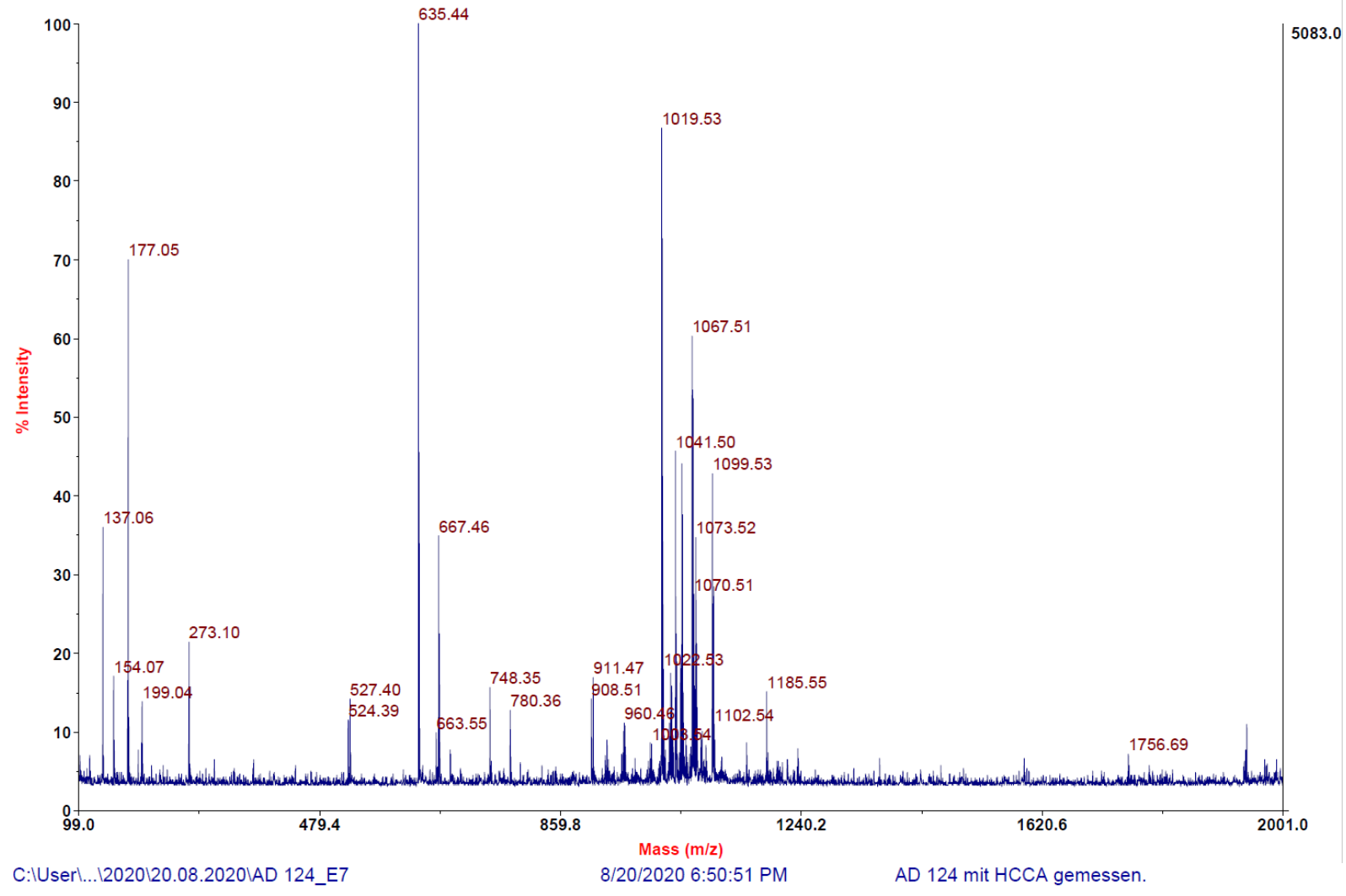

AD 124_E7 \#1-7 RT: 0.00-0.74 AV: 7 NL: 4.05E5

T: FTMS + p MALDI Full ms [700.00-1700.00]

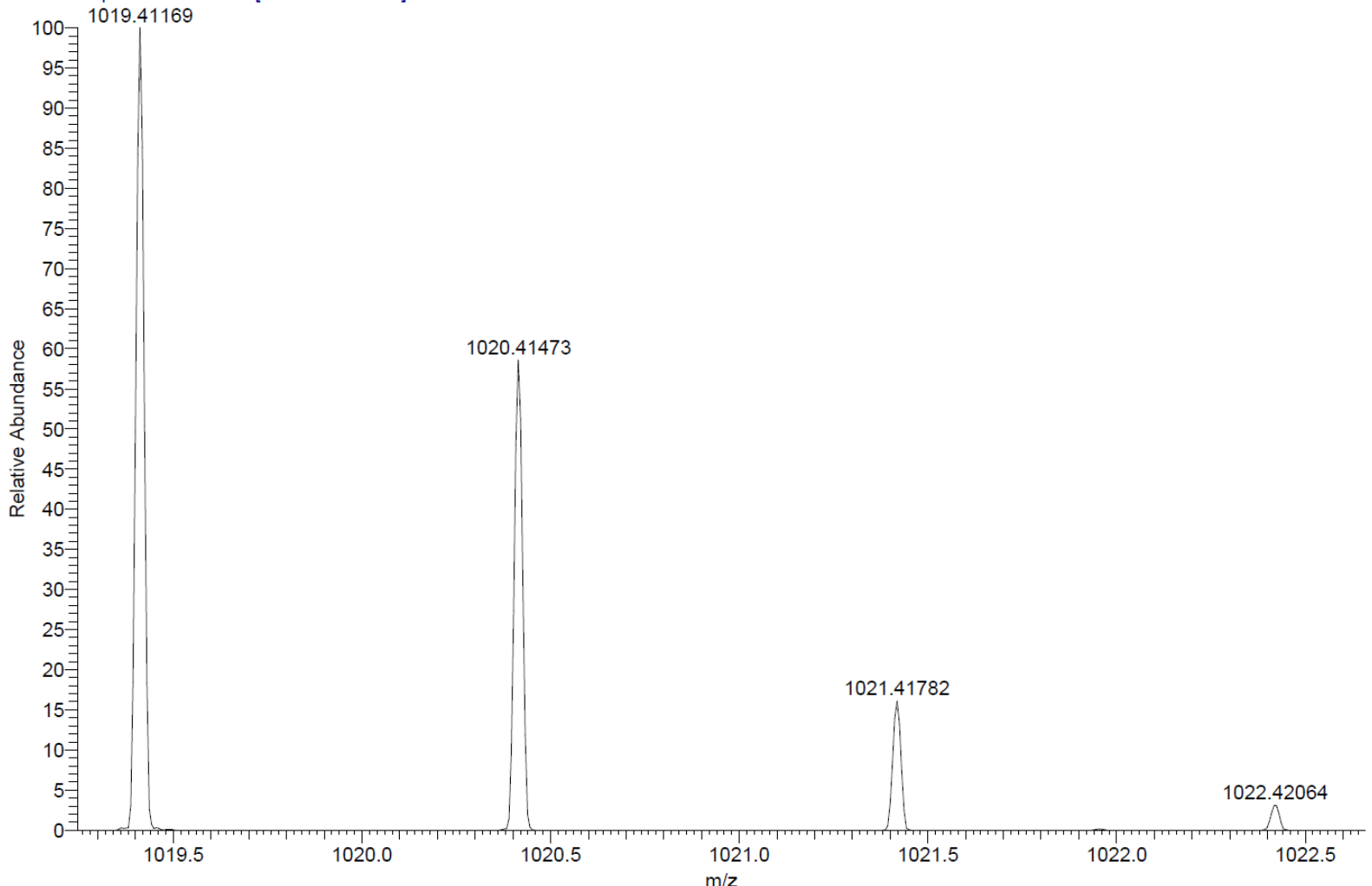

Signal: $\quad$ MWD1 A, Sig=254,4 Ref=off 


$\begin{array}{crrrr}\text { RT [min] Type } & \text { Width [min] } & \text { Area } & \text { Height } & \text { Area\% } \\ 11.593 \text { VV } & 0.2390 & 18262.3613 & 1133.0564 & 100.0000 \\ & \text { Sum } & 18262.3613 & & \end{array}$

Signal: $\quad$ MWD1 B, Sig=280,4 Ref=off

$\begin{array}{crrrr}\text { RT [min] Type } & \text { Width [min] } & \text { Area } & \text { Height } & \text { Area\% } \\ 11.593 \text { VV } & 0.2389 & 22847.2031 & 1418.2609 & 100.0000 \\ & \text { Sum } & 22847.2031 & & \end{array}$

Signal: $\quad$ MWD1 F, Sig=260,4 Ref=off

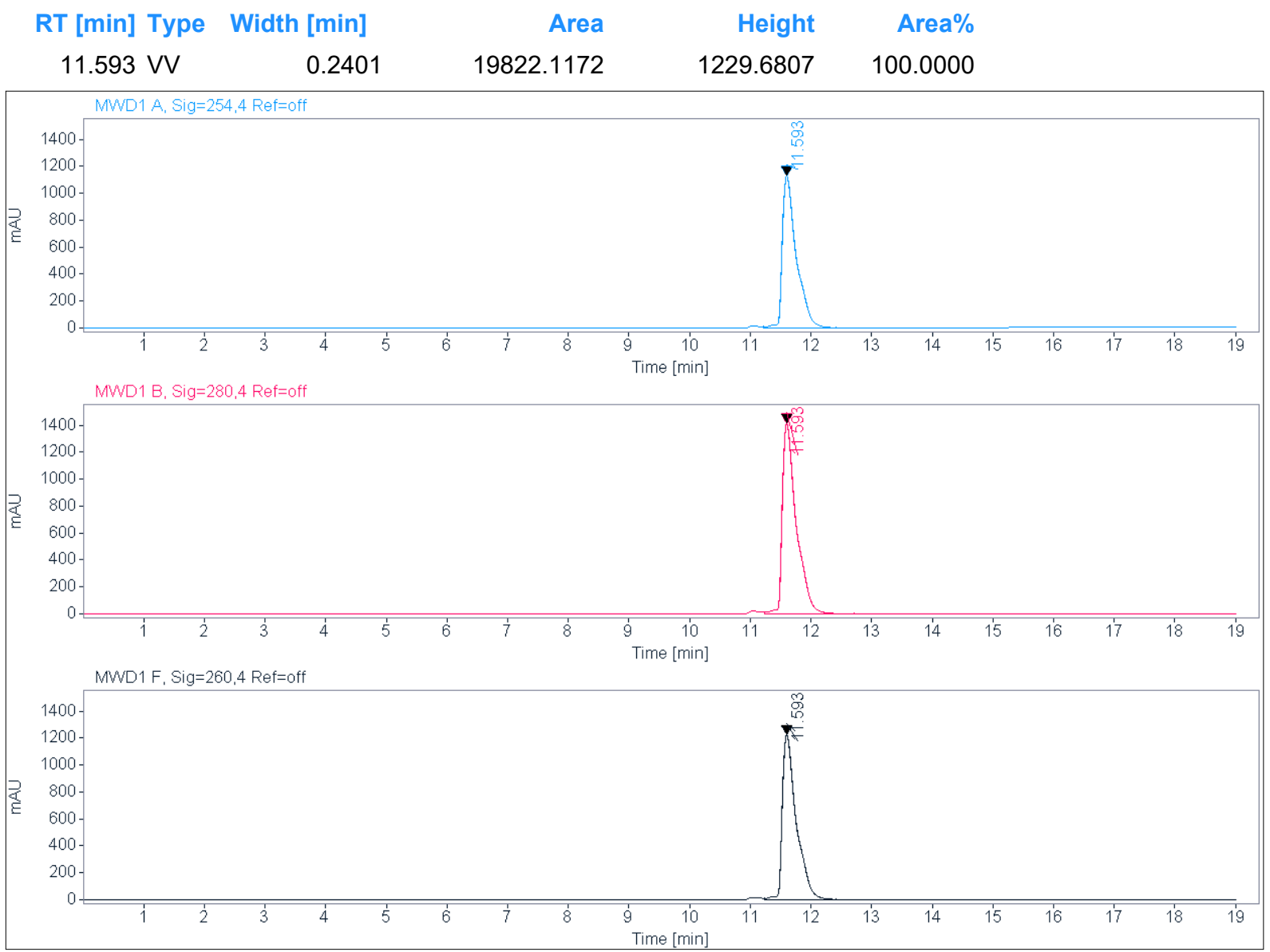



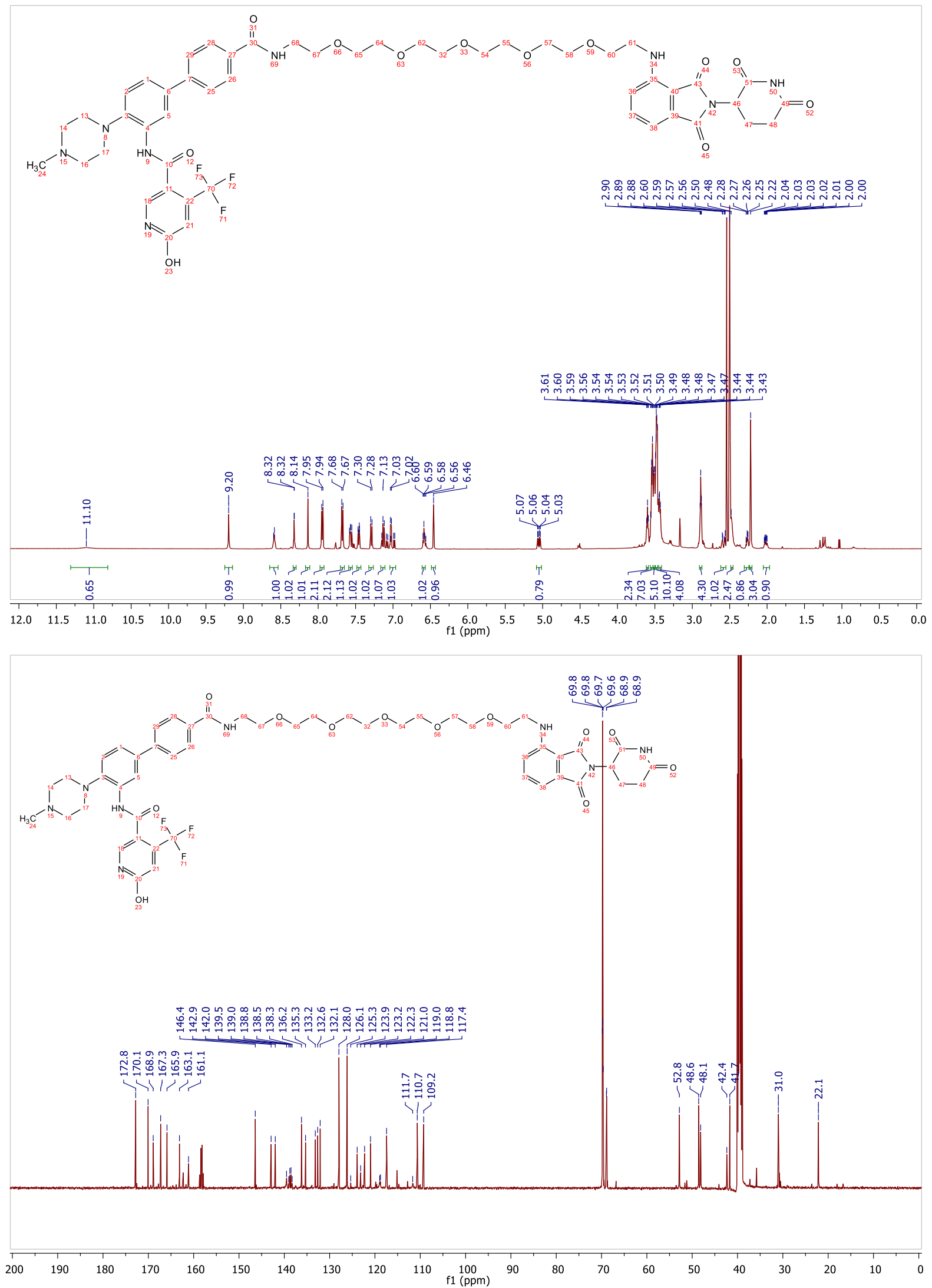

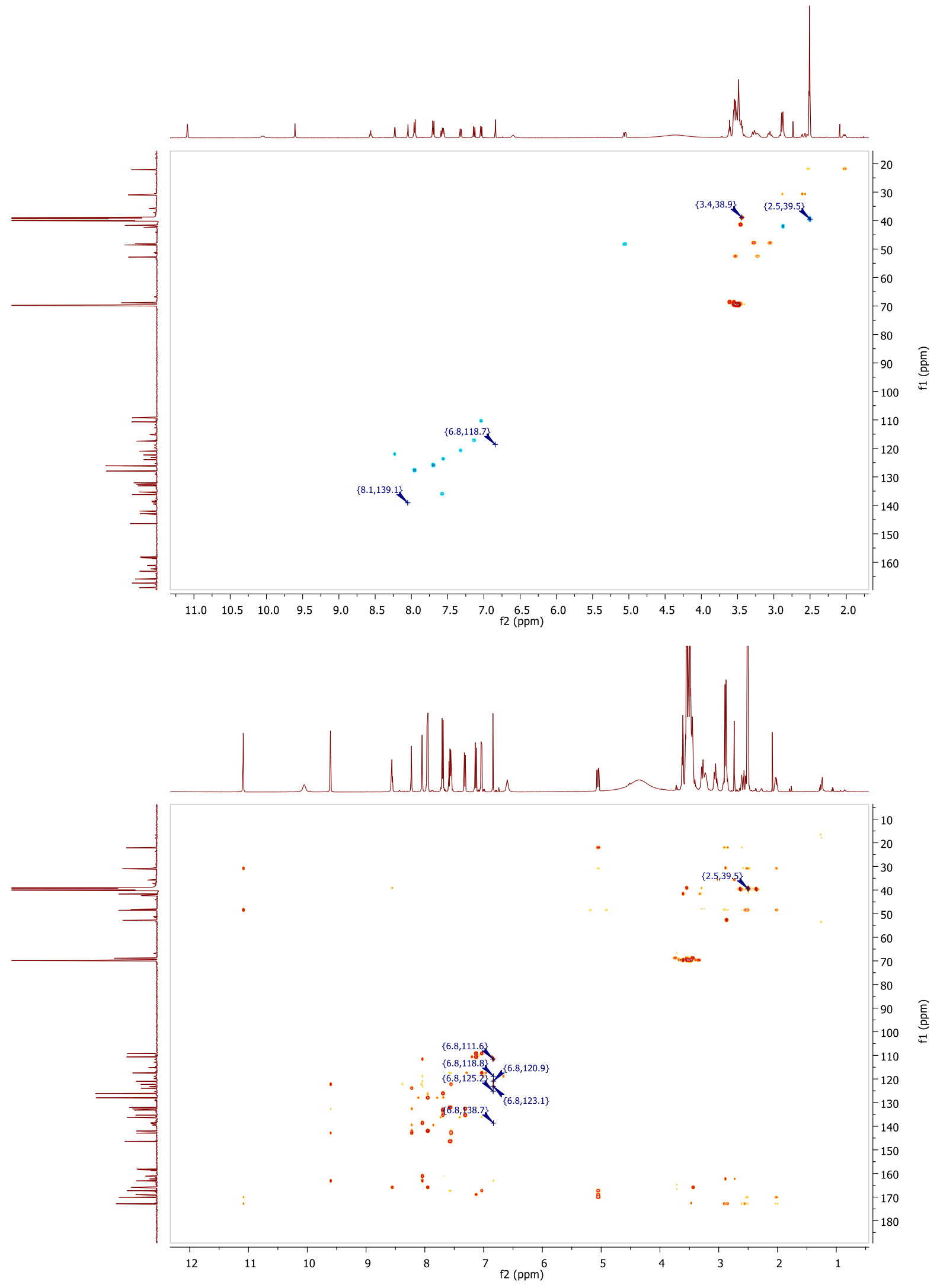
HRMS, MALDI, HPLC, ${ }^{1} \mathrm{H}-\mathrm{NMR},{ }^{13} \mathrm{C}-\mathrm{NMR},{ }^{1} \mathrm{H}-{ }^{13} \mathrm{C}-\mathrm{HSQC},{ }^{1} \mathrm{H}-{ }^{13} \mathrm{C}-\mathrm{HMBC}$ of $\mathrm{N}-\left(4{ }^{1}-((23-)((2-)(2,6-\right.$ dioxopiperidin-3-yl)-1,3-dioxoisoindolin-4-yl)amino)-3,6,9,12,15,18,21-

heptaoxatricosyl)carbamoyl)-4-(4-methylpiperazin-1-yl)-[1,1'-biphenyl]-3-yl)-6-hydroxy-4-

(trifluoromethyl)nicotinamide (7d)

C:IUserl...12020120.08.2020\AD 125_E8

AD 125_E8 \#1-6 RT: 0.01-0.47 AV: 6 NL: 4.52E6

T: FTMS + p MALDI Full ms [700.00-1700.00]
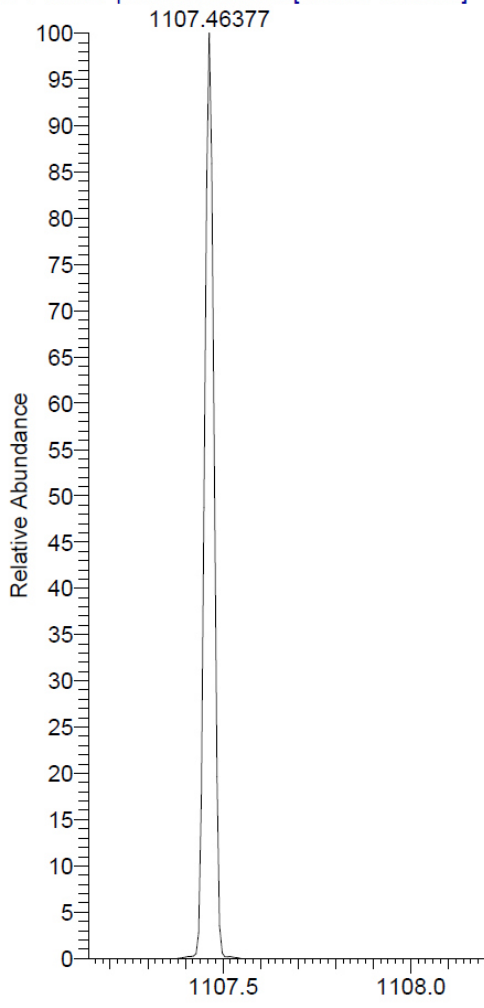

$$
1108.46703
$$
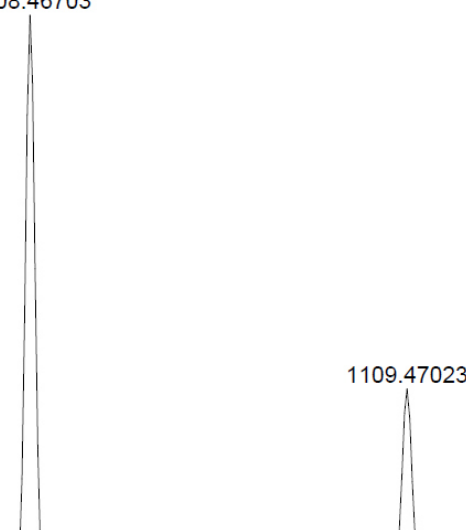

(1)
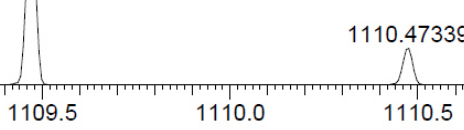

1110.0

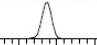

1111.0

Voyager Spec \#1[BP = 1107.5, 56192]

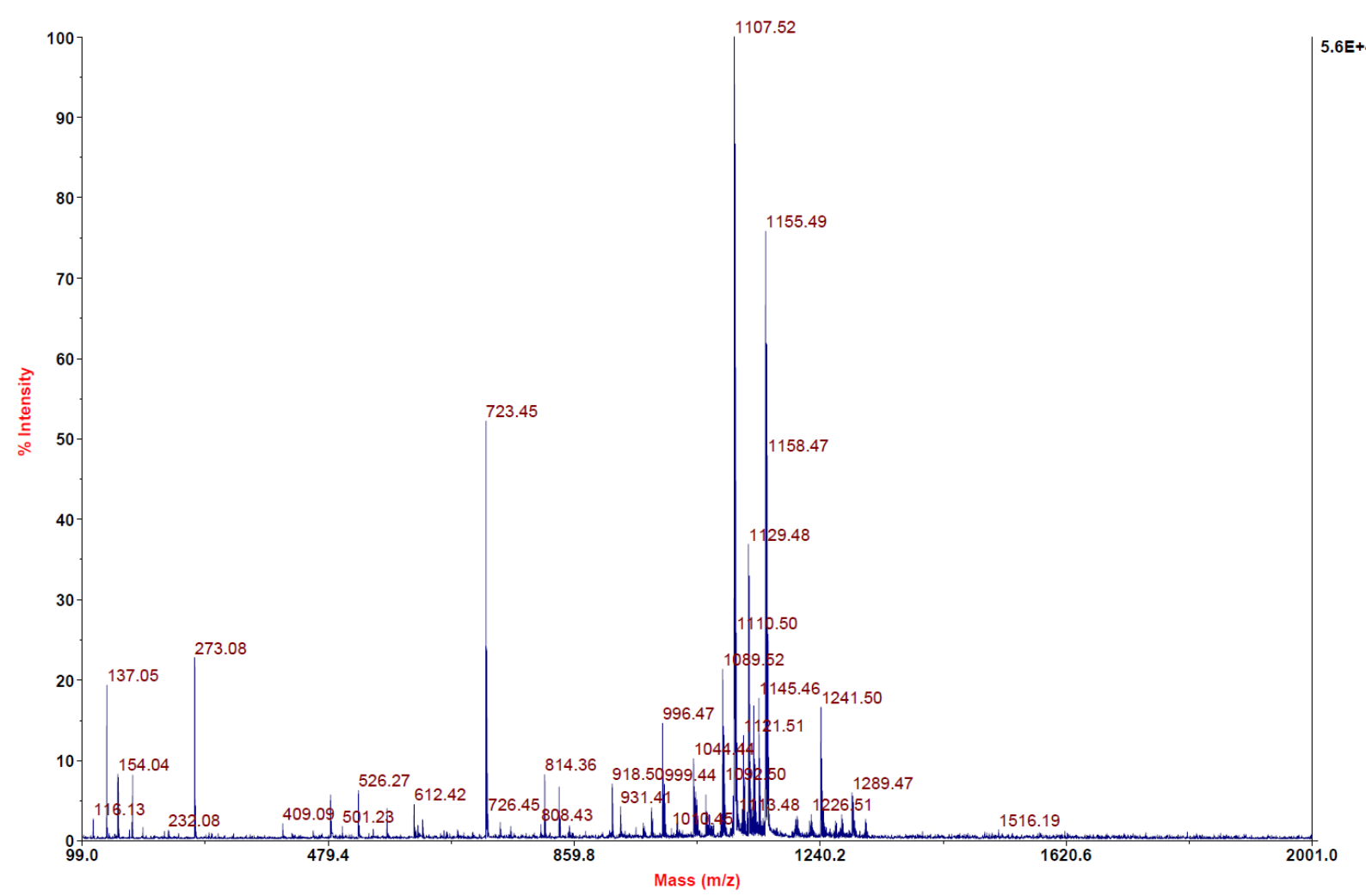



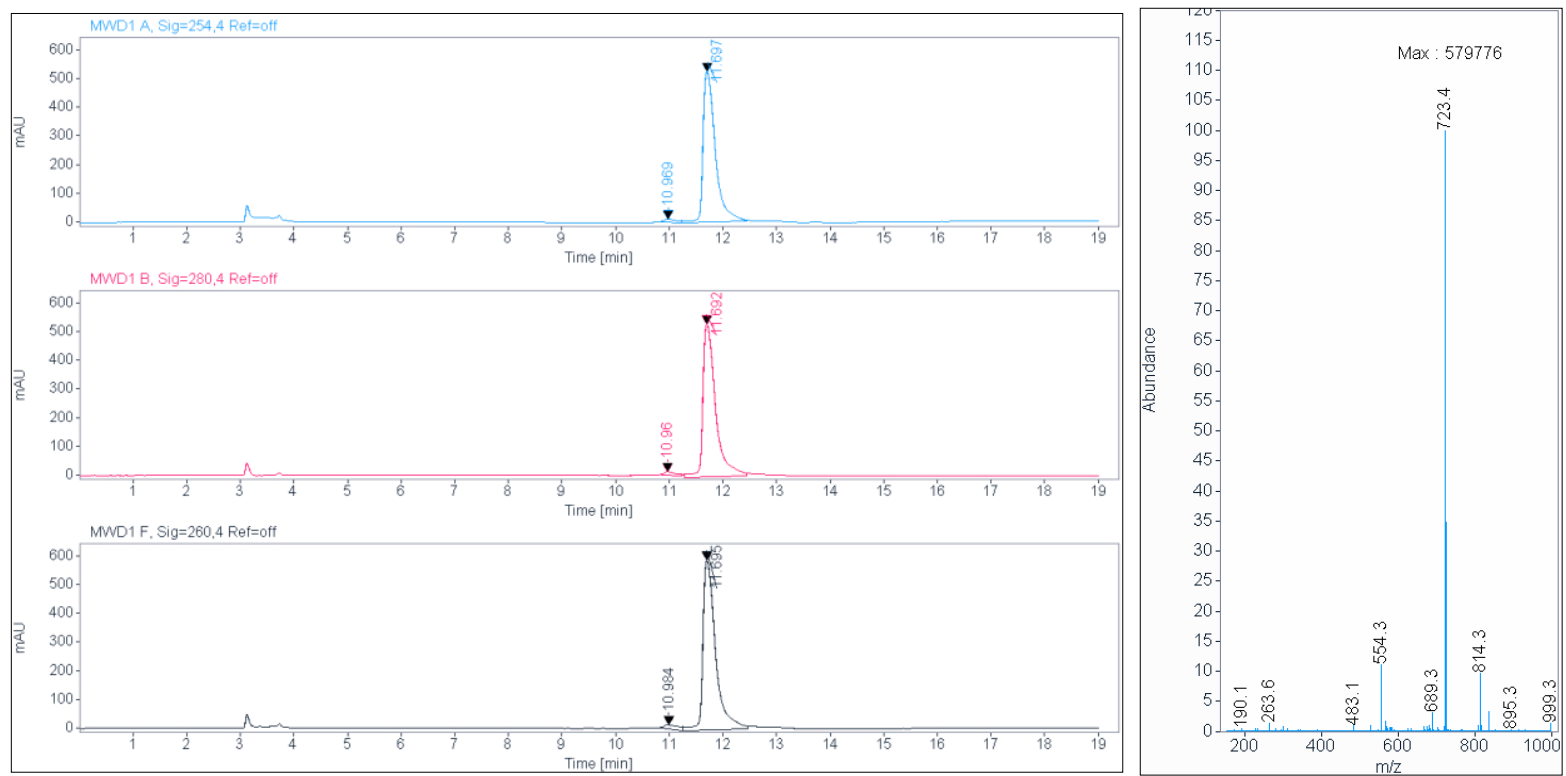

Signal: $\quad$ MWD1 A, Sig=254,4 Ref=off

$\begin{array}{crrr}\text { RT [min] Type } & \text { Width [min] } & \text { Area } & \text { Height Area\% } \\ 10.969 \mathrm{MM} & 0.3015 & 220.6292 & 12.19712 .5413 \\ 11.697 \mathrm{MM} & 0.2683 & 8461.0498 & 525.601697 .4587 \\ & \text { Sum } & 8681.6790 & \end{array}$

Signal: $\quad$ MWD1 B, Sig $=280,4$ Ref $=$ off

$\begin{array}{crrr}\text { RT [min] Type } & \text { Width [min] } & \text { Area } & \text { Height Area\% } \\ 10.960 \mathrm{MM} & 0.2779 & 234.3564 & 14.05302 .5827 \\ 11.692 \mathrm{MM} & 0.2764 & 8839.7959 & 533.001897 .4173 \\ & \text { Sum } & 9074.1523 & \end{array}$

Signal: $\quad$ MWD1 F, Sig $=260,4$ Ref $=$ off

$\begin{array}{rrrr}\text { RT [min] Type } & \text { Width [min] } & \text { Area } & \text { Height Area\% } \\ 10.984 \mathrm{MM} & 0.3388 & 313.1158 & 15.40243 .0819 \\ 11.695 \mathrm{MM} & 0.2776 & 9846.8340 & 591.277296 .9181\end{array}$


Appendix

297
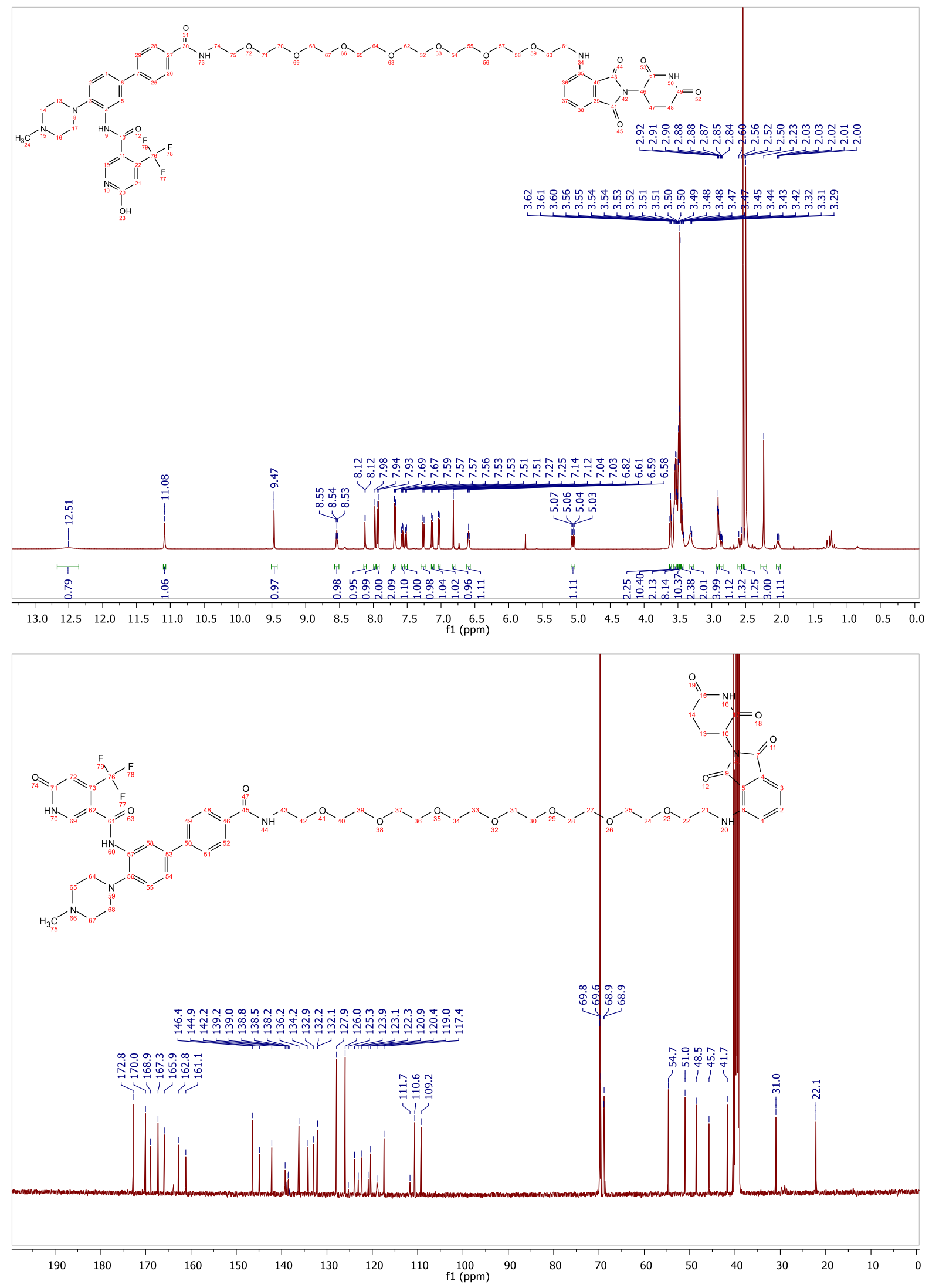

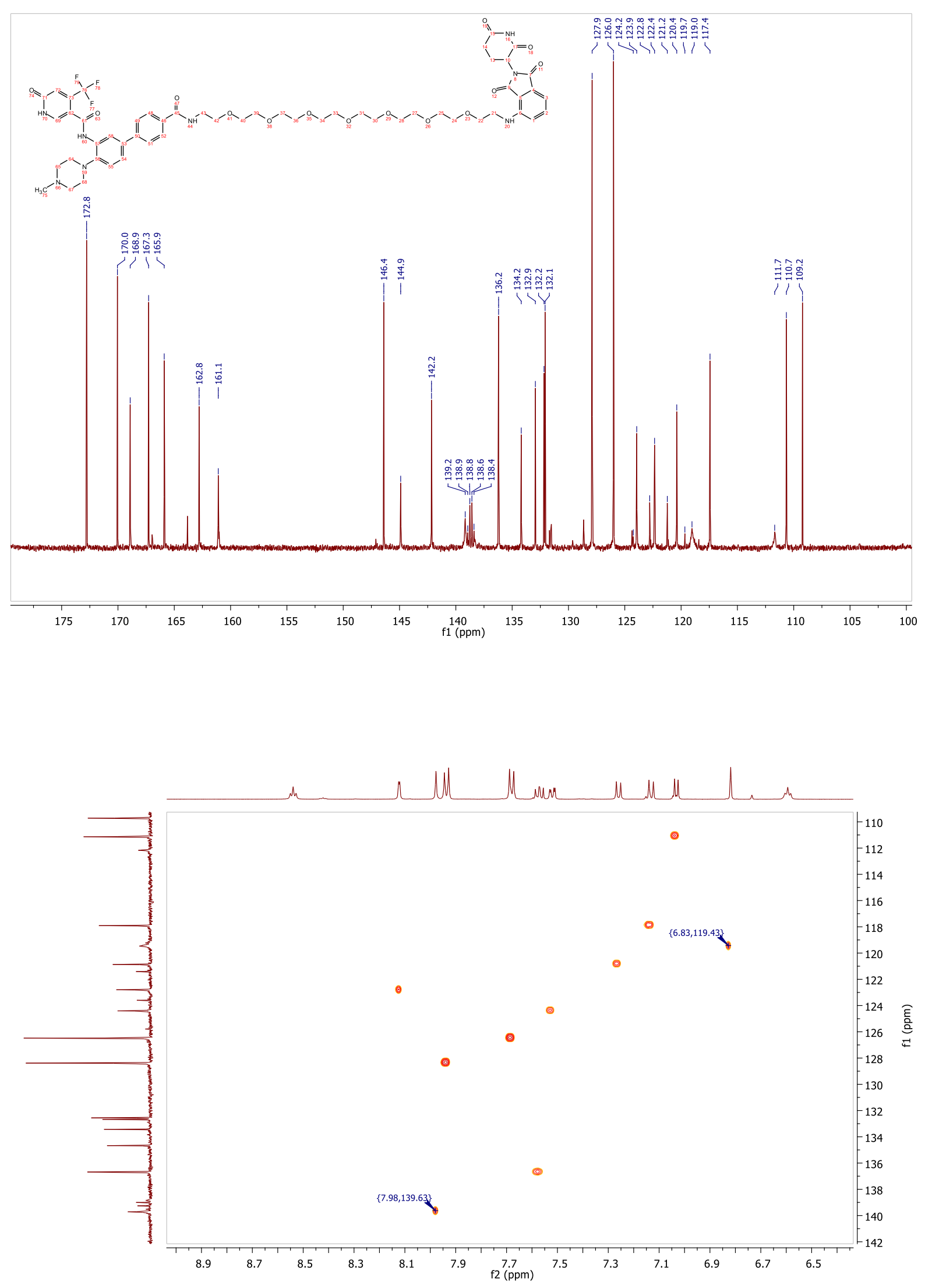


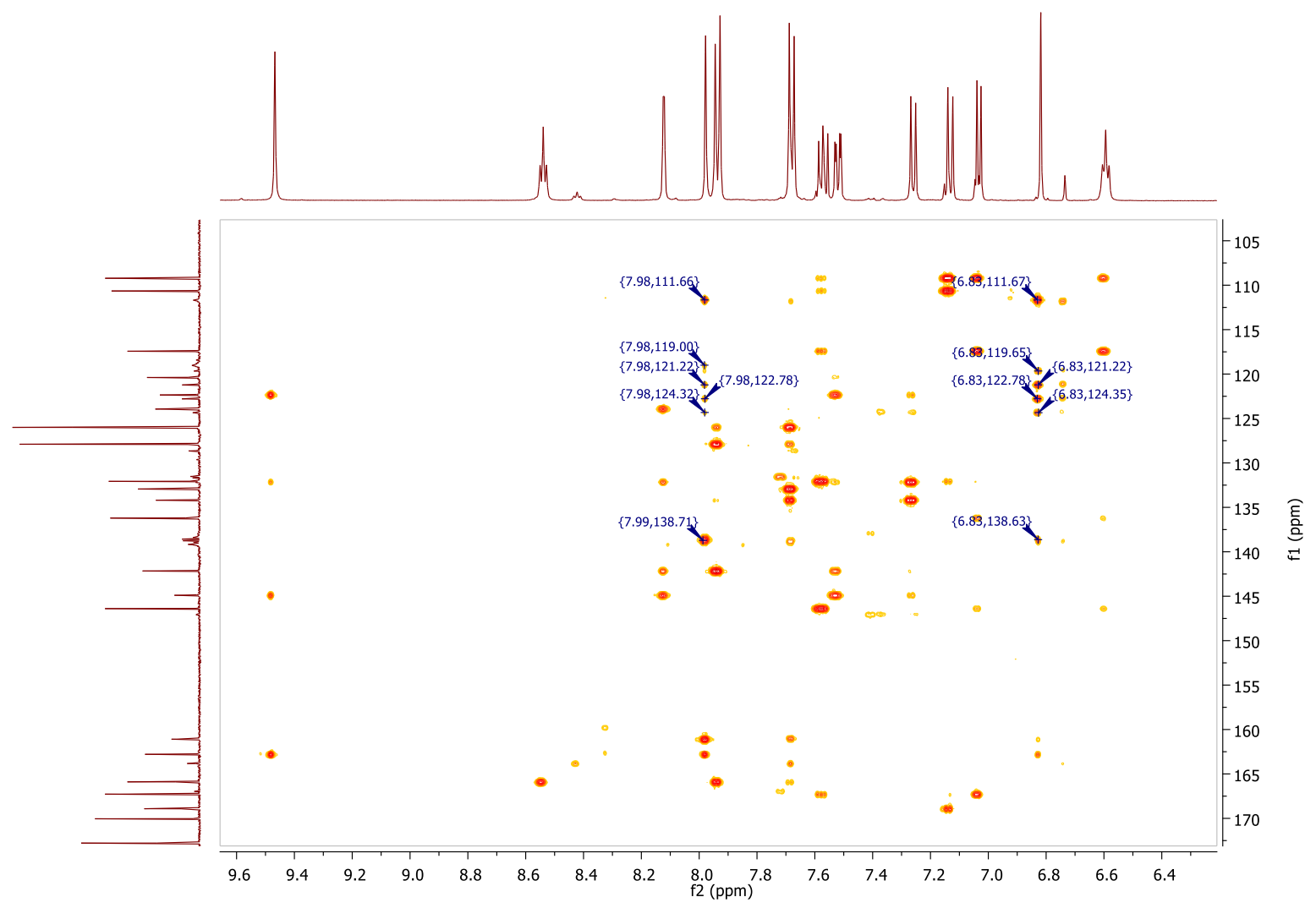


MALDI, HRMS, ${ }^{1} \mathrm{H}-\mathrm{NMR},{ }^{13} \mathrm{C}-\mathrm{NMR},{ }^{1} \mathrm{H}-{ }^{13} \mathrm{C}-\mathrm{HSQC},{ }^{1} \mathrm{H}-{ }^{13} \mathrm{C}-\mathrm{HMBC}$ and HPLC of $\mathrm{N}-(4 \mathrm{-}-((4-)((2-(2,6-$ dioxopiperidin-3-yl)-1,3-dioxoisoindolin-4-yl)amino)methyl)benzyl)carbamoyl)-4-(4methylpiperazin-1-yl)-[1,1'-biphenyl]-3-yl)-6-hydroxy-4-(trifluoromethyl)nicotinamide (7e)

Voyager Spec \#1[BP = 897.2, 20813]

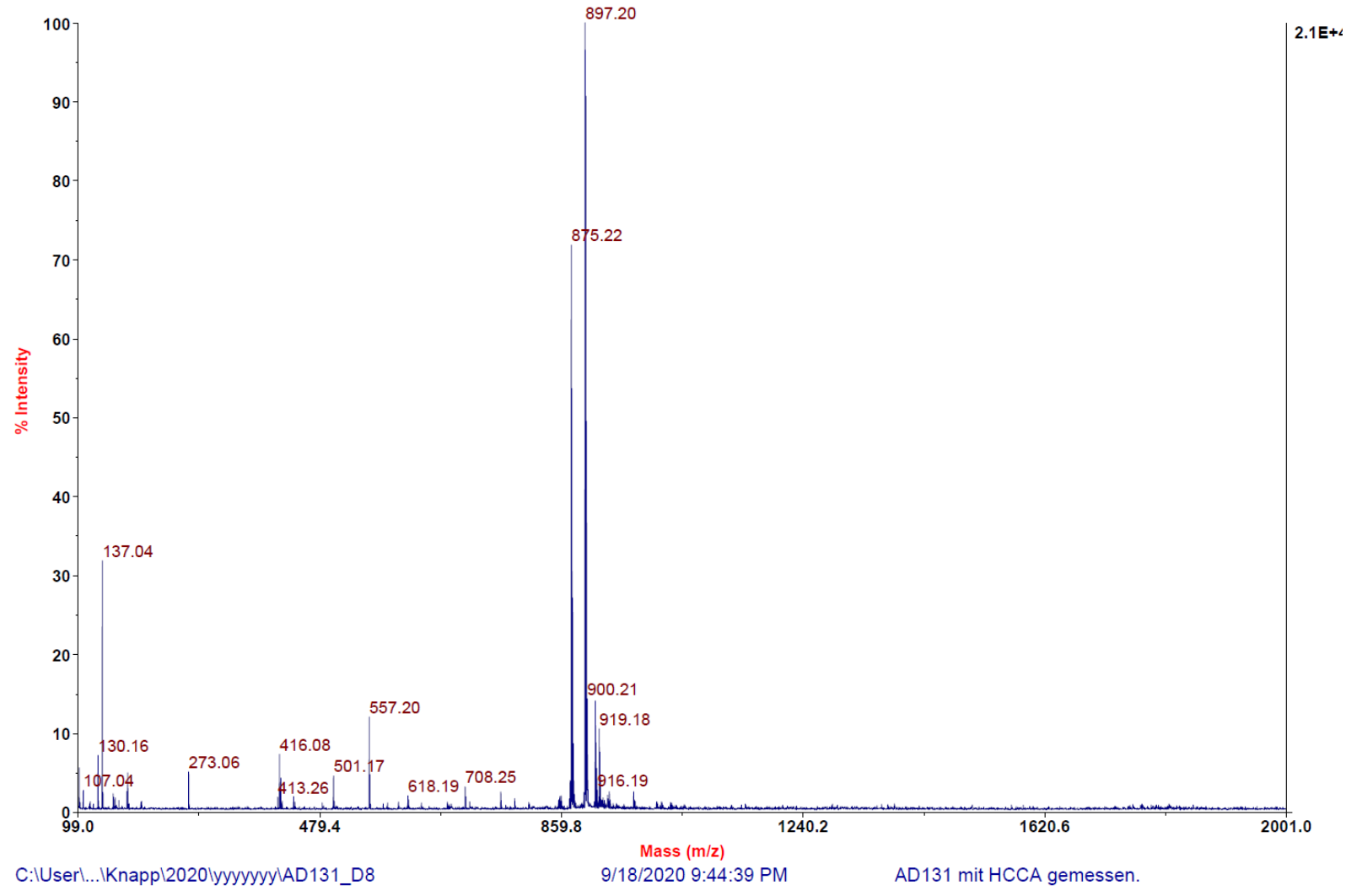

AD131_D8 \#1-7 RT: 0.01-0.27 AV: 7 NL: 3.49E6

T: FTMS + p MALDI Full ms [300.00-950.00]

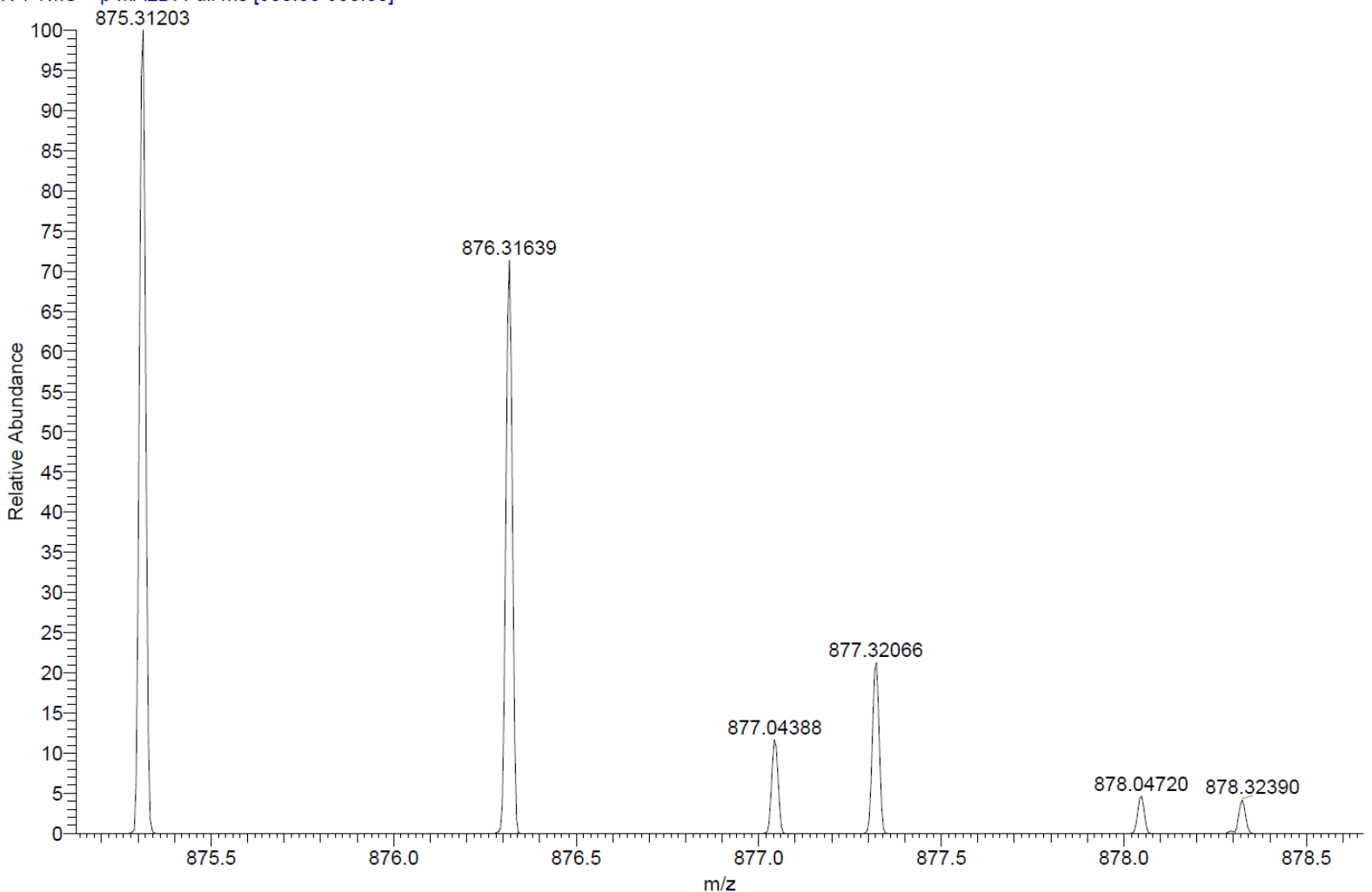



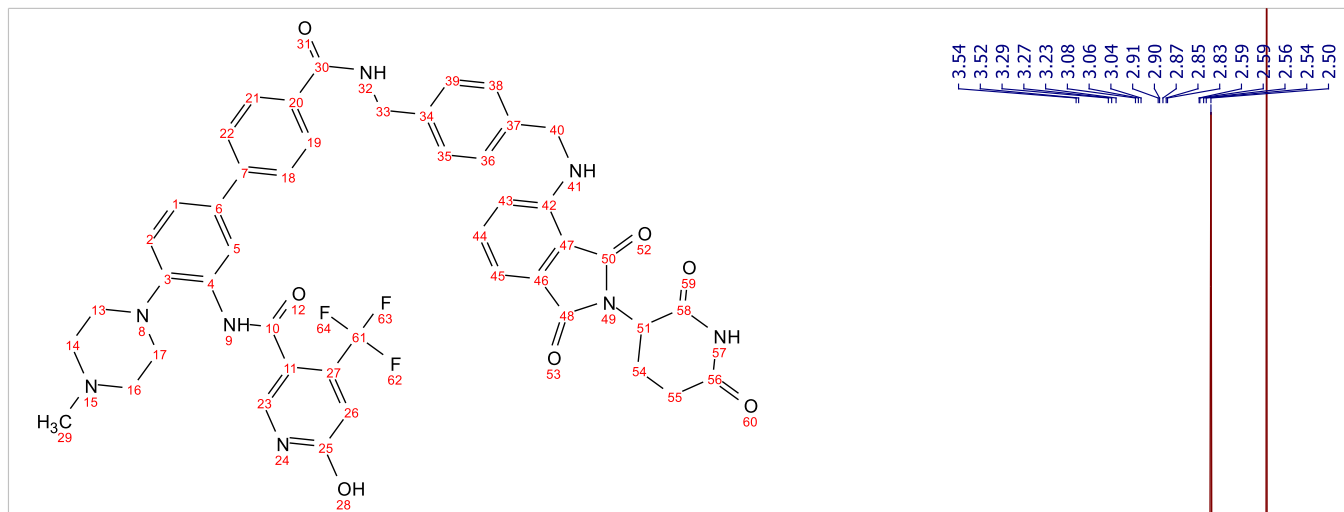

mimmmmm N
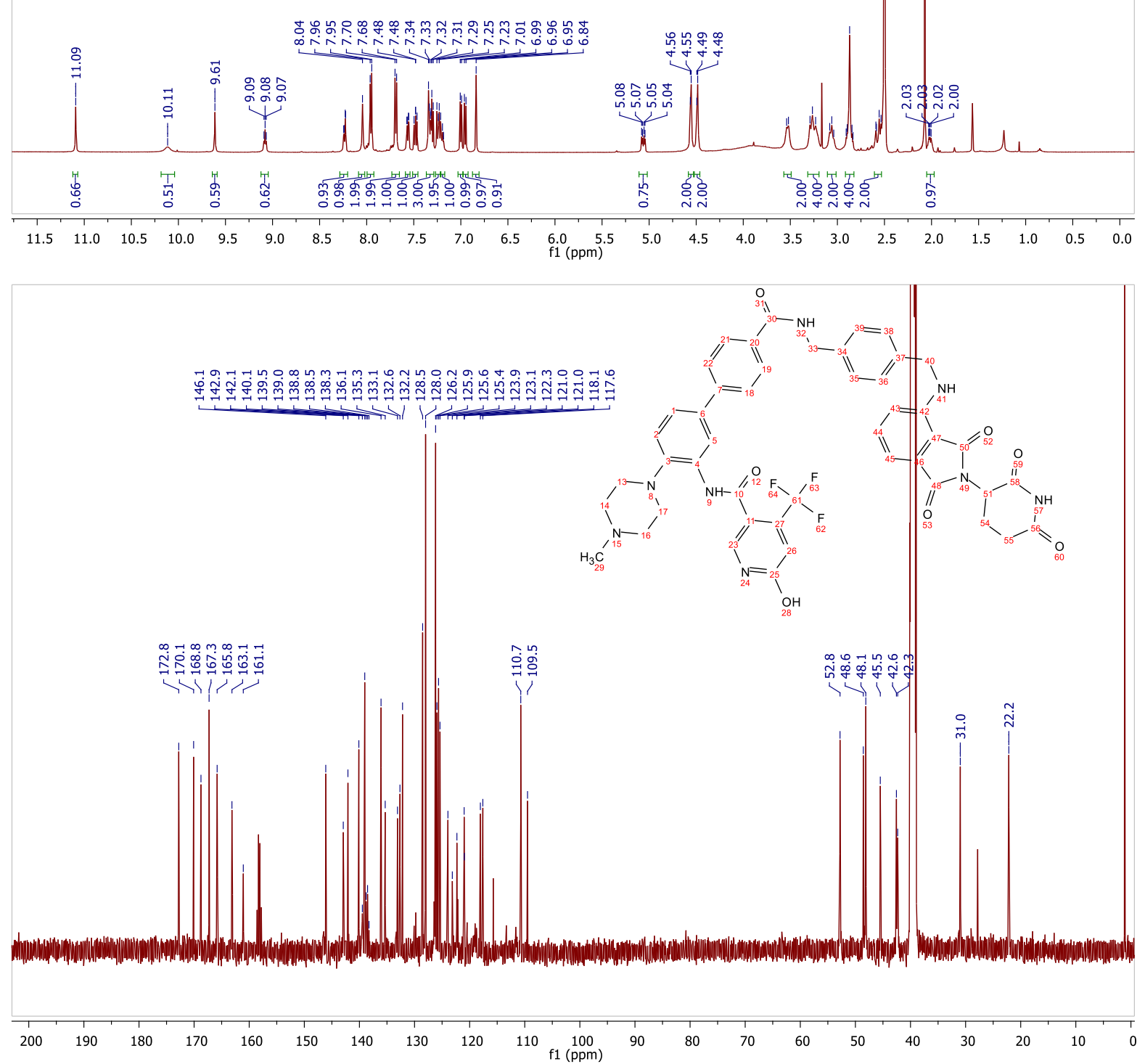


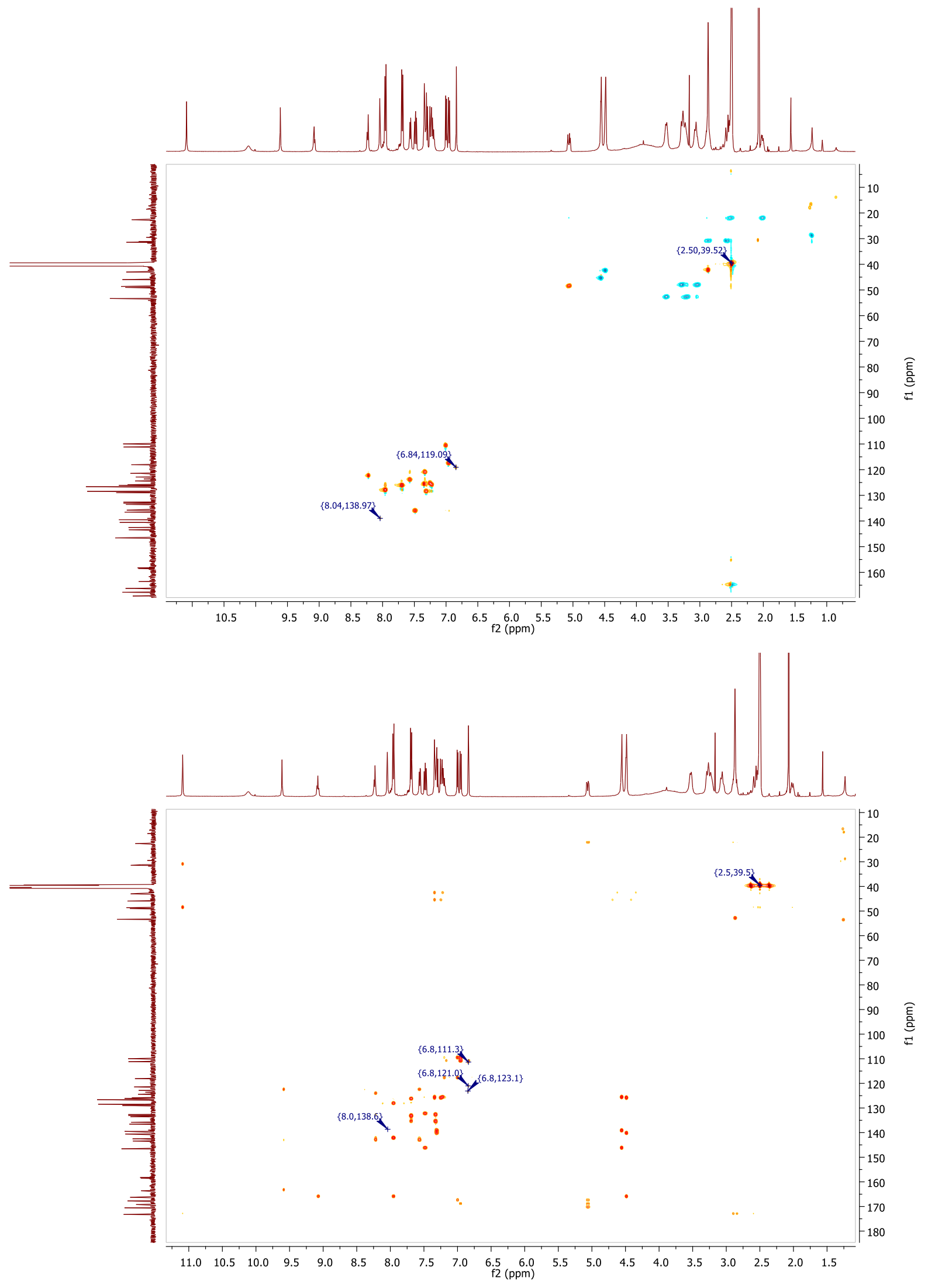

Signal: $\quad$ MWD1 A, Sig $=254,4$ Ref $=$ off

$\begin{array}{ccrr}\text { RT [min] Type } & \text { Width [min] } & \text { Area } & \text { Height Area\% } \\ 11.654 \mathrm{MM} & 0.1599 & 142.2890 & 14.83301 .5723\end{array}$




$\begin{array}{rrrr}12.065 \mathrm{MM} & 0.2775 & 8600.4414 & 516.458695 .0367 \\ 12.892 \mathrm{MM} & 0.2353 & 306.8708 & 21.73803 .3910 \\ & \text { Sum } & 9049.6012 & \end{array}$

Signal: $\quad$ MWD1 B, Sig=280,4 Ref=off

$\begin{array}{crrr}\text { RT [min] Type } & \text { Width [min] } & \text { Area } & \text { Height Area\% } \\ 11.659 \text { MM } & 0.1161 & 118.6666 & 12.66001 .3609 \\ 12.065 \mathrm{MM} & 0.2644 & 8392.1240 & 529.016796 .2442 \\ 12.895 \mathrm{MM} & 0.1790 & 208.8267 & 19.44802 .3949 \\ & \text { Sum } & 8719.6173 & \end{array}$

Signal: $\quad$ MWD1 F, Sig=260,4 Ref=off

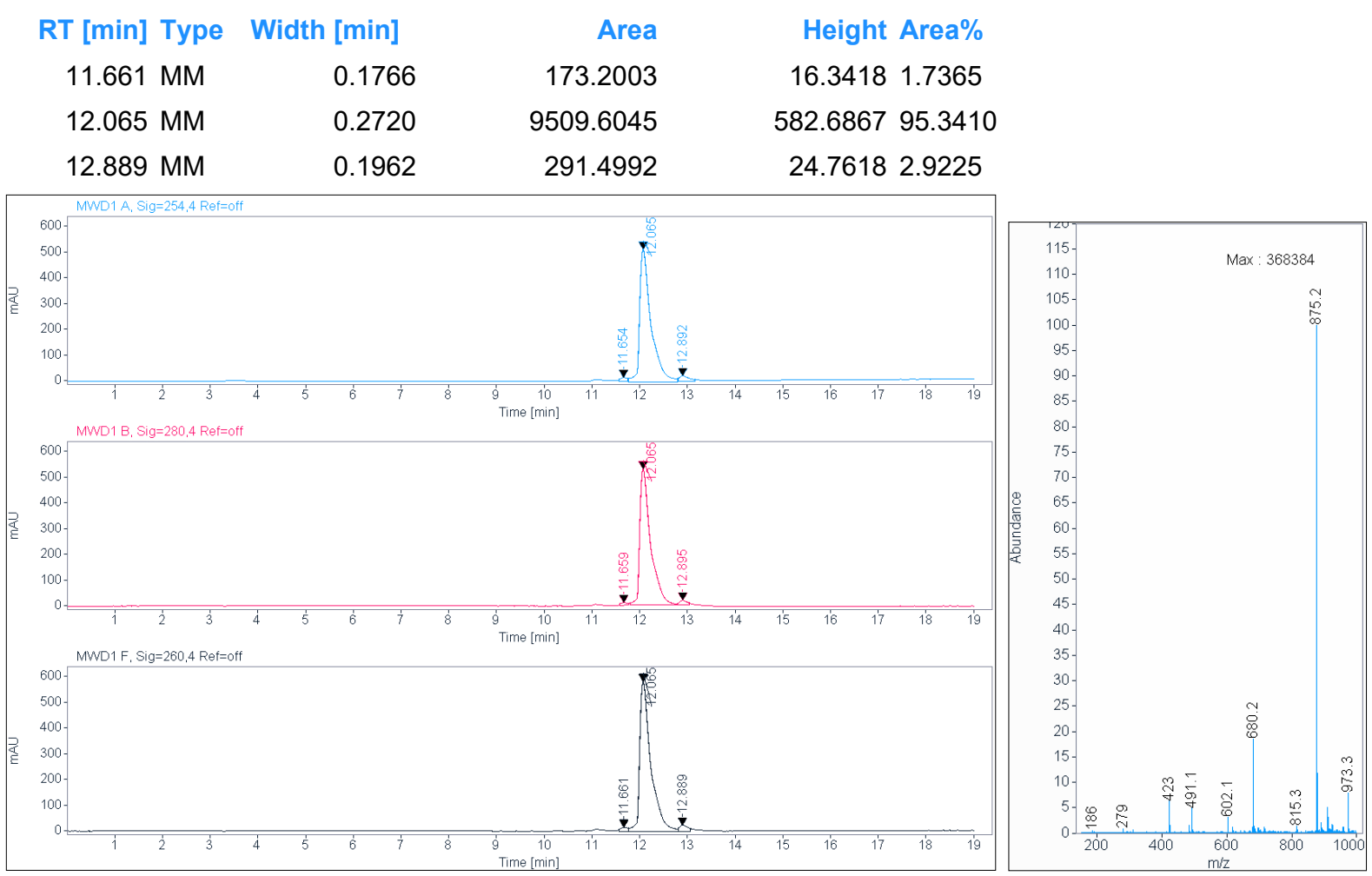


MALDI, HRMS, HPLC, ${ }^{1} \mathrm{H}-\mathrm{NMR},{ }^{13} \mathrm{C}-\mathrm{NMR},{ }^{1} \mathrm{H}-{ }^{13} \mathrm{C}-\mathrm{HSQC}$ and ${ }^{1} \mathrm{H}-{ }^{13} \mathrm{C}-\mathrm{HMBC}$ of 6 -hydroxy-N-(4'-((2-(3(((S)-1-((2S,4R)-4-hydroxy-2-((4-(4-methylthiazol-5-yl)benzyl)carbamoyl)pyrrolidin-1-yl)-3,3dimethyl-1-oxobutan-2-yl)amino)-3-oxopropoxy)ethyl)carbamoyl)-4-(4-methylpiperazin-1-yl)-[1,1'biphenyl]-3-yl)-4-(trifluoromethyl)nicotinamide (8a)

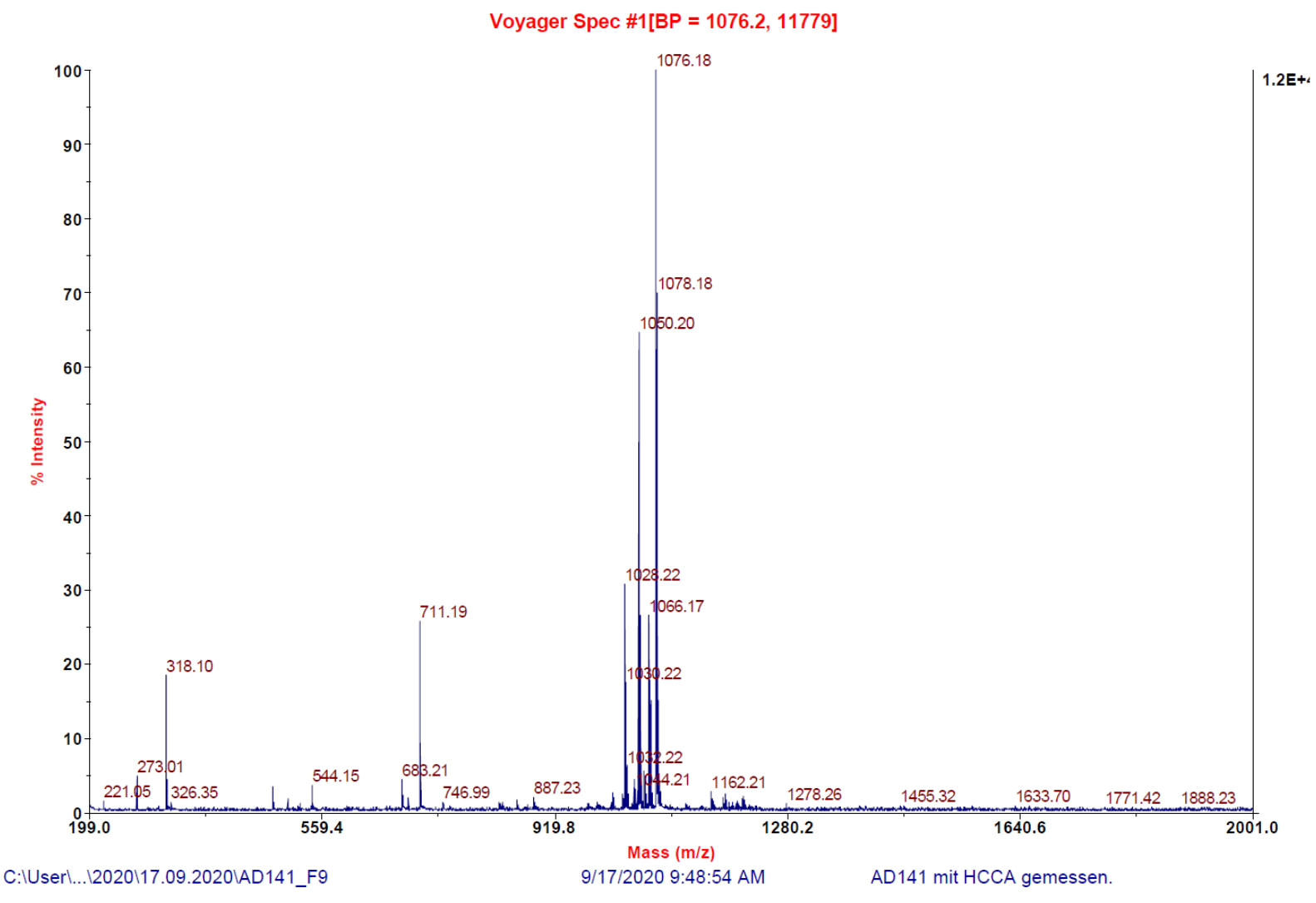

AD141_F9 \#1-20 RT: 0.01-0.87 AV: 20 NL: 5.57E6

T: FTMS + p MALDI Full ms [1000.00-1250.00]

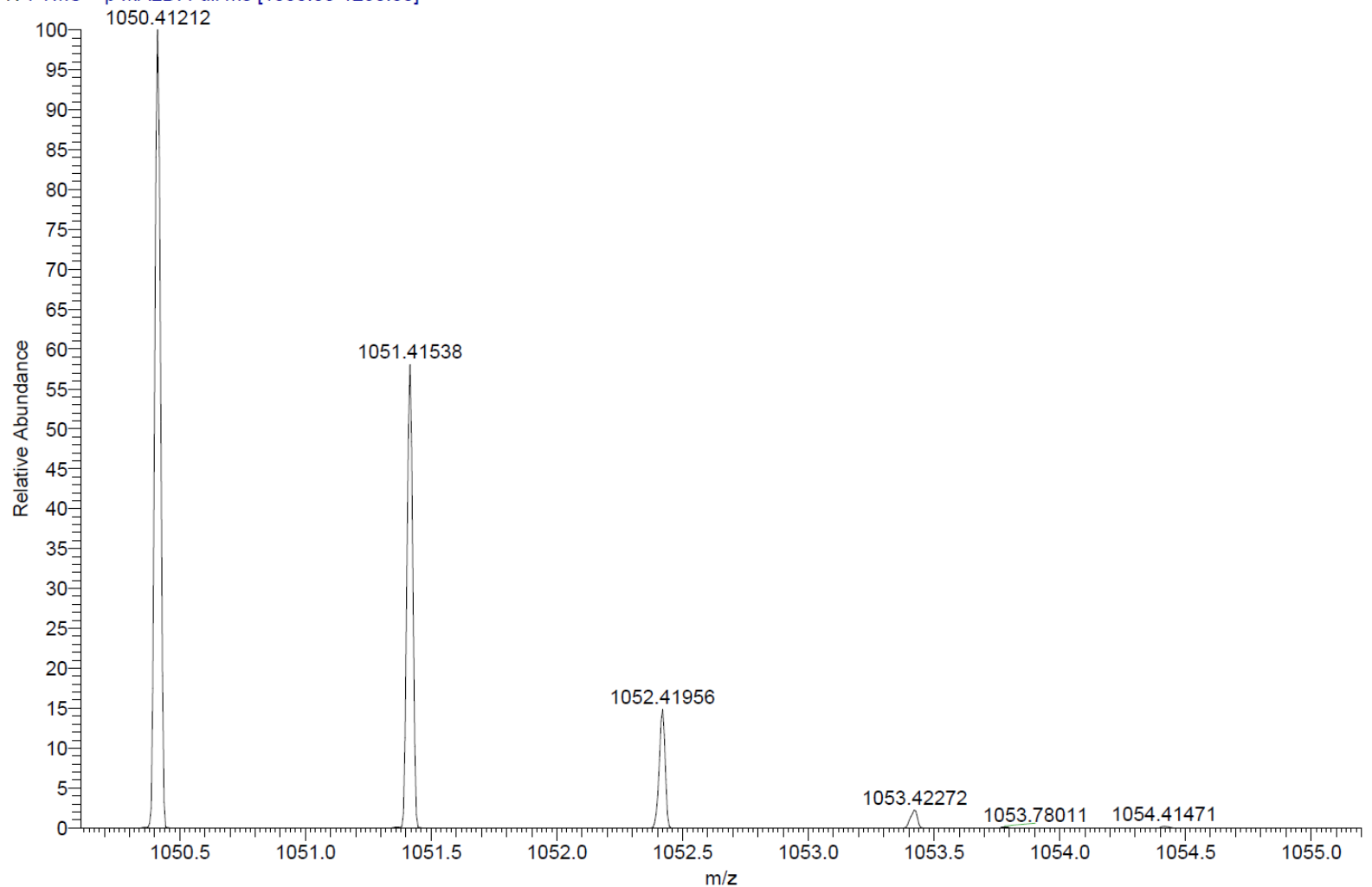

Signal: $\quad$ MWD1 A, Sig=254,4 Ref=off 


$\begin{array}{crrrr}\text { RT [min] Type } & \text { Width [min] } & \text { Area } & \text { Height } & \text { Area\% } \\ 11.278 \text { VV } & 0.2050 & 7216.8145 & 486.5691 & 97.2523 \\ 11.786 \text { MM } & 0.1465 & 203.8960 & 23.2009 & 2.7477 \\ & \text { Sum } & 7420.7104 & & \end{array}$

Signal: $\quad$ MWD1 B, Sig=280,4 Ref=off

$\begin{array}{crrrr}\text { RT [min] Type } & \text { Width [min] } & \text { Area } & \text { Height } & \text { Area\% } \\ 11.278 \text { VV } & 0.2150 & 9186.0752 & 582.5713 & 100.0000 \\ & \text { Sum } & 9186.0752 & & \end{array}$

Signal: $\quad$ MWD1 F, Sig=260,4 Ref=off

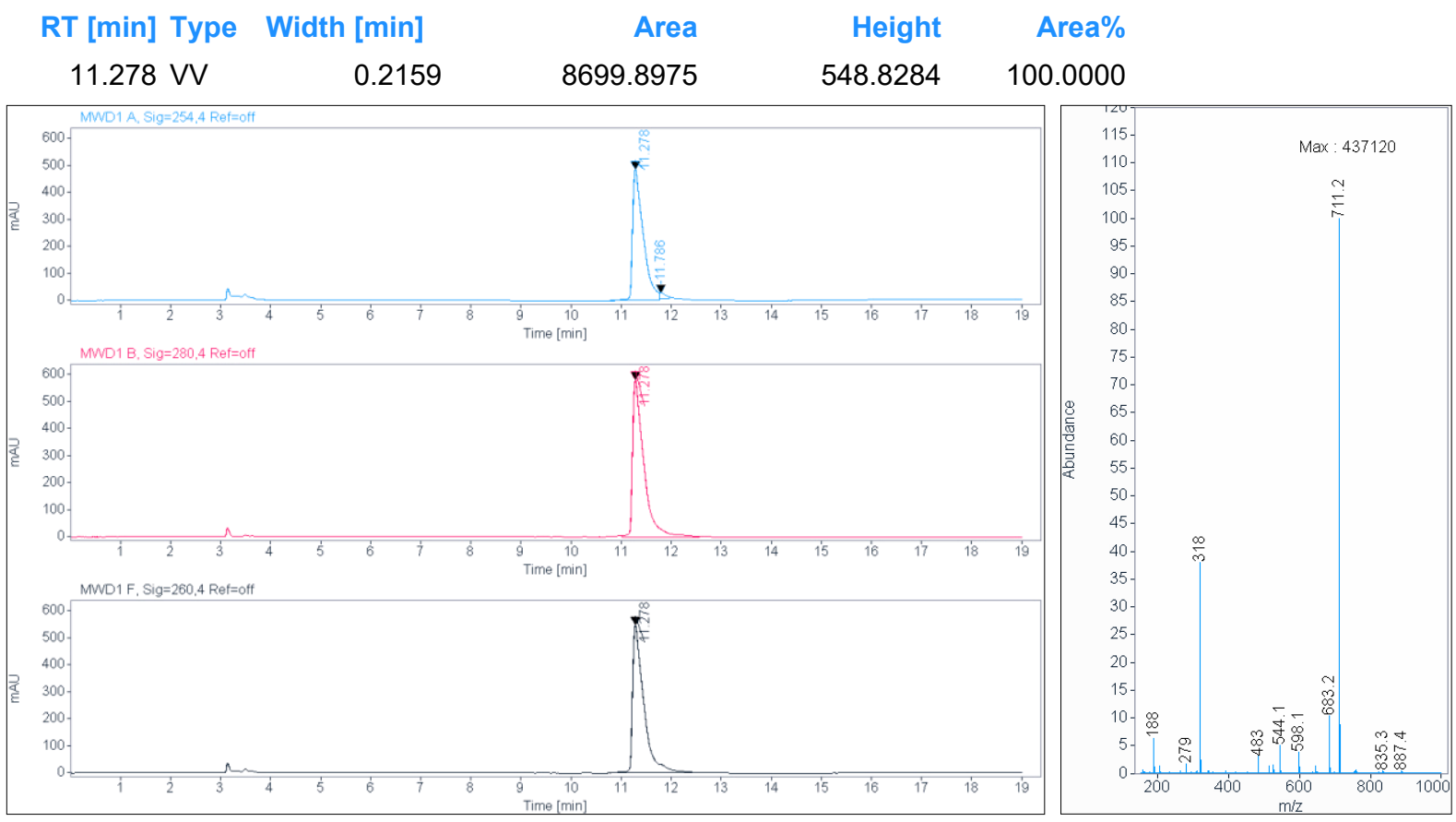



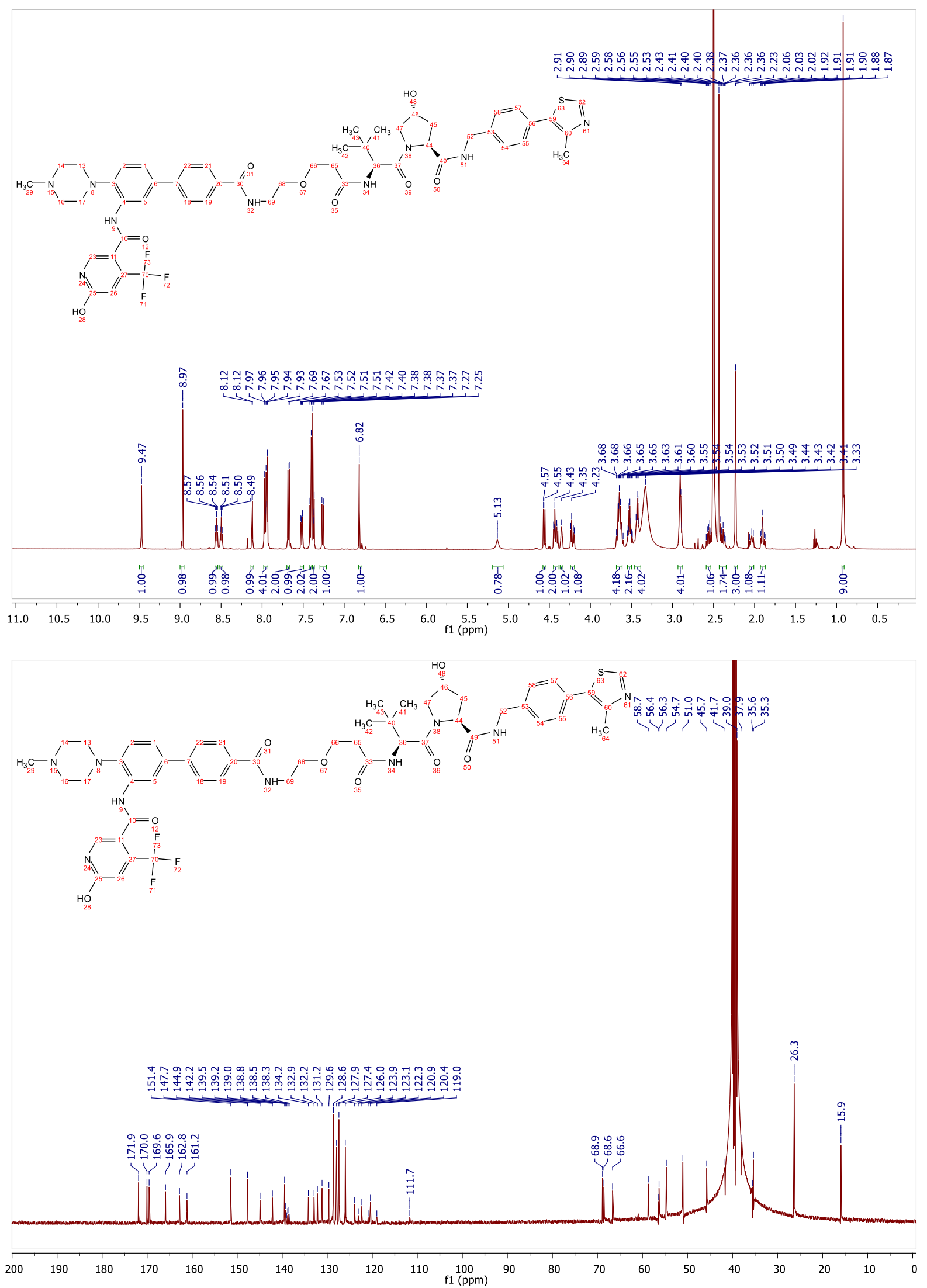

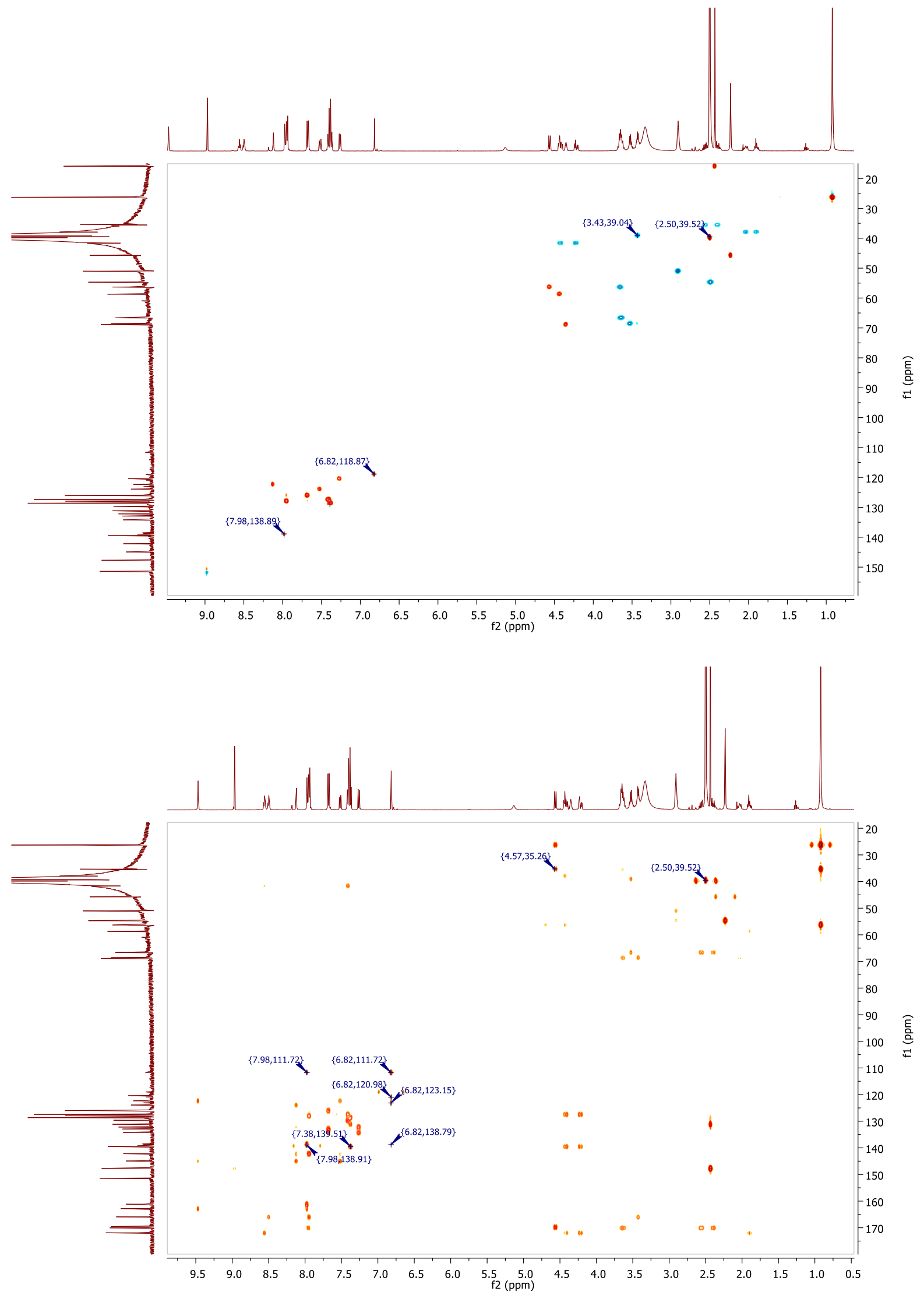
MALDI, HRMS, HPLC, ${ }^{1} \mathrm{H}-\mathrm{NMR}$ and ${ }^{13} \mathrm{C}-\mathrm{NMR}$ of 6-hydroxy-N-(4'-((2-)(2-(3-)((S)-1-((2S,4R)-4-hydroxy-2((4-(4-methylthiazol-5-yl)benzyl)carbamoyl)pyrrolidin-1-yl)-3,3-dimethyl-1-oxobutan-2-yl)amino)-3oxopropoxy)ethoxy)ethyl)carbamoyl)-4-(4-methylpiperazin-1-yl)-[1,1'-biphenyl]-3-yl)-4-

(trifluoromethyl)nicotinamide (8b)

Voyager Spec \#1[BP = 1094.4, 1696]

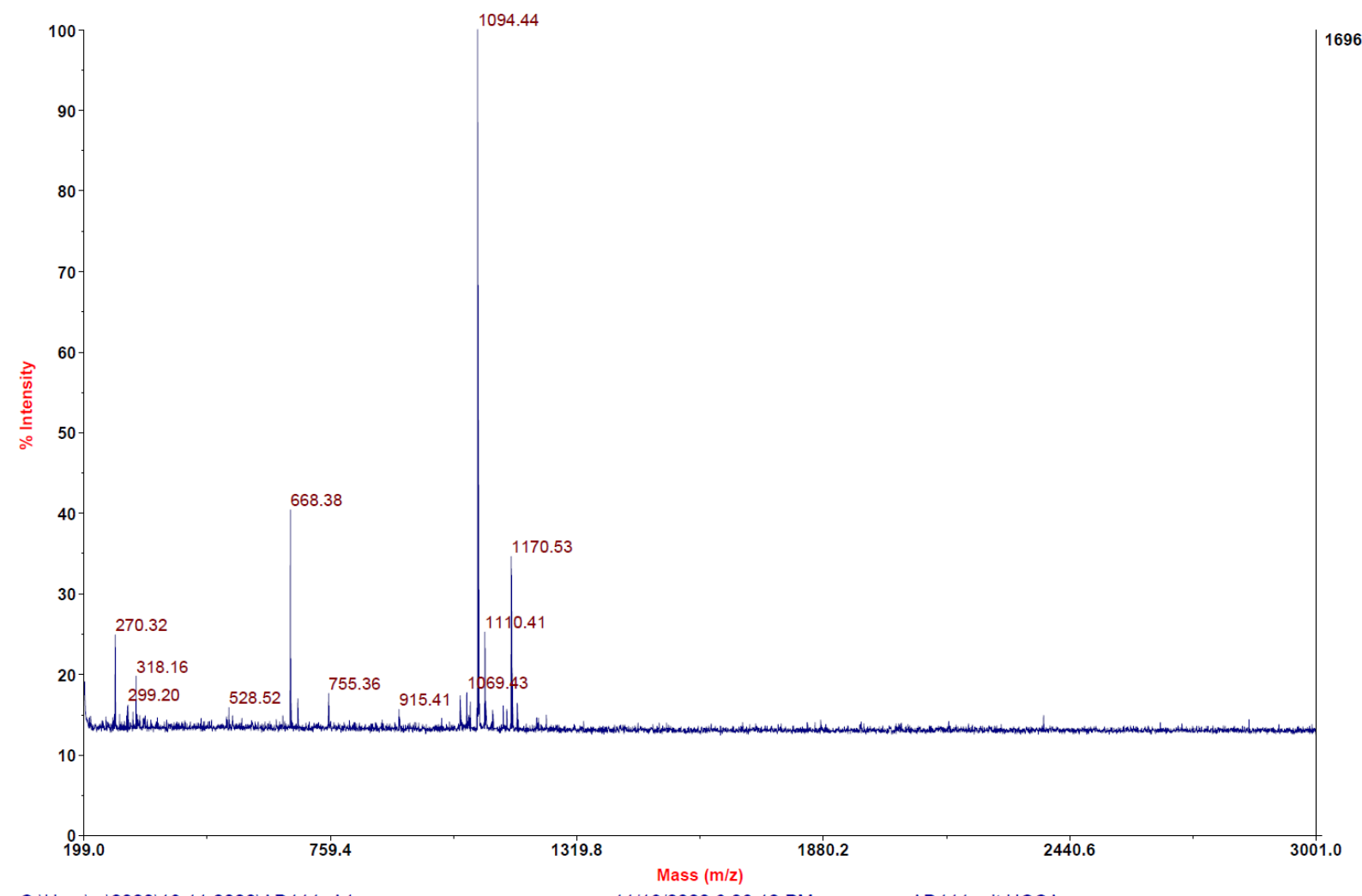

C:IUserl...|2020\19.11.2020\AD111_A1

11/19/2020 6:08:12 PM

AD111 mit HCCA gemessen.

AD111_A1\#1-4 RT: 0.00-0.27 AV: 4 NL: 3.82E6

T: FTMS + p MALDI Full ms [800.00-1400.00]

1094.43840

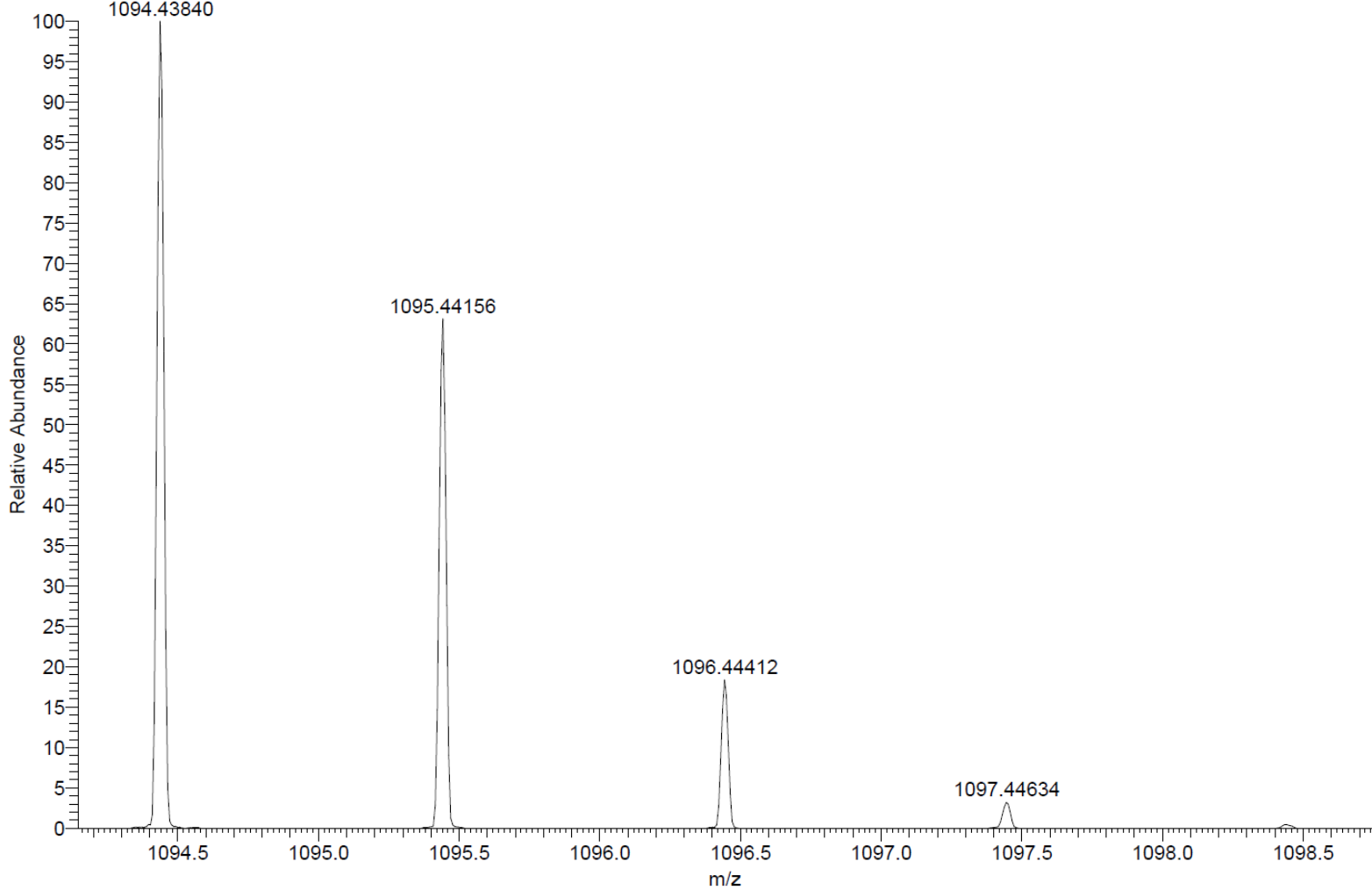

Signal: $\quad$ MWD1 A, Sig $=254,4$ Ref $=$ off 


$\begin{array}{crrrr}\text { RT [min] Type } & \text { Width [min] } & \text { Area } & \text { Height } & \text { Area\% } \\ 11.319 \text { VV } & 0.2071 & 54018.9102 & 3087.1851 & 100.0000 \\ & \text { Sum } & 54018.9102 & & \end{array}$

Signal: $\quad$ MWD1 B, Sig=280,4 Ref=off

$\begin{array}{crrrr}\text { RT [min] Type } & \text { Width [min] } & \text { Area } & \text { Height } & \text { Area\% } \\ 11.316 \text { VV } & 0.2211 & 60787.4297 & 3251.7109 & 100.0000 \\ & \text { Sum } & 60787.4297 & & \end{array}$

Signal: $\quad$ MWD1 F, Sig=260,4 Ref=off

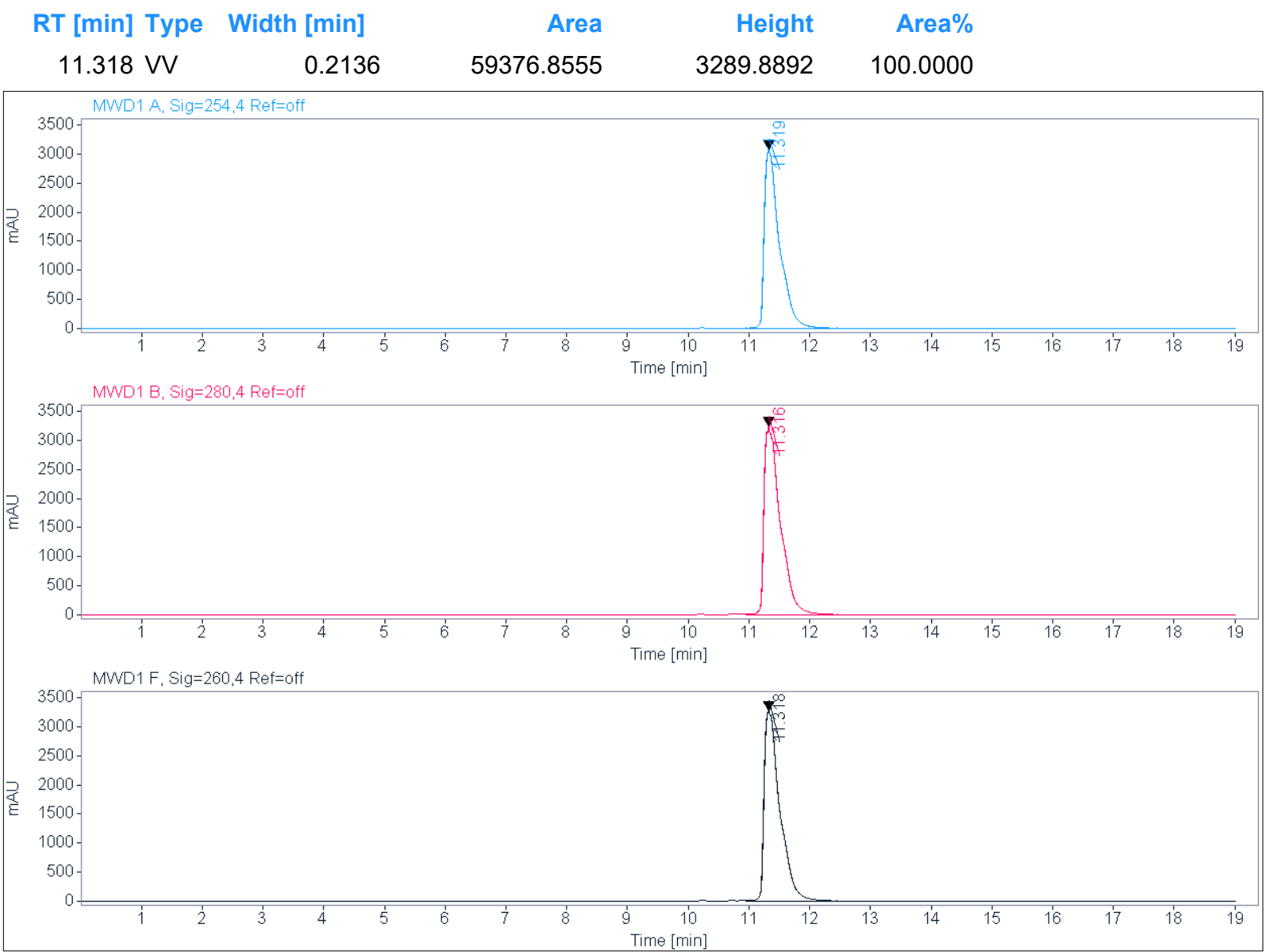



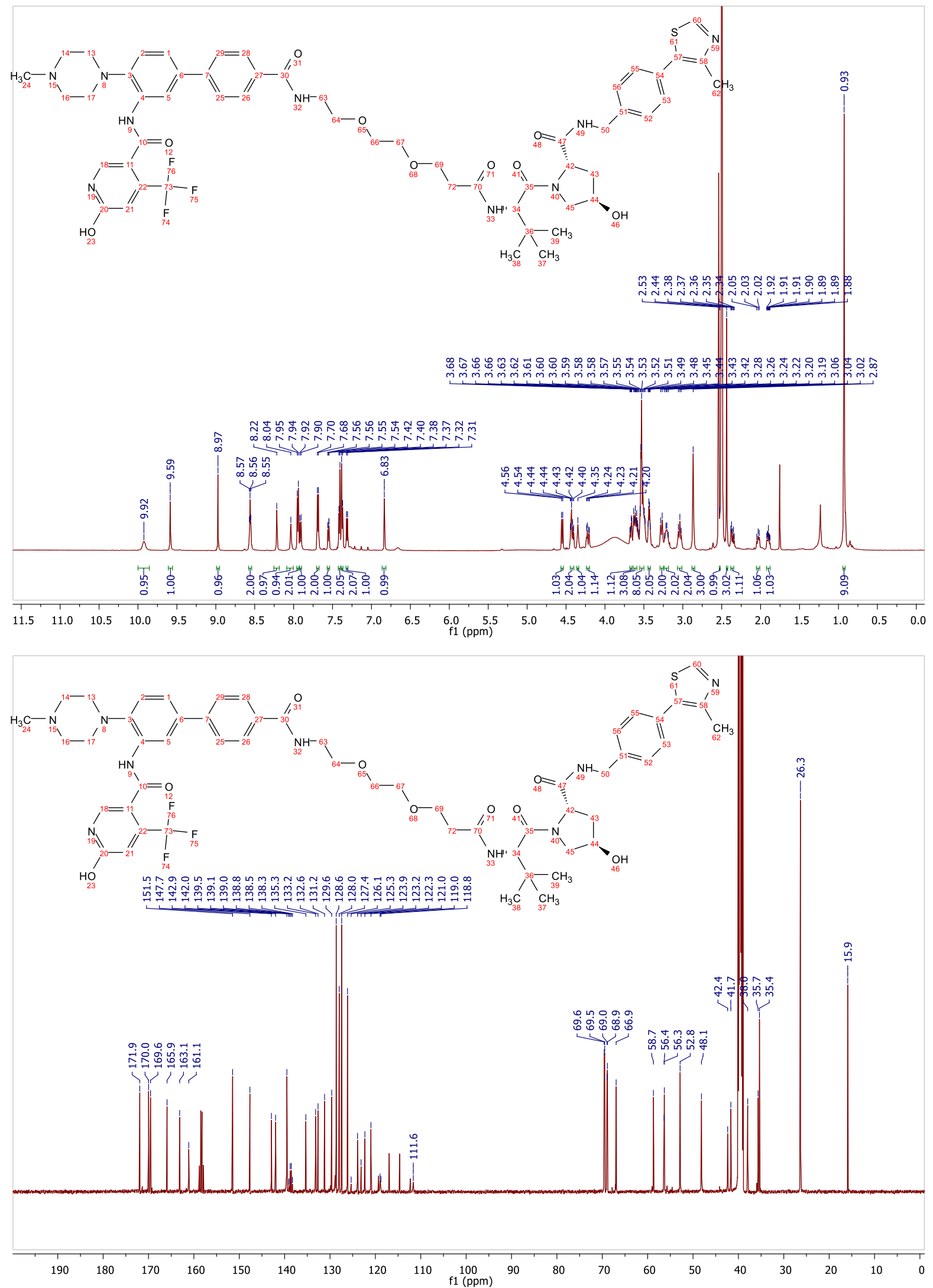
HRMS, MALDI, HPLC, ${ }^{1} \mathrm{H}-\mathrm{NMR}$ and ${ }^{13} \mathrm{C}-\mathrm{NMR}$ of 6-hydroxy-N-(4'-(((S)-17-((2S,4R)-4-hydroxy-2-((4-(4methylthiazol-5-yl)benzyl)carbamoyl)pyrrolidine-1-carbonyl)-18,18-dimethyl-15-oxo-3,6,9,12tetraoxa-16-azanonadecyl)carbamoyl)-4-(4-methylpiperazin-1-yl)-[1,1'-biphenyl]-3-yl)-4(trifluoromethyl)nicotinamide (8c)

C:IUserl...12020117.09.2020\AD112_F8

AD112_F8 \#1-14 RT: 0.01-0.60 AV: 14 NL: 9.41E6

T: FTMS + p MALDI Full ms [1000.00-1250.00]

$100 \quad 1182.49132$

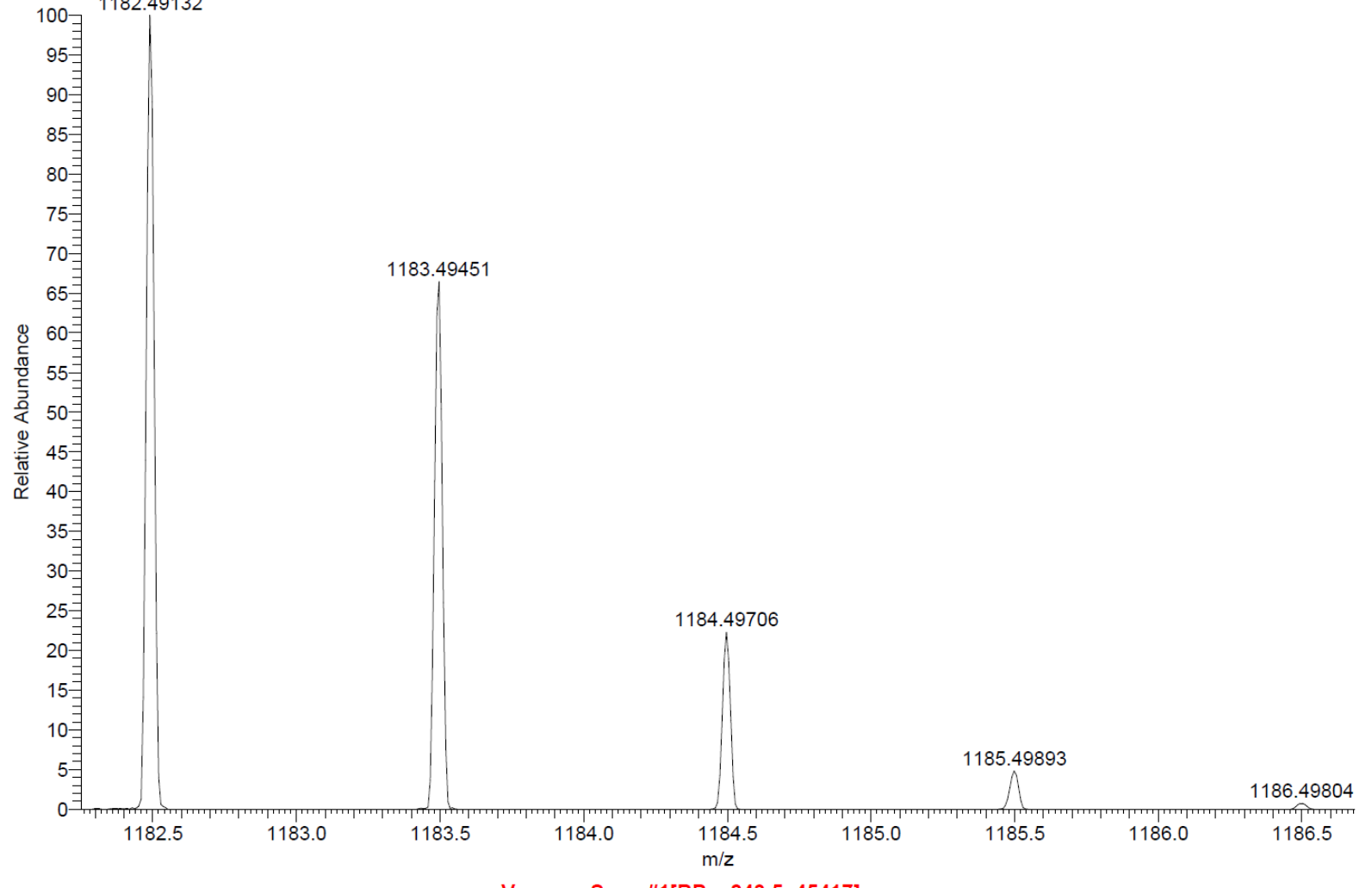

Voyager Spec \#1[BP = 843.5, 45417]

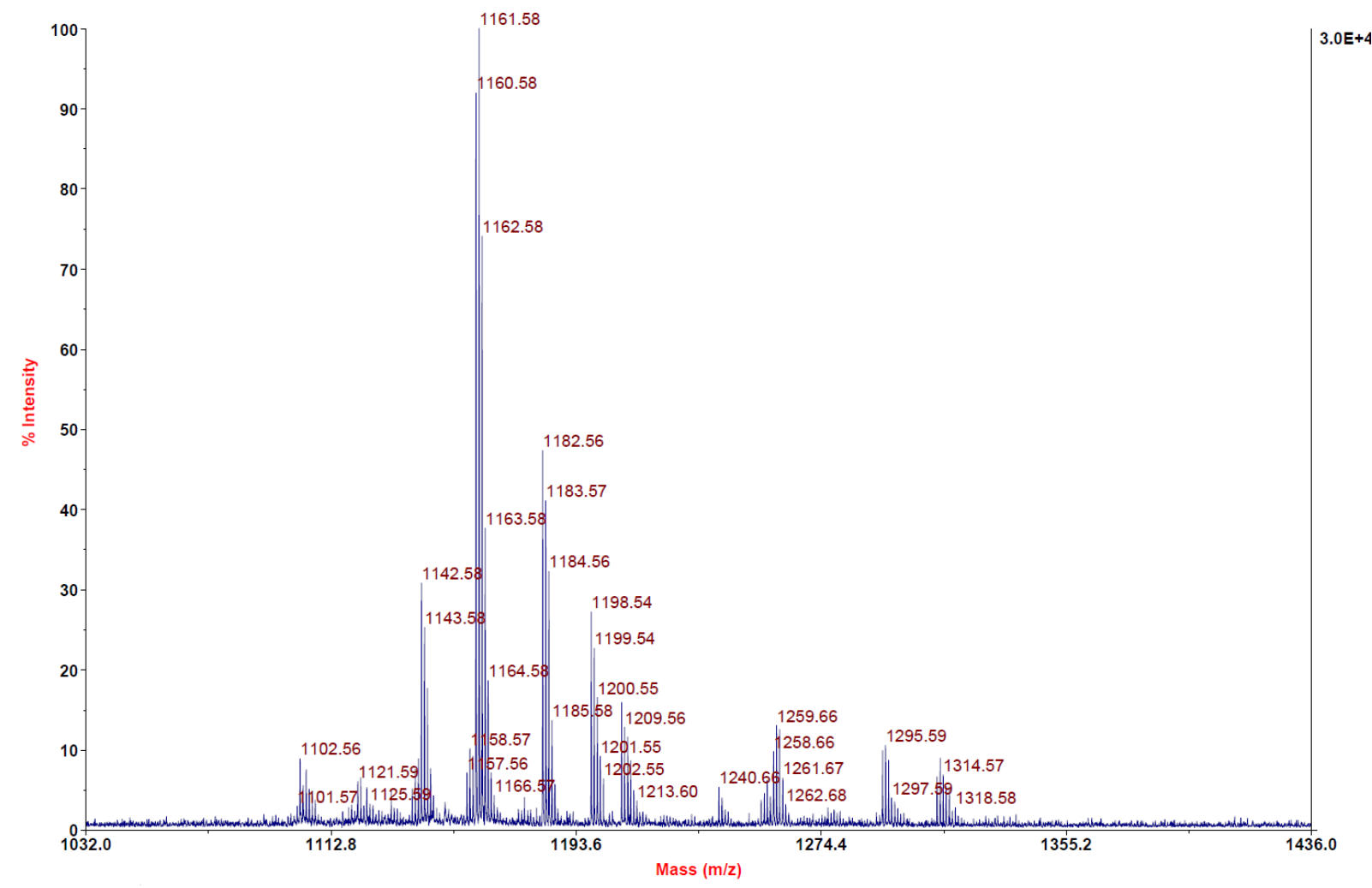

Signal: $\quad$ MWD1 A, Sig $=254,4$ Ref $=$ off 


$\begin{array}{crrrr}\text { RT [min] Type } & \text { Width [min] } & \text { Area } & \text { Height } & \text { Area\% } \\ 11.445 \text { VV } & 0.2008 & 1150.3380 & 79.0589 & 98.6354 \\ 12.949 \mathrm{MM} & 0.1184 & 15.9151 & 2.2397 & 1.3646 \\ & \text { Sum } & 1166.2531 & & \end{array}$

Signal: $\quad$ MWD1 B, Sig=280,4 Ref=off

$\begin{array}{crrrr}\text { RT [min] Type } & \text { Width [min] } & \text { Area } & \text { Height } & \text { Area\% } \\ 11.445 \text { VV } & 0.2055 & 1419.3114 & 94.8723 & 100.0000 \\ & \text { Sum } & 1419.3114 & & \end{array}$

Signal: $\quad$ MWD1 F, Sig=260,4 Ref=off

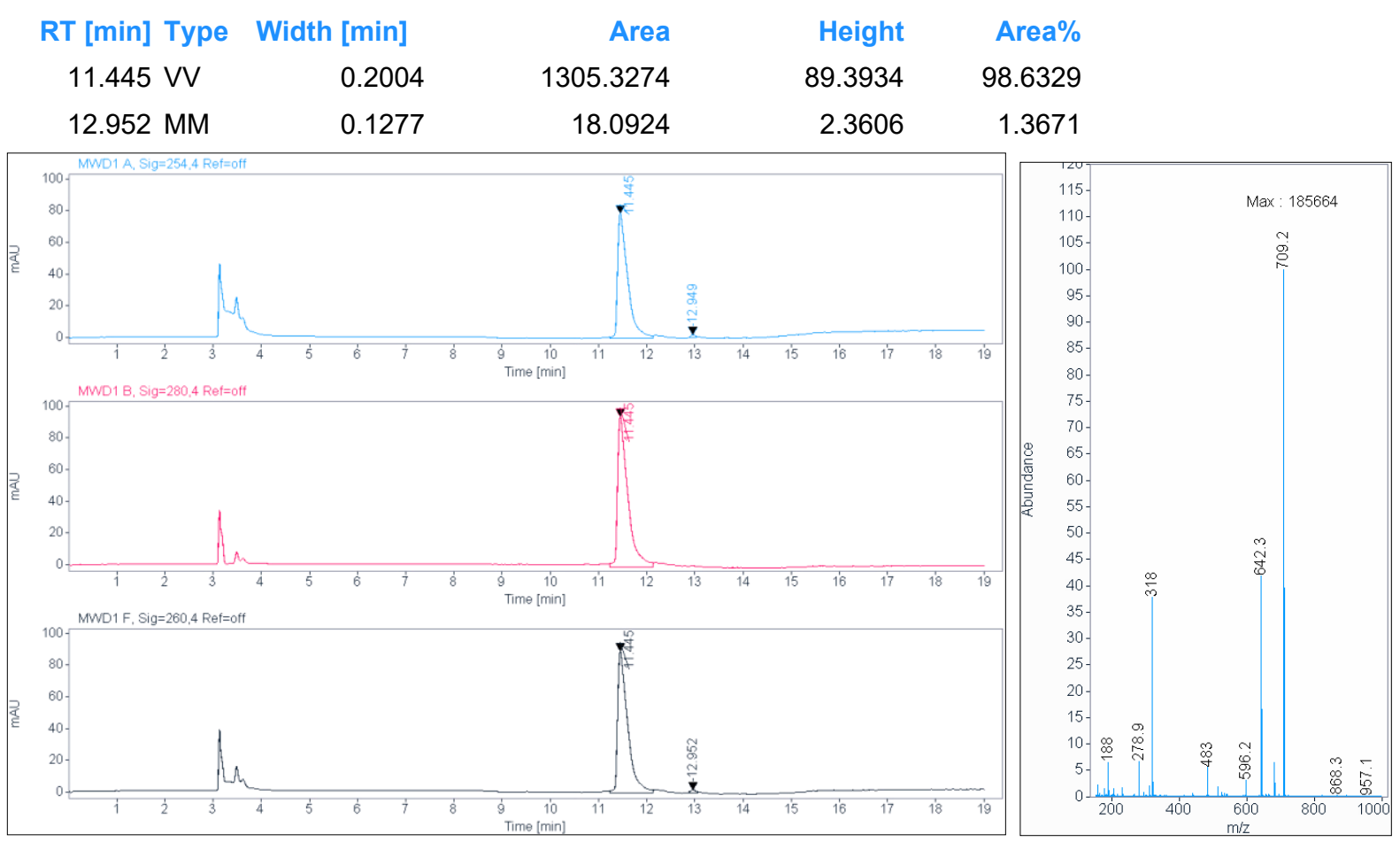



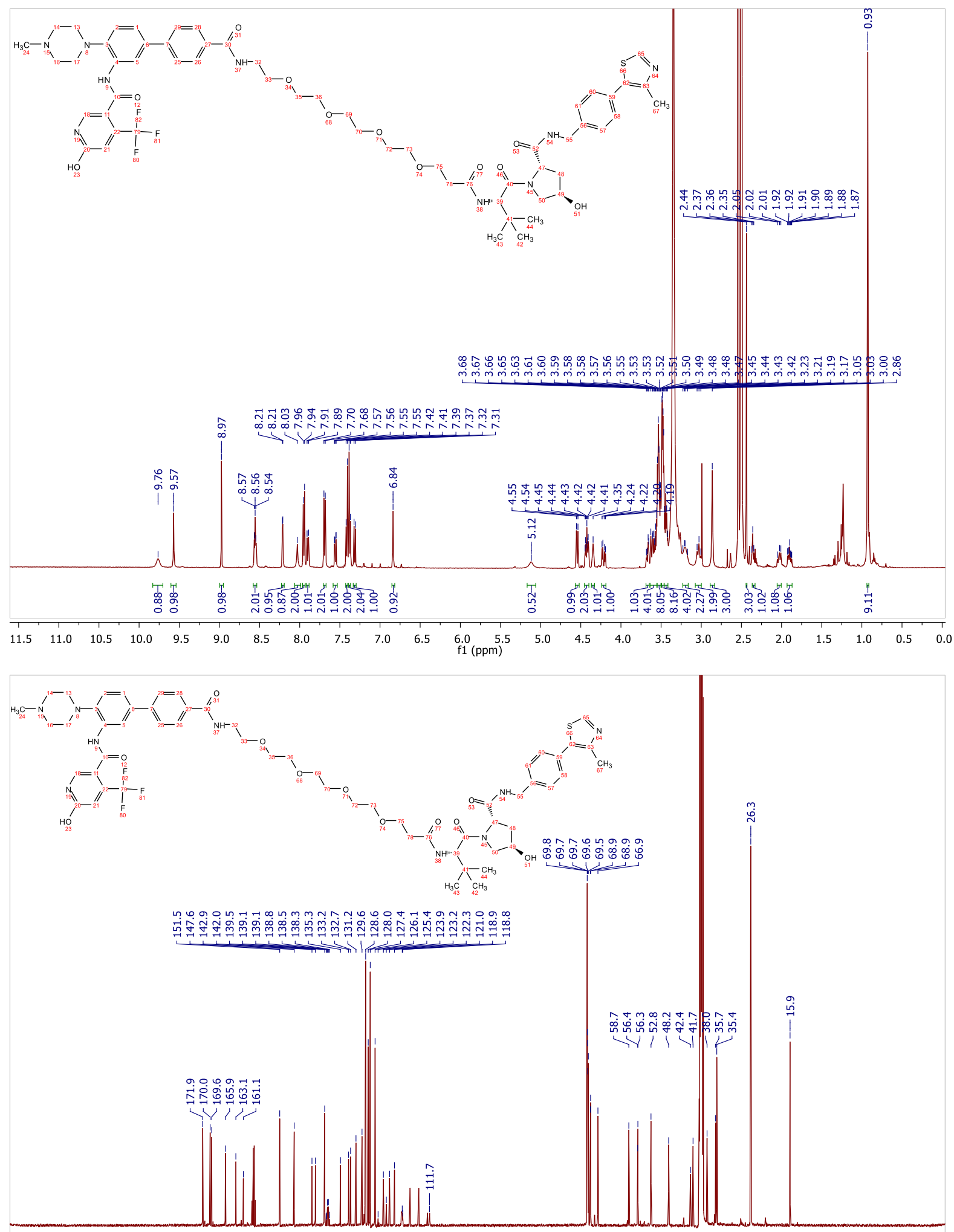

$\begin{array}{llllllllllllllllllllllllllllll}220 & 210 & 200 & 190 & 180 & 170 & 160 & 150 & 140 & 130 & 120 & 110 & \begin{array}{c}100 \\ \mathrm{f} 1(\mathrm{ppm})\end{array} & 90 & 80 & 70 & 60 & 50 & 40 & 30 & 20 & 10 & 0 & -10 & -20\end{array}$ 
ESI, MALDI, HRMS, HPLC, ${ }^{1} \mathrm{H}-\mathrm{NMR},{ }^{13} \mathrm{C}-\mathrm{NMR},{ }^{13} \mathrm{C}-\mathrm{NMR}$ and ${ }^{1} \mathrm{H}-{ }^{13} \mathrm{C}-\mathrm{HMBC}$ of 6 -hydroxy-N-(4'-(( (S)-1((2S,4R)-4-hydroxy-2-((4-(4-methylthiazol-5-yl)benzyl)carbamoyl)pyrrolidin-1-yl)-3,3-dimethyl-1oxobutan-2-yl)carbamoyl)-4-(4-methylpiperazin-1-yl)-[1,1'-biphenyl]-3-yl)-4-

(trifluoromethyl)nicotinamide (8d)

C:IXcaliburldata|AD158-1

10/15/2020 6:50:44 AM

AD158-1 \#34-42 RT: 0.60-0.74 AV: 9 SB: 11 0.02-0.20 NL: $1.85 E 3$

$\mathrm{T}:\{0,0\}+\mathrm{c} E \mathrm{SI}$ !corona sid=75.00 det=1306.00 Full ms [200.00-1500.00]

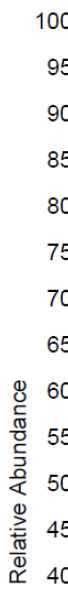

100

95

85

75

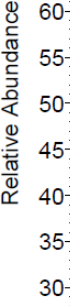

30

$20 \exists \quad 775.88 \quad 792.05$

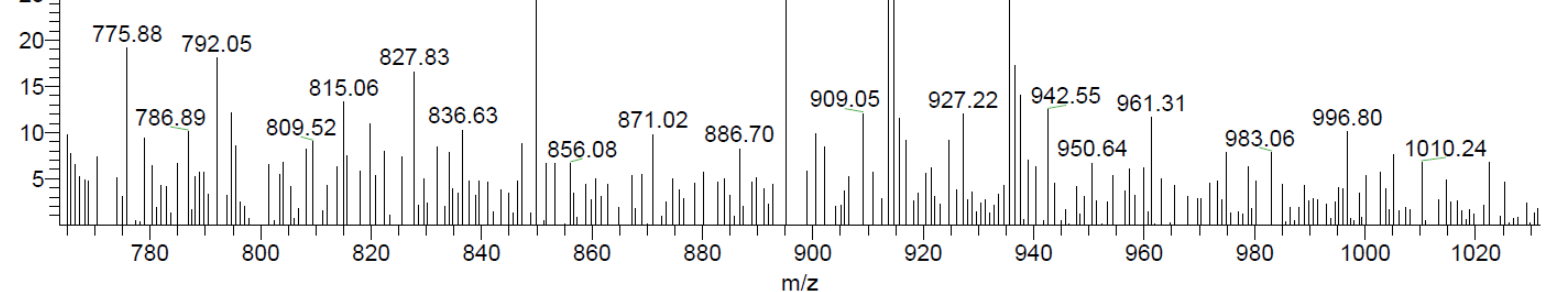

Voyager Spec \#1[BP = 318.1, 4476]

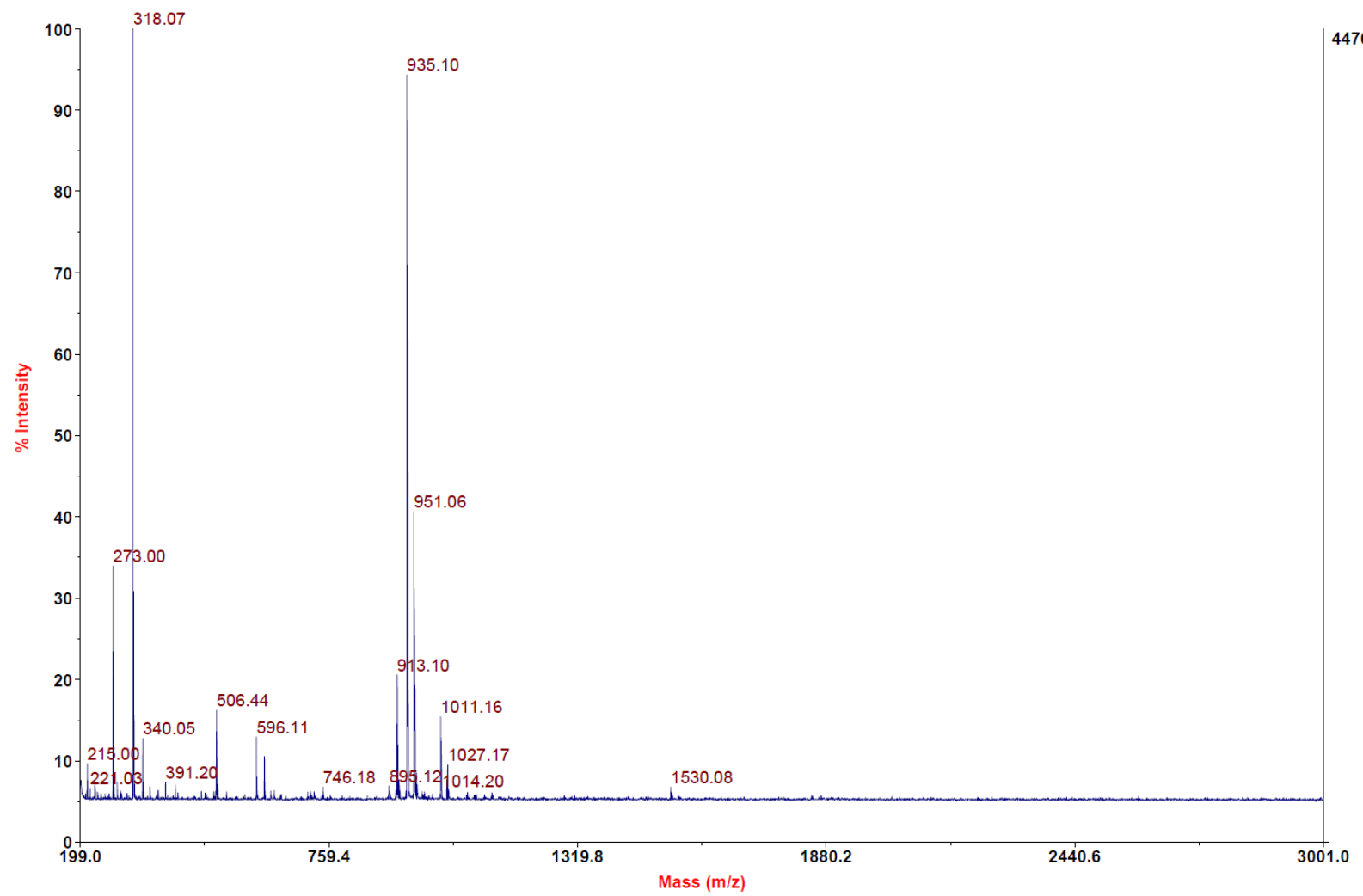




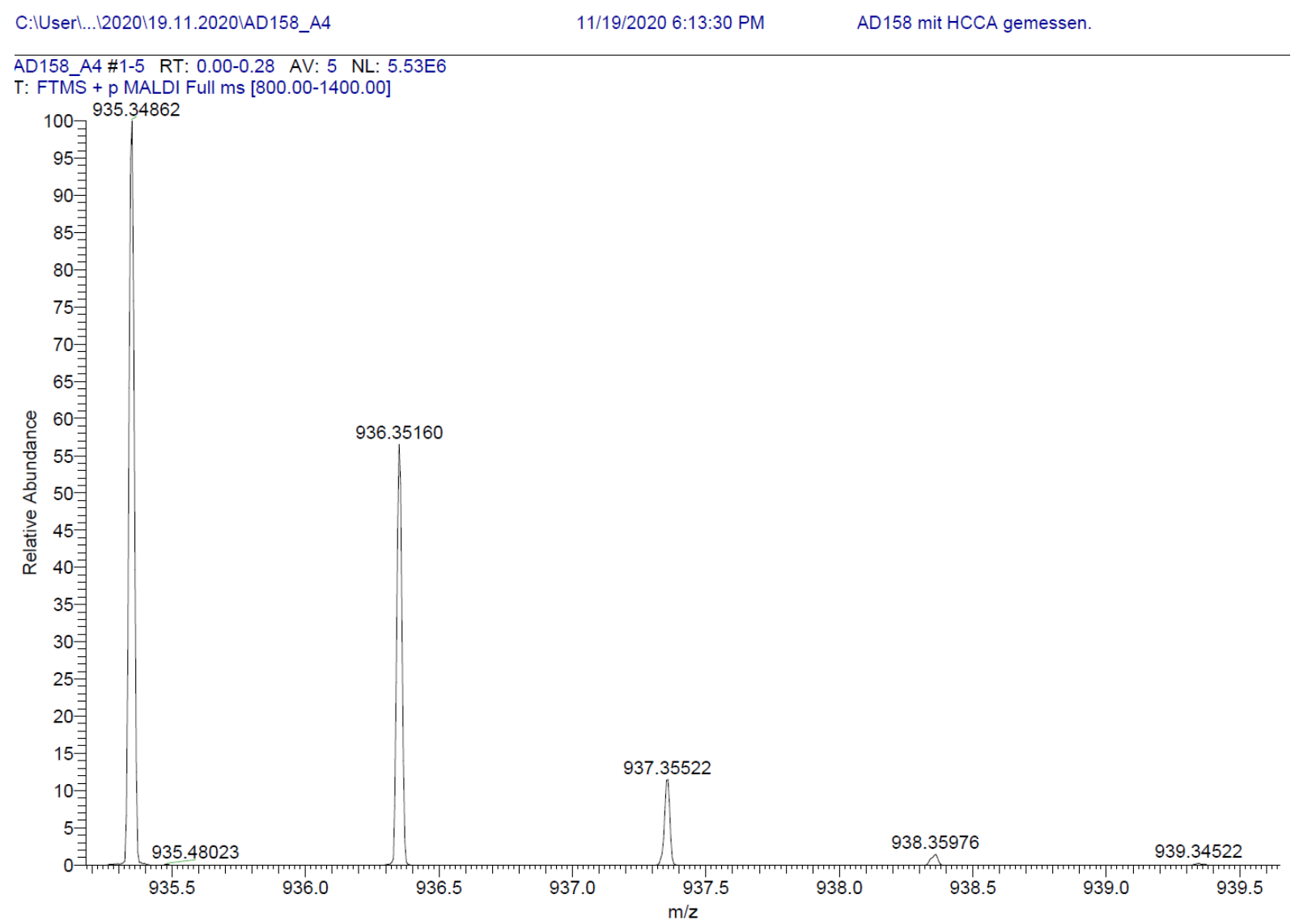

$\begin{array}{crrr}\text { RT [min] Type } & \text { Width [min] } & \text { Area } & \text { Height Area\% } \\ 10.184 \mathrm{VV} & 0.2023 & 725.7850 & 53.83511 .6325 \\ 11.567 \mathrm{VV} & 0.2195 & 42875.0313 & 2667.234996 .4396 \\ 12.127 \mathrm{VV} & 0.1624 & 857.0851 & 76.67091 .9279 \\ & \text { Sum } & 44457.9013 & \end{array}$

Signal: $\quad$ MWD1 B, Sig=280,4 Ref=off

$\begin{array}{crrr}\text { RT [min] Type } & \text { Width [min] } & \text { Area } & \text { Height Area\% } \\ 10.181 \mathrm{VV} & 0.2005 & 1076.0703 & 79.68962 .0573 \\ 11.558 \mathrm{VV} & 0.1974 & 50273.9727 & 3016.563296 .1188 \\ 12.123 \mathrm{VV} & 0.1574 & 953.9785 & 88.03501 .8239 \\ & \text { Sum } & 52304.0214 & \end{array}$

Signal: $\quad$ MWD1 F, Sig=260,4 Ref=off

$\begin{array}{crrr}\text { RT [min] Type } & \text { Width [min] } & \text { Area } & \text { Height Area\% } \\ 10.184 \text { VV } & 0.2011 & 783.1274 & 58.15241 .5765 \\ 11.563 \text { VV } & 0.2178 & 47934.4141 & 2930.301096 .4971 \\ 12.127 \text { VV } & 0.1643 & 956.9185 & 85.66131 .9264\end{array}$



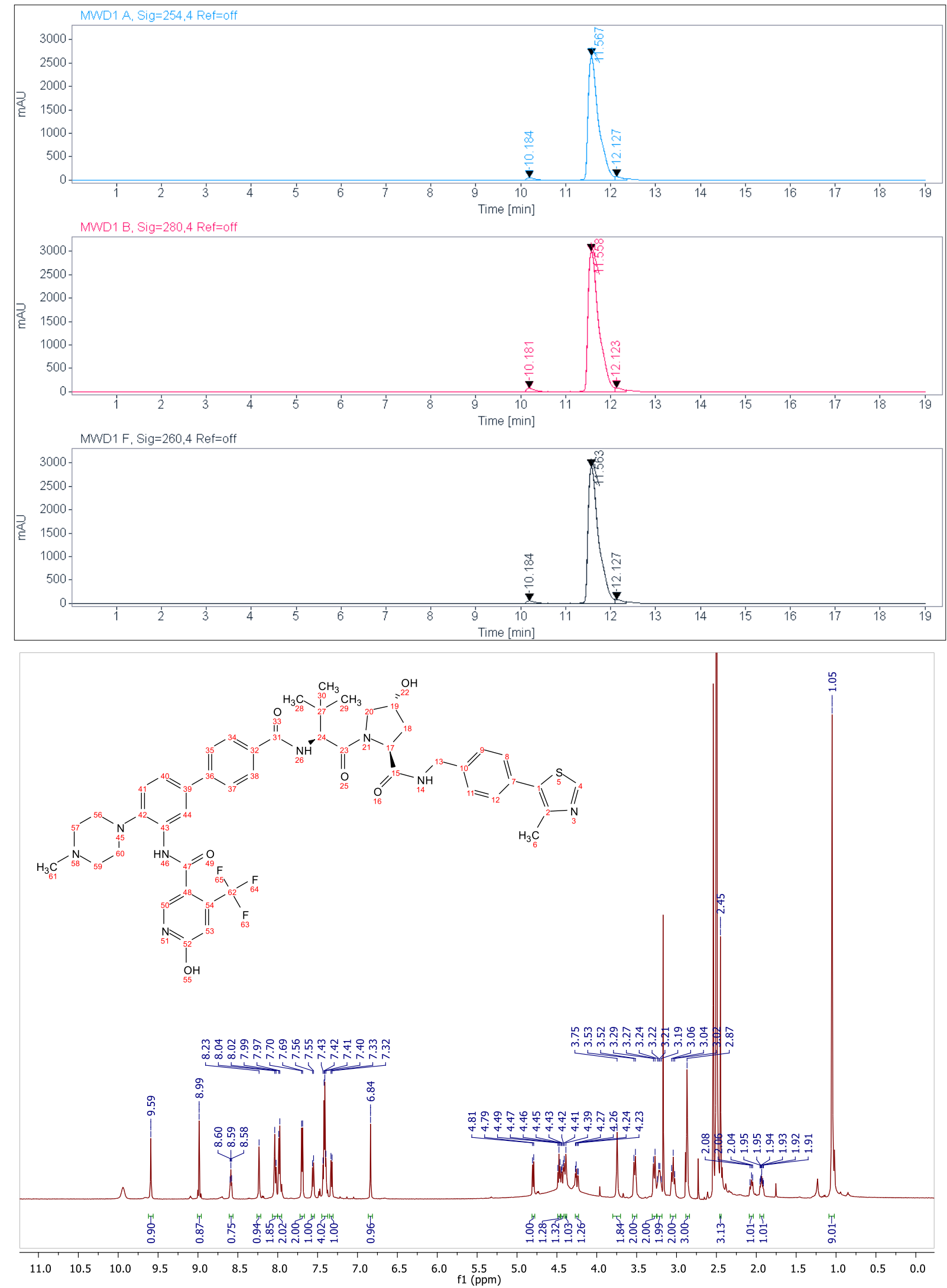

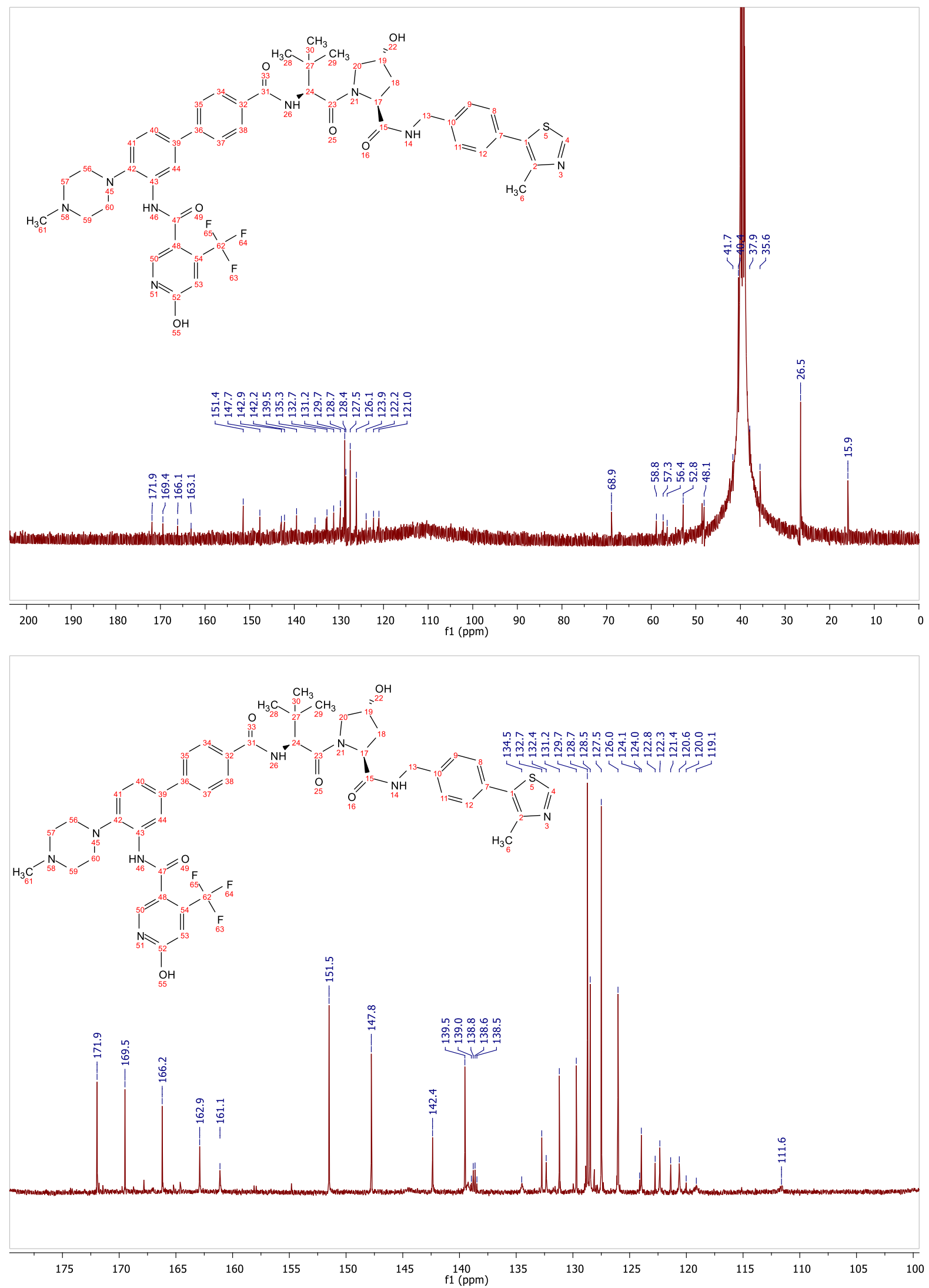


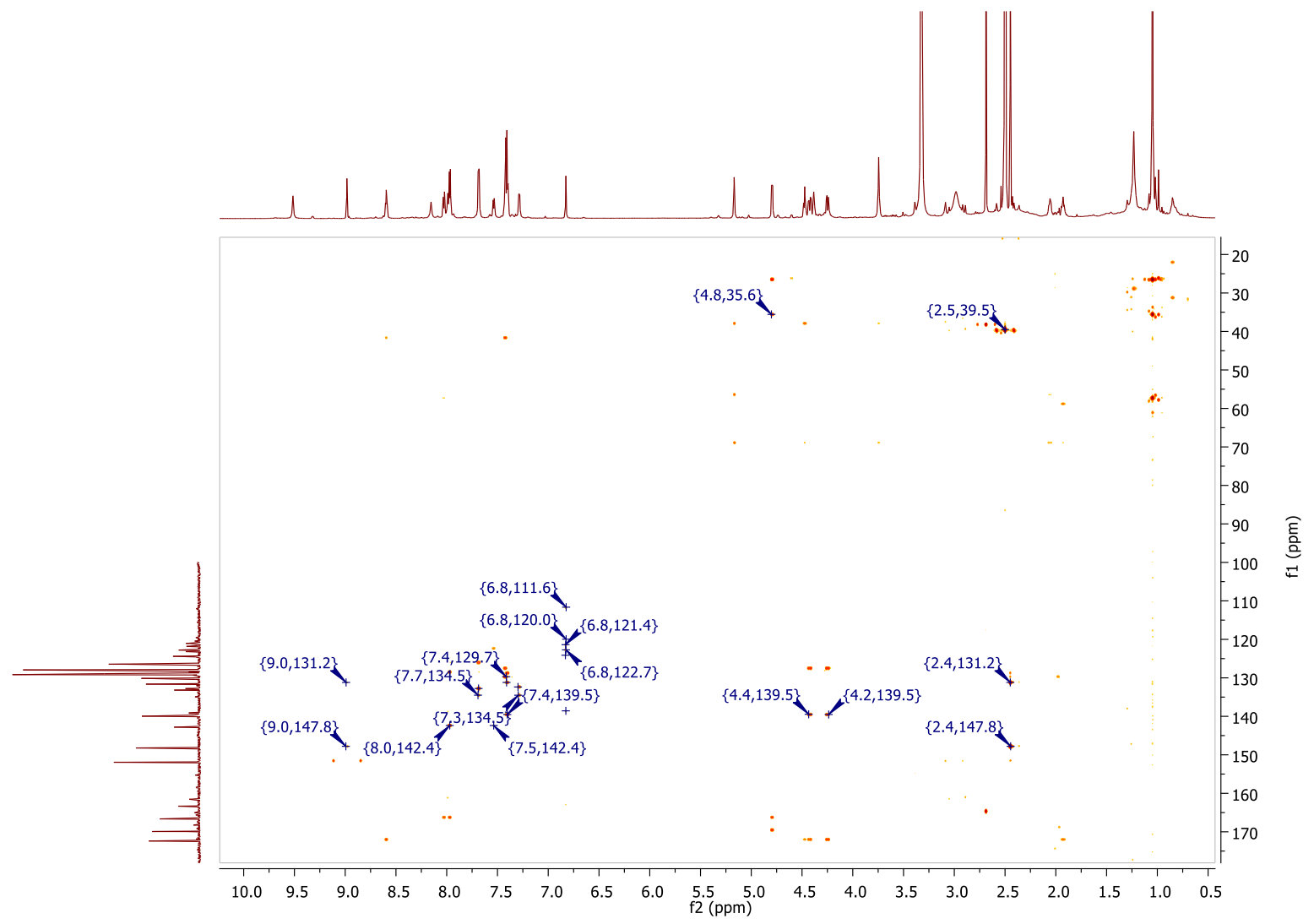


MALDI, HRMS, HPLC, ${ }^{1} \mathrm{H}-\mathrm{NMR}$ and ${ }^{13} \mathrm{C}-\mathrm{NMR}$ of 6-hydroxy-N-(4'-((3-)((S)-1-((2S,4R)-4-hydroxy-2-((4(4-methylthiazol-5-yl)benzyl)carbamoyl)pyrrolidin-1-yl)-3,3-dimethyl-1-oxobutan-2-yl)amino)-3oxopropyl)carbamoyl)-4-(4-methylpiperazin-1-yl)-[1,1'-biphenyl]-3-yl)-4-

(trifluoromethyl)nicotinamide (8e)

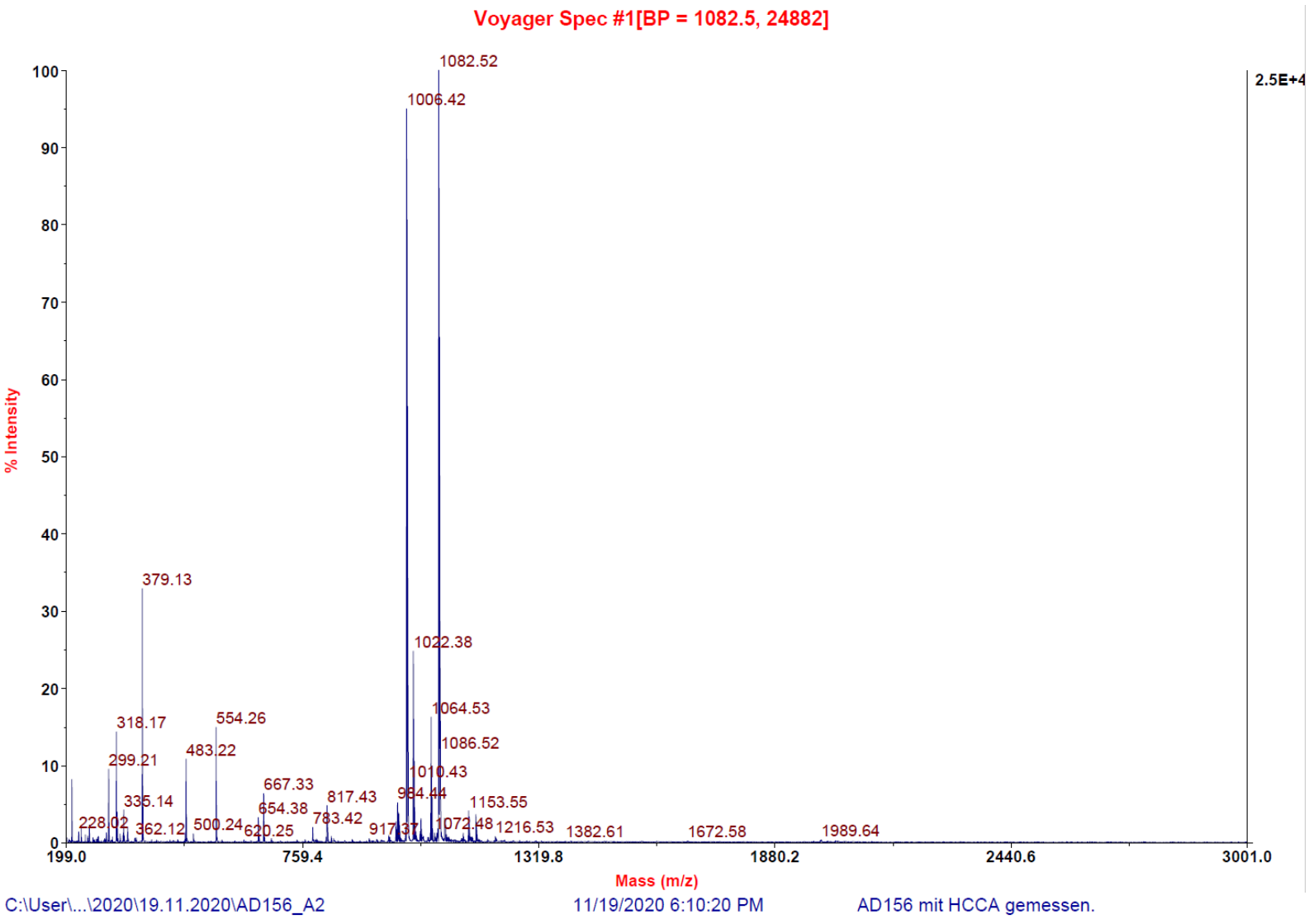

AD156_A2 \#1-11 RT: 0.00-1.06 AV: 11 NL: 6.24E4

T: FTMS + p MALDI Full ms [800.00-1400.00]

1001006.38702

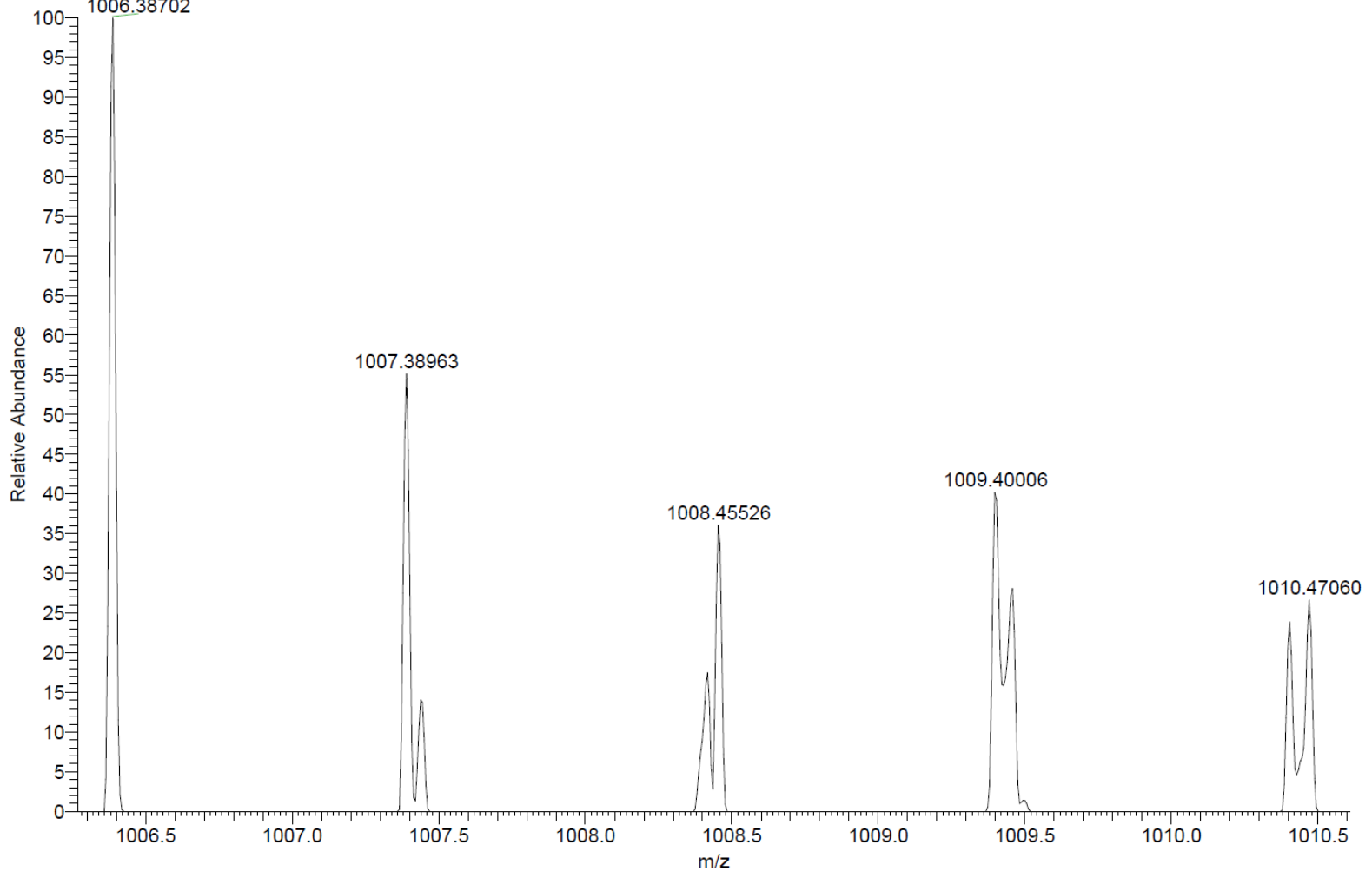

Signal: $\quad$ MWD1 A, Sig $=254,4$ Ref $=$ off 


$\begin{array}{crrrr}\text { RT [min] Type } & \text { Width [min] } & \text { Area } & \text { Height } & \text { Area\% } \\ 11.239 \text { VV } & 0.2244 & 10229.9883 & 672.8884 & 100.0000 \\ & \text { Sum } & 10229.9883 & & \end{array}$

Signal: $\quad$ MWD1 B, Sig=280,4 Ref=off

$\begin{array}{crrrr}\text { RT [min] Type } & \text { Width [min] } & \text { Area } & \text { Height } & \text { Area\% } \\ 11.239 \text { VV } & 0.2255 & 12384.9932 & 809.5344 & 100.0000 \\ & \text { Sum } & 12384.9932 & & \end{array}$

Signal: $\quad$ MWD1 F, Sig=260,4 Ref=off

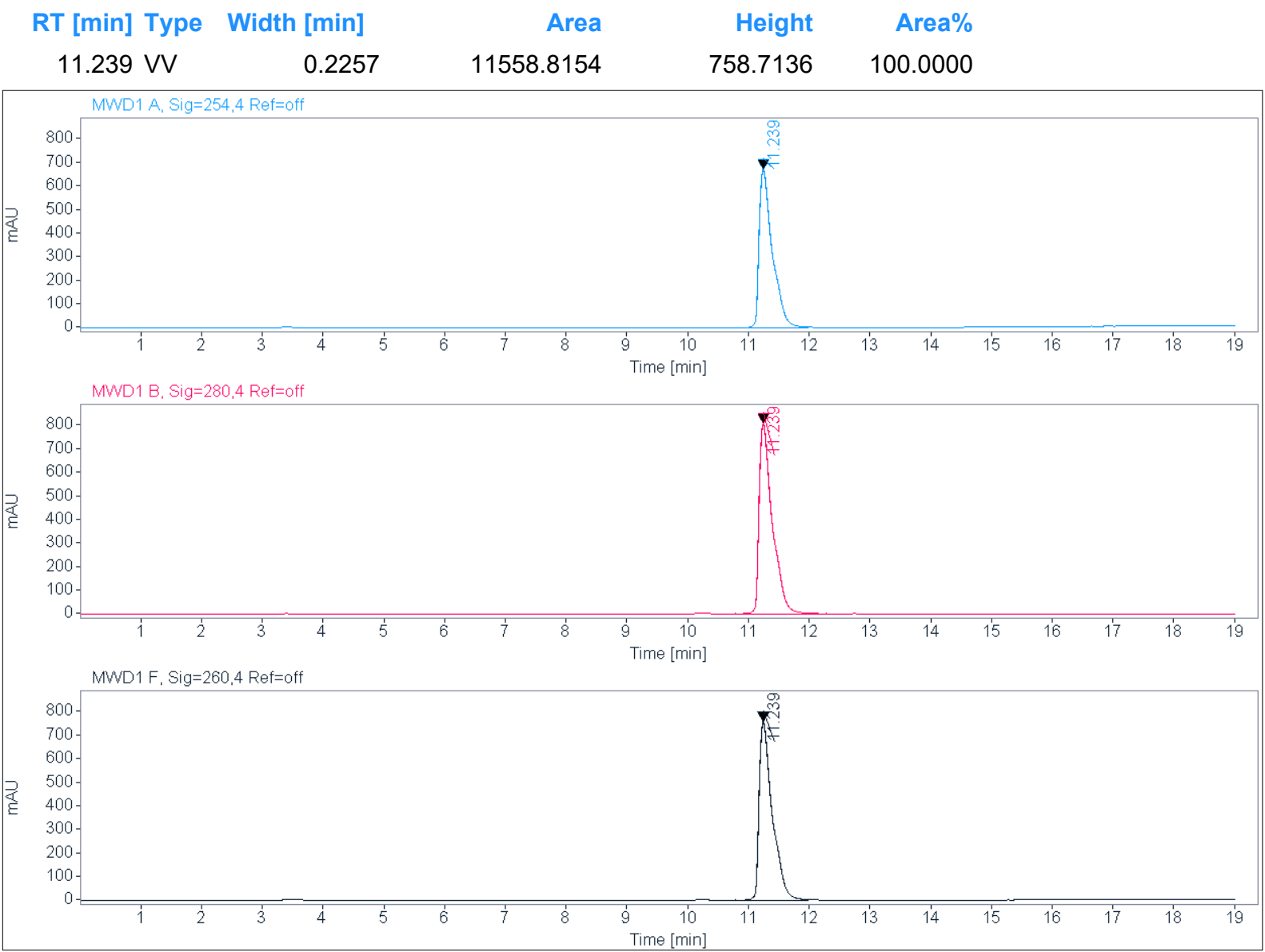



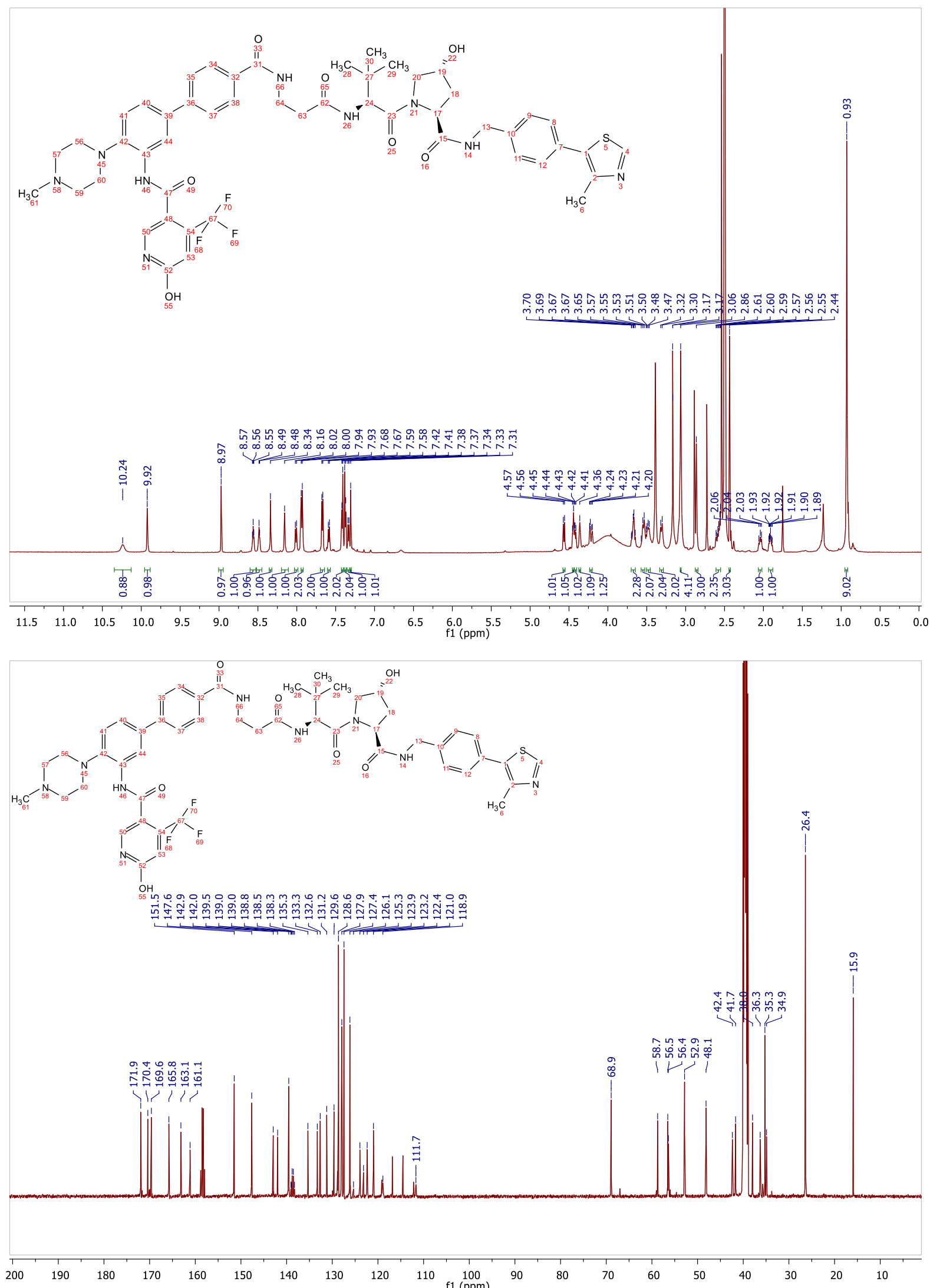
ESI, MALDI, HRMS, HPLC, ${ }^{1} \mathrm{H}-\mathrm{NMR},{ }^{13} \mathrm{C}-\mathrm{NMR},{ }^{1} \mathrm{H}-{ }^{13} \mathrm{C}-\mathrm{HSQC}(2 \mathrm{x})$ and ${ }^{1} \mathrm{H}^{-13} \mathrm{C}-\mathrm{HMBC}(2 \mathrm{x})$ of 6-hydroxy-N(4'-((4-)((S)-1-((2S,4R)-4-hydroxy-2-((4-(4-methylthiazol-5-yl)benzyl)carbamoyl)pyrrolidin-1-yl)-3,3dimethyl-1-oxobutan-2-yl)amino)-4-oxobutyl)carbamoyl)-4-(4-methylpiperazin-1-yl)-[1,1'biphenyl]-3-yl)-4-(trifluoromethyl)nicotinamide (8f)

C:IXcaliburldataIAD157-2 10/15/2020 7:10:15 AM

AD157-2 \#36-42 RT: 0.63-0.74 AV: 7 SB: 8 0.04-0.16 NL: 1.56E3

$\mathrm{T}:\{0,0\}+\mathrm{c}$ ESI !corona sid=75.00 det=1306.00 Full ms [200.00-1500.00]

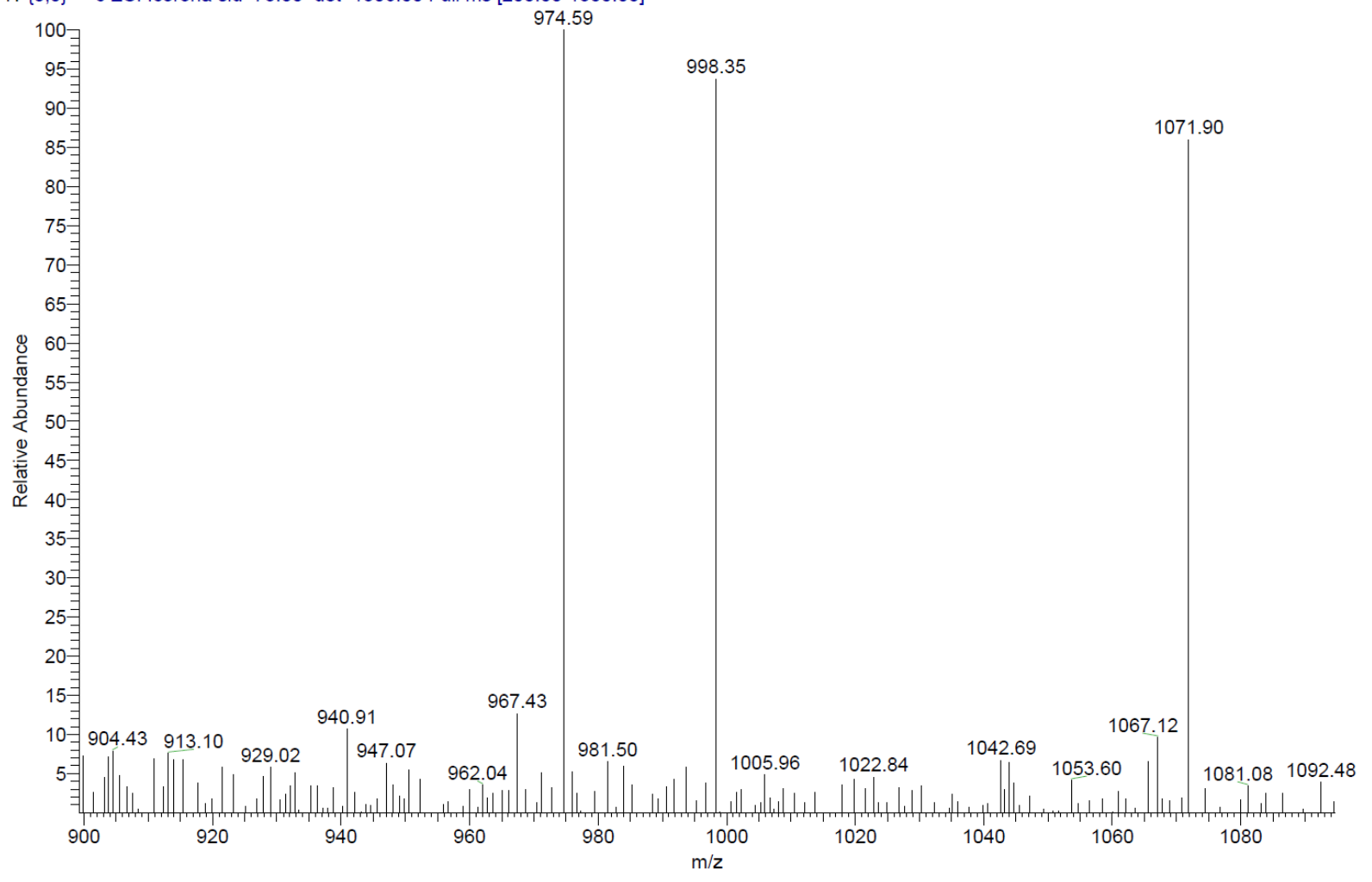

Voyager Spec \#1[BP = 1020.4, 34690]

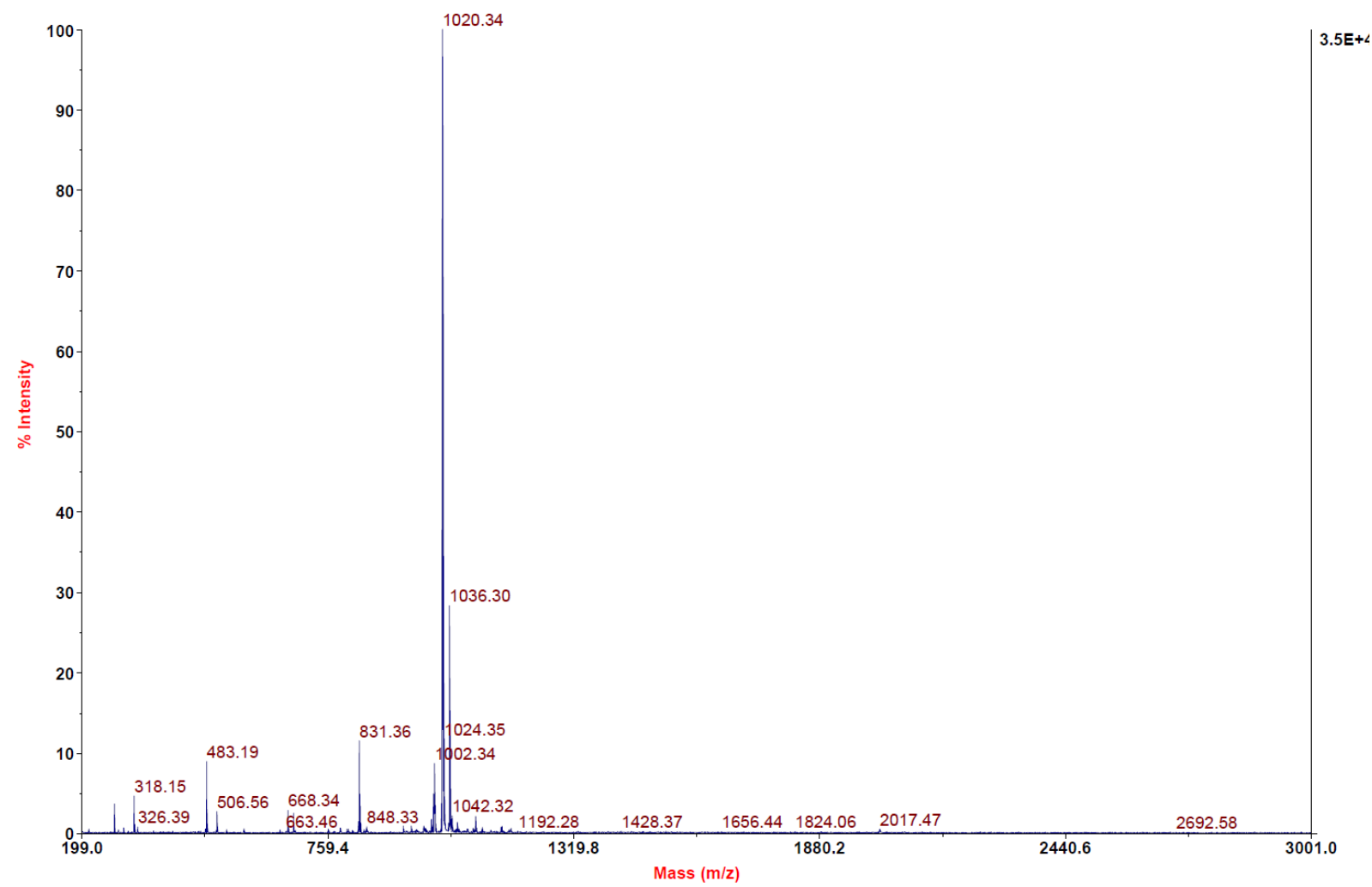


C:IUser|...12020119.11.2020\AD157_A3

AD157_A3 \#1-4 RT: 0.00-0.34 AV: 4 NL: 1.61E6

T: FTMS + p MALDI Full ms [800.00-1400.00]

$100 \quad 1020.40101$
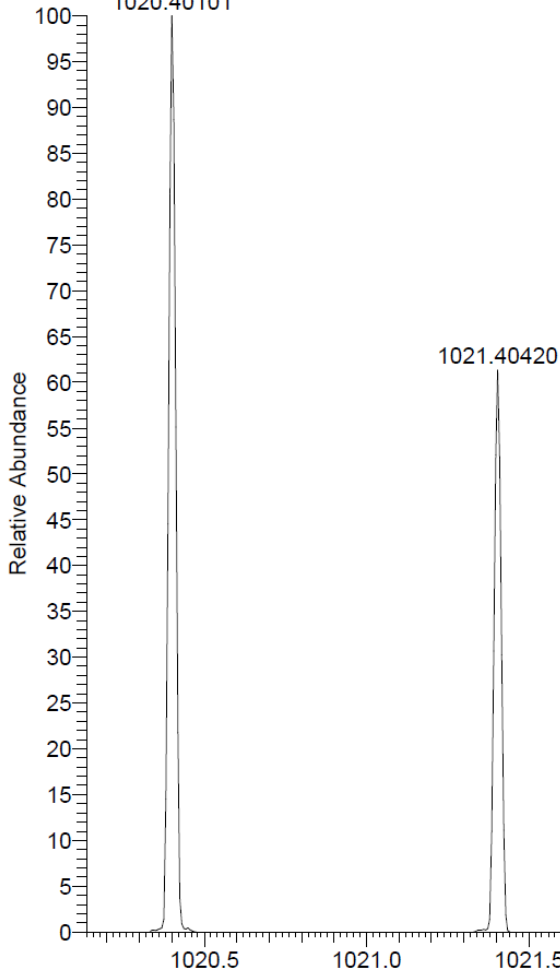

021.40420

Signal: $\quad$ MWD1 A, Sig=254,4 Ref=off

RT [min] Type Width [min]

11.279 VV

0.2370

Sum
Area

9289.1738

9289.1738
1022.40677

1023.40929

$\begin{array}{lll}1023.5 & 1024.0 & 1024.5\end{array}$

Signal: $\quad$ MWD1 B, Sig=280,4 Ref=off

$\begin{array}{crrrr}\text { RT [min] Type } & \text { Width [min] } & \text { Area } & \text { Height } & \text { Area\% } \\ 11.279 \text { VV } & 0.2382 & 10607.9912 & 664.5951 & 100.0000 \\ & \text { Sum } & 10607.9912 & & \end{array}$

Signal: $\quad$ MWD1 F, Sig $=260,4$ Ref $=$ off

$\begin{array}{crrrr}\text { RT [min] Type } & \text { Width [min] } & \text { Area } & \text { Height } & \text { Area\% } \\ 11.279 \mathrm{VV} & 0.2376 & 10198.9717 & 641.1873 & 100.0000\end{array}$



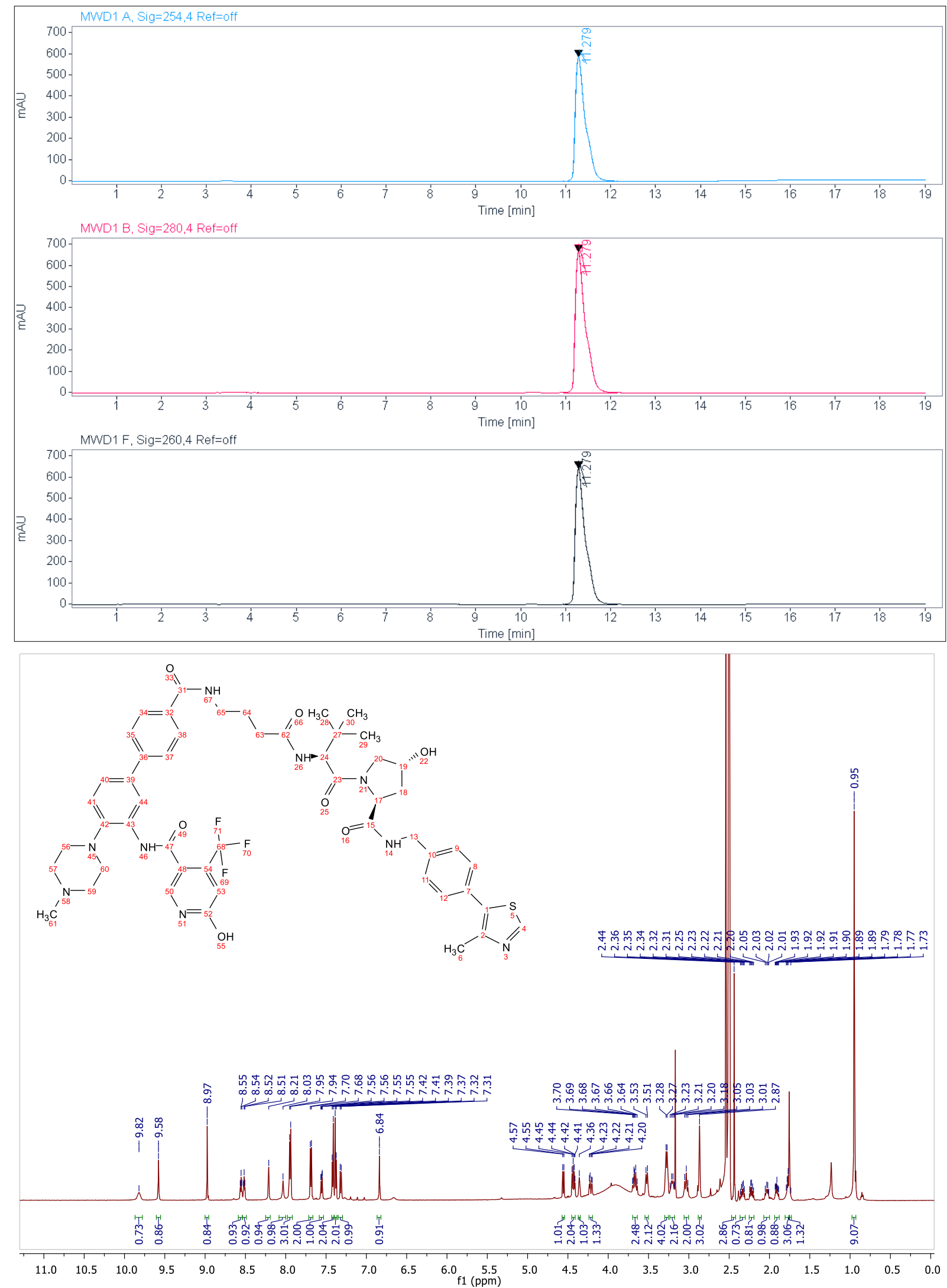

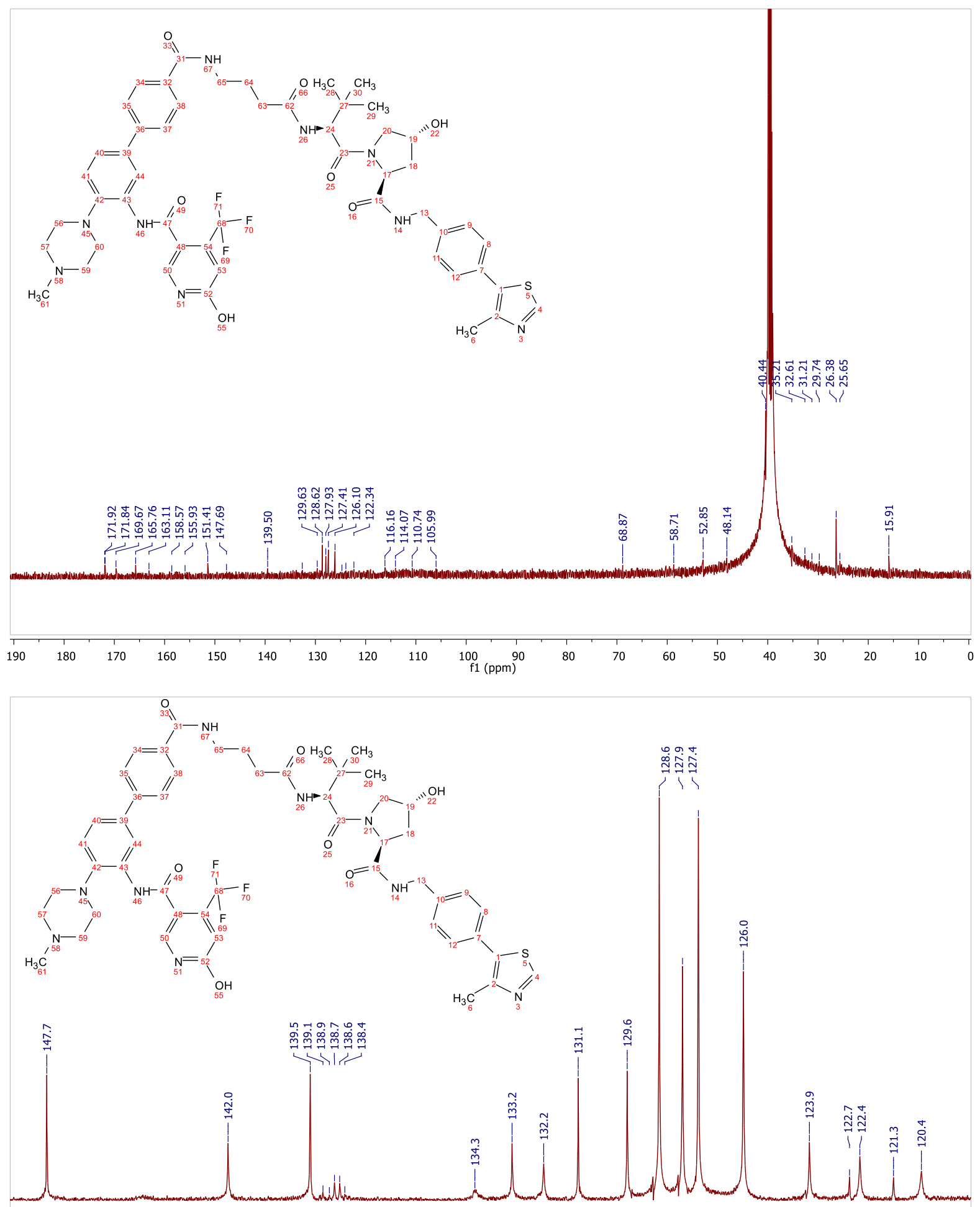

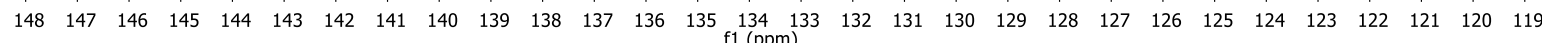



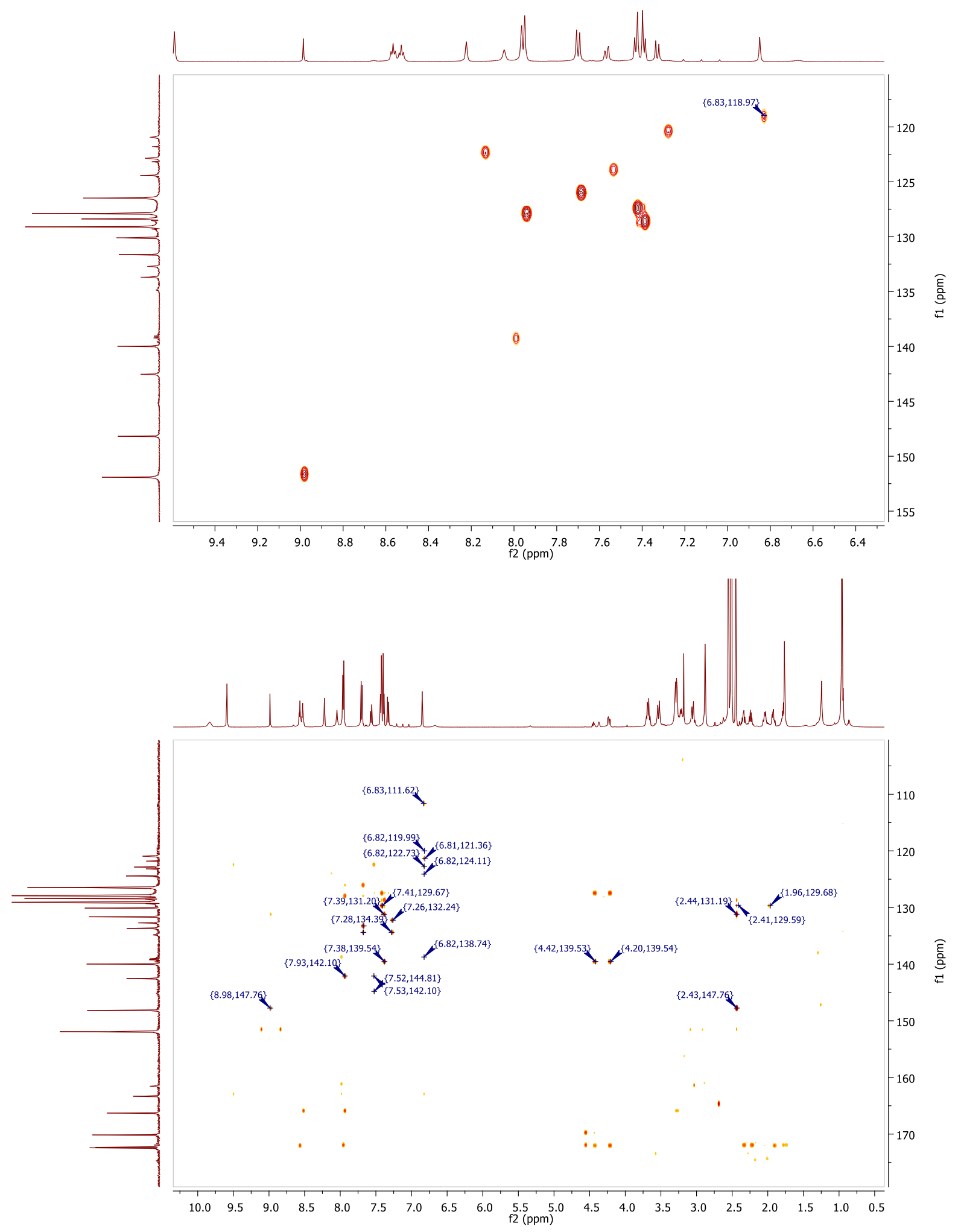

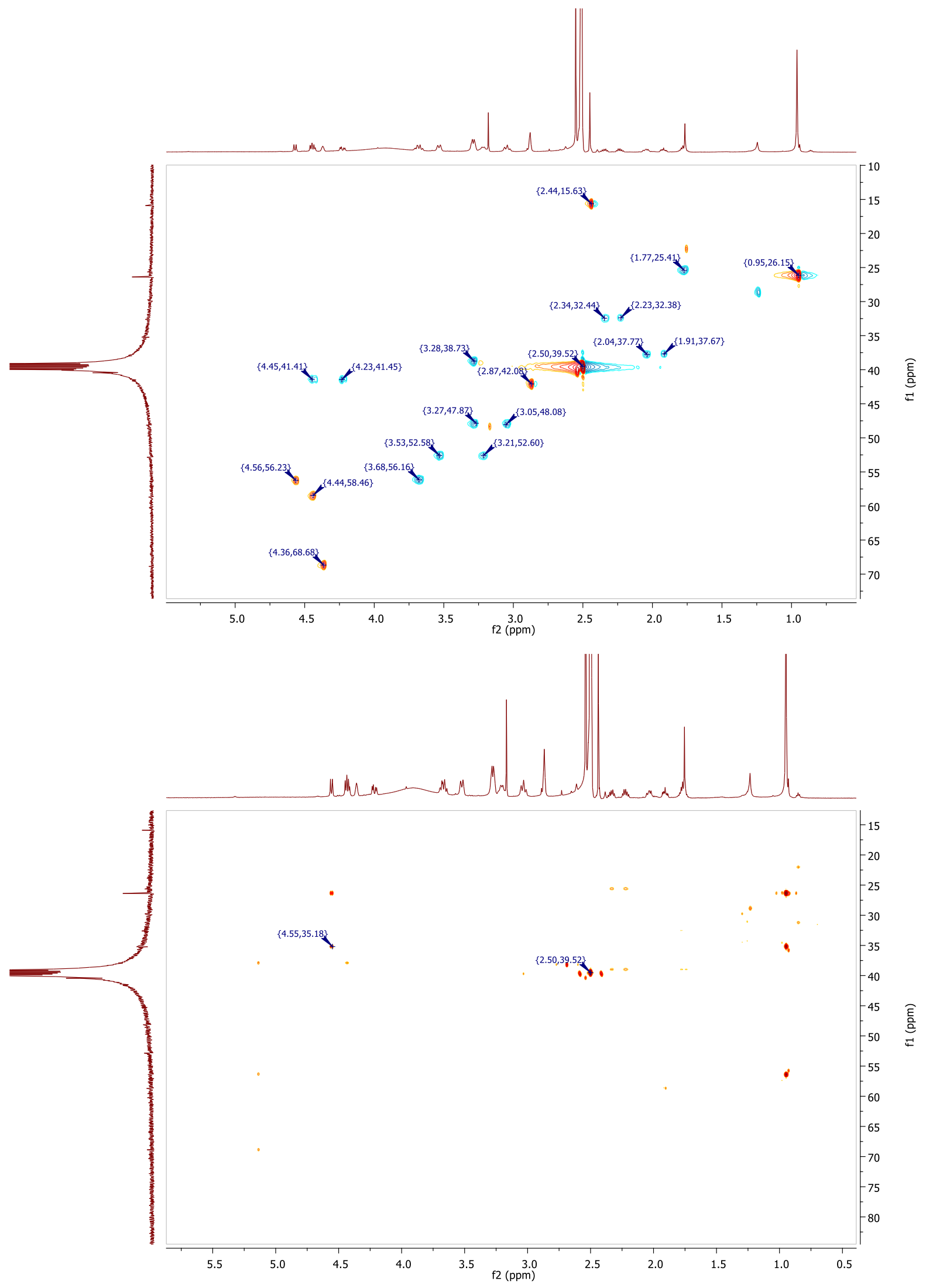
ESI, HRMS, HPLC, ${ }^{1} \mathrm{H}-\mathrm{NMR},{ }^{13} \mathrm{C}-\mathrm{NMR},{ }^{13} \mathrm{C}-\mathrm{NMR},{ }^{1} \mathrm{H}-{ }^{13} \mathrm{C}-\mathrm{HSQC}$ and ${ }^{1} \mathrm{H}-{ }^{13} \mathrm{C}-\mathrm{HMBC}(2 \mathrm{x})$ of 6-hydroxy-N-(4'((5-(((S)-1-((2S,4R)-4-hydroxy-2-((4-(4-methylthiazol-5-yl)benzyl)carbamoyl)pyrrolidin-1-yl)-3,3dimethyl-1-oxobutan-2-yl)amino)-5-oxopentyl)carbamoyl)-4-(4-methylpiperazin-1-yl)-[1,1'biphenyl]-3-yl)-4-(trifluoromethyl)nicotinamide (8g/ Homer)

AD122-6 \#9-11 RT: 0.54-0.68 AV: 3 SB: 5 0.07-0.34 NL: $2.78 E 6$

$\mathrm{T}:\{0,0\}+\mathrm{c}$ ESI !corona sid=75.00 det=1600.00 Full ms [200.00-1500.00]

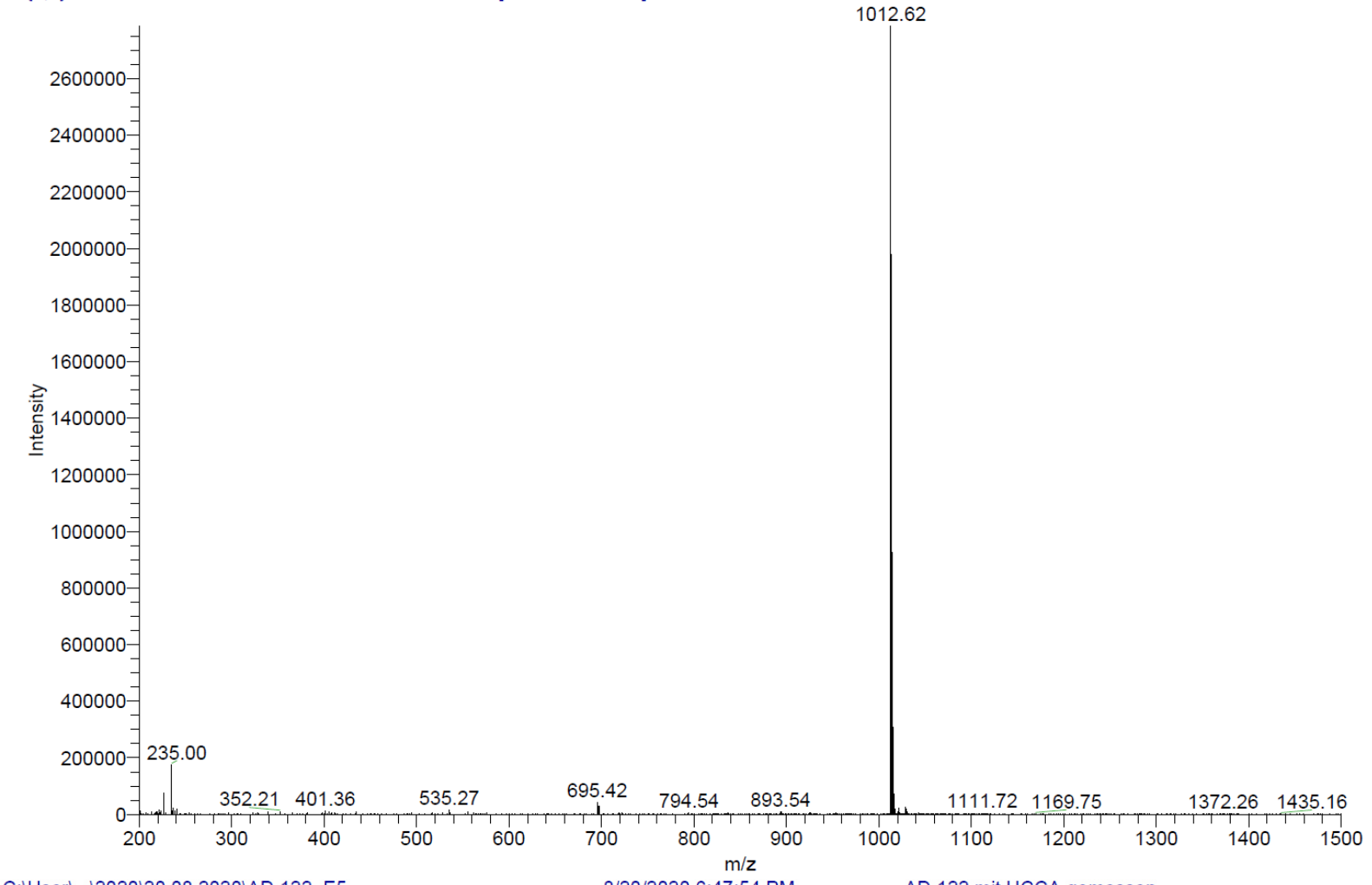

C:IUserl...12020120.08.20201AD 122_E5

AD 122_E5 \#1-5 RT: 0.00-0.41 AV: 5 NL: 4.58E6

T: FTMS + p MALDI Full ms [700.00-1700.00]

$100 \quad 1034.41709$

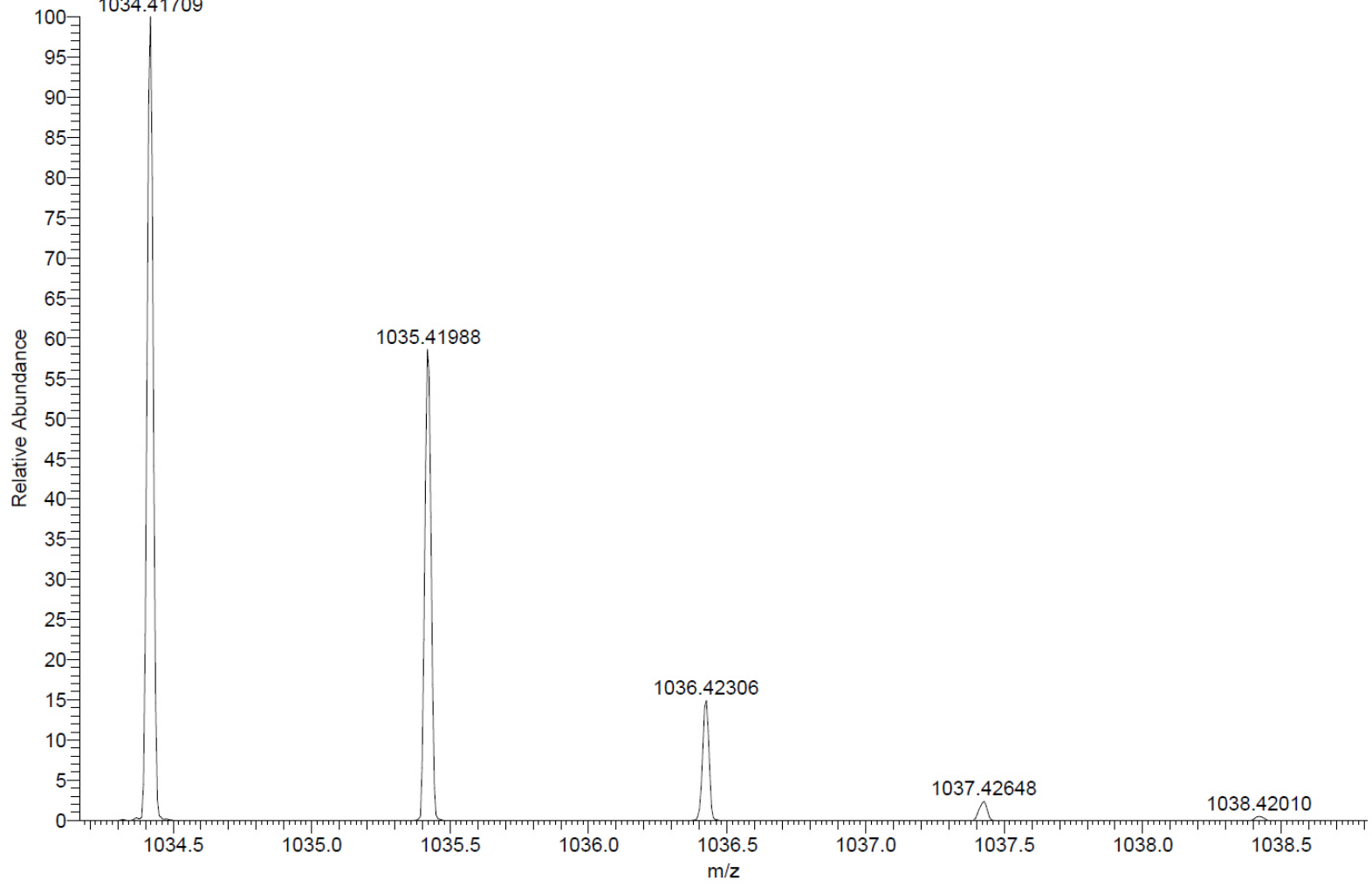




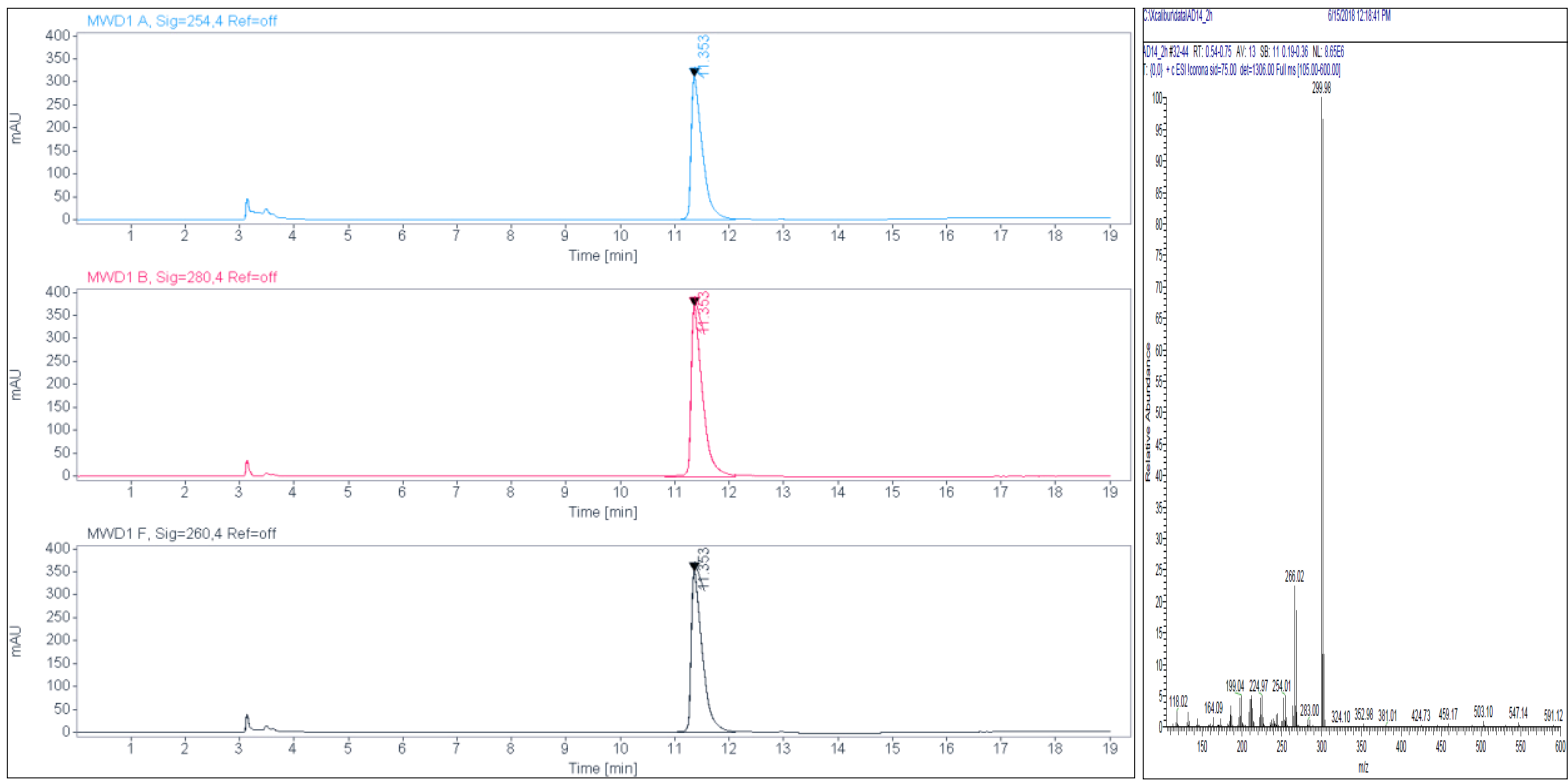

Signal: $\quad$ MWD1 A, Sig=254,4 Ref=off

$\begin{array}{crrrr}\text { RT [min] Type } & \text { Width [min] } & \text { Area } & \text { Height } & \text { Area\% } \\ 11.353 \text { VV } & 0.2008 & 4513.2095 & 311.9199 & 100.0000 \\ & \text { Sum } & 4513.2095 & & \end{array}$

Signal: $\quad$ MWD1 B, Sig=280,4 Ref=off

$\begin{array}{crrrr}\text { RT [min] Type } & \text { Width [min] } & \text { Area } & \text { Height } & \text { Area\% } \\ 11.353 \text { VV } & 0.2029 & 5449.8301 & 372.0602 & 100.0000 \\ & \text { Sum } & 5449.8301 & & \end{array}$

Signal: $\quad$ MWD1 F, Sig=260,4 Ref $=$ off

$\begin{array}{crrrr}\text { RT [min] Type } & \text { Width [min] } & \text { Area } & \text { Height } & \text { Area\% } \\ 11.353 \text { VV } & 0.2021 & 5101.9272 & 352.0205 & 100.0000\end{array}$



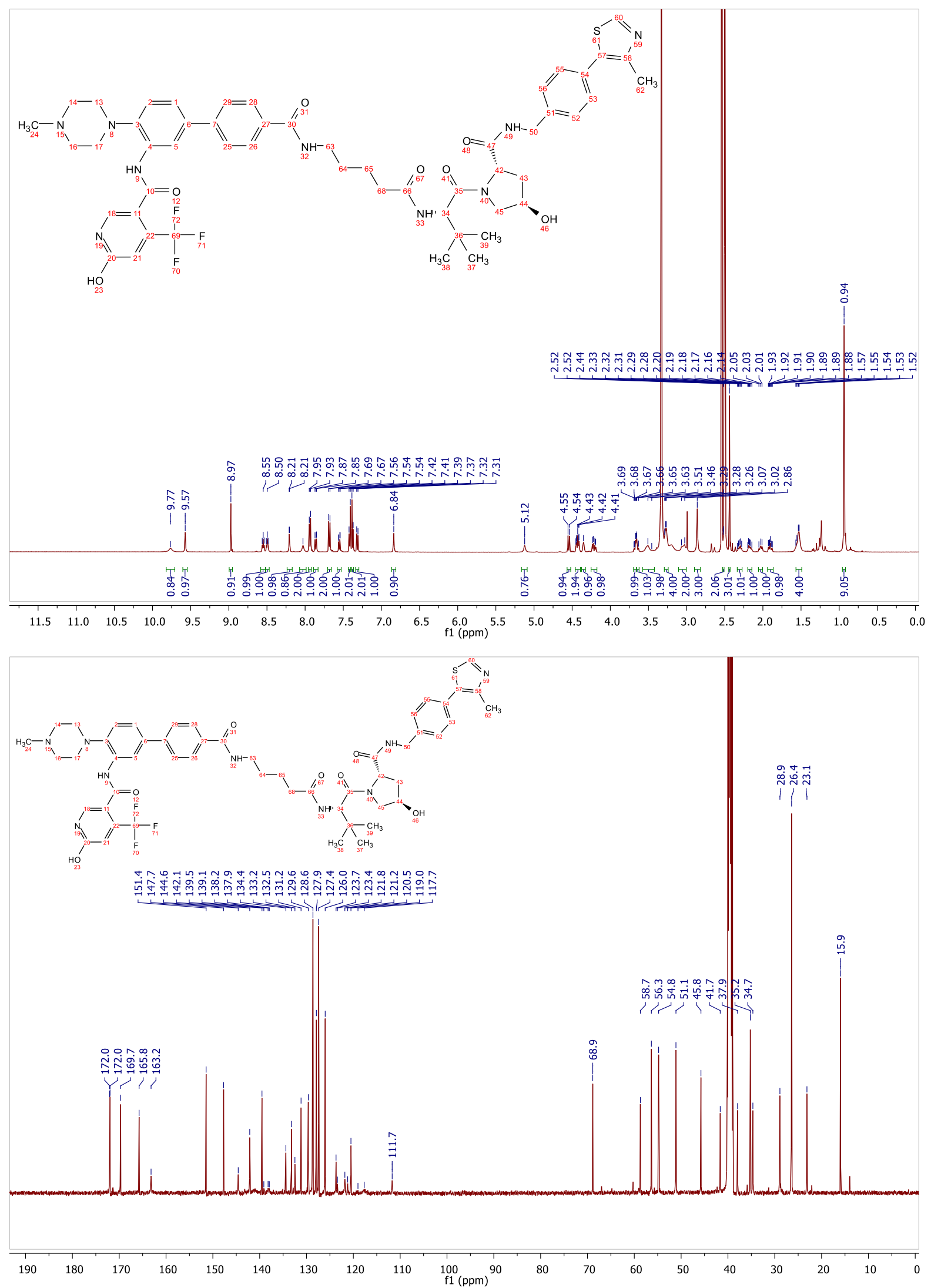

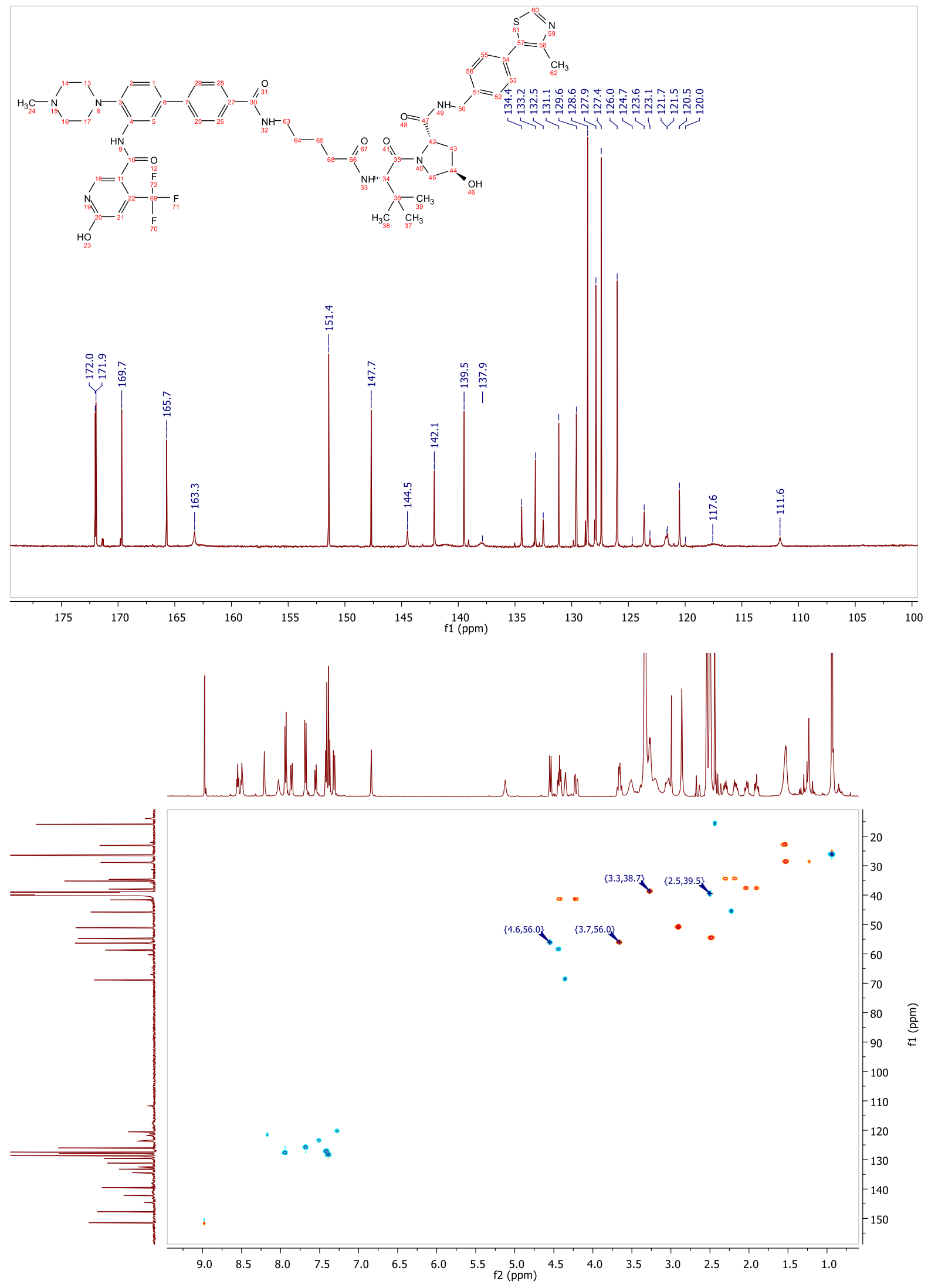


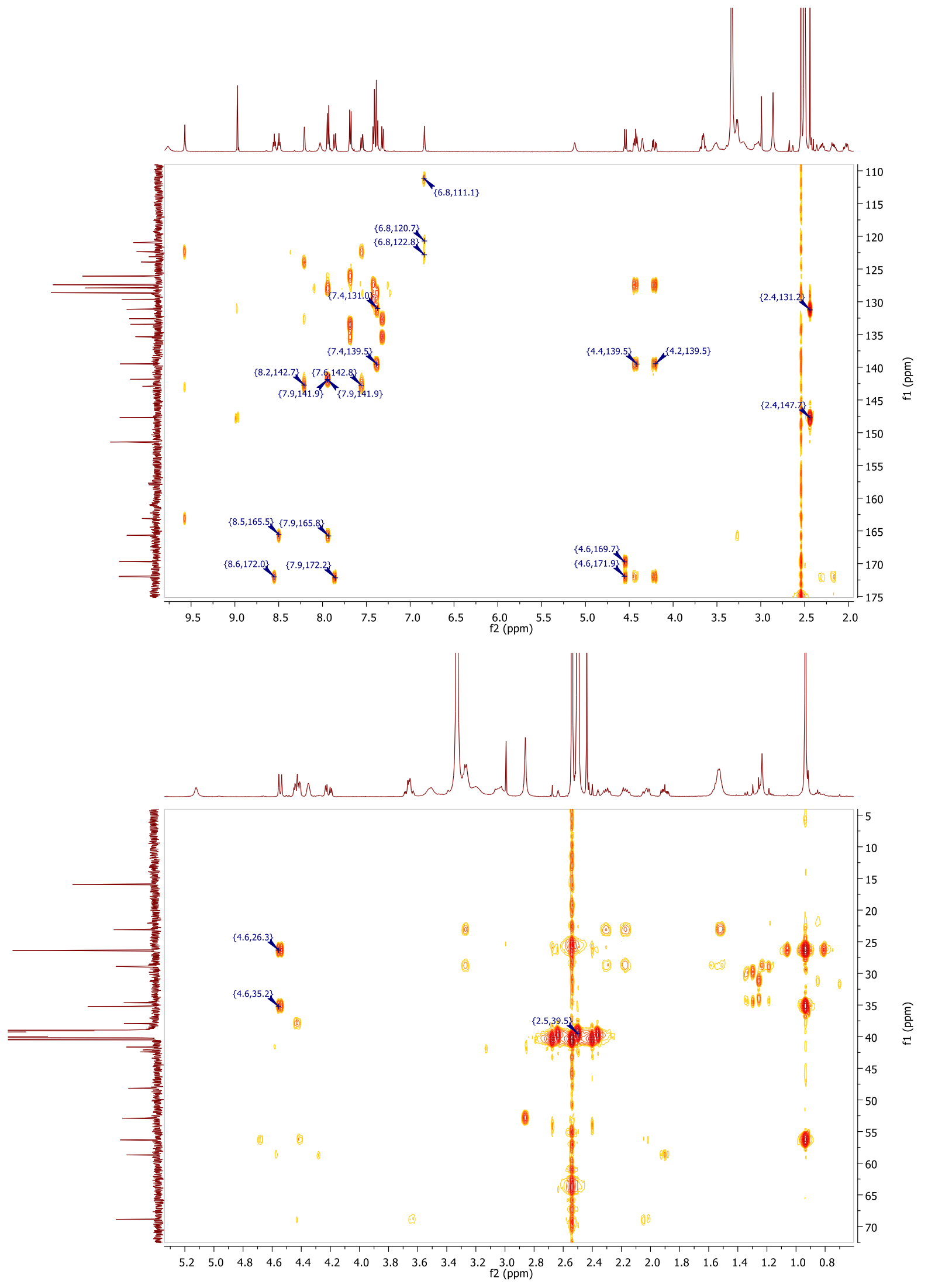


MALDI, HRMS, HPLC, ${ }^{1} \mathrm{H}-\mathrm{NMR}$ and ${ }^{13} \mathrm{C}-\mathrm{NMR}$ of 6-hydroxy-N-(4'-((6-)((S)-1-((2S,4R)-4-hydroxy-2-((4-(4methylthiazol-5-yl)benzyl)carbamoyl)pyrrolidin-1-yl)-3,3-dimethyl-1-oxobutan-2-yl)amino)-6oxohexyl)carbamoyl)-4-(4-methylpiperazin-1-yl)-[1,1'-biphenyl]-3-yl)-4-

(trifluoromethyl)nicotinamide (8h)

Voyager Spec \#1[BP = 137.0, 55410]

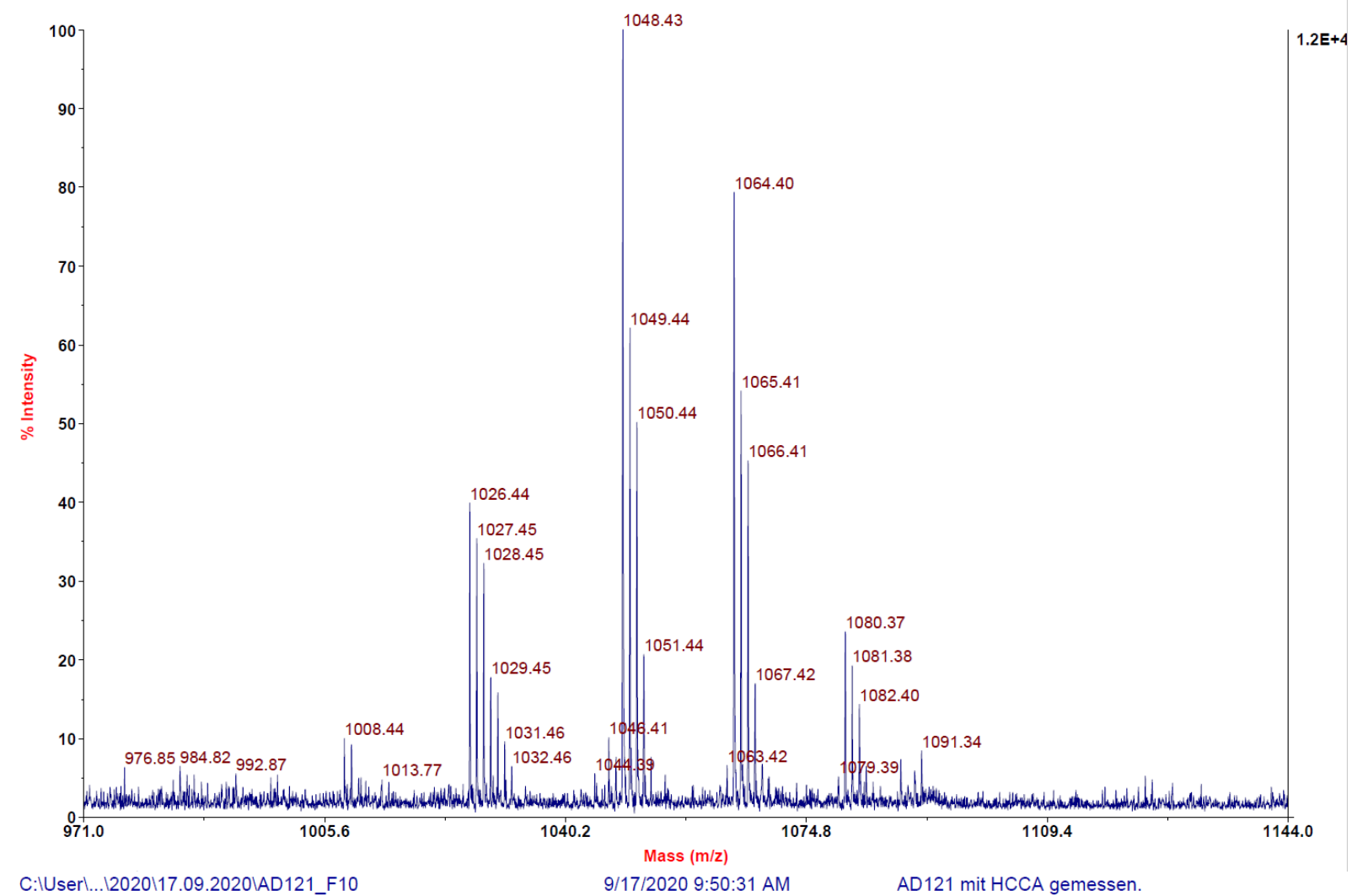

C:IUserl...I2020117.09.20201AD121_F10

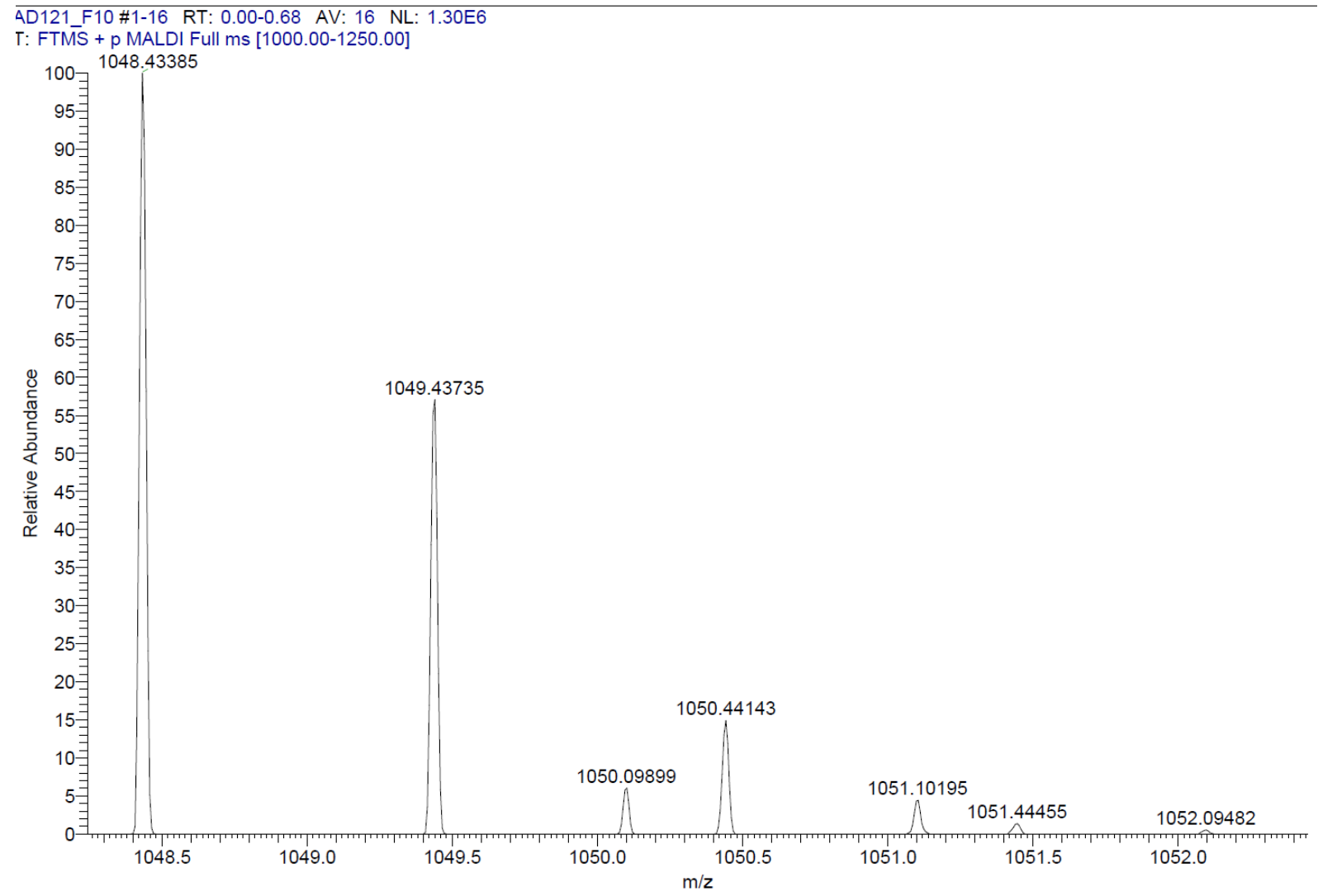

Signal: $\quad$ MWD1 A, Sig=254,4 Ref=off 


$\begin{array}{crrrr}\text { RT [min] Type } & \text { Width [min] } & \text { Area } & \text { Height } & \text { Area\% } \\ 11.385 \text { VV } & 0.2094 & 4595.4873 & 303.9242 & 100.0000 \\ & \text { Sum } & 4595.4873 & & \end{array}$

Signal: $\quad$ MWD1 B, Sig=280,4 Ref=off

$\begin{array}{crrrr}\text { RT [min] Type } & \text { Width [min] } & \text { Area } & \text { Height } & \text { Area\% } \\ 11.385 \text { VV } & 0.2101 & 5472.0347 & 360.5278 & 100.0000 \\ & \text { Sum } & 5472.0347 & & \end{array}$

Signal: $\quad$ MWD1 F, Sig=260,4 Ref=off

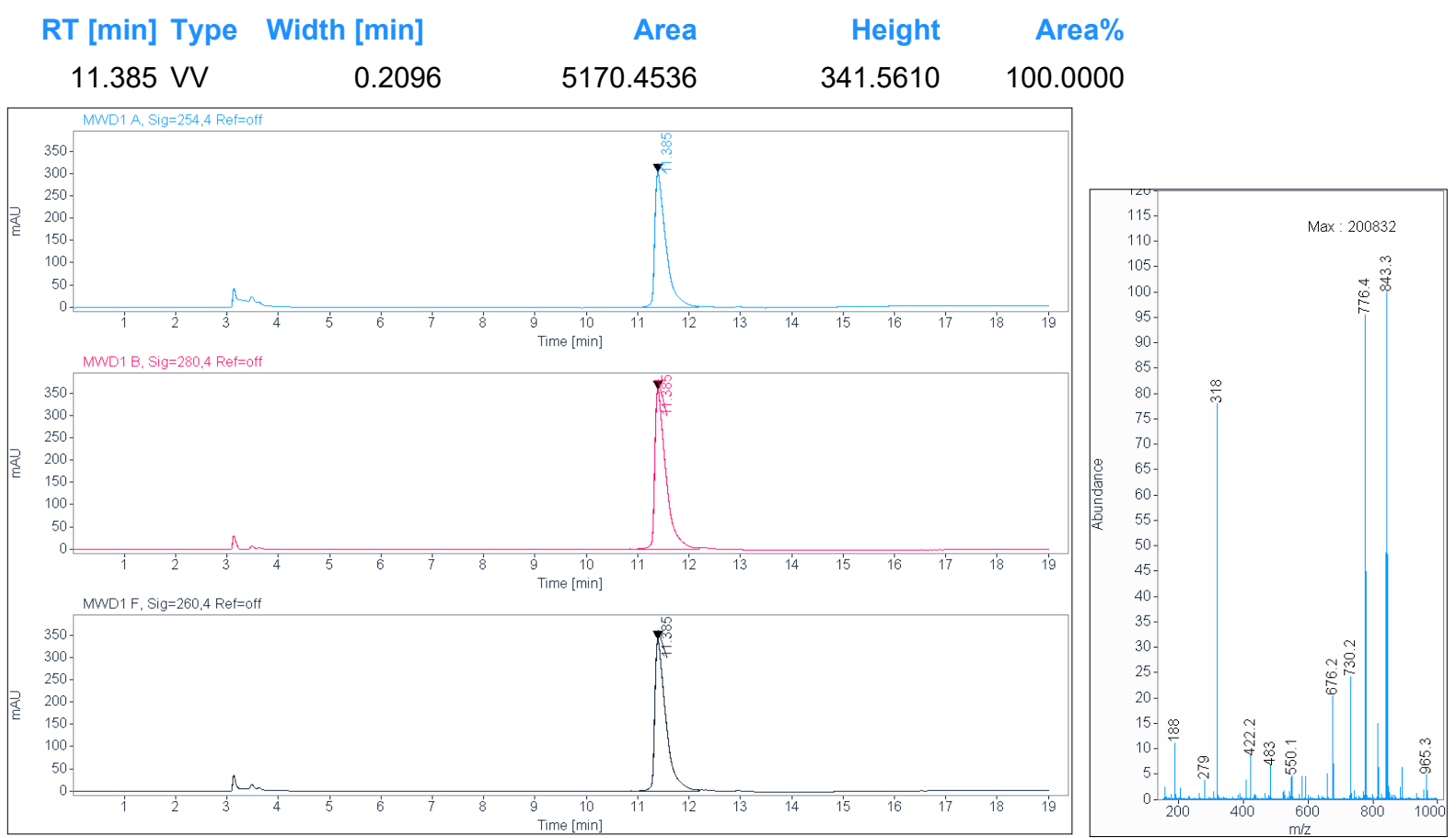



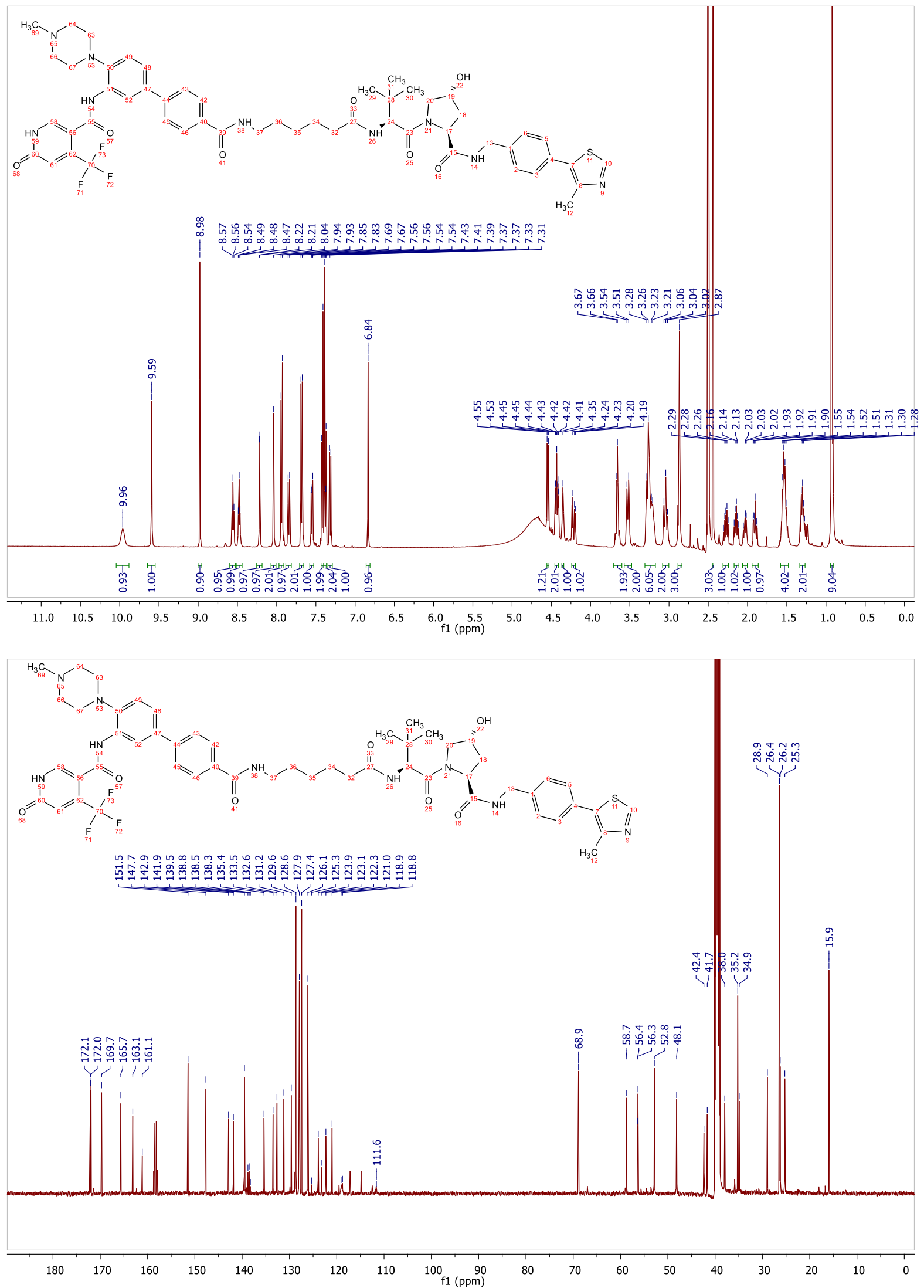
HPLC, MALDI, HRMS, ${ }^{1} \mathrm{H}-\mathrm{NMR},{ }^{13} \mathrm{C}-\mathrm{NMR},{ }^{1} \mathrm{H}-{ }^{13} \mathrm{C}-\mathrm{HSQC}$ and ${ }^{1} \mathrm{H}-{ }^{13} \mathrm{C}-\mathrm{HMBC}$ of 6 -hydroxy-N-(4'-((7-)((S)1-((2S,4R)-4-hydroxy-2-((4-(4-methylthiazol-5-yl)benzyl)carbamoyl)pyrrolidin-1-yl)-3,3-dimethyl-1oxobutan-2-yl)amino)-7-oxoheptyl)carbamoyl)-4-(4-methylpiperazin-1-yl)-[1,1'-biphenyl]-3-yl)-4(trifluoromethyl)nicotinamide (8i)
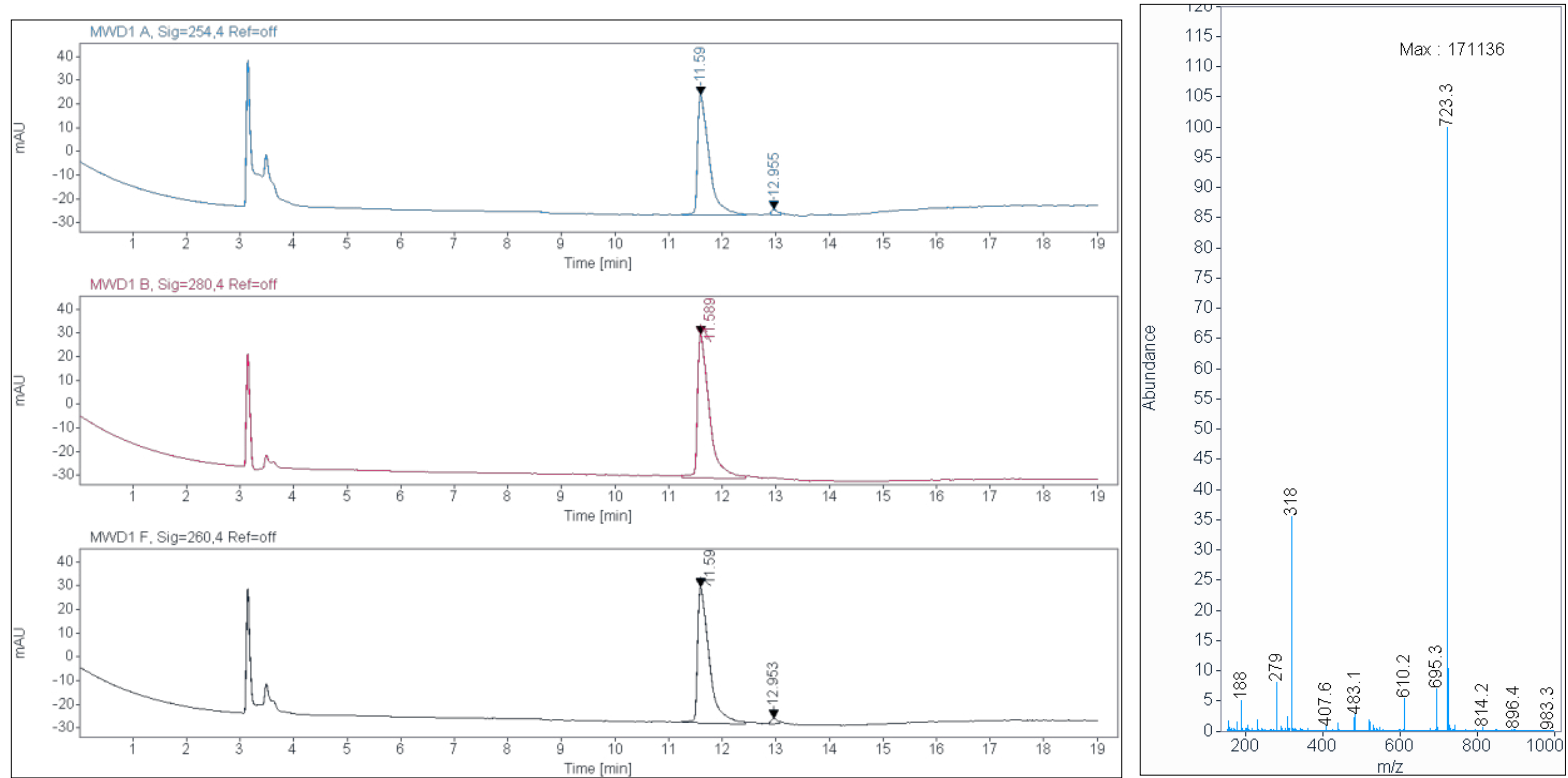

Signal: $\quad$ MWD1 A, Sig=254,4 Ref=off

$\begin{array}{crrrr}\text { RT [min] Type } & \text { Width [min] } & \text { Area } & \text { Height } & \text { Area\% } \\ 11.590 \text { VV } & 0.2077 & 772.4310 & 50.7114 & 97.5053 \\ 12.955 \text { MM } & 0.1361 & 19.7630 & 2.4203 & 2.4947 \\ & \text { Sum } & 792.1941 & & \end{array}$

Signal: $\quad$ MWD1 B, Sig=280,4 Ref=off

$\begin{array}{crrrr}\text { RT [min] Type } & \text { Width [min] } & \text { Area } & \text { Height } & \text { Area\% } \\ 11.589 \text { VV } & 0.2115 & 947.8234 & 60.6039 & 100.0000 \\ & \text { Sum } & 947.8234 & & \end{array}$

Signal: $\quad$ MWD1 F, Sig=260,4 Ref=off

$\begin{array}{crrrr}\text { RT [min] Type } & \text { Width [min] } & \text { Area } & \text { Height } & \text { Area\% } \\ 11.590 \mathrm{VV} & 0.2078 & 879.9773 & 57.4239 & 97.6449 \\ 12.953 \mathrm{MM} & 0.1394 & 21.2239 & 2.5383 & 2.3551\end{array}$


Voyager Spec \#1 [BP = 1041.3, 13031]
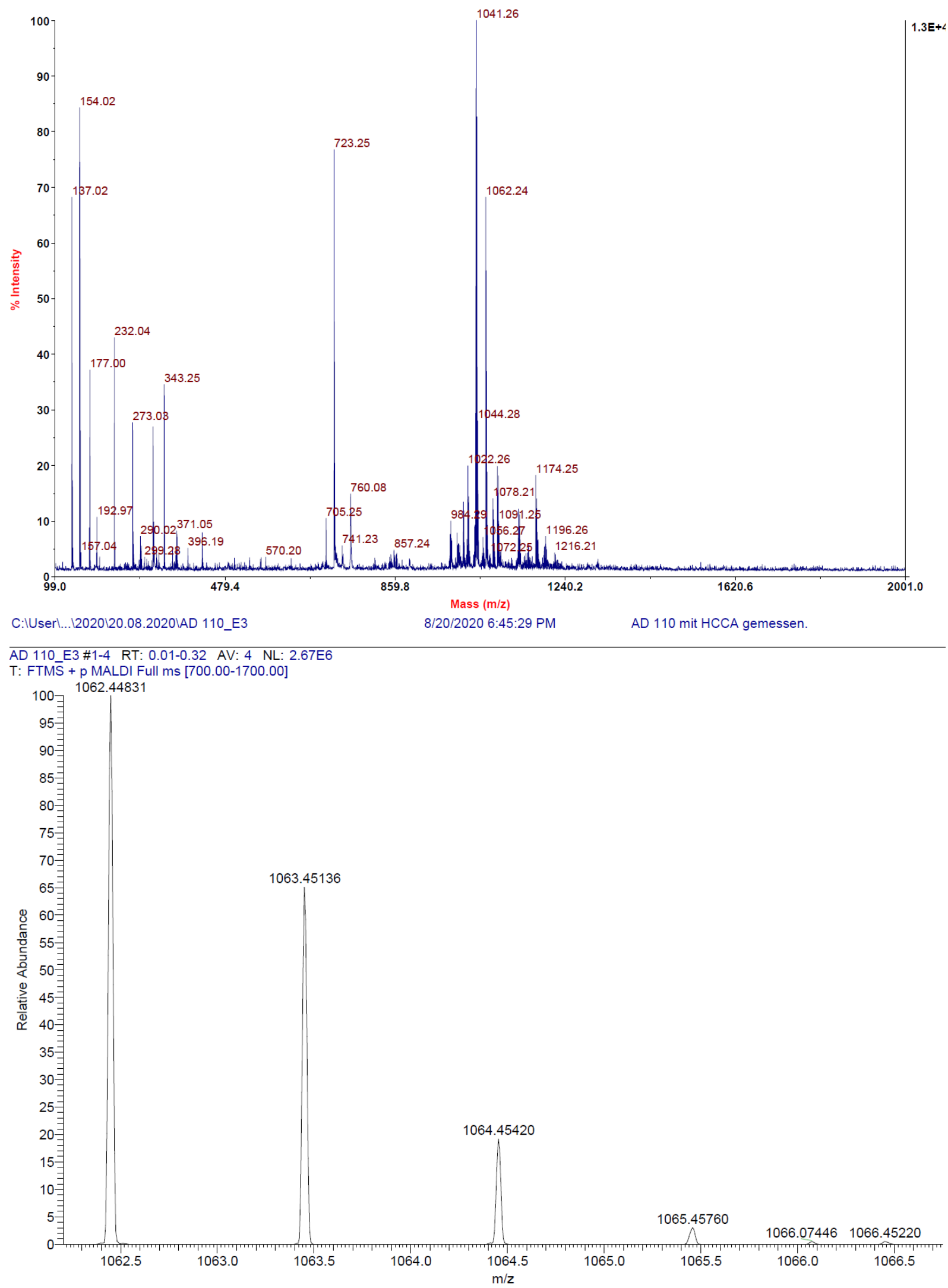

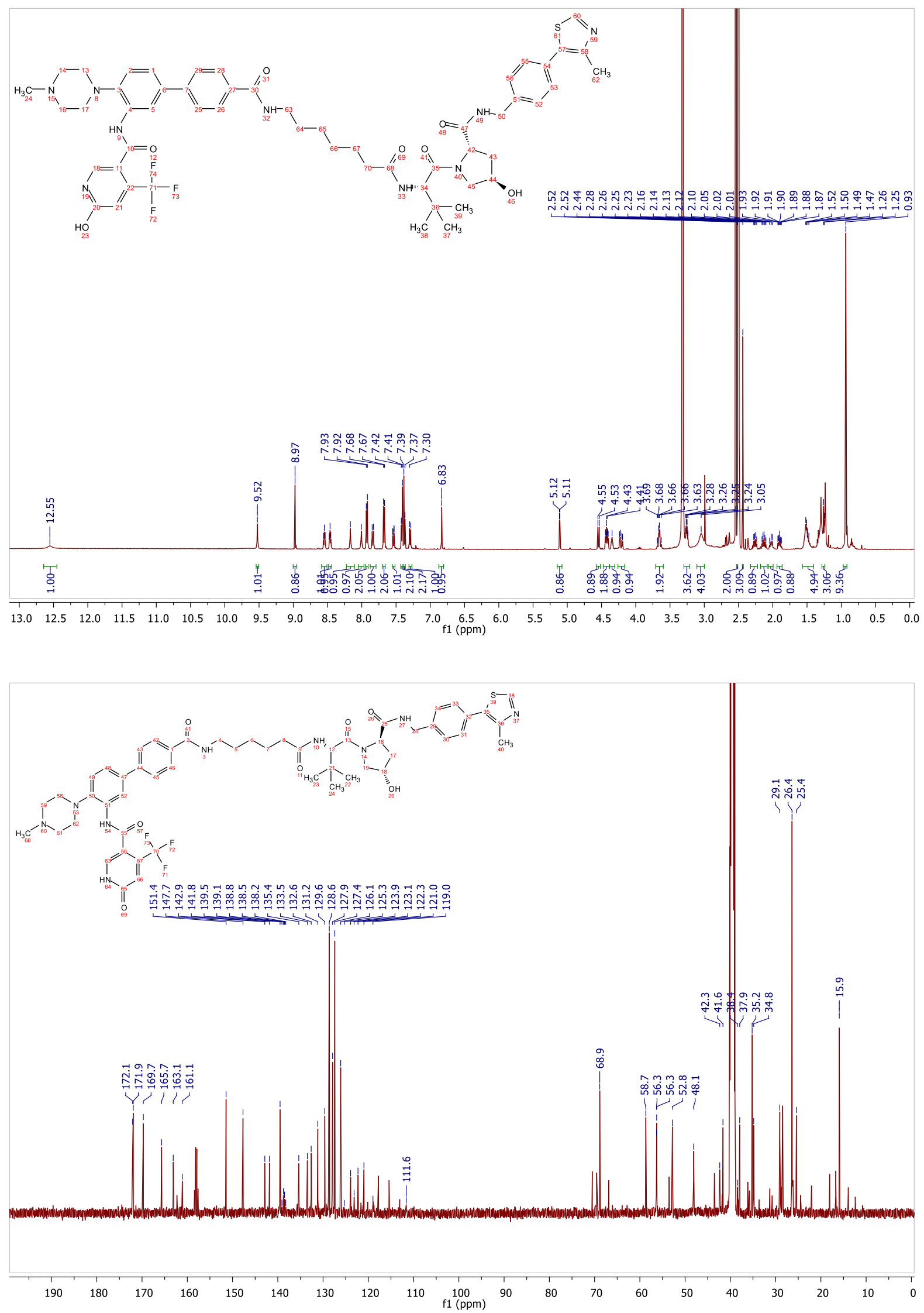

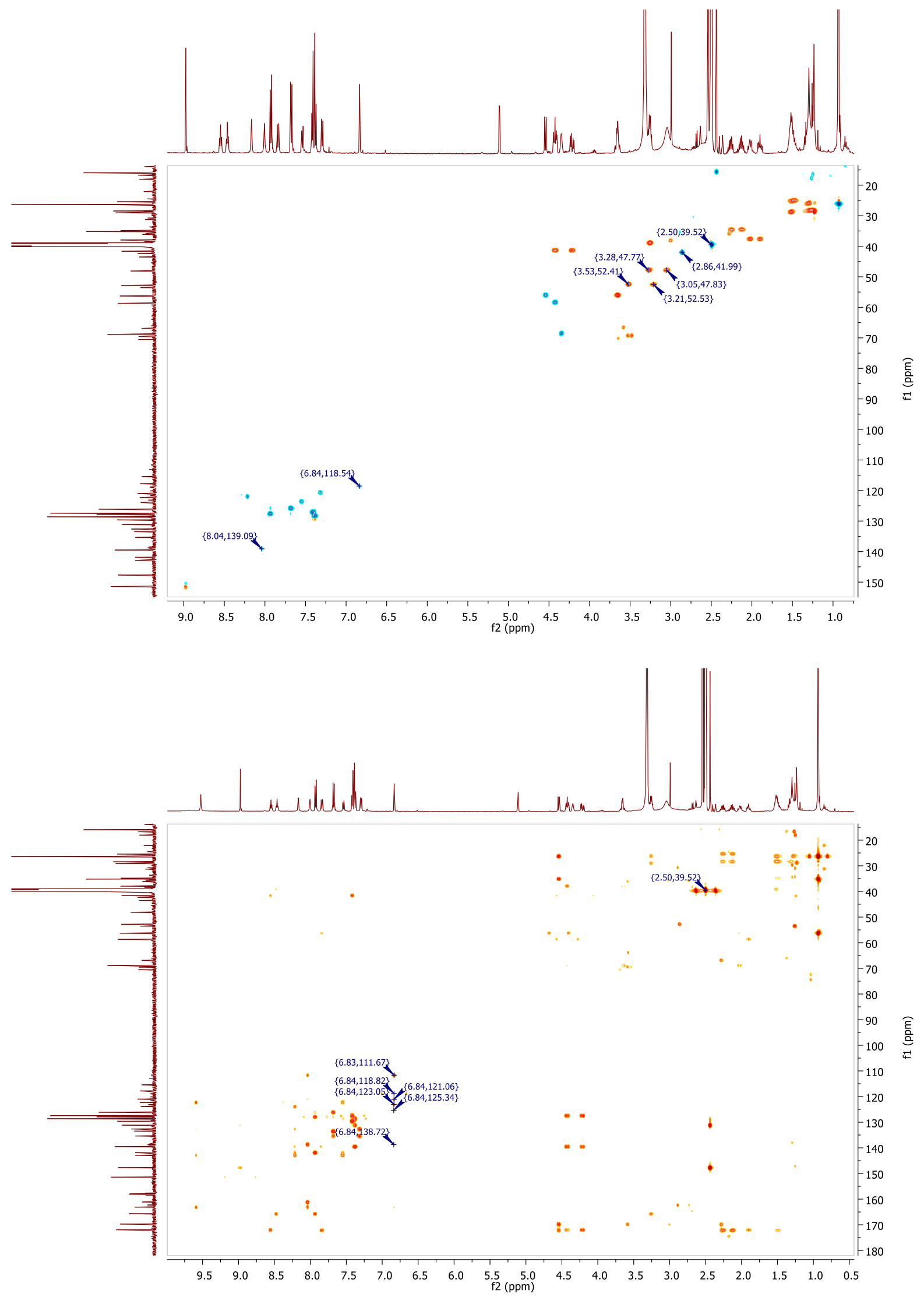
MALDI, HRMS, HPLC, ${ }^{1} \mathrm{H}-\mathrm{NMR},{ }^{13} \mathrm{C}-\mathrm{NMR},{ }^{1} \mathrm{H}-{ }^{13} \mathrm{C}-\mathrm{HSQC}$ and ${ }^{1} \mathrm{H}-{ }^{13} \mathrm{C}-\mathrm{HMBC}$ of 6-hydroxy-N-(4'-((4-)(2(((S)-1-((2S,4R)-4-hydroxy-2-((4-(4-methylthiazol-5-yl)benzyl)carbamoyl)pyrrolidin-1-yl)-3,3dimethyl-1-oxobutan-2-yl)amino)-2-oxoethyl)benzyl)carbamoyl)-4-(4-methylpiperazin-1-yl)-[1,1'biphenyl]-3-yl)-4-(trifluoromethyl)nicotinamide (8j)
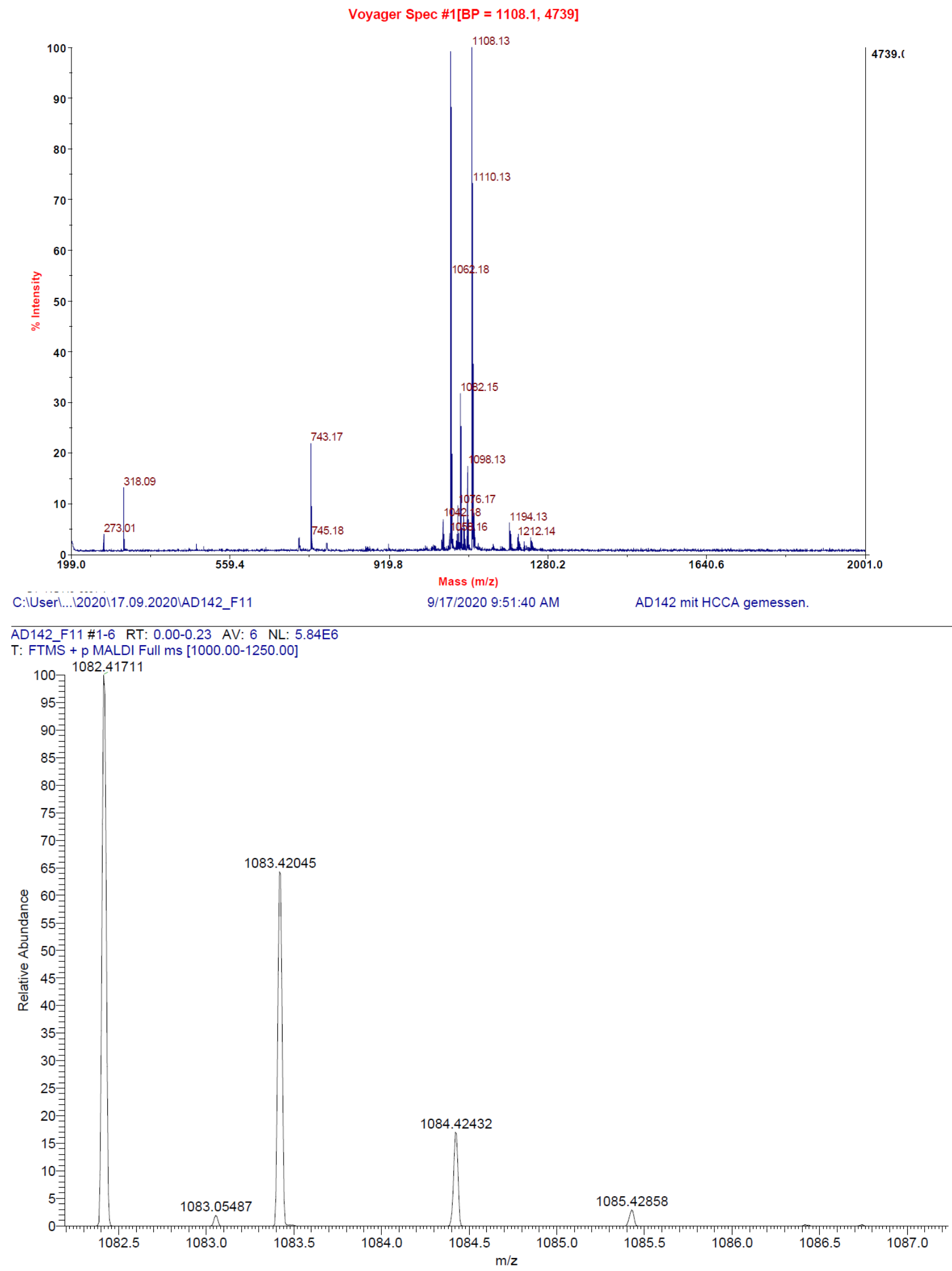
Signal: $\quad$ MWD1 A, Sig=254,4 Ref=off

$\begin{array}{crrrr}\text { RT [min] Type } & \text { Width [min] } & \text { Area } & \text { Height } & \text { Area\% } \\ 11.560 \text { VV } & 0.2134 & 4870.5161 & 308.0758 & 100.0000 \\ & \text { Sum } & 4870.5161 & & \end{array}$

Signal: $\quad$ MWD1 B, Sig=280,4 Ref=off

$\begin{array}{crrrr}\text { RT [min] Type } & \text { Width [min] } & \text { Area } & \text { Height } & \text { Area\% } \\ 11.560 \mathrm{VV} & 0.2151 & 5846.5635 & 366.4924 & 100.0000 \\ & \text { Sum } & 5846.5635 & & \end{array}$

Signal: $\quad$ MWD1 $\mathrm{F}, \mathrm{Sig}=260,4 \mathrm{Ref}=\mathrm{off}$

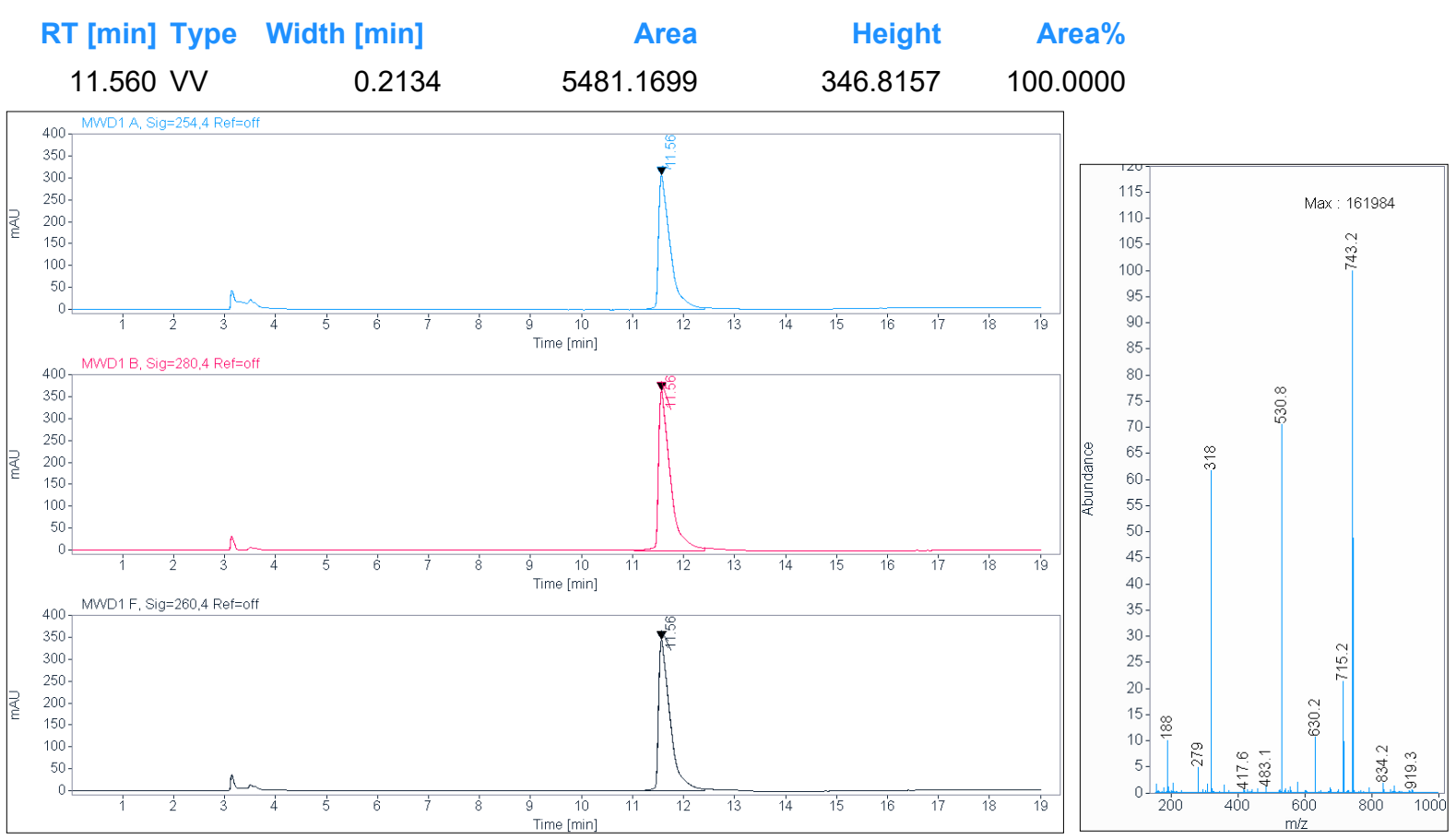



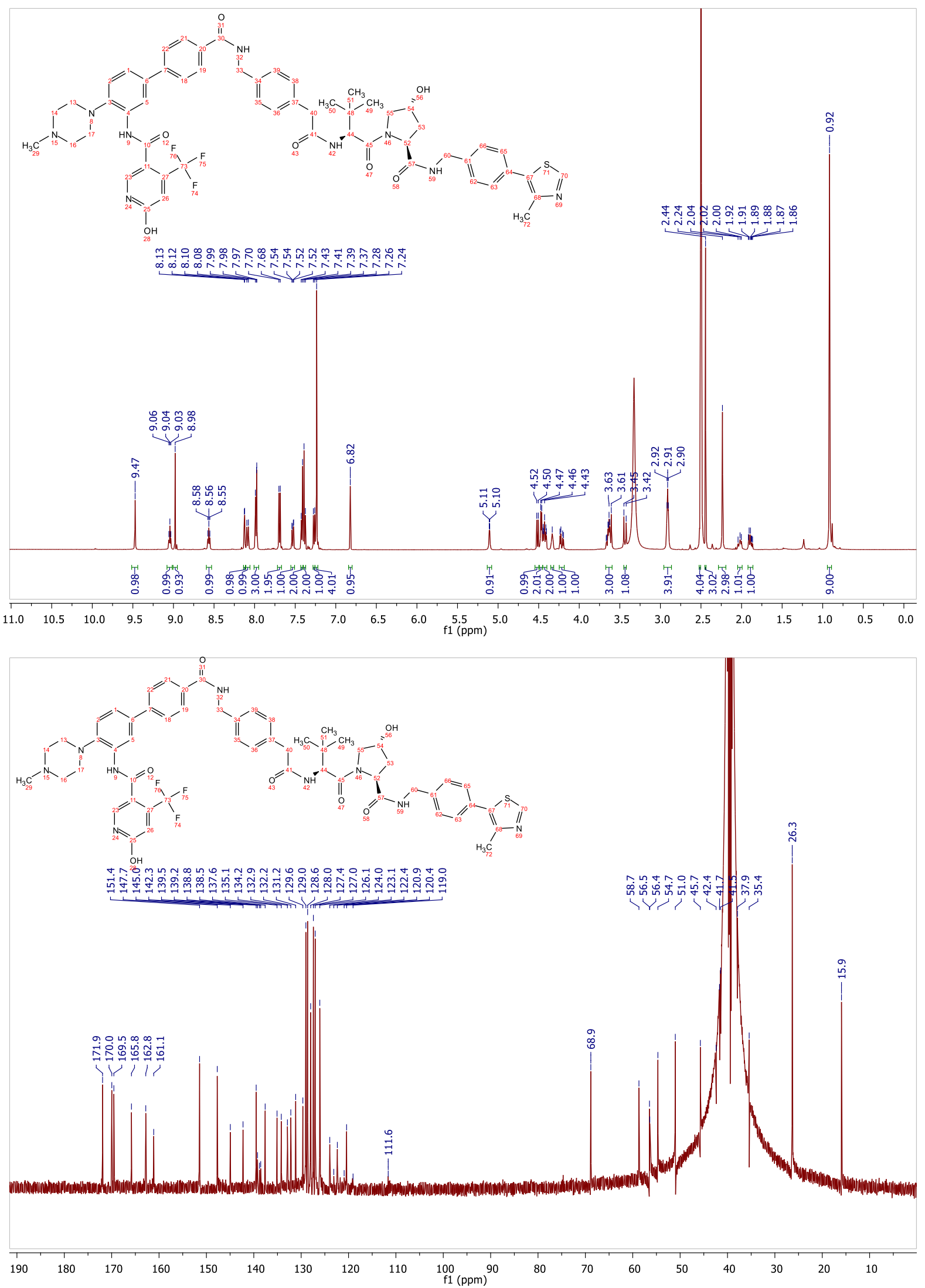

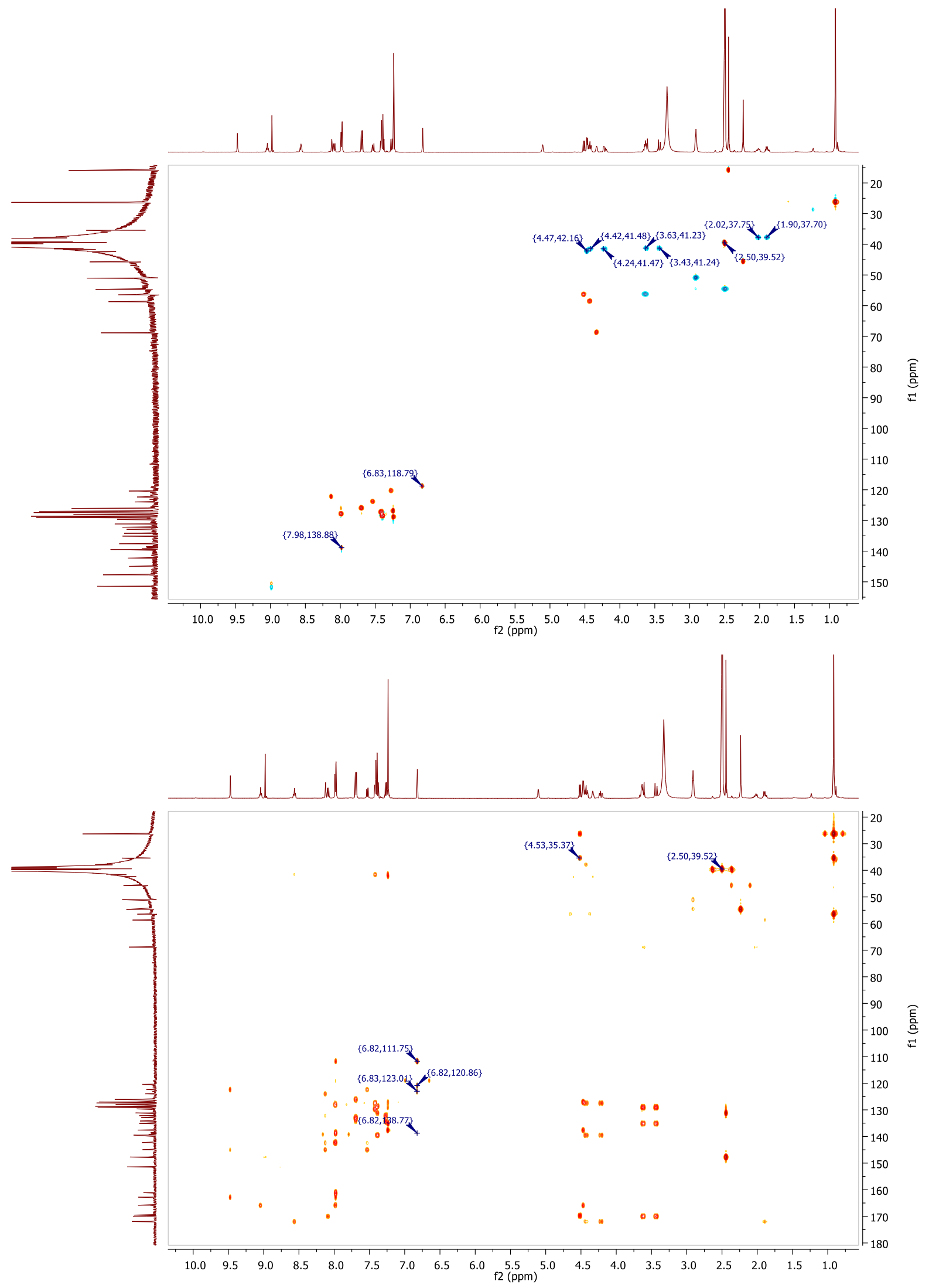
MALDI, HRMS, HPLC and ${ }^{1} \mathrm{H}-\mathrm{NMR}$ of $\mathrm{N}-(4$ '-((2-(2-(4-)((2R,3S,4R,5S)-3-(3-chloro-2-fluorophenyl)-4-(4chloro-2-fluorophenyl)-4-cyano-5-neopentylpyrrolidine-2-carboxamido)-3methoxybenzamido)ethoxy)ethyl)carbamoyl)-4-(4-methylpiperazin-1-yl)-[1,1'-biphenyl]-3-yl)-6hydroxy-4-(trifluoromethyl)nicotinamide (9a)

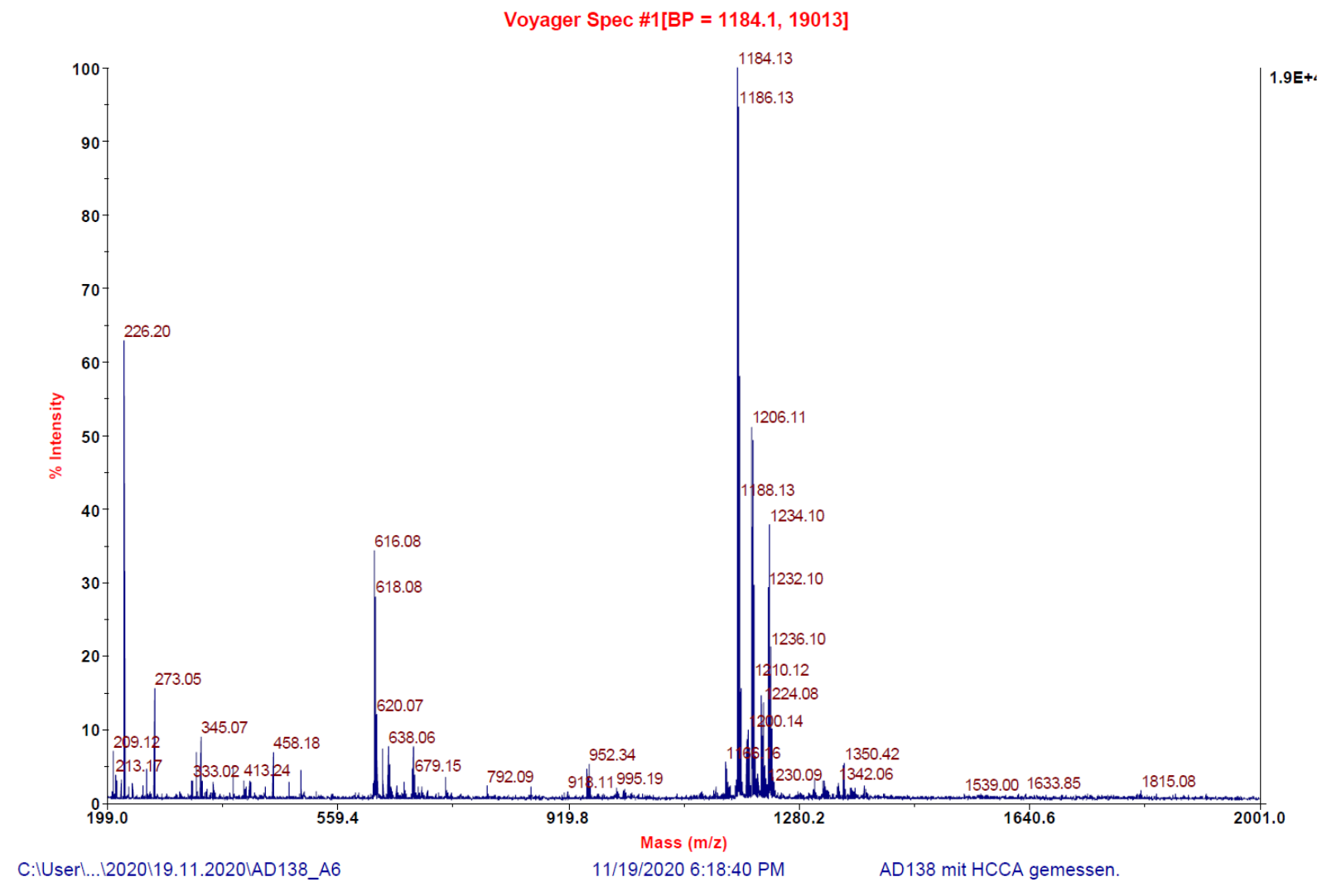

AD138_A6 \#1-20 RT: 0.00-1.29 AV: 20 NL: 4.85E4

T: FTMS + p MALDI Full ms [800.00-1500.00]

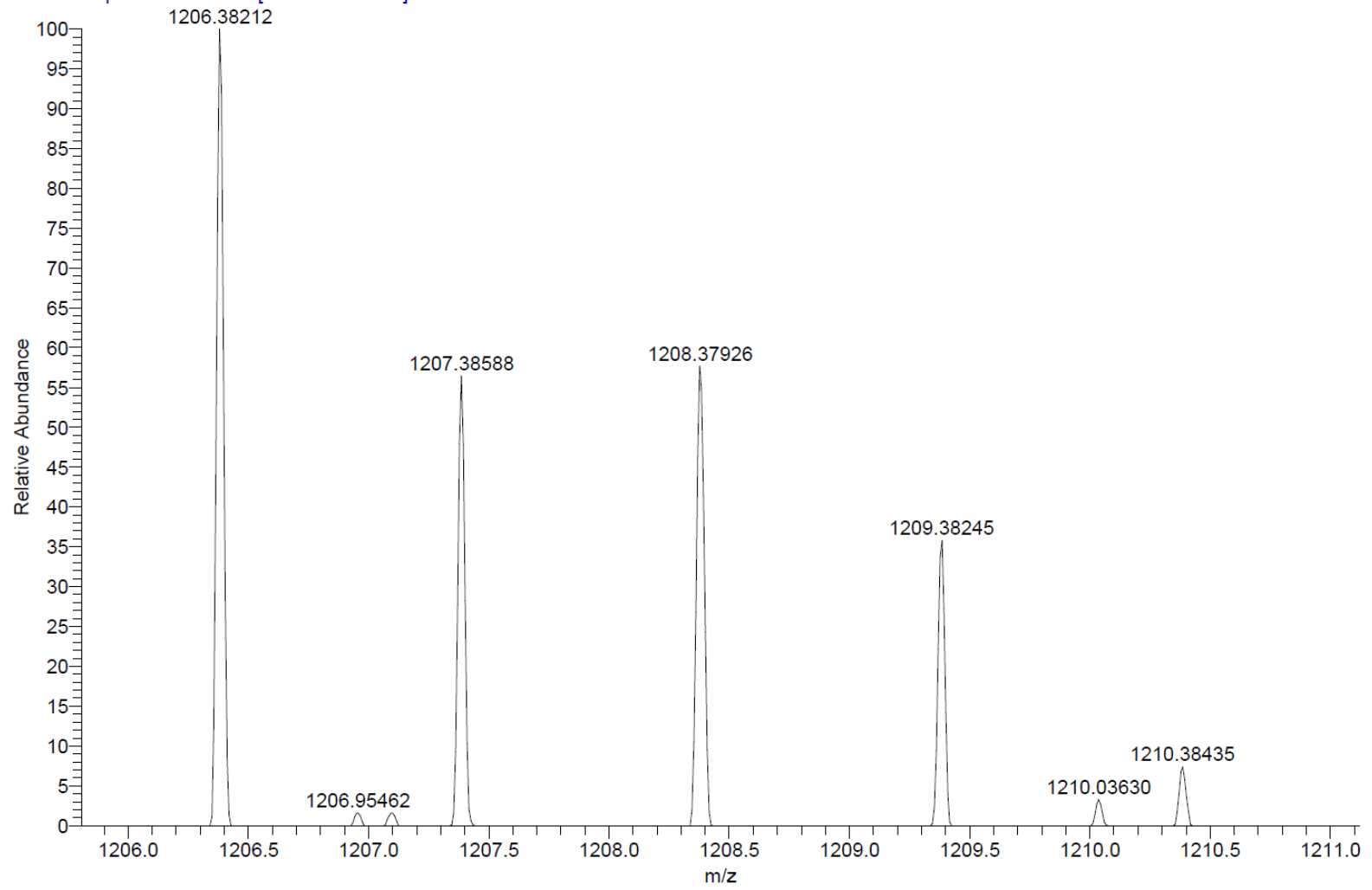

Signal: $\quad$ MWD1 A, Sig $=254,4$ Ref $=$ off 


$\begin{array}{crrr}\text { RT [min] Type } & \text { Width [min] } & \text { Area } & \text { Height Area\% } \\ 12.966 \mathrm{MM} & 0.1809 & 45.8666 & 4.22691 .5833 \\ 14.363 \mathrm{VV} & 0.2753 & 2851.0457 & 147.633598 .4167 \\ & \text { Sum } & 2896.9123 & \end{array}$

Signal: $\quad$ MWD1 B, Sig=280,4 Ref=off

$\begin{array}{crrr}\text { RT [min] Type } & \text { Width [min] } & \text { Area } & \text { Height Area\% } \\ 12.960 \mathrm{MM} & 0.2518 & 15.9346 & 1.05460 .5114 \\ 14.363 \mathrm{VV} & 0.2623 & 3099.7249 & 162.661699 .4886 \\ & \text { Sum } & 3115.6594 & \end{array}$

Signal: $\quad$ MWD1 F, Sig=260,4 Ref=off

$\begin{array}{crrr}\text { RT [min] Type } & \text { Width [min] } & \text { Area } & \text { Height Area\% } \\ 12.962 \mathrm{MM} & 0.2060 & 54.8831 & 4.44131 .5802 \\ 14.363 \mathrm{VV} & 0.2919 & 3418.3169 & 178.098098 .4198\end{array}$

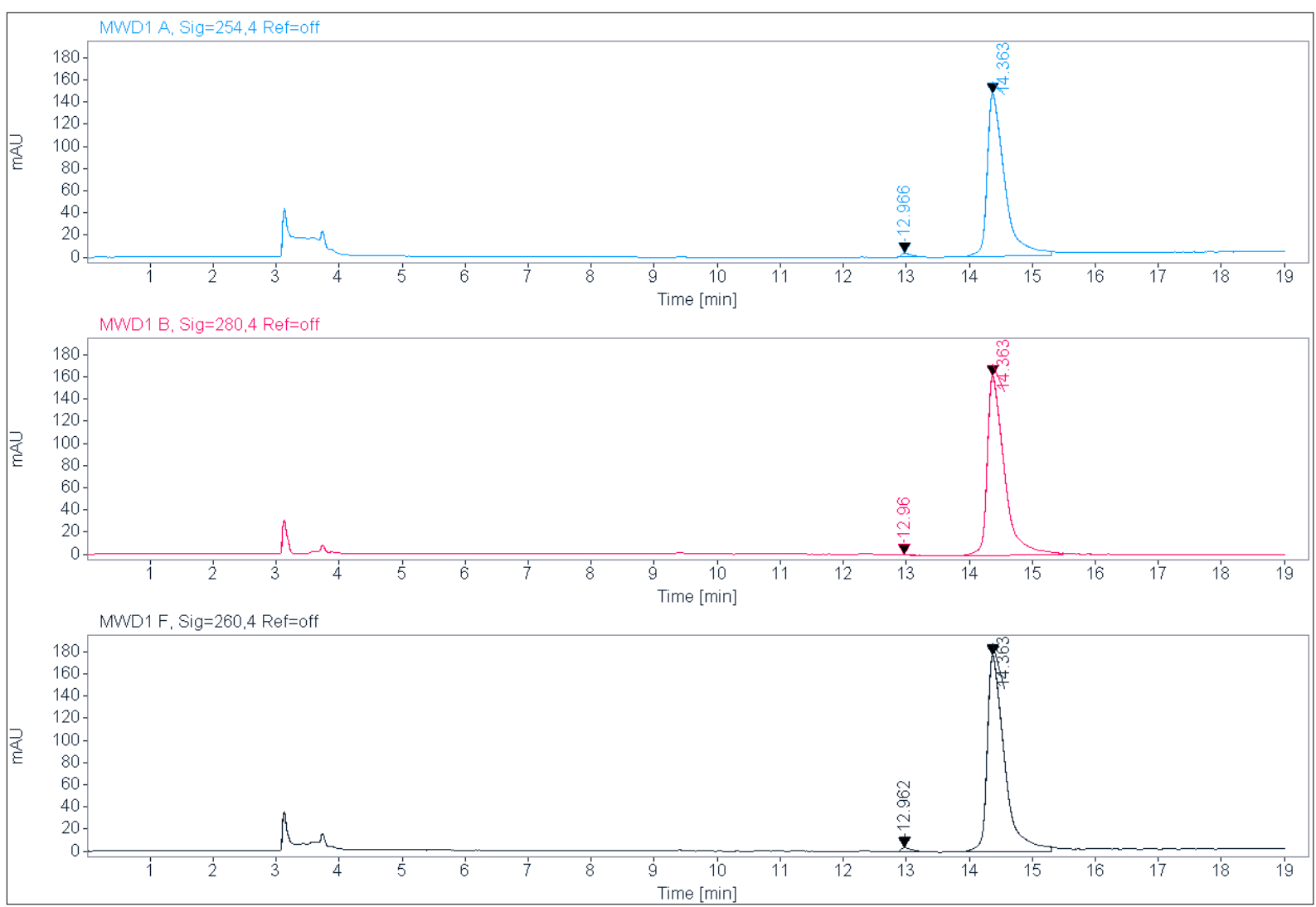




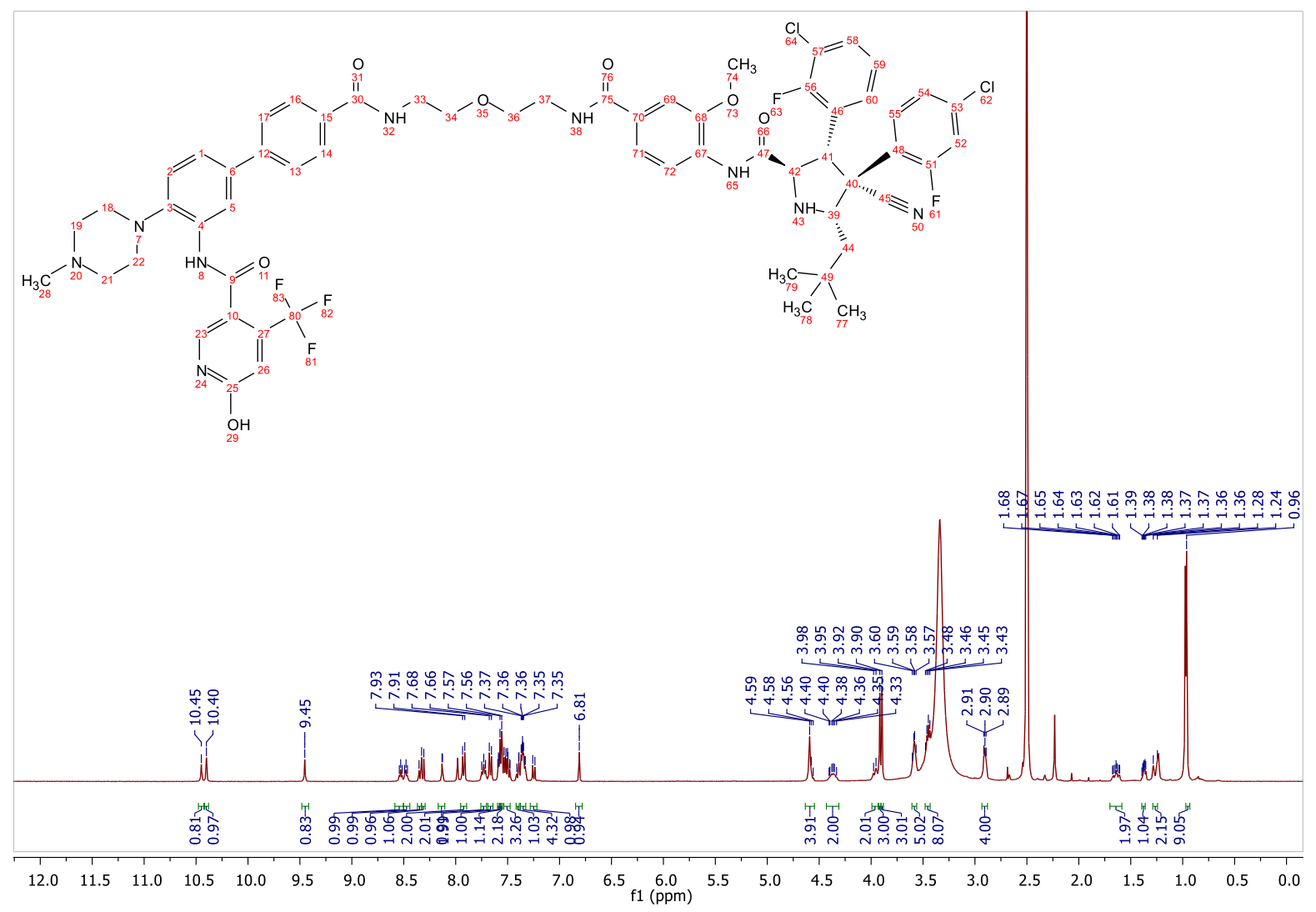


MALDI, HRMS, HPLC and ${ }^{1} \mathrm{H}-\mathrm{NMR}$ of $\mathrm{N}$-(4'-((1-(4-)((2R,3S,4R,5S)-3-(3-chloro-2-fluorophenyl)-4-(4chloro-2-fluorophenyl)-4-cyano-5-neopentylpyrrolidine-2-carboxamido)-3-methoxyphenyl)-1-oxo$5,8,11,14,17,20,23$-heptaoxa-2-azapentacosan-25-yl)carbamoyl)-4-(4-methylpiperazin-1-yl)-[1,1'biphenyl]-3-yl)-6-hydroxy-4-(trifluoromethyl)nicotinamide (9b)

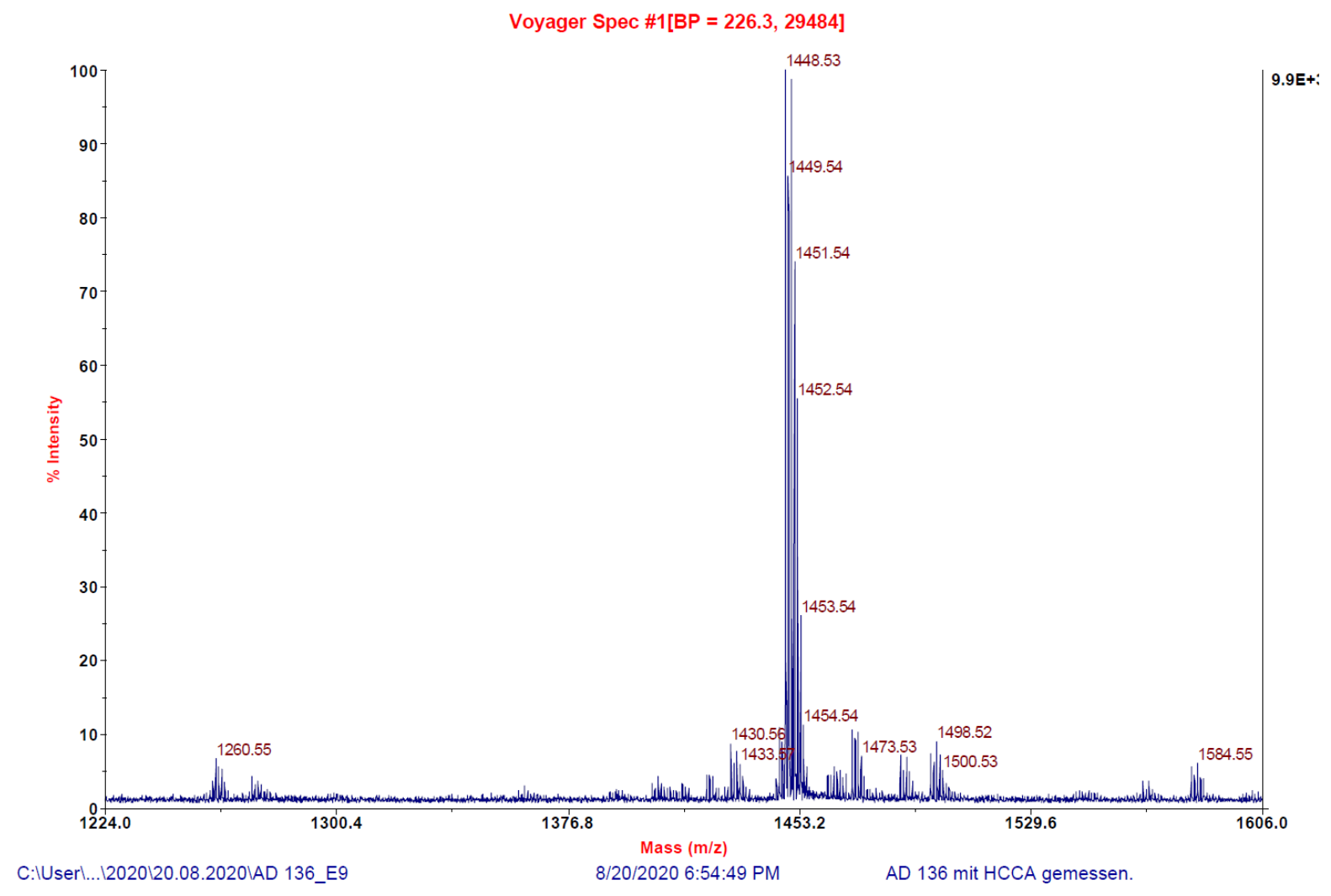

AD 136_E9 \#1-16 RT: 0.00-1.43 AV: $16 \quad$ NL: 8.09E5

T: FTMS + p MALDI Full ms [700.00-1700.00]

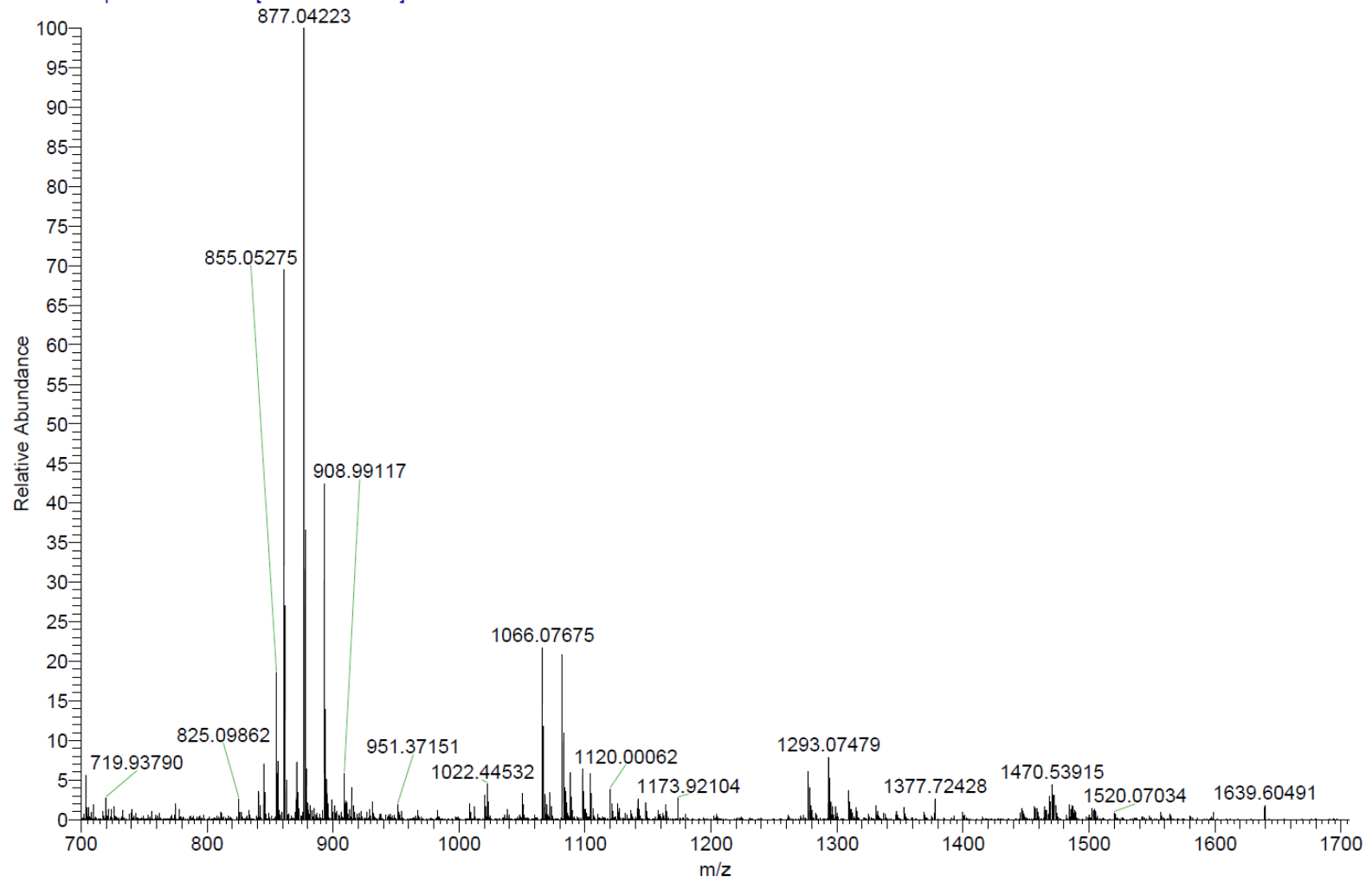


C:IUserl...12020120.08.2020\AD 136_E9

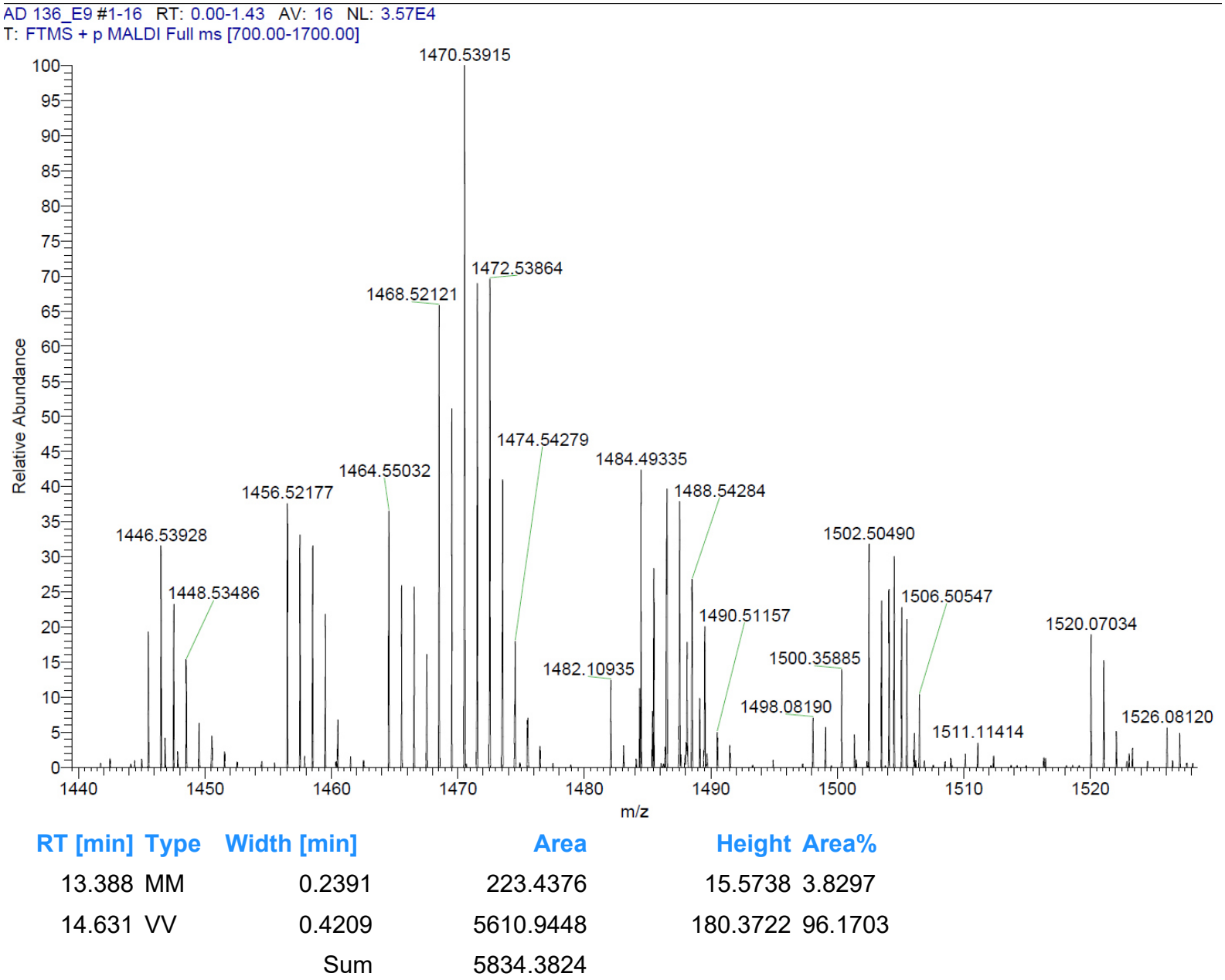

Signal: $\quad$ MWD1 B, Sig=280,4 Ref=off

$\begin{array}{crrr}\text { RT [min] Type } & \text { Width [min] } & \text { Area } & \text { Height Area\% } \\ 13.385 \mathrm{MM} & 0.1997 & 178.9707 & 14.93363 .1186 \\ 14.630 \mathrm{VV} & 0.3882 & 5559.8906 & 195.256996 .8814 \\ & \text { Sum } & 5738.8614 & \end{array}$

Signal: $\quad$ MWD1 F, Sig $=260,4$ Ref $=$ off

$\begin{array}{ccrr}\text { RT [min] Type } & \text { Width [min] } & \text { Area } & \text { Height Area\% } \\ 13.386 \mathrm{MM} & 0.2384 & 306.7542 & 21.44234 .6729 \\ 14.631 \mathrm{VV} & 0.4019 & 6257.7744 & 214.151295 .3271\end{array}$



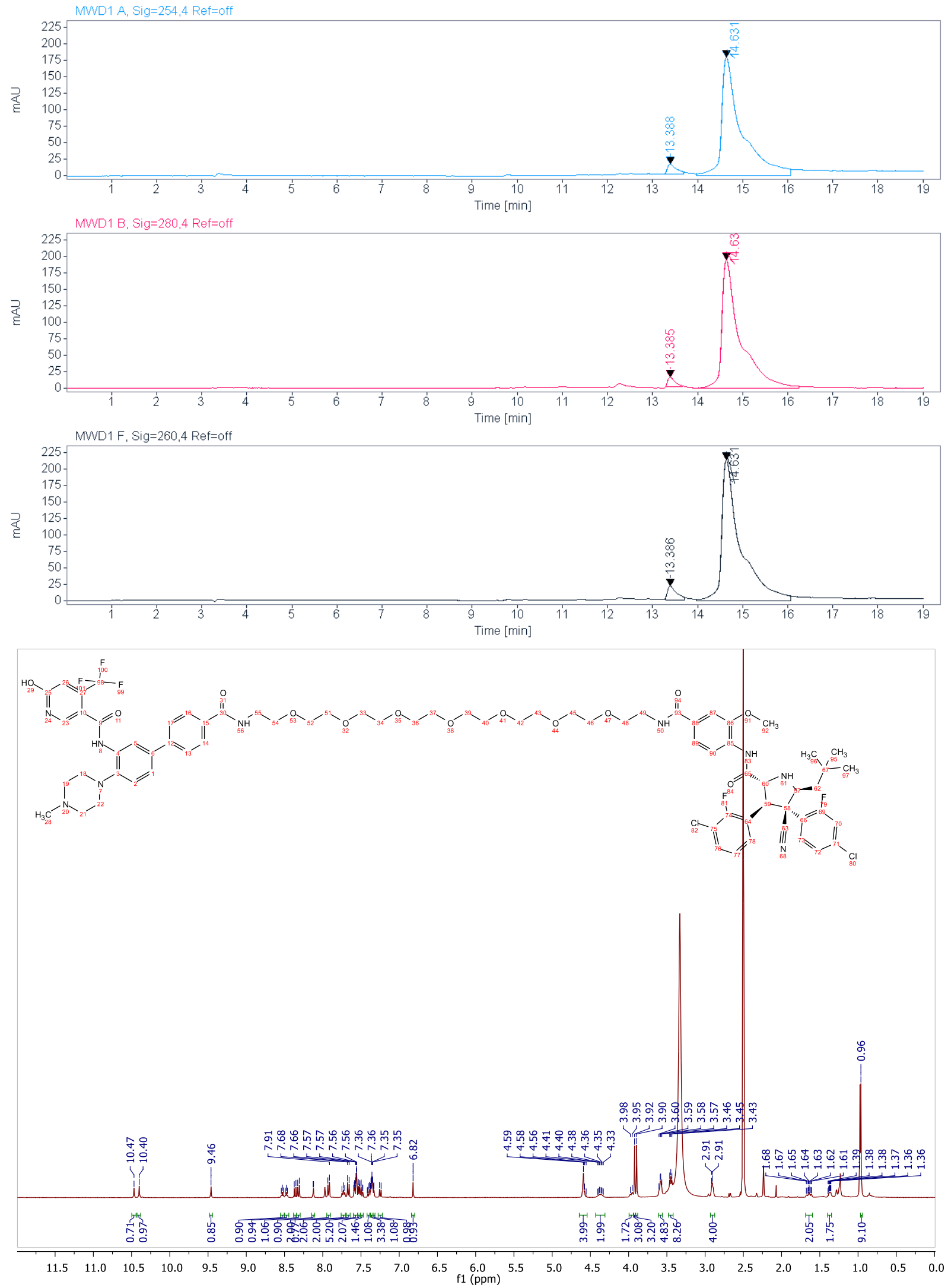
MALDI, HRMS, HPLC, ${ }^{1} \mathrm{H}-\mathrm{NMR}$ of N-(4'-((4-)((4-)((2R,3S,4R,5S)-3-(3-chloro-2-fluorophenyl)-4-(4-chloro2-fluorophenyl)-4-cyano-5-neopentylpyrrolidine-2-carboxamido)-3methoxybenzamido)methyl)benzyl)carbamoyl)-4-(4-methylpiperazin-1-yl)-[1,1'-biphenyl]-3-yl)-6hydroxy-4-(trifluoromethyl)nicotinamide (9c)

Voyager Spec \#1 [BP = 242.3, 58541]

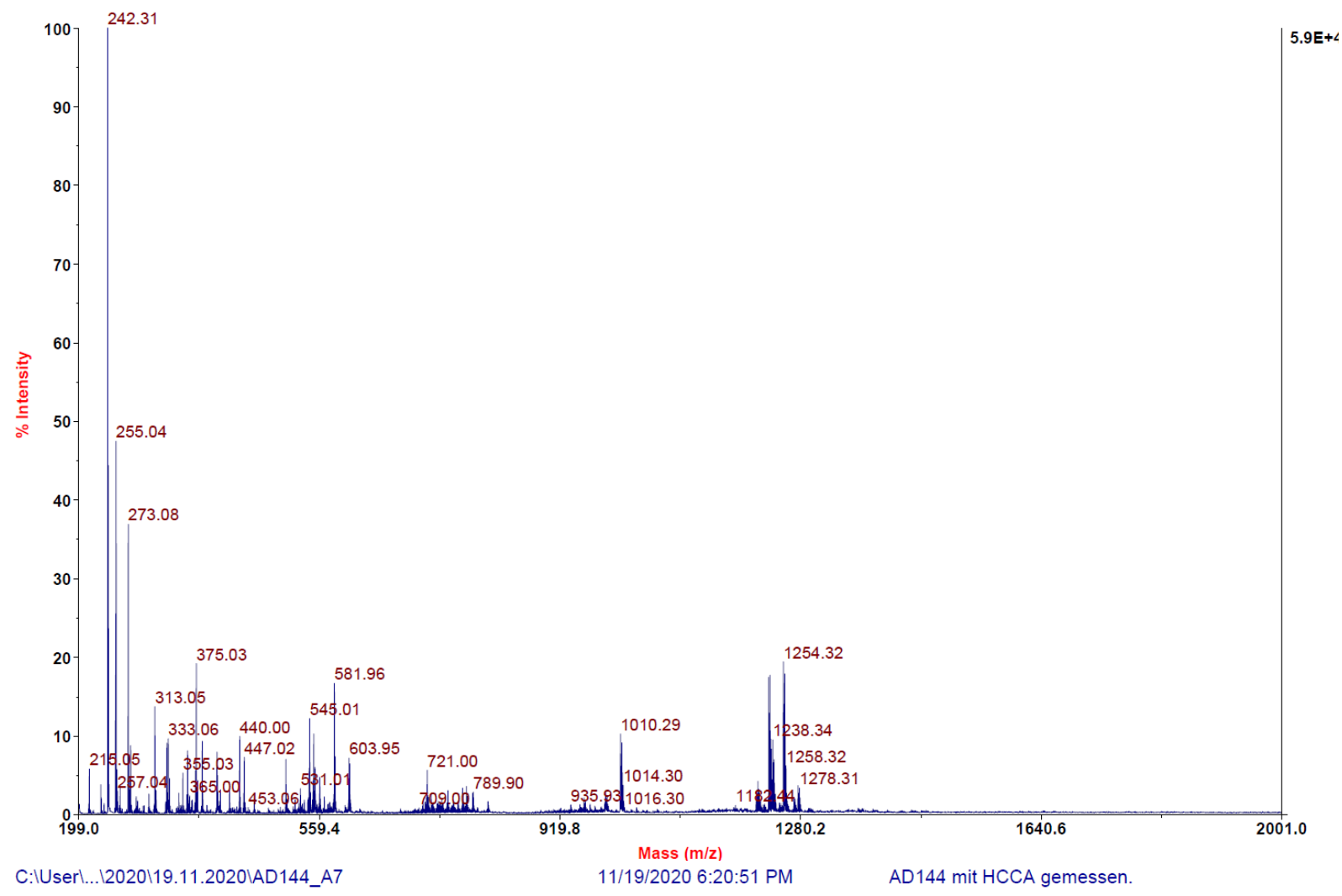

AD144_A7 \#1-19 RT: 0.01-0.96 AV: 19 NL: 5.84E4

AD144_A7 \#1-19 RT: $0.01-0.96$ AV: 19 NL:
T: FTMS + p MALDI Full ms [800.00-1500.00]

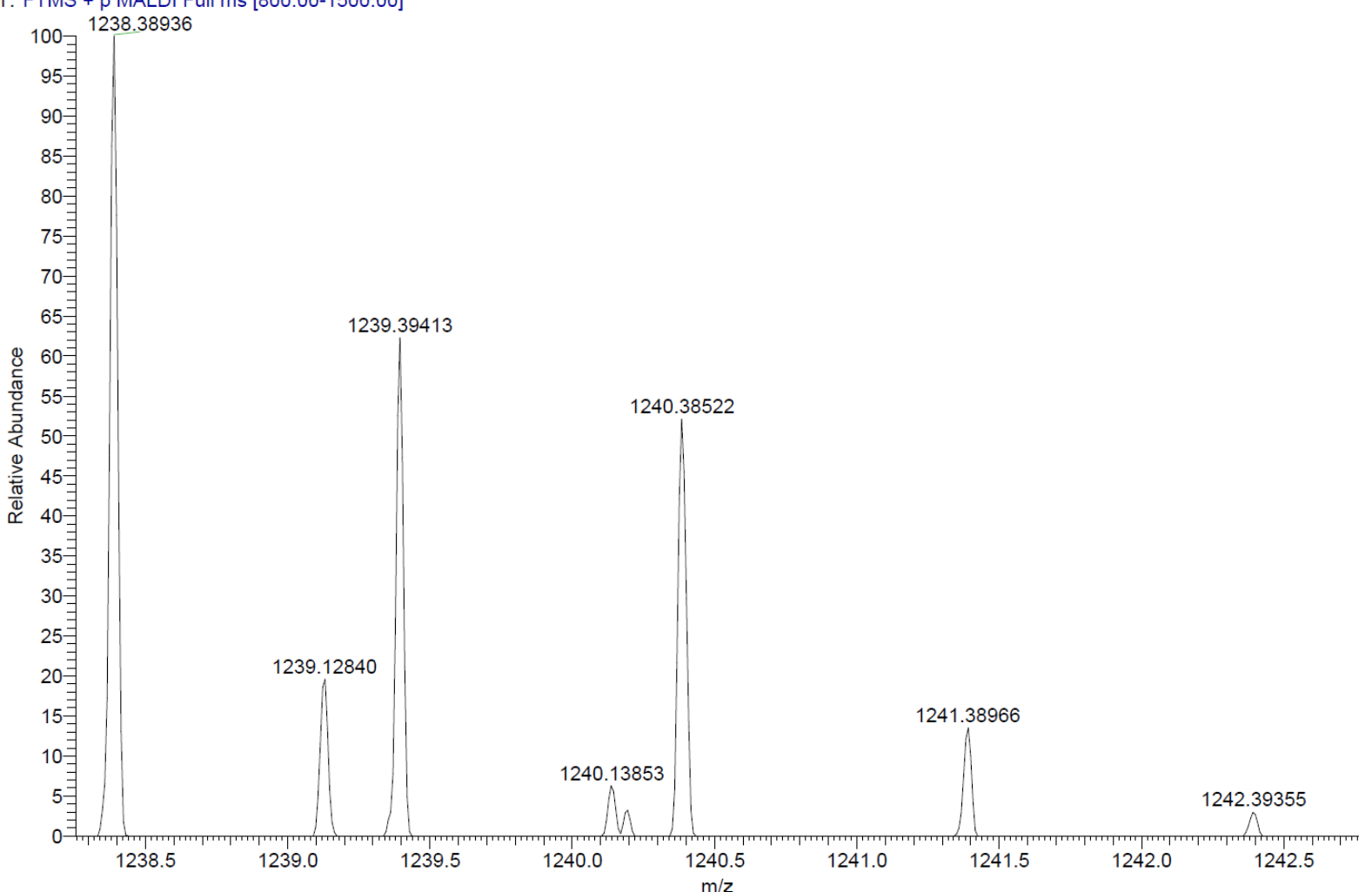

Signal: $\quad$ MWD1 A, Sig=254,4 Ref=off 


$\begin{array}{crrrr}\text { RT [min] Type } & \text { Width [min] } & \text { Area } & \text { Height } & \text { Area\% } \\ 12.962 \mathrm{MM} & 0.1568 & 35.1322 & 3.7335 & 1.8300 \\ 14.724 \mathrm{VV} & 0.3252 & 1884.6840 & 75.5157 & 98.1700 \\ & \text { Sum } & 1919.8162 & & \end{array}$

Signal: $\quad$ MWD1 B, Sig=280,4 Ref=off

$\begin{array}{crrrr}\text { RT [min] Type } & \text { Width [min] } & \text { Area } & \text { Height } & \text { Area\% } \\ 14.722 \text { VV } & 0.3226 & 2160.1218 & 87.6254 & 100.0000 \\ & \text { Sum } & 2160.1218 & & \end{array}$

Signal: $\quad$ MWD1 F, Sig=260,4 Ref=off

$\begin{array}{crrrr}\text { RT [min] Type } & \text { Width [min] } & \text { Area } & \text { Height } & \text { Area\% } \\ 12.967 \text { MM } & 0.1599 & 33.1664 & 3.4567 & 1.4688 \\ 14.724 \text { VV } & 0.3246 & 2224.9216 & 89.0201 & 98.5312\end{array}$

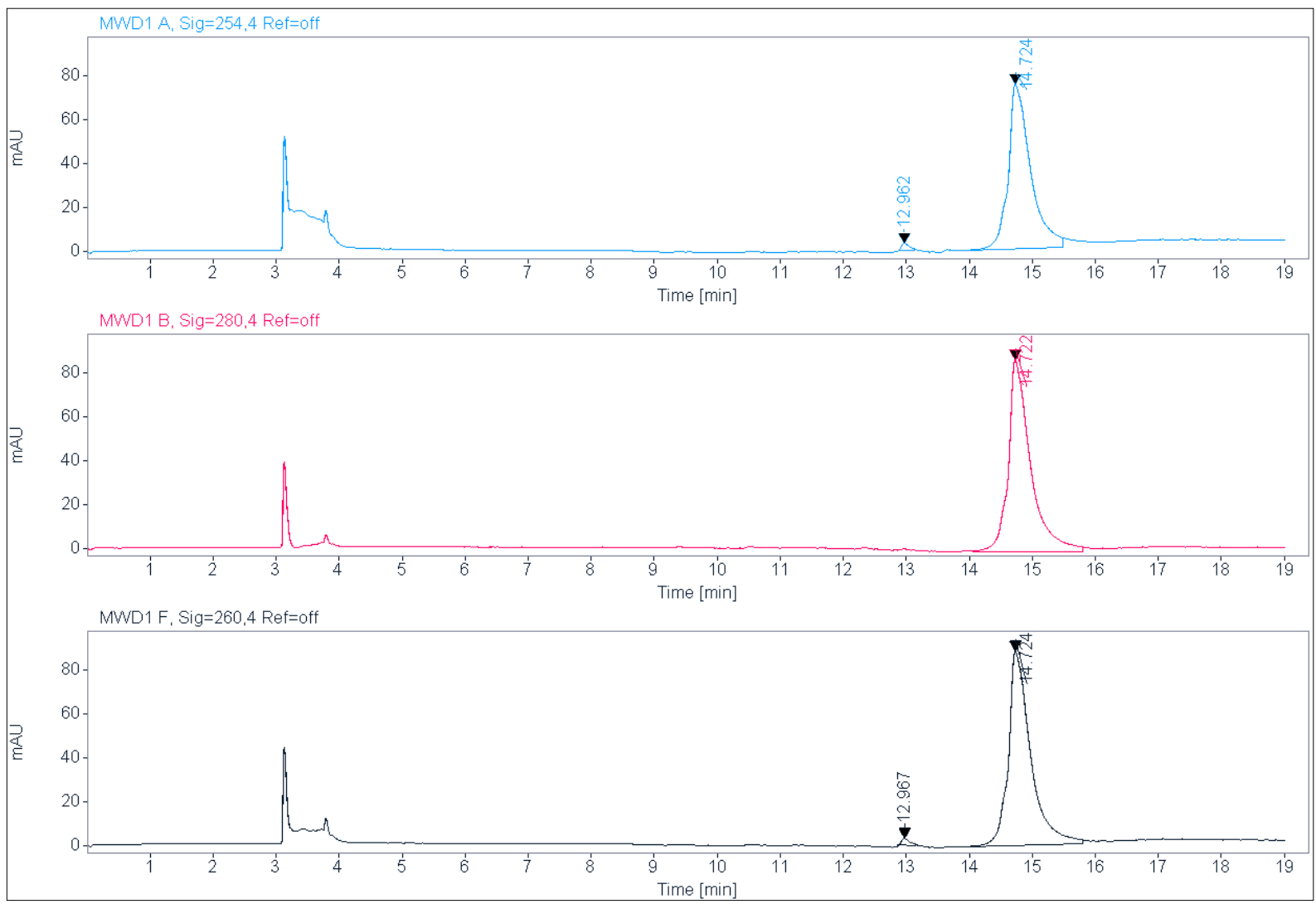




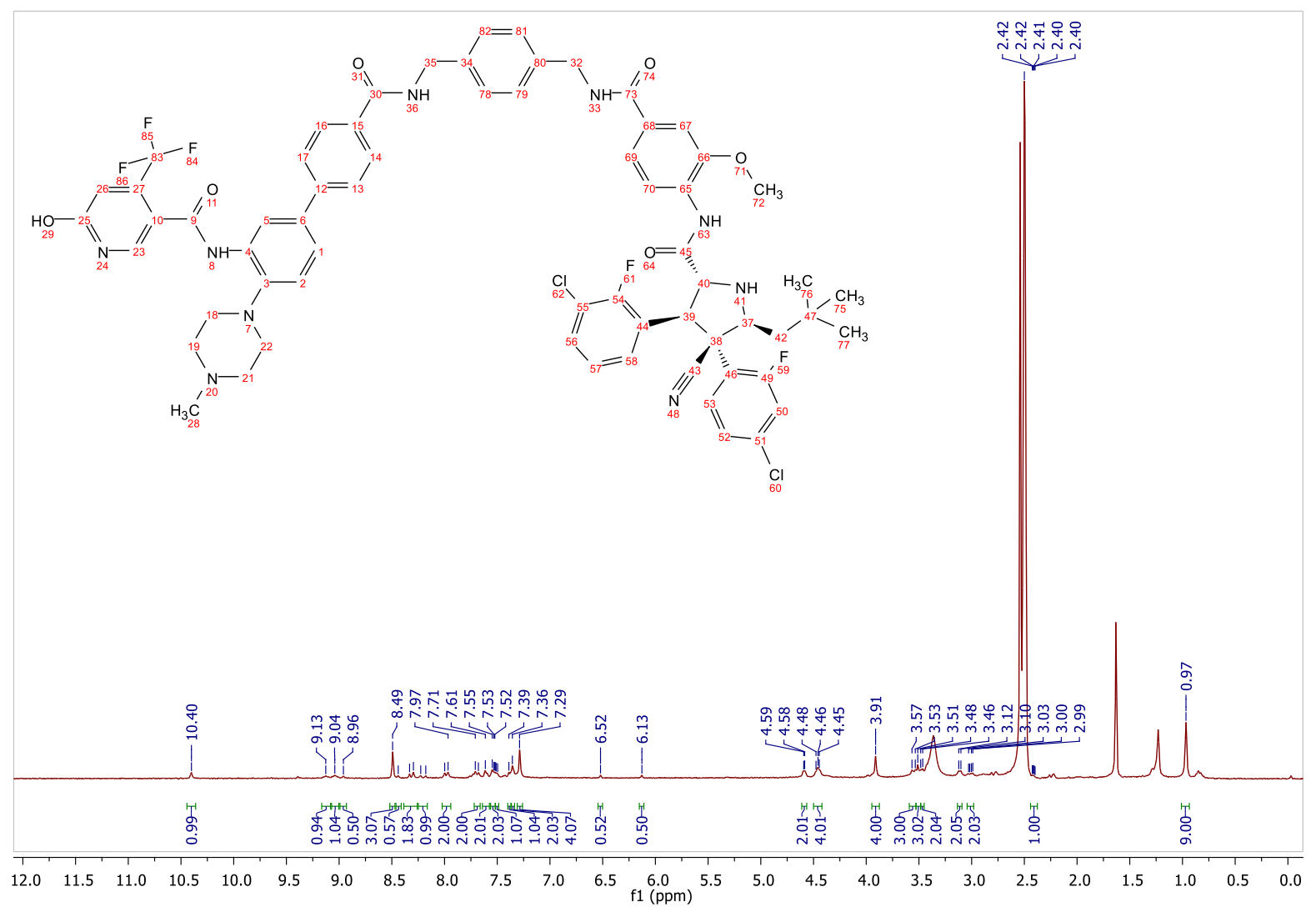


MALDI, HRMS, HPLC and ${ }^{1} \mathrm{H}-\mathrm{NMR}$ of 6 -hydroxy-N-(4'-((5-)((S)-1-((2S,4S)-4-hydroxy-2-((4-)(4methylthiazol-5-yl)benzyl)carbamoyl)pyrrolidin-1-yl)-3,3-dimethyl-1-oxobutan-2-yl)amino)-5oxopentyl)carbamoyl)-4-(4-methylpiperazin-1-yl)-[1,1'-biphenyl]-3-yl)-4-

(trifluoromethyl)nicotinamide (22/ nc_VHL)

Voyager Spec \#1 [BP = 941.4, 31125]

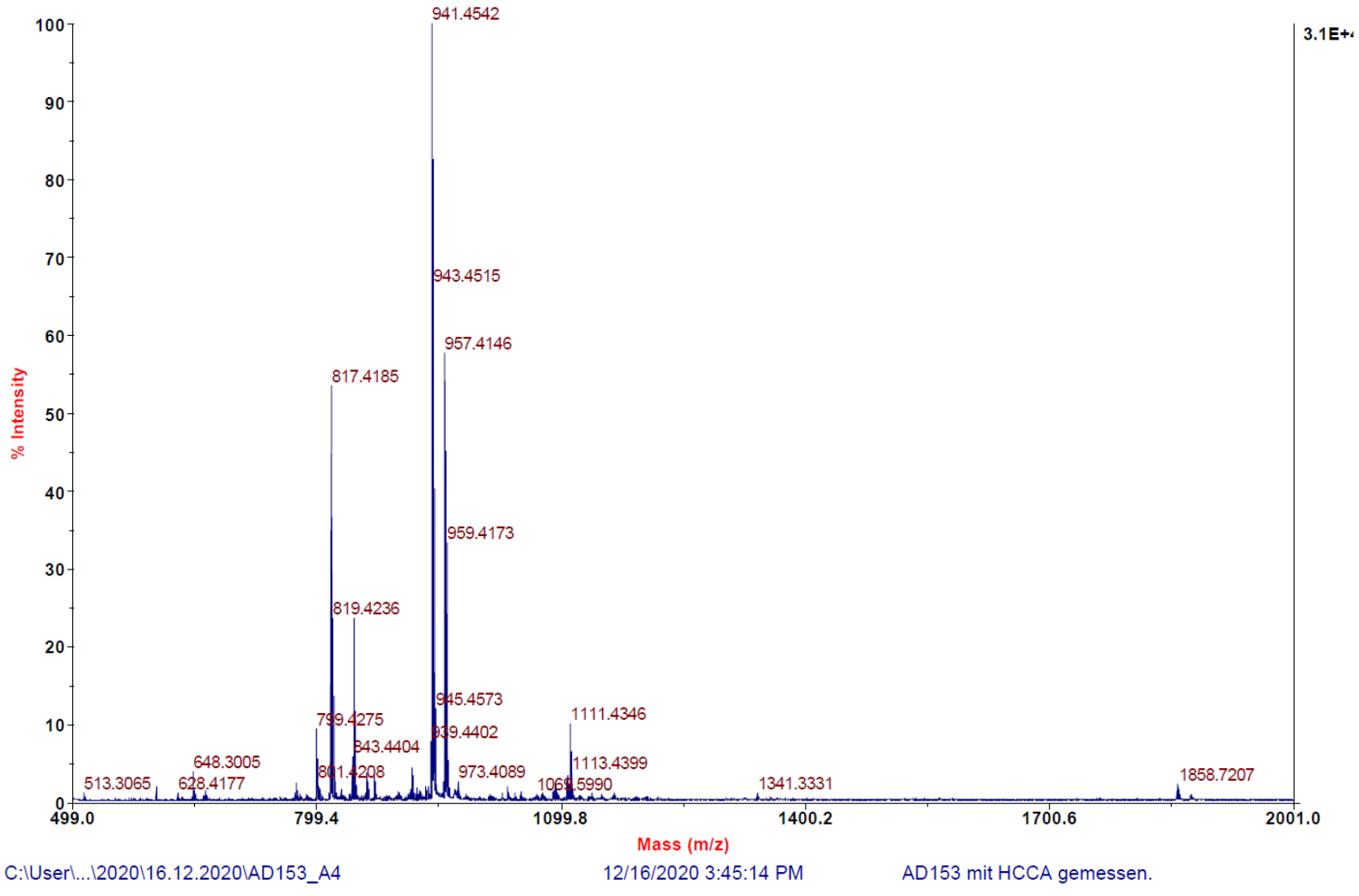

AD153_A4 \#1-13 RT: 0.00-0.55 AV: 13 NL: 2.10E7

T: FTMS + p MALDI Full ms [250.00-1100.00]

$100 \quad 1034.41691$
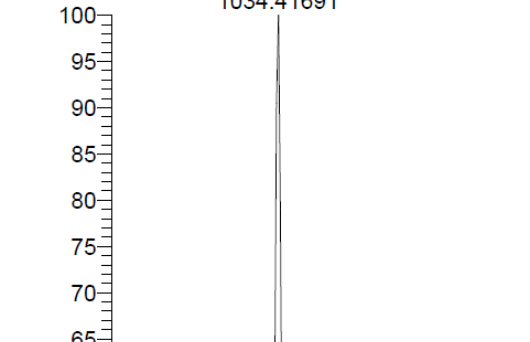

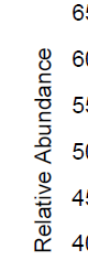

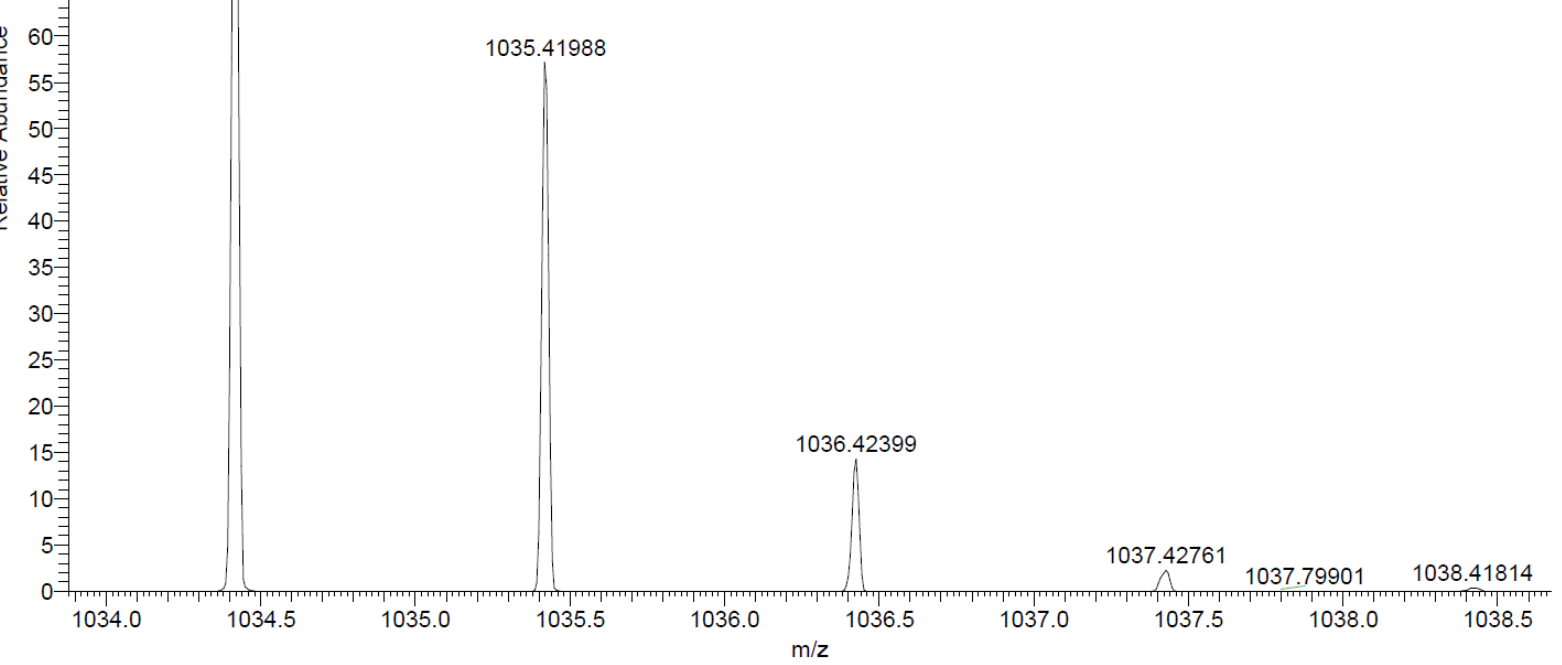

Signal: $\quad$ MSD1 TIC, MS File 


$\begin{array}{crrrr}\text { RT [min] Type } & \text { Width [min] } & \text { Area } & \text { Height } & \text { Area\% } \\ 11.580 \text { BB } & 0.3359 & 26619110.0000 & 1236768.2500 & 58.3972 \\ 18.536 \text { BBA } & 0.3762 & 18963760.0000 & 840934.5625 & 41.6028 \\ & \text { Sum } & 45582870.0000 & & \end{array}$

Signal: $\quad$ MWD1 A, Sig=254,4 Ref=off

$\begin{array}{crrrr}\text { RT [min] Type } & \text { Width [min] } & \text { Area } & \text { Height } & \text { Area\% } \\ 11.353 \text { VV } & 0.2008 & 4513.2095 & 311.9199 & 100.0000 \\ & \text { Sum } & 4513.2095 & & \end{array}$

Signal: $\quad$ MWD1 B, Sig=280,4 Ref=off

$\begin{array}{crrrr}\text { RT [min] Type } & \text { Width [min] } & \text { Area } & \text { Height } & \text { Area\% } \\ 11.353 \text { VV } & 0.2029 & 5449.8301 & 372.0602 & 100.0000 \\ & \text { Sum } & 5449.8301 & & \end{array}$

Signal: $\quad$ MWD1 F, Sig=260,4 Ref=off
RT [min] Type Width [min]
Area
Height
Area\%
$11.353 \mathrm{VV}$
0.2021
5101.9272
352.0205
100.0000
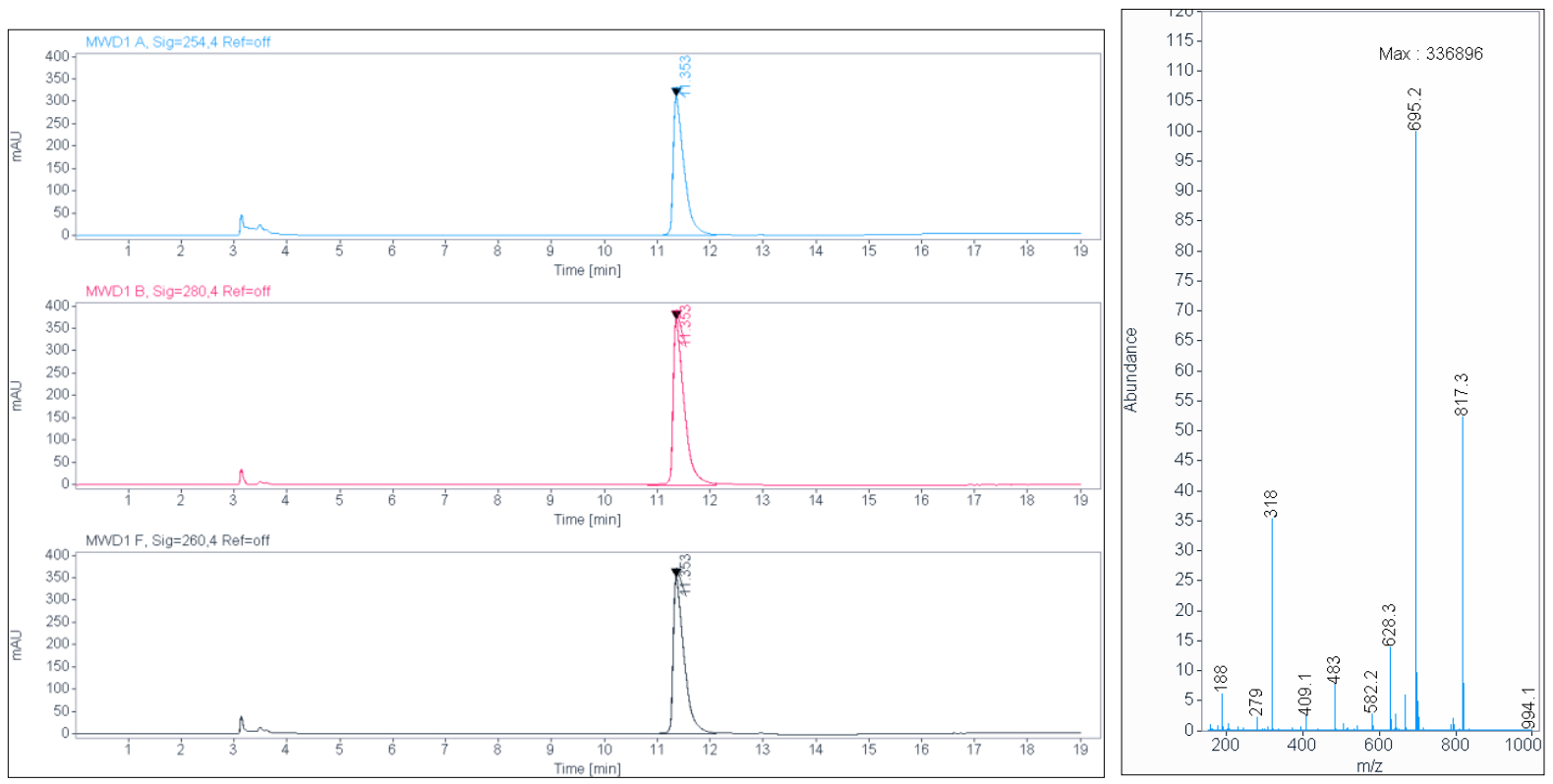


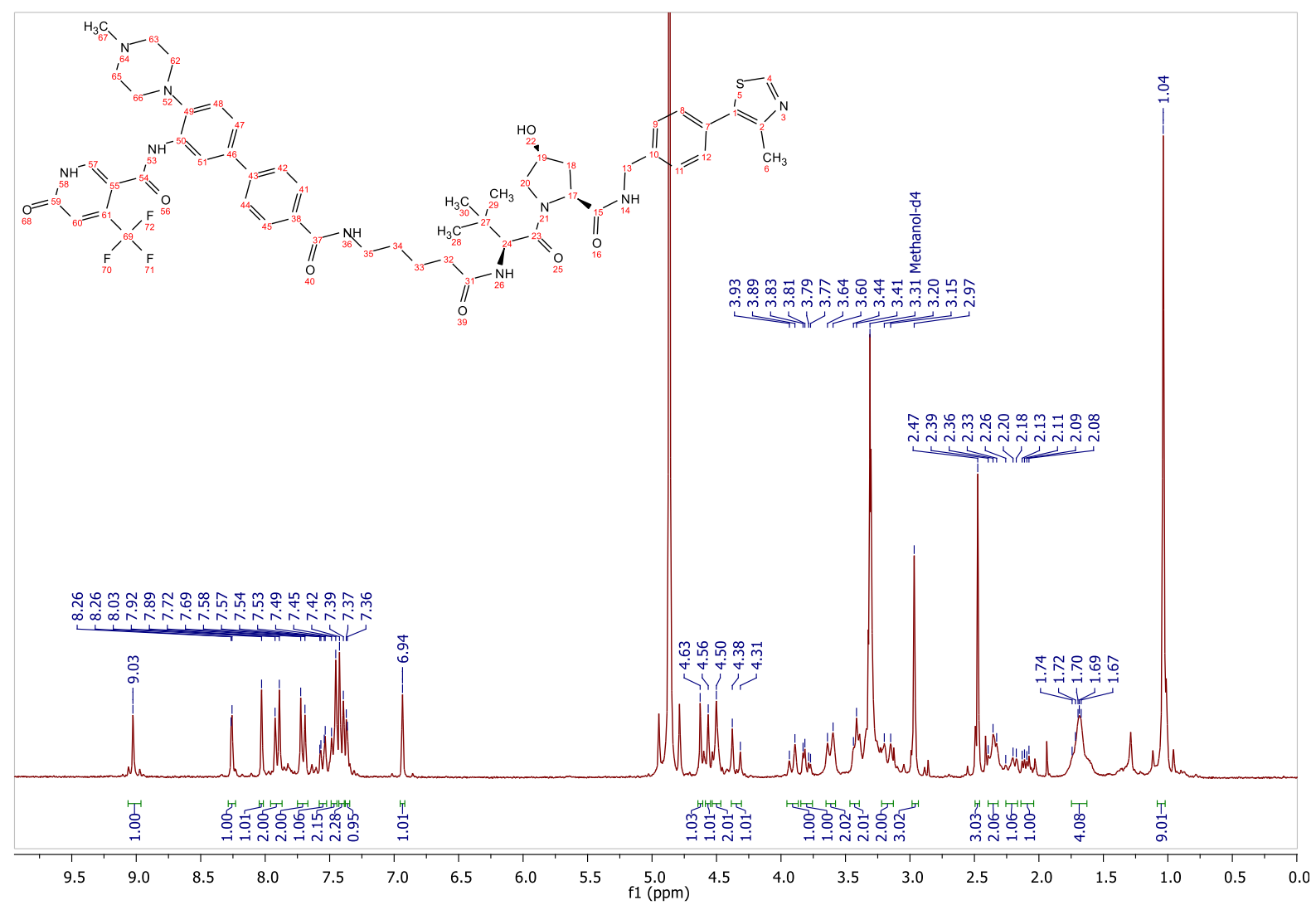


ESI, MALDI, HRMS, ${ }^{1} \mathrm{H}-\mathrm{NMR},{ }^{13} \mathrm{C}-\mathrm{NMR}$ and ${ }^{1} \mathrm{H}-{ }^{13} \mathrm{C}-\mathrm{HMBC}$ 6-hydroxy-N-(4'-((5-)((S)-1-((2S,4R)-4hydroxy-2-((4-(4-methylthiazol-5-yl)benzyl)carbamoyl)pyrrolidin-1-yl)-3,3-dimethyl-1-oxobutan-2yl)amino)-5-oxopentyl)carbamoyl)-4-morpholino-[1,1'-biphenyl]-3-yl)-4-

(trifluoromethyl)nicotinamide (23/ nc_WDR5)

C:IXcaliburldatalad167

2/17/2021 10:34:14 AM
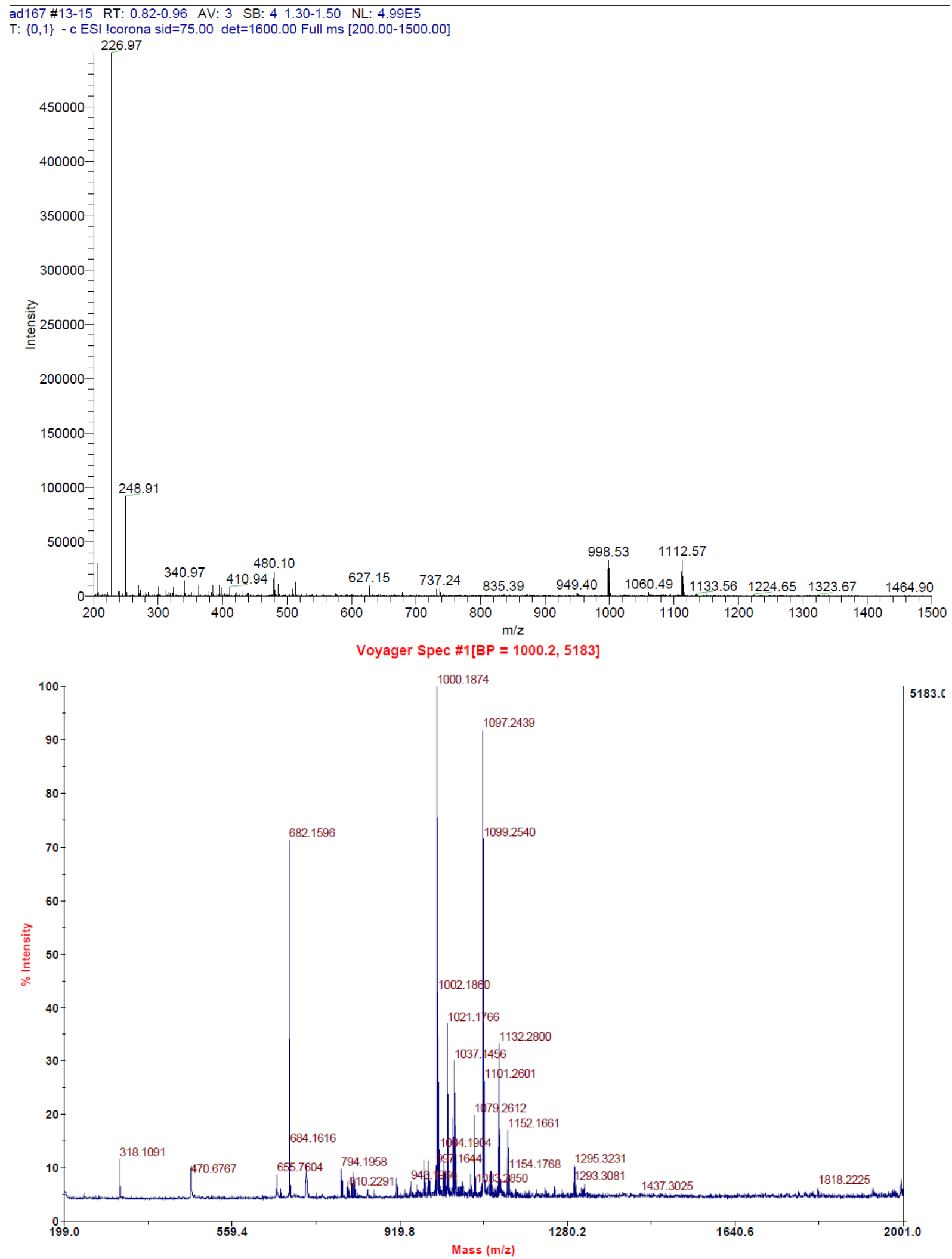
C:IUserl...12021|25.06.2021\AD167_E9

AD167 mit CICCA gemessen

AD167_E9 \#1-6 RT: 0.00-0.22 AV: 6 NL: 3.92E7

T: FTMS + p MALDI Full ms [350.00-1100.00]
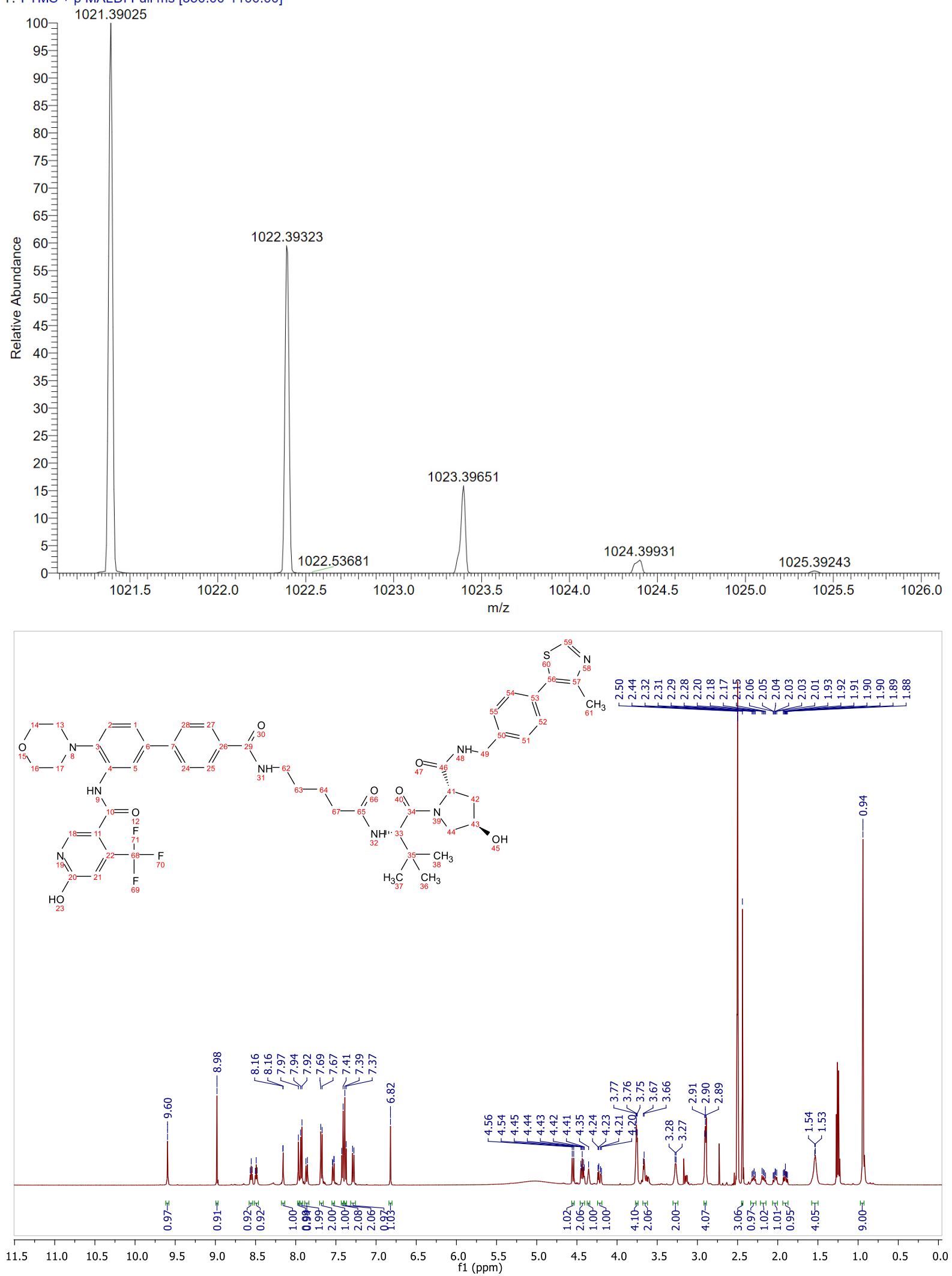

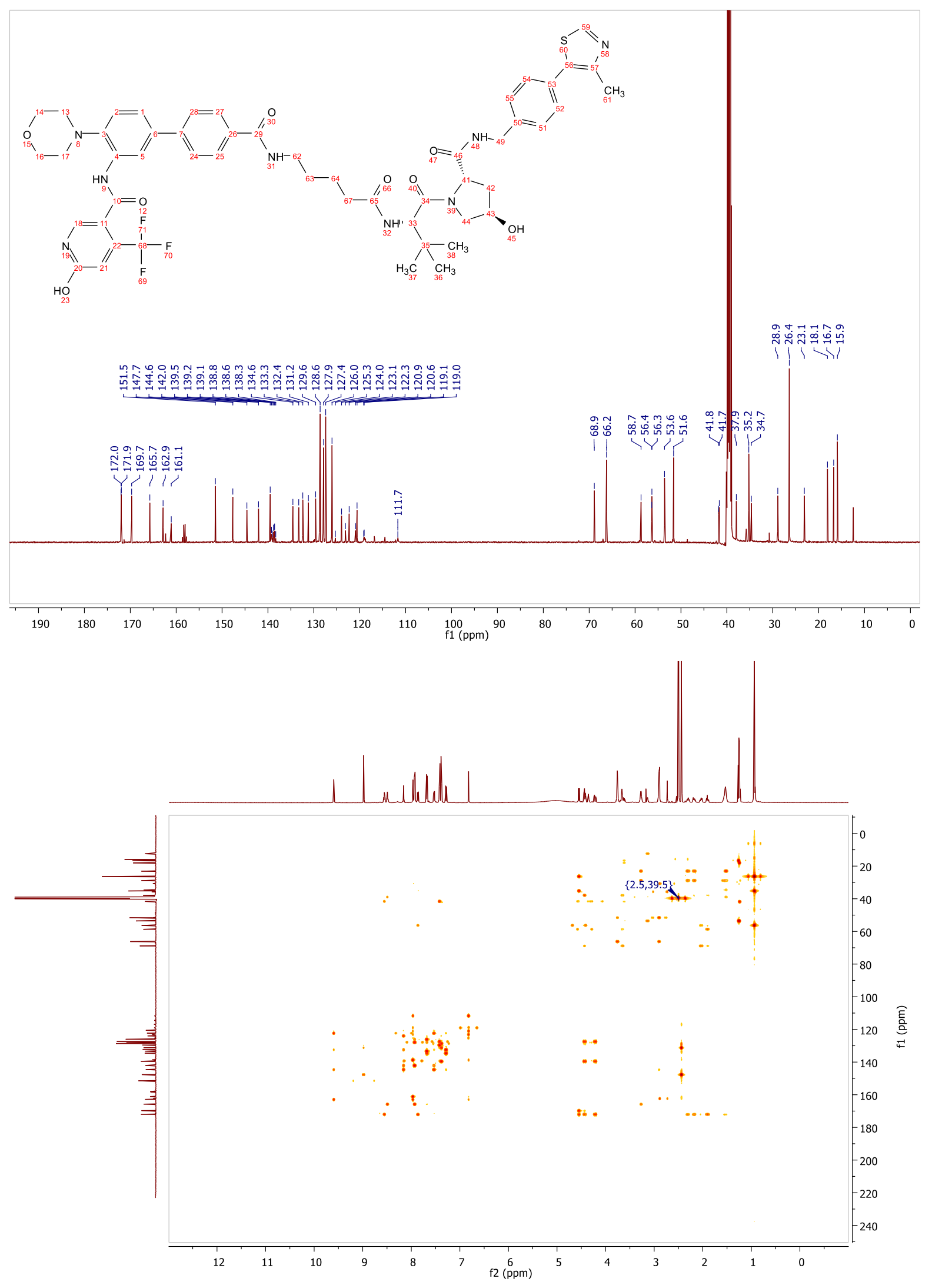


\section{List of figures}

Figure 1: Histone proteins interact with the DNA to form nucleosomes.

Figure 2: Cryo-EM structure of the MLL1 KMT complex in complex with DNA and a histone octamer.

Figure 3: Apo crystal structure of WDR5. Like all WD40 proteins, WDR5 consists out of a seven-bladed propeller of beta sheets.

Figure 4: Crystal structure of the MYC-MAX heterodimer in complex with DNA.

Figure 5: Schematic illustration of the MYC protein structure with its protein-protein interaction domains.

Figure 6: Ribosomogram, displaying ribosome subunits of small and large subunit ribosomal protein genes (RPGs).

Figure 7: Proposed mechanism of Win site inhibition by Aho et al.

Figure 8: WDR5 phenocopies by WIN site inhibition and WDR5 depletion.

Figure 9: Mechanism of action (MoA) of proteolysis targeting chimeras (PROTACs).

Figure 10: Model of a crystal structure of the CRL4 ${ }^{\mathrm{CRBN}}$ E3 ligase in complex with thalidomide.

Figure 11: WDR5 Win site (grey) interaction of OICR-9429.

Figure 12: Exit vectors out of the WDR5 Win site binding pocket.

Figure 13: ITC curves for VHL-addressing PROTACs (8g) and (8i).

Figure 14: Cellular permeability and target engagement studies were performed with the BRET assay.

Figure 15: Cellular degradation studies on WDR5.

Figure 16: Cellular degradation studies on WDR5.

Figure 17: Immunoblot of WDR5. MV4-11 $1^{\text {WDR5-HiBiT }}$ cells were treated with different concentration of (8g) for $24 \mathrm{~h}$ and compared with DMSO treated or naive MV4-11 cells.

Figure 18: Degrader-induced depletion of WDR5 depends on the ubiquitin system.

Figure 19: Volcano plot exhibiting global proteomics change upon PROTAC treatment.

Figure 20: Volcano plot exhibiting global proteomics change upon Win site ligand binding.

Figure 21: Crystal structure of the WDR5/ (8g)/ VHL ternary complex.

Figure 22: ITC curves of OICR-9429 derived molecule (6d) and degraders (7a), (8a), (8e-j). 
360 List of figures 


\section{List of schemes}

Scheme 1: Chemical structures of WDR5 ligands.

Scheme 2: Schematic drawing of the chemical structures of the heterobifunctional molecules.

Scheme 3: Retrosynthetic approach for addressing WDR5 with OICR-9429 derived scaffolds.

Scheme 4: Synthetic procedure to obtain WDR5 ligands (6a-d).

Scheme 5: Steps a) and b) to synthesise precursor 5-bromo-2-(4-methylpiperazin-1-yl)aniline (4).

Scheme 6: Steps c) and d) to synthesize piperazine-based WDR5 ligands tert-butyl 5-(3-(3methylbenzamido)-4-(4-methylpiperazin-1-yl)phenyl)-1H-indole-1-carboxylate (6a), tert-butyl 5-(3-(5amino-2-chloro-4-fluoro-3-methylbenzamido)-4-(4-methylpiperazin-1-yl)phenyl)-1H-indole-1-

carboxylate (6b) and tert-butyl 5-(4-(4-methylpiperazin-1-yl)-3-(6-oxo-4-(trifluoromethyl)-1,6dihydropyridine-3-carboxamido)phenyl)-1H-indole-1-carboxylate (6c).

Scheme 7: Steps c) and d) to synthesize the piperazine-based WDR5 ligand tert-butyl 4'-(4methylpiperazin-1-yl)-3'-(6-oxo-4-(trifluoromethyl)-1,6-dihydropyridine-3-carboxamido)-[1,1'biphenyl]-4-carboxylate (6d).

Scheme 8: Isolated side products of WDR5 ligands $(6 c, d)$, obtained in yields of approx. $25 \%$.

Scheme 9: Synthesis of E3 ligase ligand pomalidomide (LO) and E3 ligase linkers (L1-L5) addressing the E3 ligase Cereblon (CRBN).

Scheme 10: Synthesis procedure of E3 ligase linkers (L6-L14) addressing the E3 ligase Von-HippelLindau (VHL).

Scheme 11: Synthesis scheme of WDR5 degraders (7a-e) based on ligand (6d) addressing E3 ligase CRBN.

Scheme 12: Chemical structures and obtained yield of WDR5 degraders (8a-j) addressing the E3 ligase Von-Hippel-Lindau (VHL). The synthesis steps start from intermediate (6d).

Scheme 13: Synthesis route of WDR5 degraders (9a-c) addressing the E3 ligase MDM2. The synthesis steps start from intermediate $(\mathbf{6 d})$.

Scheme 14: Synthesis of WDR5 degraders $(\mathbf{1 6} \mathbf{a}, \mathbf{b})$ addressing the E3 ligase CRBN.

Scheme 15: Synthesis of WDR5 degraders (20a-g) addressing the E3 ligase VHL.

Scheme 16: Synthesis of NanoBRET ${ }^{\mathrm{TM}}$ tracer molecules (21a-c) via intermediates $(\mathbf{6 e}, \mathbf{h}, \mathbf{i})$. 
362 List of schemes 


\section{List of tables}

Table 1: Examples of epigenetic protein complexes and epigenetic mutations that are now known to be perturbed in cancer and affect gene expression and genomic stability.

Table 2: DSF and NanoBRET ${ }^{\mathrm{TM}}$ data for WDR5 ligands (6a-d).

Table 3: Thermal shift experiments of OICR-9429 derived molecules (6d-g), degraders (7a-e), (8a-j), (9a-c) and the negative controls (nc_VHL) and (nc_WDR5).

Table 4: Thermodynamic properties of OICR-9429 derived molecule (6d) and degraders (7a), (8a), (8ej).

Table 5. In cellulo data of WDR5 antagonist OICR-9429, the modified inhibitor (6d) and degraders (7ae), (8a-j) and (9a-c).

Table 6. HiBiT data of WDR5 ligand (6d) and degraders (7a-e) and (8a-j).

Table 7. Biophysical and biological data of the negative controls of PROTAC (8g).

Table 8: Probe criteria for the WDR5 PROTAC probe Homer.

Table 9: Synthesis of 1-(4-bromo-2-nitrophenyl)-4-methylpiperazine (3).

Table 10: Synthesis of 1-(4-bromo-2-nitrophenyl)-4-methylpiperazine (4).

Table 11: Synthesis of tert-butyl 5-(3-amino-4-(4-methylpiperazin-1-yl)phenyl)-1H-indole-1carboxylate (5b).

Table 12: Synthesis of tert-butyl 5-(3-(2-chloro-4-fluoro-3-methyl-5-nitrobenzamido)-4-(4methylpiperazin-1-yl)phenyl)-1H-indole-1-carboxylate.

Table 13: Synthesis of tert-butyl 5-(3-(6-hydroxy-4-(trifluoromethyl)nicotinamido)-4-(4methylpiperazin-1-yl)phenyl)-1H-indole-1-carboxylate (6c).

Table 14: Synthesis 3'-amino-4'-(4-methylpiperazin-1-yl)-[1,1'-biphenyl]-4-carboxylate (5b).

Table 15: Synthesis of tert-butyl 3'-(6-hydroxy-4-(trifluoromethyl)nicotinamido)-4'-(4methylpiperazin-1-yl)-[1,1'-biphenyl]-4-carboxylate (6d).

Table 16: Thermal shift experiments of OICR-9429 derived molecules (6a-g), degraders (7a-e), (8a-j), (9a-c) and the negative controls (nc_VHL) and (nc_WDR5).

Table 17: Thermodynamic properties of OICR-9429 derived molecule (6d), degraders (7a), (8a), (8e-j) and pyrroloimidazole-based inhibitor (17) and degrader (20b). 
364 List of tables 


\section{Acknowledgements}

First of all, I would like to thank Prof. Dr. Stefan Knapp for being a member of his working group in Frankfurt, for the free creation of the topic and for the many helpful discussions. I would also like to thank Stefan for allowing me to participate in the open-science project of the Structural Genomics Consortium (SGC), the latter enabled me to build a variety of collaborations.

Special thanks to Bikash Adhikari and Elmar Wolf from the University of Würzburg for their collaboration and their investigative testing of my compounds in cells and on the other hand for their willingness to help and drive this project forward.

My very special thanks therefore go to my colleagues at the SGC in Frankfurt. Thanks to Andreas Krämer for crystalizing the ternary complex crystal structure, for providing the proteins and assisting with the biophysical assays. I would like to thank Lena Marie Berger for the cellular assay data.

Beside the people who helped on the PROTAC project, I would like to thank Jakob Gebel and Volker Dötsch from the University of Frankfurt for their help and guidance on the NMR-based fragment screen. Without the providing of peptide and NMR measurement time and know-how, this exciting side-project would not have been possible. Thanks to Frank Löhr who later on helped alongside with Jakob during the revision process.

I would also like to thank the research group of Cheryl Arrowsmith at the University of Toronto, especially Dalia Baryste-Lovejoy and Magda Szwenczy for providing cloning constructs for the BRET assay system.

At the University of Frankfurt, I would like to thank the service unit mass spectrometry for the numerous measured mass spectra and especially Uwe Hener and Andreas Münch for his constant helpfulness. Many thanks to the NMR service department for the possibility to record my spectra on site. Without these analytical departments this work would not have been possible.

Another special thank goes to my interns Jenny Adam, Lennart Laube and Nina Krause, to my Bachelor student Achim Glaesmann, and to my master students Dominic Löw and Janik Weckesser who provided time and motivation for this project. 
366 Acknowledgements

I would like to thank Andreas Krämer and Marek Wanior in particular for their competent lecturing skills, the quick review of my doctoral thesis and constant helpfulness. Furthermore, many thanks to Nadine Russ, Marcel Rak, Marek Wanior and Janik Weckesser for the excellent working atmosphere in the laboratory and on the other hand for their willingness to discuss, help and support in solving problems as well as all other colleagues of the working group.

Finally, I would like to thank my friends and my family who always supported me. 


\section{Publications}

Dölle, Adhikari, et al., "Design, Synthesis and Evaluation of WD40-repeat containing protein 5 (WDR5) degrader", J. Med. Chem. 2021, 64 (15), 10682-10710. 
368 Publications 


\section{Eidesstattliche Erklärung}

\section{Erklärung}

Ich erkläre hiermit, dass ich mich bisher keiner Doktorprüfung im MathematischNaturwissenschaftlichen Bereich unterzogen habe.

Frankfurt am Main, den

(Unterschrift)

\section{Versicherung}

Ich erkläre hiermit, dass ich die vorgelegte Dissertation über

selbständig angefertigt und mich anderer Hilfsmittel als der in ihr angegebenen nicht bedient habe, insbesondere, dass alle Entlehnungen aus anderen Schriften mit Angabe der betreffenden Schrift gekennzeichnet sind. Ich versichere, die Grundsätze der guten wissenschaftlichen Praxis beachtet, und nicht die Hilfe einer kommerziellen Promotionsvermittlung in Anspruch genommen zu haben.

Frankfurt am Main, den

(Unterschrift) 
370 Eidesstattliche Erklärung 
